\title{
Syntheses and Structures of Functionalized Cycloparaphenylenes and Fused Cycloparaphenylene Precursors.
}

\author{
Changfeng Huang
}

Follow this and additional works at: https://researchrepository.wvu.edu/etd

\section{Recommended Citation}

Huang, Changfeng, "Syntheses and Structures of Functionalized Cycloparaphenylenes and Fused Cycloparaphenylene Precursors." (2016). Graduate Theses, Dissertations, and Problem Reports. 8177. https://researchrepository.wvu.edu/etd/8177

This Dissertation is protected by copyright and/or related rights. It has been brought to you by the The Research Repository @ WVU with permission from the rights-holder(s). You are free to use this Dissertation in any way that is permitted by the copyright and related rights legislation that applies to your use. For other uses you must obtain permission from the rights-holder(s) directly, unless additional rights are indicated by a Creative Commons license in the record and/ or on the work itself. This Dissertation has been accepted for inclusion in WVU Graduate Theses, Dissertations, and Problem Reports collection by an authorized administrator of The Research Repository @ WVU.

For more information, please contact researchrepository@mail.wvu.edu. 


\title{
Syntheses and Structures of Functionalized Cycloparaphenylenes and Fused Cycloparaphenylene Precursors
}

\section{Changfeng Huang}

\author{
Dissertation submitted to the Fberly College of Arts and Sciences \\ at West Virginia University \\ in partial fulfillment of the requirements \\ for the degree of
}

Doctoral of Philosophy

in

Organic Chemistry

\author{
Kung K. Wang, Ph.D., Chair \\ Jeffrey L. Petersen, Ph.D. \\ Brian V. Popp, Ph.D. \\ Carsten Milsmann, Ph.D. \\ Yon Rojanasakul, Ph.D.
C. Eugene Bennett Department of Chemistry
Morgantown, West Virginia \\ 2016
}

Keywords: Diels-Alder Reaction, Cycloparaphenylenes, Fused Carbon Nanohoops, Homocoupling Reaction, Oxidative Aromatization

$$
\text { Copyright } 2016 \text { Changfeng Huang }
$$




\title{
ABSTRACT \\ Syntheses and Structures of Functionalized Cycloparaphenylenes and Fused Cycloparaphenylene Precursors
}

\author{
Changfeng Huang
}

A synthetic pathway of preparing functionalized [9]cycloparaphenylene ([9]CPP) bearing three evenly spaced 5,8-dimethyoxynaphth-1,4-diyl units and two macrocyclic [6]CPP precursors has been developed. The key steps included the Diels-Alder reaction between ( $E . E$ )-1,4-bis(4-bromophenyl)-1,3-butadiene and I,4-benzoquinone followed by methylation to produce an L-shaped building block with two 4-bromophenyl groups cis to each other exclusively, the nickel-mediated homocoupling reactions to construct the macrocyclic dimers and trimers, and mild, efficient oxidative aromatization by $2,3-$ dichloro-5,6-dicyano-1,4-benzoquinone (DDQ) to furnish the functionalized [9]CPP.

A synthetic pathway for constructing fused carbon nanohoops with the two nanohoops in nanotube-like connection has also been developed. The key intermediate with all four 4-bromophenyl groups cis to one another in an L-shaped building block was prepared by two consecutive Diels-Alder reactions, followed by methylation. The $\mathrm{Ni}(\operatorname{cod})_{2}$-mediated homocoupling reactions produced the molecules that contained either two hydrogenated [6]CPP, or two hydrogenated [9]CPPs fused through 1,4dimethoxybenzene moieties and other bent and fused nanostructures with subunits of differing sizes of CPPs. The structure of a fused carbon nanohoop containing two hydrogenated [6]CPP units was established by X-ray structure analysis and a stepwise synthetic sequence. The structure of a molecule containing two fused carbon nanohoops 
comprised of two hydrogenated [9]CPPs was also established by a similar stepwise synthesis.

In addition, a synthetic sequence to a functionalized [10]CPP has also heen established. This synthetic pathway is being explored for the construction of fused carbon nanohoops containing two fully aromatized [10]CPPs. 


\section{DEDICATED TO}

My wife, Hongmei Yan, My sons, Xinnan Huang, Yanis Huang And My Parents 


\section{Acknowledgement}

First and foremost I wish to express my cordial gratitude to my advisor Dr. Kung K. Wang, Eberly Distinguished Professor of Chemistry and Department Chair. You have been so relentlessly supportive of me both academically and personally for almost every possible chance from the early hardship to the finishing of the thesis. Your guidance, patience, motivation, profound knowledge, concentration on research all has set an example of excellence. I have been so lucky to have such an advisor and mentor as you!

I would like to thank my committee members, Professors Dr. Jeffrey L. Petersen, Dr. Brian V. Popp, Dr. Carsten Milsmann and Dr. Yon Rojanasakul for taking time to serve as my committee member. Special thanks go to tough and nice Dr. Petersen for your challenging but benign suggestions and comments and your tremendous help in X-ray structure analyses. I would also thank Dr. Popp for your great help in DFT calculations and my knowledge expansion of NMR theory and skills. I also would like to thank Dr. Milsmann for broadening my horizon in inorganic chemistry. In addition I wish to thank Dr. Rojanasakul for his brilliant advice and amiable reminding.

I would also be indebted to the other faculties and staffs in Chemistry Department for their help and assistance in fulfillment of the research and the thesis. In particular I would like to express my profound appreciation to Dr. Novruz. Akhmedov for his NMR experiments for structural elucidation, patient teaching in NMR techniques and awesome work in high-profile NMR spectra. As well special gratitude goes to Dr. Stephen J. 
Valentine and his group members for their assistance in acquiring high-resolution mass spectra.

I will also be thankful to my former and current group members in Dr. Wang's group for their help, aids and happy time together. Special thanks to Dr. Bo Wen for leading me to organic chemistry field. Much appreciation to Dr. Mingde Shan for your time and every long discussion and instructions. Of course I will never forget my brother and friend Dr. Haibing Guo who brought me here and opened a new area in life for me.

Last I would like to thank my parents and my sister for their countless love and support. Especially I wish to acknowledge my beloved wife Hongmei Yan for her selfless sacrifices, constant contribution, comprehensive care and unconditional love. And thank you for bringing me two lovely boys, Xinnan and Yanis, two happiness and surprise sources. Finally I wish I could dedicate this thesis to my Grandma, who passed away during my study here. Even though I could not see you for the last time, Grandma still lives with me forever. 


\section{TABLE OF CONTENTS}

\section{Chapter 1. Syntheses and Structures of Functionalized}

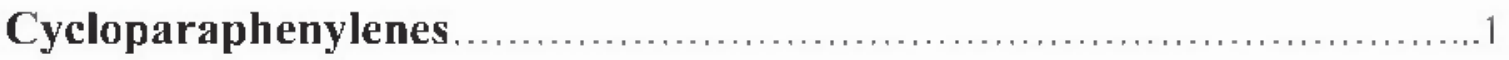

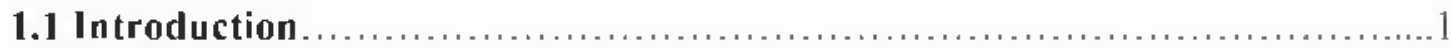

1.2 Characteristics of different growth-on-template strategies $\ldots \ldots \ldots \ldots \ldots \ldots \ldots \ldots \ldots$

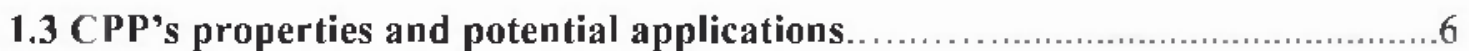

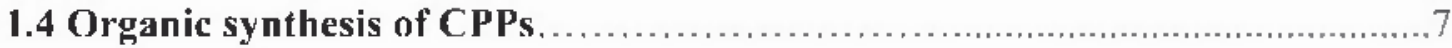

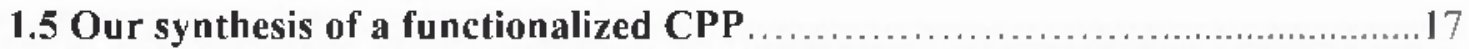

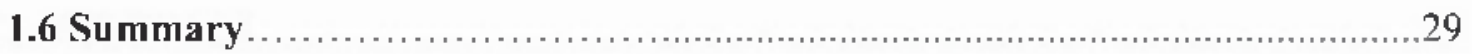

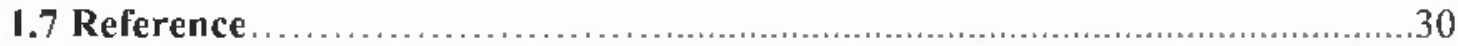

Chapter 2. Syntheses and Structures of Bent and Fused

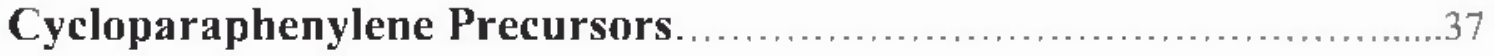

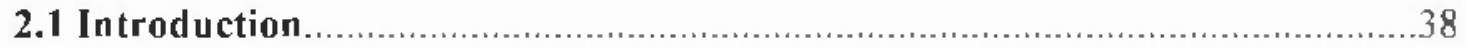

2.2 Our synthesis of a nanotube-like dimer, trimer and related bent and fused

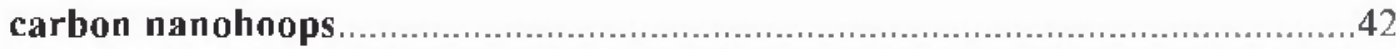

2.2.1 First route to construct nanotube-like structures $\ldots \ldots \ldots \ldots \ldots \ldots \ldots \ldots \ldots \ldots . \ldots 4$

2.2.2 Second route to construct nanotube-like structures..........................43

2.2.3 Third route to construct nanotube-like structures..............................44

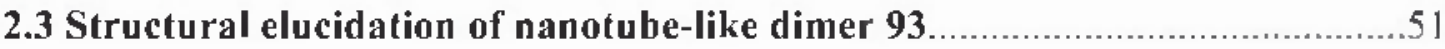

2.4 Structural elucidation of nanotube-like trimer 95a .................................53

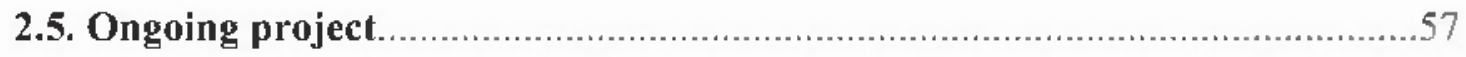


2.6. Summary.

\section{LIST OF TABLES}

Table 1. Characteristics of different templates for growth of carbon nanotubes 6

\section{LIST OF FIGURES}

Figure 1.1. Chiral index for single-wall carbon nanotubes.

Figure 1.2. Synthesis of carbon nanotubes from three endcaps, oxidized fullerenes, short $(5,5) \mathrm{CNT}$ end cap and polycyclic aromatic hydrocarbon P1 4

Figure 1.3. Types of single-wall carbon nanotubes, their smallest repeating units...........5

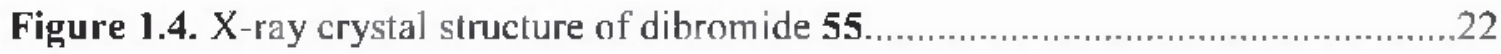

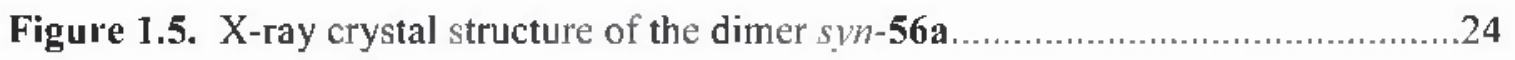

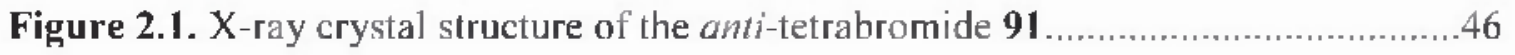

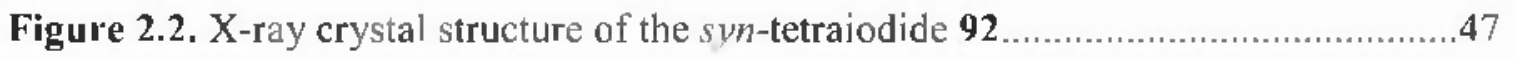

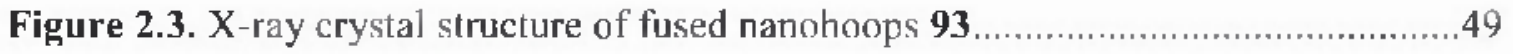




\section{LIST OF Schemes}

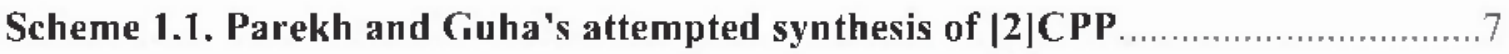

Scheme 1.2. Vogtle's two routes to CPP......................................................... 8

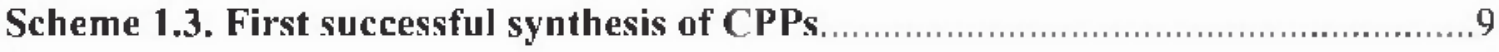

Scheme 1.4. Synthesis of |7|CPP First successful synthesis of CPPs..................... 1

Scheme 1.5. Selective Synthesis of |12]CPP ................................................. 13

Scheme 1.6. Concise Synthesis of |9] and |12|CPP ......................................... 14

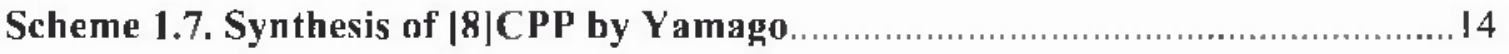

Scheme 1.8. Synthesis of Nanotube End-caps................................................. 15

Scheme 1.9. Synthesis of |5]CPP by Yamago and Jasti .................................. I7

Scheme 1.10. Synthesis of Key Intermediate to CPP ............................................. 18

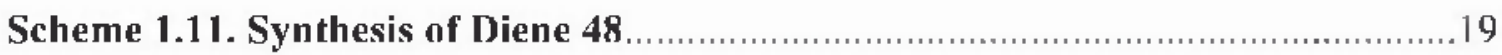

Scheme 1.12. Model Study of Diels-Alder reaction Between Diene 48 and 49........20

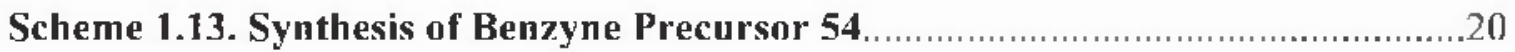

Scheme 1.14. Diels-Alder reaction Between Diene 48 and Benzyne Precursor 54_..21

Scheme 1.15. Diels-Alder reaction between 48 and 1,4 -Benzoquinone $\ldots \ldots \ldots \ldots \ldots \ldots . . .21$

Scheme 1.16. Ni(cod) 2-mediated Homocoupling Reaction with 55

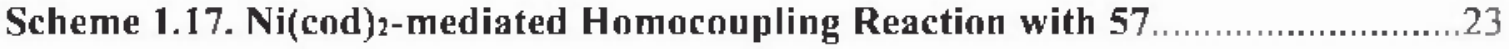

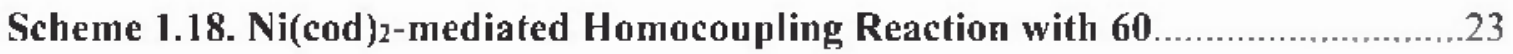

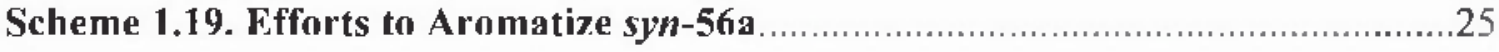

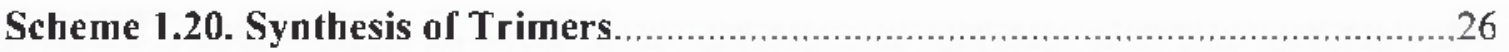

Scheme 1.21. Synthesis of 65 and Its Energy-Minimized Structure by DFT

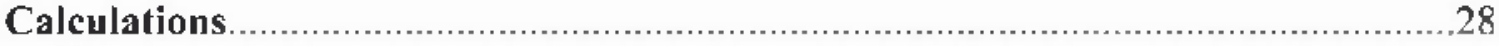


Scheme 2.1. Growth Mechanism Through Diels-Alder Reaction and

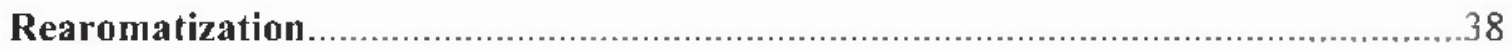

Scheme 2.2. Synthesis of Arene-Brideged [8]CPP Dimer..............................40

Scheme 2.3. Synthesis of Directly-Connected |10|CPP Dimer.............................4]

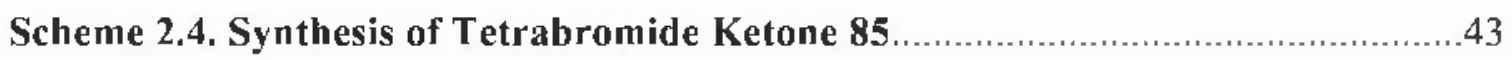

Scheme 2.5. Attempted Synthesis of Nanotube-like Structures from Cyclic Dimer..43

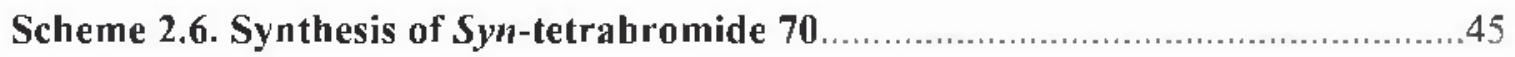

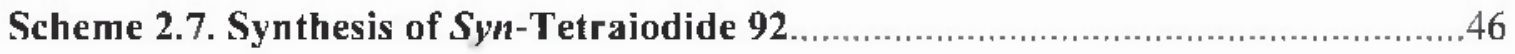

Scheme 2.8. Synthesis of Macrocyclic Dimers 93 and 94 at Iow Concentration......48

Scheme 2.9. Synthesis of Macrocyclic Trimers 95a, 95b and 95c at Higher

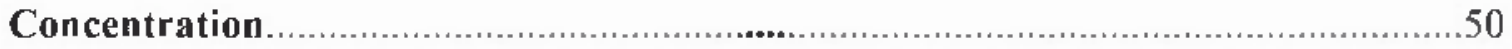

Scheme 2.10. First Attempt to Synthesize Fused Nanohoops $93 \ldots \ldots \ldots \ldots \ldots \ldots \ldots \ldots \ldots . . . \ldots$ I

Scheme 2.11. Synthesis of TMS-Protected Diene 101 ....................................52

Scheme 2.12. Elucidation of Structure of Fused Nanohoops 93..............................54

Scheme 2.13. Stepwise Synthesis of Trimer 110 and 111 and Subsequent

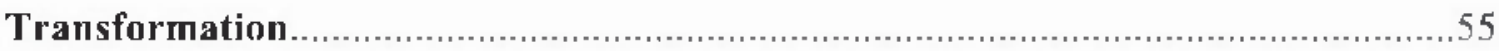

Scheme 2.14. Shotgun Synthesis of Trimer 110 and 111 and Subsequent

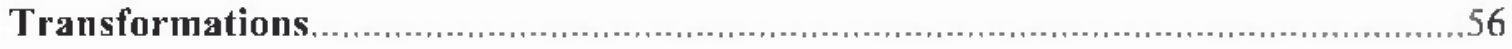

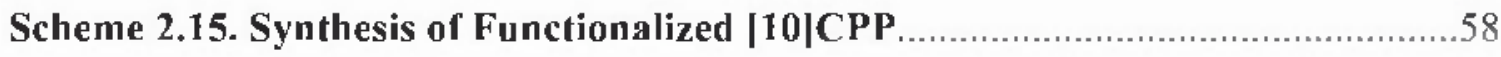

Scheme 2.16. Attempted Shotgun Synthesis of Fused [10]CPP Precursors............59

Scheme 2.17. Stepwise Synthesis toward Fused |10|CPP Precursors .......................60 


\section{Chapter 1. Syntheses and Structures of Functionalized Cycloparaphenylenes}

\subsection{Introduction}

Carbon nanotubes have been gaining extensive attention since their first report and structural analysis by lijima in 1991.' Because of their hollow cylindrical carbon nanostructures with all $\mathrm{sp}^{2}$ carbon bonding, carbon nanotubes showed unprecedented mechanical, electronic and optical properties and high potential in the application of new nanotechnologies and materials science. ${ }^{2,3,4}$ Carbon nanotubes are categorized into two types: single-wall carbon nanotubes and multi-wall nanotubes. Multi-wall carbon nanotubes can be considered as a bundle of single-wall carbon nanotubes aligned together. Single-wall carbon nanotubes are classified into three kinds: armchair, zigzag, and chiral, conceptually depending on the way the graphene is rolled to make the carbon nanotube seamlessly by a pair of chiral indices $(n, m)$, where $n>m>0$ (Figure 1.1). Two vector units are $a_{1}$ from carbon I to 3 , and $a_{2}$ from carbon 1 to 5 respectively. For example if we made a carbon nanotube by rolling the graphene from the point $\mathrm{O}$ to $\mathrm{O}^{\prime}$ in Figure 1.1 , the resultant carbon nanotube is called chiral $(4,3)$. Two extreme situations, one is when $n=m$, rolling angle $\theta=30^{\circ}$, then it is armchair type single-wall carbon nanotube, type $A$ on the right picture in Figure 1; the other is when $\mathrm{m}=0$, rolling angle $\theta=0^{\circ}$ or $60^{\circ}$, it is called zigzag type single-wall carbon nanotube, type B on the right picture. The rest of single-wall carbon nanotuhes are called chiral. One striking property of single-wall carbon nanotube is that it will shift from metallic to semi-conducting when the diameter or sidewall structure (or chirality) is slightly changed. ${ }^{4}$ So it is very important to produce single-wall carbon nanotubes with defined chirality and diameter if one wishes to study the structure-properties relationship and explore 


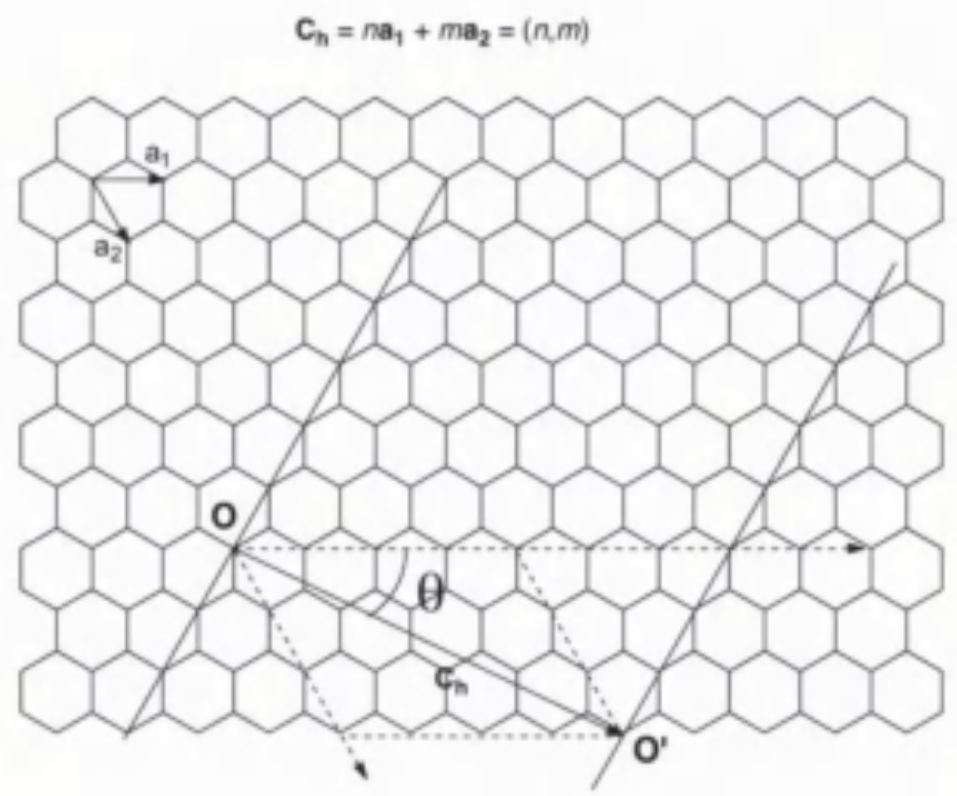

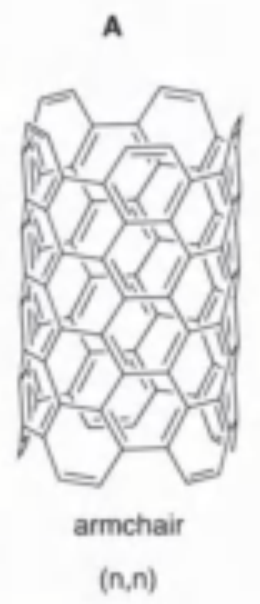

$(5,5)$

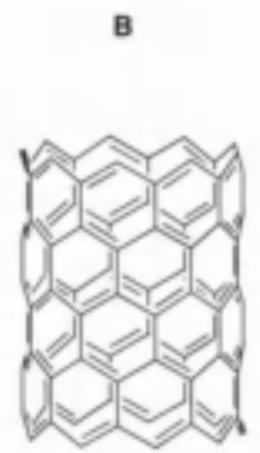

zigzag

$(n, 0)$

$(9,0)$

Figure 1.1. Chiral index for single-wall carbon nanotubes

their potential applications. However, modern methods of preparing carbon nanotubes, including arc discharge, laser ablation, and chemical vapor deposition, always produce mixtures of carbon nanotuhes. ${ }^{3}$ Some catalyst manipulations ${ }^{6,7, B, 9}$ and post synthesis purification techniques, such as the involvement of surfactant-assistant gel chromatography, ${ }^{10}$ DNA sequence, ${ }^{11}$ orthogonal iterative density gradient ultracentrifugation and electrophoresis, ${ }^{12}$ have been advanced to achieve high purity of carbon nanotubes with narrow diameter distribution. However, an effective way of producing single-chirality carbon nanotubes with predetermined type is still limited.

To address this problem, scientists proposed a bottom-up synthesis, which is a growth on template strategy. Tour and coworkers mass produced the chirality-controlled carbon nanotubes from a short pure single-wall carbon nanotube seed as a template through a complex route with $\mathrm{Fe}$ as catalyst. ${ }^{15} \mathrm{Liu}$ and coworkers expanded this cloning strategy to grow single-wall carbon nanotubes with controlled chirality on a quartz. substrate without any metal catalysts through an open-end growth mechanism using open-end single-wall carbon nanotubes as seeds. ${ }^{14}$ In this 
regard, the use of template objects that mimic part of predetermined carbon nanotubes to strictly dictate the diameter and sidewall structure during the growth of carbon nanotubes holds high promise. Progress has been made even though with limited practical success. For instance, Li and co-workers grew one kind of carbon nanotube with high purity on a W and Co alloy catalyst. They attributed the selective growth to the catalysts crystals with high melting point and their unique atomic arrangement. ${ }^{\text {th }}$ Liu and co-works generated $(5,5) \mathrm{CNT}$ s from fullerenedione that decomposed at high temperature and used the resulting material as a template. ${ }^{16} \mathrm{Zhou}$ and coworkers also prepared $(5,5)$ CNTs with extreme high purity from Scott's $(5,5) C N T$ end-cap molecule after activated with water and air at high temperature. ${ }^{17} \mathrm{~A}$ new example of controlled synthesis of CNTs with single-chirality was reported by Amsharov, Fasel and coworkers, who used polycyclic aromatic hydrocarbon $\mathrm{P} 1$ as a precursor to form carbon nanotube seed through surface-catalyzed cyclodehydrogenation, followed by growth via epitaxial elongation to form a carbon nanotube on the same catalyst (Figure 1.2). ${ }^{18}$

Another type of possible template that unambiguously mimics the chirality of single-wall carbon nanotubes is called open-end templates that are taken from the middle segments of a single-wall carbon nanotube, while still keeping the full structural information of the carbon nanotube. The smallest repeating units in these carbon nanotubes are called carbon nanorings, like one single layer left after being cut perpendicular to the main axis (Figure 1.3). Figure 1.3 shows the possible templates for growing single-chirality single-wall carbon nanotubes. The smallest carbon nanorings for armchair single-wall carbon nanotuhes are called cycloparaphenylenes (CPPs), in which all the benzene rings are connected at the para-position to form a macrocycle. The corresponding nanorings that represent zigzag type single-wall carbon 
nanotubes are named cyclacenes. However there has been no report about the successful synthesis of cyclacenes.
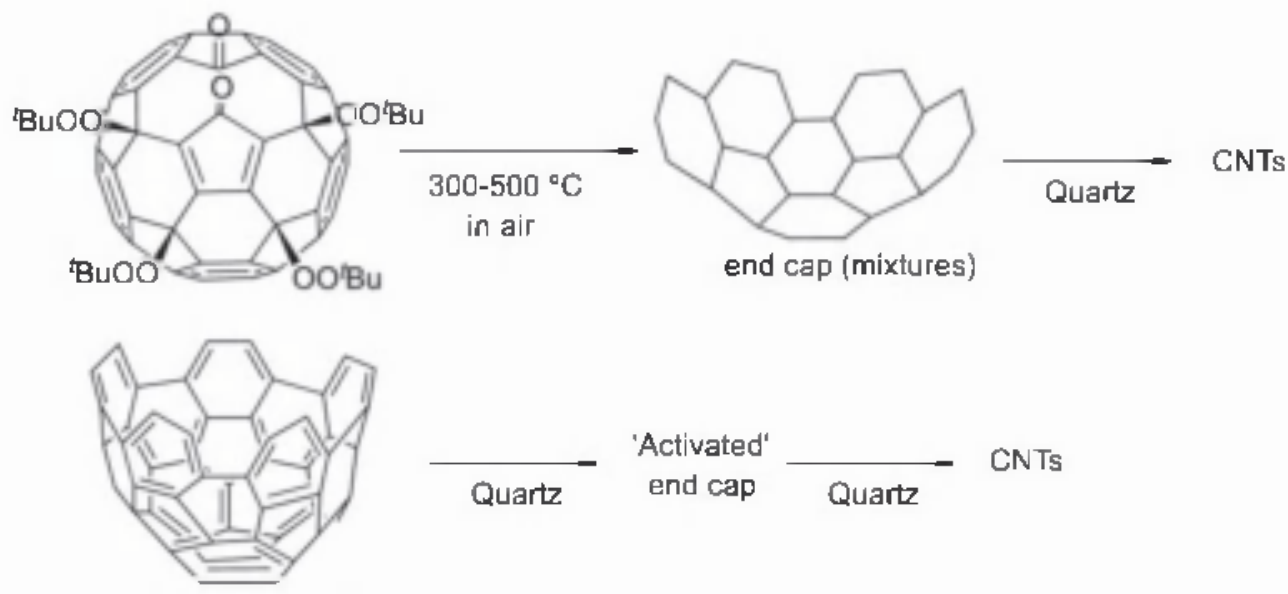

$(5,5)$ CNT end cap

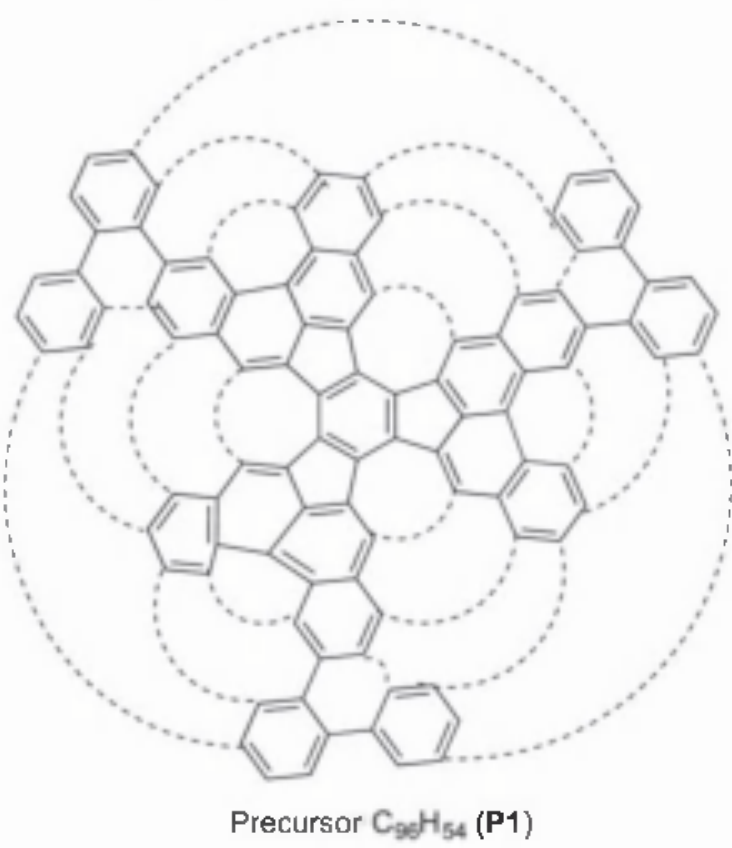

$\mathrm{CDH}$

$\mathrm{Pt}$ (6,6) SWCNT

seed

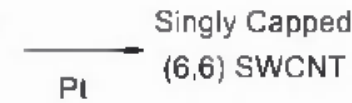

Figure 1.2. Synthesis of carbon nanotubes from three endcaps, oxidized fullerenes, short $(5,5) \mathrm{CNT}$ end cap and polycyclic aromatic hydrocarbon $\mathbf{P 1}$ 


\section{Carbon nanotubes}

Armchair

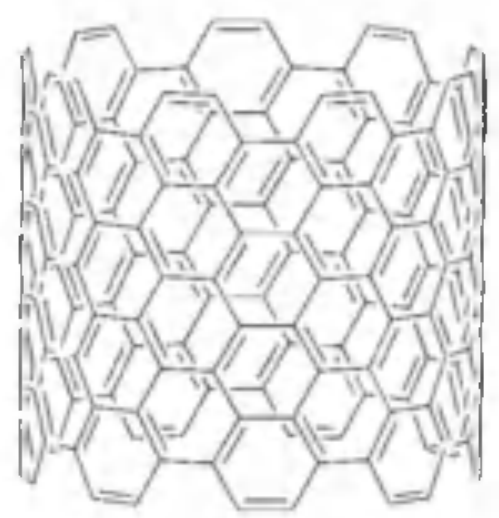

$(n, n)$ CNT
Zigrag

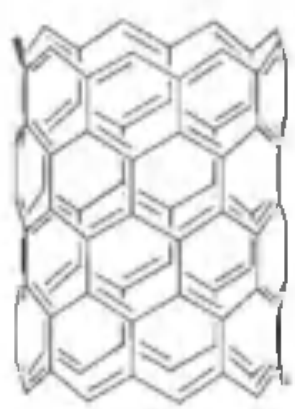

$(n, 0) \mathrm{CNT}$

\section{Carbon nanorings}

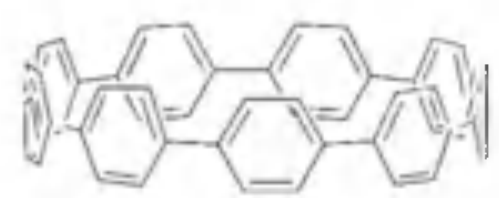

cycloparaphenylene (CPP)

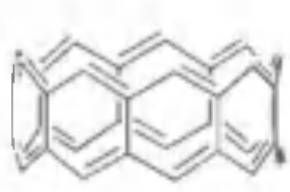

Cyclacene

Figure 1.3. Types of single-wall carhon nanotubes, their smallest repeating units ${ }^{3}$

Not only were these beautiful templates and related growth mechanisms introduced theoretically, but their growth feasibility to produce CNTs from CPPs was confirmed experimentally. In 2013, Itami and co-workers first published the metal-free CNT growth with narrow diameter distribution from [9]CPP and [12]CPP as seeds. ${ }^{19}$ The carbon nanotubes were produced with high purity when [12]CPP was coated on C-plane of sapphire wafer with a flow of ethanol as the carbon source under high temperature and high vacuum. The average diameter of resultant CNTs was similar to that of [12]CPP seeds, which supports the growth on template mechanism. 


\subsection{Characteristics of different growth-on-templates strategies}

A comparison of the different templates for carbon nanotubes growth in terms of supply quantity, product property, and mass production is summarized in Table $1 .{ }^{3}$ From Table I we can see that CPP has a great potential to act as a template over other templates. Another advantage of CPP as a potential template is that it is size-tunable, whereas other templates have very limited derivatives.

Table 1. Characteristics of different templates for growth of carbon nanotubes

\begin{tabular}{|l|l|l|l|}
\hline Template types & Template supply & Description of product & Mass production \\
\hline fullerenedione & Milligram scale & Wide diameter distribution & Scalable \\
\hline CNT endcap & Milligram scale & Mainly semiconductive & Scalable \\
\hline PAH & Milligram scale & $(6,6)$ CNT & Difficult \\
\hline CPP & & & \\
& Gram scale & Narrow diameter distribution & Scalable \\
\hline
\end{tabular}

\subsection{CPP's properties and potential applications}

Apart from serving as potential templates for the controlled synthesis of CNTs, CPPs themselves are aesthetically pleasing. The pursuit of synthesis of these unique, strained macrocycles will not only promote the discovery of new reactions and the development of novel methodology, but also will bring about new materials and applications. ${ }^{17.18 .19,20}$ Inherently interesting CPPs have shown unique size-dependent optoelectronic and redox properties. ${ }^{21}$ The 
HOMO-LUMO gaps decreases when the ring size decreases, which is opposite to that of linear oligoparaphenylenes, resulting in the longest wavelength absoiption maxima and the emission maxima being red-shifted as the ring size decreases. The oxidation potentials of CPPs decrease with the decrease of ring size. ${ }^{2\lrcorner b}$

The presence of the well-defined porous structure of CPPs makes them ideal candidates for study of host-guest interactions, ${ }^{22}$ catalysis and gas storage. ${ }^{2-1}$ Furthermore, the unusual alignment of radially oriented $\pi$-conjugated systems affords great chances to probe the effect of pyramidalization of $\mathrm{sp}^{2}$-hybridized carbons on aromaticity, ${ }^{24}$ and ring size on strain energy, ${ }^{25}$ and a variety of applications in supramolecular interactions. ${ }^{26}$

\subsection{Organic synthesis of CPPs}

Even though the CPPs' structures are relatively simple, it has been a challenge to synthesize them due to the presence of substantial strain energy in them. Early efforts include Parekh and Guha's attempted synthesis of [2]CPP in 1934 from oxidizing of 1,4-benzenedithiol to form cyclic disulfide 2 followed by copper-catalyzed desulfurization. ${ }^{27}$ However the last step did not proceed to produce any of the highly strained [2]CPPs. Cyclic disulfide 2 was not formed; instead cyclic trimer $\mathbf{4}$ or tetramer was formed (Scheme 1.1).

\section{Scheme 1.1. Parekh and Guha's attempted synthesis of $[2 \mid \mathrm{CPP}$}

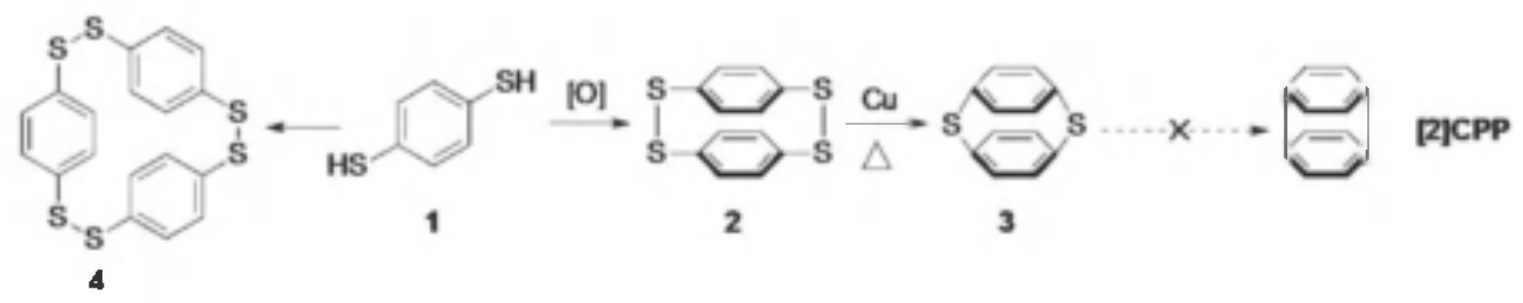


It was in 1993 when the synthesis of CPP was reported by Vogtle and co-workers in their preliminary publication, which was entirled On the Way to Macrocyclic Paraphenylenes. ${ }^{28}$ One route involved macrocyclization to form the desired ene-yne 7 from double Wittig reactions between diphosphonium 5 and dialdehyde 6. But the subsequent Diels-Alder reaction between 7 and diene equivalent did not take place to produce any CPP products.

\section{Scheme 1.2. Vogtle's two routes to CPP}

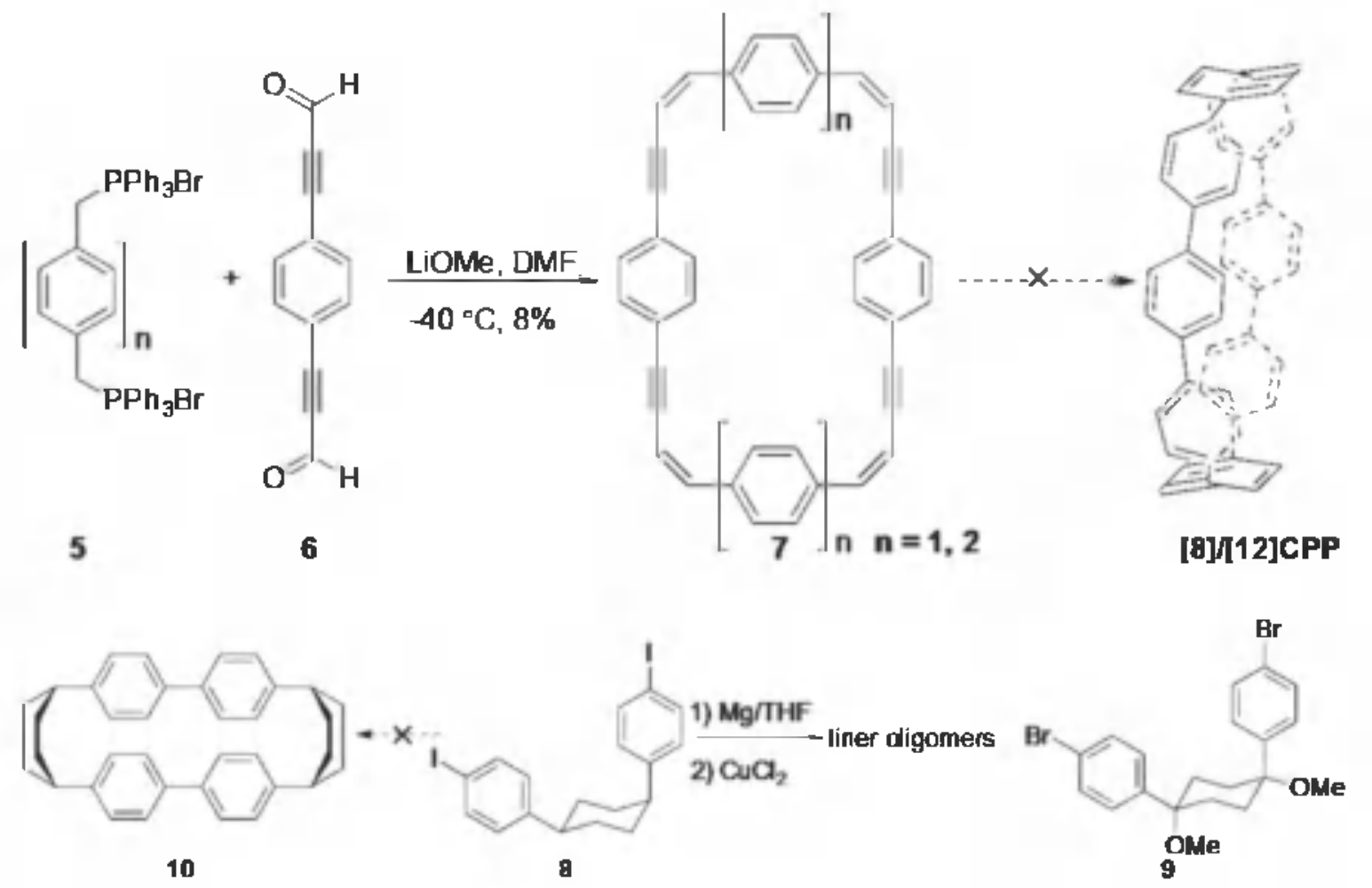

They also explored a different route starting from less strained cis-1,4-bis(4-

indophenyl)cyclohexane $\mathbf{8}$ with the hope that $s p^{3}$-hybridized carbons in macrocycle 10 would be able to accommodate the curvature and the presence of aromaticity after aromatization of the cyclohexane moiety would compensate for the buildup of the ring strain. However double copper-mediated Kumada homocoupling reaction resulted in only linear oligomers. Similarly the 
more rigid building block 9 also did not help in macrocyclization. After these attempts, he commented that the most promising pathway to make CPPs would be from 1,4-disubstituted cyclohexane huilding blocks.

The first successful synthesis of CPPs was achieved by Jasti and Bertozzi in 2008, almost 15 years later (Scheme 1.3) ${ }^{29 a}$ Since then several synthetic pathways for constructing CPP with different sizes ranging from the smallest with 5 benzene units to the largest 18 benzenes were developed. ${ }^{29}$ In the first successful synthesis the key intermediate iodidephenyl substituted 1,4 cis-dimethoxy-2,5-cyclohexadiene 11 was formed more favorably by two consecutive nucleophilic addition reactions of lithium reagents, produced from 1,4-diiodobenzene, to 1,4benzoquinone. Then 11 was converted to borylated partner 12 for macrocyclization. The SuzukiMiyaura cross-coupling reaction between 11 and 12 afforded a mixture of the CPP precursors, which were transformed successfully to CPPs under a reductive aromatization condition with lithium naphthalenide.

\section{Scheme 1.3. First successful synthesis of CPPs}
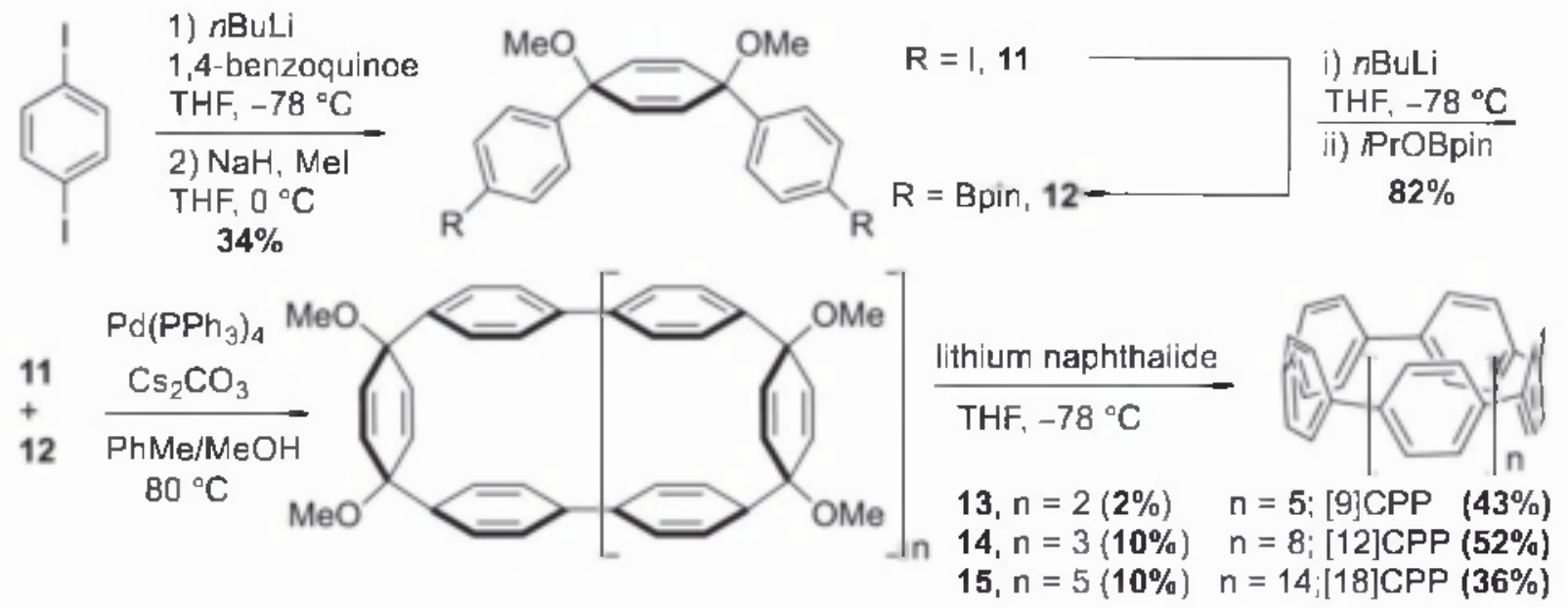

I.ater, both Jasti's group ${ }^{295.29 \mathrm{~g}, 30,31}$ and Itami's group ${ }^{29 \mathrm{~h}, 29 \mathrm{~d}, 32,33}$ expanded this methodology to selective synthesis of CPP with different sizes. The differences between these groups lie in 
two aspects. Jasti expanded the difuntionalized L-shaped building blocks either by taking advantage of different reactivity of aryl bromides and chlorides or by sequential oxidative dearomatization of $\mathbf{4}$-hydroxybiphenyl/nucleophilic addition, while Itami used the excess of one partner in the Suzuki-Miyaura cross-coupling reaction. Further Jasti prepared CPPs from reductive aromatization starting with diaryl substituted cyclohexadienes, whereas ltami produced CPPs with oxidative aromatization beginning with diaryl-substituted cyclohexanes. In Jasti's synthesis of [7]CPP (Scheme 1.4), he started with oxidative dearomatization of 16 by phenyliodonium diacetate (PIDA) to afford $\mathbf{1 7}$, which is equivalent to one nucleophilic addition of 1,4-benzoquinone with the corresponding lithium reagent. A modified procedure was adopted to prepare the unsymmetrical L-shaped building block 19 with high diastereoselectivity, which later became the standard protocol for making this kind of cis L-shape building block. Compound 17 was deprotonated with $\mathrm{NaH}$, followed by the nucleophilic addition of 4cholorbenzenelithium from the side opposite to the alkoxide due to static repulsion to afford $\mathbf{1 8}$, in high diastereoselectivity ( cis: $\operatorname{trans}=19: 1$ ). Subsequent methylation led to the unsymmetrical L-shaped building block 19. Still a portion of 19 was borylated to yield 20 . The subsequent orthogonal Suzuki-Miyaura cross-coupling reactions provided the macrocyclic CPP precursor 22, which was aromatized to afford [7]CPP. 


\section{Scheme 1.4. Synthesis of $|7| C P P$}

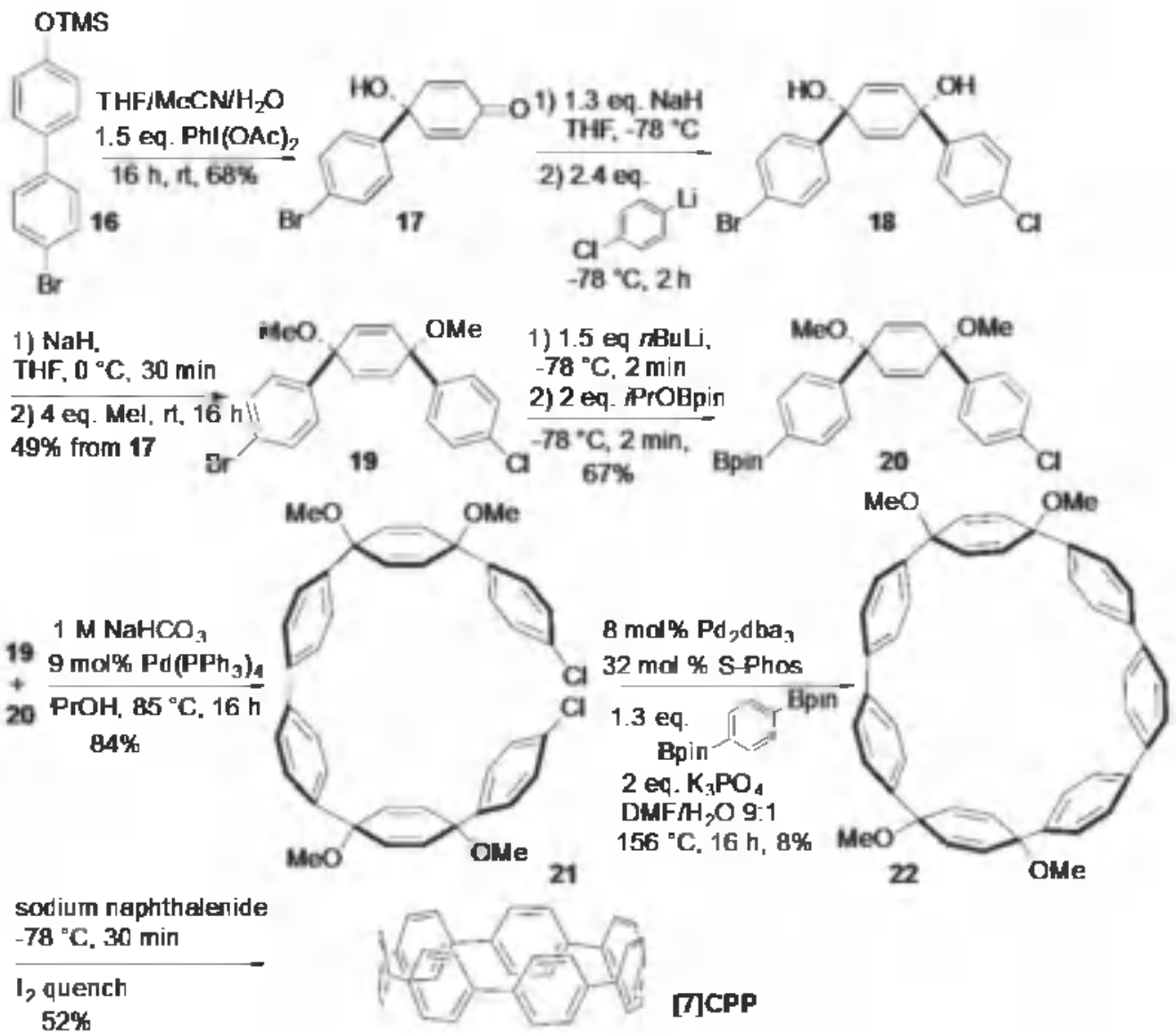

Itami and co-workers at the same time adopted a different strategy of selectively

preparing CPPs (Scheme I.5). In their first report of selective synthesis of CPPs, they used 24a recommended by Vogtle, as the L-shaped building block, which was synthesized from I,4diiodohenzene and cyclohexane-1,4-dione. They cleverly converted $\mathbf{2 3 a}$ to borylated $\mathbf{2 3 b}$ without protection of hydroxyl groups, which later proved a nice way for better purification. Then by using an excess of $\mathbf{2 4 a}$ to react with $\mathbf{2 3 \mathrm { h }}$ in two consecutive Suzuki-Miyaura crosscoupling reactions delivered the difunctionalized 25, which underwent another Suzuki-Miyaura cross-coupling with $\mathbf{2 3 b}$ to furnish the macrocycle $\mathbf{2 6}$. All the cyclohexane units in the macrocycle 26 were aromatized with $p$-toluenesulfonic acid $(\mathrm{TsOH})$ and microwave irradiation to afford [12]CPP. 
In 2011 , Itami and co-workers reported a nice way of macrocyclization: shotgun synthesis of [9] and [12]CPPs through the Ni(cod) 2 -mediated homocoupling reactions (Scheme 1.6). ${ }^{29,34}$ This time they adopted an improved procedure to increase the diastereoselectivity and the yield of double nucleophilic addition of the lithium reagent to cyclohexane- I,4-dione with the help of cerium and lithium salts, which suppressed the monoaddition product and the formation of the trans isomer. In doing so the L-shaped building block 27 was prepared in 7 I$84 \%$ yield. The subsequent $\mathrm{Ni}(\operatorname{cod})_{2}$-mediated homocoupling reations furnished the macrocyclic trimer $\mathbf{2 8}$ and tetramer $\mathbf{2 9}$ in a one-pot operation. Improved oxidative aromatization of $\mathbf{2 8}$ and $\mathbf{2 9}$ by sodium hydrogen sulfate in m-xylene/water under air afforded [9]CPP and [12]CPP, respectively. 


\section{Scheme 1.5. Selective Synthesis of |12|CPP}

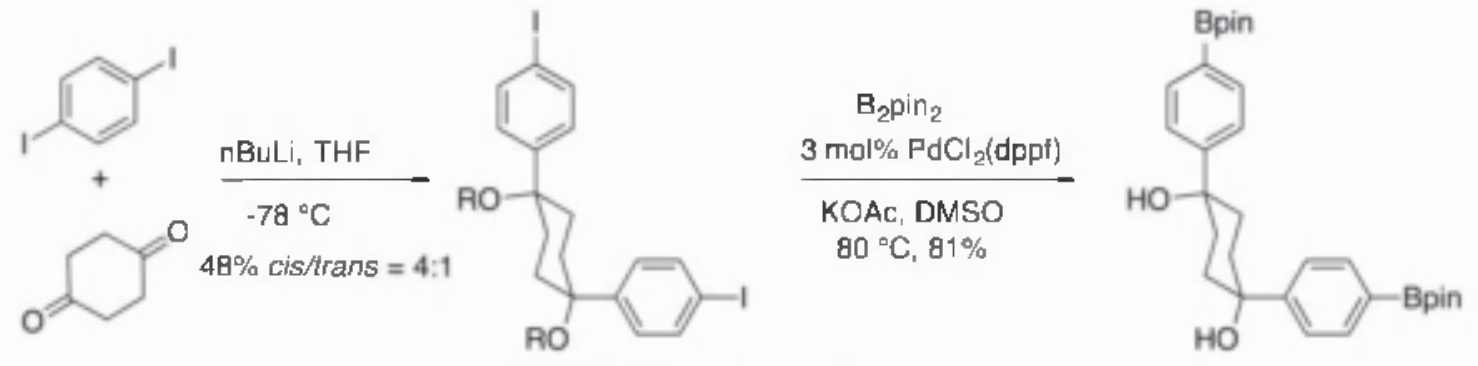

$23 a(\mathrm{P}=\mathrm{H})$

24a $(\mathrm{R}=\mathrm{MOM})=98 \% \quad \begin{aligned} & \mathrm{MOMCl}_{1} \text { PrNEt } \\ & \mathrm{CH}_{2} \mathrm{Cl}_{21} \mathrm{r}, 19 \mathrm{~h}\end{aligned}$
$23 b$
$24 a+23 b$

$10: 1$

$10 \mathrm{~mol} \% \mathrm{PdCl}_{2}$ (dppf)

5 eq. $\mathrm{NaOH}$

$16 \mathrm{eq} \cdot \mathrm{H}_{2} \mathrm{O}$

1,4-dioxane, $2 \mathrm{mM}$ $80{ }^{\circ} \mathrm{C}, 24 \mathrm{~h}, 81 \%$

14 eq. 23b

$20 \mathrm{~mol} \% \mathrm{Pd}(\mathrm{OAc})_{2}$ $20 \mathrm{~mol} \% \mathrm{X}$-Phos

5.0 eq. $\mathrm{NaOH}_{1} 26$ eq. $\mathrm{H}_{2} \mathrm{O}$ 1,4-dioxane, $2 \mathrm{mM}, 80{ }^{\circ} \mathrm{C}$ 24 h, $51 \%$
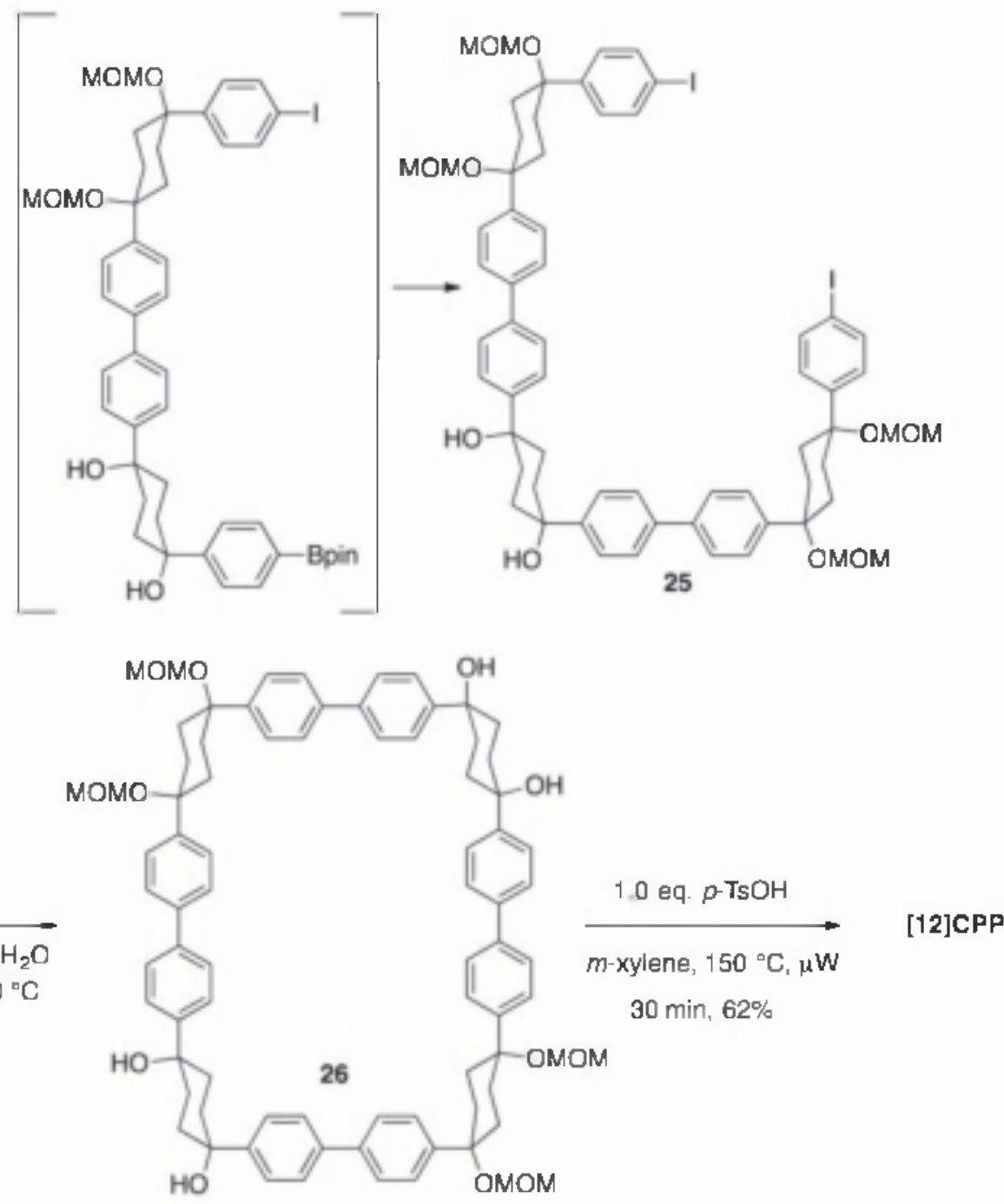
Scheme 1.6. Concise Synthesis of $[9]$ and $\mid 12] \mathrm{CPP}$

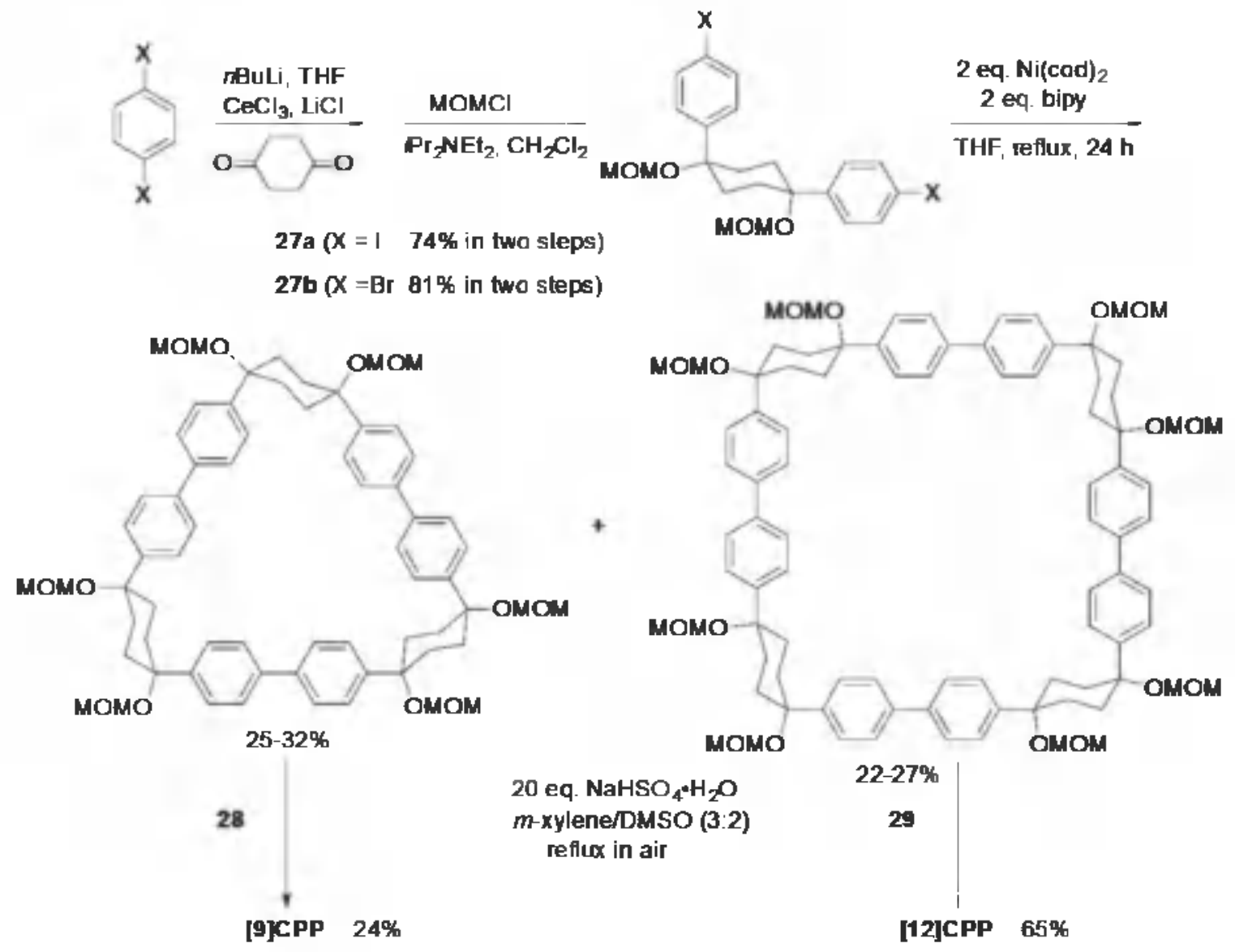

Another strategy of overcoming the ring strain during the synthesis of CPPs directly from benzene units was reported by Yamago ${ }^{290}$ through reductive elimination (Scheme 1.7) and

Scheme 1.7. Synthesis of |8|CPP by Yamago

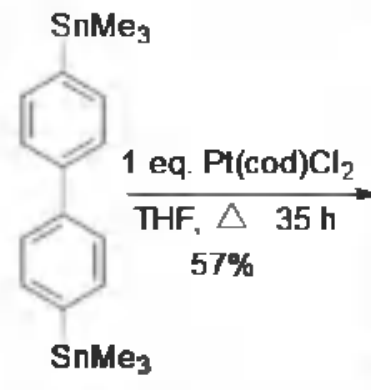

30

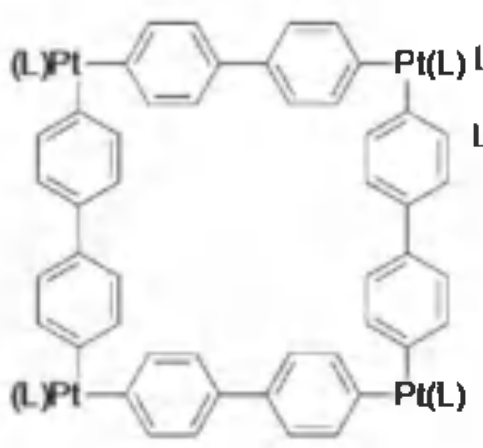

31

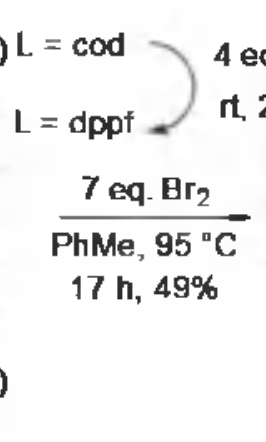

4 eq. dppf, $\mathrm{CH}_{2} \mathrm{Cl}_{2}$

it, $20 \mathrm{~h}, 91 \%$

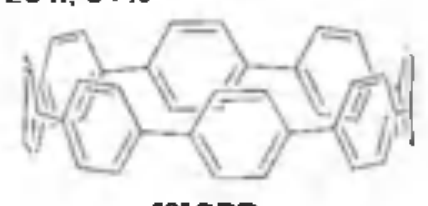

[8]CPP 
by Stępien ${ }^{29 p}$ through template effect. Yamago and co-workers found that transmetallation between ditin $\mathbf{3 0}$ and $\mathrm{Pt}(\operatorname{cod}) \mathrm{Cl}_{2}$ afforded the less strained macrocyclic $\mathrm{Pt}$ complex $\mathbf{3 1}$.

Subsequently, the ligand exchange and reductive elimination, promoted by oxidation with bromine, afforded the [8]CPP without any aromatization step as the smallest CPP at that time.

Stępien and coworkers synthesized two nanotube endcaps through a completely different but concise approach (Scheme 1.8). Strain-free precursors $\mathbf{3 2}$ and $\mathbf{3 4}$ in which three 2,7dibromocarbonzoles or derivatives were connected by a mesitylene unit underwent three-fold nickel-mediated homocoupling reactions to deliver nanotube endcaps $\mathbf{3 3}$ and $\mathbf{3 5}$ respectively, in one step. This approach features a one step macrocyclization and very high ring strain buildup,

Scheme 1.8. Synthesis of Nanotuhe End-caps

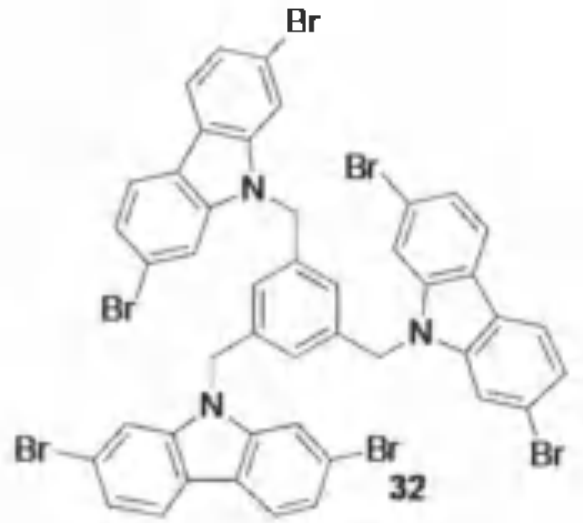

$$
\begin{aligned}
& \underset{\text { eq. } \mathrm{Ni}(\operatorname{cod})_{2}}{\text { bipy }} \\
& \frac{\text { DMF, } 100^{\circ} \mathrm{C}_{2} 24 \mathrm{~h}}{29 \%}
\end{aligned}
$$
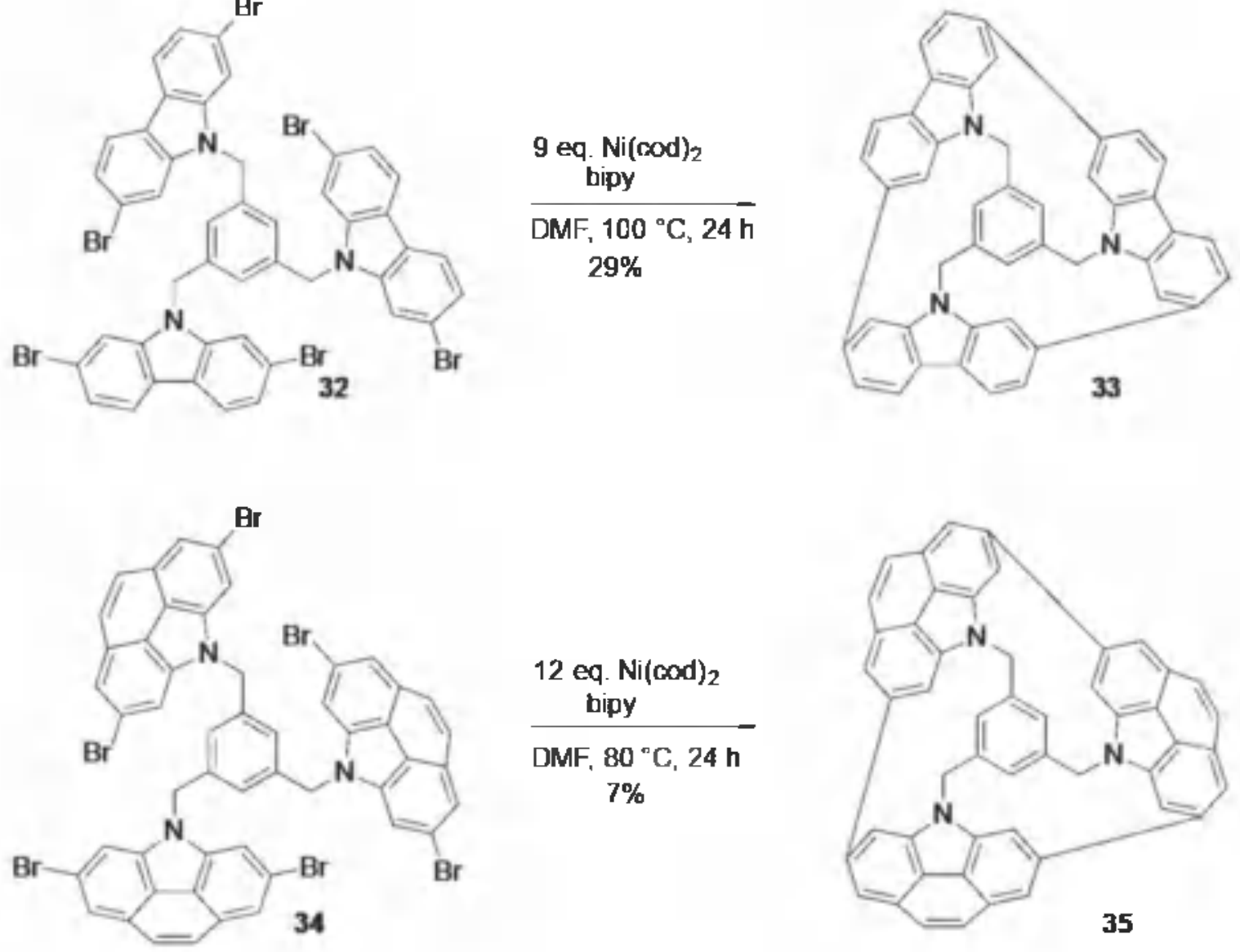
which are quite different from other strategies. One can anticipate that future strategy for preparing CPPs could involve with removable tethers.

The pursuit of synthesis of CPPs gained more attention in 2014 when Jasti's group ${ }^{29 m}$ and Yamago's group ${ }^{29 \mid}$ independently published almost simultaneously the synthesis of smallest [5]CPP (Scheme 1.9). Yamago and co-workers prepared the macrocyclic [5]CPP precursor 38 through reductive elimination of a $\mathrm{Pt}$ complex and Ni-mediated homocoupling respectively. However, they could not proceed further to aromatize it with the widely used reductive aromatization method due to the low reactivity of methoxy groups and high ring strain. But stannane-mediated reductive aromatization took place to furnish [5]CPP when the highly reactive free hydroxyl-substituted precursor 39, obtained by removal of the TES groups, were used. Jasti's group discovered the existence of macrocyclic precursor $\mathbf{4 I}$ when they tried to prepare a [10]CPP precursor through Suzuki-Miyaura cross-coupling reaction. Optimization of the reaction conditions led to the formation of $\mathbf{4 1}$ in $44 \%$ yield through the palladium-catalyzed homocoupling of aryl boronic esters at room temperature under air. In order to circumvent the limitation of the reductive power of sodium naphthalenide, they quenched the reaction mixture with methanol to form the partial reduced product $\mathbf{4 2}$. Following the LDA induced Elcb elimination at room temperature, the desired [5] CPP was obtained. 


\section{Scheme 1.9. Synthesis of |5|CPP hy Yamago and Jasti}

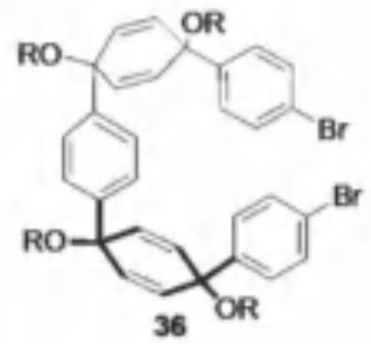

$R=$ TES $3863 \%$

- $\mathrm{R}=\mathrm{H} \quad 39$ quantitative
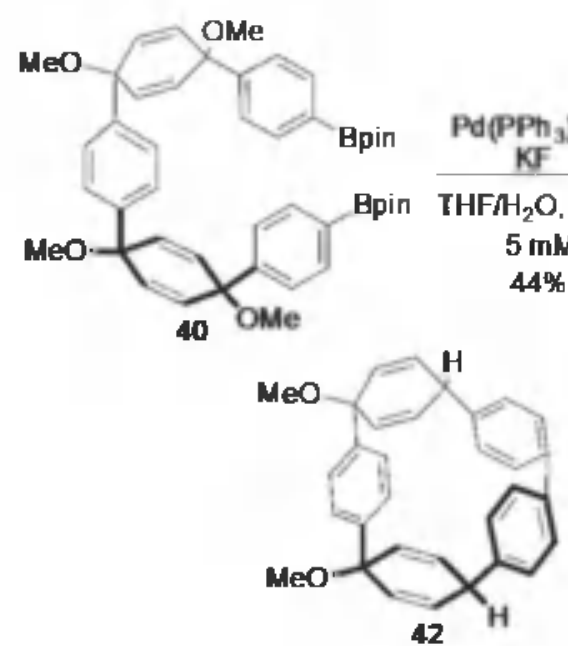

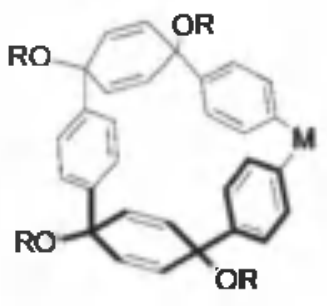

37

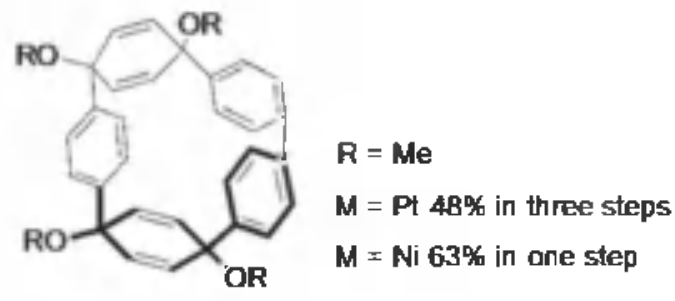

38

$\mathrm{SnCl}{ }_{2} \cdot \mathrm{H}_{2} \mathrm{O}$, THF, $60^{\circ} \mathrm{C}, 6 \mathrm{~h}$

[5]CPP

$58 \%$

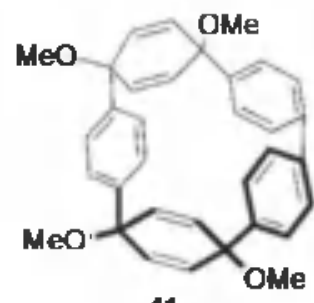

41

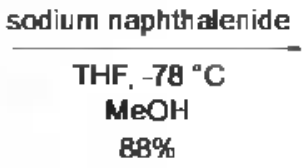

LDA, toluene, $25^{\circ} \mathrm{C}$

$69 \%$

[5]CPP

\subsection{Our synthesis of a functionalized CPP}

It is apparent from the work of other research groups that the efficient synthesis of the intermediate cis-difunctionalized aryl-substituted cyclohexadienes or cyclohexanes, is crucial to the successful synthesis of CPPs. However, the current methods of preparing them involved multiple steps with precise sequence of addition and quantity (Scheme 1.10). In the first report of CPP synthesis cis-diiodide aryl-substituted cyclohexadiene 11 was prepared by two consecutive nucleophilic additions of a lithium reagent to 1,4-benzoquinone in $34 \%$ yield. Oxidative dearomatization with a hypervalent iodide compound was employed to eliminate one of the two steps of nucleophilic addition reaction. Deprotonation of free hydroxyl group greatly improved 
the diastereoselectivity of the next nucleophilic addition reaction of an aryllithium reagent from 3:1 to 19:1 in favor of the desired cis product. This was attributed to ionic repulsion that anionic nucleophilic lithium reagent would attack from the opposite side. ${ }^{291}$ This procedure was adopted as a standard method for preparing cis-difunctionalized aryl-substituted cyclohexadienes or cyclohexanes. Later Itami developed a procedure that used the cerium ion to coordinate with the oxygen atom of the hydroxyl group in order to block one side of the cyclohexyl system from the nucleophilic attack to achieve high diastereoselectivity with high yield. ${ }^{29 \mathrm{e}}$

\section{Scheme 1.10. Synthesis of Key Intermediate to CPP}
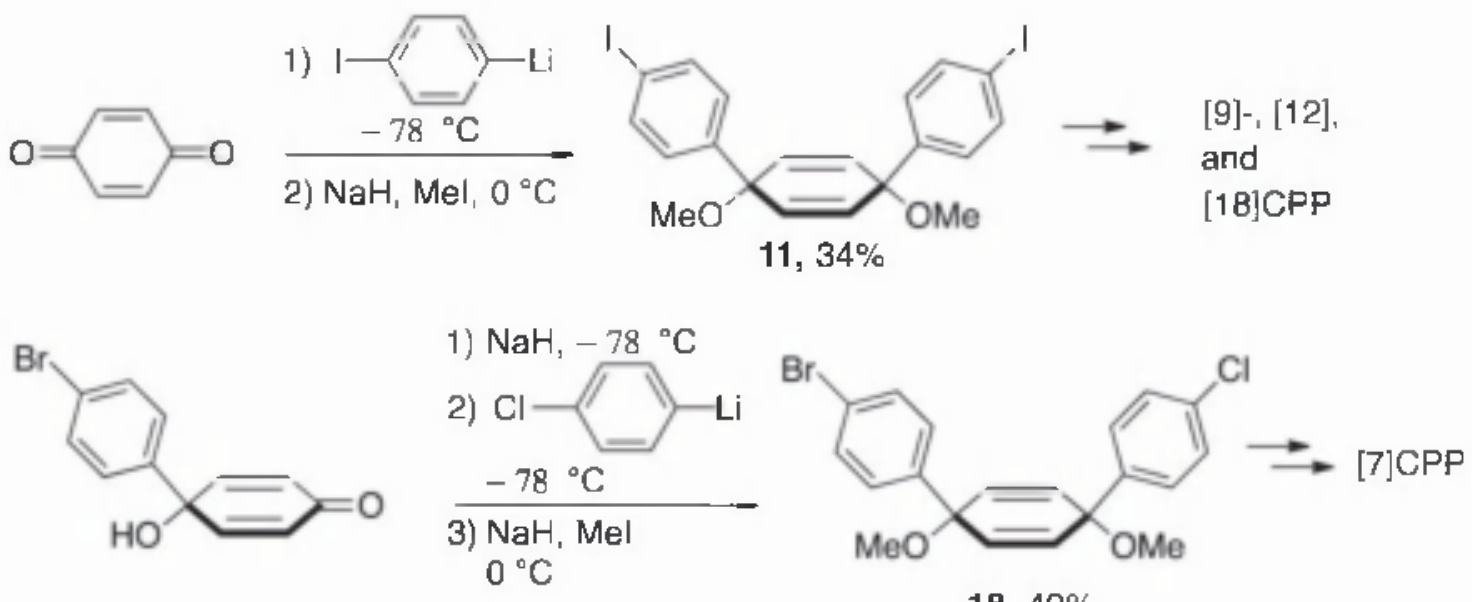

$18,49 \%$

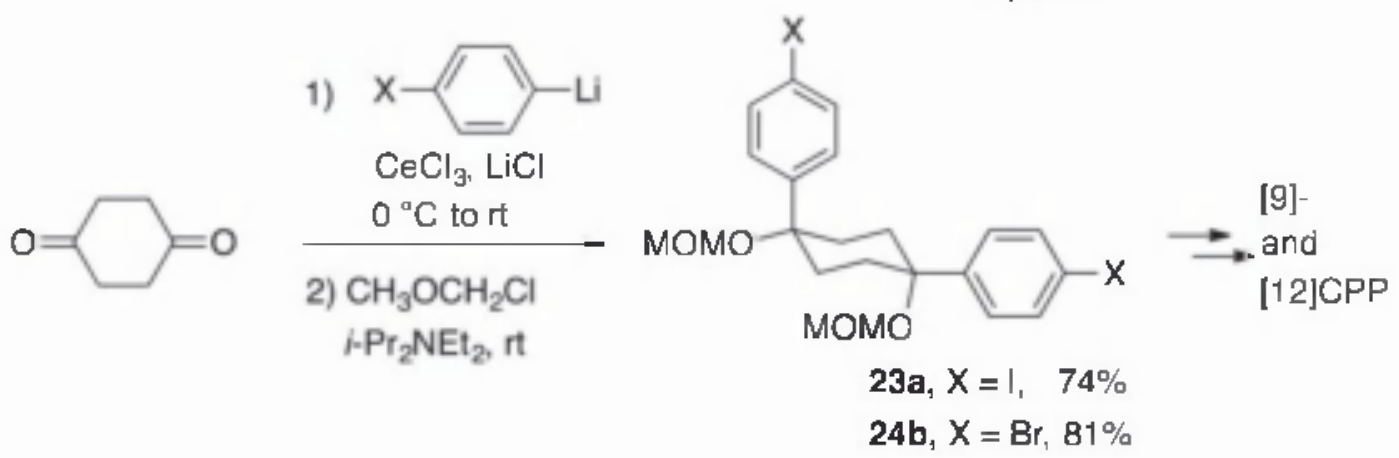

We envisioned that the possibility of taking advantage of the inherent high stereoselectivity of the Diels-Alder reaction to form the desired CPP precursors. The first step of our approach involved the synthesis of difunctionalized dienes. The simplest difunctionalized diene would be 1,4-bis(4-bromophenyl)-1,3-butadiene. Using Davis's procedure (Scheme 
I.11), ${ }^{30}$ we were able to make large quantities of difunctionalized diene $\mathbf{4 8}$ from $\mathbf{4}$-bromocinnaic acid $\mathbf{4 3}$ with a sequence of reactions that involved esterification, reduction, oxidation, and the Horner-Wadsworth-Emmons reaction with diethyl 4-bromobenzylphosphonate 47, which was prepared by the Arbuzov reaction with 4-bromobenzyl bromide and triethyl phosphite.

Scheme 1.11. Synthesis of Diene 48

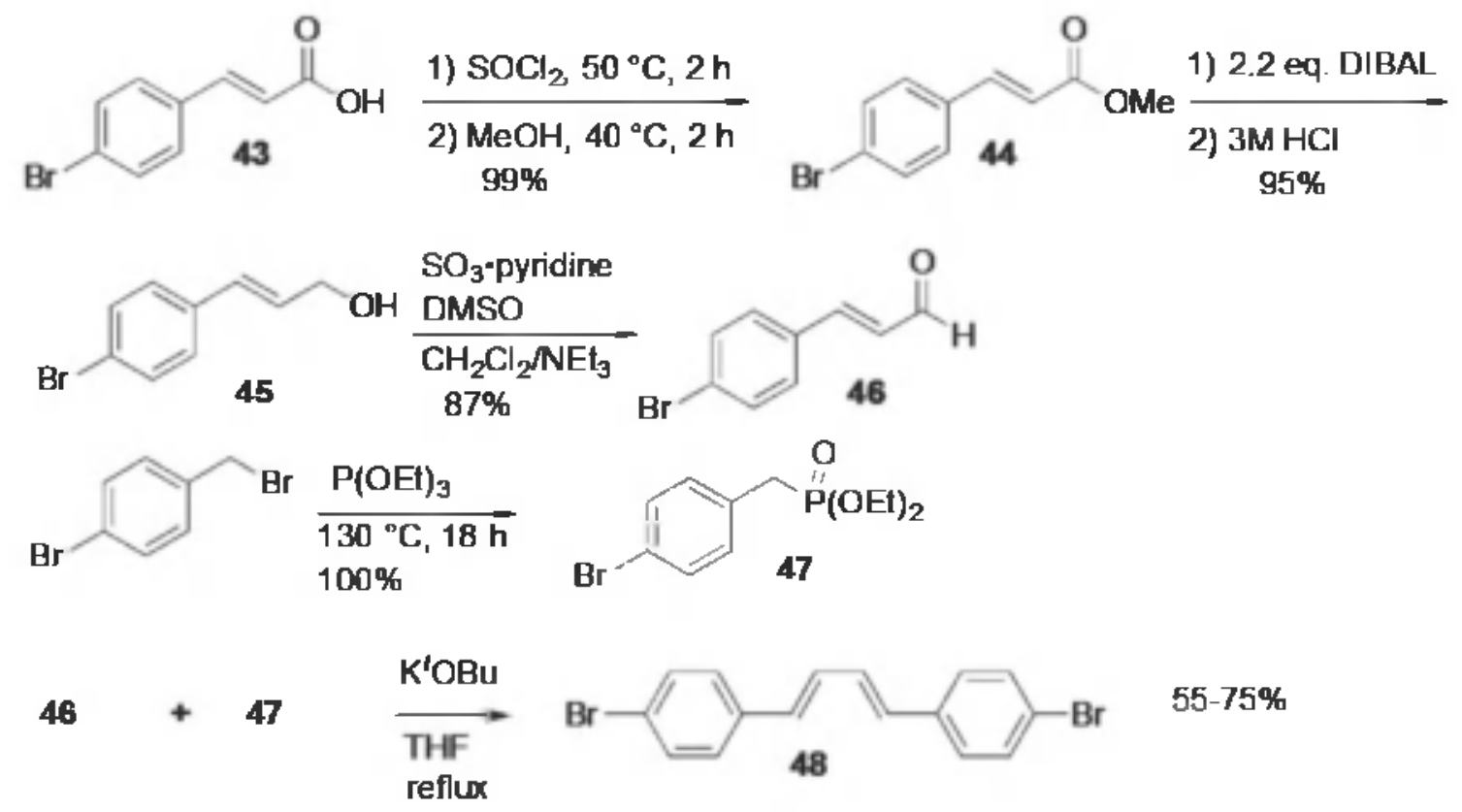

With large amounts of diene $\mathbf{4 8}$ at hand, we next investigated the feasibility of using $\mathbf{4 8}$ for the Diels-Alder reaction with benzyne as reactive dienophiles. First we tried a model study the Diels-Alder reaction between diene $\mathbf{4 8}$ and the in-situ generated benzyne from the commercial available triflate $\mathbf{4 9}$ using KF to induce hezyne generation (Scheme 1. I2). We were very pleased to observe the formation of the desired adduct $\mathbf{5 0}$ by the 'H NMR spectrum. ${ }^{31}$ 


\section{Scheme 1.12. Model Study of Diels-Alder reaction Between Diene 48 and 49}

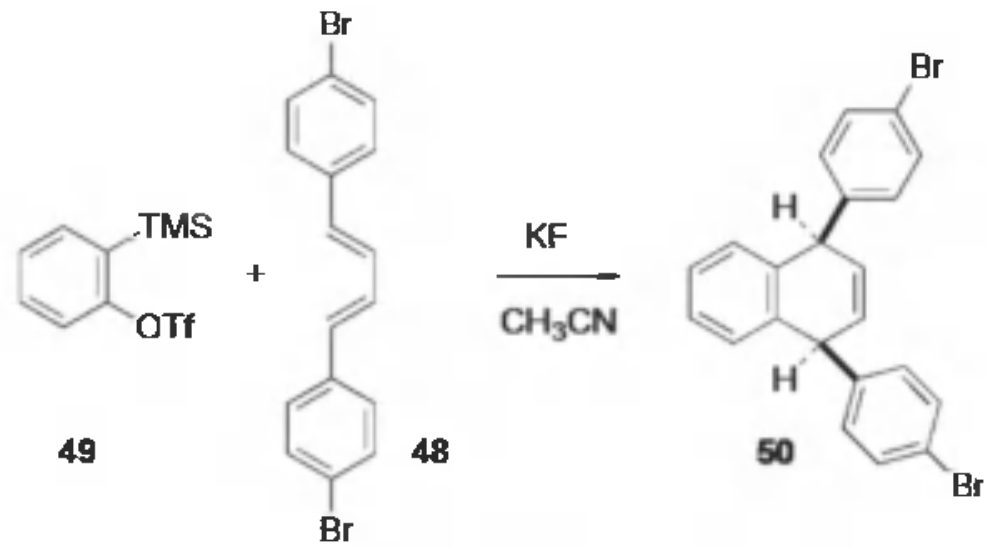

Then we turned our attention to explore the Diels-Alder reaction between 3,6-

dimethoxybenzyne and diene 48 . The precursor triflate 54 to 3,6-dimethoxybenzyne was synthesized from the commercially available 2,5-dimethoxybenzaldehyde through BaeyerVilliger oxidation, TMS protection, lithiation followed by quenching with TMSCI, deprotection, and trifluoromethylsulfonation in high yield (Scheme 1.13). However, the following Diels-Alder reaction between diene $\mathbf{4 8}$ and 3,6-dimethoxybenzyne, generated from 54, did not take place, with a variety of reaction conditions including different fluoride sources, addition sequences, temperatures, and solvents (Scheme I.14).

\section{Scheme 1.13. Synthesis of Benzyne Precursor 54}
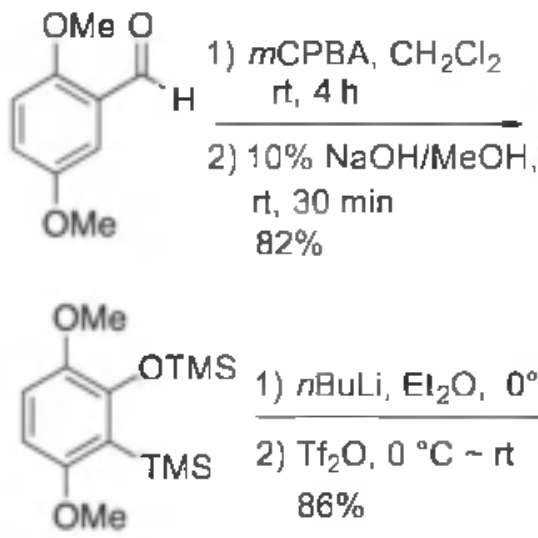

1) $n \mathrm{BuLi}, \mathrm{El}_{2} \mathrm{O}, 0^{\circ} \mathrm{C}$

53

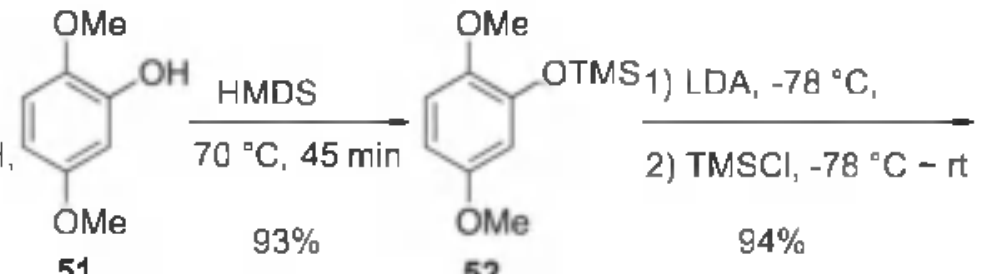

51

52<smiles>CCCOc1c(OC)ccc(OC)c1S(C)(=O)=O</smiles> 


\section{Scheme 1.14. Diels-Alder reaction Between Diene 48 and Benzyne Precursor 54}

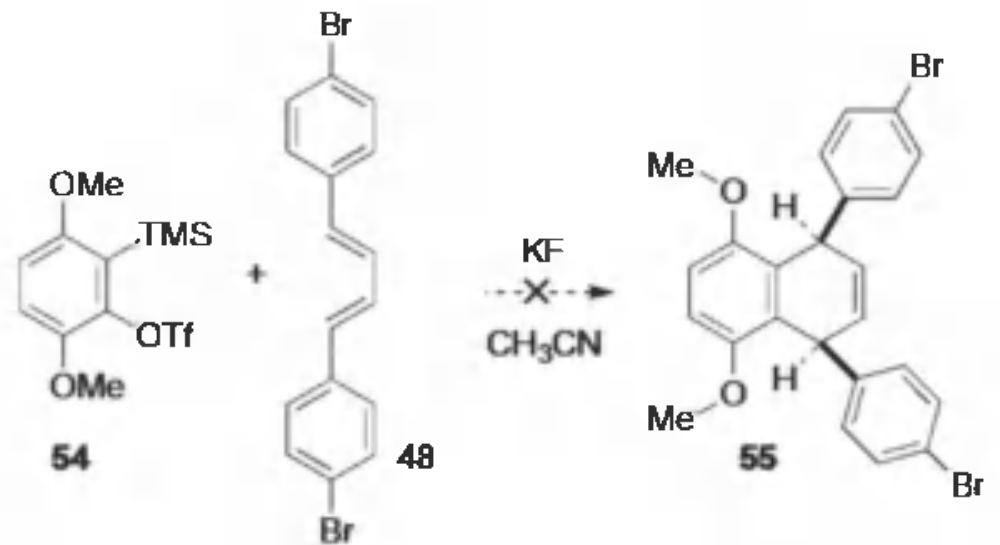

Then we turned to the Lewis-acid catalyzed Diels-Alder reaction between diene $\mathbf{4 8}$ and 1,4-benzoquinone (Scheme 1.15). Gratifyingly, the dihydronaphthanene derivative $\mathbf{5 5}$ was achieved in $92 \%$ yield by a one-pot, two-step reaction using $\mathrm{BF}_{3} \cdot \mathrm{OEt}_{2}$ to catalyze the Diels-Alder reaction between $\mathbf{4 8}$ and 1,4-benzoquinone, followed by methylation. A single crystal of $\mathbf{5 5}$ suitable for X-ray diffraction was obtained from by recrystallization from dichloromethane/hexanes. The X-ray structure analysis clearly showed that the two 4bromophenyl substituents were situated cis to each other exclusively (Figure 1.4).

Scheme 1.15. Diels-Alder reaction between 48 and 1,4-Benzoquinone<smiles>CCOC(C)(Br)Br</smiles><smiles>C[R](C)(C)C(C)(C)C</smiles>

$91 \%$ yield over two steps 


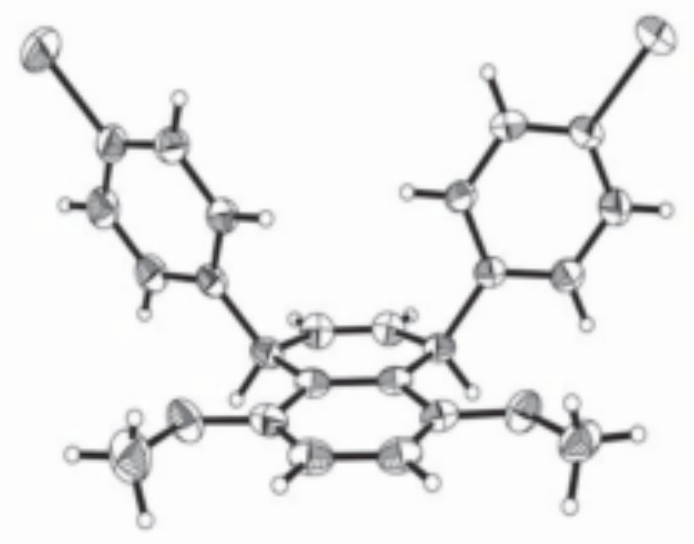

Figure 1.4. X-ray crystal structure of dibromide $\mathbf{5 5}$

We then used 55 to undergo the $\mathrm{Ni}(\mathrm{cod})_{2}$-mediated homocoupling reaction in the presence of 2,2'-bipyridyl (bipy) to promote the macrocyclic ring formation (Scheme 1.16). At 5 $\mathrm{mM}$ concentration of $\mathbf{5 5}$, the cyclic dimer syn-56a and $a n t i \mathbf{- 5 6} \mathbf{b}$ were produced in $21 \%$ and $6 \%$ yields, respectively. This was the first example of producing the cyclic dimers from the L-shaped building block, which was in sharp contrast to the reports in which only a trimer or a tetramer or a mixture of both was obtained from the L-shaped building blocks. ${ }^{29 e, 34}$ One year later, Yamago and co-workers reported a second example of cyclic dimer formation in $11 \%$ yield from the $\mathrm{L}$ shaped building block $\mathbf{5 7}$ (Scheme 1.17). ${ }^{35}$ More recently our group expanded the reaction scope with other dienophiles (Scheme 1.18). ${ }^{36}$ The Diels-Alder reaction between diene $\mathbf{4 8}$ and dimethyl acetylenedicarboxylate again afforded the I-shaped building block 60 in $85 \%$ yield. The $\mathrm{Ni}(\operatorname{cod})_{2}$-mediated homocoupling reactions produced the cyclic dimer $\mathbf{6 I}$ in less than $5 \%$ yield and the cyclic trimer $\mathbf{6 2}$ as the major product in $18 \%$ yield. 
Scheme 1.16. Ni(cod)2-mediated Homocoupling Reaction with 55

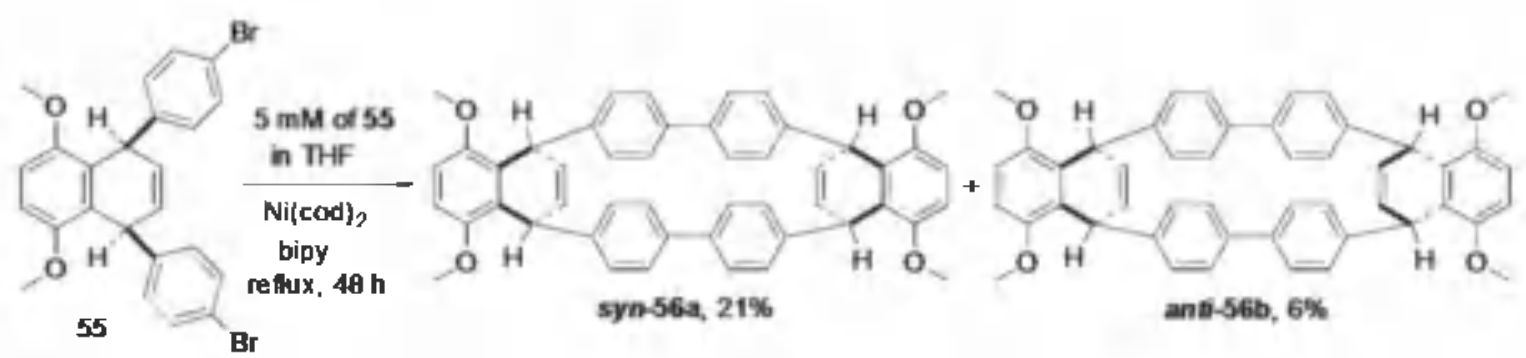

Scheme 1.17. Ni(cod)2-mediated Homocoupling Reaction with 57

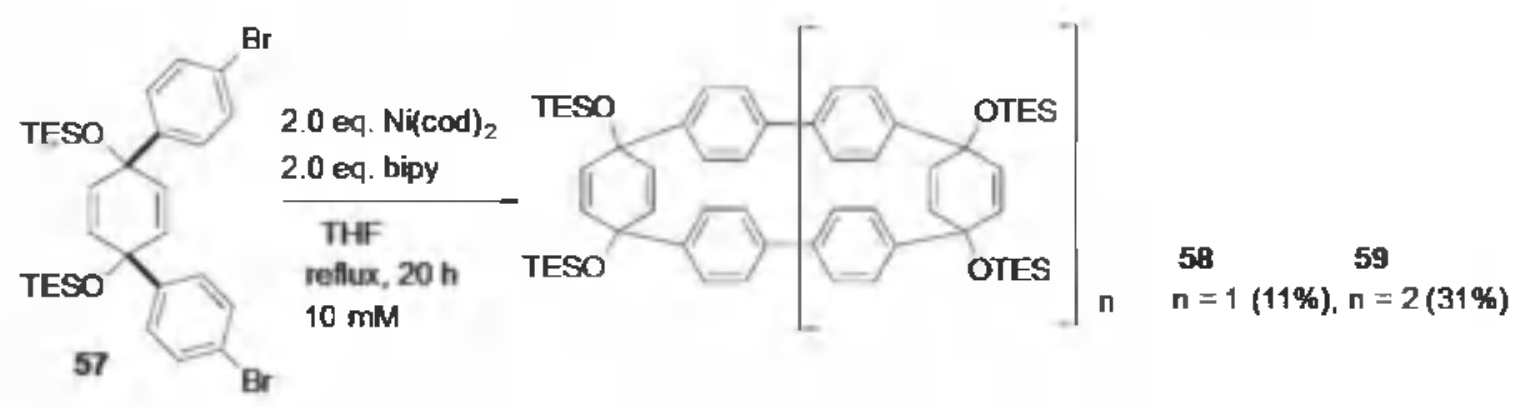

Scheme 1.18. Ni(cod)2-mediated Homocoupling Reaction with 60

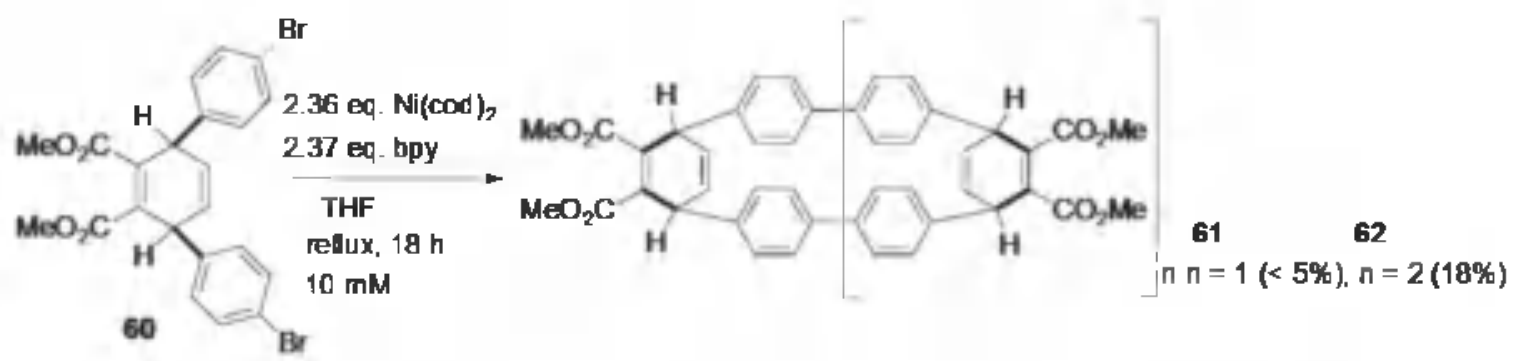

The structure of the dimer $s y n-56 a$ was characterized by the ${ }^{1} \mathrm{H}$ and ${ }^{15} \mathrm{C}$ NMR

spectroscopy and high-resolution mass spectroscopy. In the 'H NMR spectrum, all the aromatic proton signals in the biphenyl segments are shifted upfield to 6.6-6.7 $\mathrm{ppm}$. The signal of the allylic hydrogens occurred at $5.2 \mathrm{ppm}$, while the signal of the vinylic hydrogens occurred at 6.8 ppm. Recrystallization of $s y n-\mathbf{5 6 a}$ from dichloromethane and hexanes provided suitable crystals for X-ray diffraction (Figure 1.5). The X-ray structure analysis not only unambiguously confirmed its structure, but also showed that the dimer is oval-shaped, and the two biphenyl segments lies close to each other within a distance ranging from ca. 3.42 to $4.10 \AA$. The two 


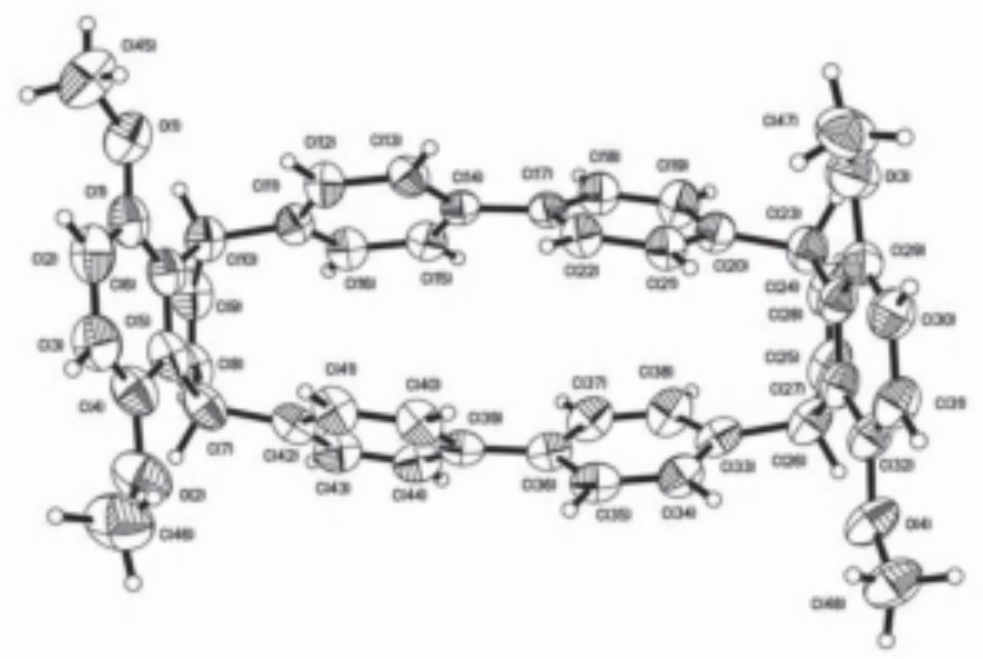

Figure 1.5. X-ray crystal structure of the dimer syn-56a

phenyl rings in the biphenyl segments are twisted with a dihedral angle around $49^{\circ}$. This may account for the upfield shift of the aromatic proton signals in the biphenyl segments, which are located in the magnetically shielded region of the aromatic ring current of the phenyl groups on the opposite side.

Vogtle predicted that rigid building blocks would facilitate the formation of macrocyclic rings. ${ }^{2 R}$ The X-ray structure of our L-shaped building block $\mathbf{5 5}$ clearly showed that the included angle between the two 4-bromophenyl groups is $73.6^{\circ}$ in the crystal lattice, smaller than that of $23 \mathrm{a}$ and $24 \mathrm{~b}$ at ca. $80^{\circ}$ and similar to that of 60 at $73.5^{\circ}$. The conformational analysis of $1,4-$ cyclohexadienes by Marshall and coworkers ${ }^{i 7}$ suggests that the small '.J coupling constant of 4.7 Hz between allylic protons and vinylic protons in $\mathbf{5 5}$ indicates that the dihydronaphthalene ring is only slightly puckered $\left(<10^{\circ}\right)$ from planarity, with a flattened-boat structure in solution as in crystal lattice. This smaller included angle between the two 4-bromophenyl groups in $\mathbf{5 5}$ than in 23a and 24h may be responsible for channeling the second $\mathrm{Ni}(\operatorname{cod})_{2}$ - mediated homocoupling reaction toward the formation of the cyclic dimers $s y n-\mathbf{5 6}$ and anfi-56b as the major products. 
The allylic 1,3-strain between the methoxy groups and the two 4-bromophenyl groups in $\mathbf{5 5}$ may he responsible for the smaller included angle between the two 4-bromophenyl groups and thus favor dimer formation. However these interactions are not severe in $\mathbf{6 0}$ or in Itami and Yamago's cases. Instead, those I_-shaped building blocks are more in favor of the formation of trimers under the $\mathrm{Ni}(\operatorname{cod})_{2}$-mediated homocoupling condition.

We first tried to brominate the allylic hydrogens in hope of using LDA for aromatization as reported by Jasti's group in their synthesis of [5]CPP. ${ }^{29 m}$ Although we did observe the formation of some brominated products, the LDA induced elimination did not occur. Other aromatization methods were also unsuccessful (Scheme 1.19).

Scheme 1.19. Efforts to Aromatize syn-56a

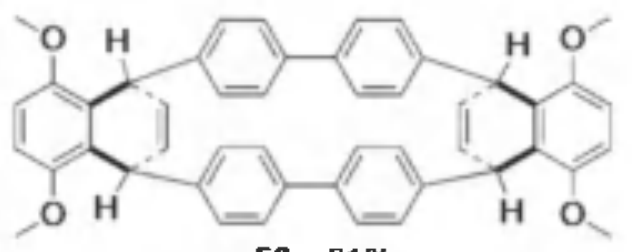

syn-56a, 21\% $\begin{array}{ccc}\text { DDQ, } & T \leq 150^{\circ} \mathrm{C} & \text { recovered starting material } \\ \triangle & T=160^{\circ} \mathrm{C} & \text { decomposed }\end{array}$

Pd/C anly recovered starting material

CH activation (bromination) brominated mixtures after treated with LDA, no discemible products strong oxidants decomposed frustrated lewis acid only recovered starting material

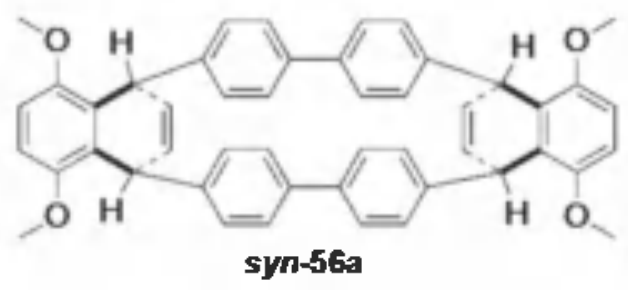

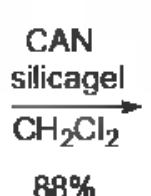

$88 \%$

trealed with WA, still only recovered slarting material 63

We also attempted to aromatize the cyclic dimers by treatment with 2,3-dichloro-5,6dicyano- I,4-benzoquinone (DDQ) at temperatures ranging from 25 to $150^{\circ} \mathrm{C}$, but were not successful. Perhaps DDQ's oxidation power is not strong enough to overcome the developing ring strain in the functionalized [6]CPP. 
We also tried to oxidize the cyclic dimer $s y n-56 a$ to quinone 63 , hoping that the baseinduced tautomerization would compensate for the ring strain to form the anion of the corresponding hydroquinone[6]CPP. The oxidation proceeded well with CAN in silica gel in 15 min. However, the base-induced tautomerization did not take place, and $\mathbf{6 3}$ was recovered.

We then turned our attention to exploring the aromatization of functionalized [9]CPPs with less ring strain by synthesizing the trimers from the $\mathrm{Ni}(\operatorname{cod})_{2}$-mediated homocoupling reactions with L-shaped building block $\mathbf{5 5}$. After careful and tedious flash column

\section{Scheme 1.20. Synthesis of Trimers}

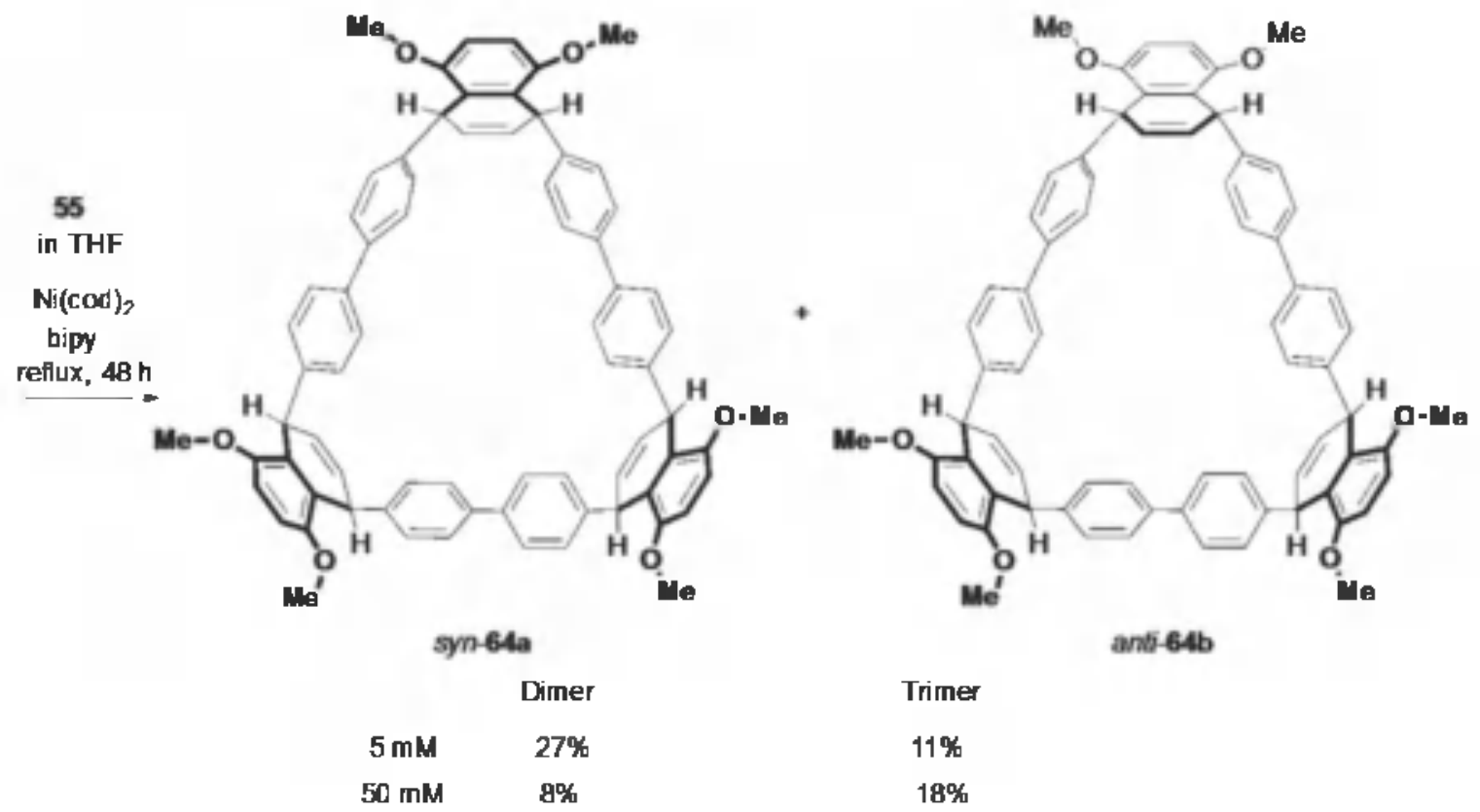

chromatography of the reaction mixture of $\mathrm{Ni}(\mathrm{cod})_{2}$-mediated homocoupling reaction of $\mathbf{5 5}$, we are able to isolate the cyclic trimers syn-64a and anti-64b (syn-64a: anti-64b $=1: 3)$ in a $11 \%$ combined yield. At higher $50 \mathrm{mM}$ concentration of $\mathbf{5 5}$, we obtained the cyclic dimers in $8 \%$ combined yield ( $(s y n-56 \mathbf{a}:$ anti-56b $=3: 1)$ and the cyclic trimers in $18 \%$ combined yield $(s y n-64 a$ : anti-64b = 1:3) (Scheme 1.20). 
Again the structures of the cyclic trimers were characterized by the ${ }^{1} \mathrm{H}$ and ${ }^{13} \mathrm{C}$ NMR spectroscopy and high-resolution mass spectroscopy. With syn-64a possessing three planes of symmetry and one $C_{3}$ axis of symmetry; and anti-64h containing only one plane of symmetry, it is relatively easy to identify them from the NMR spectroscopy. Only one singlet from the methoxy groups, one set of doublets with $\mathrm{AB}$ pattern from the aromatic protons in the biphenyl segments, a doublet from the allylic protons and a doublet from the vinylic protons were observed in syn-64a ${ }^{1} \mathrm{H}$ NMR spectrum. The anti-64b 'H spectrum showed three singlets with equal intensity for the methoxy groups, three sets of doublets with $\mathrm{AB}$ pattem from the aromatic protons in the biphenyl segments, one singlet and one AB pattern from the aromatic protons in the dimethoxybenzene parts, one set of two doublets and one set of ABXY spin system from the allylic and vinylic protons in the cyclohexadiene strucutures. These signals fall within the normal chemical shift range of these types of protons. Unlike the dimers, the aromatic signals are not shifted upfield to ca. $6.6 \mathrm{ppm}$. Another difference in chemical shifts of the ${ }^{1} \mathrm{H}$ NMR spectrum between the dimers and trimers is that vinylic signals of the dimers occurred at $6.8 \mathrm{ppm}$, whereas that of the trimers occurred at around $6.0 \mathrm{ppm}$.

We were delighted to observe the disappearance of the doublets for the allylic and vinylic protons in the cyclohexadiene units when the trimers were treated with DDQ for aromatization. After extensive screening of the oxidants, solvents, reaction temperature, and reaction time, we were able to aromatize the trimer with $\mathrm{DDQ}{ }^{18}$ cleanly in $88 \%$ yield at $70{ }^{\circ} \mathrm{C}$ in chlorobenzene for 2 hrs. It was interesting to note that both syn-64a and anti-64h underwent fully aromatization to form the functionalized [9]CPP $\mathbf{6 5}$ with three evenly spaced 5,8 dimethoxynaphth-1,4-diyl units (Scheme 1.21). This mild aromatization protocol with excellent 
yield is in contrast with Itami's oxidative aromatization of [9]CPP at higher temperature $\left(150^{\circ} \mathrm{C}\right)$ and longer time $(48 \mathrm{~h})$ in $24 \%$ yield..$^{29.34}$

Scheme 1.21. Synthesis of 65 and Its Energy-Minimized Structure hy DFT Calculations
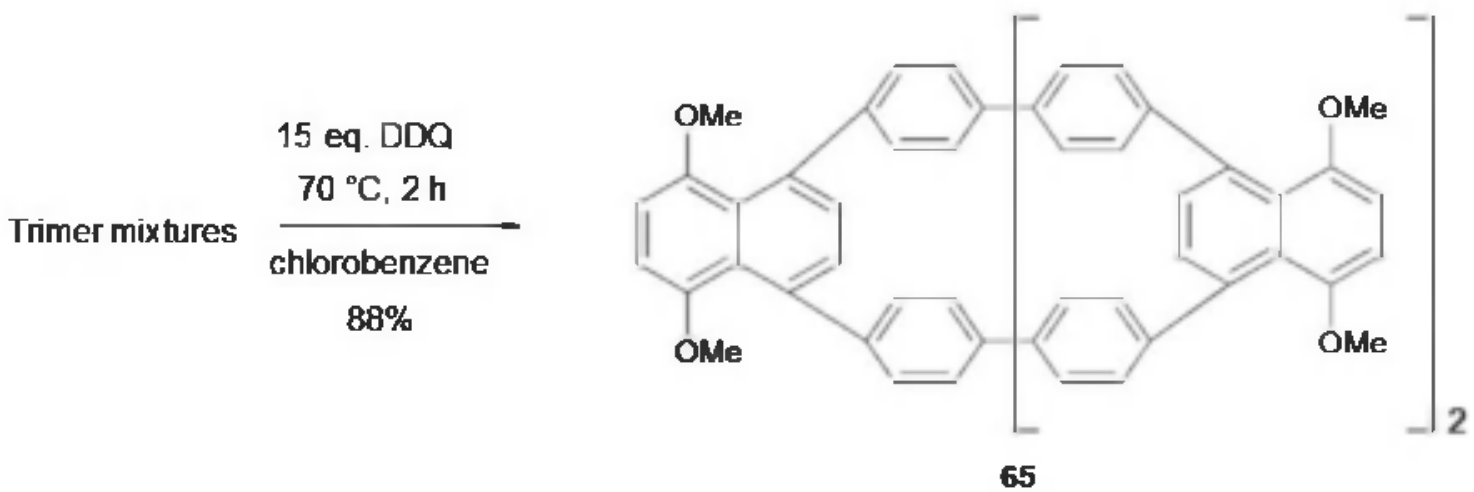

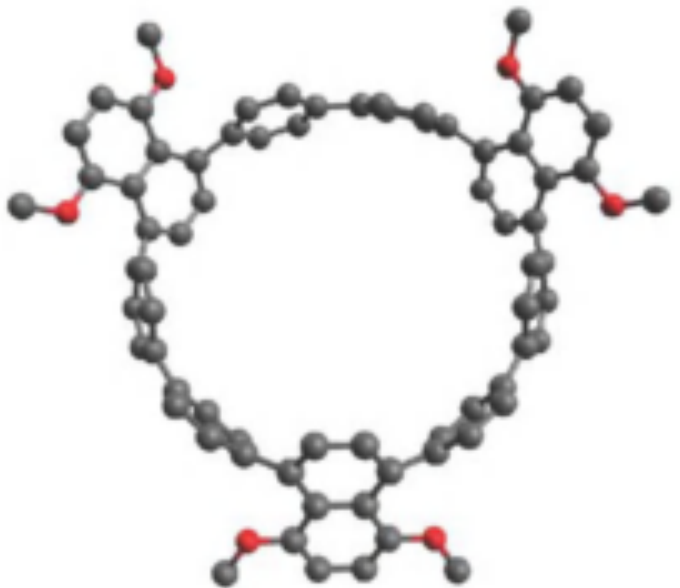

65

aerial view

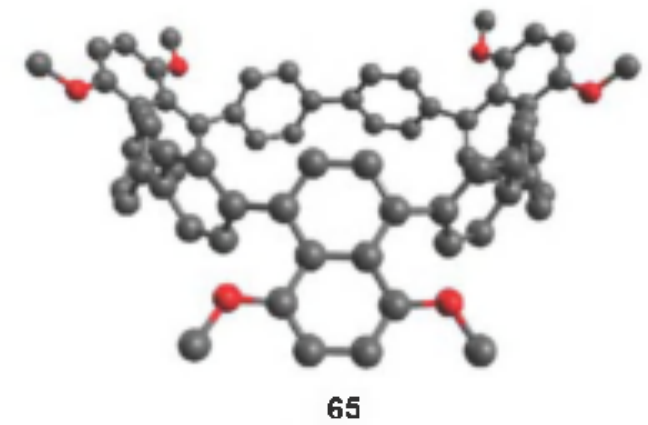

perspective view

We also conducted DFT calculations of the functionalized [9] CPP 65. The most stable conformer indicates that the 5,8-dimethoxynaphth-1,4-diyl units cant away from the inner plane of the [9]CPP circle at an angle of $133.8^{\circ}$ on average with two of the units tilting above the ring and the third tilting below the ring, which is similar to the X-ray structure confirmed by the crystal structure of the aromatized product of $\mathbf{6 2} .^{36}$ The three biphenyl moieties of $\mathbf{6 5}$ have three unique torsional angles of $19.5^{\circ}, 36.5^{\circ}$, and $-37.5^{\circ}$. The diameter of the ring is calculated to be 
12.4 $\AA$ as reported previously for the parent $[9] \mathrm{CPP} .{ }^{29_{a}}$ However, the NMR spectrum of $\mathbf{6 5}$ at $25^{\circ} \mathrm{C}$ showed only one set of signals, including one singlet for the methoxy groups, two singlets for the hydrigens on the naphthyl systems, and one set of two coupling doublets for the hydrogens on biphenyl segments. Conducting the NMR experiment at $-50{ }^{\circ} \mathrm{C}$ did not show any significant change with regard to the broadness of the signals. In conjunction with the DFT calculations, the observation of only one set of NMR signals for $\mathbf{6 5}$ suggests rapid arene rotations on the NMR time scales.

The UV-vis spectrum of 65 showed a major absorption maximum at $362 \mathrm{~nm}$ with a molecular absorption coefficient $(\varepsilon)$ of $5.2 \times 10^{4} \mathrm{~cm}^{-1} \mathrm{M}^{-1}$, which lay between those of the parent [9]CPP $(340 \mathrm{~nm})$ and [9]cyclo-1,4-naphthalene $(378 \mathrm{~nm})$. The fluorescence maximum $\left(\lambda_{\mathrm{em}}\right)$ was observed at $512 \mathrm{~nm}$, indicating a red shift compared to those of the parent [9]CPP $(494 \mathrm{~nm})$ and [9]cyclo-1,4-naphthalene $(491 \mathrm{~nm}){ }^{39}$

\subsection{Summary}

In summary, we have developed an efficient pathway of preparing functionalized [9]CPP bearing three evenly spaced 5,8-dimethoxynaphth-1,4-diyl units and macrocyclic dimer [6]CPP precursors. This pathway features a key step involving a Diels-Alder reaction to construct the $\mathrm{cis}$ L-shaped building block, nickel-mediated homocoupling to build macrocycles efficiently, and mild aromatization methods with DDQ. The high stereoselectivity and versatility of the Diels-Alder reaction between a variety of readily available dienes and dienophiles bearing diverse structural features make our synthetic pathway especially attractive for the synthesis of functionalized CPPs. ${ }^{40}$ Moreover, the presence of 5,8-dimethoxynaphth-1,4-diyl units in $\mathbf{6 5}$ affords additional handles for subsequent synthetic elaborations. ${ }^{4}$ 


\subsection{Reference}

(1) lijima, S. Nature 1991, 354, 56-58.

(2) Basu-Dutt, S.; Kumar, S. .J. Chem. Educ. 2012, 89, 22 I-229.

(3) Jorio, A.; Dresselhaus, G.; Dresselhaus, M. S. Carbon Nanotubes: Advanced Topics in the Synthesis, Structure, Properties, and Applications; Springer, 2008.

(4) Jariwala, D.; Sangwan, V. K.; Lauhon, L. J.; Marks, T. J.; Hersam, M. C. Chem. Soc. Rev. 2013, 42, 2824-2860.

(5) Segawa, Y.; Ito, H.; Itami, K. Nat. Rev. Mater. 2016, l, 15002-15017.

(6) Wang, H.; Wang, B.; Quek, X.; Wei, L.; Zhao, J.; Li, L.; Chan-Park, M.; Yang, Y.; Chen, Y. J. Am. Chem. Soc. 2010, 132, 16747-16749.

(7) He, M.; Chemov, A.; Fedotov, P.; Obraztsova, E.; Rikkinen, E.; Zhu, Z.; Sainio, J.; Jiang, H.; Nasibulin, A.; kauppinen, E.; Niemela, M.; Krause, A. Chem. Commun. 2011, 47, $1219-1221$

(8) Chiang, W.; Mohan Sankaran, R. Nature Mater, 2009, 8, 882-886.

(9) Hong, G.; Chen, Y.; Zhang, J.; Carhon 2012, 50, 2067-2082.

(10) Liu, H.; Nishide, D.; Tanaka, T.; kataura, H. Nature Commun. 2011, 2, 309-316.

(11) Tu, X.; Manohar, S.; Jagota, A.; Zheng, M. Nature 2009, 460, 250-253.

(12) Hersam, M. Nature Nanotechnol. 2008, 3, 387-394.

(13) Smalley, R.; Li, Y.; Moore, V.; Price, K.; Colorado, R.; Schmidt, H.; Hauge, R.; Barron, A.; Tour, J. J. Am. Chem. Soc. 2006, 128, 15824-15829.

(14) Yan, Y.; Feng, C.; Zhang, J.; Liu, Z. Nano Lett. 2009. 9, I673-1677. 
(15) Yang, Y.; Wang, D.; Zhang, Q.; Yang, J.; I.uo, D.; Xu, Z.; Wei, J.; Wang, J.; Xu, Z.;

Peng, F.; Li, X.; Li, R.; Li, Y.; Li, M.; Bai, X.; Ding, F.; Li, Y. Nature, 2014. 510, $522-$ 524.

(16) Yu, X.; Zhang, J.; Choi, W.; Choi, J.; Kim J.; Gan, L.; Liu, Z. Nano Lett. 2010, 10, $3343-$ 3349.

(17) Liu, B.; Liu, J.; Li, H.; Bhola, R.; Jackson, E.; Scott, L.; Page, A.; Irle, S.; Morokuma, K.; Zhou, C. Nano Lett. 2015, 15, 586-595.

(18) Valencia J.; Dienel T.; Grōning, O.; Shorubalko, 1.; Mueller, A.; Jansen, M.; Amsharov, K.; Ruffieux, P.; Fasel, R. Nature 2014, 512, 61-64.

(19) Omachi, H.; Nakayama, T.; Takahashi, E.; Segawa, Y.; Itami, K. Nat.Chem. 2013, 5, $572-576$.

(20) (a) Jasti, R.; Bertozzi, C. R. Chem. Phys. Leff. 2010, 494, 1-7. (b) Fort, E. H.; Donovan, P. M.; Scott, L. T. J. Am. Chem. Soc. 2009. 131, 16006-16007. (c) Steinberg, B. D.; Scott, L. T. Angew. Chem., Int. Ed. 2009, 48, 5400-5402. (d) Memer, B. L.; Dawe, L. N.;

Bodwell, G. J. Angew. Chem.. Int. Ed. 2009, 48, 5487-5491. (e) Bodwell, G. J. Nat. Nanotechnol. 2010, 5, 103-I04. (f) Fort, E. H.; Scott, L. T. Angew. Chem., Int. Ed. 2010, 49, 6626-6628. (g) Fort, E. H.; Scott, L. T. J. Mater. Chem. 2011, 21, 1373-1381. (h) Scott, L. T.; Jackson, E. A.; Zhang, Q.; Steinherg, B. D.; Bancu, M.; Li, B. .J. Am. Chem. Soc. 2012, 134, 107-110. (i) Schrettl, S.; Frauenrath, H. Angew. Chem.. Int. Ed. 2012, 51, 6569-657I. (j) Bunz, U. H. F.; Menning, S.; Martín, N. Angew. Chem., Int. Ed. 2012, 51, 7094-7101. (k) Merner, B. L.; Unikela, K. S.; Dawe, L. N.; Thompson, D. W.; Bodwell, G. J. Chem. Commun 2013, 49, 5930-5932. (1) Li, H.- B.; Page, A. J.; Irle, S.; 
Morokuma, K. J. Phys. Chem. Lett. 2013, 4, 3176-3180. (m) Sisto, T. J.; Zakharov, L. N.; White, B. M.; Jasti, R. Chem. Sci. DOI: 10.1039/c5sc04218f.

(2I) (a) Segawa, Y.; Fukazawa, A.; Matsuura, S.; Omachi, H.; Yamaguchi, S.; Irle, S.; Itami, K. Org Biomol. Chem. 2012, 10, 5979-5984. (b) Matsui, K.; Segawa, Y.; Itami, K. Org. Letl. 2012, 14, 1888- 189I. (c) Fujitsuka, M.; Cho, D. W.; Iwamoto, T.; Yamago, S.; Majima, T. Phys. Chem. Chem. Phys. 2012, 14, 14585-I4588. (d) Nishihara, T.; Segawa, Y.; Itami, K.; Kanemitsu, Y. J. Phys. Chem. Lett. 2012, 3, 3125-3128. (e) Camacho, C.; Niehaus, T. A.; Itami, K.; Irle, S. Chem. Sci. 2013, 4. I87-195. (f) Golder, M. R.; Wong, B. M.; Jasti, R. Chem. Sci. 2013, 4, 4285-429l. (g) Hines, D. A.; Darzi, E. R.; Jasti, R.; Kamat, P. V. J. Phys. Chem. A 2014, 118, I595-I600. (h) Fujitsuka, M.; Tojo, S.; Iwamoto, T.; Kayahara, E.; Yamago, S.; Majima, T. J. Phys. Chem. Letl. 2014, 5, 2302-2305. (i) Fuj itsuka, M.; Lu, C.; Iwamoto, T.; Kayahara, E.; Yamago, S.; Majima, T. J. Phys. Chem. A 2014, 1/8, 4527-4532. (j) Reddy, V. S.; Camacho, C.; Xia, J.; Jasti, R.; Irle, S. J. Chem. Theory Comput. 2014, 10, 4025-4036. (k) Toriumi, N.; Muranaka, A.; Kayahara, E.; Yamago, S.; Uchiyama, M. J. Am. Chem. Soc. 2015, 137, 82-85. (1) Chen, H.; Golder, M. R.; Wang, F.; Doom, S. K.; Jasti, R.; Tretiak, S.; Swan, A. K. J. Phys. Chem. C 2015, 119, 2879-2887. (m) Kuwabara, T.; Orii, J.; Segawa, Y.; Itami, K. Angew. Chem., Int. Ed. 2015, 54, 9646-9649. (n) Darzi, E. R.; Hirst, E. S.; Weber, C. D.; Zakharov, L. N.; Lonergan, M. C.; Jasti, R. ACS Cent. Sci. 2015, 1, 335-342. (o) Talipov, M. R.; Jasti, R.; Rathore, R. J. Am. Chem. Soc. 2015, 137, 14999-I5006. (p) Kayahara, E.; Kouyama, T.; Kato, T.; Yamago, S. J. Am. Chem. Soc. 2016, 138, 338-344. (q) Van Raden, J. M.; Darzi, E. R.; Zakharov, L. N.; Jasti, R. Org. Biomol Chem. DOl: $10.1039 / \mathrm{c} 6 \mathrm{ob} 00133 \mathrm{e}$ 
(22) (a) Jwamoto, T.; Watanabe, Y.; Sadahiro, T.; Haino, T.; Yamago, S. Angew. Chem., Int. Ed. 2011, 50, 8342-8344. (b) Xia, J.; Bacon, J. W.; Jasti, R. Chem. Sci. 2012, 3, 3018-3021. (c) Isobe, H.; Hitosugi, S.; Yamasaki, T.; lizuka, R. Chem. Sci. 2013, 4. 1293-1297. (d) Hitosugi, S.; lizuka, R.; Yamasaki, T.; Zhang, R.; Murata, Y.; Isobe, H. Org. Lett. 2013, 15, 3199-3201. (e) Iwamoto, T.; Watanabe, Y.; Takaya, H.; Haino, T.; Yasuda, N.; Yamago, S. Chem. - Eur. J. 2013, 19, I406I-14068. (f) Nakanishi, Y.; Omachi, H.; Matsuura, S.; Miyata, Y.; Kitaura, R.; Segawa, Y.; Itami, K.; Shinohara, H. Angew. Chem., Int. Ed. 2014, 53,3102-3106. (g) Alvarez, M. P.; Murtezo, P. M.; Iwamoto, T.; Qiu, L.; Kertesz, M.; Taravillo, M.; Baonza, V. G.; Navarrete, J. T. L.; Yamago, S.; Casado, J. Faraday Discuss. 2014, 173, 157-171. (h) Iwamoto, T.; Slanina, Z.; Mizorogi, N.; Guo, J.; Akasaka, T.; Nagase, S.; Takaya, H.; Yasuda, N.; Kato, T.;

Yamago, S. Chem - Eur. J. 2014, 20, I4403-14409. (i) Ueno, H.; Nishihara, T.; Segawa, Y.; Itami, K. Angew. Chem., Int. Ed. 2015, 54, 3707-371 J. (j) Hitosugi, S.; Ohkubo, K.; Kawashima, Y.; Matsuno, T.; Kamata, S.; Nakamura, K.; Kono, H.; Sato, S.; Fukuzumi, S.; Isobe, H. Chem. - Asian J. 2015, 10, 2404-2410.

(23) Sakamoto, H.; Fjimori, T.; Li, X.; Kaneko, K.; Kan, K.; Ozaki, N.; Hijikata, Y.; Irle, S.; Itami, K. Chem. Sci. 2016, DOI:10.1039/C6SC00092D

(24) (a) Fujitsuka, M.; Iwamoto, T.; Kayahara, E.; Yamago, S.; Majima, T. ChemPhysChem 2013. 14. 1570-1572. (b) Kubota, N.; Segawa, Y.; Itami, K. J. Am. Chem. Soc. 2015, 137. 1356-136I. (c) Peña-Alvarez, M.; Qiu, L.; Taravillo, M.; Baonza, V. G.; Delgado, M. C. R.; Yamago, S.; Jasti, R.; Navarrete, J. T. L.; Casado, J.; Kertesz, M. Phys. Chem. Chem. Phys. DOI: 10.1039/c5cp05500h. (d) Kayahara, E.; Kouyama, T.; Kato, T.; Yamago, S.; J. Am. Chem. Soc. 2016, 138, 338-344. 
(25) (a) Segawa, Y.; Omachi, H.; Itami, K. Org. Lett. 2010, 12, 2262-2265. (b) Iwamoto, T.; Watanabe, Y.; Sakamoto, Y.; Suzuki, T.; Yamago, S. J. Am. Chem. Soc. 2011, 133, 8354-8361. (c) Segawa, Y.; Yagi, A.; Ito, H.; Itami, K. Org. Lett. 2016, 18, 1430-1433.

(26) (a) Hirst, E. S.; Jasti, R. J. Org. Chem. 2012, 77, 10473-10478. (b) Omachi, H.; Segawa, Y.; Itami, K. Acc. Chem. Res. 2012, 45, 1378- 1389. (c) Itami, K. Pure Appl. Chem. 2012, 84, 907-916. (d) Yamago, S.; Kayahara, E.; Iwamoto, T. Chem. Rec. 2014, 14, 84-100. (e) Tran-Van, A.-F.; Wegner, H. A. Beilstein J. Nanotechnol. 2014, 5, 1320-1333. (f) Golder, M. R.; Jasti, R. Acc. Chem. Res. 2015, 48, 557-566. (g) Lewis, S. E. Chem. Soc. Rev. 2015, 44, 222I-2304. (h) Darzi, E. R.; Jasti, R. Chem. Soc. Rev. 2015, 44, 640I-6410. (i) Golling, F. E.; Osella, S.; Quemheim, M.; Wagner, M.; Beljonne, D.; Mülen, K. Chem. Sci. 2015, 6, 7072-7078. (j) Segawa, Y,; Yagi, A.; Matsui, K.; Itami, K. Angew. Chem., Int. Ed. 2016, 55, 2-25. (k) Hammer, B. A. G.; Müllen, K. Chem. Rev. 2016, 116, 2103-2140.

(27) Parekh, V.; Guha, P. J. Indian Chem. Soc. 1934, 11, 95-100.

(28) Friederich, R.; Nieger, M.; Vogtle, F. Chem.Ber. 1993, 126, I723-1732.

(29) (a) Jasti, R.; Bhattacharjee, J.; Neaton, J. B.; Bertozzi, C. R. J. Am. Chem. Soc. 2008, 130, 17646-17647. (b) Takaba, H.; Omachi, H.; Yamamoto, Y.; Bouffard, J.; Itami, K. Angew. Chem., Int. Ed. 2009, 48, 6112-61।6. (c) Yamago, S.; Watanabe, Y.; Iwamoto, T. Angew. Chem., Int. Ed. 2010, 49, 757-759. (d) Omachi, H.; Matsuura, S.; Segawa, Y.; Itami, K. Angew. Chem., Int. Ed. 2010, 49, 10202-10205. (e) Segawa, Y.; Miyamoto, S.; Omachi, H.; Matsuura, S.; Šenel, P.; Sasamori, T.; Tokitoh, N.; Itami, K. Angew. Chem.. Int. Ed. 2011, 50, 3244-3248. (f) Sisto, T. J.; Golder, M. R.; Hirst, E. S.; Jasti, R. J. Am. Chem. Soc. 2011, 133, 15800-15802. (g) Xia, J.; Jasti, R. Angew. Chem., Int. Ed. 2012, 
5l, 2474-2476. (h) Nishiuchi, T.; Feng, X.; Enkelmann, V.; Wagner, M.; Mullen, K.

Chem. - Eur. J. 2012, 18, 16621-I6625. (i) Kayahara, E.; lwamoto, T.; Suzuki, T.;

Yamago, S. Chem. Lett. 2013, 42,621-623. (j) Batson, J. M.; Swager, T. M. Synlett 2013, 24, 2545-2549. (k) Golling, F. E.; Quemheim, M.; Wagner, M.; Nishiuchi, T.; Müllen, K. Angew. Chem., Int. Ed. 2014. 53, 1525-1528. (I) Kayahara, E.; Patel, V. K.; Yamago, S. J. Am. Chem. Soc. 2014, 136, 2284-2287. (m) Evans, P. J.; Darzi, E. R.; Jasti, R. Nat. Chem. 2014, 6, 404-408. (n) Tran-Van, A.-F.; Huxol, E.; Basler, J. M.; Neuburger, M.; Adjizian, J.-J.; Ewels, C. P.; Wegner, H. A. Org. Lett. 2014, 16, I594-I597. (o) Huang, C.; Huang, Y.; Akhmedov, N. G.; Popp, B. V.; Petersen, J. L.; Wang, K. K. Org. Lett. 2014, 16 , 2672-2675. (p) Mysliwiec, D.; Kondratowicz, M.; Lis, T.; Chmielewski, P. J.; Stępien, M. J. Am. Chem. Soc. 2015, 137, 1643-1649. (q) Patel, V. K.; Kayahara, E.; Yamago, S. Chem. - Eur. J. 2015, 21, 5742-5749. (r) Sarkar, P.; Sato, S.; Kamata, S.; Matsuno, T.; Isobe, H. Chem. Lett. 2015, 44, 1581-1583. (s) Miyauchi, Y.; Johmoto, K.; Yasuda, N.; Uekusa, H.; Fujii, S.; Kiguchi, M.; Ito, H.; Itami, K.; Tanaka, K. Chem.-Eur. J. 2015, 21, 18900-18904. (t) Liu, Y.-Y.; Lin, J.-Y.; Bo, Y.-F.; Xie, L.- H.; Yi, M.-D.; Zhang, X.-W.; Zhang, H.-M.; Loh, T.-P.; Huang, W. Org. Lett. 2016, 18, I72-175.

(30) Davis, M. C.; Groshens, T. J. Synth. Commun. 2011, 41, 206-208.

(31) Huang, Y. 2013, MA thesis. West Virginia Universtiy.

(32) Darzi, E.; Sisto, T.; Jasti, R. J. Org. Chem. 2012, 77, 6624-6628.

(33) Xia, J.; Bacon J.; Jasti, R. Chem. Sci. 2012, 3, 3018-3021.

(34) Ishii, Y.; Nakanishi, Y.; Omachi, H.; Matsura, S.; Matsui, k.; Shinohara, H.; Segawa, Y.; Itami, K. Chem. Sci. 2012, 3, 2340-2345. 
(35) Sibbel, F.; Matsui, K.; Segawa, Y.; Studer, A.; Itami, K. Chem. Commun. 2014, 50, 954956.

(36) Segawa, Y.; Senel, P.; Matsuura, S.; Omachi, H.; Itami, K. Chem. Lett. 2011, 40, 423425.

(37) Kayahara, E.; Patel V.; Xia, J.; Jasti, R.; Yamago, S. Synlent 2015, 26, 1615-1619.

(38) Li, S.; Huang, C.; Thakellapalli, H.; Farajidizaji, B.; Popp, B.; Petersen, J.; Wang, K. Org. Lett. 2016, 18, 2268-2271.

(39) Marshall, J.; Faehl, L.; McDaniel, C.; Ledford, N.; ,J. Am. Chem. Soc. 1977, 99, 32 I-325.

(40) Carreño, M.; García-Cerrada, S.; Urbano, A. Chem. Eur. J. 2003, 9, $4118-4131$.

(4I) Yagi, A.; Segawa, Y.; Itami, K. J. Am. Chem. Soc. 2012, 134, 2962-2965.

(42) (a) Coleman, R. S.; Walczak, M. C. Org. Lett. 2005, 7, 2289-2291. (b) Takahashi, T.;

Tsai, F. -Y.; Li, Y. Chem. Letr. 1999, 1173- I174. (c) Ananikov, V. P.; Kashin, A. S.;

Hazipov, O. V.; Beletskaya, 1. P.; Starikova, Z. A. Synlett 2011, 2021-2024. (d)

Molander, G. A.; Zinke, P. W. Organometallics 1986, 5, 216I-2162.

(43) Ali, M. H.; Niedbalski, M.; Bohnert, G.; Rryant, D. Synth. Commun. 2006, 36, $175 \mathrm{I}-1759$. 


\section{Chapter 2. Syntheses and Structures of Bent and Fused Cycloparaphenylene Precursors}

\subsection{Introduction}

In his pioneering paper, Itami conceptually demonstrated the first diameter-controlled synthesis of CNTs from cycloparapheneylenes as templates without any metal catalysts. ' It is considered a great advance toward controlled synthesis of CNTs with uniform structure and defined diameter using a bottom-up synthesis, growth-on template strategy and exemplify the synthetic potential of CPP-based templates for such purpose. In the end, he mentioned that for perfect diameter and sidewall structure control, robust substrates and modified equipment are needed. Toward this end, a number of substituted or $\pi$-extended CPP derivatives have been prepared, such as Itami's [9]cyclo- 1,4-naphthalene, ${ }^{2}$ Jasti's tetraphenyl substituted [ 12]CPP, Swager's naphthalene-spaced [12]CPP, ${ }^{4}$ Müllen's 3D $\pi$-extended polyphenylene cylinders, ${ }^{5}$ Wegner's substituted [8]CPP, ${ }^{6}$ and Yamago "s [4]cyclo-2,7-pyrenylene. ${ }^{7}$ However, if one reexamines the growth mechanism of CNTs, other issues need to be resolved before the bottomup synthetic strategy becomes practical in producing CNTs.

In addition to the hypothesis by Irle and Morokuma from DFT calculations that CNTs growth can be induced by ethynyl radical addition on CPP substrates, ${ }^{8}$ there are two popular mechanisms on the CNT growth. The first was put forward by Scott, suggesting that repetitive Diels-Alder reactions between the substrate's bay area and acetylene or acetylene equivalents, followed by aromatization by release of $\mathrm{H}_{2}$ at that thermal conditions could regenerate bay area for the next Diels-Alder reactions for tube growth (Scheme 2.1). ${ }^{9-13}$ Clearly this method will only elongate the substrate, keeping the growing CNTs with the same structure as the substrate. However it is well known and experimentally established that CPP substrates will not undergo 
Diels-Alder reaction with dienophiles in the bay area, ${ }^{14,15,16}$ similar to the observation that biphenyl or terphenyl will not take part in any Diels-Alder reaction. One possible solution to this problem, suggested by Scott, was to use longer PAH segments, such as hisanthene or perylene with suitable dienophiles, ${ }^{9-13}$ which were proved to be reactive by lasti's group. ${ }^{14-13} \mathrm{~A}$ straightforward method to incorporate the longer PAH segments in those aryl-substituted CPPs is through Scholl reaction. However, halogenation, ${ }^{16}$ structural rearrangement $^{17}$ including I,2phenyl shift ${ }^{16,18}$ or strain release ring opening ${ }^{16}$ in these strained macrocycles is always a concern. It is still a challenge to synthesize these larger $\pi$-extended CPP derivatives ${ }^{19,20}$ and no reports about larger fully $\pi$-extended CPPs such as 66 have been published. Also no experimental conditions to demonstrate the growth of CNTs through this mechanism have been reported.

Scheme 2.1. Growth Mechanism Through Diels-Alder Reaction and Rearomatization
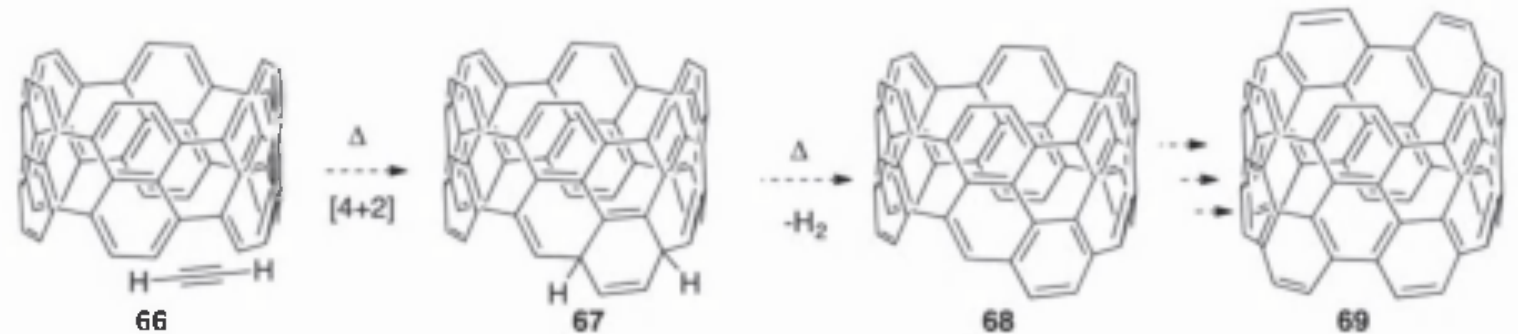

68

69

A second carbon nanotuhes growth mechanism with CPPs as the growth templates was proposed by ltami through CPP radicals under chemical vapor deposition (CVD) method with carbon sources. ${ }^{\prime}$ At high temperature, CPP radicals, generated from homolytic $\mathrm{C}-\mathrm{H}$ hond cleavage, annulated with unsaturated $\mathrm{C} 2$ species from ethanol as the carbon source to produce CNTs. Because the bond dissociation energies of aryl $\mathrm{C}-\mathrm{H}$ bond $(\mathrm{I} 13 \mathrm{kcal} / \mathrm{mol})$ and aryl C-C bond ( $114 \mathrm{kcal} / \mathrm{mol}$ ) are almost the same, it is likely that at high temperature both aryl C-H bond cleavage and aryl $\mathrm{C}-\mathrm{C}$ bond cleavage will take place, thus destroying the $\mathrm{CPP}$ template. This 
may account for the formation of oligophenylenes, multi-wall CNTs, low yield of the process, and the imperfect diameter distribution of the resulting CNTs. However if the template is longer with fully $\pi$-extended CPP such as $\mathbf{6 6}$, even though one layer of the CPP is broken, the whole template is not totally destroyed and there is still opportunity for recombination. From both aspects of growth mechanisms, Jasti proposed that the $\pi$-extended CPP may be important for generating CNTs in high yield by using CVD method. ${ }^{16}$ The simplest of the $\pi$-extended CPPs would be CPP dimers. So far there are only two reports of the synthesis of CPP dimers. ${ }^{21.22}$ The first synthesis of arene-bridged CPP dimer was reported by Jasti group in 2012 (Scheme 2.2). ${ }^{2 \mid}$ The synthetic sequence used the cis-bromo-containing diiodide 72, prepared from two consecutive nucleophilic additions of lithium reagent to a bromo-containing protected dieneone, as a key intermediate. The coupling partner, diboronic ester 73, was obtained in large quantity during the exploration of the synthesis of $[6] \mathrm{CPP}^{23}$ and the large-scale synthesis of [8] and $[10] \mathrm{CPPS}^{24}$ by the oxidative dearomatization/addition sequence. Suzuki-Miyaura crosscoupling reactions between the bromo-containing diiodide 72 and 73 effectively delivered the bromo-containing macrocyclic [8]CPP precursor $\mathbf{7 4}$ in 30\% yield. The subsequent SuzukiMiyaura cross-coupling reaction between $\mathbf{7 4}$ and 1,4-bisborylbenzene, 1,5-diborylnaphthalene afforded the arene-bridged CPP dimer precursors 75 and $\mathbf{7 6}$, respectively. Lastly reductive aromatization by sodium nanphthalenide furmished the arene-bridged CPP dimers, which according to DFT calculations adopted the cis conformation in the gas phase and in solution, but trans conformation in solid phase. To form the nanotube-like structures, additional carboncarbon bond connection to form the fixed cis structure will be needed. 


\section{Scheme 2.2. Synthesis of Arene-Brideged |8|CPP Dimer}

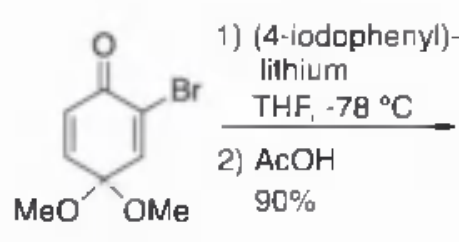

70

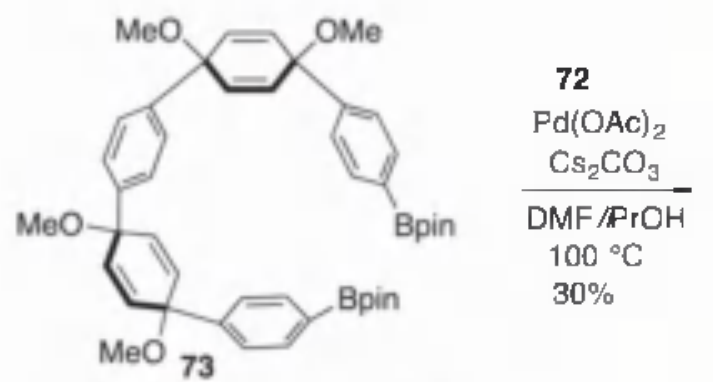

$\mathrm{r}, 16 \mathrm{~h} 78 \%$

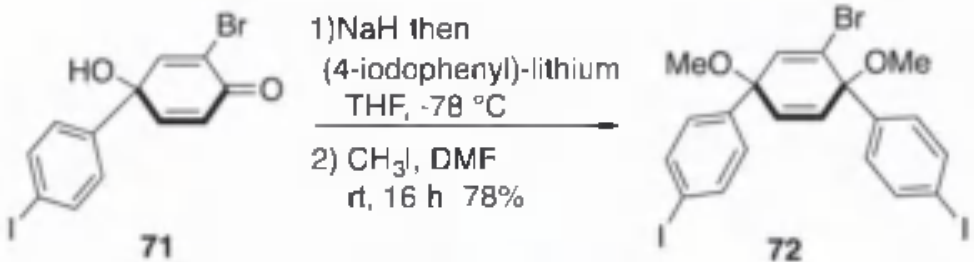

72
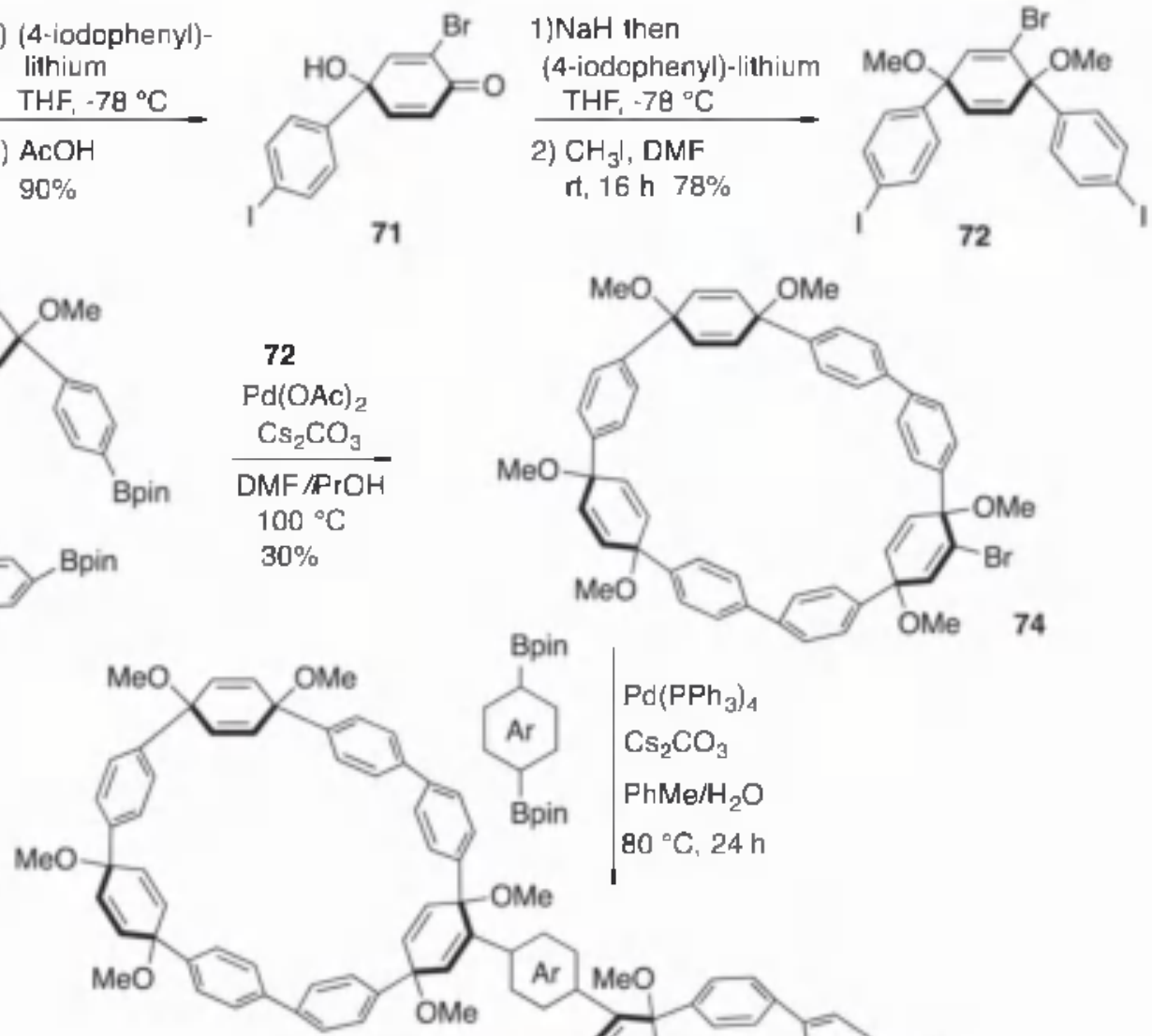

Bpin

$\mathrm{Pd}\left(\mathrm{PPh}_{3}\right)_{4}$

$\mathrm{Cs}_{2} \mathrm{CO}_{3}$

$\mathrm{PhMe} / \mathrm{H}_{2} \mathrm{O}$

$80^{\circ} \mathrm{C}, 24 \mathrm{~h}$

Ar $=1,4$-benzene, $77,75 \%$

1) sodium naphthalenide

THF, $-78^{\circ} \mathrm{C}, 2 \mathrm{~h}$

1,5-naphlhalene, $\mathbf{7 8 , 4 8 \%}$

2) $I_{2}$
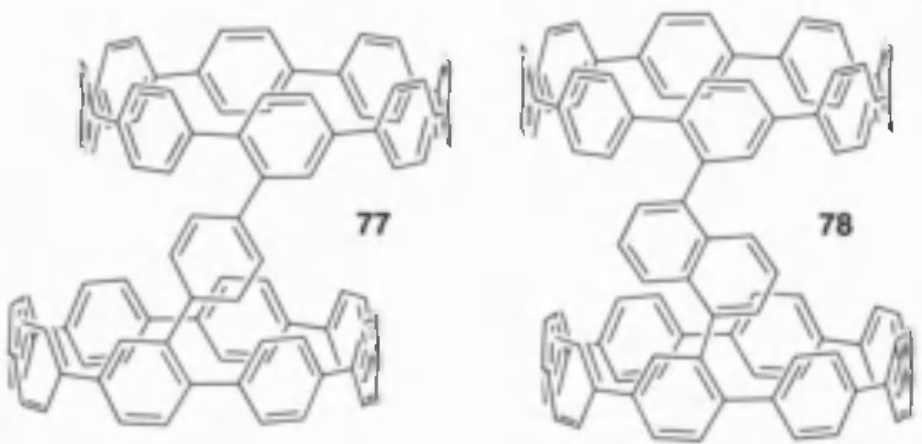

Itami group later published the synthesis of the directly connected [10]CPP dimer 84

through a single $\mathrm{C}-\mathrm{C}$ bond by the nickel-mediated homocoupling through a chloro-containing [10]CPP 83, which was produced from the acid-mediated oxidative aromatization of chloro- 
containing [10]CPP precursor $\mathbf{8 2} .^{22}$ Triangle-shaped chloro-containing $\mathbf{8 2}$ was prepared by a 9+1-type cyclization between $\mathbf{8 1}$ and the C-shaped $\mathbf{8 0}$ (Scheme 2.3).

Scheme 2.3. Synthesis of Directly-Connected |10|CPP Dimer
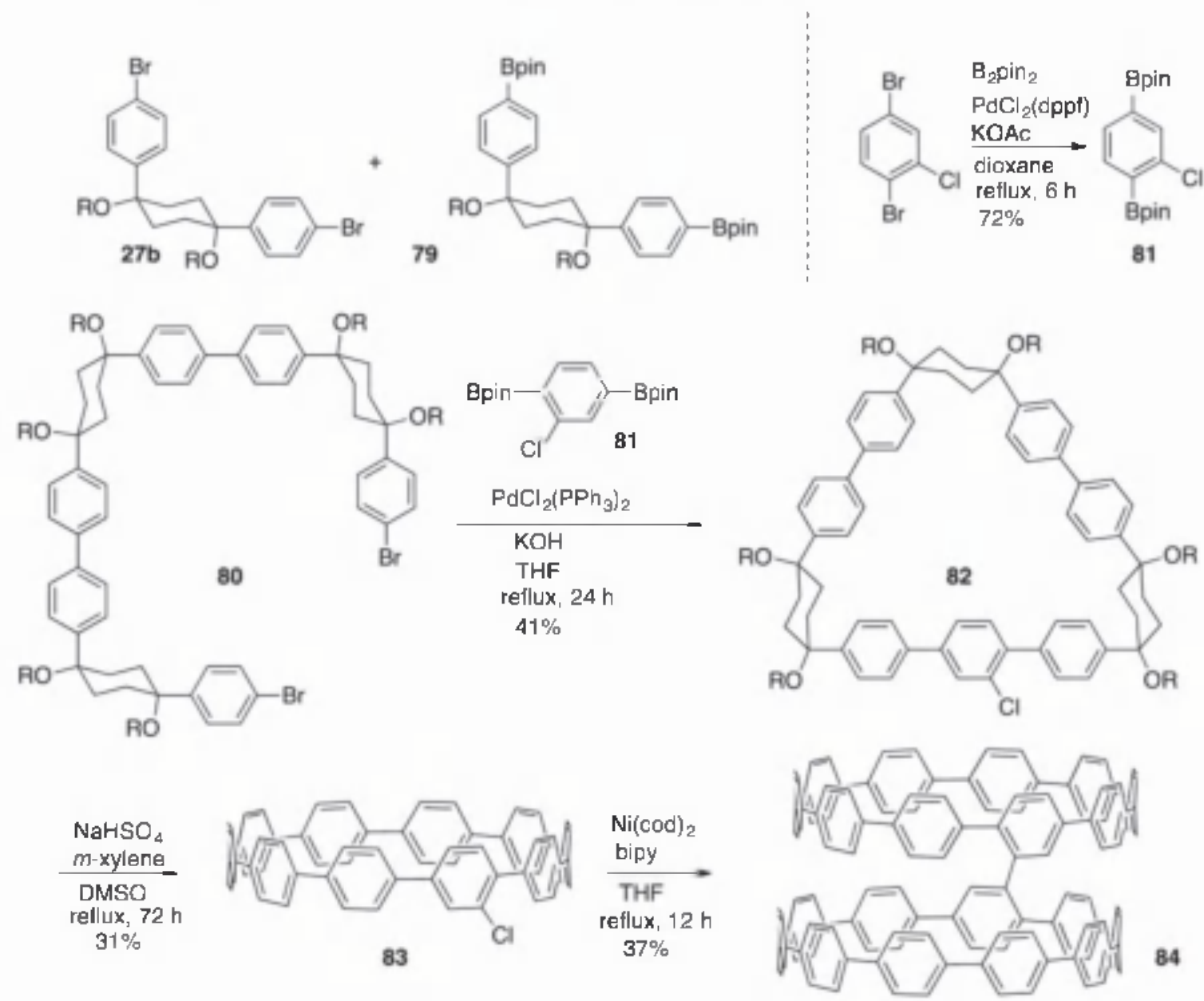

Compound 80 was prepared from two I -shaped building blocks $27 \mathbf{b}$ and 79 using well established procedures for the selective synthesis of [9]-[11] and [13]CPP. ${ }^{25}$ The chlorocontaining linear unit $\mathbf{8 1}$ was synthesized by the palladium-catalyzed borylations of 1,4-dihromo2-chlorobenzene. ${ }^{26}$ They showed that the stable conformation of $\mathbf{8 4}$ is the structure in which one CPP unit sits directly above the other CPP unit. 


\subsection{Our synthesis of a nanotube-like dimer, trimer and related bent and fused carbon}

nanohoops

As these CPP dimers are connected through a single $\mathrm{C}$ - $\mathrm{C}$ bond, there are always problems of free rotation and confonmational conversion. So they are not considered as ideal templates for CNT growth according to the analysis from above two growth mechanisms. However, our functionalized CPPs and precursors can be easily oxidized to quinone forms and act as good dienophiles. If the second Diels-Alder reaction takes place on the same side as the macrocylces, then after a second nickel-mediated homocoupling reaction, the nanotube-like structures could be constructed, which can serve as potential templates for the CNTs growth.

\subsubsection{First route to construct nanotube-like structures}

The idea that the second Diels-Alder reaction may proceed to form an adduct with all four 4-bromophenyl groups cis to each other was first observed by Yiwei Huang ${ }^{27}$ during our $^{2}$ pursuit to find an efficient Lewis acid-catalyzed Diels-Alder reaction. In compound $\mathbf{8 5}$, all the four 4-bromophenyl groups are located on the same side (Scheme 2.4). The structure of $\mathbf{8 5}$ was established by $\mathrm{X}$-ray structure analysis. However the $\mathrm{Ni}(\mathrm{cod})_{2}$-mediated homocoupling reaction of 85 did not produce any discernable products. 
Scheme 2.4. Synthesis of Tetrabromide Ketone 85
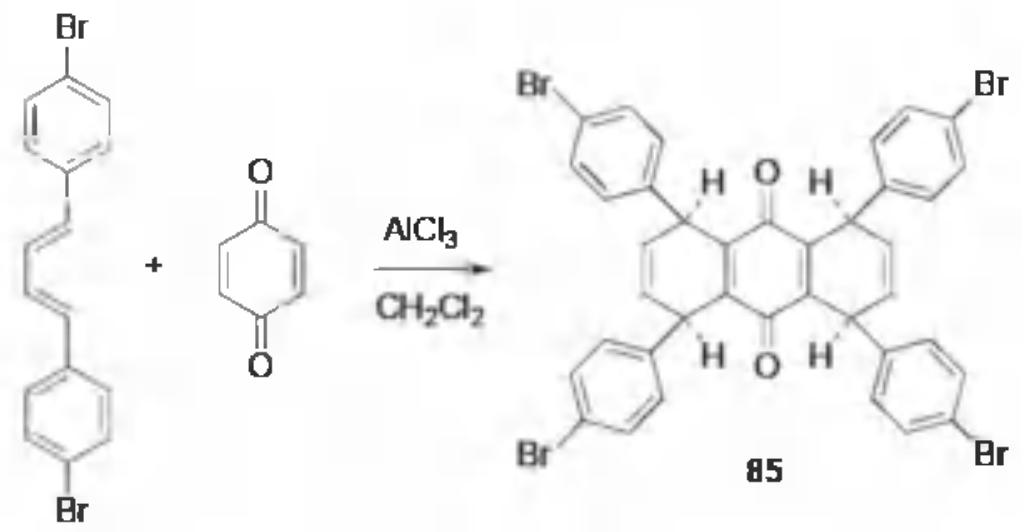

\subsubsection{Second route to construct nanotube-like structures}

Scheme 2.5. Attempted Synthesis of Nanotube-like Structures from Cyclic Dimer

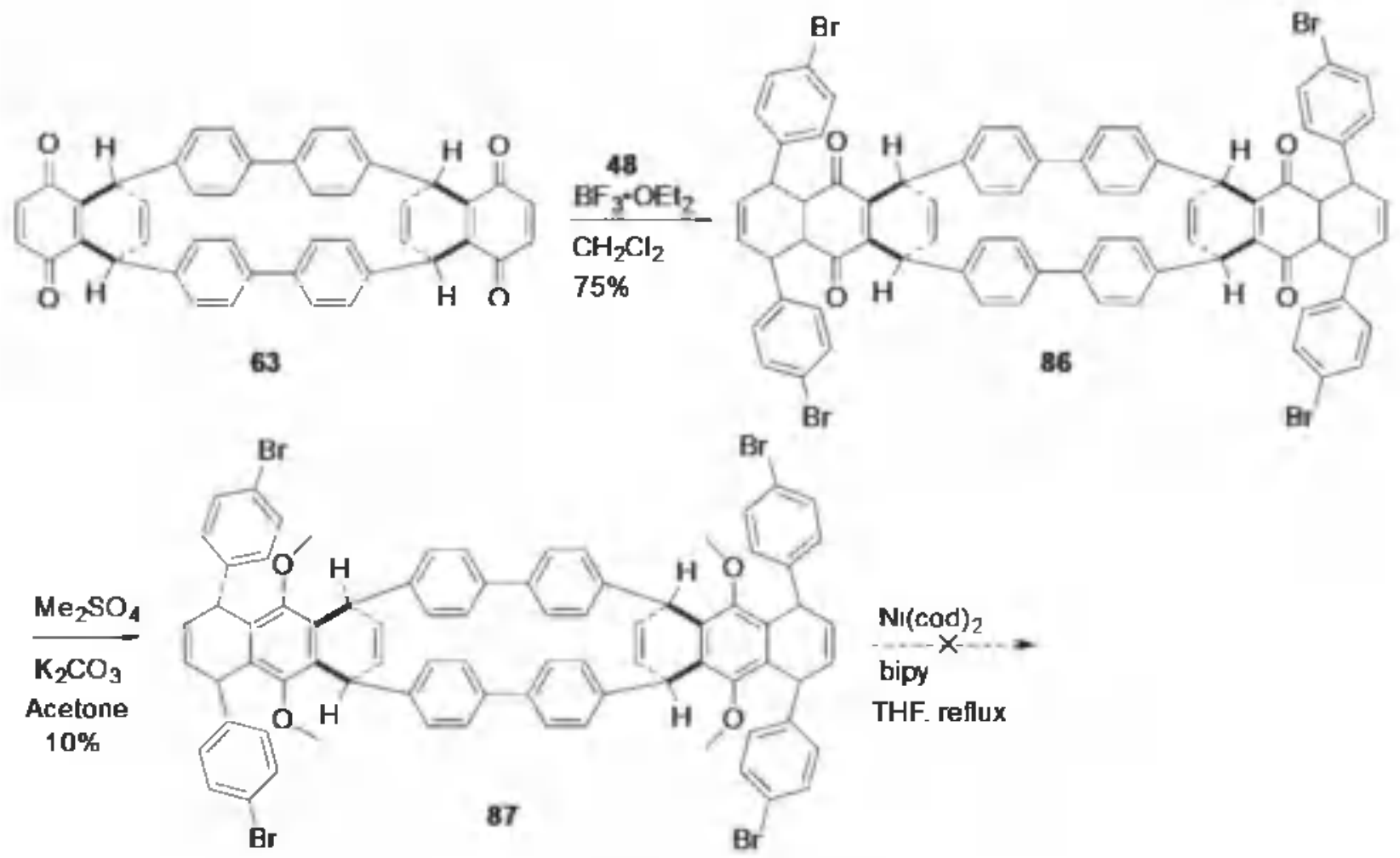

With the idea that the second Diels-Alder reaction may form the all cis product, we explored the possibility of using the nxidized cyclic dimer $\mathbf{6 3}$ for the second Diels-Alder reaction with diene 48 promoted by $\mathrm{BF}_{3}{ }^{\circ} \mathrm{OEt}_{2}$ (Scheme 2.5 ). The second Diels-Alder reaction 
went smoothly to afford the DA adduct $\mathbf{8 6}$ in $75 \%$ yield. However, the following methylation reaction did not proceed well, producing the methylated product 87 in only $10 \%$ yield. We suspect that $\mathbf{8 6}$ may have the anti structure, which is more difficult for methylation hecause the resulting methoxyl groups suffer from more severe steric interactions with either the 4bromophenyl groups or the biphenyl bridges. Further indication of the anti structure came from the fact that no intramolecular homocoupling product from the $\mathrm{Ni}(\mathrm{cod})_{2}$-mediated homocoupling reaction was obtained.

\subsubsection{Third route to construct nanotube-like structures}

We then tumed our attention to investigating how the second Diels-Alder reaction took place. As we increase the amount of diene $\mathbf{4 8}$ in the Diels-Alder reaction with I,4benzoquinone in the presence of $\mathrm{BF}_{3} \bullet \mathrm{OEt}_{2}$, no double DA adducts were observed. However, when I,4-hydrobenzoquinone $\mathbf{8 8}$ was oxidized to I,4-benzoquinone $\mathbf{8 9}$, it underwent the second Diels-Alder reaction to deliver, after methylation, the syn-tetrabromide $\mathbf{9 0}$ as the major product in $68 \%$ yield and the anti-tetrabromide 91 as the minor product (syn-tetrabromide 90 :antitetrabromde $91=8: 1$ ) (Scheme 2.6). The structure of anti-tetrabromide $\mathbf{9 1}$ was unambiguously established by $\mathrm{X}$-ray structure analysis. We also grew crystals of syn-tetrabromide $\mathbf{9 0}$ from dichloromethane/hexanes, although an X-ray structure of 91 was obtained (Figure 2.1), the quality of the X-ray structure of $\mathbf{9 0}$ was poor. Fortunately, we were able to obtain crystals suitable for X-ray structure analysis, when syn-tetrabromide $\mathbf{9 0}$ was converted to the corresponding syn-tetraiodide 92 (Scheme 2.7). The X-ray structure of 92 (Figure 2.2) clearly indicates that the four 4-iodophenyl groups are on the same side exclusively and are in close 
proximity to each other, resulting in upfield shift of the 'H NMR signals of phenyl protons to less than $7.0 \mathrm{ppm}$.

Scheme 2.6. Synthesis of $s y n$-Tetrabromide 70<smiles>O=C1C=CC(=O)C(/C=C/C=C\c2ccc(Br)cc2)=C1</smiles>

$\mathrm{Br}$.

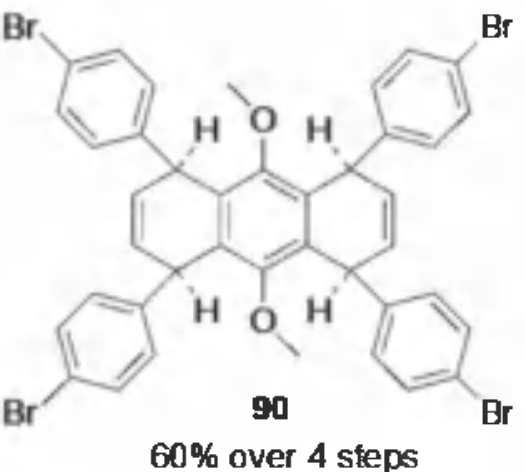

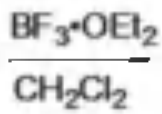<smiles>Oc1ccc(O)c2c1[C@H](c1ccc(Br)cc1)C=C[C@H]2c1ccc(Br)cc1</smiles>

88

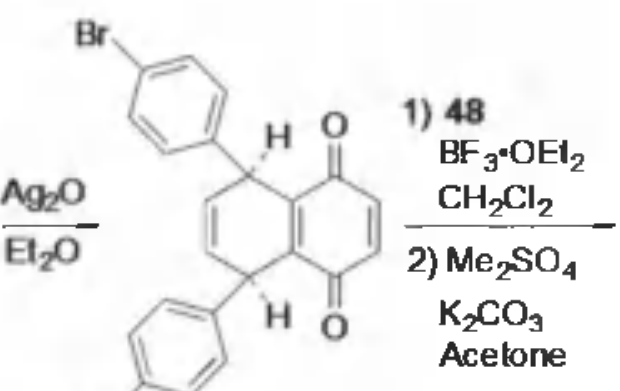

89

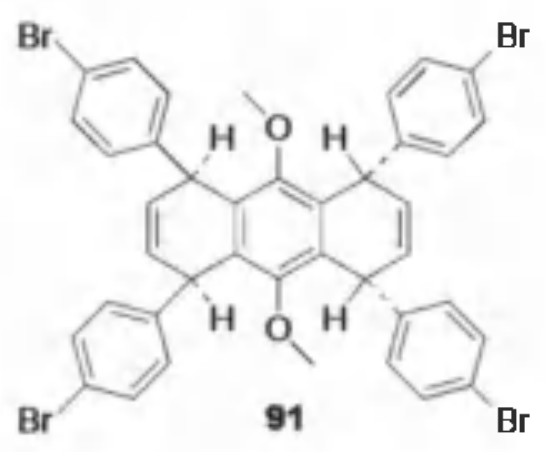




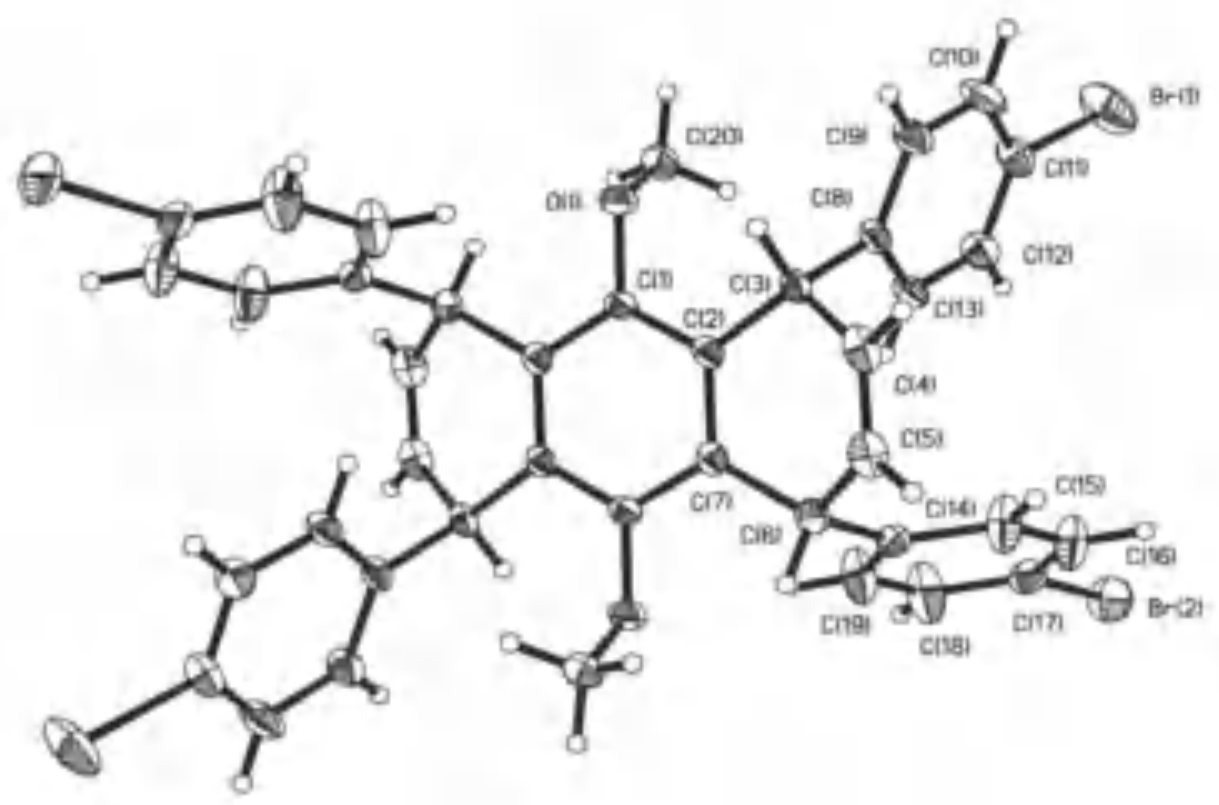

Figure 2.1. X-ray crystal structure of the anti-tetrabromide 91

Scheme 2.7. Synthesis of $s y n$-Tetraiodide 92

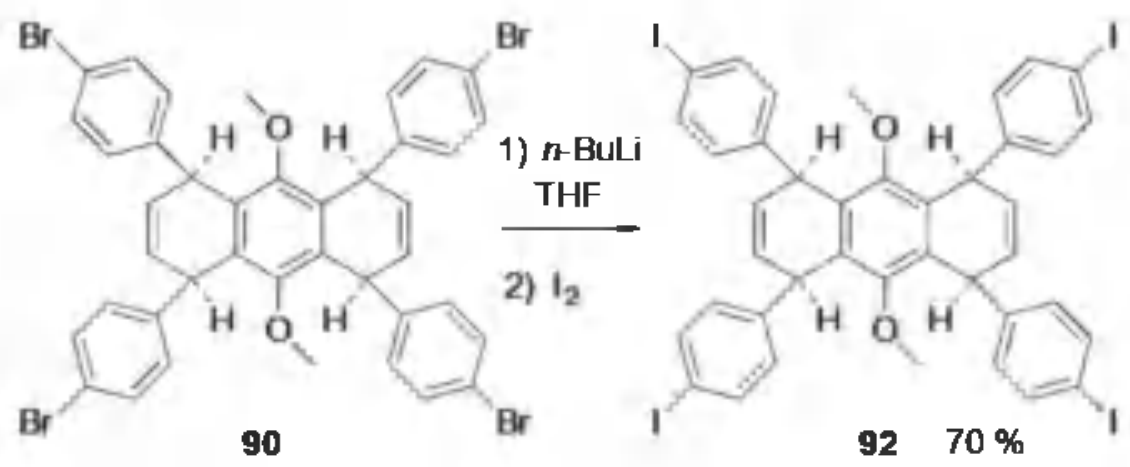

In comparison the 'H NMR signals of phenyl protons in the anti-tetrabromide 91 occur in the normal aromatic region higher than $7.0 \mathrm{ppm}$. These characteristic features of proton signals in the 'H NMR spectra allow easy differentiation of the syn adduct from the anti adduct. 


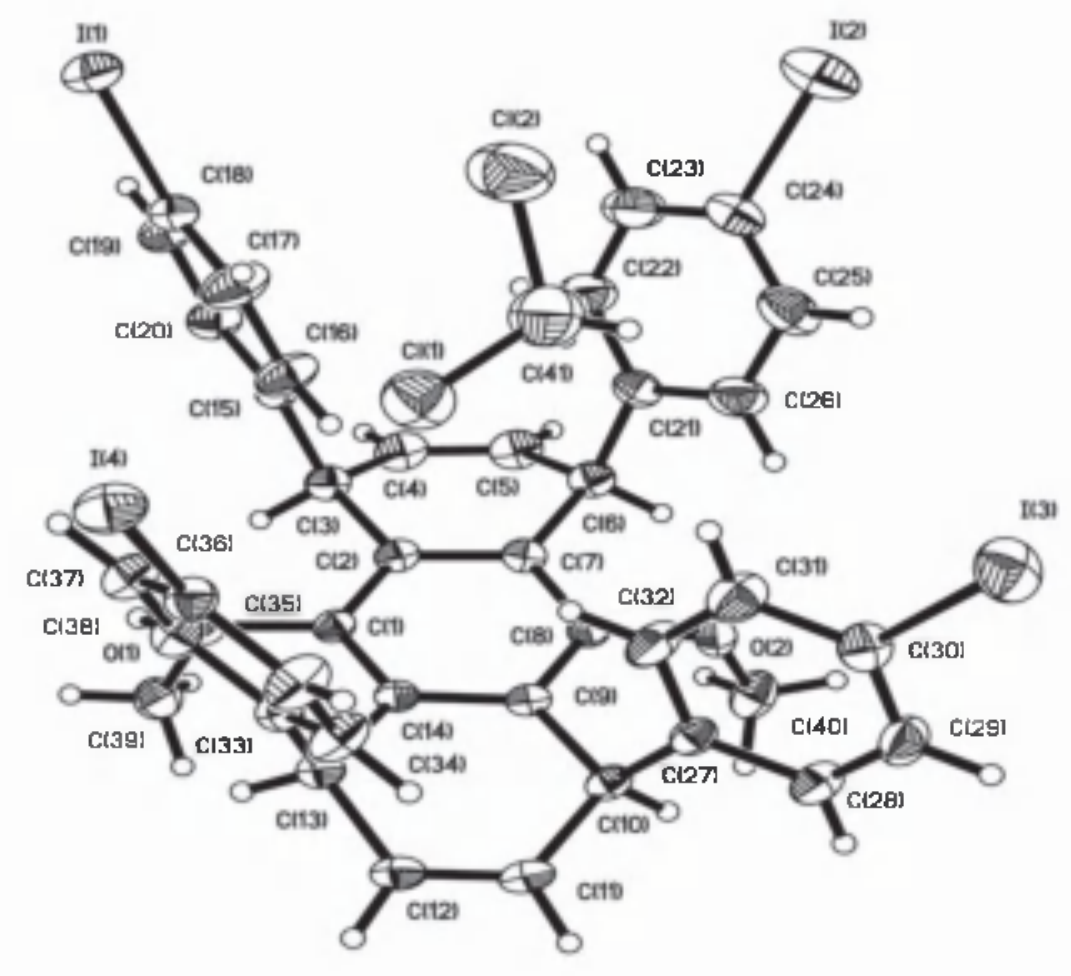

Figure 2.2. X-ray crystal structure of the syn-tetraiodide $\mathbf{9 2}$

With large quantity of the syn-tetrabromide $\mathbf{9 0}$ at hand, we applied the same protocol of the $\mathrm{Ni}(\operatorname{cod})_{2}$-mediated homocoupling reaction of syn-tetrabromide $\mathbf{9 0}$ in the presence of 2,2 'bipyridyl (Schemes 2.8 and 2.9). We were able to isolate the macrocyclic dimers $\mathbf{7 3}$ and $\mathbf{7 4}$ in $5 \%$ and $1 \%$ yield, respectively, when the reaction was run at I $\mathrm{mM}$ concentration. The structure of $\mathbf{9 3}$ was established by X-ray structure analysis (Figure 2.3). It is very interesting to note that dimer 93 has a nanotube-like structure in which two [6]CPP precursors are fused through two 1,4-dimethoxybenzene units. The structure of $\mathbf{9 3}$ represents the first example of fusing two nanohoops together with the holes facing each other in a nanotube-like arrangement. The dimer 94 contains a bent [12]CPP precursor which is fused within itself with two 1,4- 
dimethoxybenzene units. Dimer $\mathbf{9 3}$ and $\mathbf{9 4}$ display similar chemical shifts and splitting patterns as one-layer dimer syn-56a, one upfield AB pattern for the aromatic protons, one downfield pseudo quartet with equal intensity for the vinylic protons, and a downfield "hump" for allylic protons. The high-resolution MS spectra also indicate that 93 and 94 are dimers.

\section{Scheme 2.8. Synthesis of Macrocyclic Dimers 93 and 94 at Low Concentration}
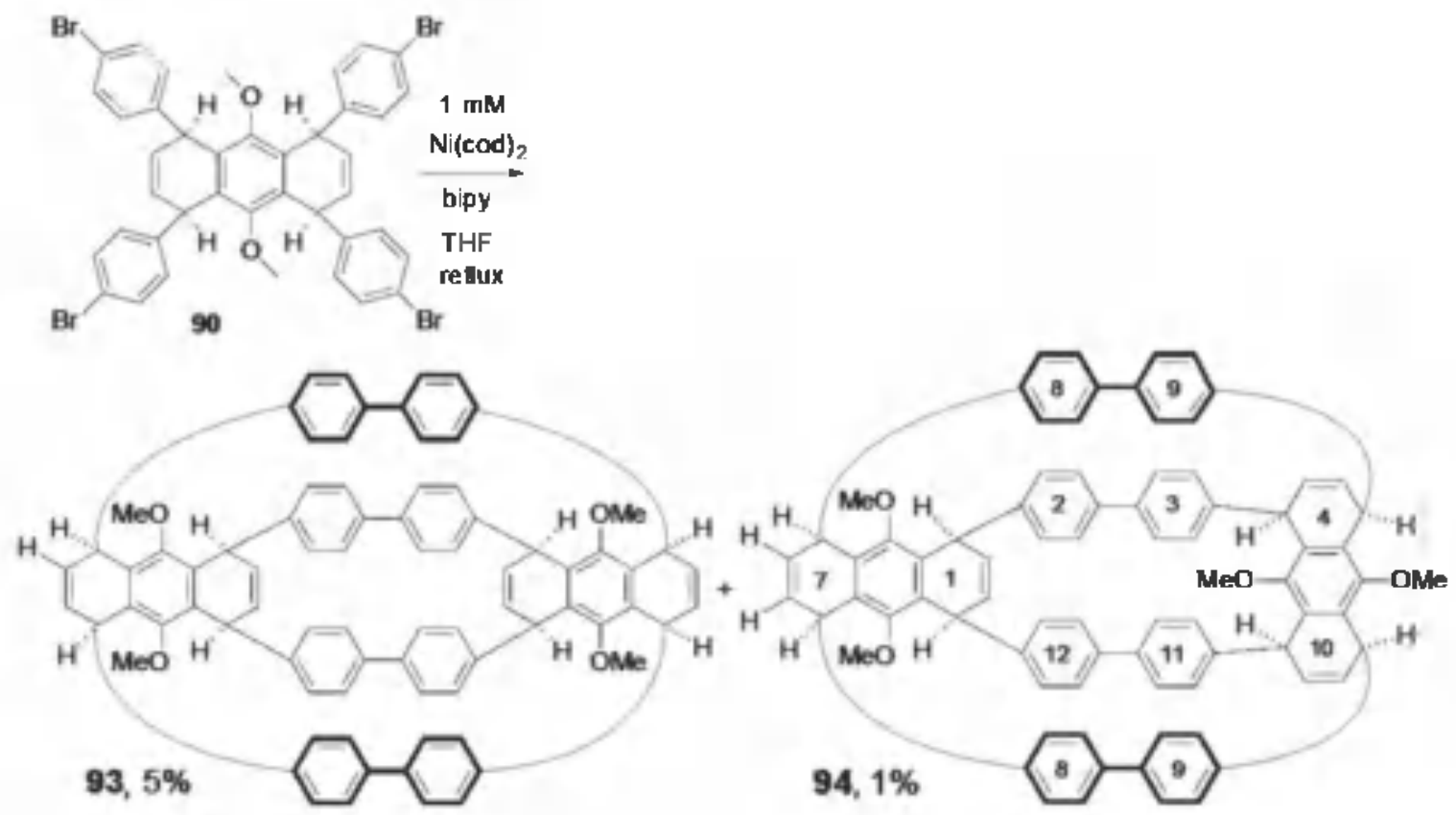

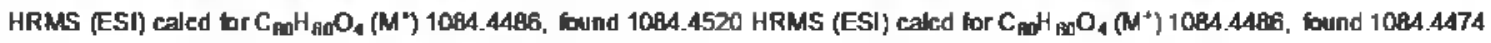




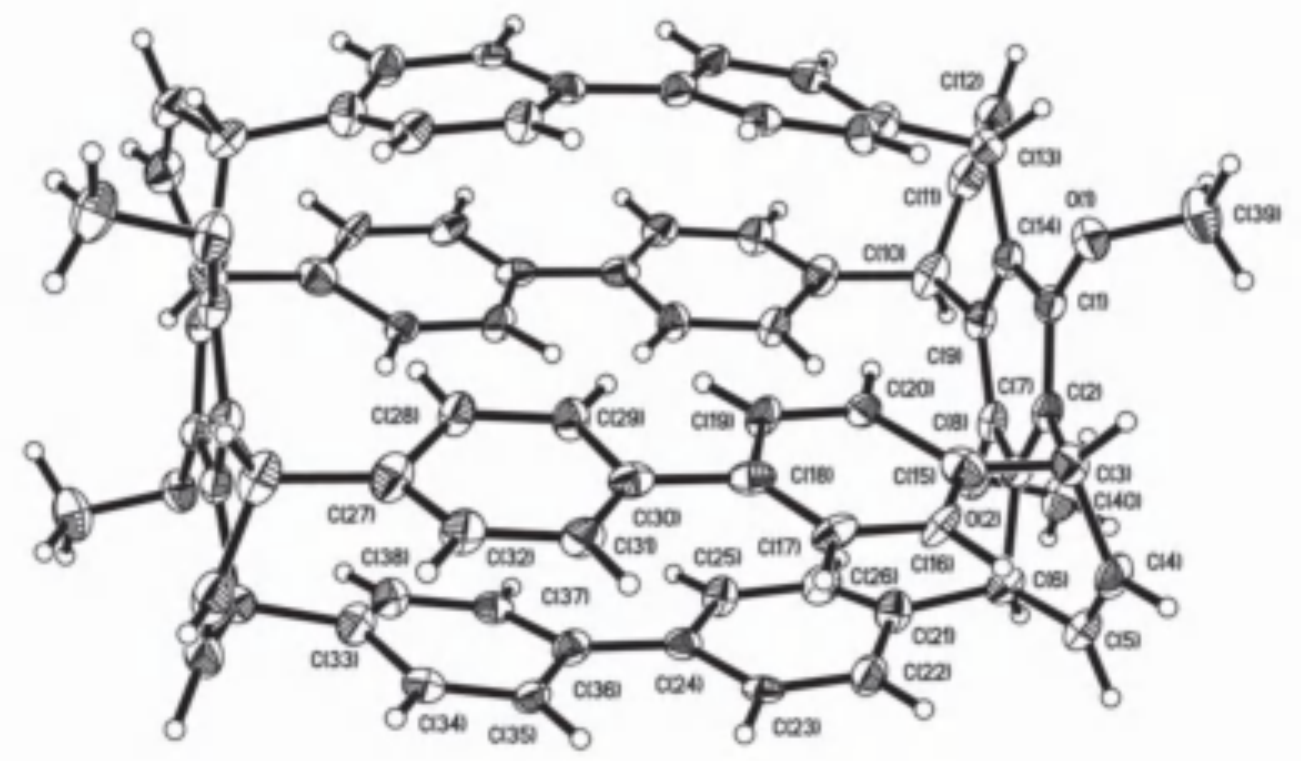

Figure 2.3. X-ray crystal structure of fused nanohoops 93

In addition, we were also able to purify and identify three $(95 a, 95 b$, and $95 c)$ of the four possible trimers by NMR and high-resolution MS techniques when the homocoupling reaction were carried out at $50 \mathrm{mM}$ concentration. It is worth noting that 95a contains two fused [9]CPP precursors in a nanotube-like structure, $\mathbf{9 5} \mathbf{h}$ contains a hent $[18] \mathrm{CPP}$ precursor macrocyclic ring, and 95c contains fused [12]CPP and [6]CPP precursors (Scheme 2.9). 
Scheme 2.9. Synthesis of Macrocyclic Trimers 95a, 95b, and 95c at Higher Concentration
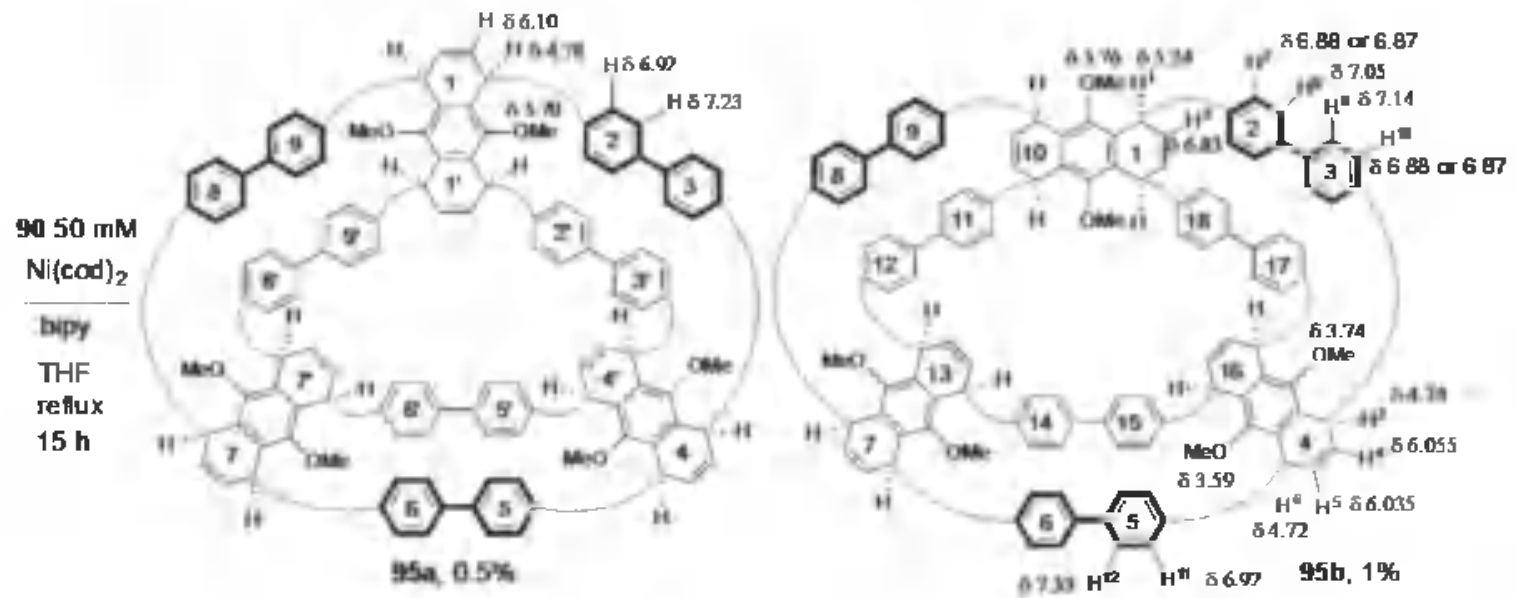

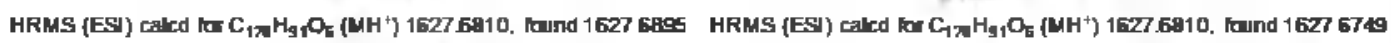

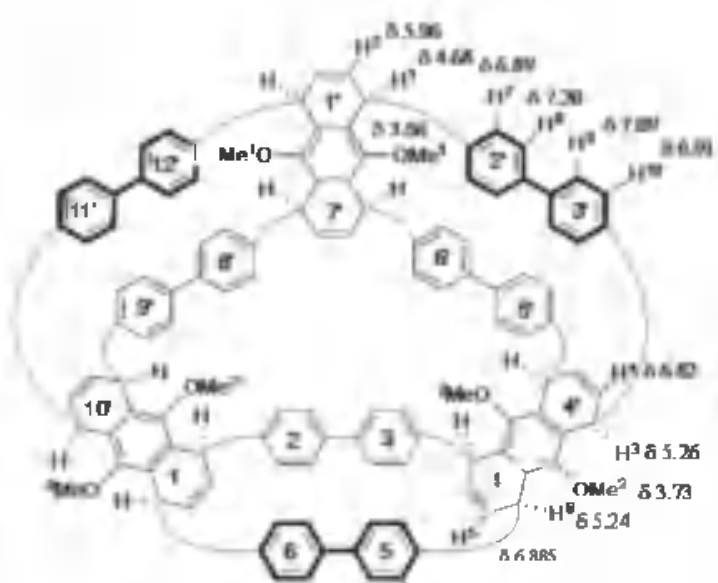

95c, $1 \%$

5680.1062

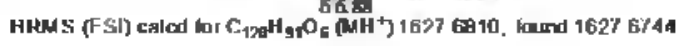

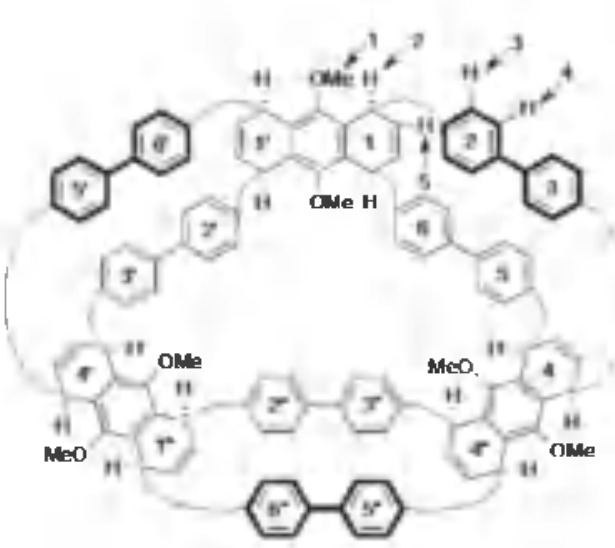

95id

On the basis of the NMR analysis, we were also able to differentiate the three trimers

from the ${ }^{~} \mathrm{H}$ NMR spectra. It is easiest to discern 95a, which has a $D_{3 \mathrm{~h}}$ symmetry and shows only one AB pattern for the aromatic protons, a set of two doublets coupled to each other for the vinylic protons and allylic protons, and a singlet for the methoxy protons. The most obvinus difference between $\mathbf{9 5 b}$ and $\mathbf{9 5}$ c is that $\mathbf{9 5 b}$ exhibits three singlets of equal intensity for the three different types of methoxy groups, whereas 95 exhibits two singlets in 2:1 ratio for the two different types of methoxy groups. In addition $\mathbf{9 5 b}$ has one set of vinylic protons and allylic protons that show a pseudo quartet with equal intensity and a "hump" respectively, but 95c has two sets of those spin systems. Moreover, 95h has an ABXY spin system in which $\mathrm{H}^{3}, \mathrm{H}^{4}, \mathrm{H}^{3}$, 
$\mathrm{H}^{6}$ are chemical nonequivalent and four distinct signals were observed, whereas $95 \mathrm{c}$ does not. Unfortunately we were not able to isolate the trimer 95d, which would be expected to show upfield shifted aromatic signals as observed in the spectrum of $\mathbf{9 3}$.

\subsection{Structural elucidation of nanotube-like dimer 93}

Before the X-ray structure became available for 93, we undertook the development of a stepwise synthesis of $\mathbf{9 3}$ to allow the elucidation of its structure. First we prepared the dibromide diboronic ester 97 that has a coupling partner on each side. It was anticipated that the SuzukiMiyaura cross-coupling reactions of two molecules of $\mathbf{9 7}$ could lead to dimer $\mathbf{9 3}$. The Diels-Alder reaction between dibromide 89 and diboryl diene 96, which was prepared by the palladium-catalyzed borylation of diene $\mathbf{4 8}$ in excellent yield with Buchwald's catalyst system, ${ }^{28}$ afforded, after methylation, the syn-dibromide diboronic ester $\mathbf{9 7}$ in $55 \%$ yield.

Scheme 2.10. First Attempt to Synthesize Fused Nanohoops 93

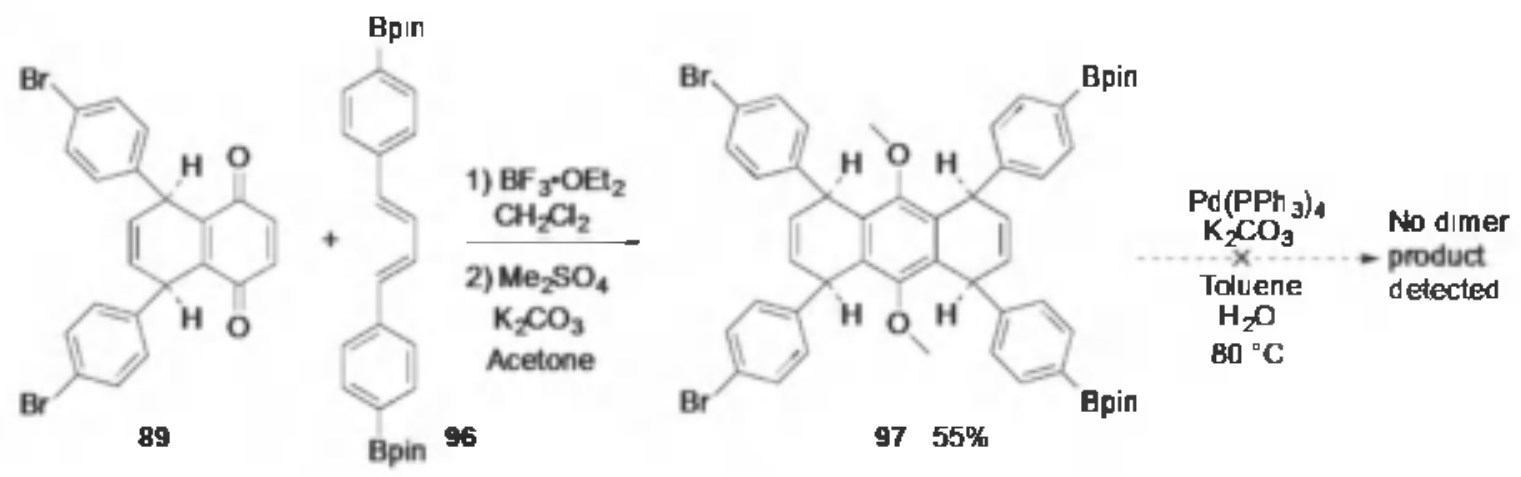

However, the subsequent Suzuki-Miyaura cross-coupling reaction between two molecules of 97 did not produce the desired dimer 93 (Scheme 2.10), as we did not observe any upfield-shifted signals around 6.4-6.8 ppm.

Then we switched to another strategy, with a stepwise synthetic sequence for $\mathbf{9 3}$ by constructing one ring first followed by an intramolecular ring formation for the second ring. It is 
well known aryltrimethylsilanes can act as masked aryl halides, ${ }^{27,28}$ and they do not interfere with the nickel-mediated homocoupling reaction. So we started with the synthesis of the trimethylsilyl (TMS)-protected diene 101 (Scheme 2.11).

\section{Scheme 2.11. Synthesis of TMS-Protected Diene 101}

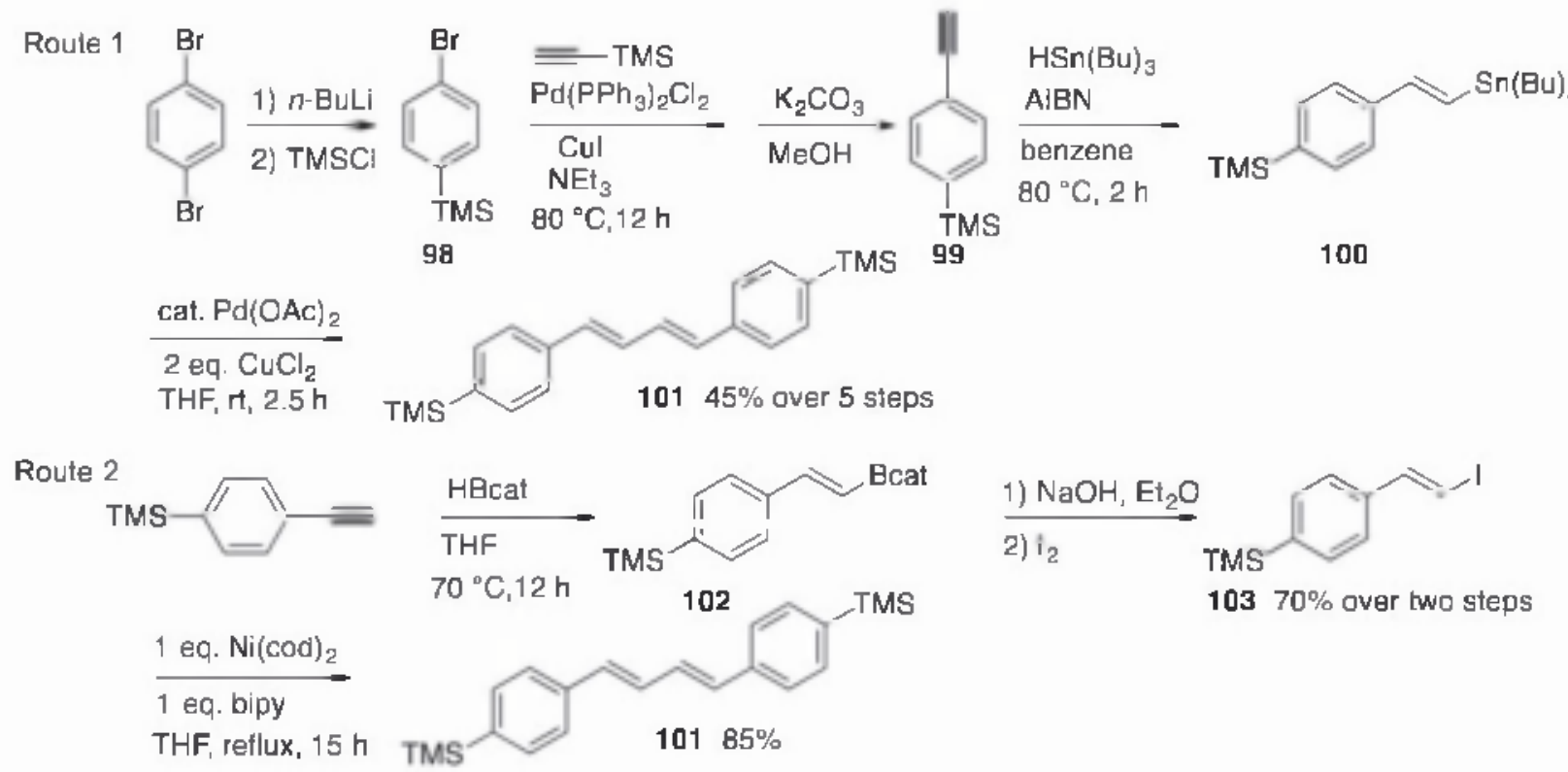

We tried two routes to make TMS-protected diene 101. The first route made use of the palladium catalyzed homocoupling reaction of vinylic stannane $\mathbf{1 0 0}$ assisted by copper, ${ }^{29}$ which was prepared from 2,2'-azobis(2-methylpropionitrile) (AIBN)-mediated alkyne hydrostannylation under air with alkyne $99 .{ }^{30}$ The terminal alkyne 99 was prepared from monolithiation of $\mathbf{1 , 4 -}$ dibromobenzene, silylation with TMSCl, Sonogashira coupling with (trimethylsilyl)ethyne, and desilylation of the terminal acetylenic silane. However vinylic stannane $\mathbf{1 0 0}$ was not stable on a silica gel column and was easily hydrolyzed.

We then tried another route starting with hydroboration of terminal alkyne $\mathbf{9 9}$, followed hy base-induced iodination afforded alkenyl iodide $\mathbf{1 0 3},{ }^{31}$ which underwent the $\mathrm{Ni}(\operatorname{cod})_{2}$ mediated homocoupling reaction to produce the desired TMS-protected diene $\mathbf{1 0 1}$. 
With TMS-protected diene $\mathbf{1 0 1}$ in hand, we performed the Diels-Alder reaction between quinone dibromide 89 and diene 101 using $\mathrm{AlCl}_{3}$ as the catalyst instead of $\mathrm{BF}_{3} \cdot \mathrm{OEt}_{2}$ to avoid any desilylation induced by fluoride, followed by methylation. This one-pot sequence successfully provided the desired syn-TMS-protected dibromide 104 in 55\% yield (Scheme 2.12). The $\mathrm{Ni}(\operatorname{cod})_{2}$-mediated homocoupling reaction of $\mathbf{1 0 4}$ at low concentration produced cyclic dimers syn-105 in 30\% yield and anti-106 in 30\% yield. Both 105 and 106 were treated with $\mathrm{ICl}$ to form the corresponding iodides, which were treated with $\mathrm{Ni}(\operatorname{cod})_{2}$ for homocoupling reactions at low conceniration. As can be expected only 105 produced 93 in $8 \%$ yield from 104 . The ${ }^{\prime} \mathrm{H}$ NMR spectrum of $\mathbf{9 3}$ from this procedure matches exactly with that of $\mathbf{9 3}$ obtained from tetrabromide 90. This stepwise synthetic sequence further confirmed the structure of $\mathbf{9 3}$ apart from its $\mathrm{X}$-ray structure.

\subsection{Structural elucidation of nanotube-like trimer 95a}

The structure of 95a was further elucidated by a similar stepwise synthetic pathway developed for structural clarification of $\mathbf{9 3}$. We designed two ways for preparing the key intermediates, syn- and anti-macrocyclic trimers 110 and 111. The first step of the stepwise pathway involved selective Suzuki-Miyaura cross-coupling reactions between TMS-protected diboronic ester 107 and TMS-protected monochloroindide 108 to produce 109, followed by the intramolecular $\mathrm{Ni}(\mathrm{cod})_{2}$-mediated homocoupling reaction of $\mathbf{1 0 9}$ to form syn-trimer $\mathbf{1 1 0}$ and anti-trimer 111 (Scheme 2.13). 
Scheme 2.12. Elucidation of Structure of Fused Nanohoops 93

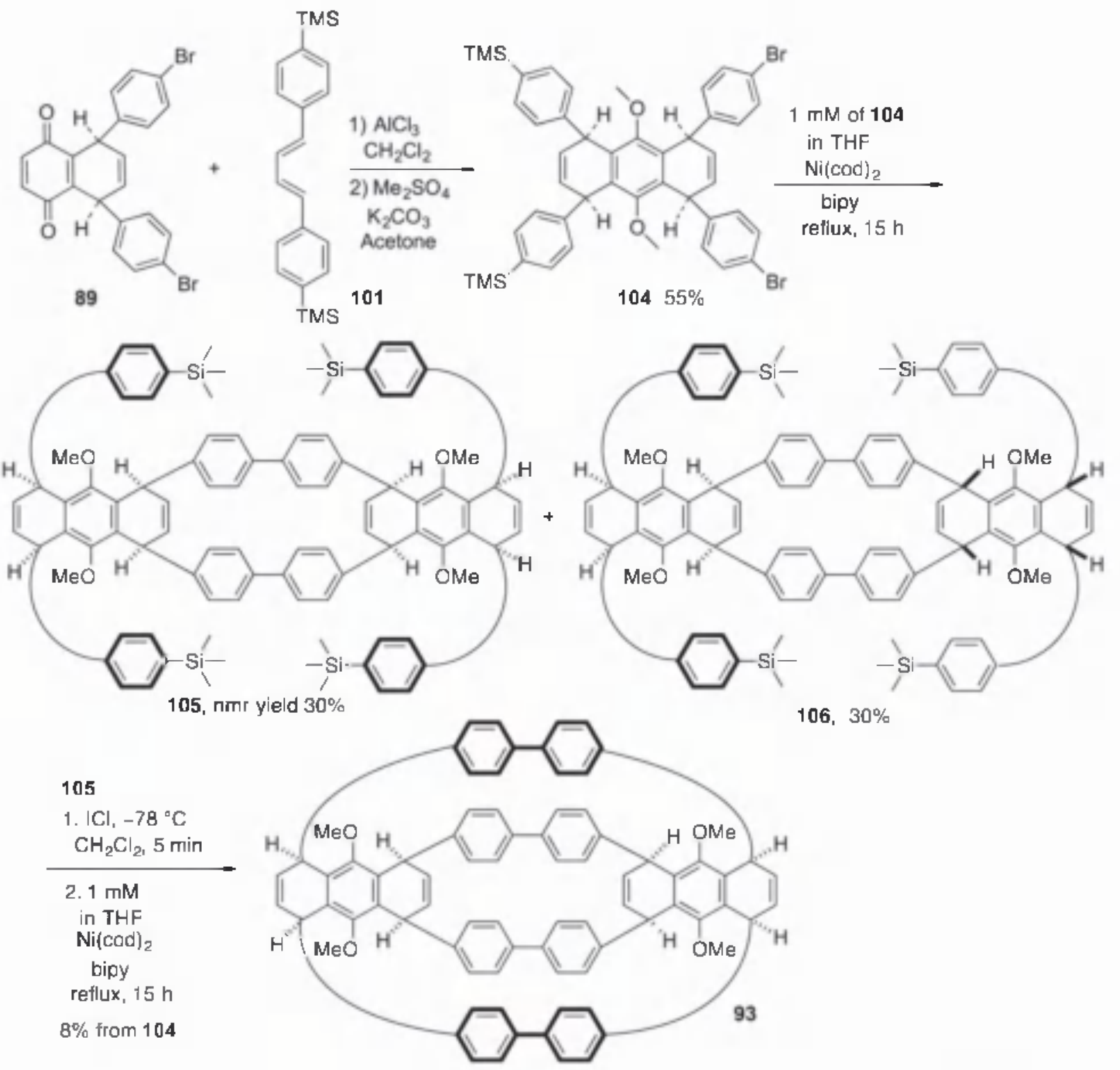

Compound 107 was prepared by borylation of 104 and compound 108 was made by a procedure similar to that of $\mathbf{1 0 4}$ but by starting from 1-(4-chlorophenyl)-4-(4-iodophenyl)-1,3-butadiene.

Later we developed a concise pathway to syn-and anti-macrocyclic trimers 110 and 111 from a shotgun approach (Scheme 2.14). At a $50 \mathrm{mM}$ concentration, TMS-protected dibromide 104 also underwent the $\mathrm{Ni}(\mathrm{cod})_{2}$-mediated homocoupling reactions to produce trimers $\mathbf{1 1 0}$ and 111 (syn-trimer 110:anti-trimer $\mathbf{1 1 1}=\mathbf{1}: 3$ ). Trimer $\mathbf{1 1 0}$ was treated with $\mathrm{ICl}$ to produce the 
Scheme 2.13. Stepwise Synthesis of Trimers 110 and 111 and Subsequent Transformations
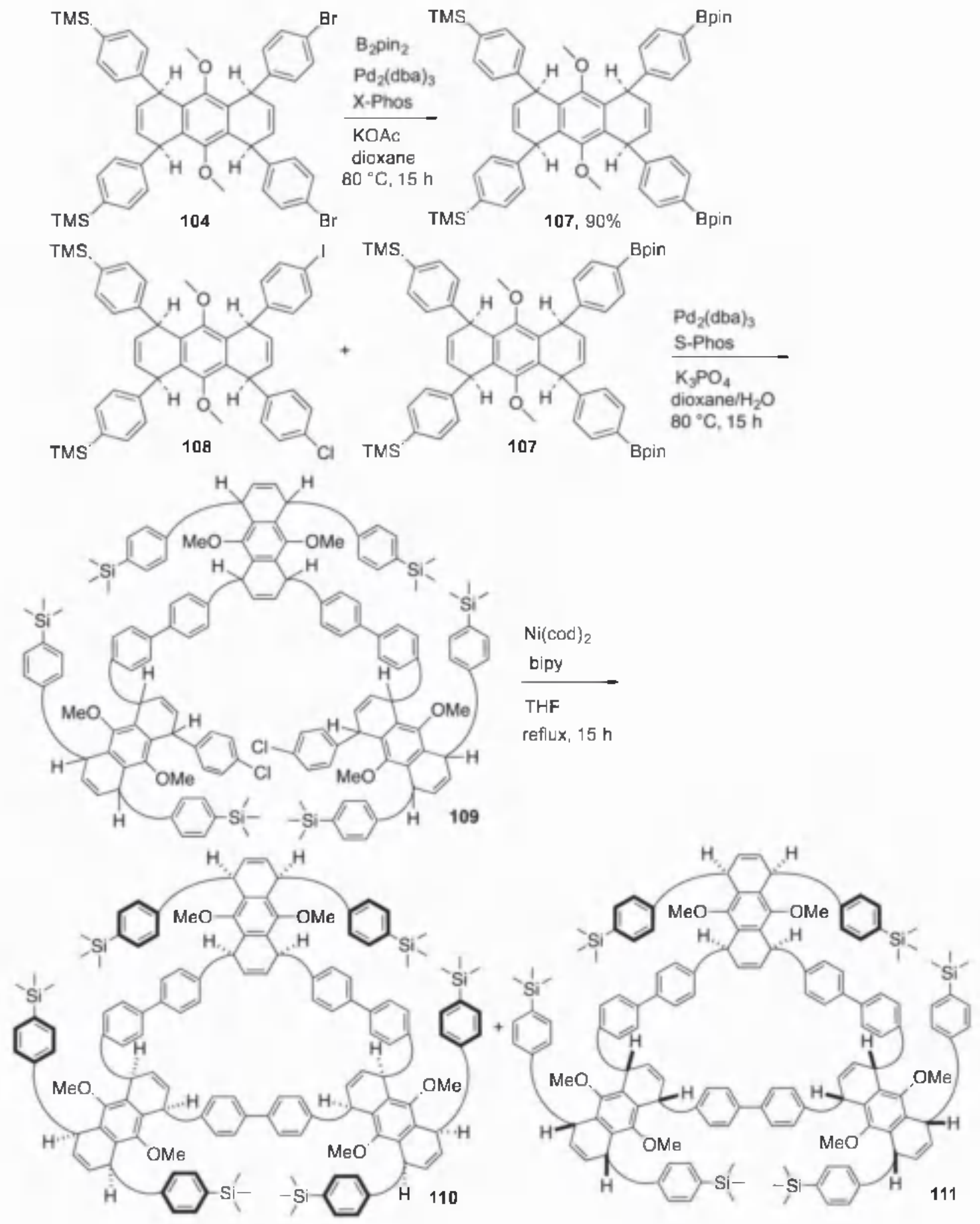


\section{Scheme 2.14. Shotgun Synthesis of Trimer 110 and 111 and Subsequent Transformations}
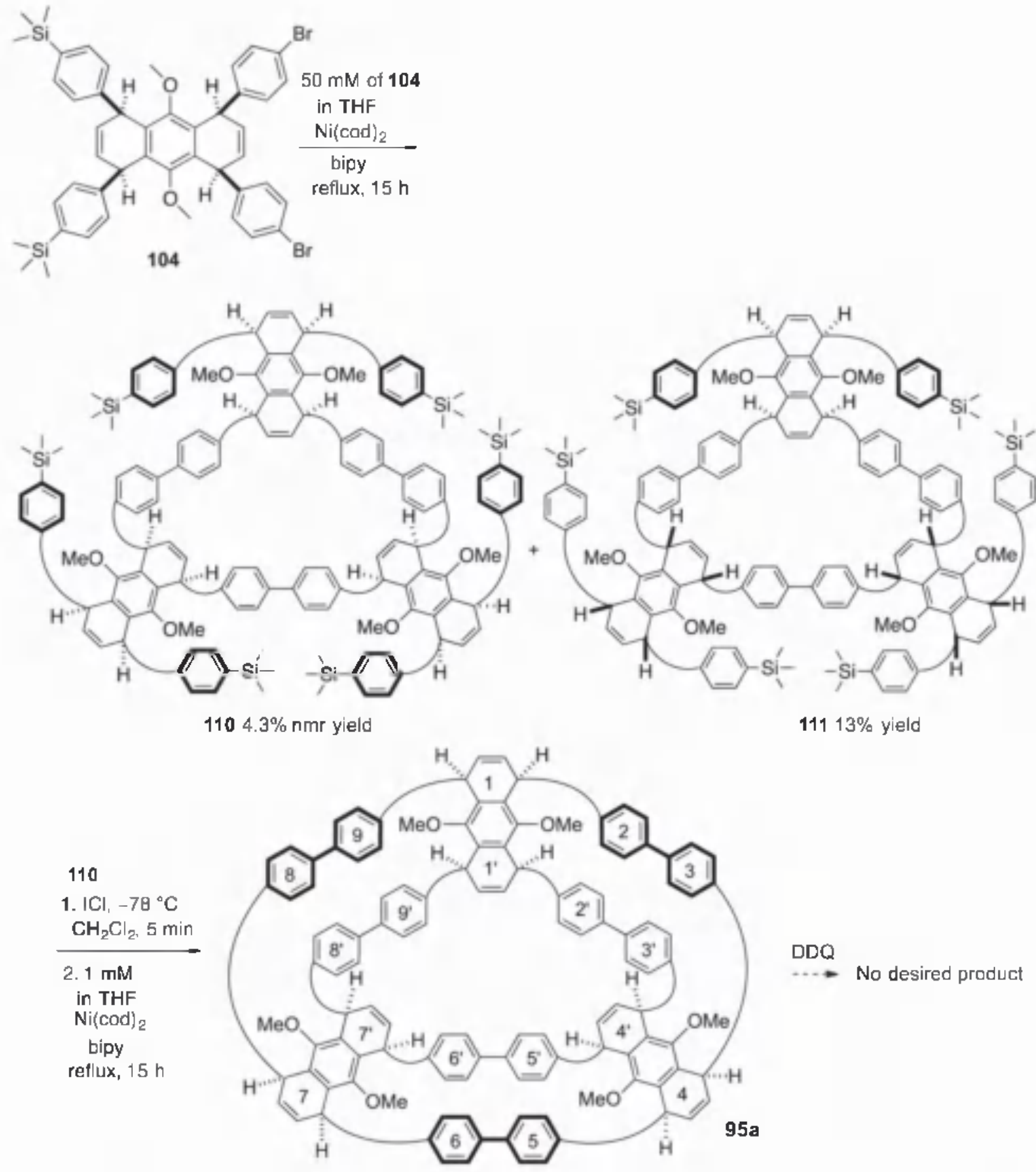

corresponding hexaiodide, which on exposure to $\mathrm{Ni}(\operatorname{cod})_{2}$ in the presence of bipy produce trimer

95a, because the crude 'H NMR spectrum had identical peaks as in that of $95 \mathbf{a}$ obtained in Scheme 2.9. 
However attempts to perform oxidative aromatization of 95 a with DDQ at temperatures ranging from ambient temperature to $70^{\circ} \mathrm{C}$ failed to produce the fully aromatized product. But we do observe partial removal of the allylic hydrogens in 95a initially. Forcing the reaction to go to completion resulted in decomposition.

\subsection{Ongoing project}

As larger rings should have less ring strain, we began to develop a synthetic pathway for the functionalized [10]CPP and the corresponding fused two-nanohoop system by using the pathway developed for stepwise synthesis of $\mathbf{9 3}$. Compared to the synthesis of functionalized $[9] \mathrm{CPP}$, the functionalized $[10] \mathrm{CPP}$ can be prepared from aromatization of the dimer of the Lshaped building block $\mathbf{1 1 3}$ with an extended biphenyl system prepared from dibromide $\mathbf{5 5}$ (Scheme 2.15). The synthesis started with borylation of the L-shaped building block syndibromide 55 with the $\mathrm{Pd}_{2}(\mathrm{dba})_{3} / \mathrm{X}$-Phos catalyst to produce 112 in excellent yield. However, the next step for the extension of one benzene ring with normal procedures, ${ }^{21,26}$ afforded the desire product either in low yield or a mixture that was hard to isolate the desired product. After extensive screening, we were able to prepare the one phenyl ring extended L-shaped building block 113 in $76 \%$ yield with the $\mathrm{Pd}\left(\mathrm{PPh}_{3}\right)_{4} / \mathrm{Ag}_{2} \mathrm{CO}_{3}$ in refluxing tetrahydrofuran (THF) for a longer time. ${ }^{32}$ The same $\mathrm{Ni}(\mathrm{cod})_{2}$-mediated homocoupling reactions of $\mathbf{1 1 3}$ in dilute condition yielded the desired dimer $\mathbf{1 1 4}$ in $10 \%$ yield. We did not know the exact stereochemistry of the isolated dimer at this stage. However, based on the observation during the silica gel column purification of $s y n-\mathbf{5 6 a}$ and $a n t i-\mathbf{5 6 h}$, the $s y n$-isomer was eluted first from the column, and so we suspect that the obtained sample is the syn-isomer depicted in $\mathbf{1 1 4}$. It was very surprising to observe that the attempted aromatization by DDQ at $70^{\circ} \mathrm{C}$ did not deliver any of the desired 
product 115. Only 114 was recovered. However, upon increasing the reaction temperature to $150^{\circ} \mathrm{C}$, the fully aromatized 115 having a functionalized [10]CPP structure was obtained in $65 \%$ yield.

\section{Scheme 2.15. Synthesis of Functionalized |10|CPP}
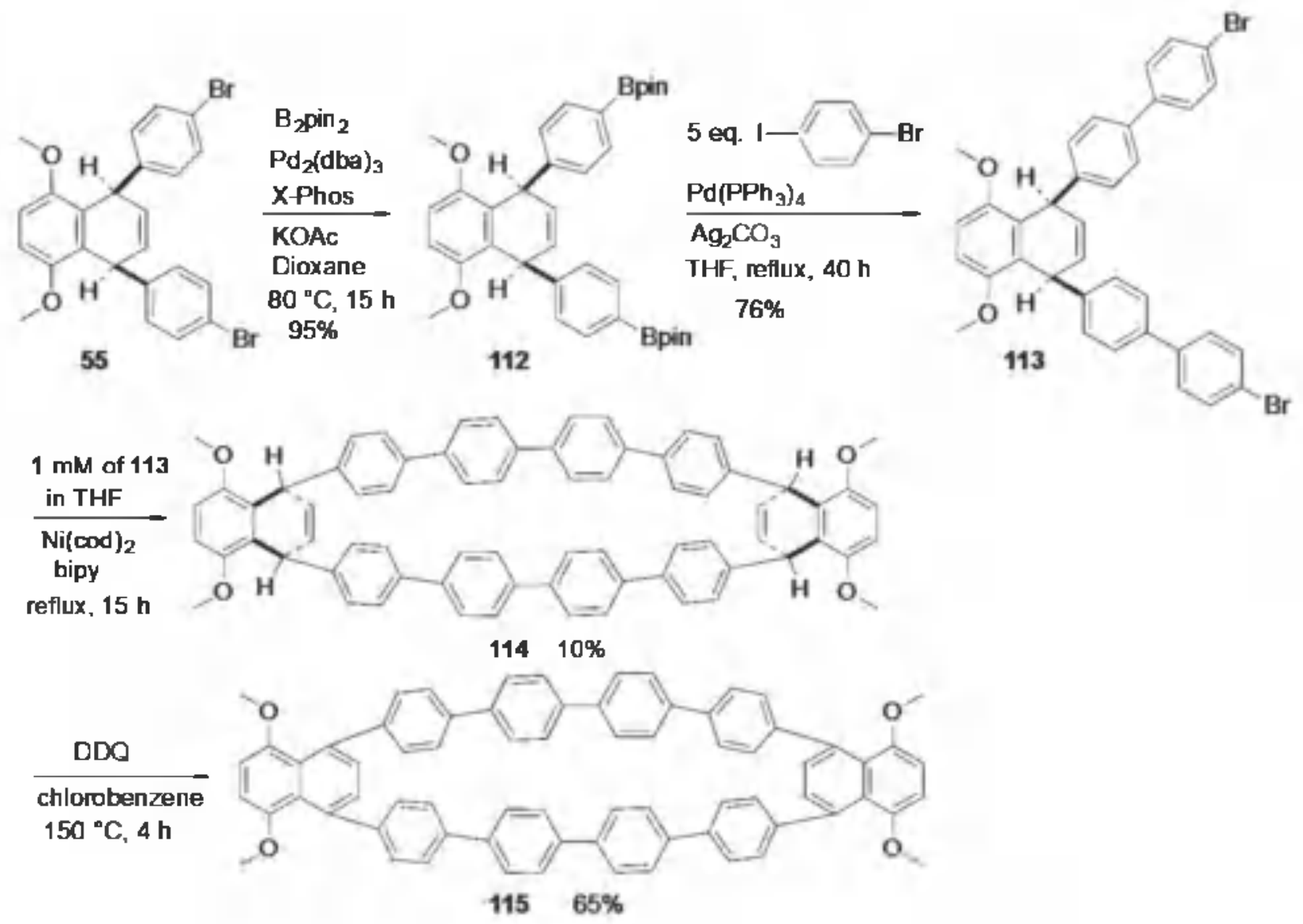

With the model study of the functionalized [10]CPP 115 being accomplished, we targeted our next goal to the preparation of fused nanohoops containing two units of the [10]CPP precursor. First we tried the shotgun approach used previously for the synthesis of fused two layer nanohoops 93 . Using the same sequences, including borylation and one phenyl ring extension, compound 117 was prepared in $42 \%$ yield in two steps from tetrabromide $\mathbf{9 0}$ (Scheme 2.16). However, we did not observe any dimer products upon the Ni(cod) $)_{2}$-mediated homocoupling reactions. 


\section{Scheme 2.16. Attempted Shotgun Synthesis of Fused |10|CPP Precursors}
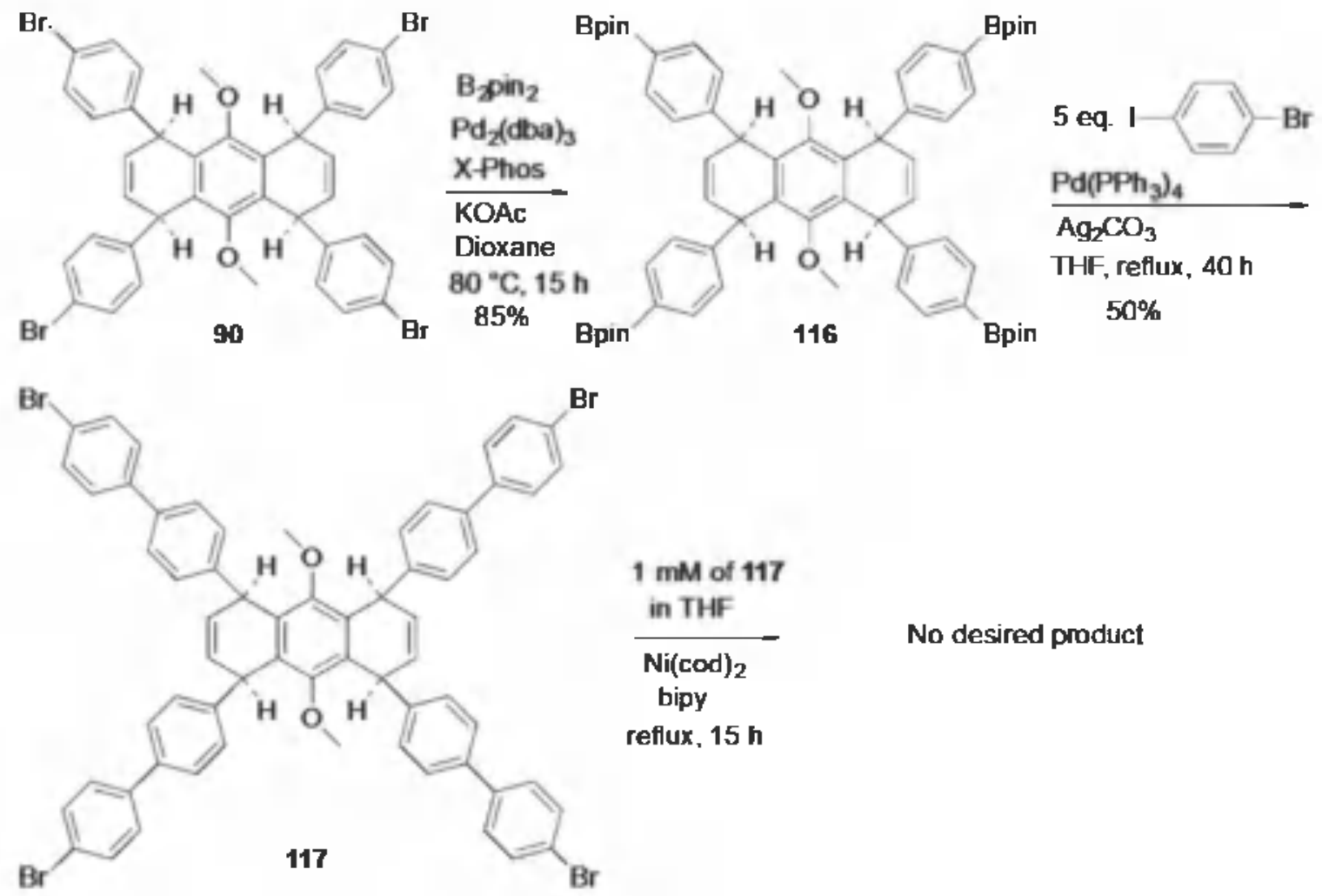

Then we turned to the stepwise sequence to try to construct a molecule containing two fused [10]CPP precursors (Scheme 2.17). As the solubility of the desired benzene extended dibromide diene is very poor, we proceeded with one benzene extended TMS-protected diene 118 by the Suzuki-Miyaura cross-couplings between 96 and 98 . The $\mathrm{AlCl}_{3}$-catalyzed Diels-Alder reaction between $\mathbf{1 1 8}$ and quinone dibromide 89, afforded, after methylation, 119 in $60 \%$ yield. Borylation, followed by one-benzene extension produced the TMS-protected dibromde $\mathbf{1 2 0}$ in $54 \%$ yield. The $\mathrm{Ni}(\operatorname{cod})_{2}$-mediated homocoupling reactions of 120 under dilute concentration formed the expected syn-dimer 121 in 15\% yield and anti-dimer 122 in $15 \%$ yield. Iodination followed by the $\mathrm{Ni}(\operatorname{cod})_{2}$-mediated homocoupling with a small amount of syn-dimer 121 provided very encouraging results. We observed a set of doublets ${ }^{~} H$ NMR signals in the upfileld-shifted region around 6.4-6.8ppm, suggesting that intramolecular homocoupling 
Scheme 2.17. Stepwise Synthesis toward Fused |10|CPP Precursors

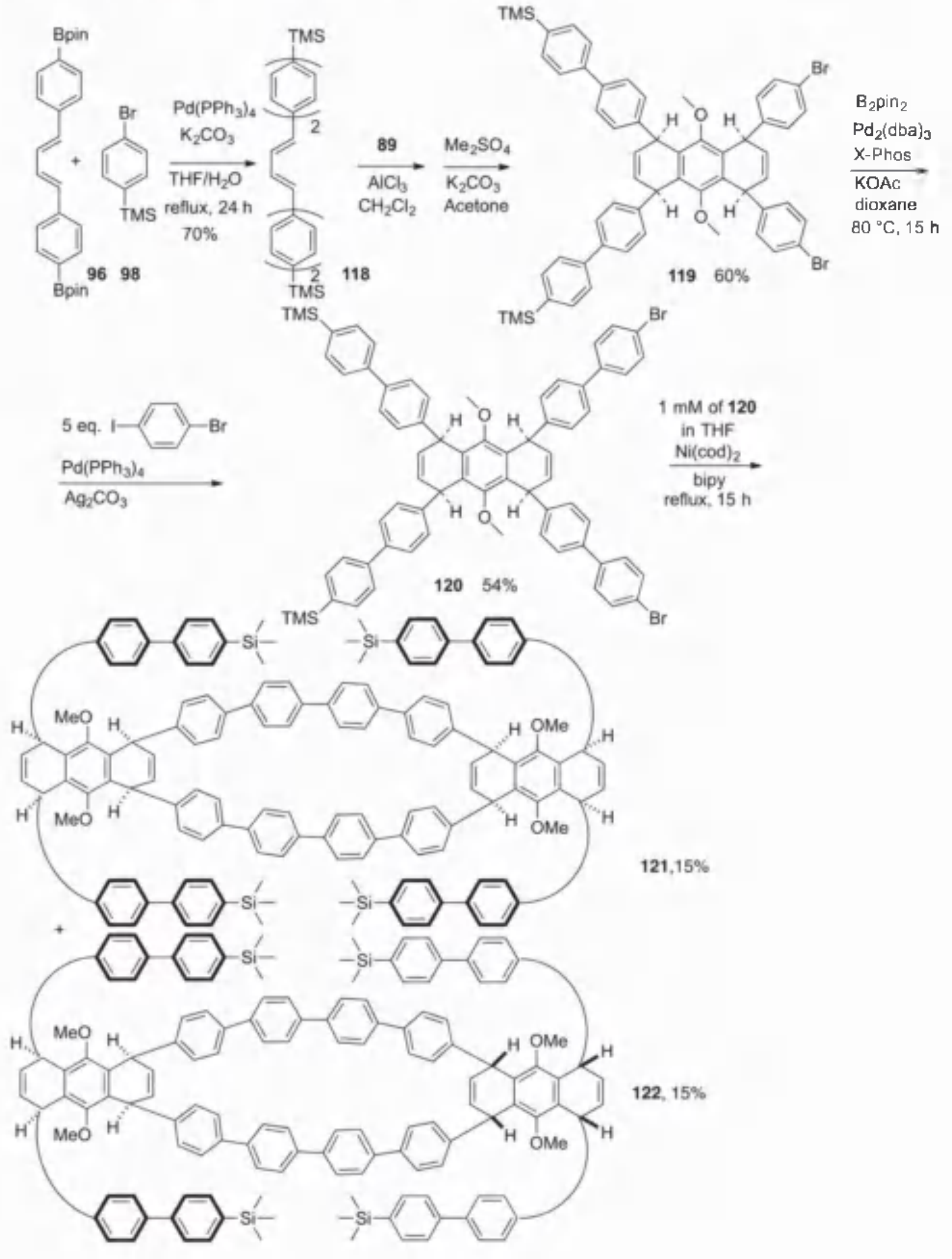


occurred to form a molecule that contained two units of the [10]CPP precursor. Unfortunately, we need more materials to finish the project for the subsequent oxidative aromatization. Nevertheless, this effort provides the foundation for the construction of a structure containing two fused and fully aromatized [10]CPP units.

\subsection{Summary}

We have developed pathways for preparing fused two-layer [6] and [9]CPP precursors in a nanotube-like arrangement either via shotgun synthsis or via stepwise synthesis. The shotgun synthesis involved $\mathrm{Ni}(\mathrm{cod})_{2}$-mediated homocoupling reaction of all syn-tetrabromide building blocks. The stepwise pathway, in which the rings were constructed consecutively, not only confirmed the structures of the isomers from shotgun synthesis, but also improved the overall yield of 93 containing two [6]CPP precursors and it provides possibilities of preparing two-layer CPP precursors with different sizes. These nanotube-like structures may serve as potential templates for the controlled growth of armchair type single-wall carbon nanotubes. 


\subsection{Reference}

(1) Omachi, H.; Nakayama, T.; Takahashi, E.; Segawa, Y.; Itami, K. Nat.Chem. 2013, 5, $572-576$

(2) Yagi, A.; Segawa, Y.; Itami, K. J. Am. Chem. Soc. 2012, 134, 2962-2965.

(3) Sisto, T.; Tian, X.; Jasti, R. J. Org. Chem. 2012, 77, 5857-5860.

(4) Batson, J.; Swager, T. Synlett 2013, 24, 2545-2549.

(5) Golling, F.; Quemheim, M.; Wagner, M.; Nishiuchi, T.; Mülen, K. Angew. Chem. Int. Ed. 2014, 53, 1525-1528.

(6) Tran-Van, A.; Huxol E.; Basler, J.; Neuburger, M.; Adjizian, J.; Ewels, C.; Wegner H. Org. Lell. 2014, 16, 1594-1597.

(7) Iwamoto, T.; Kayahara, E.; Yasuda, N.; Suzuki, T.; Yamago, S. Angew. Chem. Int. Ed. 2014, $53,6430-6434$.

(8) Li, H.; Page, A.; irle, S.; Morokuma, K. J. Am. Chem. Soc. 2012, 134, I5887-I 5896.

(9) Fort, E.; Donovan, P.; Scott, L. J. Am. Chem. Soc. 2009, 131, 16006-16007.

(10) Fort, E.; Scott, L. Angew. Chem. Int. Ed. 2010, 49, 6626-6628.

(1 I) Fort, E.; Scott, L. J. Mater. Chem. 2011, 21, 1373-1381.

(12) Fort, E.; Scott, L. Tetrahedron Lett. 2011, 52, 2051-2053.

(13) Fort, E.; Jeffreys, M.; Scott, L. Chem. Commun. 2012, 48, $8102-8104$.

(14) Li, P.; Wong, B.; Zakharov, L.; Jasti, R. Org. Lett. 2016, 18,1574-1577.

(15) Jackson, E.; Sisto, T.; Darzi, E.; Jasti, R. Tetrahedron 2016, 72, 3754-3758.

(16) Sisto, T.; Zakharnv, L.; White, B.; Jasti, R. Chem. Sci. 2016, 7, 368I-3688.

(17) Golling, F.; Osella, S.; Quernheim, M.; Wagner, M.; Bejjonne, D.; Müllen, K. Chem. Sci. $\mathbf{2 0 1 5}, 6,7072-7078$. 
(18) Golling, F.; Quernheim, M.; Wagner, M.; Nishiuchi, T.; Mullen, K. Angew. Chem. Int. Ed. 2014, 53, 1525-1528.

(19) Quernheim, M.; Golling, F.; Zhang, W.; Wagner M.; Rader, H.; Nishiuchi, T.; Mullen, K. Angew. Chem. Int. Ed. 2015, 54, 10341-10346.

(20) Golder, M.; Colwell, C.; Wong, B.; Zakharov, L.; Zhen, J; Jasti, R. J. Am. Chem. Soc. 2016, $738,6577-6582$.

(2I) Xia, J.; Golder, M.; Foster, M.; Wong, B.; Jasti, R. J. Am. Chem. Soc. 2012, 134, 1970919715.

(22) Ishii, Y.; Matsuura, S.; Segawa, Y.; Itami, K. Org. Lett. 2014, 16, 2174-2176.

(23) Xia, J.; Jasti, R. Angew. Chem. Int. Ed. 2012, 51, 2474-2476.

(24) Xia, J.; Bacon, J. W.; Jasti, R. Chem. Sci. 2012, 3, 30I8-302I.

(25) Ishii, Y.; Nakanishi, Y.; Omachi H.; Matsuura, S.; Matsui K.; Shinohara, H.; Segawa, Y.; Itami, K. Chem. Sci. 2012, 3, 2340-2345.

(26) Ishiyama, T.; Murata, M.; Miyaura, N. J. Org. Chem. 1995, 60, 7508-75I0.

(27) Huang, Y. 2013, MA thesis. West Virginia Universtiy.

(28) Matsui, K.; Segawa, Y.; Itami, K. J. Am. Chem. Soc. 2014, 136, I6452-16458.

(29) Wilson, S.; Jacob, L. J. Org. Chem. 1986, 51, 4833-4836.

(30) Jacob, L.; Chen, B.; Stec, D. Synthesis 1993, 6, 61 I-614.

(3I) Parrish, J.; Flanders, V.; Floyd, R.; Jung, K. Tetrahedron Lett. 2001, 42, 7729-7731.

(32) Oderinde, M.; Froese, R.; Organ, M. Angew. Chem. Int. Ed. 2013, 52, 11334-11338.

(33) Brown, H.; Hamaoka, T.; Ravindran, N. J. Am. Chem. Soc. 1973, 95, 5786-5788.

(34) Sinclai, D.; Sherburn, M. J. Org. Chem. 2005, 70, 3730-3733. 


\section{Chapter 3. Experiment Section}

\section{General Experimental Methods}

All reactions were conducted in oven-dried $\left(120^{\circ} \mathrm{C}\right)$ glassware under a nitrogen atmosphere. Diethyl ether and tetrahydrofuran (THF) was distilled from benzophenone ketyl prior to use. Commercial available chemicals were of reagent grade and were used as received without further purification. Purification by column chromatography was performed using 32-65 $\mu \mathrm{m}$ silica gel. Melting points were uncorrected. High resolution mass spectra were obtained on a hybrid linear ion trap Fourier transform mass spectrometer system equipped with an ion source. The UV-vis absorption spectrum was recorded on a Shimadzu UV-I800 spectrophotometer with a $\mathbf{l - n m}$ resolution, and the baseline was corrected with a solvent filled square quartz cell. The fluorescence spectrum was recorded on a Shimadzu Rf-530I PC spectrofluorophotometer with a 2-nm resolution. (E,E)-1,4-Bis(4-bromophenyl)-1,3-butadiene (48) was prepared according to the reported procedure. ${ }^{\$ 1}$ 
Experimental Procedure for 2,5-Dimethoxyphenol 51. To an ice-cold solution of $19.7 \mathrm{~g}$ of $70 \% \mathrm{~m}$-chloroperbenzoic acid in $100 \mathrm{~mL}$ of dichloromethane was added dropwise a solution of $10.2 \mathrm{~g}$ of 2,5 -dimethoxybenzaldehyde $(61.4 \mathrm{mmol})$ in $50 \mathrm{~mL}$ of dicholoromethane. After the addition was finished, the ice bath was removed and the reaction mixture was stirred for $4 \mathrm{~h}$ at room temperature. Then the reaction mixture was washed consecutively with aqueous saturated sodium bicarbonate $(3 \times 30 \mathrm{~mL})$ and $100 \mathrm{~mL}$ of $10 \%$ sodium thiosulfate. The organic layer was concentrated and dissolved in methanol, and stirred with excess $10 \%$ aqueous sodium hydroxide for $30 \mathrm{~min}$. The reaction mixture was acidified to $\mathrm{pH} 1$ with $3 \mathrm{~N} \mathrm{HCl}$ solution and extracted with ethyl acetate $(3 \times 60 \mathrm{~mL})$. The organic layers were collected, dried and concentrated via high vacuo to give $7.80 \mathrm{~g}$ of $51(82 \%)$ as yellow liquid: ' $\mathrm{H} \mathrm{NMR}\left(\mathrm{CDCl}_{3}, 400 \mathrm{MHz}\right) \delta 6.74(\mathrm{I} \mathrm{H}, \mathrm{d}, . J$ $=8.4 \mathrm{~Hz}), 6.63(\mathrm{I} \mathrm{H}, \mathrm{d}, J=3.2 \mathrm{~Hz}), 6.42(\mathrm{I} \mathrm{H}, \mathrm{br}), 6.39(1 \mathrm{H}, \mathrm{dd}, J=8.8,3.2 \mathrm{~Hz}), 3.75(3 \mathrm{H}, \mathrm{s})$, $3.72(3 \mathrm{H}, \mathrm{s}) ;{ }^{13} \mathrm{C} \mathrm{NMR}\left(\mathrm{CDCl}_{3}, 100 \mathrm{MHz}\right) \delta 154 . \mathrm{I}, 146.2,140.9, \mathrm{I} 1 \mathrm{I} .4,103.8,101.7,56.0$, 55.0.

Experimental Procedure for Silane 52. To the mixture of $1.90 \mathrm{ml}$ of hexamethyldisilazane (9.06 mmol) and $4.82 \mathrm{~g}$ of $\mathrm{LiClO}_{4}(4.53 \mathrm{mmol})$ was added $1.37 \mathrm{~g}$ of $\mathbf{5 1}(8.88 \mathrm{mmol})$ and the reaction mixture was stirred at room temperature for $4 \mathrm{~h}$ under an inert atmosphere. Then the reaction mixture was diluted with dichloromethane, filtered and concentrated under high vacuum to yield $1.88 \mathrm{~g}$ of $\mathbf{5 2}(8.30 \mathrm{mmol}, 93 \%)$ as yellow liquid: ${ }^{1} \mathrm{H} \mathrm{NMR}\left(\mathrm{CDCl}_{3}, 400 \mathrm{MHz}\right) \delta 6.76(1$ $\mathrm{H}, \mathrm{d}, J=8.8 \mathrm{~Hz}), 6.50(1 \mathrm{H}, \mathrm{d}, J=3.2 \mathrm{~Hz}), 6.44(1 \mathrm{H}, \mathrm{dd}, J=9.2,3.2 \mathrm{~Hz}), 3.74(3 \mathrm{H}, \mathrm{s}), 3.70(3$ $\mathrm{H}, \mathrm{s}), 0.27(9 \mathrm{H}, \mathrm{s}) ;{ }^{13} \mathrm{C}$ NMR $\left(\mathrm{CDCl}_{3}, 100 \mathrm{MH}\right) \delta 153.9,145 . \mathrm{I}, \mathrm{I} 45.0, \mathrm{I} 12.7,108.0,105.4$, $55.8,55.1,0.04$

Experimental Procedure for Silane 53. To the solution of $1.88 \mathrm{~g}$ of $\mathbf{5 2}(8.30 \mathrm{mmol})$ in $30 \mathrm{~mL}$ THF at $-78{ }^{\circ} \mathrm{C}$ was added $5.4 \mathrm{~mL}$ of $2 \mathrm{M}$ LDA (10.8 mmol) solution dropwise. After the reaction 
mixture was kept at $-78{ }^{\circ} \mathrm{C}$ for $2 \mathrm{~h}, 1.6 \mathrm{~mL}$. of trimethylsilyl chloride (12.5 mmol) was added dropwise at this temperature. Then the reaction mixture was allowed to warm to room temperature for overnight. Water was added and extracted with diethyl ether. The organic layers was collected, dried and concentrated. The residue was purified by a short silica gel column chromatography to give $2.35 \mathrm{~g}$ of $\mathbf{5 3}(7.87 \mathrm{mmol}, 94 \%)$ as yellow liquid: ' $\mathrm{H}$ NMR (CDCl 3,400 $\mathrm{MHz}) \delta 7.0 \mathrm{I}(1 \mathrm{H}, \mathrm{d}, J=8.8 \mathrm{~Hz}), 6.62(\mathrm{I} \mathrm{H}, \mathrm{d}, J=8.4 \mathrm{~Hz}), 3.97(3 \mathrm{H}, \mathrm{s}), 3.94(3 \mathrm{H}, \mathrm{s}), 0.68(9$ $\mathrm{H}, \mathrm{s}), 0.61(9 \mathrm{H}, \mathrm{s}) ;{ }^{13} \mathrm{C} \mathrm{NMR}\left(\mathrm{CDCl}_{3}, 100 \mathrm{MHz}\right) \delta 158.6,150.0,143.6,118.0,112.3,102.4$ $55.0,54.5,1.68,1.55$

Experimental Procedure for Trillate 54. To the solution of $2.35 \mathrm{~g}$ of $53(7.87 \mathrm{mmol})$ in $50 \mathrm{~mL}$ of diethyl ether at $0{ }^{\circ} \mathrm{C}$ was added $5.90 \mathrm{~mL}$ of I.6M $\mathrm{n}$-BuLi $(9.44 \mathrm{mmol})$ in hexanes dropwise. The reaction mixture was stirred at $0{ }^{\circ} \mathrm{C}$ for $30 \mathrm{~min}$, then $2.7 \mathrm{~mL}$ of triflate anhydride (15.8 mmol) was added to the reaction mixture. After it was allowed to warm to room temperature for two more hours, it was quenched with $\mathrm{NaHCO}_{3}$ solution $(50 \mathrm{~mL})$ and extracted with diethyl ether $(3 \times 40 \mathrm{~mL})$. The combined organic layers were dried, concentrated and purified by silica gel column chromatography to afford $2.44 \mathrm{~g}$ of $\mathbf{5 4}(6.81 \mathrm{mmol}, 86 \%)$ as yellow liquid: ' $\mathrm{H}$ NMR $\left(\mathrm{CDCl}_{3}, 400 \mathrm{MHz}\right) \delta 6.96(\mathrm{I} \mathrm{H}, \mathrm{d}, J=9.2 \mathrm{~Hz}), 6.78(1 \mathrm{H}, \mathrm{d}, J=9.2 \mathrm{~Hz}), 3.80(3 \mathrm{H}, \mathrm{s}), 3.77(3 \mathrm{H}$, s), $0.38(9 \mathrm{H}, \mathrm{s}) ;{ }^{13} \mathrm{C} \mathrm{NMR}\left(\mathrm{CDCl}_{3}, 100 \mathrm{MHz}\right) \delta 158.1,145.1,142.6,123.7,120.5,117.3,114.1$, $110.0,55.9,55.7,0.66$

Experimental Procedure for CPP Precursor Dibromides 55. To a mixture of $1.000 \mathrm{~g}$ of diene $48(2.75 \mathrm{mmol})$ and $0.360 \mathrm{~g}$ of 1,4 -henzoquinone $(3.33 \mathrm{mmol})$ in $150 \mathrm{~mL}$ of anhydrous dichloromethane under a nitrogen atmosphere was added by using a syringe $0.61 \mathrm{~mL}$ of boron trifluoride diethyl etherate $(4.94 \mathrm{mmol})$. The reaction mixture was stirred at room temperature for $20 \mathrm{~h}$. Distilled water $(20 \mathrm{~mL})$ was added slowly, and the organic layer was separated. The 
aqueous layer was back extracted with $40 \mathrm{~mL}$ of dichloromethane. The combined organic layers were dried over sodium sulfate and concentrated to afford a brown/yellow solid. The crude solid was used without further purification.

To the brown/yellow solid and $5.315 \mathrm{~g}$ of potassium carbonate $(38.5 \mathrm{mmol})$ in $200 \mathrm{~mL}$ of anhydrous acetone under a nitrogen atmosphere was added $2.6 \mathrm{~mL}$ of dimethyl sulfate $(27.5$ mmol) by using a syringe, and the reaction mixture was heated under reflux for $18 \mathrm{~h}$. Distilled water $(40 \mathrm{~mL})$ was added and the reaction mixture was extracted with ethyl acetate $(3 \times 40 \mathrm{~mL})$ and then dichloromethane $(40 \mathrm{~mL})$. The combined organic layers were dried over sodium sulfate and concentrated. The solid residue was purified by flash column chromatography (silica gel/ethyl acetate:hexanes $=1: 5)$ to produce $1.248 \mathrm{~g}$ of $\mathbf{5 5}(2.49 \mathrm{mmol}, 91 \%$ yield $)$ as a white solid: mp 203-204 ${ }^{\circ} \mathrm{C}$; IR $1483,1257,811 \mathrm{~cm}^{-1}$; 'H NMR $\left(\mathrm{CDCl}_{3}, 600 \mathrm{MHz}\right) \delta 7.33(4 \mathrm{H}, \mathrm{d}, J=$ $7.8 \mathrm{~Hz}), 7.05(4 \mathrm{H}, \mathrm{d}, J=8.4 \mathrm{~Hz}), 6.73(2 \mathrm{H}, \mathrm{s}), 5.95(2 \mathrm{H}, \mathrm{d}, J=3.0 \mathrm{~Hz}), 4.83(2 \mathrm{H}, \mathrm{d}, J=3.0$ $\mathrm{Hz}), 3.61(6 \mathrm{H}, \mathrm{s}) ;{ }^{13} \mathrm{C} \mathrm{NMR}\left(\mathrm{CDCl}_{3}, 150 \mathrm{MHz}\right) \delta 151.2,143.7,131.0,129.9,127.7,127.0$, I 19.5, 109.2, 55.7, 40.6; HRMS (ESI) calcd for $\mathrm{C}_{24} \mathrm{H}_{20} \mathrm{Br}_{2} \mathrm{O}_{2}\left(\mathrm{M}^{+}\right)$497.9825, 499.9804, 501.9784 , found $497.9818,499.9798,501.9777$. Recrystallization of $\mathbf{5 5}$ from chloroform/hexanes produced a single crystal suitable for $\mathrm{X}$-ray structure analysis.

Experimental Procedure for Cyclic Dimers syn-56a and anti-56b and Cyclic Trimers syn-

64a and anti-64b. To a $500 \mathrm{~mL}-f l a s k$ were added $0.650 \mathrm{~g}$ of $55(1.30 \mathrm{mmol})$ and $0.490 \mathrm{~g}$ of $2,2^{\prime}-$ bipyridyl ( $3.14 \mathrm{mmol})$. The flask was flushed with nitrogen and placed in a glovebox under a nitrogen atmosphere before $0.870 \mathrm{~g}$ of $\mathrm{Ni}(\operatorname{cod})_{2}(3.16 \mathrm{mmol})$ was added. The flask was fitted with a condenser, rubber septa, and then removed from the glovebox before $260 \mathrm{~mL}$ of THF was introduced via cannula. The reaction mixture was heated at reflux for $48 \mathrm{~h}$ before it was allowed to cool to room temperature. Then the reaction mixture was passed through a short pad of silica 
gel and eluded with ethyl acetate $(200 \mathrm{~mL})$ and dichloromethane $(200 \mathrm{~mL})$. The combined eluents were concentrated, and the residue was purified by flash column chromatography (silica gel/ dichloromethane:hexanes $=1: 5$ to $\mathrm{I}: 1)$ to produce $0.091 \mathrm{~g}$ of $s y n-56 \mathrm{a}(0.134 \mathrm{mmol}, 2 \mathrm{I} \%$ yield) as a white solid, $0.025 \mathrm{~g}$ of anti-56b $(0.037 \mathrm{mmol}, 6 \%$ yield $)$ as a white solid, and $0.050 \mathrm{~g}$ of a mixture of $s y n-64 \mathbf{a}$ and anti-64b $(0.049 \mathrm{mmol}, 11 \%$ combined yield, syn-64a:anit-64b $=$ I:3) as a white solid. A small fraction of the mixture of syn-64a and anti-64b was further purified by preparative TLC to afford pure samples of syn-64a and anti-64b for structural elucidation. At $50 \mathrm{mM}$ concentration of $\mathbf{5 5}$, the cyclic dimers syn-56a and anti-56b were isolated in $8 \%$ combined yield $(s y n-56 \mathbf{a}: a n t i-\mathbf{5 6} \mathbf{b}=3: 1)$, and the cyclic trimers syn-64a and anti-64b were isolated in $18 \%$ combined yield $($ syn-64a:anti-64b $=1: 3)$. syn-56a: $\mathrm{mp}>310^{\circ} \mathrm{C}$; IR 1483 , $1253 \mathrm{~cm}^{-1}$; 'H NMR (CDCl $\left.3,400 \mathrm{MHz}\right) \delta 6.90(4 \mathrm{H}, \mathrm{s}), 6.78(4 \mathrm{H}, \mathrm{dd}, J=4.4,2.4 \mathrm{~Hz}), 6.67(8$ $\mathrm{H}, \mathrm{d}, J=8.4 \mathrm{~Hz}), 6.60(8 \mathrm{H}, \mathrm{d}, J=7.6 \mathrm{~Hz}), 5.17(4 \mathrm{H}, \mathrm{m}), 3.84(12 \mathrm{H}, \mathrm{s}) ;{ }^{13} \mathrm{C} \mathrm{NMR}(\mathrm{CDCl}, 100$ MHz) $\delta 150.9,140.2,138.4,133.3,131.4,127.4,125.9,108.5,55.9,37.3 ;$ HRMS (ESI) calcd for $\mathrm{C}_{48} \mathrm{H}_{40} \mathrm{O}_{4} \quad\left(\mathrm{M}^{*}\right)$ 680.292I, found 680.2923. Recrystallization of syn-56a from dichloromethane/hexanes produced a single crystal suitable for $\mathrm{X}$-ray structure analysis. anti56b: IR 1488, $1260 \mathrm{~cm}^{-1}$; ' $\mathrm{H}$ NMR $\left(\mathrm{CDCl}_{3}, 600 \mathrm{MHz}\right) \delta 6.9 \mathrm{I}(4 \mathrm{H}, \mathrm{s}), 6.77(4 \mathrm{H}, \mathrm{dd}, J=4.4,2.6$ $\mathrm{Hz}), 6.67(8 \mathrm{H}, \mathrm{d}, J=8.5 \mathrm{~Hz}), 6.60(8 \mathrm{H}, \mathrm{d}, J=7.9 \mathrm{~Hz}), 5.17(4 \mathrm{H}, \mathrm{m}), 3.85(12 \mathrm{H}, \mathrm{s}) ;{ }^{13} \mathrm{C} \mathrm{NMR}$ $\left(\mathrm{CDCl}_{3}, 150 \mathrm{MHz}\right) \delta$ I51.0, I40.3, 138.4, I33.2, 131.4, 127.4, 125.9, 108.6, 55.9, 37.3; HRMS (ESI) calcd for $\mathrm{C}_{48} \mathrm{H}_{40} \mathrm{O}_{4}\left(\mathrm{M}^{+}\right)$680.2921, found 680.2926. syn-64a: IR 1476, $1256 \mathrm{~cm}^{-1}$; ${ }^{1} \mathrm{H}$ NMR $\left(\mathrm{CDCl}_{3}, 600 \mathrm{MHz}\right) \delta 7.50(12 \mathrm{H}, \mathrm{d}, J=8.5 \mathrm{~Hz}), 7.29(12 \mathrm{H}, \mathrm{d}, J=8.4 \mathrm{~Hz}), 6.73(6 \mathrm{H}, \mathrm{s})$, $5.97(6 \mathrm{H}, \mathrm{d}, J=3.1 \mathrm{~Hz}), 4.91(6 \mathrm{H}, \mathrm{d}, J=2.9 \mathrm{~Hz}), 3.60(18 \mathrm{H}, \mathrm{s}) ;{ }^{13} \mathrm{C} \mathrm{NMR}\left(\mathrm{CDCl}_{3}, 150 \mathrm{MHz}\right)$ $\delta 151.5,143.7,137.9,128.5,127.7,127.6,126.2,109.1,55.8,40.9$; HRMS (ESI) calcd for $\mathrm{C}_{72} \mathrm{H}_{60} \mathrm{O}_{6}\left(\mathrm{M}^{+}\right)$1020.4384, found 1020.4356. anti-64b: IR 1477, $1255 \mathrm{~cm}^{-1}$; 'H NMR (CDCl ${ }_{3}$, 
$600 \mathrm{MHz}) \delta 7.54(4 \mathrm{H}, \mathrm{d}, J=8.4 \mathrm{~Hz}), 7.430(4 \mathrm{H}, \mathrm{d}, J=8.4 \mathrm{~Hz}), 7.426(4 \mathrm{H}, \mathrm{d}, J=8.4 \mathrm{~Hz}), 7.34$ $(4 \mathrm{H}, \mathrm{d}, J=7.8 \mathrm{~Hz}), 7.27(4 \mathrm{H}, \mathrm{d}, J=8.4 \mathrm{~Hz}), 7.25(4 \mathrm{H}, \mathrm{d}, J=8.4 \mathrm{~Hz}), 6.76(2 \mathrm{H}, \mathrm{d}, J=8.9 \mathrm{~Hz})$, $6.74(2 \mathrm{H}, \mathrm{s}), 6.73(2 \mathrm{H}, \mathrm{d}, J=8.9 \mathrm{~Hz}), 6.10(2 \mathrm{H}, \mathrm{ddd}, J=9.8,4.8,1.0 \mathrm{~Hz}), 6.00(2 \mathrm{H}, \mathrm{d}, J=3.0$ $\mathrm{Hz}), 5.96(2 \mathrm{H}, \mathrm{ddd}, J=9.8,4.7, \mathrm{I} .2 \mathrm{~Hz}), 4.95(2 \mathrm{H}, \mathrm{t}, J=4.5 \mathrm{~Hz}), 4.933(2 \mathrm{H}, \mathrm{d}, J=3.0 \mathrm{~Hz})$, $4.928(2 \mathrm{H}, \mathrm{t}, J=4.5 \mathrm{~Hz}), 3.72(6 \mathrm{H}, \mathrm{s}), 3.63(6 \mathrm{H}, \mathrm{s}), 3.57(6 \mathrm{H}, \mathrm{s}) ;{ }^{13} \mathrm{C} \mathrm{NMR}\left(\mathrm{CDCl}_{3}, \mathrm{I} 50 \mathrm{MHz}\right)$ $\delta$ I5I.47, I5I.45, 151.43, 143.8, I43.4, I43.0, 138.7, I38.6, I37.5, 129.0, I28.43, 128.34, 128.2, I28.1, I27.8, 127.58, 127.56, I27.0, I26.6, I26.5, I26.1, I09.1, I08.9, 55.81, 55.75, 40.86, 40.83, 40.77; HRMS (ESI) calcd for $\mathrm{C}_{72} \mathrm{H}_{60} \mathrm{O}_{6}\left(\mathrm{M}^{+}\right)$I020.4384, found 1020.4379 .

Experimental Procedure for Quinone 63. To a 100-mL flask were added $600 \mathrm{mg}$ silica gel and a solution of $275 \mathrm{mg}$ of ceric ammonium nitrate $(5.07 \mathrm{mmol})$ in $5 \mathrm{~mL} \mathrm{\textrm {H } _ { 2 }} \mathrm{O}$ was added dropwise while stirring. After $5 \mathrm{~min}, 20 \mathrm{~mL}$ of dichloromethane was added to the mixture. Then a solution of $56 \mathrm{mg}$ of syn-56a $(0.082 \mathrm{mmol})$ in $15 \mathrm{~mL}$ of dichloromethane was added to the reaction mixture. $15 \mathrm{~min}$ later the whole reaction mixture was filtered and washed with $100 \mathrm{~mL}$ of dichloromethane. The combined solvents were concentrated and the residue was purified by silica gel column chromatography (hexanes:dichloromethane:diethyl ether $=10: 3: 3$ ) to give 45 mg of $63(0.072 \mathrm{mmol}, 88 \%)$ as orange solid: ${ }^{1} \mathrm{H} \mathrm{NMR}\left(\mathrm{CDCl}_{3}, 400 \mathrm{MHz}\right) \delta 6.95(4 \mathrm{H}, \mathrm{s}), 6.7 \mathrm{I}$ $(4 \mathrm{H}, \mathrm{m}), 6.70(8 \mathrm{H}, \mathrm{d}, J=8.4 \mathrm{~Hz}), 6.57(8 \mathrm{H}, \mathrm{d}, J=7.2 \mathrm{~Hz}), 5.02(4 \mathrm{H}, \mathrm{m}) ;{ }^{13} \mathrm{C} \mathrm{NMR}\left(\mathrm{CDCl}_{3}\right.$, $150 \mathrm{MHz}) \delta 187.0,146.0,138.9,137.8,136.3,131.9,127.2,126.4,36.9$.

Experimental Procedure for the Functionalized [9]CPP 65. To a 200-mL flask were added $0.076 \mathrm{~g}(0.074 \mathrm{mmol})$ of a mixture of $64 \mathbf{a}$ and $64 \mathrm{~h}$ and $0.255 \mathrm{~g}(1.12 \mathrm{mmol})$ of DDQ. The flask was flushed with nitrogen at $0{ }^{\circ} \mathrm{C}$ and then $30 \mathrm{~mL}$ of chlorobenzene was introduced by using a syringe. The reaction mixture was heated to $70^{\circ} \mathrm{C}$. After $2 \mathrm{~h}$ at $70^{\circ} \mathrm{C}$, the reaction mixture was allowed to cool to room temperature, diluted with $100 \mathrm{~mL}$ of dichloromethane and immediately 
passed through a basic aluminum oxide column ( $4 \mathrm{~cm}$ high, $2.5 \mathrm{~cm}$ in diameter). The column was eluded with an additional $300 \mathrm{~mL}$ of dichloromethane. The combined eluents were concentrated in vacuo immediately to afford $65(0.066 \mathrm{~g}, 0.065 \mathrm{mmol}, 88 \%$ yield $)$ as a yellow solid: IR 1459, $1255 \mathrm{~cm}^{-1}$; ${ }^{1} \mathrm{H}$ NMR $\left(\mathrm{CDCl}_{3}, 600 \mathrm{MHz}\right) \delta 7.45(12 \mathrm{H}, \mathrm{d}, J=8.7 \mathrm{~Hz}), 7.24(12 \mathrm{H}$, d, $J=8.7 \mathrm{~Hz}), 6.99(6 \mathrm{H}, \mathrm{s}), 6.97(6 \mathrm{H}, \mathrm{s}), 3.81(18 \mathrm{H}, \mathrm{s}) ;{ }^{13} \mathrm{C}$ NMR $\left(\mathrm{CDCl}_{3}, 150 \mathrm{MHz}\right) \delta 150.8$, $142.9,137.4,135.8,132.5,127.5,126.0,124.6,107.1,56.0$; HRMS (ESI) calcd for $\mathrm{C}_{72} \mathrm{H}_{54} \mathrm{O}_{6}$ $\left(\mathrm{M}^{+}\right)$1014.3915, found 1014.3940 .

Experimental Procedure for DA Adduct 86. To a mixture of $45 \mathrm{mg}$ of diene $48(0.12 \mathrm{mmol})$ and $22 \mathrm{mg}$ of quinone $63(0.035 \mathrm{mmol})$ in $10 \mathrm{~mL}$ of anhydrous dichloromethane under a nitrogen atmosphere was added by using a syringe $0.10 \mathrm{~mL}$ of boron trifluoride diethyl etherate $(0.8$ mmol). The reaction mixture was stirred at room temperature for $20 \mathrm{~h}$. Distilled water $(20 \mathrm{~mL})$ was added slowly, and the organic layer was separated. The aqueous layer was back extracted with $40 \mathrm{~mL}$ of dichloromethane. The combined organic layers were dried and concentrated. The residue was purified by silica gel column chromatography (hexanes:dichloromethane:diethyl ether $=10: 2: 2)$ to give $36 \mathrm{mg}$ of $\mathbf{8 6}(0.027 \mathrm{mmol}, 75 \%)$ as white solid: ' $\mathrm{H}$ NMR $\left(\mathrm{CDCl}_{3}, 600\right.$ $\mathrm{MHz}) \delta 7.37(8 \mathrm{H}, \mathrm{d}, J=7.8 \mathrm{~Hz}), 7.11(8 \mathrm{H}, \mathrm{d}, . J=7.2 \mathrm{~Hz}), 6.55(8 \mathrm{H}, \mathrm{d}, J=8.4 \mathrm{~Hz}), 6.28(12$ $\mathrm{H}, \mathrm{m}), 6.18(4 \mathrm{H}, \mathrm{m}), 4.45(4 \mathrm{H}, \mathrm{m}), 4.00(4 \mathrm{H}, \mathrm{d}, J=6.0 \mathrm{~Hz}), 3.96(4 \mathrm{H}, \mathrm{d}, J=6.0 \mathrm{~Hz}) ;{ }^{13} \mathrm{C}$ NMR $\left(\mathrm{CDCl}_{3}, 150 \mathrm{MHz}\right) \delta 138.9,138.7,137.8,131.2,130.7,130.5,128.7,126.8,126.3,121.3$, $41.4,36.9,29.7$.

Experimental Procedure for Tetrahromide 87. To $23 \mathrm{mg}$ of potassium carbonate $(0.17 \mathrm{mmol})$ and $36 \mathrm{mg}$ of $86(0.027)$ in $20 \mathrm{~mL}$ of anhydrous acetone under a nitrogen atmosphere was added $0.012 \mathrm{~mL}$ of dimethyl sulfate $(0.13 \mathrm{mmol})$ by using a syringe, and the reaction mixture was heated under reflux for $18 \mathrm{~h}$. Distilled water $(40 \mathrm{~mL})$ was added and the reaction mixture was 
extracted with dichloromethane $(3 \times 40 \mathrm{~mL})$. The combined organic layers were dried over sodium sulfate and concentrated. The solid residue was purified by flash column chromatography (silica gel/ dichloromethane:hexanes $=1: 4)$ to produce $4 \mathrm{mg}$ of $87(0.003 \mathrm{mmol}$, $10 \%$ yield) as a white solid: ' $\mathrm{H}$ NMR $\left(\mathrm{CDCl}_{3}, 600 \mathrm{MHz}\right) \delta 7.39(8 \mathrm{H}, \mathrm{d}, . J=8.4 \mathrm{~Hz}), 7.17(8 \mathrm{H}$, $\mathrm{d}, J=8.4 \mathrm{~Hz}), 6.80(4 \mathrm{H}, \mathrm{m}), 6.64(8 \mathrm{H}, \mathrm{d}, J=8.4 \mathrm{~Hz}), 6.60(8 \mathrm{H}, \mathrm{d}, J=7.8 \mathrm{~Hz}), 6.01(4 \mathrm{H}, \mathrm{d}$, $J=3.0 \mathrm{~Hz}), 5.05(8 \mathrm{H}, \mathrm{m}), 3.26(12 \mathrm{H}, \mathrm{s}) ;{ }^{13} \mathrm{C} \mathrm{NMR}\left(\mathrm{CDCl}_{3}, 150 \mathrm{MHz}\right) \delta 152.3, \mathrm{I} 44 . \mathrm{I}, 139.9$, $138.6,134.9,133.2,131.5,130.2,130.0,127.8,126.1,120.1,61.5,41.6,38.3$.

Experimental Procedure for Dibromide 89. To a mixture of $3.64 \mathrm{~g}$ of diene 48 (10.0 mmol) and $1.30 \mathrm{~g}$ of 1,4 -benzoquinone $(12.0 \mathrm{mmol})$ in $200 \mathrm{~mL}$ of anhydrous dichloromethane under a nitrogen atmosphere was added by using a syringe $1.00 \mathrm{~mL}$ of boron trifluoride diethyl etherate $(8.10 \mathrm{mmol})$. The reaction mixture was stirted at room temperature for $20 \mathrm{~h}$. The reaction mixture was then passed through a short silica gel column, washed with dichloromethane, and concentrated to afford a brown/yellow solid. The crude solid was used without further purification.

To the brown/yellow solid and $2.78 \mathrm{~g}$ of silver oxide $(12.0 \mathrm{mmol})$ under a nitrogen atmosphere was added $100 \mathrm{~mL}$ of anhydrous diethyl ether. The reaction mixture was stirred ovemight and passed through a short silica gel column, washed with diethyl ether, and concentrated. The residue was recrystallized from diethyl ether to afford $3.76 \mathrm{~g}$ of $\mathbf{8 9}(8.0 \mathrm{mmol}, 80 \%$ yield) as a brown solid. 'H NMR $\left(\mathrm{CDCl}_{3}, 600 \mathrm{MHz}\right) \delta 7.43(4 \mathrm{H}, \mathrm{d}, J=8.4 \mathrm{~Hz}), 7.15(4 \mathrm{H}, \mathrm{d}, J=8.4 \mathrm{~Hz})$, $6.60(2 \mathrm{H}, \mathrm{s}), 5.9 \mathrm{I}(2 \mathrm{H}, \mathrm{d}, J=2.7 \mathrm{~Hz}), 4.72(2 \mathrm{H}, \mathrm{d}, J=2.7 \mathrm{~Hz}),{ }^{13} \mathrm{C} \mathrm{NMR}\left(\mathrm{CDCl}_{3}, 150 \mathrm{MHz}\right) \delta$ $186.0,141.6,140.2 .0,136.4,131.8,130.2,126.5,121.0,40.0 ;$ HRMS (ESI) calcd for $\mathrm{C}_{22} \mathrm{H}_{14} \mathrm{Br}_{2} \mathrm{O}_{2}\left(\mathrm{M}^{+}\right)$467.9355, found 467.9342 . 
Experimental Procedure for Tetrabromides syn-90 and anti-91. To a mixture of $3.64 \mathrm{~g}$ of diene $48(10.0 \mathrm{mmol})$ and $4.70 \mathrm{~g}$ of dibromoquinone $89(10.0 \mathrm{mmol})$ in $200 \mathrm{~mL}$ of anhydrous dichloromethane under a nitrogen atmosphere was added by using a syringe $1.00 \mathrm{~mL}$ of boron trifluoride diethyl etherate $(8.10 \mathrm{mmol})$. The reaction mixture was stirred at room temperature for $20 \mathrm{~h}$. The reaction mixture was passed through a short silica gel column, washed with dichloromethane, and concentrated to afford a yellow solid. The crude solid was used without further purification. To the yellow solid and $5.52 \mathrm{~g}$ of potassium carbonate in dry acetone (200 $\mathrm{mL}$ ) was added $2.40 \mathrm{~mL}$ of dimethyl sulfate $(25.4 \mathrm{mmol})$ via a syringe, and the reaction mixture was heated at reflux for $3 \mathrm{~h}$. The reaction mixture was then allowed to cool to rt and concentrated. Distilled water (100 mL) was added, and the reaction mixture was extracted with dichloromethane $(3 \times 100 \mathrm{~mL})$. The combined organic layers were dried over sodium sulfate and concentrated. Recrystallization of the crude solid from chloroform produced $5.86 \mathrm{~g}$ of the methylated tetrabromide $s y n-90(6.80 \mathrm{mmol}, 68 \%$ yield) as a white solid and anti-91 (NMR yield $8.5 \%$ ) as white solid. syn-90: mp: $174-176{ }^{\circ} \mathrm{C}$; IR 1703, I668, 1484, $817 \mathrm{~cm}^{-1}$; ${ }^{1} \mathrm{H}$ NMR $\left(\mathrm{CDCl}_{3}, 600 \mathrm{MHz}\right) \delta 7.22(8 \mathrm{H}, \mathrm{d}, J=8.4 \mathrm{~Hz}), 6.8 \mathrm{I}(8 \mathrm{H}, \mathrm{d}, J=8.4 \mathrm{~Hz}), 6.09(4 \mathrm{H}, \mathrm{d}, J=2.8$ $\mathrm{Hz}), 4.81(4 \mathrm{H}, \mathrm{d}, J=3.2 \mathrm{~Hz}), 3.53(6 \mathrm{H}, \mathrm{s}) ;{ }^{13} \mathrm{C} \mathrm{NMR}(\mathrm{CDCl}, \mathrm{I} 50 \mathrm{MHz}) \delta 152.1, \mathrm{I} 42.6,131.2$, 129.2, 128.1, 119.9, 60.4, 40.4; HRMS (ESI) calcd for $\mathrm{C}_{40} \mathrm{H}_{30} \mathrm{Br}_{4} \mathrm{O}_{2}\left(\mathrm{M}^{+}\right) 859.8953,861.8933$, 863.8912, found 859.8940, 861.8930, 863.8901. anti-91: ' $\mathrm{H} \mathrm{NMR}\left(\mathrm{CDCl}_{3}, 400 \mathrm{MHz}\right) \delta 7.36(8$ $\mathrm{H}, \mathrm{d}, J=8.8 \mathrm{~Hz}), 7.06(8 \mathrm{H}, \mathrm{d}, J=8.8 \mathrm{~Hz}), 5.86(4 \mathrm{H}, \mathrm{d}, J=2.8 \mathrm{~Hz}), 4.82(4 \mathrm{H}, \mathrm{d}, J=2.8 \mathrm{~Hz})$, $2.97(6 \mathrm{H}, \mathrm{s}) ;{ }^{13} \mathrm{C}$ NMR $\left(\mathrm{CDCl}_{3}, 100 \mathrm{MH}\right)$ ) $\delta 152.7,143.6,131.5,131.0,129.6,127.3,120.1$, $60.2,41.0$

Experimental Procedure for Tetraidodie 92. To $1.0 \mathrm{I} \mathrm{g}$ of Tetrabromide $s y n-90(1.17 \mathrm{mmol})$ in $50 \mathrm{~mL}$ dry THF at $-78^{\circ} \mathrm{C}$ was added dropwise $3.2 \mathrm{~mL}$ of a $1.6 \mathrm{M}$ solution of $n$-butyllithium $(5.12$ 
mmol) in hexanes. After $2 \mathrm{~h}$ at $-78^{\circ} \mathrm{C}$, a solution of $1.48 \mathrm{~g}$ of iodine $(5.83 \mathrm{mmol})$ in $50 \mathrm{~mL}$ of THF was added dropwise. Then the reaction mixture was stirred at room temperature overnight. Distilled water (100 mL) was added, and the reaction mixture was extracted with diethyl ether (3 $\times 50 \mathrm{~mL})$ and dichloromethane $(100 \mathrm{~mL})$. The combined organic layers were dried over magnesium sulfate and concentrated. The crude residue was purified by flash column chromatography (silica gel/ dichloromethane:hexanes $=1: 4)$ to produce $0.86 \mathrm{~g}$ of $92(0.82$ mmol, 70\%). Recrystallization of the residue from dichloromethane/hexanes produced a single crystal of 92 suitable for X-ray structure analysis: ' $\mathrm{H}$ NMR (CDCl, $400 \mathrm{MHz}) \delta 7.42(8 \mathrm{H}, \mathrm{d}, . J$ $=8.8 \mathrm{~Hz}), 6.67(8 \mathrm{H}, \mathrm{d}, J=8.4 \mathrm{~Hz}), 6.06(4 \mathrm{H}, \mathrm{d}, J=3.6 \mathrm{~Hz}), 4.77(4 \mathrm{H}, \mathrm{d}, J=2.8 \mathrm{~Hz}) ;{ }^{13} \mathrm{C}$ NMR $\left(\mathrm{CDCl}_{3}, 150 \mathrm{MHz}\right) \delta 152.1,143.3,137.1,131.0,129.5,128.0,91.4,60.4,40.5$.

Experimental Procedure for Fused Nanostructures Dimers 93 and 94 and Trimers 95a, 95b and 95c. To an oven-dried $2 \mathrm{~L}$-flask was added $1.26 \mathrm{~g}$ of tetrabromide $s y n-90$ (1.46 mmol) and $1.00 \mathrm{~g}$ of 2,2'-bipyridyl (6.42 mmol). Then the flask was flushed with nitrogen and moved into a glove box, where I.64 $\mathrm{g}$ of bis(1,5-cyclooctadiene)nickel(0) (6.42 mmol) was added. The flask was fitted with a condenser, rubber septa, and then removed from the glove box before $1500 \mathrm{~mL}$ of THF was transferred via cannula under a nitrogen atmosphere. Then the reaction mixture was heated at reflux for $15 \mathrm{~h}$. After the reaction mixture was allowed to cool to room temperature, it was passed through a short silica gel column and eluted with dichloromethane. The combined eluents were concentrated, and the residue was purified by flash column chromatography (silica gel, dichloromethane:hexanes $=1: 5$ to $2: \mathrm{I})$ to produce $0.039 \mathrm{~g}$ of $93(0.036 \mathrm{mmol}, 5 \%$ yield $)$ as a white solid, and $0.008 \mathrm{~g}$ of $94(0.007 \mathrm{mmol}, 1 \%$ yield $)$ as a white solid. At $50 \mathrm{mM}$ concentration, $1.96 \mathrm{~g}$ of tetrabromide $s y n-90$ produced $6 \mathrm{mg}$ of $95 \mathrm{a}(0.004 \mathrm{mmol}, 0.5 \%$ yield) as a white solid, $12 \mathrm{mg}$ of $95 \mathrm{~b}(0.007 \mathrm{mmol}, 1 \%$ yield $)$ as a white solid, and $14 \mathrm{mg}$ of $95 \mathrm{c}(0.009 \mathrm{mmol}, 1 \%$ 
yield) as a white solid were isolated. 93: ' $\mathrm{H}$ NMR $\left(\mathrm{CDCl}_{3}, 400 \mathrm{MHz}\right) \delta 6.94(8 \mathrm{H}, \mathrm{m}), 6.68(16$ $\mathrm{H}, \mathrm{d}, J=8.0 \mathrm{~Hz}), 6.54(16 \mathrm{H}, \mathrm{d}, J=8.0 \mathrm{~Hz}), 5.17(8 \mathrm{H}, \mathrm{m}), 3.83(12 \mathrm{H}, \mathrm{s}) ;{ }^{13} \mathrm{C} \mathrm{NMR}\left(\mathrm{CDCl}_{3}\right.$, $100 \mathrm{MHz}) \delta 151.4,139.8,139.7,134.0,132.1,127.7,126.8,62.0,38.3$; HRMS (ESI) calcd for $\mathrm{C}_{89} \mathrm{H}_{60} \mathrm{O}_{4}\left(\mathrm{M}^{+}\right)$I084.4486, found 1084.4509. Recrystallization of $\mathbf{9 3}$ from dichloromethane/hexanes produced a single crystal suitable for $\mathrm{X}$-ray structure analysis. 94: 'H NMR $\left(\mathrm{CDCl}_{3}, 400 \mathrm{MHz}\right) \delta 6.71(8 \mathrm{H}, \mathrm{m}), 6.50(16 \mathrm{H}, \mathrm{d}, J=8.4 \mathrm{~Hz}), 6.44(16 \mathrm{H}, \mathrm{d}, J=8.4 \mathrm{~Hz})$, $5.09(8 \mathrm{H}, \mathrm{m}), 3.86(12 \mathrm{H}, \mathrm{s})$; HRMS (ESI) calcd for $\mathrm{C}_{80} \mathrm{H}_{60} \mathrm{O}_{4}\left(\mathrm{M}^{+}\right)$1084.4486, found 1084.4494. 95a: 'H NMR (CDCl, $600 \mathrm{MHz}) \delta 7.24(12 \mathrm{H}, \mathrm{d}, J=8.0 \mathrm{~Hz}), 6.92(12 \mathrm{H}, \mathrm{d}, J=8.0$ H7), $6.1 \mathrm{I}(\mathrm{I} 2 \mathrm{H}, \mathrm{d}, J=2.4 \mathrm{~Hz}), 4.79(12 \mathrm{H}, \mathrm{d}, J=2.4 \mathrm{~Hz}), 3.71(18 \mathrm{H}, \mathrm{s}) ;{ }^{13} \mathrm{C}$ NMR $\left(\mathrm{CDCl}_{3}, \mathrm{I} 50\right.$ MHz) $\delta 152.7,142.8,138.3,130.7,127.8,127.4,126.5,60.2,40.5$; HRMS (ESI) calcd for $\mathrm{C}_{120} \mathrm{H}_{91} \mathrm{O}_{6}\left(\mathrm{MH}^{+}\right) 1627.6810$, found $1627.6895 .95 \mathbf{b}:{ }^{\prime} \mathrm{H} \mathrm{NMR}\left(\mathrm{CDCl}_{3}, 600 \mathrm{MHz}\right) \delta 7.33(8 \mathrm{H}, \mathrm{d}$, $J=8.0 \mathrm{~Hz}), 7.14(8 \mathrm{H}, \mathrm{d}, J=8.4 \mathrm{~Hz}), 7.05(8 \mathrm{H}, \mathrm{d}, J=8.4 \mathrm{~Hz}), 6.91(8 \mathrm{H}, \mathrm{d}, J=8.0 \mathrm{~Hz}), 6.88(8$ $\mathrm{H}, \mathrm{d}, J=8.4 \mathrm{~Hz}), 6.87(8 \mathrm{H}, \mathrm{d}, J=8.0 \mathrm{~Hz}), 6.84(4 \mathrm{H}, \mathrm{m}), 6.05(8 \mathrm{H}, \mathrm{t}, J=3.2 \mathrm{~Hz}), 5.24(4 \mathrm{H}$, $\mathrm{m}), 4.73(8 \mathrm{H}, \mathrm{t}, J=2.4 \mathrm{~Hz}), 3.75(12 \mathrm{H}, \mathrm{s}), 3.6 \mathrm{l}(6 \mathrm{H}, \mathrm{s}) ;{ }^{13} \mathrm{C} \mathrm{NMR}\left(\mathrm{CDCl}_{3}, 150 \mathrm{MHz}\right) \delta 152.9$, $152.8,151.3,143.2,142.8,140.6,138.0,137.8,137.5,133.9,132.9,130.8,129.9,127.8,127.7$, 127.6, 127.5, 126.4, I26.3, I26.2, 125.8, 61.8, 60.6, 59.7, 40.8, 40.6, 38,8; HRMS (ESI) calcd for $\mathrm{C}_{120} \mathrm{H}_{91} \mathrm{O}_{6}\left(\mathrm{MH}^{+}\right)$1627.6810; found 1627.6744. 95c: ' $\mathrm{H}$ NMR $\left(\mathrm{CDCl}_{3}, 600 \mathrm{MHz}\right) \delta 7.20(8$ $\mathrm{H}, \mathrm{d}, J=8.4 \mathrm{~Hz}), 7.09(8 \mathrm{H}, \mathrm{d}, J=8.4 \mathrm{~Hz}), 6.91(8 \mathrm{H}, \mathrm{d}, J=7.2 \mathrm{~Hz}), 6.89(8 \mathrm{H}, \mathrm{d}, J=8.4 \mathrm{~Hz})$, $6.88(8 \mathrm{H}, \mathrm{d}, J=8.4 \mathrm{~Hz}), 6.83(16 \mathrm{H}, \mathrm{m}), 5.98(4 \mathrm{H}, \mathrm{d}, J=2.4 \mathrm{~Hz}), 5.25(4 \mathrm{H}, \mathrm{m}), 5.23(4 \mathrm{H}, \mathrm{m})$, $4.68(4 \mathrm{H}, \mathrm{d}, J=2.4 \mathrm{~Hz}), 3.73(12 \mathrm{H}, \mathrm{s}), 3.66(6 \mathrm{H}, \mathrm{s}) ;{ }^{13} \mathrm{C} \mathrm{NMR}\left(\mathrm{CDCl}_{3}, 150 \mathrm{MHz}\right) \delta 152.9$, $151.6,143.4,140.6,140.4,138.6,137.7,137.4,134.2,133.8,133.0,130.4,127.70,127.65$, $127.58,127.23,126.8,126.2,126.1,125.6,61.9,60.0,40.9,38.8,38.7$; HRMS (ESI) calcd for $\mathrm{C}_{120} \mathrm{H}_{91} \mathrm{O}_{6}\left(\mathrm{MH}^{+}\right) 1627.6810$ found 1627.6744. 
Experimental Procedure for Boronic Ester 96. To a mixture of $3.64 \mathrm{~g}$ of diene $\mathbf{4 8}$ (10 mmol), $6.09 \mathrm{~g}$ of bis(pinacolato)diboron $(24 \mathrm{mmol}), 5.89 \mathrm{~g}$ of potassium acetate $(60 \mathrm{mmol}), 3 \mathrm{I} 0 \mathrm{mg}$ of tris(dibenzylideneacetone)dipalladium(0)-chloroform adduct $(0.3 \mathrm{mmol})$ and $238 \mathrm{mg}$ of XPhos (0.5 mmol) under a Argon atmosphere was added $300 \mathrm{~mL}$ of anhydrous dioxane. Then the reaction mixture was heated at $80^{\circ} \mathrm{C}$ for $12 \mathrm{~h}$. After the reaction was complete, the reaction mixture was allowed to cool to room temperature and filtered through a short pad of silica gel, washed with diethyl ether $100 \mathrm{~mL}$. The combined organic solvents were collected and concentrated via high vacuo. The solid was washed with hexanes and to give $4.22 \mathrm{~g}$ of boronic ester 96 (9.2 mmol, 92\%) as grey solid: 'H NMR (CDCl $3,400 \mathrm{MHz}) \delta 7.79(4 \mathrm{H}, \mathrm{d}, J=8.0 \mathrm{~Hz})$, $7.45(8 \mathrm{H}, \mathrm{d}, J=8.0 \mathrm{~Hz}), 7.03(2 \mathrm{H}, \mathrm{m}), 6.70(2 \mathrm{H}, \mathrm{m}), 1.36(24 \mathrm{H}, \mathrm{s}) ;{ }^{13} \mathrm{C} \mathrm{NMR}\left(\mathrm{CDCl}_{3}, 100\right.$ $\mathrm{MHz} \delta 139.9,135.1,133.3,130.1,125.7,83.7,24.8$.

Experimental Procedure for Diboronic ester 97. To a mixture of $4.58 \mathrm{~g}$ of 96 (10.0 mmol) and $4.72 \mathrm{~g}$ of dibromoquinone $89(10.0 \mathrm{mmol})$ in $200 \mathrm{~mL}$ of anhydrous dichloromethane under a nitrogen atmosphere was added by using a syringe $1.00 \mathrm{~mL}$ of boron trifluoride diethyl etherate $(8.10 \mathrm{mmol})$. The reaction mixture was stirred at room temperature for $20 \mathrm{~h}$. The reaction mixture was passed through a short silica gel column, washed with dichloromethane, and concentrated to afford a yellow solid. The crude solid was used without further purification. To the yellow solid and $5.52 \mathrm{~g}$ of potassium carbonate in dry acetone $(200 \mathrm{~mL})$ was added $2.40 \mathrm{~mL}$ of dimethyl sulfate $(25.4 \mathrm{mmol})$ via a syringe, and the reaction mixture was heated at reflux for 3 h. The reaction mixture was then allowed to cool to rt and concentrated. Distilled water ( $100 \mathrm{~mL})$ was added, and the reaction mixture was extracted with dichloromethane $(3 \times 50 \mathrm{~mL})$. The combined organic layers were dried over sodium sulfate and concentrated. Recrystallization of the crude solid from ether produced $5.25 \mathrm{~g}$ of $97(5.50 \mathrm{mmol}, 55 \%$ yield) as yellow solid: ' $\mathrm{H}$ 
$\operatorname{NMR}\left(\mathrm{CDCl}_{3}, 400 \mathrm{MHz}\right) \delta 7.6 \mathrm{I}(4 \mathrm{H}, \mathrm{d}, J=8.0 \mathrm{~Hz}), 7.18(4 \mathrm{H}, \mathrm{d}, J=8.4 \mathrm{~Hz}), 7.06(4 \mathrm{H}, \mathrm{d}, J=$ $8.4 \mathrm{~Hz}), 6.78(4 \mathrm{H}, \mathrm{d}, J=8.4 \mathrm{~Hz}), 6.12(2 \mathrm{H}, \mathrm{d}, J=3.6 \mathrm{~Hz}), 6.03(2 \mathrm{H}, \mathrm{d}, J=2.8 \mathrm{~Hz}), 4.87(2 \mathrm{H}$, $\mathrm{d}, J=2.8 \mathrm{~Hz}), 4.82(2 \mathrm{H}, \mathrm{d}, J=2.8 \mathrm{~Hz}), 3.46(6 \mathrm{H}, \mathrm{s}), 1.34(24 \mathrm{H}, \mathrm{s}) ;{ }^{3} \mathrm{C} \mathrm{NMR}\left(\mathrm{CDCl}_{3}, 100\right.$ $\mathrm{MHz}) \delta 152.3,147.3,142.5,134.7, \mathrm{I} 31.4,131.1,131.0,129.2,128.4,127.6,126.9,119.7,83.5$, $60.5,41.5,40.2,24.9,24.8$

Experimental Procedure for Vinyl Idodide 103. To a mixture of $3.48 \mathrm{~g}$ of 4trimethylsilylphenylacetylene $(20.0 \mathrm{mmol})$ and in $20 \mathrm{~mL}$ of anhydrous THF under a nitrogen atmosphere was added by using a syringe $24.0 \mathrm{~mL}$ of catecholborane. The reaction mixture was stirred for $12 \mathrm{~h}$ at $70^{\circ} \mathrm{C}$. Then the reaction mixture was concentrated under high vacuum. Distilled water $(20 \mathrm{~mL})$ was added to the solid residue. After the reaction mixture was stirred at $90^{\circ} \mathrm{C}$ for $2 \mathrm{~h}$, it was filtered and washed with cold water. Then the solid residue was dissolved in $50 \mathrm{~mL}$ of diethyl ether and cooled to $0^{\circ} \mathrm{C}$. $20 \mathrm{~mL}$ of $3 \mathrm{M}$ aqueous $\mathrm{NaOH}$ solution was added to the mixture, followed by addition of $6.10 \mathrm{~g}$ of iodine in $150 \mathrm{~mL}$ of diethyl ether. After $2 \mathrm{~h}$, the reaction mixture was added $100 \mathrm{~mL}$ of water and extracted with diethyl ether $(3 \times 40 \mathrm{~mL})$. The combined organic layers were washed with water, dried over sodium sulfate and concentrated. The residue was purified with silica gel column chromatography (hexanes as eluents) to give $4.23 \mathrm{~g}$ of vinyl iodide $\mathbf{1 0 3}$ (14 mmol, 70\%) as yellow liquid: ${ }^{1} \mathrm{H}$ NMR (CDCl $\left.3,400 \mathrm{MHz}\right) \delta 7.64$ $(2 \mathrm{H}, \mathrm{d}, J=8.4 \mathrm{~Hz}), 7.57(1 \mathrm{H}, \mathrm{d}, J=14.8 \mathrm{~Hz}), 7.39(2 \mathrm{H}, \mathrm{d}, J=8.0 \mathrm{~Hz}), 6.99(1 \mathrm{H}, \mathrm{d}, J=14.8$ $\mathrm{Hz}), 0.45(9 \mathrm{H}, \mathrm{s}) ;{ }^{13} \mathrm{C} \mathrm{NMR}\left(\mathrm{CDCl}_{3}, 100 \mathrm{MHz}\right) \delta 144.9,140.7,137.8,133.6,125.2,77.2,-1.13$. Experimental Procedure for TMS-Protected Diene 101. To a $500 \mathrm{~mL}$-flask were added 4.23 $\mathrm{g}$ of $103(14.0 \mathrm{mmol})$ and $2.94 \mathrm{~g}$ of 2,2'-bipyridyl $(16.8 \mathrm{mmol})$. The flask was flushed with nitrogen and placed in a glovebox under a nitrogen atmosphere before $4.62 \mathrm{~g}$ of $\mathrm{Ni}(\operatorname{cod})_{2}(\mathrm{I} 6.8$ mmol) was added. The flask was fitted with a condenser, rubber septa, and then removed from 
the glovebox before $150 \mathrm{~mL}$ of THF was introduced via cannula. The reaction mixture was heated at reflux for $48 \mathrm{~h}$ before it was allowed to cool to room temperature. Then the reaction mixture was passed through a short pad of silica gel and eluded with dichloromethane $(200 \mathrm{~mL})$. The combined eluents were concentrated, and the residue was purified by flash column chromatography (silica gel/hexanes), and then recrystallized with hexanes to produce $2.10 \mathrm{~g}$ of $101(6.0 \mathrm{mmol}, 85 \%$ yield $)$ as a white solid: ${ }^{1} \mathrm{H} \mathrm{NMR}\left(\mathrm{CDCl}_{3}, 400 \mathrm{MHz}\right) \delta 7.51(4 \mathrm{H}, \mathrm{d}, J=8.0$ $\mathrm{Hz}), 7.43(8 \mathrm{H}, \mathrm{d}, J=8.0 \mathrm{~Hz}), 7.00(2 \mathrm{H}, \mathrm{m}), 6.69(2 \mathrm{H}, \mathrm{m}), 0.29(18 \mathrm{H}, \mathrm{s}) ;{ }^{13} \mathrm{C} \mathrm{NMR}\left(\mathrm{CDCl}_{3}\right.$, $100 \mathrm{MHz}) \delta 140.0,137.7,133.7,132.9,129.5,125.7,-1.1$.

Experimental Procedure for Dibromide 104. To a mixture of $3.50 \mathrm{~g}$ of diene 101 (10.0 mmol), $4.70 \mathrm{~g}$ of dibromoquinone 89 ( $10.0 \mathrm{mmol})$ and $0.34 \mathrm{~g}$ of anhydrous $\mathrm{AlCl}_{3}(2.5 \mathrm{mmol}), 200 \mathrm{~mL}$ of anhydrous dichloromethane was added in glove box. The reaction mixiure was stirred at room temperature for $20 \mathrm{~h}$. The whole mixture was passed through a short pad of silica gel, washed with dichloromethane and concentrated to afford a yellow solid. The crude solid was used without further purification. To the yellow solid and $5.52 \mathrm{~g}$ of potassium carbonate $(40 \mathrm{mmol})$ in $200 \mathrm{ml}$ of dry acetone was added $2.4 \mathrm{~mL}$ of dimethyl sulfate $(25.4 \mathrm{mmol})$ via a syringe, and the reaction mixture was heated under reflux for $3 \mathrm{~h}$. After that the reaction mixture was allowed to cool to room temperature and concentrated. Then distilled water $(100 \mathrm{~mL})$ was added and the reaction mixture was extracted with dichloromethane $(3 \times 100 \mathrm{~mL})$. The combined organic layers were dried over sodium sulfate and concentrated. The residue was purified carefully by flash column chromatography (silica gel, dichloromethane:hexanes $=1: 4$ to $1: 2$ ) to produce 4.67 g of 104 (5.5 mmol, $55 \%$ yield) as a yellow solid: $\mathrm{mp} 96.6-97.9{ }^{\circ} \mathrm{C} ;{ }^{\prime} \mathrm{H} \mathrm{NMR}\left(\mathrm{CDCl}_{3}, 600 \mathrm{MHz}\right)$ $\delta 7.26(4 \mathrm{H}, \mathrm{d}, J=8.0 \mathrm{~Hz}), 7.17(4 \mathrm{H}, \mathrm{d}, J=8.8 \mathrm{~Hz}), 6.95(4 \mathrm{H}, \mathrm{d}, J=8.0 \mathrm{~Hz}), 6.79(4 \mathrm{H}, \mathrm{d}, J=$ $8.4 \mathrm{~Hz}), 6.11(2 \mathrm{H}, \mathrm{d}, J=3.6 \mathrm{~Hz}), 6.02(2 \mathrm{H}, \mathrm{d}, J=3.2 \mathrm{~Hz}), 4.81(2 \mathrm{H}, \mathrm{d}, J=2.8 \mathrm{~Hz}), 4.80(2 \mathrm{H}$, 
d, $J=2.8 \mathrm{~Hz}), 3.54(6 \mathrm{H}, \mathrm{s}), 0.23(18 \mathrm{H}, \mathrm{s}) ;{ }^{13} \mathrm{C} \mathrm{NMR}\left(\mathrm{CDCl}_{3}, \mathrm{I} 50 \mathrm{MHz}\right) \delta \mathrm{I} 22.3,144.6,142.7$, $137.4,133.1,131.4,131.0,130.6,129.2,128.2,127.7,119.7,60.3,41.2,40.2$

Experimental Procedure for Stepwise Synthesis of Fused Nanotube-like Dimer 93. To an oven-dried $1000 \mathrm{~mL}$ flask was added $0.57 \mathrm{~g}$ of Dibromide $104(0.67 \mathrm{mmol})$ and $0.25 \mathrm{~g} \mathrm{of} \mathrm{2,2'-}$ bipyridyl ( $0.44 \mathrm{mmol})$. Then the whole flask was flushed with nitrogen and moved into a glove box, where $0.44 \mathrm{~g}$ of bis(cyclooctadiene)nickel $(0)(1.60 \mathrm{mmol})$ was added and a water condenser was fitted. Outside of the glove box, $700 \mathrm{~mL}$ of THF was transferred under a nitrogen atmosphere. Then the reaction mixture was heated at reflux for I 5 hours. After the mixture was allowed to cool to room temperature, the reaction mixture was passed through a short pad of silica gel and flushed with dichloromethane. The combined solvents were concentrated and the residue was purified by flash column chromatography ( silica gel, dichloromethane:hexanes $=1: 6$ to $1: 3$ ) to produce $0.220 \mathrm{~g}$ of $\mathbf{1 0 5}$ mixture contaminated with a few trimers as a white solid, 0.137 g of $106(0.0074 \mathrm{mmol}, 30.0 \%$ yield $)$ as a white solid. One third of 105 mixture contaminated with trimers was dissolved in $20 \mathrm{~mL}$ of dichloromethane under a nitrogen atmosphere and cooled to $-78^{\circ} \mathrm{C}$. To the solution at $-78^{\circ} \mathrm{C}, 0.2 \mathrm{~mL}$ of a solution of $1 \mathrm{M} \mathrm{ICl}$ in dichloromethane was added dropwise. After 5 min at $-78^{\circ} \mathrm{C}$, the solution was quenched with a saturated sodium thiosulfate solution and diluted with $30 \mathrm{~mL}$ of water. The mixture was extracted with dichloromethane $(3 \times 20 \mathrm{~mL})$, dried over sodium sulfate and concentrated. To the crude mixture was added $0.4 \mathrm{l} \mathrm{g}$ of $2,2^{\prime}$-bipyridyl $(0.26 \mathrm{mmol})$ in a $250 \mathrm{~mL}$-flask. Then the whole flask was flushed with nitrogen and moved into a glove box, where $0.73 \mathrm{~g}$ of bis(cyclooctadiene)nickel( 0$)$ $(0.26 \mathrm{mmol})$ was added and a water condenser was fitted. The flask was then removed from the glove box and $100 \mathrm{~mL}$ of THF was added via cannula under a nitrogen atmosphere. Then the reaction mixture was heated at reflux for 15 hours. After the mixture was allowed to cool to 
room temperature, the reaction mixture was passed through a short silica gel column and eluted with dichloromethane. The combined eluents were concentrated, and the residue was purified by flash column chromatography (silica gel, dichloromethane:hexanes $=1: 5$ ) to produce $9 \mathrm{mg}$ of $\mathbf{9 3}$ (8.3\% yield from 104). 106: ' $\mathrm{H} \mathrm{NMR}\left(\mathrm{CDCl}_{3}, 600 \mathrm{MHz}\right) \delta 7.48(8 \mathrm{H}, \mathrm{d}, J=8.0 \mathrm{~Hz}), 7.32(8 \mathrm{H}$, $\mathrm{d}, J=8.0 \mathrm{~Hz}), 6.85(4 \mathrm{H}, \mathrm{m}), 6.64(8 \mathrm{H}, \mathrm{d}, J=8.4 \mathrm{~Hz}), 6.50(8 \mathrm{H}, \mathrm{d}, J=7.6 \mathrm{~Hz}), 6.00(4 \mathrm{H}, \mathrm{d}, J$ $=2.8 \mathrm{~Hz}), 5.00(4 \mathrm{H}, \mathrm{m}), 4.94(4 \mathrm{H}, \mathrm{d}, J=2.4 \mathrm{~Hz}), 4.80(2 \mathrm{H}, \mathrm{d}, J=2.8 \mathrm{~Hz}), 3.54(6 \mathrm{H}, \mathrm{s}), 0.25$ $(18 \mathrm{H}, \mathrm{s}) ;{ }^{13} \mathrm{C} \mathrm{NMR}\left(\mathrm{CDCl}_{3}, \mathrm{I} 50 \mathrm{MHz}\right) \delta 151.8,145.8,139.9,138.5,137.6,134.2,133.4,133.1$, $130.6,127.4,127.0,126.0,60.9,42.0,38.2,0.97 ; 105:{ }^{\prime} \mathrm{H} N M R\left(\mathrm{CDCl}_{3}, 600 \mathrm{MHz}\right) \delta 7.47(8 \mathrm{H}$, $\mathrm{d}, J=8.0 \mathrm{~Hz}), 7.34(8 \mathrm{H}, \mathrm{d}, J=8.0 \mathrm{~Hz}), 6.83(4 \mathrm{H}, \mathrm{m}), 6.67(8 \mathrm{H}, \mathrm{d}, J=8.8 \mathrm{~Hz}), 6.54(8 \mathrm{H}, \mathrm{br})$, $5.97(4 \mathrm{H}, \mathrm{d}, J=2.4 \mathrm{~Hz}), 5.02(4 \mathrm{H}, \mathrm{m}), 4.95(4 \mathrm{H}, \mathrm{d}, J=2.0 \mathrm{~Hz}), 3.46(6 \mathrm{H}, \mathrm{s}), 0.25(18 \mathrm{H}, \mathrm{s})$; ${ }^{13} \mathrm{C} \mathrm{NMR}\left(\mathrm{CDCl}_{3}, 150 \mathrm{MHz}\right) \delta 152.0,145.9,140.0,138.6,137.6,134.2,133.5,133.3,130.7$, $127.4,127.0,126.1,61.0,42.1,38.4,0.84$.

Experimental Procedure for Diboronic ester 107. To a mixture of $2.71 \mathrm{~g}$ of dibromide 104 (3.2 $\mathrm{mmol}), 1.79 \mathrm{~g}$ of bis(pinacolato)diboron $(7.0 \mathrm{mmol}), 1.90 \mathrm{~g}$ of potassium acetate $(7.0$ mmol), $30 \mathrm{mg}$ of tris(dibenzylideneacetone)dipalladium( 0$)$-chloroform adduct $(0.03 \mathrm{mmol})$ and $46 \mathrm{mg}$ of XPhos $(0.09 \mathrm{mmol})$ under a Argon atmosphere was added $200 \mathrm{~mL}$ of anhydrous dioxane. Then the reaction mixture was heated at $80^{\circ} \mathrm{C}$ for $12 \mathrm{~h}$. After the reaction was complete, the reaction mixture was allowed to cool to room temperature and filtered through a short silica gel column, washed with $100 \mathrm{~mL}$ of diethyl ether. The combined organic solvents were collected and concentrated via high vacuo. The solid was washed with hexanes to give 2.72 g of boronic ester 107 (2.9 mmol, 90\%) as white solid: ' $\mathrm{H}$ NMR (CDCl, $400 \mathrm{MHz}) \delta 7.55(4 \mathrm{H}$, $\mathrm{d}, J=8.0 \mathrm{~Hz}), 7.24(4 \mathrm{H}, \mathrm{d}, . J=8.0 \mathrm{~Hz}), 7.01(4 \mathrm{H}, \mathrm{d}, J=8.0 \mathrm{~Hz}), 6.96(4 \mathrm{H}, \mathrm{d}, J=8.0 \mathrm{~Hz}), 6.08$ $(2 \mathrm{H}, \mathrm{d}, J=3.6 \mathrm{~Hz}), 6.07(2 \mathrm{H}, \mathrm{d}, J=3.6 \mathrm{~Hz}), 4.86(2 \mathrm{H}, \mathrm{d}, J=2.8 \mathrm{~Hz}), 4.84(2 \mathrm{H}, \mathrm{d}, J=2.8 \mathrm{~Hz})$, 
$3.48(6 \mathrm{H}, \mathrm{s}), \mathrm{I} .30(24 \mathrm{H}, \mathrm{s}), 0.22(18 \mathrm{H}, \mathrm{s}) ;{ }^{3} \mathrm{C} \mathrm{NMR}\left(\mathrm{CDCl}_{3}, 100 \mathrm{MHz}\right) \delta 152.5,147.3,144.5$, $137.1,134.7,133 . \mathrm{I}, \mathrm{I} 3 \mathrm{I} .3,130.8,128.0,127.8, \mathrm{I} 26.8,83.4,60.4,41.2,4 \mathrm{I} . \mathrm{I}, 24.9,24.8,-1.0$.

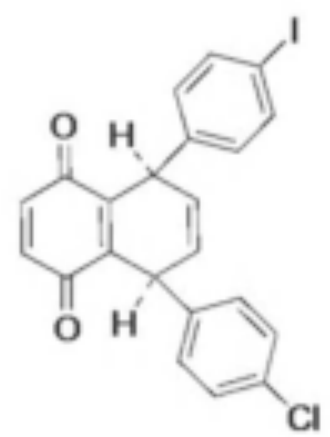

Experimental Procedure for Unsymmetrical Quinone. To a mixture of $3.67 \mathrm{~g}$ of 1-(4chlorophenyl)-4-(4-iodophenyl)-1,3-butadiene (10.0 mmol) and $1.30 \mathrm{~g}$ of 1,4-benzoquinone $(12.0 \mathrm{mmol}$ ) in $200 \mathrm{~mL}$ of anhydrous dichloromethane under a nitrogen atmosphere was added by using a syringe $1.00 \mathrm{~mL}$ of boron trifluoride diethyl etherate $(8.10 \mathrm{mmol})$. The reaction mixture was stirred at room temperature for $20 \mathrm{~h}$. The reaction mixture was then passed through a short silica gel column, washed with dichloromethane, and concentrated to afford a brown/yellow solid. The crude solid was used without further purification.

To the brown/yellow solid and $2.78 \mathrm{~g}$ of silver oxide (12.0 mmol) under a nitrogen atmosphere was added $100 \mathrm{~mL}$ of anhydrous diethyl ether. The reaction mixture was stirred overnight and passed through a short silica gel column, washed with diethyl ether, and concentrated. The residue was recrystallized from diethyl ether to afford $3.54 \mathrm{~g}$ of unsymmetric quinone (7.50 mmol, 75\% yield) as a brown solid: ' $\mathrm{H}$ NMR $\left(\mathrm{CDCl}_{3}, 600 \mathrm{MHz}\right) \delta 7.63(2 \mathrm{H}, \mathrm{d}, J=8.4 \mathrm{~Hz})$, $7.28(2 \mathrm{H}, \mathrm{d}, J=8.4 \mathrm{~Hz}), 7.23(2 \mathrm{H}, \mathrm{d}, J=8.4 \mathrm{~Hz}), 7.05(2 \mathrm{H}, \mathrm{d}, J=8.4 \mathrm{~Hz}), 6.68(2 \mathrm{H}, \mathrm{s}), 5.91$ $(2 \mathrm{H}, \mathrm{d}, J=2.4 \mathrm{~Hz}), 4.75(1 \mathrm{H}, \mathrm{dm}, J=2.4 \mathrm{~Hz}), 4.72(\mathrm{I} \mathrm{H}, \mathrm{dm}, J=1.8 \mathrm{~Hz}) ;{ }^{13} \mathrm{C} \mathrm{NMR}\left(\mathrm{CDCl}_{3}\right.$, $150 \mathrm{MHz}) \delta 185.8,141.5,141.3,140.8,139.6,137.6,136.27,136.25,132.7,130.3,129.7$, $128.7,126.5,126.3,92.4,40.0,39.7$. 
Experimental Procedure for Disilane 108: To a mixture of $1.65 \mathrm{~g}$ of diene 101 (4.7 mmol), $1.85 \mathrm{~g}$ of the unsymmetrical quinone $(3.9 \mathrm{mmol})$ and $0.13 \mathrm{~g}$ of anhydrous $\mathrm{AlCl}_{3}(1.0 \mathrm{mmol}), \mathrm{I} 00$ $\mathrm{mL}$ of anhydrous dichloromethane was added in glove hox. The reaction mixture was stirred at room temperature for $20 \mathrm{~h}$. The whole mixture was passed through a short pad of silica gel, washed with dichloromethane and concentrated to afford a yellow solid. The crude solid was used without further purification. To the yellow solid and $2.15 \mathrm{~g}$ of potassium carbonate (I5.5 mmol) in $200 \mathrm{ml}$ of dry acetone was added $0.9 \mathrm{~mL}$ of dimethyl sulfate $(9.5 \mathrm{mmol})$ via a syringe, and the reaction mixture was heated under reflux for $3 \mathrm{~h}$. After that the reaction mixture was allowed to cool to room temperature and concentrated. Then distilled water $(100 \mathrm{~mL})$ was added and the reaction mixture was extracted with dichloromethane $(3 \times 100 \mathrm{~mL})$. The combined organic layers were dried over sodium sulfate and concentrated. The residue was purified carefully by flash column chromatography (silica gel, dichloromethane:hexanes $=1: 4$ to $1: 2$ ) to produce $1.75 \mathrm{~g}$ of $108\left(2.15 \mathrm{mmol}, 55 \%\right.$ yield) as a yellow solid: ${ }^{1} \mathrm{H}$ NMR ( $\left.\mathrm{CDCl}_{3}, 400 \mathrm{MHz}\right) \delta$ $7.39(2 \mathrm{H}, \mathrm{d}, J=8.4 \mathrm{~Hz}), 7.283(2 \mathrm{H}, \mathrm{d}, J=8.0 \mathrm{~Hz}), 7.279(2 \mathrm{H}, \mathrm{d}, J=8.0 \mathrm{~Hz}), 7.03(2 \mathrm{H}, \mathrm{d}, J=$ $8.4 \mathrm{~Hz}), 6.96(4 \mathrm{H}, \mathrm{d}, J=7.6 \mathrm{~Hz}), 6.86(2 \mathrm{H}, \mathrm{d}, J=8.4 \mathrm{~Hz}), 6.69(2 \mathrm{H}, \mathrm{d}, J=8.4 \mathrm{~Hz}), 6.12(2 \mathrm{H}$, $\mathrm{d}, J=3.6 \mathrm{~Hz}), 6.04(2 \mathrm{H}, \mathrm{d}, J=2.8 \mathrm{~Hz}), 4.83(2 \mathrm{H}, \mathrm{d}, J=2.8 \mathrm{~Hz}), 4.81(\mathrm{IH}, \mathrm{m}), 4.80(1 \mathrm{H}, \mathrm{t}, 3.6$ $\mathrm{Hz}), 3.551(3 \mathrm{H}, \mathrm{s}), 3.547(3 \mathrm{H}, \mathrm{s}), 0.256(9 \mathrm{H}, \mathrm{s}), 0.243(9 \mathrm{H}, \mathrm{s}) ;{ }^{13} \mathrm{C}$ NMR $\left(\mathrm{CDCl}_{3}, 100 \mathrm{MHz}\right) \delta$ $152.3,144.61,144.59,143.5,142.2,137.41,137.39,137.0,133.15,133.14,131.54,131.49$, $131.4,130.7,130.5,129.6,128.8,128.3,128.1,128.0,127.70,127.67,126.75,126.74,91.1,60.3$, $41.18,41.16,-0.94,-1.0$.

Experimental Procedure for Stepwise Synthesis of Fused Nanotube-like Trimer 95a. To an oven-dried $200 \mathrm{~mL}$-flask was added $1.42 \mathrm{~g}$ of dibromide $104(1.67 \mathrm{mmol})$ and $0.57 \mathrm{~g}$ of 2,2”bipyridyl (3.68 mmol). Then the flask was flushed with nitrogen and moved into a glove box, 
where $1.01 \mathrm{~g}$ of bis(1,5-cyclooctadiene)nickel $(0)(3.68 \mathrm{mmol})$ was added. The flask was fitted with a condenser, a rubber septa, and then removed from the glove box before $30 \mathrm{~mL}$ of THF was transferred via cannula under a nitrogen atmosphere. Then the reaction mixture was heated at reflux for $15 \mathrm{~h}$. After the reaction mixture was allowed to cool to room temperature, it was passed through a short silica gel column and flushed with dichloromethane. The combined eluents were concentrated, and the residue was purified by flash column chromatography (silica gel, dichloromethane:hexanes $=1: 5$ to $1: 1)$ to produce $132 \mathrm{mg}$ of $\mathbf{1 0 6}(0.096 \mathrm{mmol}, 12 \%$ yield $)$ as a white solid, $0.090 \mathrm{~g}$ of $\mathbf{1 0 5}(0.066 \mathrm{mmol}, 8 \%$ yield) as a white solid, $0.140 \mathrm{~g}$ of $\mathbf{1 1 1}(0.068$ mmol, 13\% yield) as a white solid and 110 (4\% NMR yield) as a white solid. The whole 110 mixture was dissolved in $20 \mathrm{~mL}$ of dichloromethane under a nitrogen atmosphere and cooled to $-78^{\circ} \mathrm{C}$. To the solution at $-78^{\circ} \mathrm{C}, 0.15 \mathrm{~mL}$ of a solution of $1 \mathrm{M} \mathrm{ICl}$ in dichloromethane was added dropwise. After $5 \mathrm{~min}$ at $-78^{\circ} \mathrm{C}$, the solution was quenched with a saturated sodium thiosulfate solution and diluted with $30 \mathrm{~mL}$ of water. The mixture was extracted with dichloromethane $(3 \times 20 \mathrm{~mL})$, dried over sodium sulfate, and concentrated. To the crude mixture was added $30 \mathrm{mg}$ of $2,2^{\prime}$-bipyridyl $(0.19 \mathrm{mmol})$ in a $250 \mathrm{~mL}$-flask. Then the whole flask was flushed with nitrogen and moved into a glovebox, where $50 \mathrm{mg}$ of bis(1,5-cyclooctadiene)nickel $(0)(0.19 \mathrm{mmol})$ was added and a water condenser was fitted. The flask was then removed from the glove box and $100 \mathrm{~mL}$ of THF was added via cannula under a nitrogen atmosphere. Then the reaction mixture was heated at reflux for 15 hours. After the mixture was allowed to cool to room temperature, the reaction mixture was passed through a short silica gel column and eluted with dichloromethane. The combined eluents were concentrated. The crude 'H NMR spectrum clearly showed the major product was the compound 95a. 111: 'H NMR (CDCl $3,600 \mathrm{MHz}) \delta 7.37(4 \mathrm{H}, \mathrm{d}, J=8.4 \mathrm{~Hz}), 7.34(4 \mathrm{H}, \mathrm{d}, J=8.4 \mathrm{~Hz}), 7.33$ 
$(4 \mathrm{H}, \mathrm{d}, J=8.4 \mathrm{~Hz}), 7.26(4 \mathrm{H}, \mathrm{d}, J=8.4 \mathrm{~Hz}), 7.21(4 \mathrm{H}, \mathrm{d}, J=8.0 \mathrm{~Hz}), 7.13(4 \mathrm{H}, \mathrm{d}, J=8.4 \mathrm{~Hz})$,

$7.121(4 \mathrm{H}, \mathrm{d}, J=8.0 \mathrm{~Hz}), 7.117(4 \mathrm{H}, \mathrm{d}, J=8.4 \mathrm{~Hz}), 7.09(4 \mathrm{H}, \mathrm{d}, J=8.0 \mathrm{~Hz}), 6.08(4 \mathrm{H}, \mathrm{m})$, $4.90(8 \mathrm{H}, \mathrm{m}), 3.57(6 \mathrm{H}, \mathrm{s}), 3.55(6 \mathrm{H}, \mathrm{s}), 3.19(6 \mathrm{H}, \mathrm{s}), 0.18(18 \mathrm{H}, \mathrm{s}) ; 0.077(18 \mathrm{H}, \mathrm{s}) ; 0.009(18$ $\mathrm{H}, \mathrm{s}) ;{ }^{13} \mathrm{C}$ NMR $\left(\mathrm{CDCl}_{3}, 150 \mathrm{MHz}\right) \delta 152.9, \mathrm{I} 25.6,152.5,144.6,144.5,144.4,143.5,142.98$, $142.96,138.6,138.5,138.0,137.1,137.06,133.10,133.06,133.04,131.8,131.6,131.55,131.2$ $130.7,130.5,129.0,128.8,128.3,128.1,128.0,127.8,127.7,127.0,126.9,126.7,126.62$, $126.59,126.4,61.1,60.8,60.5,41.6,40.9,40.7,-1.00,-1.04,-1.09 ;$ HRMS (ESI) calcd for $\mathrm{C}_{138} \mathrm{H}_{145} \mathrm{O}_{6} \mathrm{Si}_{6}\left(\mathrm{MH}^{+}\right)$2065.9651, found 2065.9627. 110: ' $\mathrm{H} \mathrm{NMR}\left(\mathrm{CDCl}_{3}, 600 \mathrm{MHz}\right) \delta 7.44(12$ $\mathrm{H}, \mathrm{d}, J=8.4 \mathrm{~Hz}), 7.27(12 \mathrm{H}, \mathrm{d}, J=8.4 \mathrm{~Hz}), 7.18(12 \mathrm{H}, \mathrm{d}, J=7.8 \mathrm{~Hz}), 7.03(12 \mathrm{H}, \mathrm{d}, J=$ $8.4 \mathrm{~Hz}), 6.08(6 \mathrm{H}, \mathrm{d}, J=3.6 \mathrm{~Hz}), 6.00(6 \mathrm{H}, \mathrm{d}, J=3.2 \mathrm{~Hz}), 4.99(6 \mathrm{H}, \mathrm{d}, J=2.8 \mathrm{~Hz}), 4.96(6 \mathrm{H}, \mathrm{d}, J$ $=3.2 \mathrm{~Hz}), 3.22(18 \mathrm{H}, \mathrm{s}), 0.08(54 \mathrm{H}, \mathrm{s}) ;{ }^{13} \mathrm{C} \mathrm{NMR}\left(\mathrm{CDCl}_{3}, 150 \mathrm{MHz}\right) \delta 153.0,144.7,143.7$, I38.2, 137.2, 133.I, I32.0, 13I.4, 128.5, 128,4, I27.9, 127.2, 126.5, 6I.4, 4I.5, 41.4; HRMS (ESI) calcd for $\mathrm{C}_{138} \mathrm{H}_{145} \mathrm{O}_{6} \mathrm{Si}_{6}\left(\mathrm{MH}^{+}\right) 2065.9651$, found 2065.9652.

Experimental Procedure for Diboronic ester 112. To a mixture of $0.58 \mathrm{~g}$ of dibromide 55 ( 1.2 mmol), $0.71 \mathrm{~g}$ of bis(pinacolato)diboron $(2.77 \mathrm{mmol}), 0.72 \mathrm{~g}$ of potassium acetate $(6.9 \mathrm{mmol})$, $12 \mathrm{mg}$ of tris(dibenzylideneacetone)dipalladium( 0$)$-chloroform adduct $(0.01 \mathrm{mmol})$ and $30 \mathrm{mg}$ of XPhos $(0.08 \mathrm{mmol})$ under a Argon atmosphere was added $100 \mathrm{~mL}$ of anhydrous dioxane. Then the reaction mixture was heated at $80^{\circ} \mathrm{C}$ for $12 \mathrm{~h}$. After the reaction was complete, the reaction mixture was allowed to cool to room temperature and filtered through a short silica gel column, washed with diethyl ether $100 \mathrm{~mL}$. The combined organic solvents were collected and concentrated via high vacuo. The solid was washed with hexanes to give $0.65 \mathrm{~g}$ of boronic ester $112(1.1 \mathrm{mmol}, 95 \%)$ as white solid: 'H NMR ( $\left.\mathrm{CDCl}_{3}, 400 \mathrm{MH} 7\right) \delta 7.66(4 \mathrm{H}, \mathrm{d}, J=8.4 \mathrm{~Hz})$, $7.23(4 \mathrm{H}, \mathrm{d}, J=8.4 \mathrm{~Hz}), 6.71(2 \mathrm{H}, \mathrm{s}), 5.94(2 \mathrm{H}, \mathrm{d}, J=3.2 \mathrm{~Hz}), 4.88(2 \mathrm{H}, \mathrm{d}, J=2.8 \mathrm{~Hz}), 3.57$ 
$(6 \mathrm{H}, \mathrm{s}), 1.33(12 \mathrm{H}, \mathrm{s}), 1.33(12 \mathrm{H}, \mathrm{s}) ;{ }^{13} \mathrm{C}$ NMR $\left(\mathrm{CDCl}_{3}, 100 \mathrm{MHz}\right) \delta \mathrm{I} 5 \mathrm{I} .4,148.1,134.6$, $127.6,127.4,109.1,83.5,55.7,41.5,24.9,24.8$.

Experimental Procedures for Dibromide 113. To a mixture of $3.58 \mathrm{~g}$ of dibronic ester 112 (6.0 mmol), $10.2 \mathrm{~g}$ of 1-bromo-4-iodobenzene (36 mmol), $9.93 \mathrm{~g}$ of silver carbonate (36 mmol), 0.70 g of tetrakis(triphenylphosphine)palladium( 0$)(0.6 \mathrm{mmol})$ under a Argon atmosphere was added $200 \mathrm{~mL}$ of anhydrous THF. Then the reaction mixture was heated at reflux for $40 \mathrm{~h}$. After the reaction was complete, the reaction mixture was allowed to cool to room temperature and filtered through a short silica gel column, washed with $100 \mathrm{~mL}$ of diethyl ether. The combined organic solvents were collected and concentrated via high vacuo. The residue was purified by flash column chromatography (silica gel, dichloromethane:hexanes $=1: 5$ ) to produce $2.97 \mathrm{~g}$ of 113 (4.6 mmol, $76 \%$ yield) as a white solid: ' $\mathrm{H} \mathrm{NMR}\left(\mathrm{CDCl}_{3}, 400 \mathrm{MHz}\right) \delta 7.52(4 \mathrm{H}, \mathrm{d}, J=8.4 \mathrm{~Hz}$ ), $7.42(4 \mathrm{H}, \mathrm{d}, J=8.4 \mathrm{~Hz}), 7.41(4 \mathrm{H}, \mathrm{d}, J=8.4 \mathrm{~Hz}), 7.29(4 \mathrm{H}, \mathrm{d}, J=8.0 \mathrm{~Hz}), 6.77(2 \mathrm{H}, \mathrm{s}), 6.04$ $(2 \mathrm{H}, \mathrm{d}, J=3.2 \mathrm{~Hz}), 4.95(2 \mathrm{H}, \mathrm{d}, J=2.8 \mathrm{~Hz}), 3.64(6 \mathrm{H}, \mathrm{s}) ;{ }^{13} \mathrm{C} \mathrm{NMR}\left(\mathrm{CDCl}_{3}, 100 \mathrm{MHz}\right) \delta$ I5I.4, 144.4, 140.0, I37.3, I3I.7, 128.7, 128.5, I27.9, I27.5, 126.5, 121.I, I09.2, 55.8, 40.9.

Experimental Procedures for Cyclic Dimer 114. To an oven-dried $2000 \mathrm{~mL}$ flask was added $0.63 \mathrm{~g}$ of Dibromide $104(0.97 \mathrm{mmol})$ and $0.36 \mathrm{~g}$ of $2,2^{\prime}$-bipyridyl ( $\left.2.3 \mathrm{mmol}\right)$. Then the whole flask was flushed with nitrogen and moved into a glove box, where $0.64 \mathrm{~g}$ of bis(cyclooctadiene)nickel(0) (2.3 mmol) was added and a water condenser was fitted. Outside of the glove box, $1000 \mathrm{~mL}$ of THF was transferred under a nitrogen atmosphere. Then the reaction mixture was heated at reflux for 15 hours. After the mixture was allowed to cool to room temperature, the reaction mixture was passed through a short silica gel column and flushed with dichloromethane. The combined solvents were concentrated and the residue was purified by flash column chromatography (silica gel, dichloromethane:hexanes $=1: 6$ to $1: 3$ ) to produce 48 
mg of $114(0.05 \mathrm{mmol}, 10 \%$ yield $)$ as a white solid: ${ }^{1} \mathrm{H} \mathrm{NMR}\left(\mathrm{CDCl}_{3}, 400 \mathrm{MHz}\right) \delta 7.35(8 \mathrm{H}, \mathrm{d}$, $J=8.4 \mathrm{~Hz}), 7.21(8 \mathrm{H}, \mathrm{d}, J=8.4 \mathrm{~Hz}), 6.99(8 \mathrm{H}, \mathrm{d}, J=8.4 \mathrm{~Hz}), 6.92(4 \mathrm{H}, \mathrm{s}), 6.79(8 \mathrm{H}, \mathrm{d}, J=$ $8.0 \mathrm{~Hz}), 6.72(4 \mathrm{H}, \mathrm{dd}, J=4.0,2.0 \mathrm{~Hz}), 5.27(4 \mathrm{H}, \mathrm{m}), 3.88(6 \mathrm{H}, \mathrm{s}) ;{ }^{13} \mathrm{C} \mathrm{NMR}\left(\mathrm{CDCl}_{3}, 100\right.$ $\mathrm{MHz}) \delta 150.8, \mathrm{I} 4 \mathrm{I} .2,140.0,138.6,137.3132 .4,130.8,127.7,127.3,126.8,125.9,108.8,56.0$, 37.8. HRMS (ESI) calcd for $\mathrm{C}_{72} \mathrm{H}_{56} \mathrm{O}_{4}\left(\mathrm{M}^{+}\right)$984.4173, found 984.4184 .

Experimental Procedure for the Functionalized |10|CPP 115. To a 20-mL sealtube were added $0.020 \mathrm{~g}(0.02 \mathrm{mmol})$ of 114 and $0.025 \mathrm{~g}(0.12 \mathrm{mmol})$ of DDQ. The flask was flushed with nitrogen at $0{ }^{\circ} \mathrm{C}$ and then $3 \mathrm{~mL}$ of chlorobenzene was introduced by using a syringe. The reaction mixture was heated to $150^{\circ} \mathrm{C}$. After $4 \mathrm{~h}$ at $150^{\circ} \mathrm{C}$, the reaction mixture was allowed to cool to room temperature, diluted with $10 \mathrm{~mL}$ of dichloromethane and immediately passed through a basic aluminum oxide column ( $4 \mathrm{~cm}$ high, $2.5 \mathrm{~cm}$ in diameter). The column was eluded with an additional $100 \mathrm{~mL}$ of dichloromethane. The combined eluents were concentrated in vacuo immediately to afford $0.013 \mathrm{~g}$ of $115\left(0.013 \mathrm{mmol}, 65 \%\right.$ yield) as a yellow solid: ${ }^{1} \mathrm{H}$ NMR $\left(\mathrm{C}_{6} \mathrm{D}_{6}, 600 \mathrm{MHz}\right) \delta 7.49(8 \mathrm{H}, \mathrm{d}, J=8.4 \mathrm{~Hz}), 7.45(\mathrm{I} 6 \mathrm{H}, \mathrm{t}, J=8.4 \mathrm{~Hz}), 7.37(8 \mathrm{H}, \mathrm{d}, J=8.4 \mathrm{~Hz})$, $7.00(4 \mathrm{H}, \mathrm{s}), 6.70(4 \mathrm{H}, \mathrm{s}), 3.45(12 \mathrm{H}, \mathrm{s}) ;{ }^{13} \mathrm{C} \mathrm{NMR}\left(\mathrm{C}_{6} \mathrm{D}_{6}, 150 \mathrm{MHz}\right) \delta 151.0,143.8,139.2$, I38.2, 137.6, I36.2, 132.8.0, 127.2, I26.7, 124.9, I06.7, 55.1; HRMS (ESI) calcd for $\mathrm{C}_{72} \mathrm{H}_{52} \mathrm{O}_{4}$ $\left(\mathrm{M}^{+}\right) 980.3860$, found 980.3878 .

Experimental Procedure for Tetraboronic Ester 116. To a mixture of $1.50 \mathrm{~g}$ of tetrabromide $90(1.74 \mathrm{mmol}), 2.12 \mathrm{~g}$ of bis(pinacolato)diboron $(8.35 \mathrm{mmol}), 2.05 \mathrm{~g}$ of potassium acetale $(20.9$ mmol), $32 \mathrm{mg}$ of tris(dibenzylideneacetone)dipalladium(0)-chloroform adduct (0.035 mmol) and $83 \mathrm{mg}$ of XPhos $(0.17 \mathrm{mmol})$ under a Argon atmosphere was added $150 \mathrm{~mL}$ of anhydrous dioxane. Then the reaction mixture was heated at $80{ }^{\circ} \mathrm{C}$ for $12 \mathrm{~h}$. After the reaction was complete, the reaction mixture was allowed to cool to room temperature and filtered through a 
short silica gel column, washed with $100 \mathrm{~mL}$ of diethyl ether. The combined organic solvents were collected and concentrated via high vacuo. The solid was washed with hexanes to give 1.55 $\mathrm{g}$ of boronic ester $116(1.48 \mathrm{mmol}, 85 \%)$ as a white solid: ${ }^{1} \mathrm{H}$ NMR (CDCl $\left.3,400 \mathrm{MHz}\right) \delta 7.59(8$ $\mathrm{H}, \mathrm{d}, J=8.4 \mathrm{~Hz}), 7.09(8 \mathrm{H}, \mathrm{d}, J=8.4 \mathrm{~Hz}), 6.05(4 \mathrm{H}, \mathrm{d}, J=3.2 \mathrm{~Hz}), 4.92(4 \mathrm{H}, \mathrm{d}, J=2.8 \mathrm{~Hz})$, $3.25(6 \mathrm{H}, \mathrm{s}), 1.32(48 \mathrm{H}, \mathrm{s}) ;{ }^{13} \mathrm{C} \mathrm{NMR}\left(\mathrm{CDCl}_{3}, 100 \mathrm{MHz}\right) \delta 152.7,147.4,134.8,131.4,128.0$, $127.0,83.4,60.9,41.5,24.84,24.81$.

Experimental Procedure for Tetrabromide 117. To a mixture of $1.19 \mathrm{~g}$ of dibronic ester 116 (1.13 mmol), $3.84 \mathrm{~g}$ of 1-bromo-4-iodobenzene (13.6 mmol), $3.74 \mathrm{~g}$ of silver carbonate (13.6 mmol), $0.26 \mathrm{~g}$ of tetrakis(triphenylphosphine)palladium( 0$)(0.23 \mathrm{mmol})$ under an argon atmosphere was added $200 \mathrm{~mL}$ of anhydrous THF. Then the reaction mixture was heated at reflux for $40 \mathrm{~h}$. After the reaction was complete, the reaction mixture was allowed to cool to room temperature and filtered through a short silica gel column, washed with $100 \mathrm{~mL}$ of diethyl ether. The combined organic solvents were collected and concentrated via high vacuo. The residue was purified by flash column chromatography (silica gel, dichloromethane:hexanes $=1: 5$ to $1: 3)$ to produce $0.92 \mathrm{~g}$ of $117\left(0.79 \mathrm{mmol}, 70 \%\right.$ yield) as a white solid: ' $\mathrm{H}$ NMR ( $\mathrm{CDCl}_{3}, 400$ $\mathrm{MHz}) \delta 7.42(8 \mathrm{H}, \mathrm{d}, J=8.8 \mathrm{~Hz}), 7.25(8 \mathrm{H}, \mathrm{d}, J=8.0 \mathrm{~Hz}), 7.24(8 \mathrm{H}, \mathrm{d}, J=8.4 \mathrm{~Hz}), 7.04(8 \mathrm{H}$, d, $J=8.0 \mathrm{~Hz}), 6.15(4 \mathrm{H}, \mathrm{d}, J=2.8 \mathrm{~Hz}), 4.90(4 \mathrm{H}, \mathrm{d}, J=2.8 \mathrm{~Hz}), 3.64(6 \mathrm{H}, \mathrm{s}) ;{ }^{13} \mathrm{C}$ NMR $\left(\mathrm{CDCl}_{3}, 100 \mathrm{MHz}\right) \delta 152.5,143.6,139.7,137.3,131.7,131.1,128.4,128.04,127.97,126.4$, , 121.1, 60.4, 40.7. HRMS (ESI) calcd for $\mathrm{C}_{64} \mathrm{H}_{46} \mathrm{Br}_{4} \mathrm{O}_{2}\left(\mathrm{M}^{+}\right) 1164.0205,1165.0239,1166.0185$, 1167.0218 , found $1164.0239,1165.0191,1166.0219$, and 1167.0242 .

Experimental Pracedures for Diene 118. To a mixture of $1.52 \mathrm{~g}$ of dibronic ester 96 (3.32 mmol), $1.70 \mathrm{~g}$ of bromide $98(7.42 \mathrm{mmol}), 2.00 \mathrm{~g}$ of potassium carbonate $(14.6 \mathrm{mmol}), 0.12 \mathrm{~g}$ of tetrakis(triphenylphosphine)palladium( 0$)(0.10 \mathrm{mmol})$ under a Argon atmosphere was added 150 
$\mathrm{mI}$ of anhydrous THF and $50 \mathrm{~mL}$ of degased $\mathrm{H}_{2} \mathrm{O}$. Then the reaction mixture was heated at reflux for 40 h. After the reaction was complete, the hot reaction mixture was quickly filtered. $100 \mathrm{~mL}$ of diethyl ether and $50 \mathrm{~mL}$ of $\mathrm{H}_{2} \mathrm{O}$ was added to the mixture. Then the mixture was partitioned and extracted with $50 \mathrm{~mL}$ of diethyl ether and $100 \mathrm{~mL}$ of dichloromethane. The combined organic solvents were collected and concentrated via high vacuo. The residue was washed with water, diethyl ether and air dried to produce $1.17 \mathrm{~g}$ of 118 (2.32 mmol, $70 \%$ yield) as a yellow solid: 'H NMR $\left(\mathrm{CDCl}_{3}, 400 \mathrm{MHz}\right) \delta 7.61(8 \mathrm{H}, \mathrm{s}), 7.60(4 \mathrm{H}, \mathrm{d}, J=8.8 \mathrm{~Hz}), 7.52(4$ $\mathrm{H}, \mathrm{d}, y=8.4 \mathrm{~Hz}), 7.03(2 \mathrm{H}, \mathrm{m}), 6.73(2 \mathrm{H}, \mathrm{m}), 0.3 \mathrm{I}(18 \mathrm{H}, \mathrm{s}) ;{ }^{13} \mathrm{C} \mathrm{NMR}\left(\mathrm{CDCl}_{3}, 100 \mathrm{MHz}\right) \delta$ $141.0,140.2,139.4,136.5,133.9,132.4,129.4,127.3,126.8,126.2,-1.1$.

Experimental Procedures for Dibromide 120. To a mixture of $1.37 \mathrm{~g}$ of diene 118 (2.72 mmol), $1.21 \mathrm{~g}$ of quinone $89(2.56 \mathrm{mmol})$ and $0.085 \mathrm{~g}$ of anhydrous $\mathrm{AlCl}_{3}(0.64 \mathrm{mmol}), 100 \mathrm{~mL}$ of anhydrous dichloromethane was added in glovebox. The reaction mixture was stimed at room temperature for $20 \mathrm{~h}$. The whole mixture was passed through a short pad of silica gel, washed with dichloromethane and concentrated to afford a yellow solid. The crude solid was used without further purification. To the yellow solid and $1.41 \mathrm{~g}$ of potassium carbonate $(10.2 \mathrm{mmol})$ in $200 \mathrm{~mL}$ of dry acetone was added $0.6 \mathrm{~mL}$ of dimethyl sulfate $(6.4 \mathrm{mmol})$ via a syringe, and the reaction mixture was heated under reflux for $3 \mathrm{~h}$. After that the reaction mixture was allowed to cool to room temperature and concentrated. Then distilled water $(100 \mathrm{~mL})$ was added and the reaction mixture was extracted with dichloromethane $(3 \times 100 \mathrm{~mL})$. The combined organic layers were dried over sodium sulfate and concentrated. The residue was purified carefully by flash column chromatography (silica gel, dichloromethane:hexanes $=1: 4$ to $1: 3$ ) to produce 1.54 $\mathrm{g}$ of 119 (1.54 mmol, 60\% yield) as a yellow solid. To a mixture of $1.54 \mathrm{~g}$ of dibromide 119 (1.54 mmol), $0.80 \mathrm{~g}$ of bis(pinacolato)diboron $(3.16 \mathrm{mmol}), 0.9 \mathrm{~g}$ of potassium acetate $(9.24$ 
$\mathrm{mmol}$ ), $40 \mathrm{mg}$ of tris(dibenzylideneacetone)dipalladium( 0$)$-chloroform adduct $(0.03 \mathrm{mmol})$ and $60 \mathrm{mg}$ of XPhos $(0.15 \mathrm{mmol})$ under an argon atmosphere was added $100 \mathrm{~mL}$ of anhydrous dioxane. Then the reaction mixture was heated at $80^{\circ} \mathrm{C}$ for $12 \mathrm{~h}$. After the reaction was complete, the reaction mixture was allowed to cool to room temperature and filtered through a short silica gel column, washed with $100 \mathrm{~mL}$ of diethyl ether. The combined organic solvents were collected and concentrated via high vacuo. The residue was washed with hexanes to give the crude diboronic ester as a white solid. The crude solid was used without further purification. To a mixture of the crude dibronic ester, $2.6 \mathrm{I} \mathrm{g}$ of 1-bromo-4-iodobenzene (9.24 mmol), $2.56 \mathrm{~g}$ of silver carbonate $(9.24 \mathrm{mmol}), 0.35 \mathrm{~g}$ of tetrakis(triphenylphosphine)palladium $(0)(0.3 \mathrm{mmol})$ under an argon atmosphere was added $200 \mathrm{~mL}$ of anhydrous THF. Then the reaction mixture was heated at reflux for $40 \mathrm{~h}$. After the reaction was complete, the reaction mixture was allowed to cool to room temperature and filtered through a short silica gel column, washed with $100 \mathrm{~mL}$ of diethyl ether. The combined organic solvents were collected and concentrated via high vacuo. The residue was purified by flash column chromatography (silica gel, dichloromethane:hexanes $=1: 5)$ to produce $0.96 \mathrm{~g}$ of $120(0.83 \mathrm{mmol}, 54 \%$ yield $)$ as a white solid: ${ }^{1} \mathrm{H} \mathrm{NMR}\left(\mathrm{CDCl}_{3}, 400\right.$ MHz) $\delta 7.49(4 \mathrm{H}, \mathrm{d}, J=8.0 \mathrm{~Hz}), 7.40(4 \mathrm{H}, \mathrm{d}, J=8.4 \mathrm{~Hz}), 7.39(4 \mathrm{H}, \mathrm{d}, J=8.4 \mathrm{~Hz}), 7.29(4 \mathrm{H}$, $\mathrm{d}, J=8.4 \mathrm{~Hz}), 7.27(4 \mathrm{H}, \mathrm{d}, J=8.4 \mathrm{~Hz}), 7.26(4 \mathrm{H}, \mathrm{d}, J=7.8 \mathrm{~Hz}), 7.04(4 \mathrm{H}, \mathrm{d}, J=8.4 \mathrm{~Hz}), 7.03$ $(4 \mathrm{H}, \mathrm{d}, J=8.4 \mathrm{~Hz}), 6.18(2 \mathrm{H}, \mathrm{d}, J=3.2 \mathrm{~Hz}), 7.15(2 \mathrm{H}, \mathrm{d}, J=2.8 \mathrm{~Hz}), 7.39(4 \mathrm{H}, \mathrm{d}, J=8.4 \mathrm{~Hz})$, $4.90(4 \mathrm{H}, \mathrm{t}, J=2.8 \mathrm{~Hz}), 3.64(6 \mathrm{H}, \mathrm{s}), 0.32(18 \mathrm{H}, \mathrm{s}) ;{ }^{13} \mathrm{C} \mathrm{NMR}\left(\mathrm{CDCl}_{3}, 100 \mathrm{MHz}\right) \delta 152.5$, $143.7,143.2,141.2,139.8,138.8,138.4,137.3,133.7,131.7,131.2,130.9,128.5,128.0,127.96$, $127.90,126.6,126.5,126.2,121.0,60.4,40.73,40.69,-1.0$.

Experimental Procedures for Cyclic Dimer 121 and 122: To an oven-dried $1000 \mathrm{~mL}-\mathrm{flask}$ was added $0.96 \mathrm{~g}$ of dibromide $120(0.83 \mathrm{mmol})$ and $0.29 \mathrm{~g}$ of 2,2'-bipyridyl (1.83 mmol). Then 
the flask was flushed with nitrogen and moved into a glovebox, where $0.50 \mathrm{~g}$ of bis(1,5cyclooctadiene)nickel(0) ( $1.83 \mathrm{mmol})$ was added. The flask was fitted with a condenser, a rubber septa, and then removed from the glovebox hefore $830 \mathrm{mI}$ of THF was transferred via cannula under a nitrogen atmosphere. Then the reaction mixture was heated at reflux for $15 \mathrm{~h}$. After the reaction mixture was allowed to cool to room temperature, it was passed through a short silica gel column and eluted with dichloromethane. The combined eluents were concentrated, and the residue was purified by flash column chromatography (silica gel, dichloromethane:hexanes $=1: 5$ to $1: 2)$ to produce $0.126 \mathrm{~g}$ of $122(0.063 \mathrm{mmol}, 15 \%$ yield) as a white solid, and $0.120 \mathrm{~g}$ of 121 (0.060 mmol, $15 \%$ yield) as a white solid. Cyclic dimer 122: ${ }^{1} \mathrm{H}$ NMR $\left(\mathrm{CDCl}_{3}, 600 \mathrm{MHz}\right) \delta 7.56$ $(\mathrm{I} 6 \mathrm{H}, \mathrm{s}), 7.53(8 \mathrm{H}, \mathrm{d}, J=8.4 \mathrm{~Hz}), 7.37(8 \mathrm{H}, \mathrm{d}, J=8.4 \mathrm{~Hz}), 7.34(8 \mathrm{H}, \mathrm{d}, J=7.8 \mathrm{~Hz}), 7.22(8$ $\mathrm{H}, \mathrm{d}, J=8.4 \mathrm{~Hz}), 6.94(8 \mathrm{H}, \mathrm{d}, J=8.4 \mathrm{~Hz}), 6.80(4 \mathrm{H}, \mathrm{dd}, J=4.2, \mathrm{I} .8 \mathrm{~Hz}), 6.67(8 \mathrm{H}, \mathrm{d}, J=7.8$ $\mathrm{Hz}), 6.13(4 \mathrm{H}, \mathrm{d}, J=2.4 \mathrm{~Hz}), 5.13(4 \mathrm{H}, \mathrm{m}), 5.05(4 \mathrm{H}, \mathrm{d}, J=3.0 \mathrm{~Hz}), 3.61(\mathrm{I} 2 \mathrm{H}, \mathrm{s}), 0.28(36$ $\mathrm{H}, \mathrm{s}) ;{ }^{13} \mathrm{C} \mathrm{NMR}\left(\mathrm{CDCl}_{3}, 150 \mathrm{MHz}\right) \delta 151.7,|44.1| 41.4,140.8,139.7,138.9,138.8,$,138.6 , $137.2,133.8,133.7,132.2,130.9,128.1,127.79,127.73,127.3,127.1,126.7,126.4,125.8,61.1$, 41.5, 38.6, -1.1. Cyclic Dimer 121: 'H NMR $\left(\mathrm{CDCl}_{3}, 600 \mathrm{MHz}\right) \delta 7.56(16 \mathrm{H}, \mathrm{m}), 7.54(8 \mathrm{H}, \mathrm{d}, J$ $=8.4 \mathrm{~Hz}), 7.38(8 \mathrm{H}, \mathrm{d}, J=8.4 \mathrm{~Hz}), 7.33(8 \mathrm{H}, \mathrm{d}, J=8.4 \mathrm{~Hz}), 7.22(8 \mathrm{H}, \mathrm{d}, J=8.4 \mathrm{~Hz}), 6.94(8$ $\mathrm{H}, \mathrm{d}, J=8.4 \mathrm{~Hz}), 6.80(4 \mathrm{H}, \mathrm{dd}, J=4.8,2.4 \mathrm{~Hz}), 6.67(8 \mathrm{H}, \mathrm{d}, J=7.8 \mathrm{~Hz}), 6.13(4 \mathrm{H}, \mathrm{d}, J=3.0$ $\mathrm{Hz}), 5.12(4 \mathrm{H}, \mathrm{m}), 5.05(4 \mathrm{H}, \mathrm{d}, J=3.0 \mathrm{~Hz}), 3.61(12 \mathrm{H}, \mathrm{s}), 0.28(36 \mathrm{H}, \mathrm{s}) ;{ }^{13} \mathrm{C} \mathrm{NMR}\left(\mathrm{CDCl}_{3}\right.$, $150 \mathrm{MHz}) \delta 151.7,144.2,141.4,140.8,139.6,138.9,138.8,138.6,137.2,133.9,133.7,132.2$ $130.9,128.1,127.80,127.74,127.3,127.1,126.7,126.4,125.8,61.1,41.5,38.6,-1.08$. 


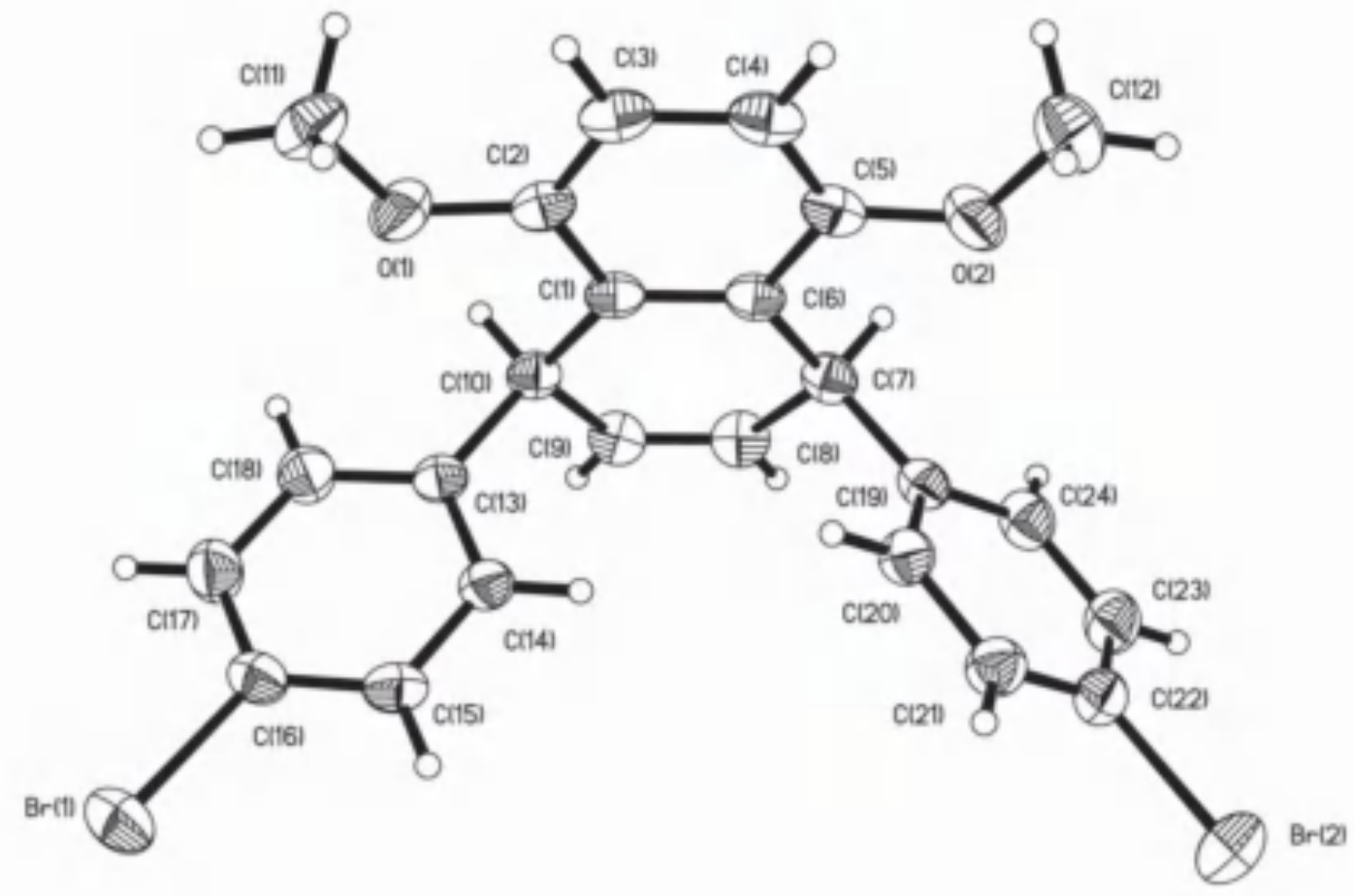

Figure S1. ORTEP Drawing of the Crystal Structure of Dibromide 55.

Perspective view of the molecular structure of $\mathbf{5 5}\left(\mathrm{C}_{24} \mathrm{H}_{20} \mathrm{Br}_{2} \mathrm{O}_{2}\right)$ with the atom labeling scheme provided for the non-hydrogen atoms. The thermal ellipsoids are scaled to enclosed $30 \%$ probability.

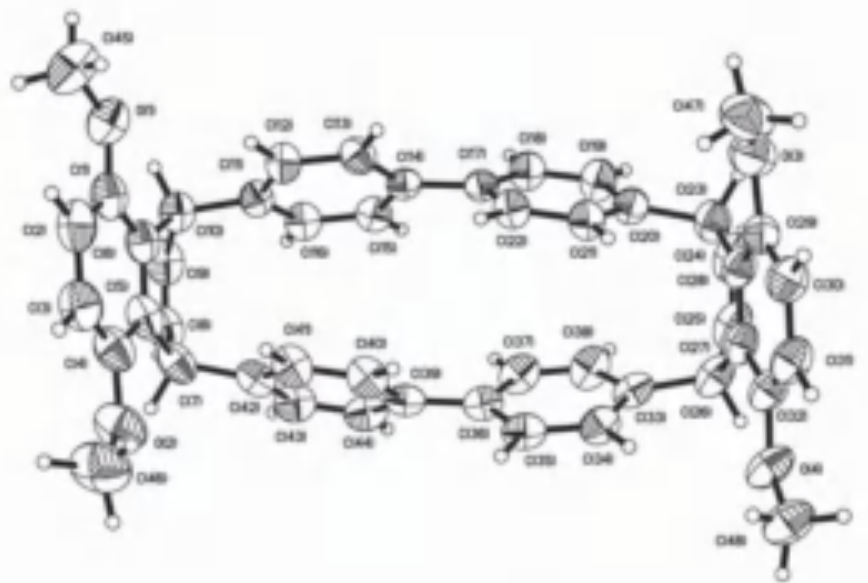


independent molecule 1

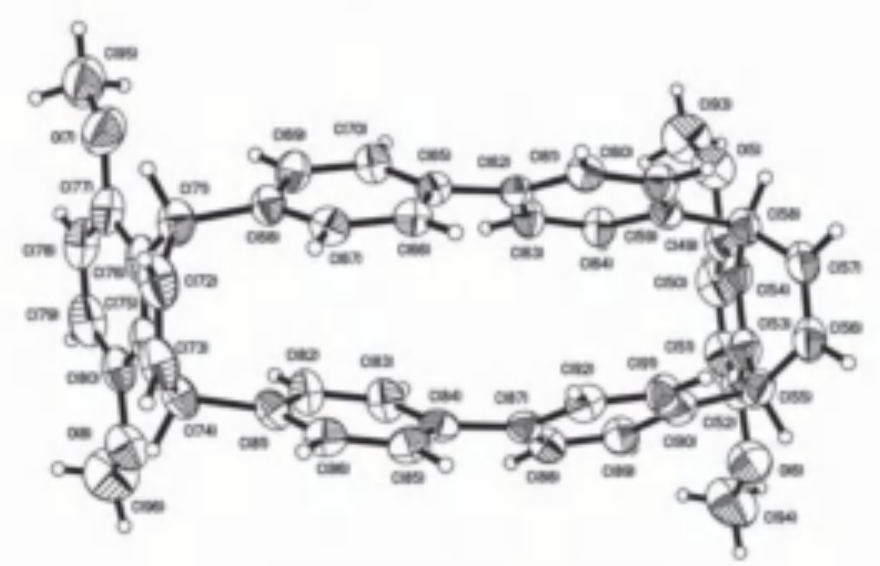

independent molecule 2

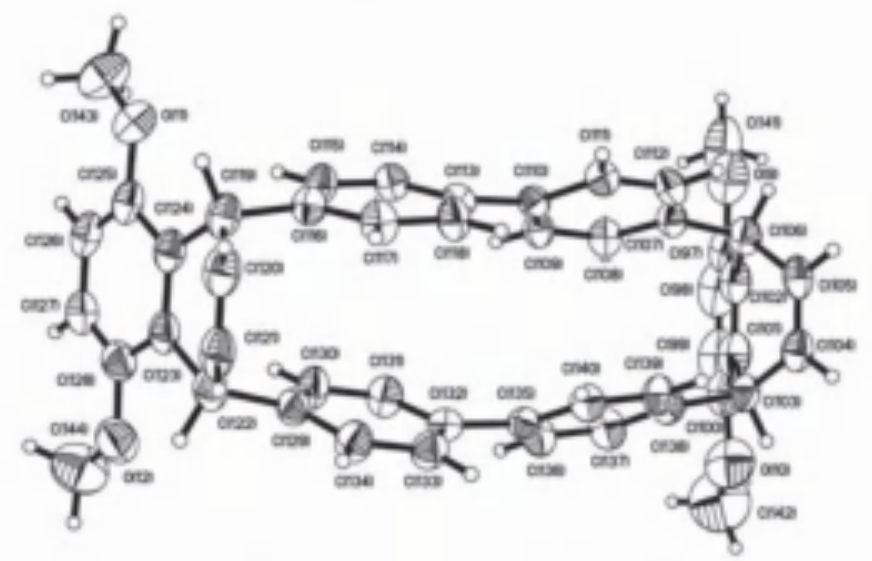

independent molecule 3

Figure S2. ORTEP Drawing of the Crystal Structure of the Cyclic Dimer syn-56a.

Perspective view of the molecular structure of syn-56a $\left(\mathrm{C}_{48} \mathrm{H}_{40} \mathrm{O}_{4}\right.$, three independent molecules] with the atom labeling scheme. The thermal ellipsoids are scaled to enclose $30 \%$ probability. 


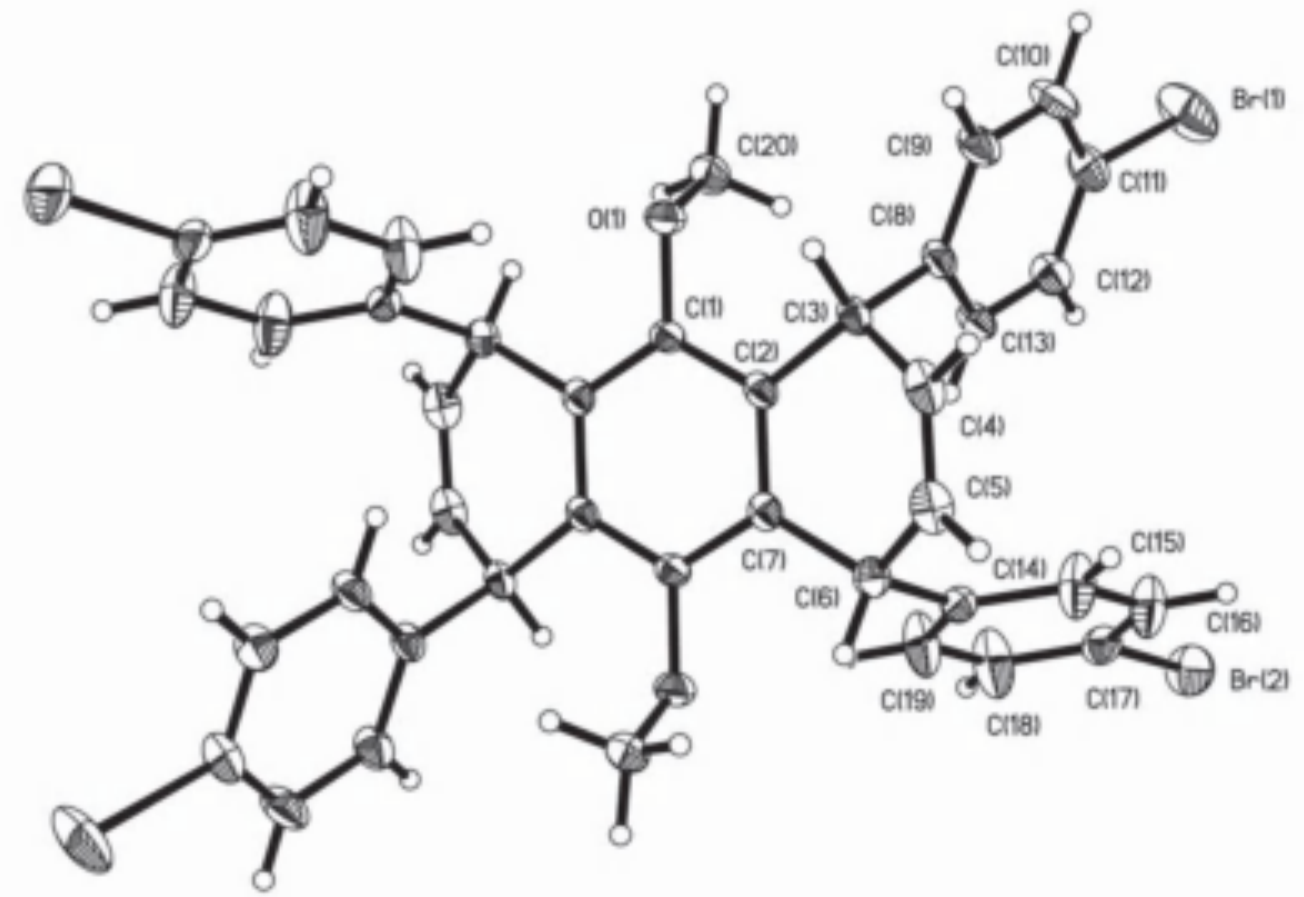

Figure S3. ORTEP Drawing of the Crystal Structure of anti-tetrabromide 91.

Perspective view of the molecular structure of anti-tetrabromide $\mathbf{9 1}$ $\left(\mathrm{C}_{40} \mathrm{H}_{30} \mathrm{Br}_{4} \mathrm{O}_{2}\right)$ with the atom labeling scheme for the independent non-hydrogen atoms. The molecule lies on a crystallographic center of inversion. The thermal ellipsoids are scaled to enclose $30 \%$ probability. 


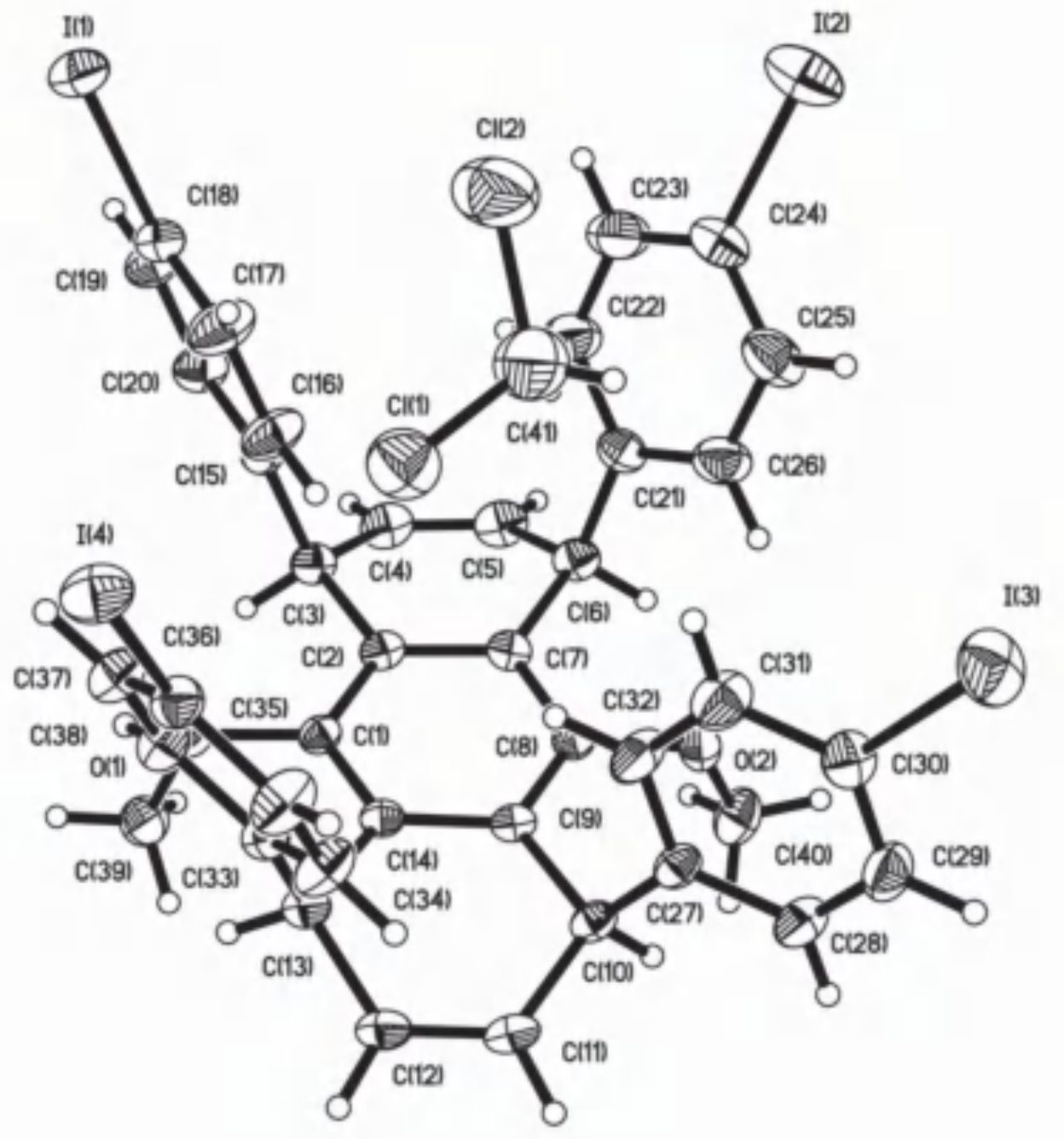

Figure S4. ORTEP Drawing of the Crystal Structure of syn-tetraiodide 92.

Perspective view of the molecular structure of syn-tetraiodide 92 $\left(\mathrm{C}_{40} \mathrm{H}_{30} \mathrm{I}_{4} \mathrm{O}_{2} \cdot \mathrm{CH}_{2} \mathrm{Cl}_{2}\right]$ with the atom labeling scheme for the independent nonhydrogen atoms. The thermal ellipsoids are scaled to enclose $30 \%$ probability. 


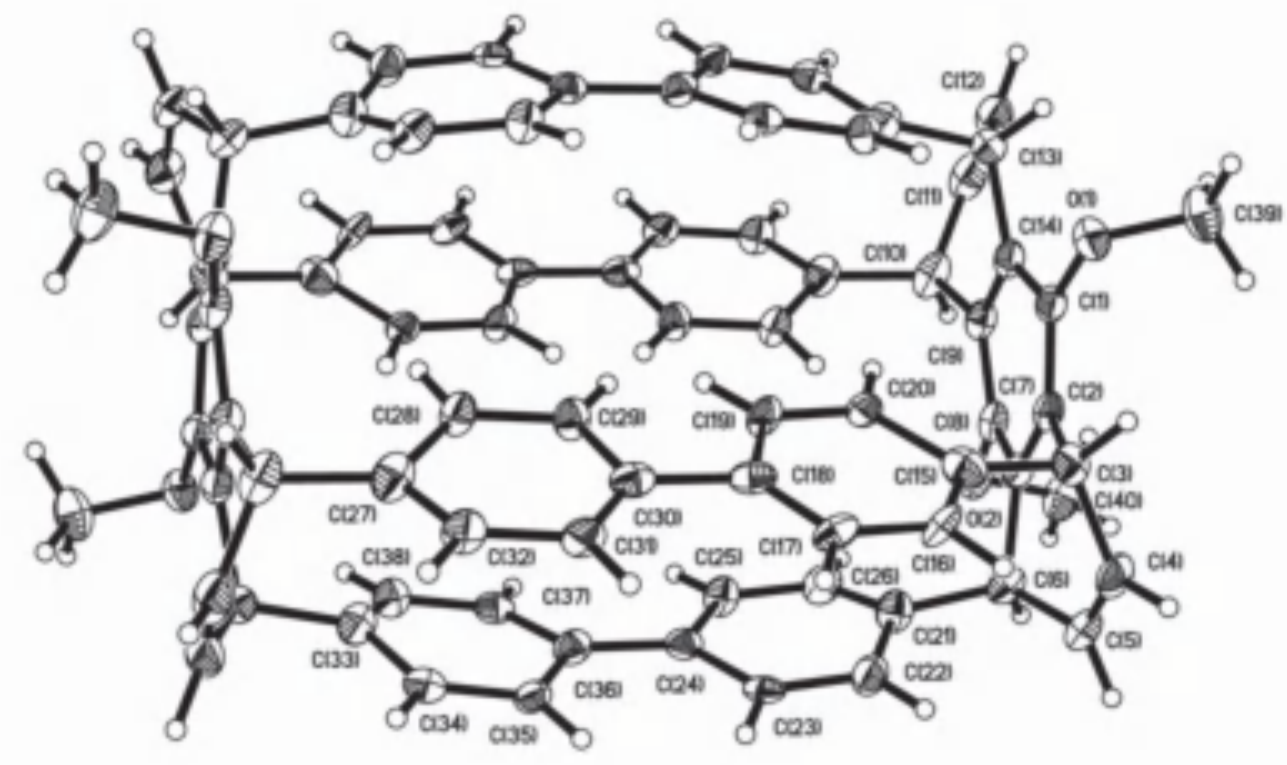

Figure S5. ORTEP Drawing of the Crystal Structure of fused nanohoops $\mathbf{9 3 .}$

Perspective view of the molecular structure of fused nanohoops $93\left(\mathrm{C}_{60} \mathrm{H}_{60} \mathrm{O}_{4}\right)$ with the atom labeling scheme for the independent non-hydrogen atoms. The thermal ellipsoids are scaled to enclose $50 \%$ probability. The molecule lies on a center of inversion. Each of the hiphenyl linkages exhibits a 50:50 two site rotational disorder. 


\section{Computational Details.}

Spin-restricted density functional theory (RDFT) calculations were performed with the Gaussian 03 (G03) program. ${ }^{\text {S2 }}$ Geometry optimization and normal mode analyses were performed using the B3LYP functional and the 6-31G(d) basis set. ${ }^{\mathrm{S}+\mathrm{S} 4}$ This functional-basis set combination was utilized in related studies, ${ }^{319.9}$ and reproduces the geometries of two X-ray crystallographic species characterized in the present study with reasonable accuracy (Table SI and S2). The identified stationary points met or exceeded all default G03 convergence criteria. Normal-modes were calculated for each optimized structure to confirm the absence of negative eigenvalues. 
Metrical Comparison of X-Ray and DFT Structure.

Table S1. Comparison of Experimental and Computational Metrical Parameters for the CPP Precursor Dibromide 55.

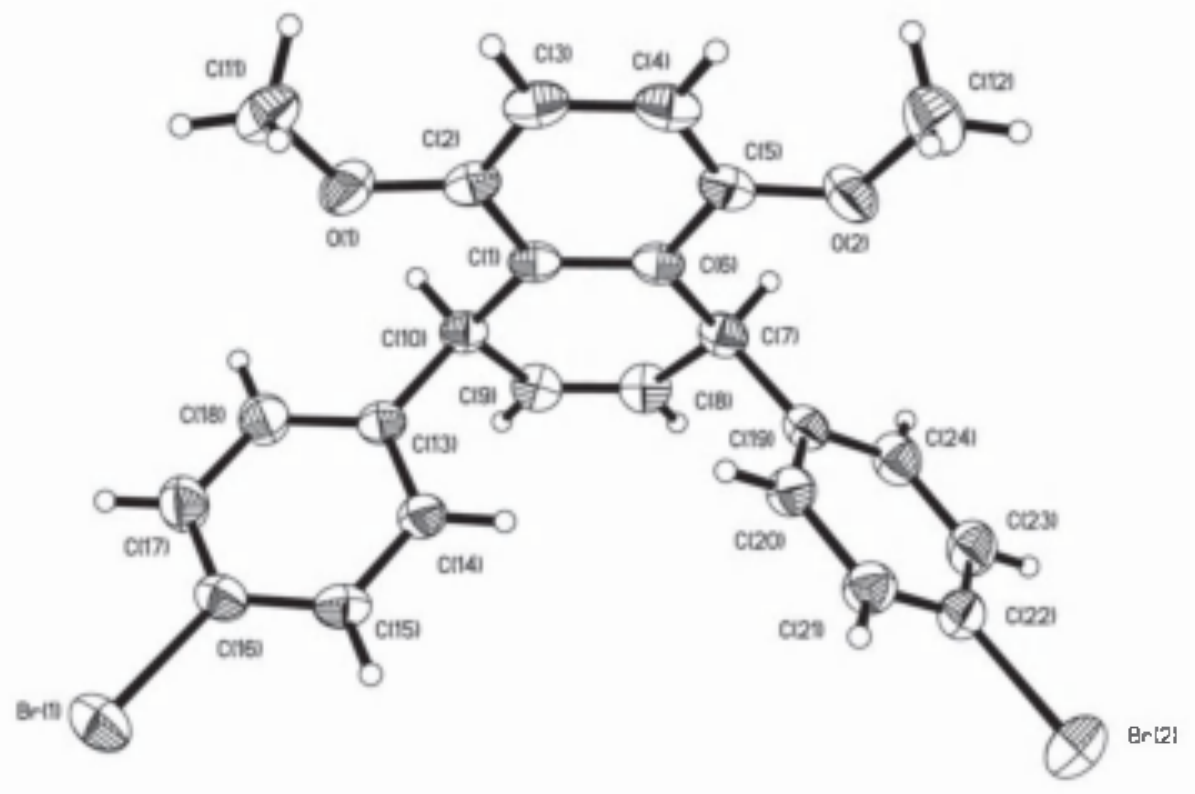

\begin{tabular}{|c|c|c|}
\hline & EXP (X-гау) & DFT (B3LYP/6-31G(d) \\
\hline C3-C4 (Ä) & $1.382(4)$ & 1.40 \\
\hline C4-C5 $(\AA)$ & $1.383(3)$ & 1.39 \\
\hline C1-C6 $(\AA)$ & $1.390(3)$ & 1.40 \\
\hline C6-C7 (A) & $1.520(3)$ & 1.52 \\
\hline C7-C8 $(\AA)$ & $1.507(3)$ & 1.51 \\
\hline $\operatorname{C8}-\mathrm{C9}(\AA)$ & $1.309(3)$ & 1.33 \\
\hline C7-C19 $[\AA]$ & $1.525(3)$ & 1.54 \\
\hline C7-C8-C9 $\left({ }^{\circ}\right)$ & $125.2(2)$ & 124.2 \\
\hline C6-C7-C19 ( & $115.0(2)$ & 114.5 \\
\hline C8-C7-C19 $\left.0^{\circ}\right)$ & $108.8(2)$ & 109.6 \\
\hline $\mathrm{C} 6-\mathrm{C} 7-\mathrm{C} 19-\mathrm{C} 20\left(^{\circ}\right)$ & $30.1(2)$ & 25.7 \\
\hline C1-C10-C13-C14 ( $\left.{ }^{\circ}\right)$ & $-58.2(2)$ & -68.2 \\
\hline
\end{tabular}


Table S2. Comparison of Experimental and Computational Metrical Parameters for the Cyclic Dimer syn-56a

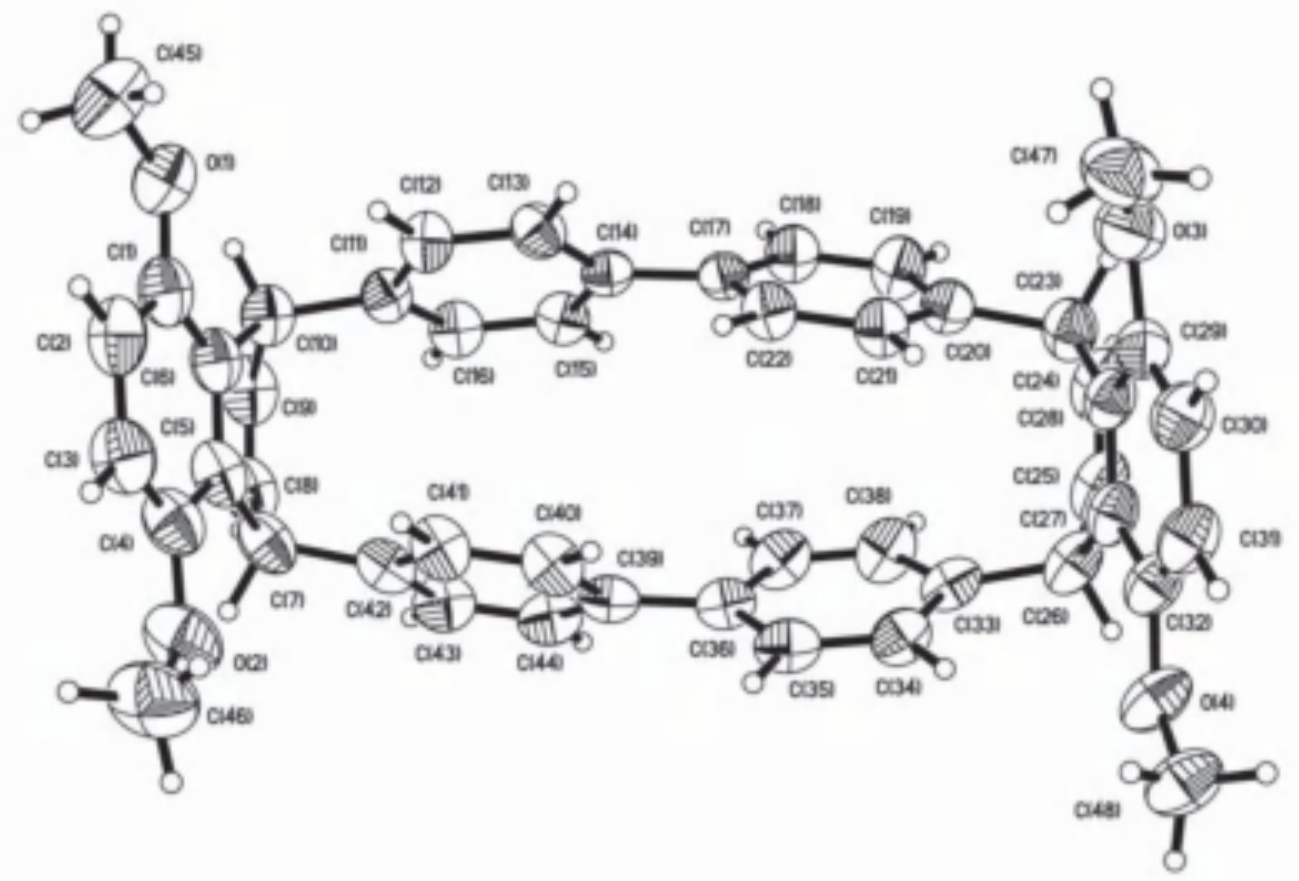

\begin{tabular}{|c|c|c|}
\hline & EXP (X-ray) & DFT (B3LYP/6-31G(d) \\
\hline $\mathrm{C} 2-\mathrm{C} 3(\AA)$ & $1.383(5)$ & 1.40 \\
\hline C3-C4 $(\AA)$ & $1.363(5)$ & 1.39 \\
\hline C5-C6 $(\AA)$ & $1.386(5)$ & 1.39 \\
\hline $\mathrm{C} 5-\mathrm{C} 7(\AA)$ & $1.504(5)$ & 1.53 \\
\hline $\operatorname{C7}-\operatorname{CB}(\AA)$ & $1.510(5)$ & 1.52 \\
\hline $\operatorname{Cg}-\operatorname{C9}(\AA)$ & $1.308(5)$ & 1.34 \\
\hline C7-C42 $(\AA)$ & $1.534(4)$ & 1.55 \\
\hline $\operatorname{C40-C41}(\AA)]$ & $1.386(4)$ & 1.40 \\
\hline $\operatorname{C36}-\operatorname{C39}(\AA)$ & $1.464(4)$ & 1.49 \\
\hline $\mathrm{C} 7-\mathrm{C} 8-\mathrm{C} 9\left(^{\circ}\right)$ & $120.6(4)$ & 121.4 \\
\hline $\mathrm{C} 5-\mathrm{C} 7-\mathrm{C} 42\left(^{\circ}\right)$ & $111.9(3)$ & 112.8 \\
\hline $\left.\mathrm{C} 8-\mathrm{C} 7-\mathrm{C} 42 \mathrm{O}^{\circ}\right)$ & $111.2(3)$ & 111.9 \\
\hline C6-C7-C19-C20 ( $)$ & $36.2(4)$ & 40.0 \\
\hline C1-C10-C13-C14 ( & $-13.4(4)$ & -6.8 \\
\hline C13-C14-C17-C22 (') & $-37.7(4)$ & -39.0 \\
\hline $\mathrm{C} 35-\mathrm{C} 38-\mathrm{C} 39-\mathrm{C} 40\left(^{\circ}\right)$ & $-38.5(4)$ & -39.0 \\
\hline
\end{tabular}




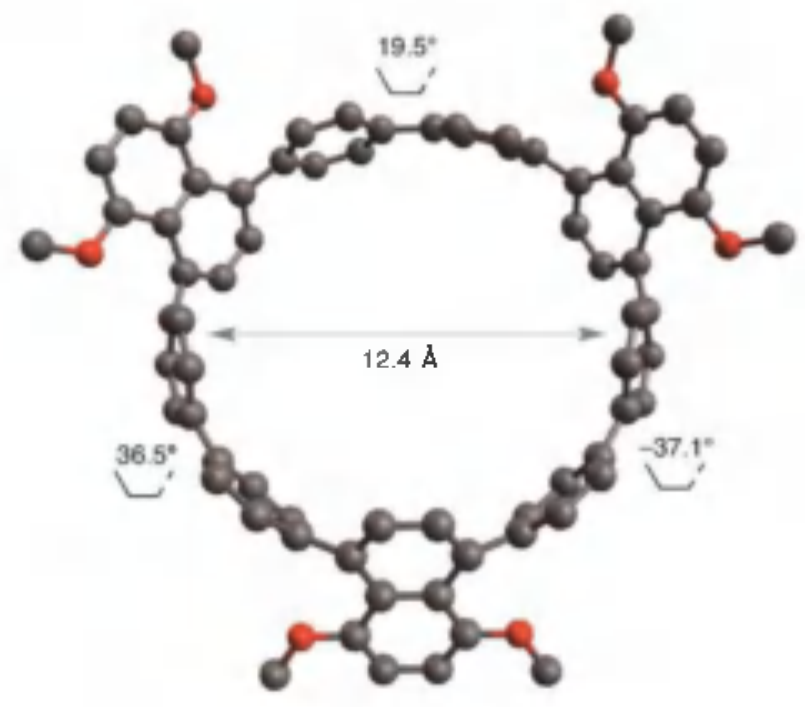

arial view

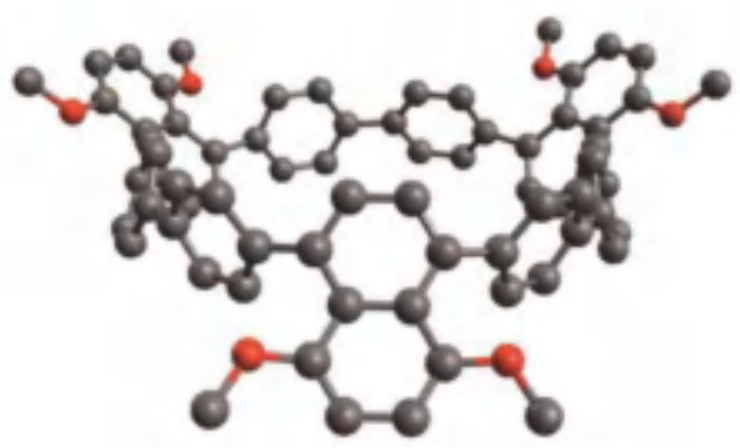

perspective view

Figure S6. Ball-and-stick model for the functionalized [9]CPP 65 derived from DFT with all aryl-aryl torsions on the top face noted. For clarity, hydrogen atoms were deleted.
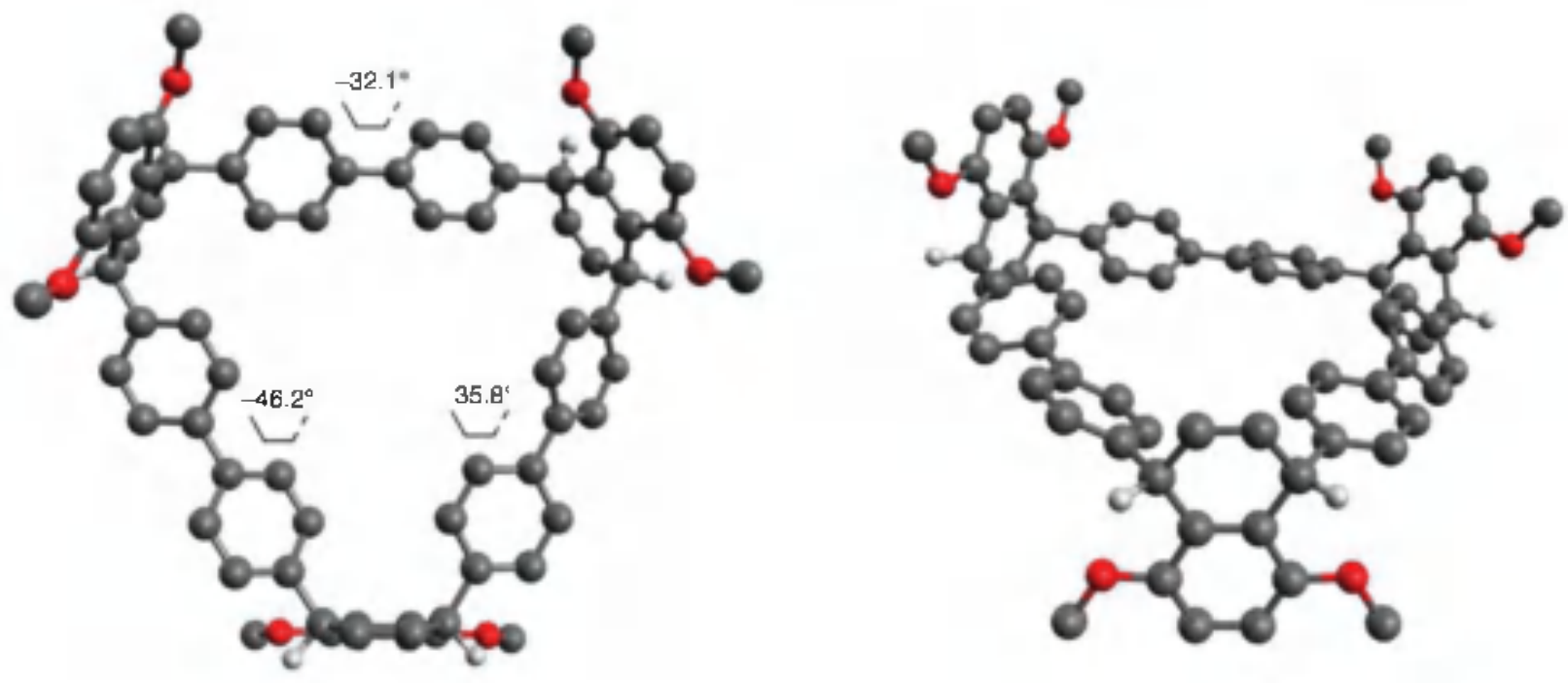

Figure S7. Ball-and-stick model for the cyclic trimer anti-64b derived from DFT with relevant metrical parameters highlighted. 
A

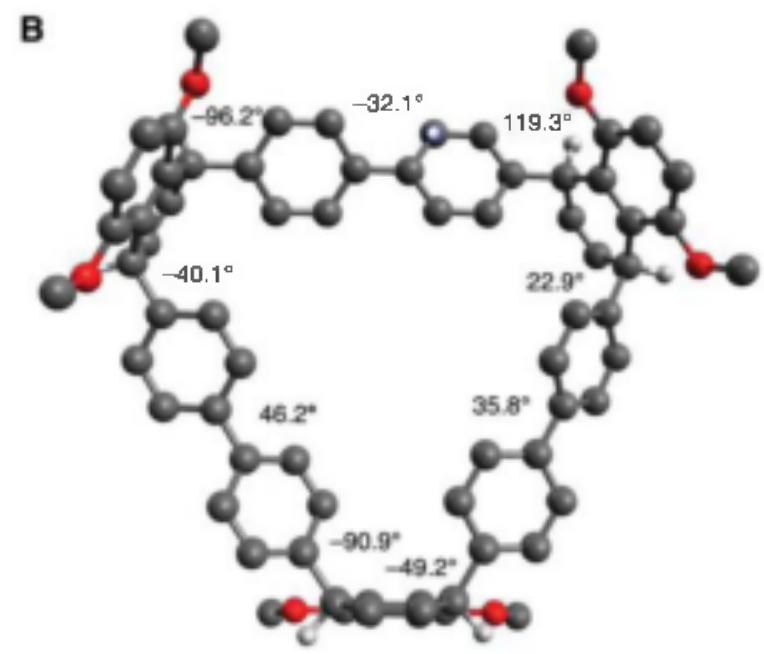

Figure S8. Aerial view of ball-and-stick models for 1 (A) and anti-64b (B) derived from DFT with aryl-aryl torsions noted. For clarity, hydrogen atoms were deleted. The dimethoxynaphthyl moieties cant away from the circle at angles of $133.8^{\circ}$ and $112.5^{\circ}$ on average for 65 and anti-64b, respectively. 


\section{Computational Coordinates and Energetic Details:}

\section{Dibromide 55}

\begin{tabular}{|c|c|c|c|}
\hline & -6.017920 & -0343981 & \\
\hline & & & \\
\hline & -6.774244 & & \\
\hline & -6.409726 & & \\
\hline & & & \\
\hline & & & \\
\hline & & & \\
\hline & $-5 B$ & & \\
\hline & & & \\
\hline & -8.5 & & \\
\hline & -6.5 & & \\
\hline & & & \\
\hline & $-6 ?$ & & \\
\hline & -7.7 & & \\
\hline & & & \\
\hline & -74 & & \\
\hline & & & \\
\hline & -7 & & \\
\hline & -9 & & \\
\hline & -7 & & \\
\hline & & & \\
\hline & -103 & & \\
\hline & & & \\
\hline & $-10 t$ & & \\
\hline & & & \\
\hline & -9.3 & & \\
\hline & -8.9 & & \\
\hline & -9.1 & & \\
\hline & -102 & & 967 \\
\hline & .11 .6 & & 2776 \\
\hline & & & 2.599101 \\
\hline & & & \\
\hline & & & \\
\hline & $-11,1$ & & \\
\hline & -120 & & 6982 \\
\hline & -12213878 & 88431 & 2.844194 \\
\hline & -10.806875 & & 6.219617 \\
\hline & -11.198456 & & \\
\hline & -11.376476 & & \\
\hline & & -2.344993 & 7.109906 \\
\hline
\end{tabular}

Calculation results at DFT-oplimized geomeiry:

Tolal Energy- -6219 80154331 AU

SCF Convergence- $0.2578 \mathrm{E}-08$

Three lowest frequencies $\left(\mathrm{cm}^{1}\right)$ : 12.4, 23.8, 295 


\section{Cyclic Dimer syn-64a}

\begin{tabular}{|c|c|c|c|}
\hline & -6.0 & -1397777 & \\
\hline & 933567 & 391161 & \\
\hline & 537049 & -0647822 & \\
\hline & -6.466337 & -0.754239 & $4 / 1550$ \\
\hline & -6413208 & 0641599 & \\
\hline & 99517 & 0.7465 & 85 \\
\hline & -6.849716 & -1.318248 & \\
\hline & -6.7 & & \\
\hline & -6.058002 & -2.762766 & 1.150240 \\
\hline & -5.851632 & 2.761172 & 1.491600 \\
\hline & -6.281693 & 3461656 & 26434 \\
\hline & .7345075 & 3282264 & 2854426 \\
\hline & -6.131291 & 4.5208 & \\
\hline & & & \\
\hline & & & \\
\hline & -6.04 & -34 & \\
\hline & -6.560867 & -4589 & 1.827 \\
\hline & -7664721 & -3294 & 23716 \\
\hline & -5.107480 & -13428 & -1.06030 \\
\hline & & & \\
\hline & & & \\
\hline & & & \\
\hline & -5488268 & -04662 & 7128 \\
\hline & -5.703229 & 1.4804 & 13937 \\
\hline & -5.77 & -09 & 0020 \\
\hline & -5.55 & & \\
\hline & & & \\
\hline & & & \\
\hline & -2.7 & -14 & \\
\hline & -29870 & -2.2 & 4666 \\
\hline & -1612007 & -2.516300 & 6534 \\
\hline & -1.42 & $-1.667 \subseteq$ & -2.1 \\
\hline & -3.2 & & \\
\hline & & & \\
\hline & & & \\
\hline & -0.8 & -13 & -3 \\
\hline & -3.5 & 186 & 3720 \\
\hline & -0.69 & 22 & -0. \\
\hline & -2.6 & & -1 \\
\hline & -2.7 & & \\
\hline & -1.3 & & \\
\hline & & & \\
\hline & -34 & 26 & -28 \\
\hline & -3.18 & 1.2 & 1074 \\
\hline & -0.7 & & 282 \\
\hline & -1.0 & & -2 s \\
\hline & 0.7 & & \\
\hline & & & \\
\hline & & & \\
\hline & 1.42 & 1.6 & -2.1 \\
\hline & 2.795401 & & -2.183468 \\
\hline & 2.98 & 2.2 & 1666 \\
\hline & 1.16 & & 58 \\
\hline & 0.82 & & \\
\hline & & & \\
\hline & & & \\
\hline & 069 & -2.20 & -0930598 \\
\hline & 3.51 & -1.86 & -0853719 \\
\hline$c$ & 1343087 & -1.778 & 3704 \\
\hline C & 14948 & -2.5 & -203 \\
\hline & 2.877 & & \\
\hline & & & 70783 \\
\hline & & & 1.102282 \\
\hline & 1.0291 & -2.9228 & -2939953 \\
\hline & & & -287 \\
\hline & 3,1826 & -1.221074 & 1.174074 \\
\hline & & & -1.060308 \\
\hline & & & \\
\hline
\end{tabular}

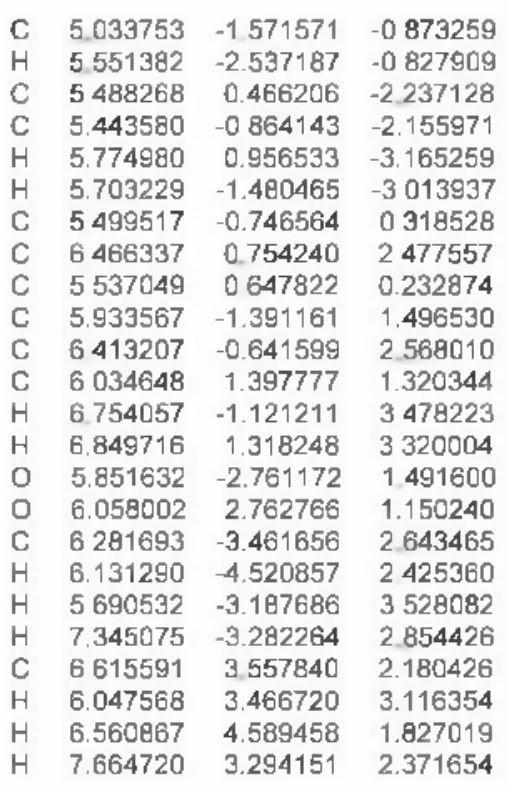

Calculation results al DFT-optimized geometry: Tolal Energy- -2153.98051611 AU SCF Convergence- 0.3572E-08 Three lowest frequencies $\left(\mathrm{cm}{ }^{1}\right.$ ): $20.4,22.1,299$ 


\section{Functionalized [9]CPP 65}

\begin{tabular}{|c|c|c|c|}
\hline C & 3.518389 & 5.396719 & 2.078322 \\
\hline C & 4.524148 & 4.429280 & 2.062281 \\
\hline$c$ & 4.713752 & 3.585033 & 0951908 \\
\hline C & 3.956143 & 3.881783 & -0.198809 \\
\hline C & 2.973194 & 4.860779 & -0.193280 \\
\hline $\mathrm{C}$ & 2673251 & 5.584960 & 0973408 \\
\hline c & 0.713076 & 7.034147 & 0.062442 \\
\hline C & 1326642 & 6.216322 & 1.074170 \\
\hline c & 0. 509077 & 5.712400 & 2075021 \\
\hline$c$ & -0.889164 & 5.718594 & 1.950403 \\
\hline $\mathrm{C}$ & -1517320 & 6243277 & 0.829901 \\
\hline C & -0.724204 & 7.061665 & -0050558 \\
\hline C & -3.800350 & 5.322600 & 1451999 \\
\hline$c$ & -2.834277 & 5.638852 & 0.481442 \\
\hline C & -2.996264 & 5.068870 & -0.794461 \\
\hline$c$ & -3.973610 & 4.112850 & -1.036759 \\
\hline C & -4.843645 & 3678870 & -0.019071 \\
\hline C & -4.783414 & 4363436 & 1207521 \\
\hline C & -6.040 & 1.961153 & -1.449133 \\
\hline C & -6.347708 & 0.620307 & -1.677101 \\
\hline C & -6.205651 & -0.340978 & -0.662228 \\
\hline C & -5.901363 & 0.126692 & 0628812 \\
\hline C & -5589778 & 1.461997 & 0854237 \\
\hline C & -5.573494 & 2397899 & -0196985 \\
\hline$c$ & -5122633 & -2070890 & -1981587 \\
\hline C & -4.423378 & -3285462 & -2.029247 \\
\hline C & -4.602325 & -4266850 & -1.065179 \\
\hline C & -5.739474 & -4137935 & -0.191621 \\
\hline C & -6.455741 & -2885671 & -0.138929 \\
\hline C & -6025627 & -1.789477 & -0966384 \\
\hline$c$ & -1.361064 & -6.107498 & -1.720799 \\
\hline C & -0.735935 & -6.00 & -0.465395 \\
\hline C & -1.543964 & -5.629003 & 0.622941 \\
\hline C & -2.958405 & -5215 & 0441 \\
\hline C & -3431489 & -5162079 & -0841583 \\
\hline C & -2679685 & -569 & -1.904516 \\
\hline$c$ & 3419756 & -5265638 & 0.289853 \\
\hline c & 2.868955 & -5.067920 & -0.988697 \\
\hline C & 1.558688 & -5.437355 & -1.269762 \\
\hline C & 0.738844 & -6031741 & -0.291735 \\
\hline C & 1.350383 & -6389412 & 0.922580 \\
\hline C & 2661397 & -6010972 & 1208390 \\
\hline c & 4.956774 & -240 & 2052 \\
\hline C & 5.950623 & -1.949758 & 1.194853 \\
\hline C & 6.474587 & -2894089 & 0.240709 \\
\hline C & 5757236 & -4122285 & -0011893 \\
\hline C & 4558105 & -4407679 & 0729781 \\
\hline C & 4.283718 & -3610362 & 1831826 \\
\hline C & 5.688435 & 1.660041 & 2256668 \\
\hline C & 6.025752 & 0.310197 & 2.320748 \\
\hline C & 6.137398 & -0.472031 & 1.158099 \\
\hline C & 6. 094397 & 0.213778 & -0070030 \\
\hline C & 5.782858 & 1.566139 & -0.131653 \\
\hline C & 5468578 & 2.305937 & 1.025839 \\
\hline C & 7.715352 & -2.669737 & -0.446598 \\
\hline C & B. 172431 & -3.571079 & -1384569 \\
\hline C & 7. 457639 & -4.757877 & -1.651602 \\
\hline C & 6294102 & -5047416 & -0970972 \\
\hline 0 & 5.608677 & -6220338 & -1.114168 \\
\hline 0 & 8 411046 & -1555590 & -0069198 \\
\hline C & 9.642722 & -1.277046 & -0.706453 \\
\hline C & 6.098059 & -7.184885 & -2.026009 \\
\hline C & 1.490366 & 7.954478 & -0.823695 \\
\hline C & 0.873668 & 8.631893 & -1.781082 \\
\hline C & -0.533548 & 8.672136 & -1879961 \\
\hline C & -1.321503 & 7928878 & -1.026565 \\
\hline 0 & 2.840497 & 7.840297 & \\
\hline C & 3.662010 & 8 636951 & -1447610 \\
\hline
\end{tabular}

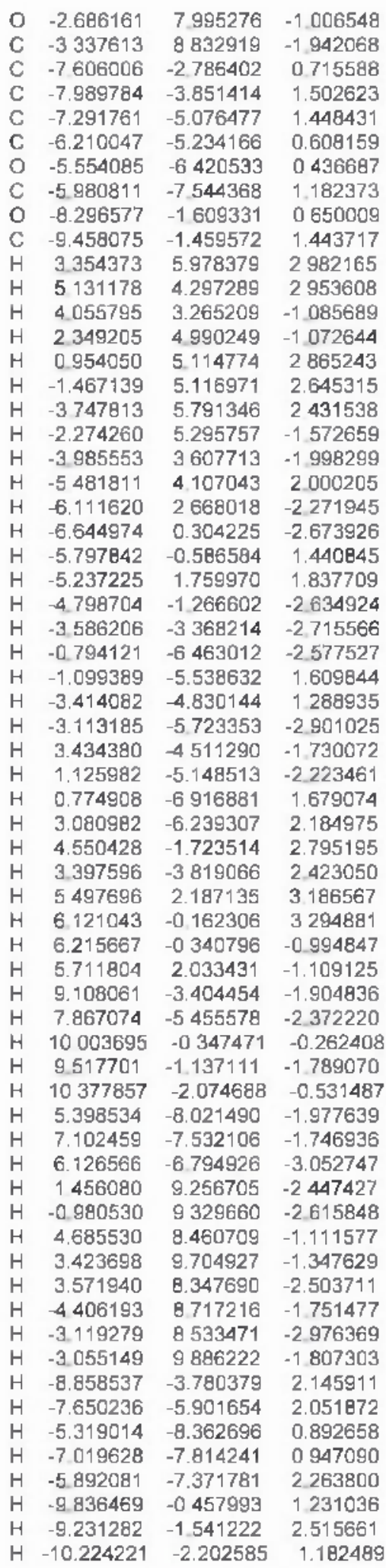

Calculation results at DFT-oplimized geometry. Tolal Energy- -3227.36894548 AU SCF Convergence- 0.1565E-08 Three lowest frequencies $\left(\mathrm{cm}^{1}\right.$ ): $16.5,18.1,18.3$ 


\section{Cyclic Trimer anti-64b}

\begin{tabular}{|c|c|c|c|}
\hline & 6.556577 & -2205917 & 1.061697 \\
\hline & 6.799340 & 0.793677 & 1.474291 \\
\hline & 7.108125 & -1.278007 & -0008760 \\
\hline & 405658 & -1.511586 & \\
\hline & 521680 & -0.196260 & 2577449 \\
\hline & 239503 & 0.101608 & 0.182736 \\
\hline & 560026 & -1.864475 & -1.216003 \\
\hline & 847290 & 0.881580 & -0832229 \\
\hline & .265379 & 0.297607 & -2.022394 \\
\hline & Q.1 & -1.080548 & \\
\hline & 7.4 & -322 & \\
\hline & 577 & 936 & .05 \\
\hline & 8.552262 & 3.056731 & -1.5 \\
\hline & 9.581682 & 2.770582 & 96944 \\
\hline & 8.554651 & & \\
\hline & 7.9 & & \\
\hline & & & \\
\hline & 72 & -34 & $-3 ?$ \\
\hline & 7.58 & -4.9 & $-2:$ \\
\hline & 8.67 & -3.74 & -2.6 \\
\hline & 6.37 & & \\
\hline & 6.1 & -2.1 & \\
\hline & 8.7 & & \\
\hline & & & \\
\hline & 5.58 & 1.7 & \\
\hline & 3.35 & 3.42 & \\
\hline & 5.7 & 3.1 & \\
\hline & 42 & & \\
\hline & 32 & & \\
\hline & & & \\
\hline & & & \\
\hline & 4.1 & 0.12 & \\
\hline & 2.2 & 1.61 & 0.7 \\
\hline & 4.80 & 5.0 & 0.9 \\
\hline & 5.2 & -28 & \\
\hline & & & \\
\hline & & & \\
\hline & & & \\
\hline & 3.0 & -2.8 & -0.2 \\
\hline & 3.72 & $-4.7 t$ & \\
\hline & 5.71 & -4.7 & \\
\hline & 442 & -12 & -0 \\
\hline & & -2 & \\
\hline & & & \\
\hline & & -4.7 & \\
\hline & 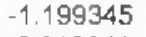 & -5.8 & -0 . \\
\hline & 0.213841 & -3.985895 & 02 \\
\hline & 1.227 & -6.0 & 22721 \\
\hline & -0.039120 & -6.6 & -06 \\
\hline & -1.0 & & \\
\hline & & & \\
\hline & & -6.6 & -0.6 \\
\hline & -0.12 & -7.6 & -09 \\
\hline & -1.929798 & -3946207 & 0265714 \\
\hline & 2201500 & 4.31 & 8162 \\
\hline & -0.047216 & 5912369 & -0190110 \\
\hline & 2.3413 & & -0298668 \\
\hline & & & \\
\hline & & & \\
\hline & 124 & 6.226469 & -0644407 \\
\hline & 3.31 & 5.694266 & -0.704067 \\
\hline & 0.77 & 3.194615 & $1.6 \subseteq$ \\
\hline & -1.161816 & 4566163 & 1. 068603 \\
\hline$n$ & & & \\
\hline & & -6473015 & -0668927 \\
\hline & -4.974494 & -4.759427 & -1406502 \\
\hline & & & \\
\hline & & -6.179839 & -2.086995 \\
\hline
\end{tabular}

\begin{tabular}{|c|c|c|c|}
\hline C & -4078435 & -5442791 & $-240918 \mathrm{~B}$ \\
\hline C & -4757722 & -5302046 & 0009647 \\
\hline & -3504956 & -6.577654 & 1.663788 \\
\hline & -5.748130 & -5064655 & 0.995169 \\
\hline & -5.591358 & -5.543519 & 2.290818 \\
\hline & -4.462884 & -6.298539 & 2.630219 \\
\hline & -2.394174 & -7349662 & 1. 885061 \\
\hline & -6855956 & -4.371301 & 0576434 \\
\hline & -7865113 & -4078733 & 1.524122 \\
\hline & -7.478996 & -3.466917 & 2.350423 \\
\hline & -8.313976 & -4.993878 & 1.934275 \\
\hline & -8.629398 & -3.514017 & 0986081 \\
\hline & -2.171100 & -7846915 & 3. 190762 \\
\hline & -2.975293 & -8524166 & 3510700 \\
\hline & -2.076089 & -7.032938 & 3.922108 \\
\hline & -1.231033 & -8.400242 & 3.144884 \\
\hline & -4.313955 & -5.259925 & -3456246 \\
\hline & -2.390075 & -6604621 & 0349 \\
\hline & -6.340086 & -5352240 & 3050302 \\
\hline & -4.363181 & -6.669739 & 3.643404 \\
\hline & -1.243957 & 6.732866 & -0.700827 \\
\hline & -3.809126 & 5.431833 & -1.671824 \\
\hline & -2.492019 & 6.647030 & 0.162319 \\
\hline & -1.517793 & 6.350330 & -2.138659 \\
\hline & -2644656 & 1658 & 141 \\
\hline & -3.675338 & 6049942 & -0.283891 \\
\hline & -2453642 & 7.245772 & 1.445399 \\
\hline & -4.816674 & 6061464 & 0.554933 \\
\hline & -4.758907 & 6.630750 & 1.821597 \\
\hline & -3.572270 & 7.224527 & 2.269967 \\
\hline & -1.262783 & 7836187 & 1.780708 \\
\hline & -5.947112 & 1429 & 1304 \\
\hline & -7.104360 & 5415825 & 0.828756 \\
\hline & -7.477930 & 6.410399 & 1.109861 \\
\hline & -6.917670 & 4832394 & 1.740485 \\
\hline & -7.857374 & 4906371 & 0224145 \\
\hline & -1.141167 & 84 & 3062853 \\
\hline & -0.121613 & 88 & 21494 \\
\hline & $-1.2 \mathrm{~s}$ & & 60852 \\
\hline & -1.850900 & 9249871 & 3.199955 \\
\hline & -2.753083 & 5.507209 & -3.611872 \\
\hline & -0.708523 & 6539807 & -2841215 \\
\hline & -5.625838 & 6635843 & 2471817 \\
\hline & -3549244 & 7.671807 & 3256891 \\
\hline & -4.004944 & 39 & 51782 \\
\hline & -4.3 & 1080738 & -1.668316 \\
\hline & -5.156503 & 3.320242 & -2.187417 \\
\hline & -3.017409 & 3059028 & -1.136975 \\
\hline & -3187811 & 1677849 & -1.134402 \\
\hline & -5.322032 & 1.935762 & -2202771 \\
\hline & -5.936634 & 3956128 & -2.599199 \\
\hline & -2.10 & 0958 & -0.732751 \\
\hline & -2.418457 & 1.052213 & -0.690992 \\
\hline & -6.215604 & 1513403 & -2654482 \\
\hline & -4.816428 & -3227577 & -1.510420 \\
\hline & -4.514496 & -0393858 & -1650551 \\
\hline & -3.557458 & -2.637816 & -1.686839 \\
\hline & -5.927316 & -2374718 & -1.457396 \\
\hline & -5.779984 & -0.989933 & -1.517110 \\
\hline & -3408796 & -1.254642 & -1.753709 \\
\hline & -2681275 & -3267886 & -1.794224 \\
\hline & -6.918244 & -2.800485 & -1.341855 \\
\hline & -6.661700 & -0360780 & -1428878 \\
\hline & -2.418295 & -0838460 & -1.914683 \\
\hline & -2.448368 & -7.560651 & -0.580387 \\
\hline & -6.018348 & -4973701 & -1.672611 \\
\hline & -4.722272 & 5846290 & $-2,120981$ \\
\hline & -0.913845 & 7.782062 & -0686620 \\
\hline & 7630163 & 1.439111 & 1.790668 \\
\hline & 7.286205 & -3.019135 & 1.187032 \\
\hline
\end{tabular}


Calculation results at DFT-oplimized geomeiry:

Tolal Energy- -3231.0035049 AU

SCF Convergence- 0.3536E-08

Three lowest frequencies (cm ${ }^{1}$ ): 11.6, 12.3, 15.5 


\section{References Cited}

(S1) Davis, M. C.; Groshens, T. J. Synth. Commun. 2011, 41, 206-208.

(S2) Frisch, M. J.; Trucks, G. W.; Schlegel, H. B.; Scuseria, G. E.; Robb, M. A.; Cheeseman, J. R.; MontgomeryJr., J. A.; Vreven, T.; Kudin, K. N.; Burant, J. C.;Millam, J. M.; lyengar, S. S.; Tomasi, J.; Berone, V.; Mennucci, B.; Cossi, M.; Scalmani, G.; Rega, N.; Petersson, G. A.; Nakatsuji, H.; Hada, M.; Ehara, M.; K. Toyota; Fukuda, R.; Hasegawa, J.; Ishida, M.; Nakajima, T.; Honda, Y.; Kitao, O.; Nakai, H.; Klene, M.; Li, X.; Knox, J. E.; Hratchian, H. P.; Cross, J. B.; Bakken, V.; Adamo, C.; Jaramillo, J.; Gomperts, R.; Stratmann, R. E.; Yazyev, O.; Austin, A. J.; Cammi, R.; Pomelli, C.; Ochterski, J. W.; Ayala, P. Y.; Morokuma, K.; Voth, G. A.; Salvador, P.; Dannenberg, J. J.; Zakrzewski, V. G.; Dapprich, S.; Daniels, A. D.; Strain, M. C.; Farkas, O.; Malick, D. K.; Rabuck, A. D.; Raghavachari, K.; Foresman, J. B.; Ortiz, J. V.; Cui, Q.; Baboul, A. G.; Clifford, S.; Cioslowski, J.; Stefanov, B. B.; Liu, G.; Liashenko, A.; Piskorz, P.; Komaromi, I.; Martin, R. L.; Fox, D. J.; Keith, T.; Al-Laham, M. A.; Peng, C. Y.; Nanayakkara, A.; Challacombe, M.; Gill, P. M. W.; Johnson, B.; Chen, W.; Wong, M. W.; Gonzalez, C.; Pople, J. A.; Gaussian, Inc.: Wallingford CT, 2004.

(S3) (a) Lee, C.; Yang, W.; Parr, R. G. Phys. Rev. B: Condens. Matter 1988, 37, 785-789. (b) Becke, A. D. J. Chem. Phys. 1993, 98, 1372-1377.

(S4) Hehre, W. J.; Radom, L.; Schleyer, P. v. R.; Pople, J. A. Ab Initio Molecular Orbital Theory; Wiley: New York, 1986. 


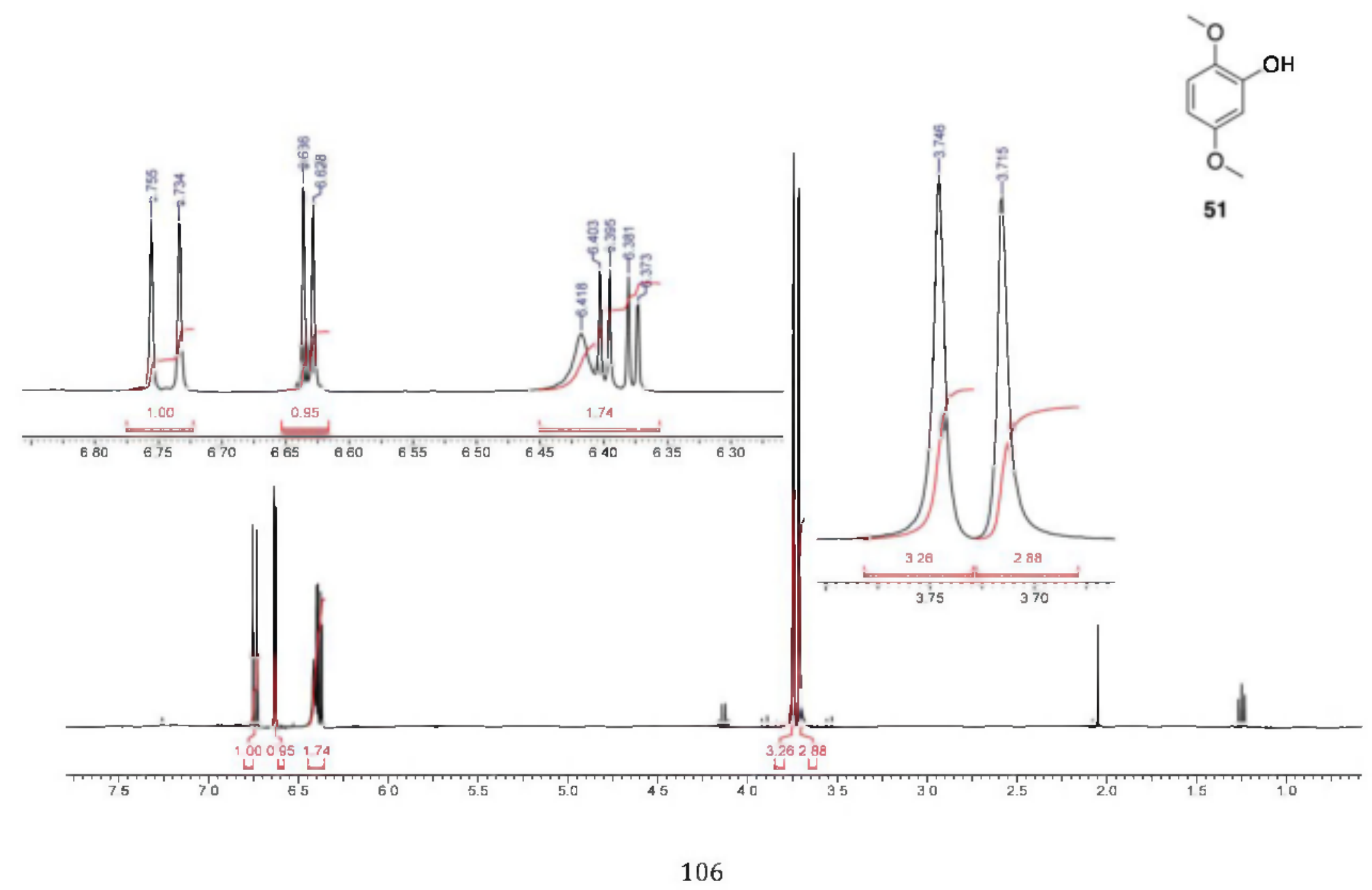




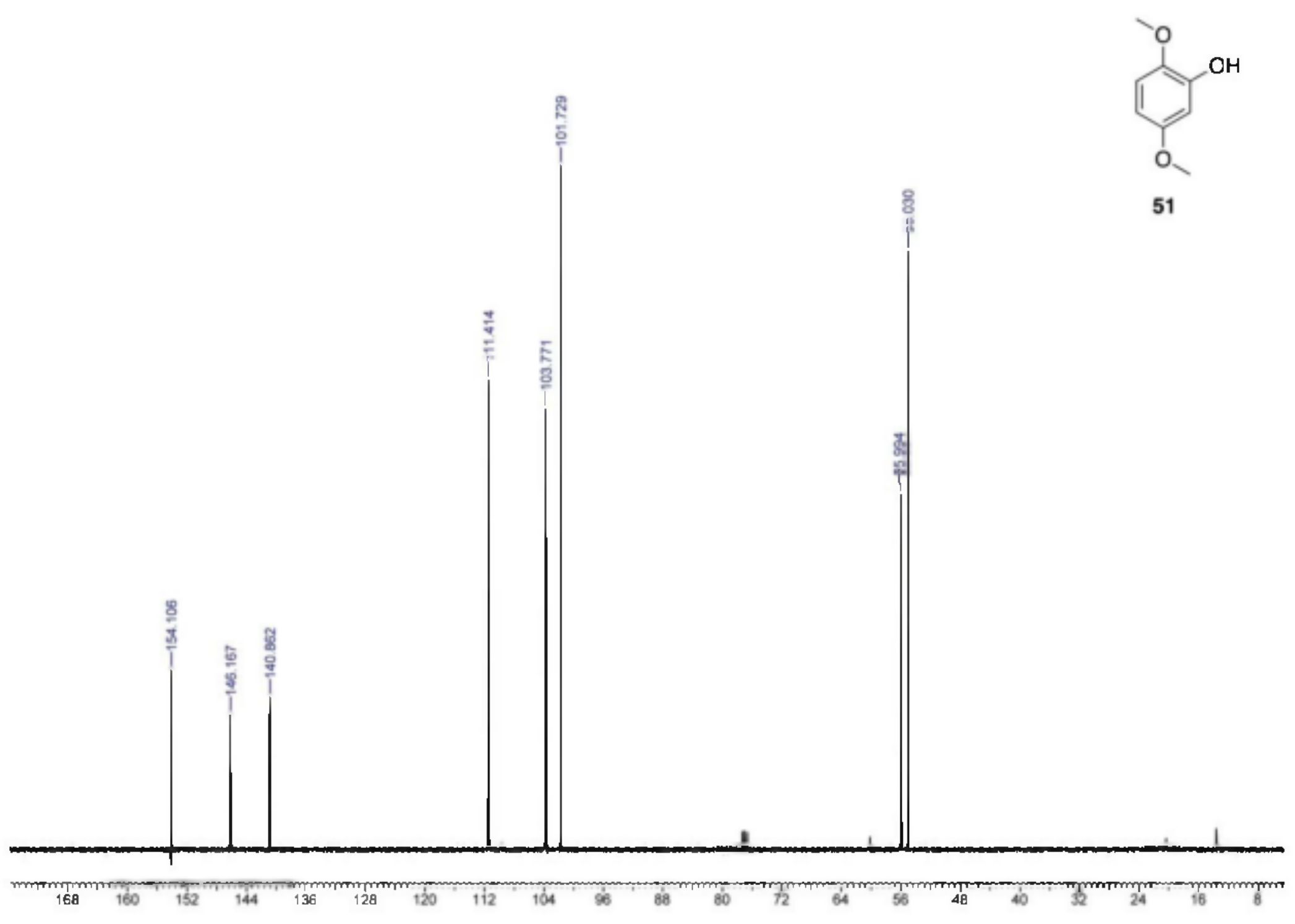




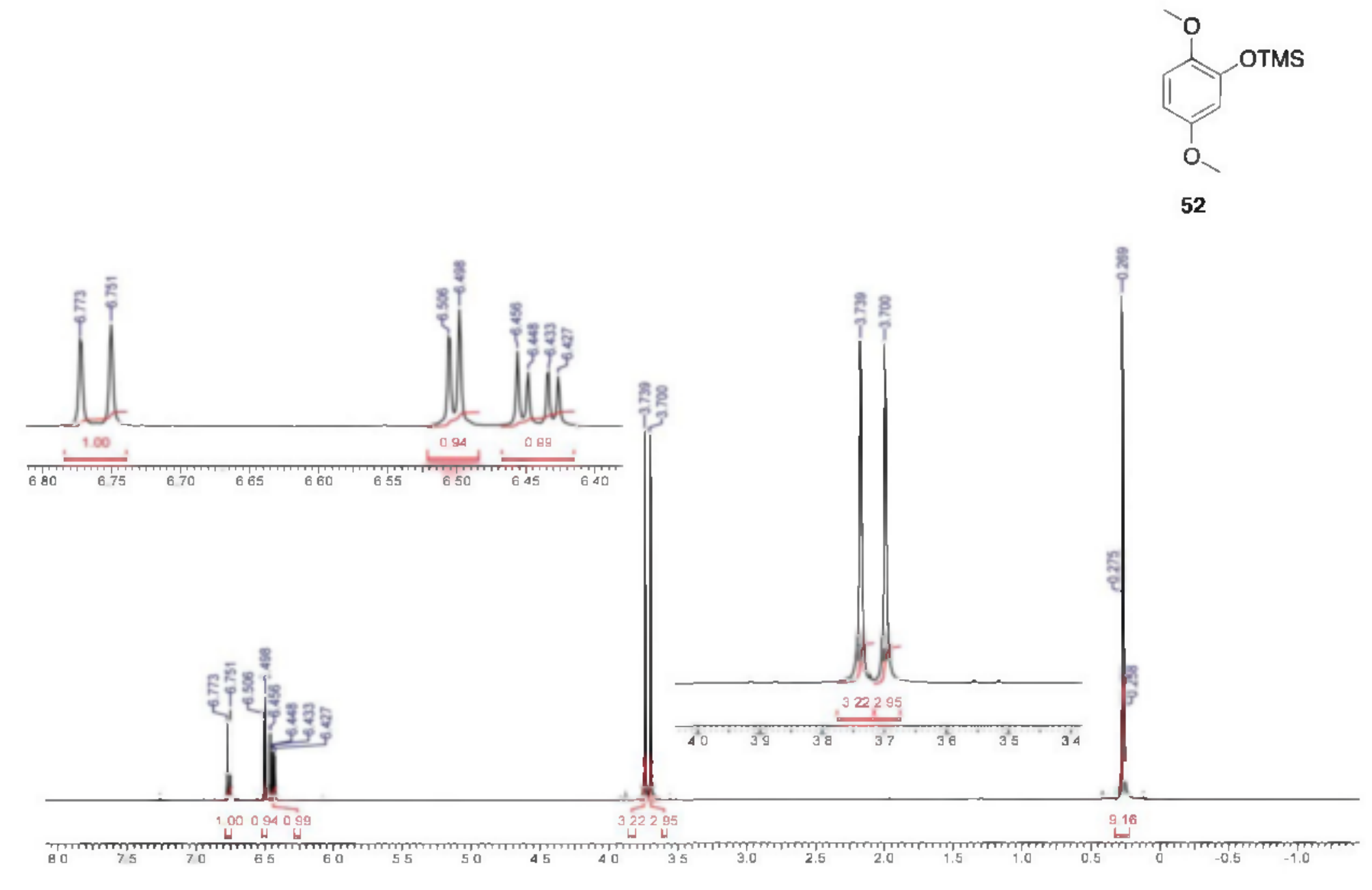




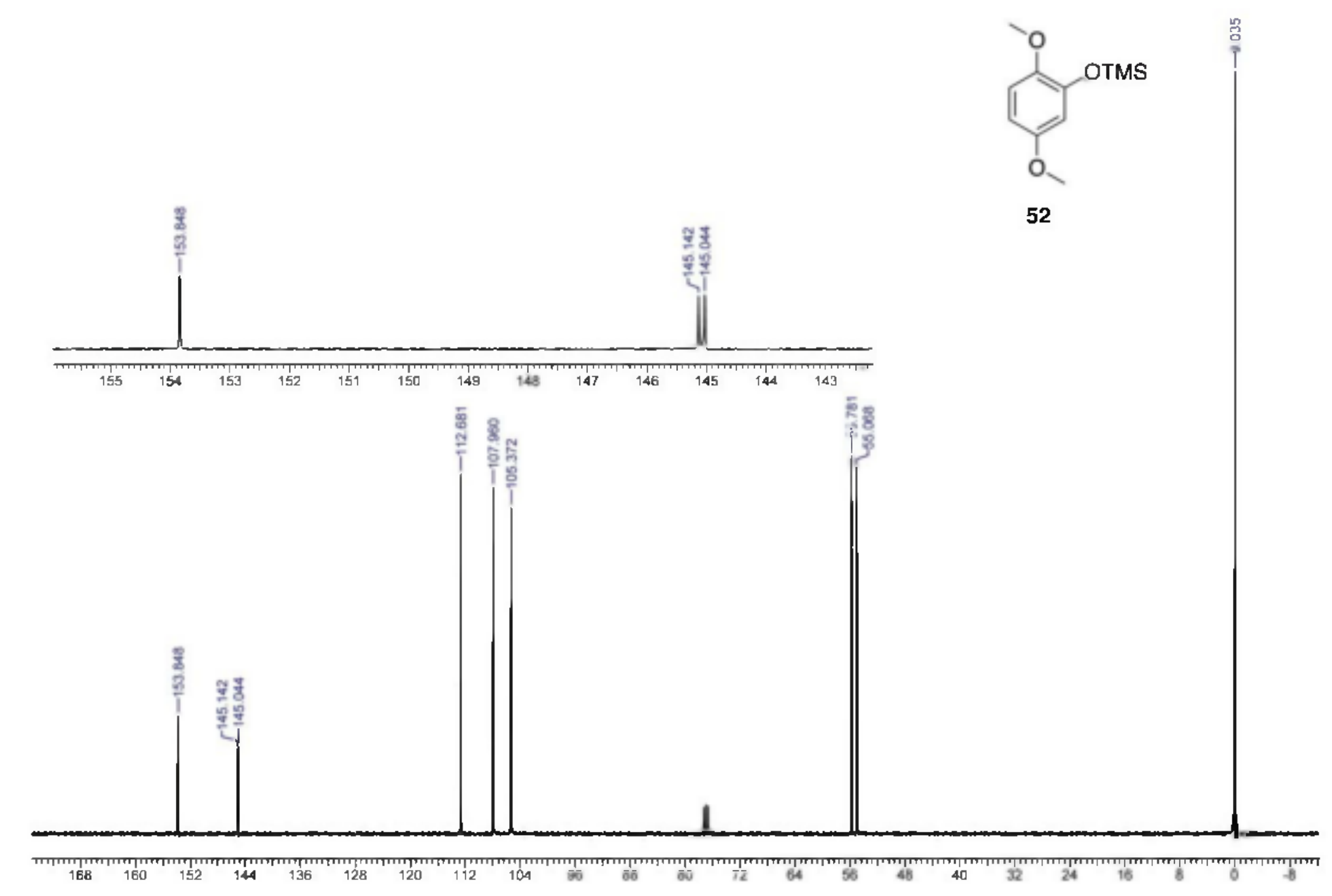



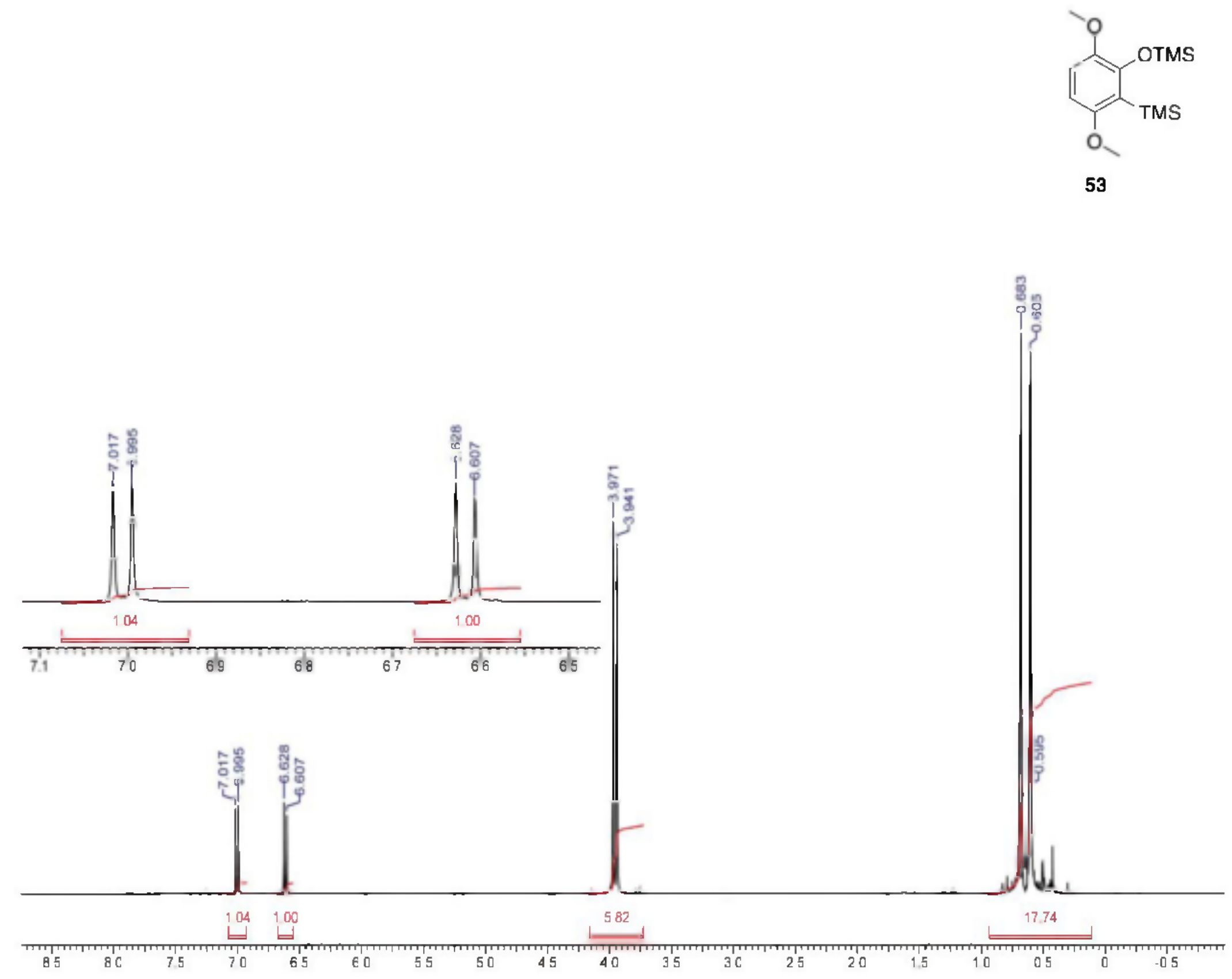


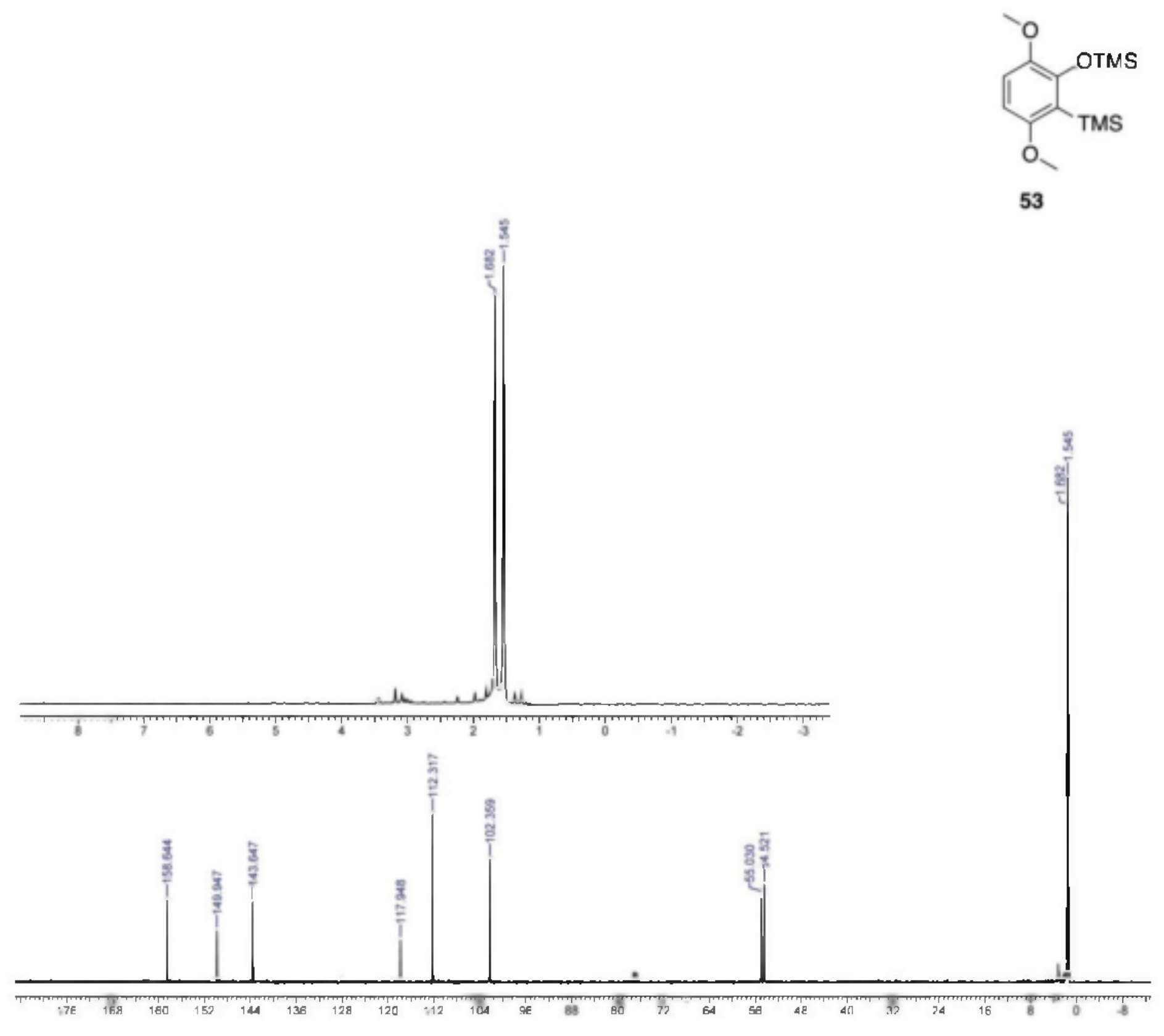




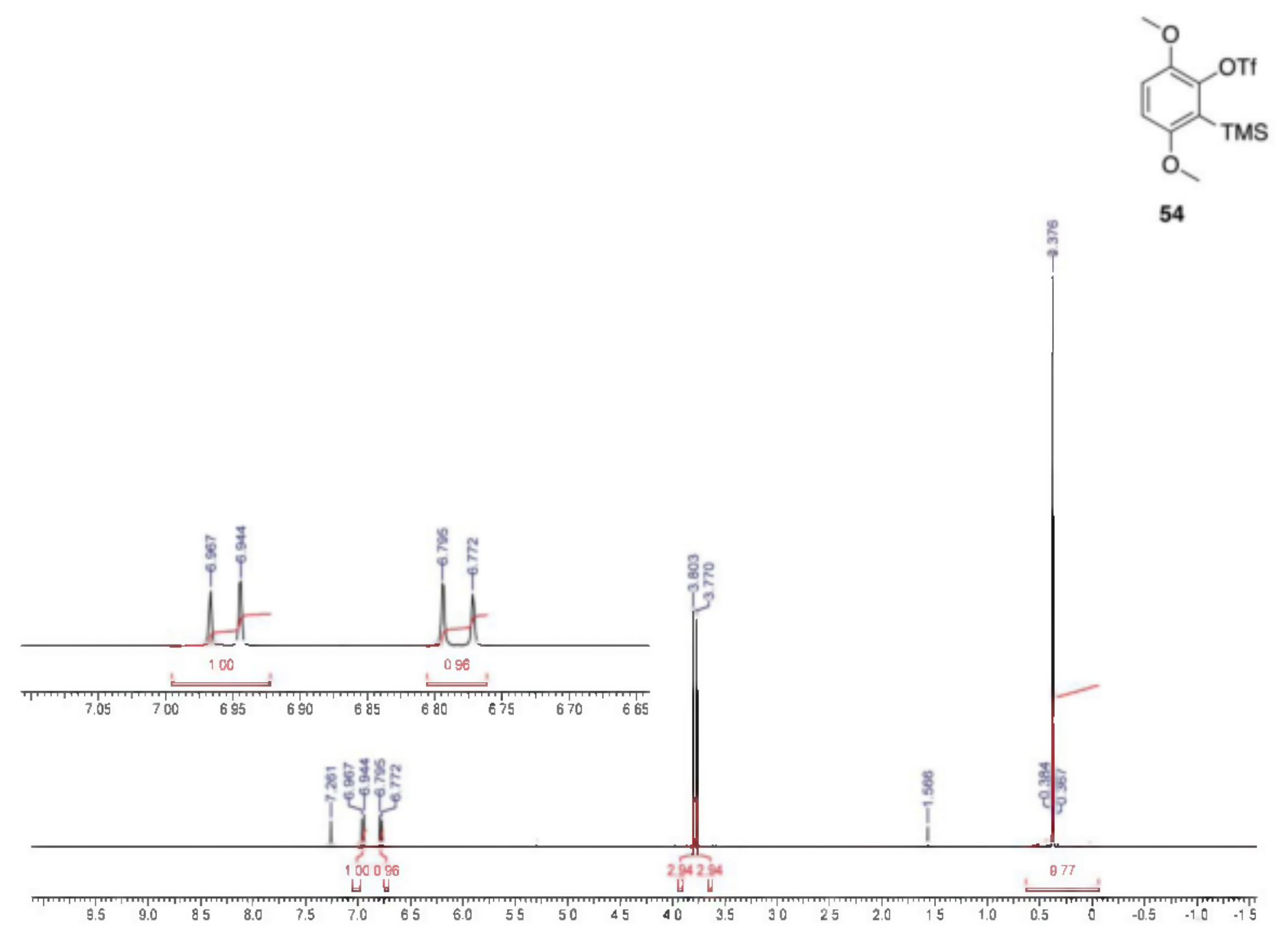




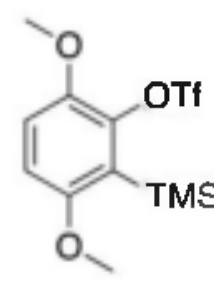

54

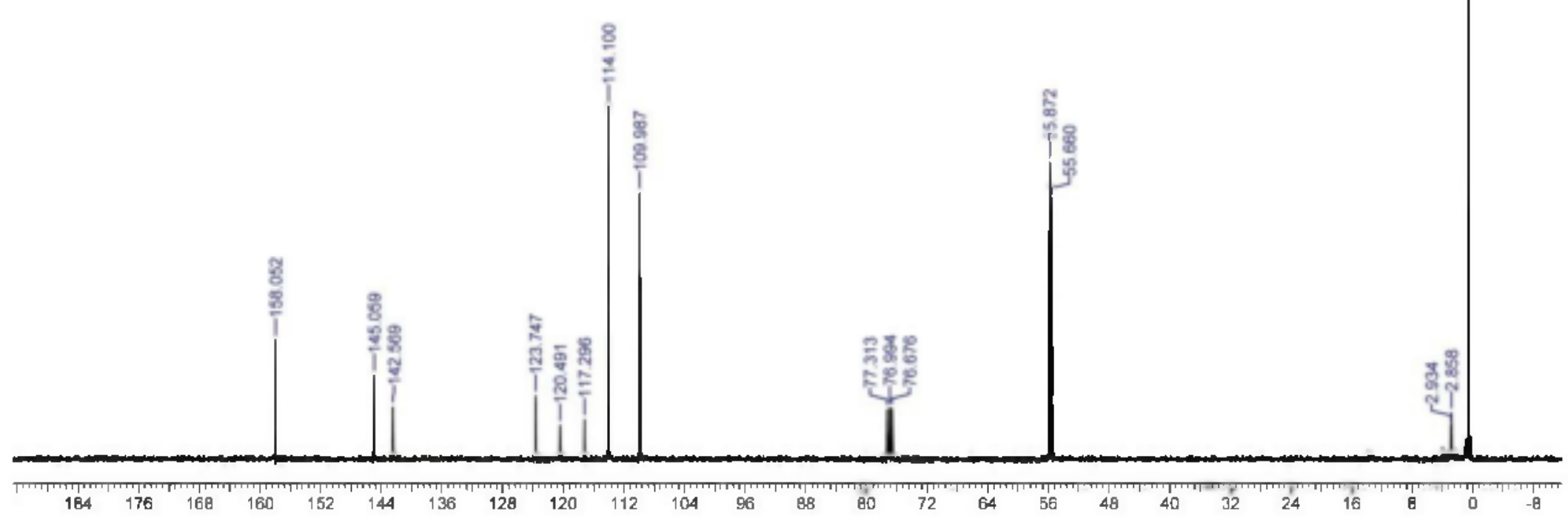



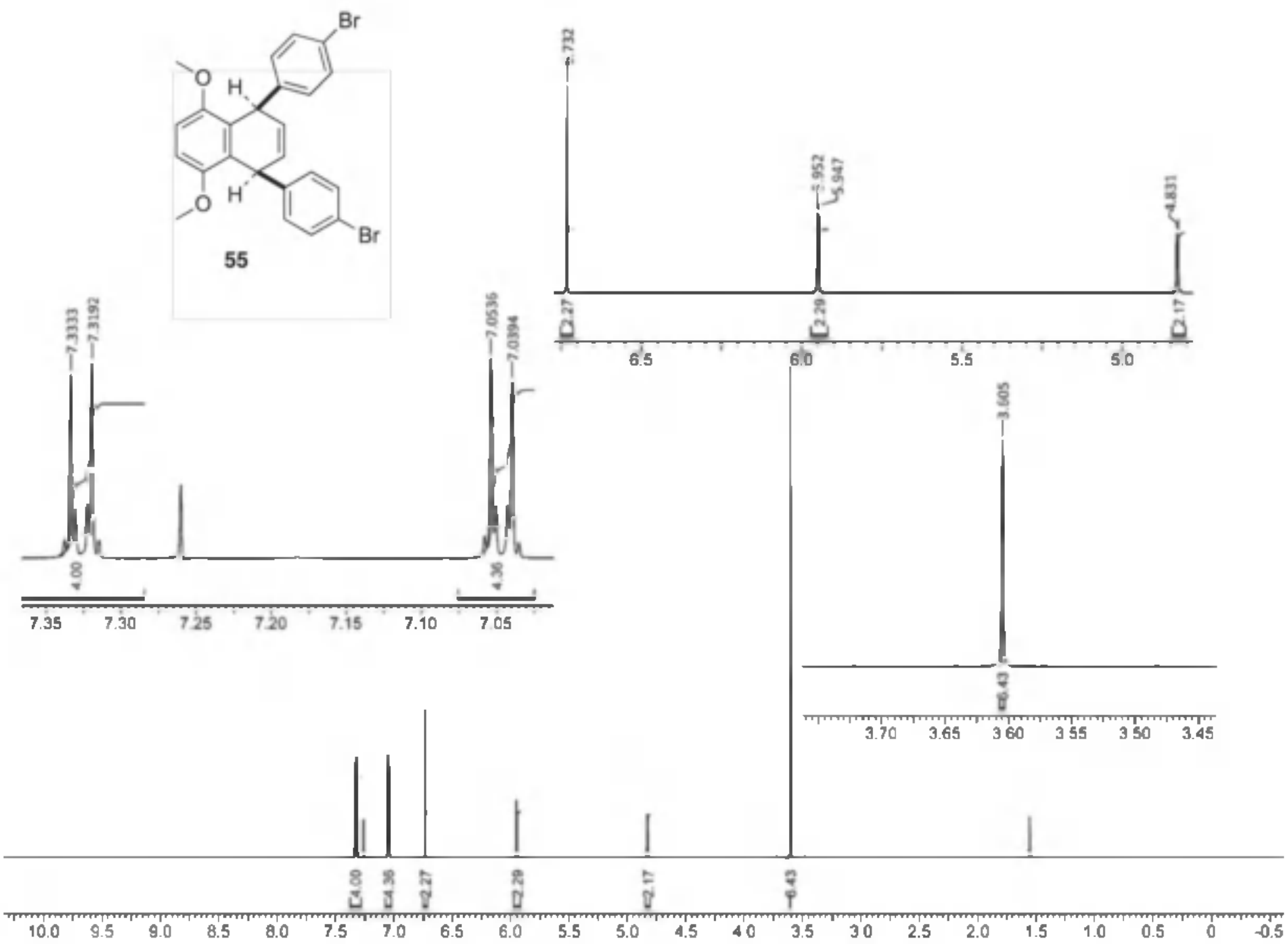


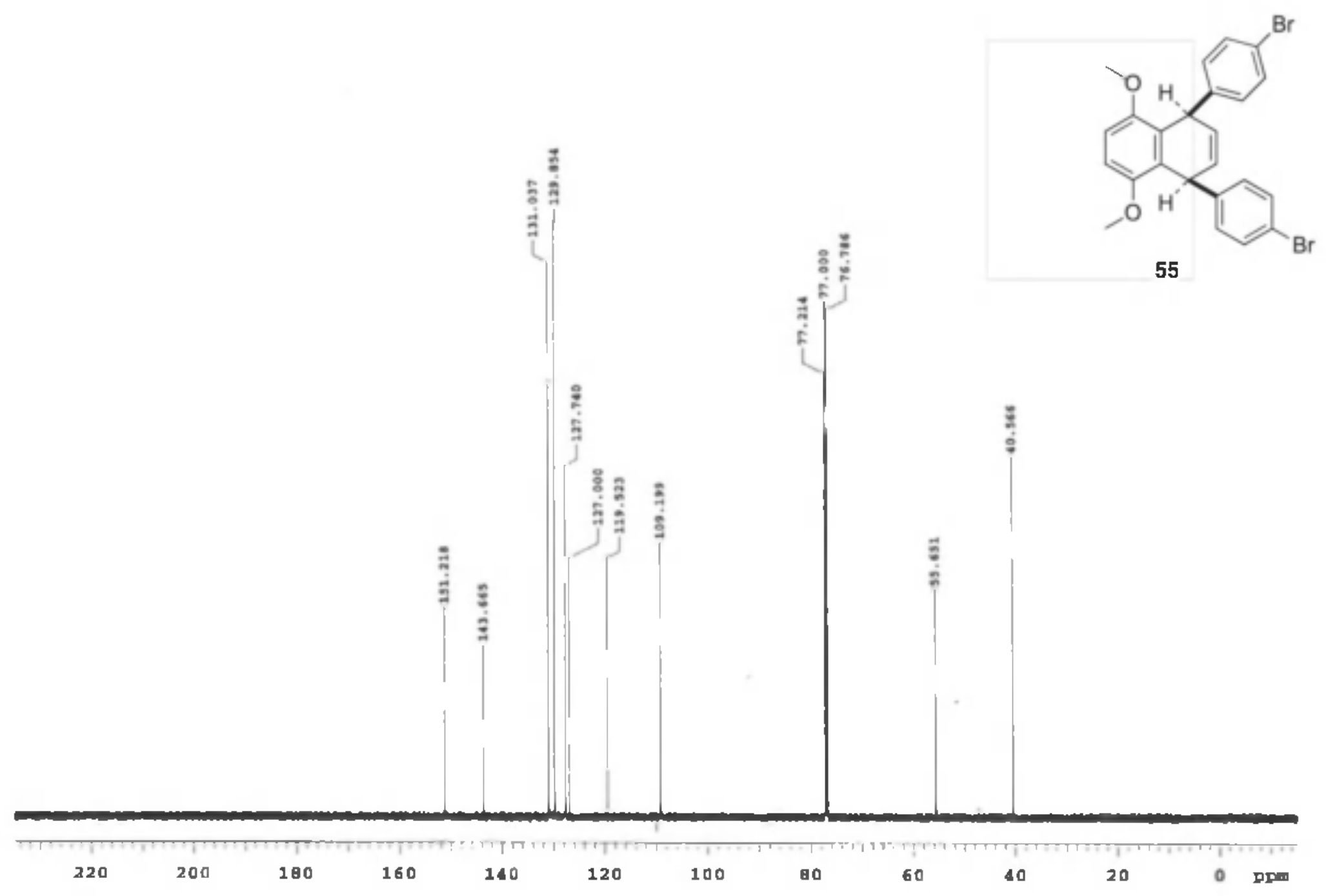




\section{Experimental (a) and calculated (b) splitting patterns}

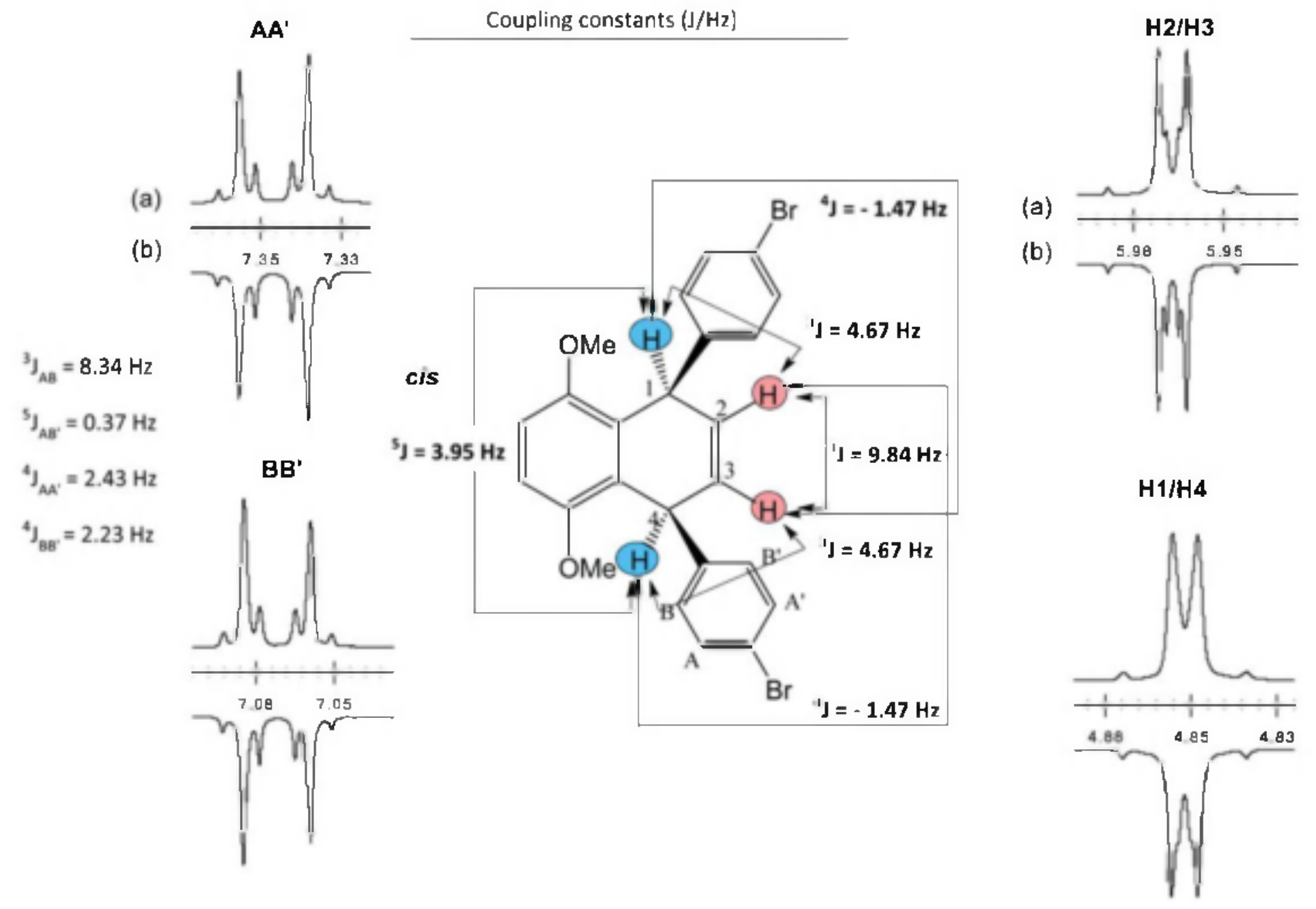




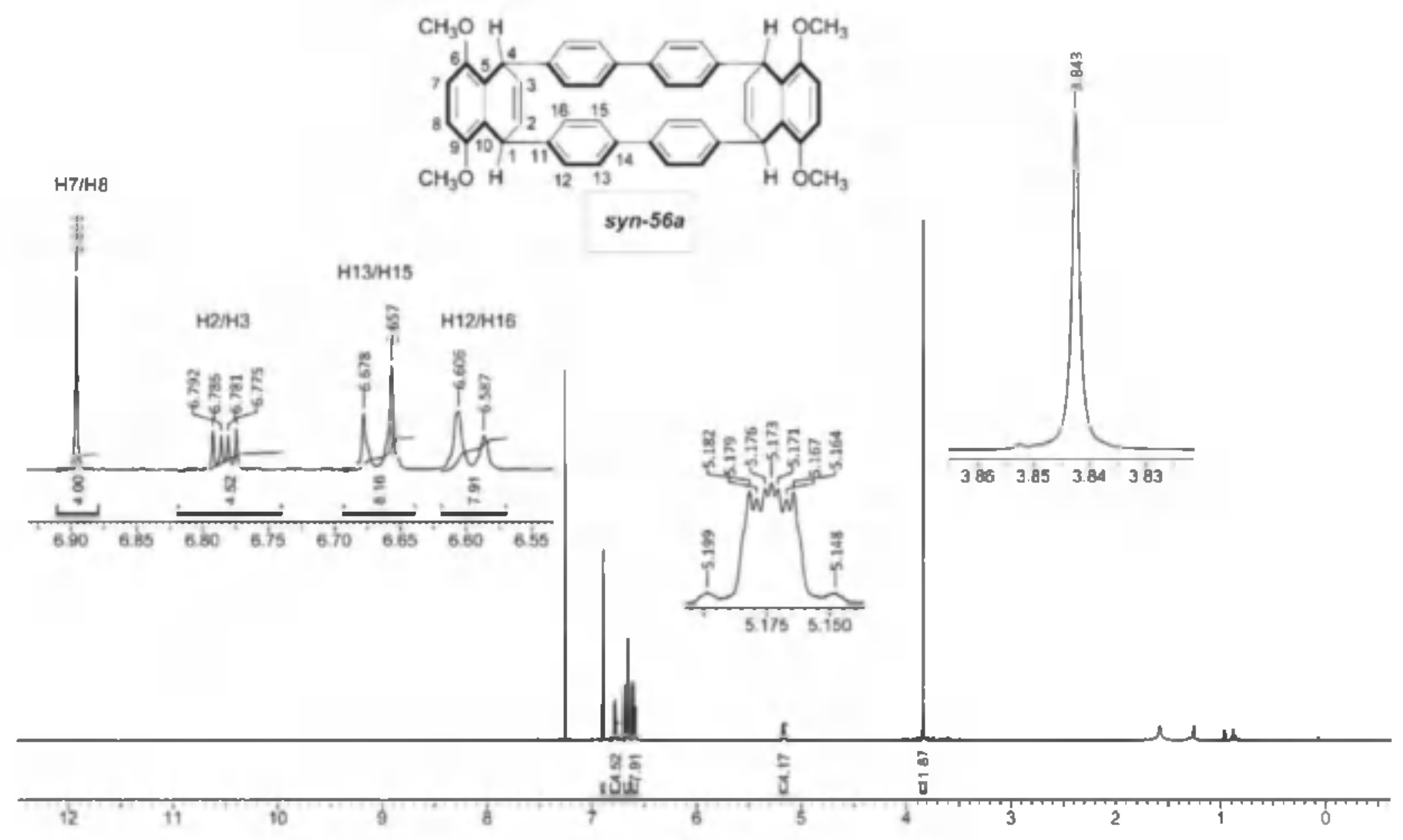


Control ' $\mathrm{H}$ NMR spectrum (a); DPFGSENOE subspectrum (b)

Selective excitation of $\mathrm{H} 1 / \mathrm{H} 4$

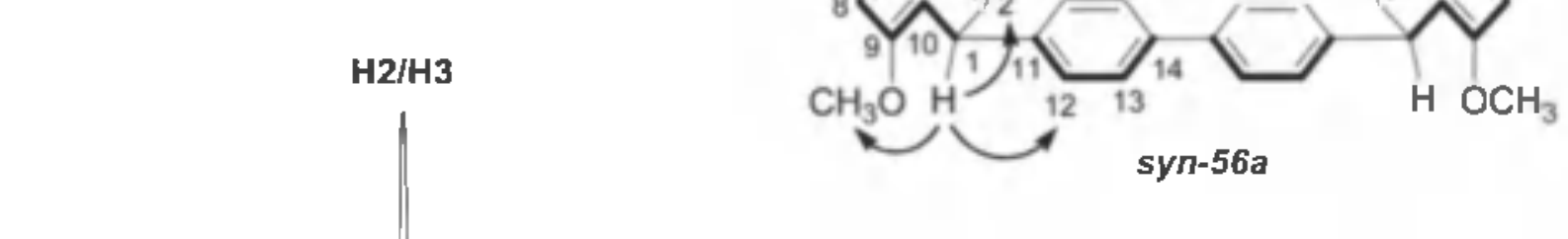

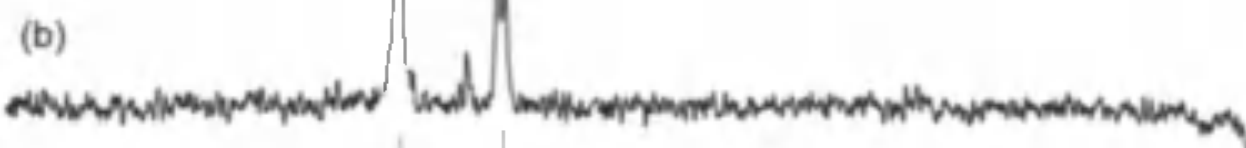

H12/H16

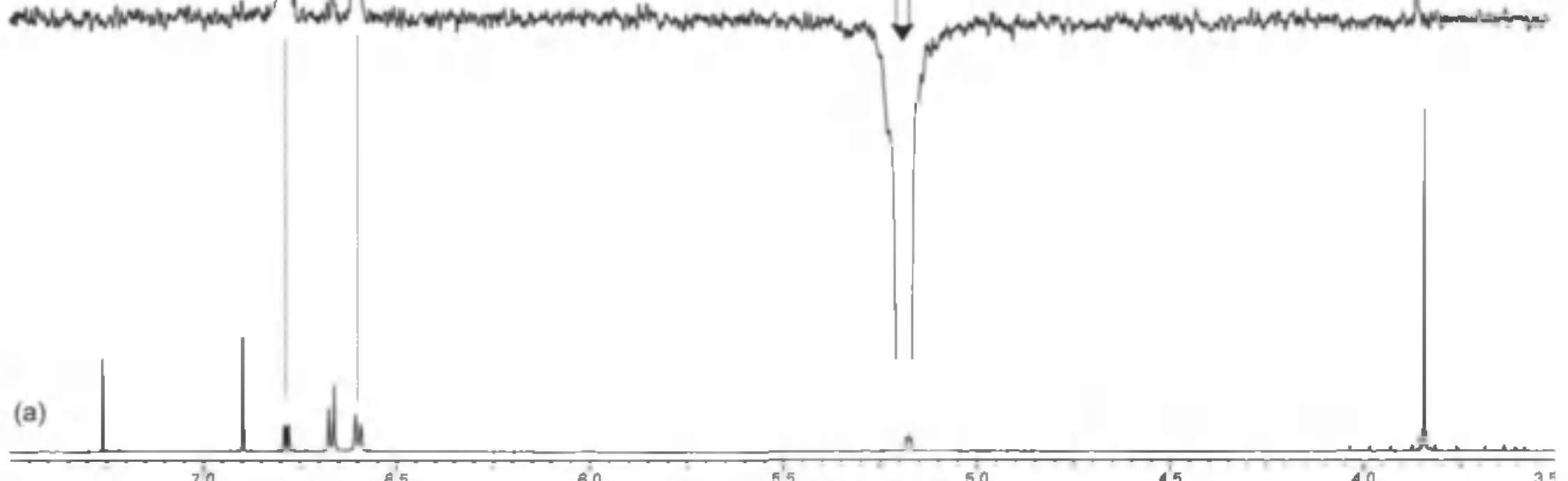

$\mathrm{H} 1 / \mathrm{H} 4 \quad 6-\mathrm{OCH}_{3}$ 9-OCH

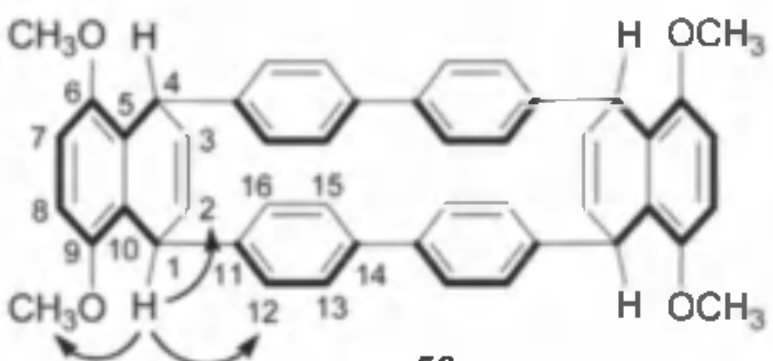


Control ${ }^{1} \mathrm{H}$ NMR spectrum (a); DPFGSENOE subspectrum (b)

Selective excitation of $\mathrm{H}_{2} / \mathrm{H} 3$
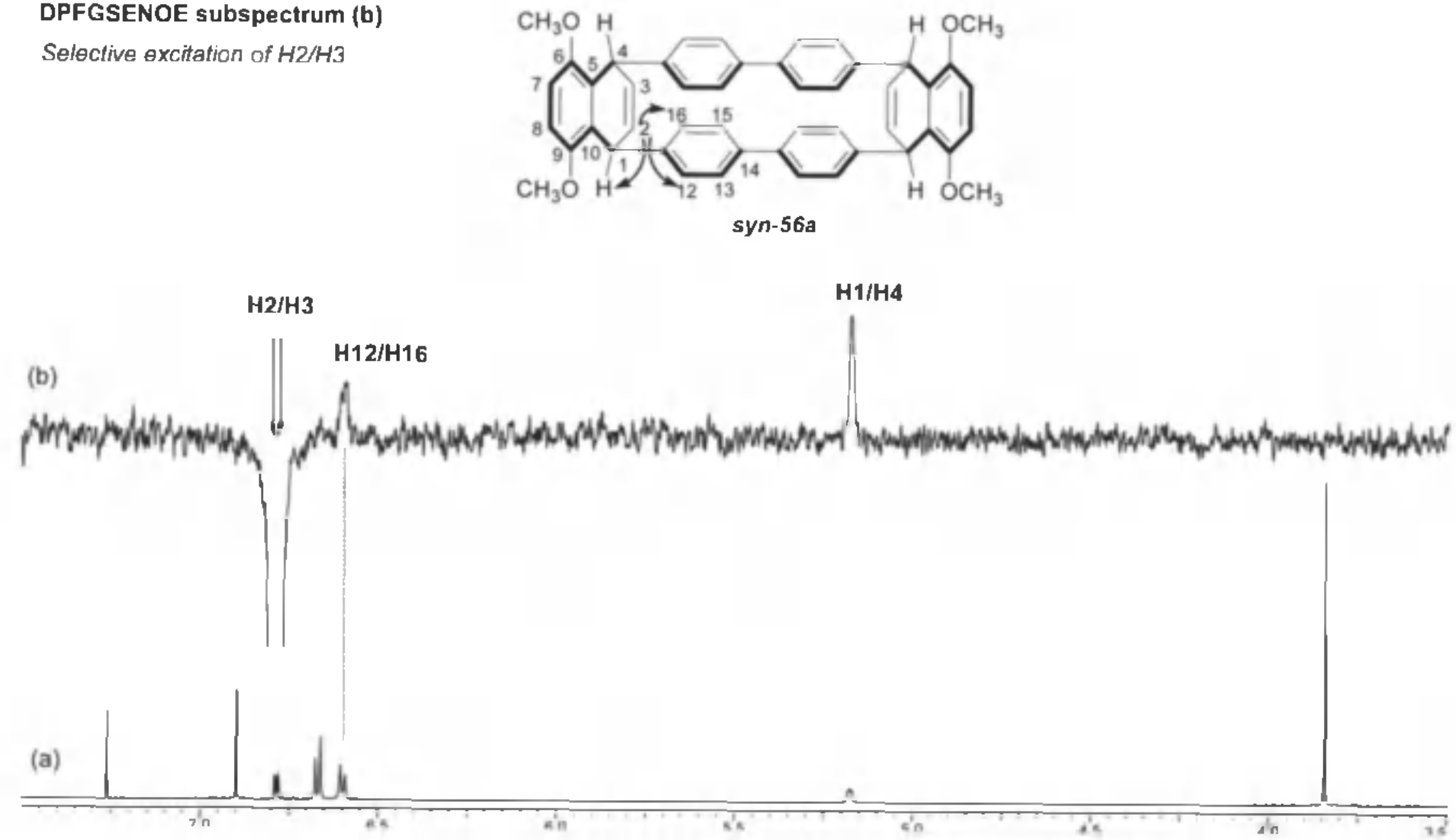
${ }^{13} \mathrm{C}$ NMR spectrum in $\mathrm{CDCl}_{3}(\mathrm{a})$

Expanded portion of the ${ }^{1} \mathrm{H}-{ }^{13} \mathrm{C}$ gHMBCAD spectrum (b)
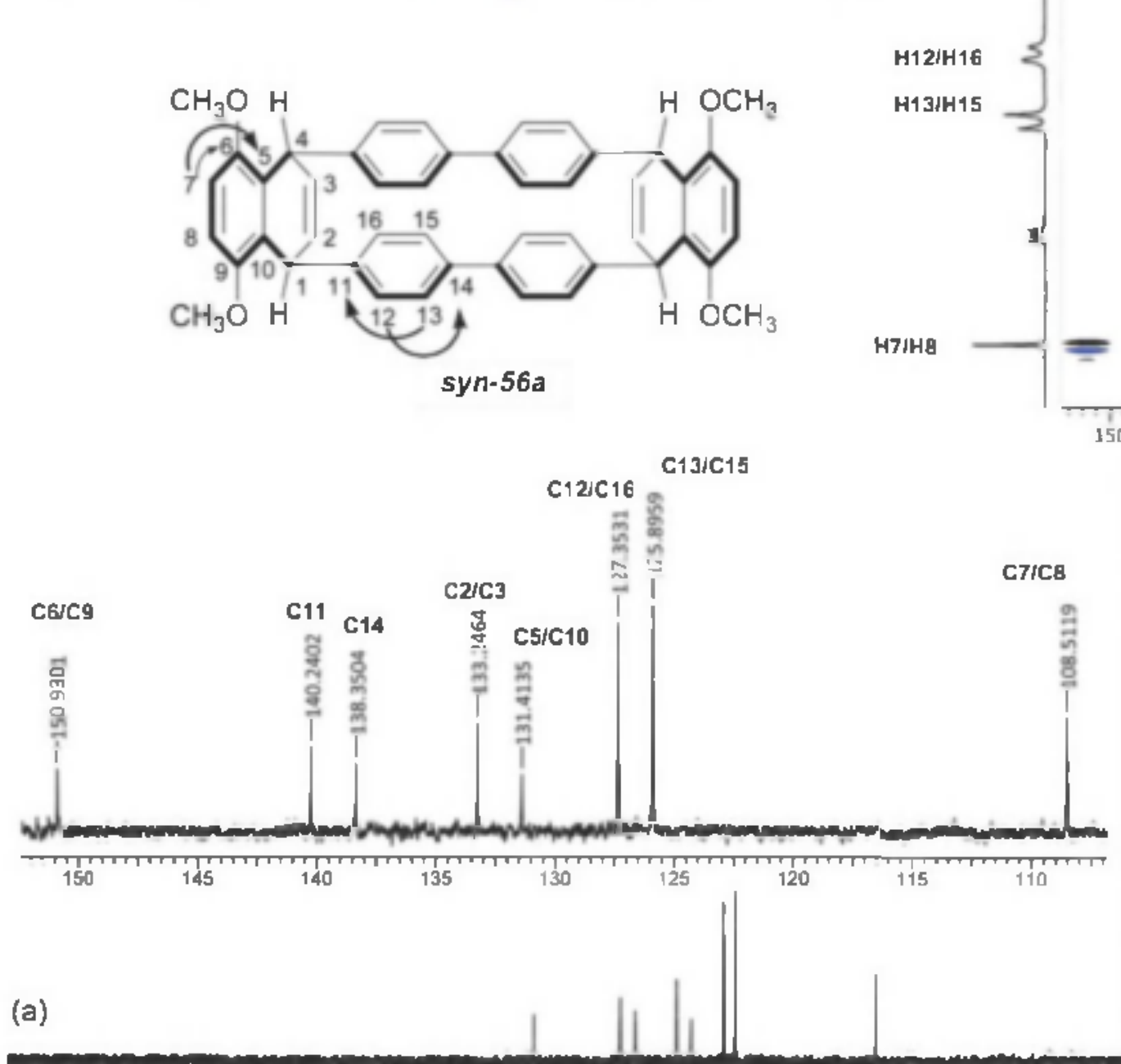

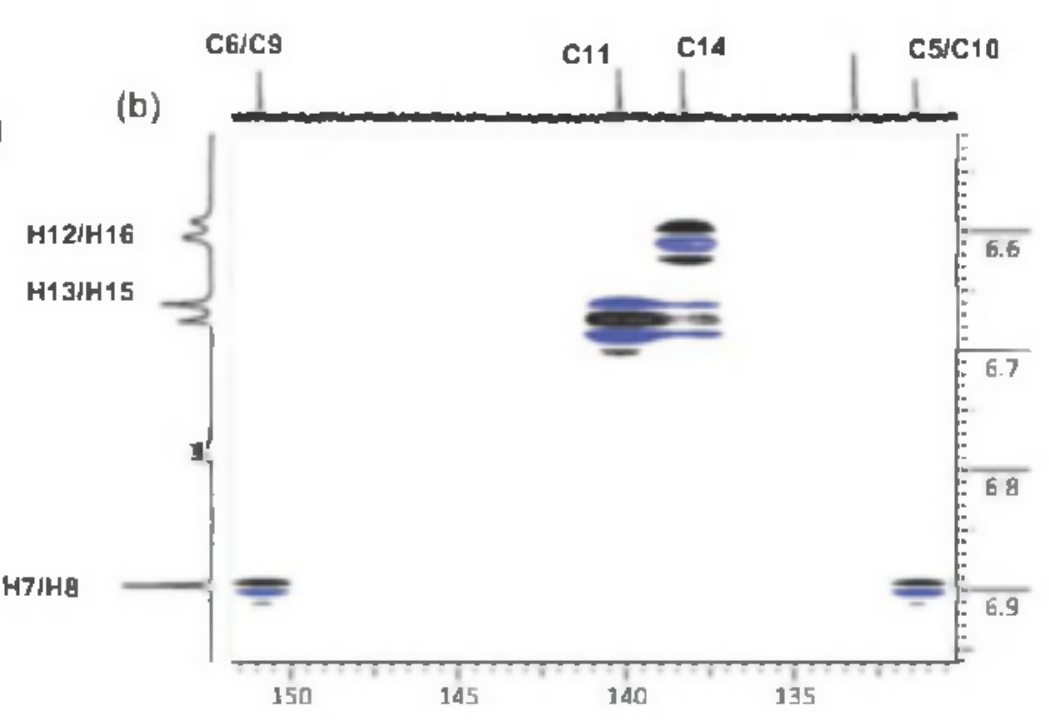

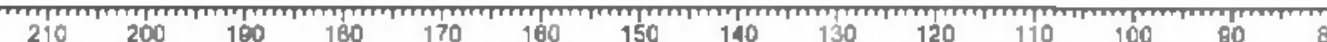
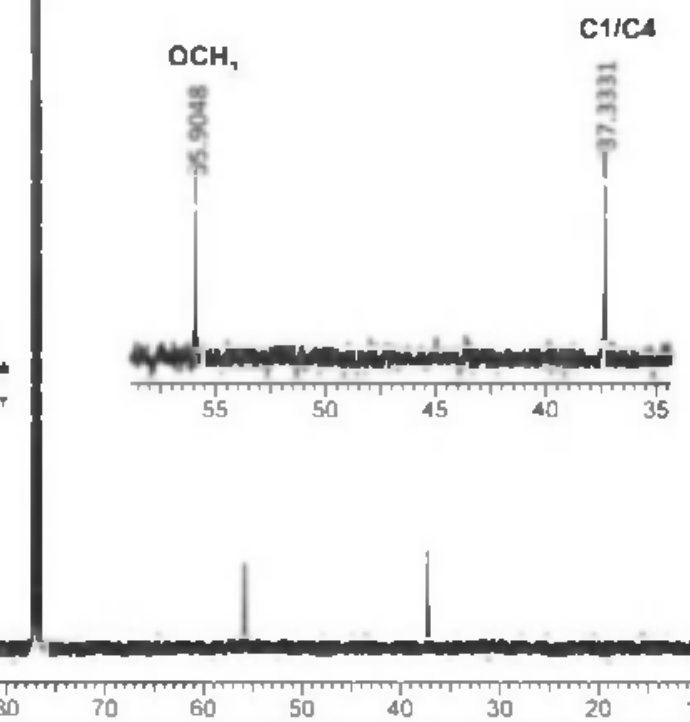

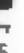
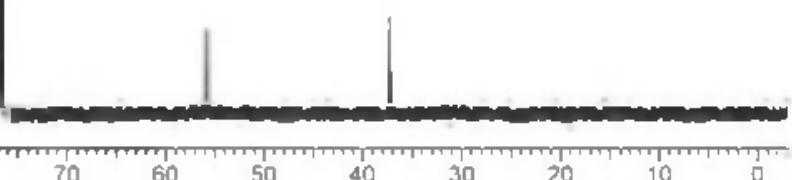
${ }^{13} \mathrm{C}$ NMR spectrum in $\mathrm{CDCl}_{3}$ (a)

Expanded portion of the ${ }^{1} \mathrm{H}-{ }^{13} \mathrm{C}$ gHMBCAD spectrum (b)
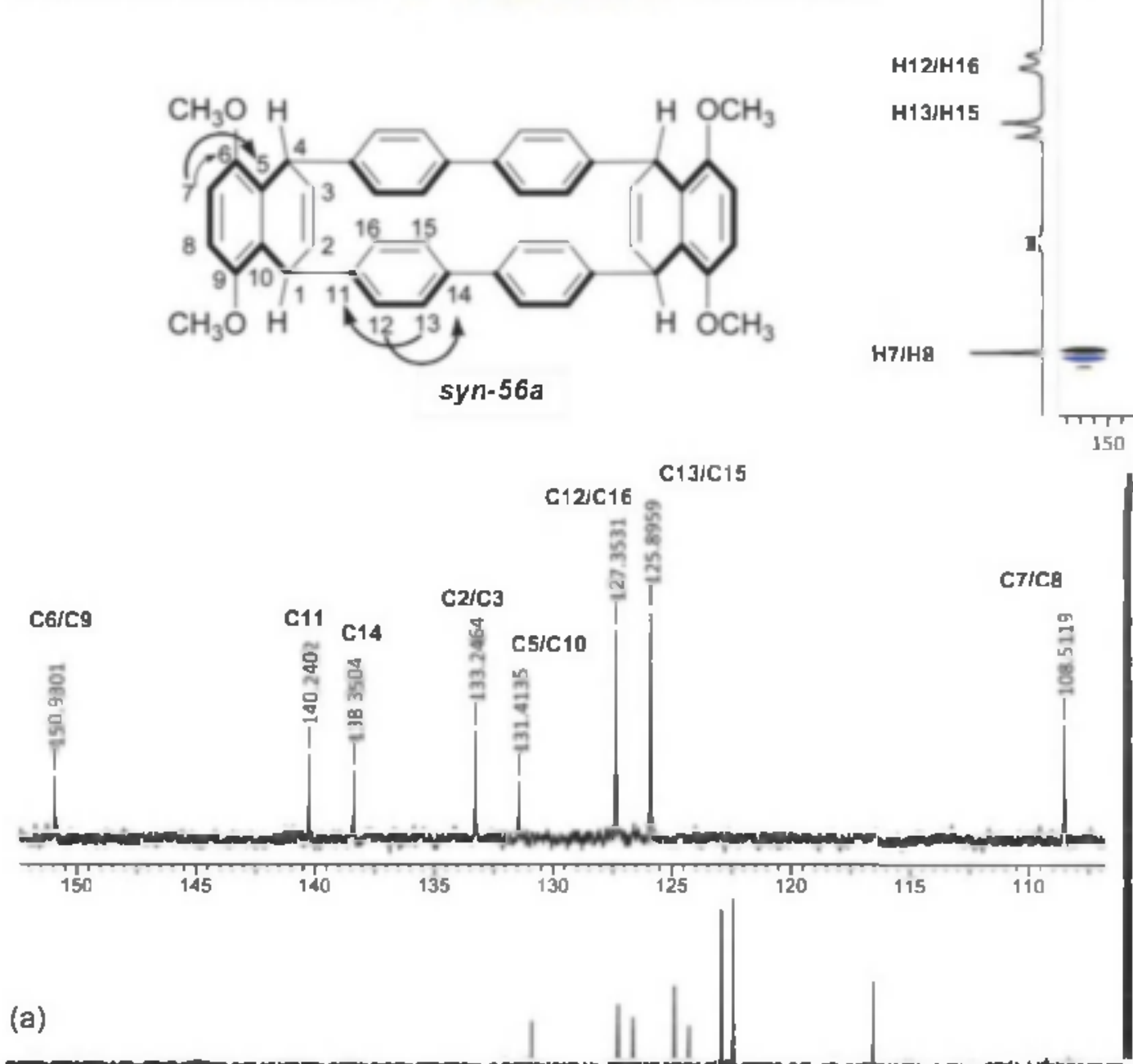
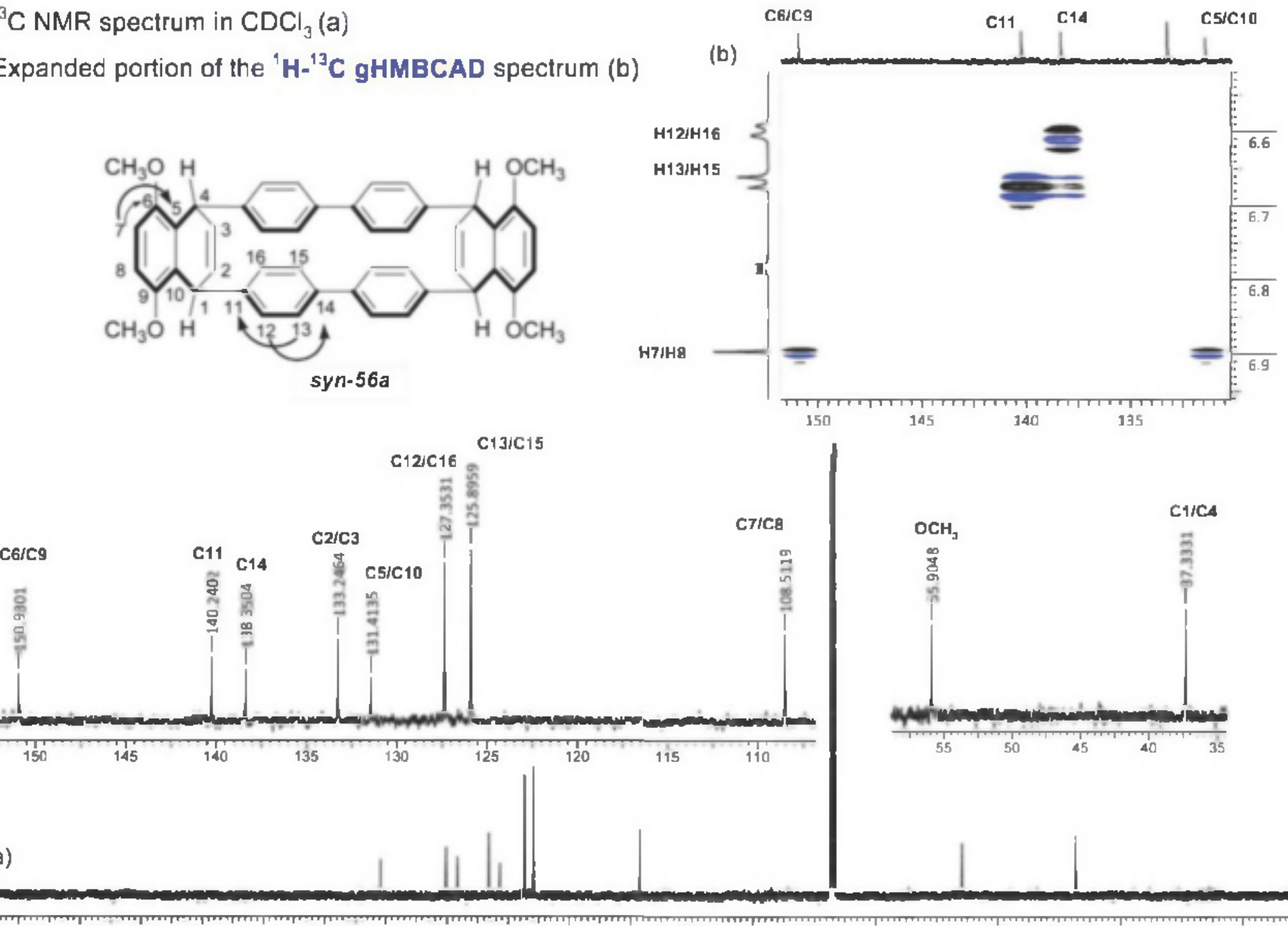

$210 \quad 200$

$180170 \quad 180$

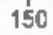

140130

$120 \quad 110$ 
Expanded portions of the ${ }^{9} \mathrm{H}-{ }^{43} \mathrm{C}$ gHMBCAD spectrum

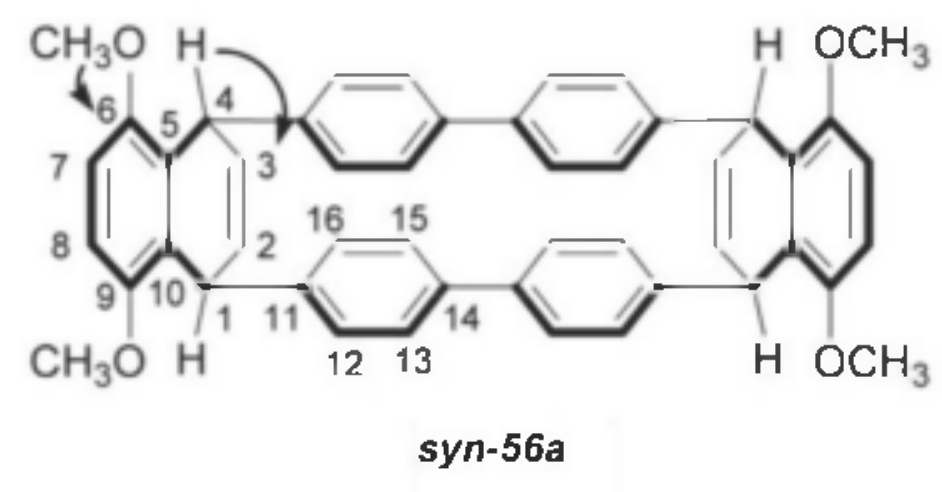

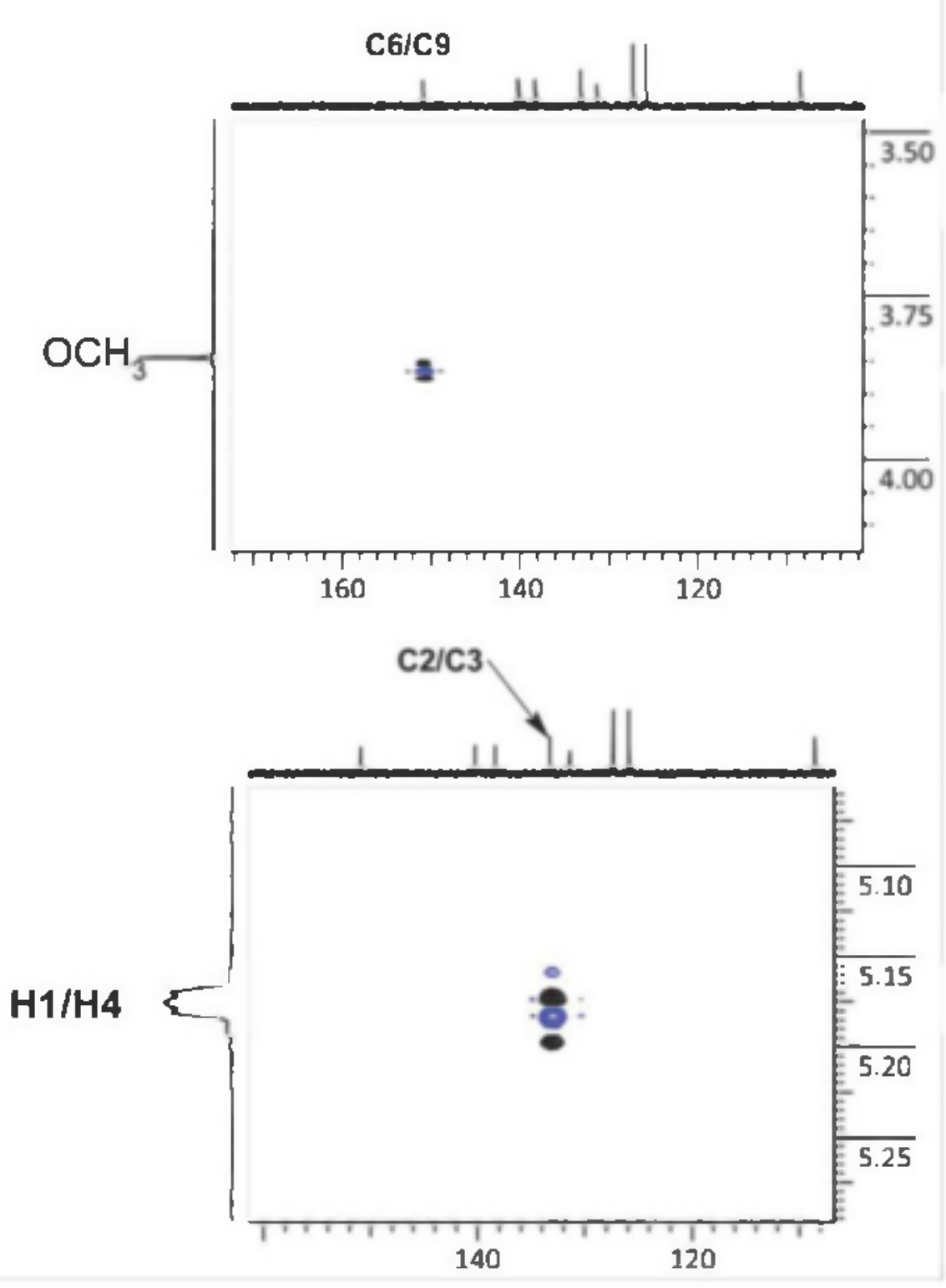




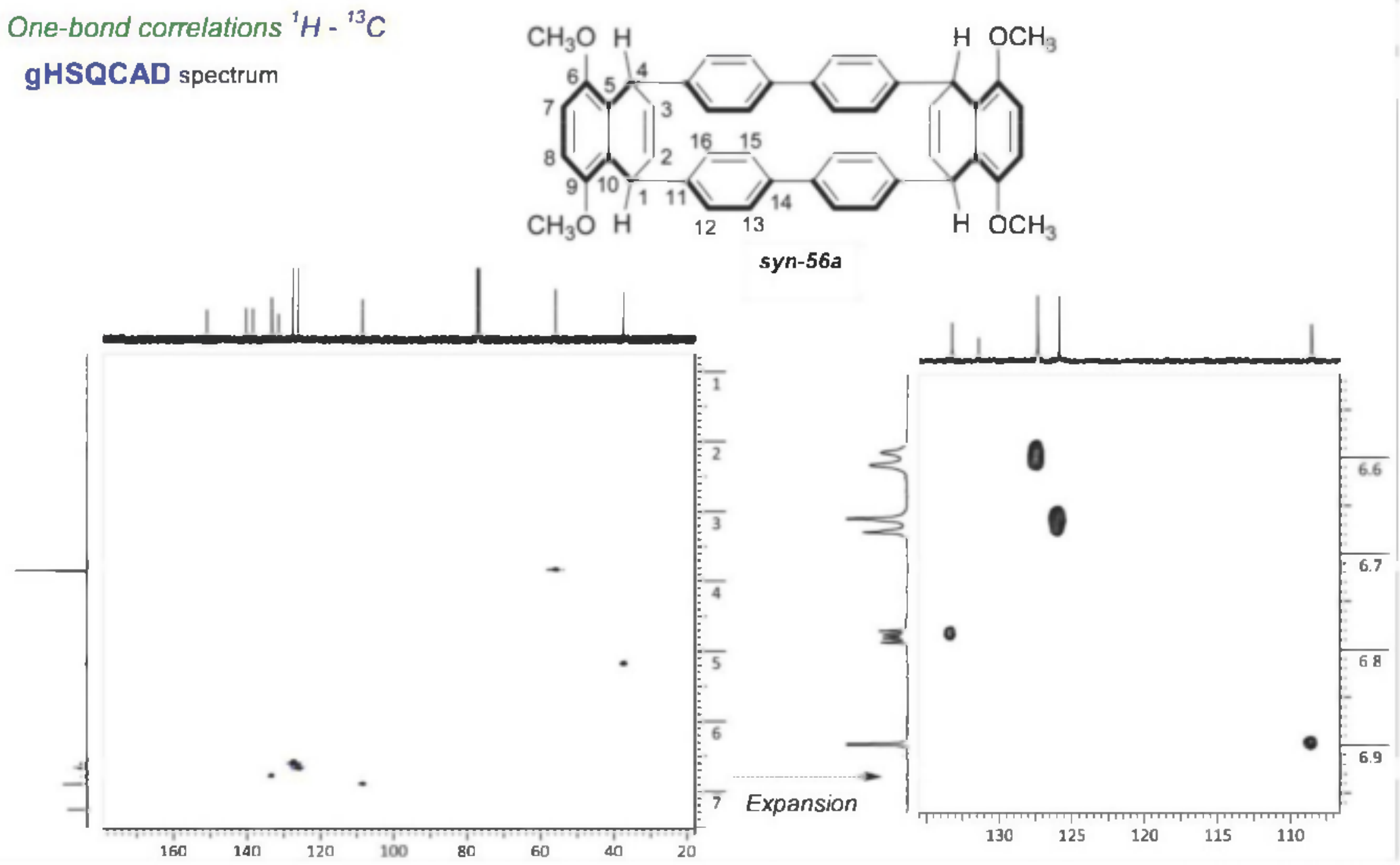




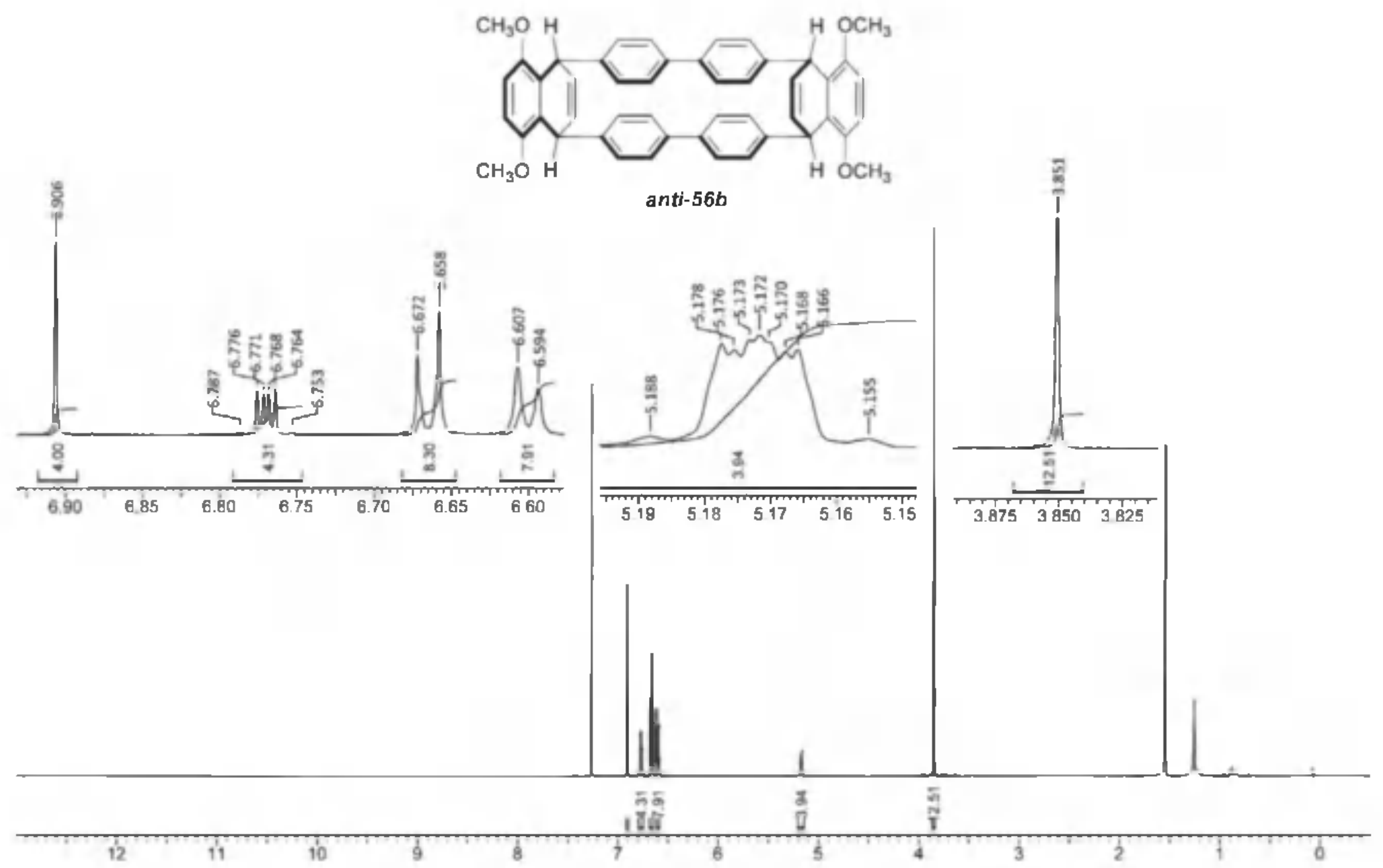




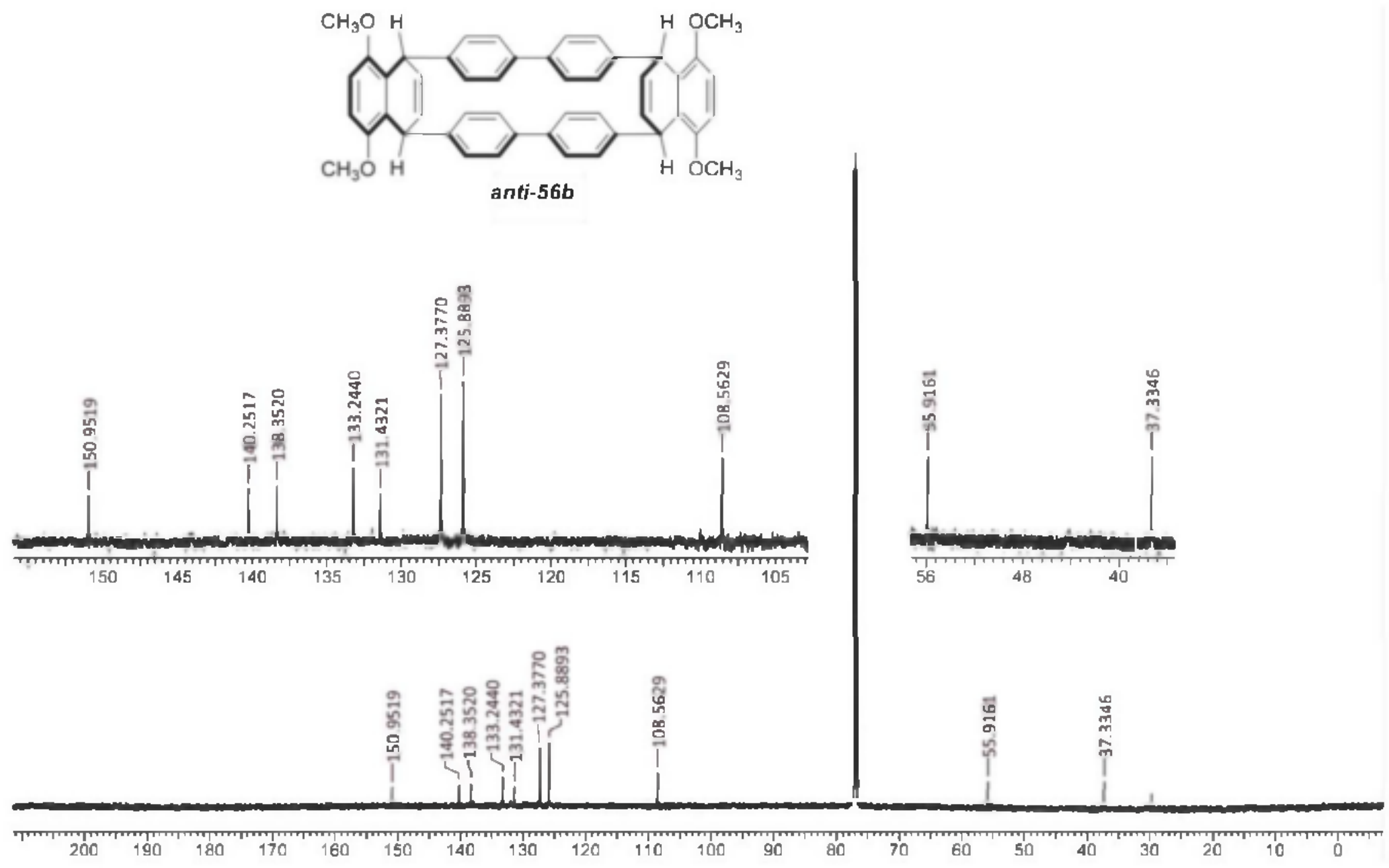




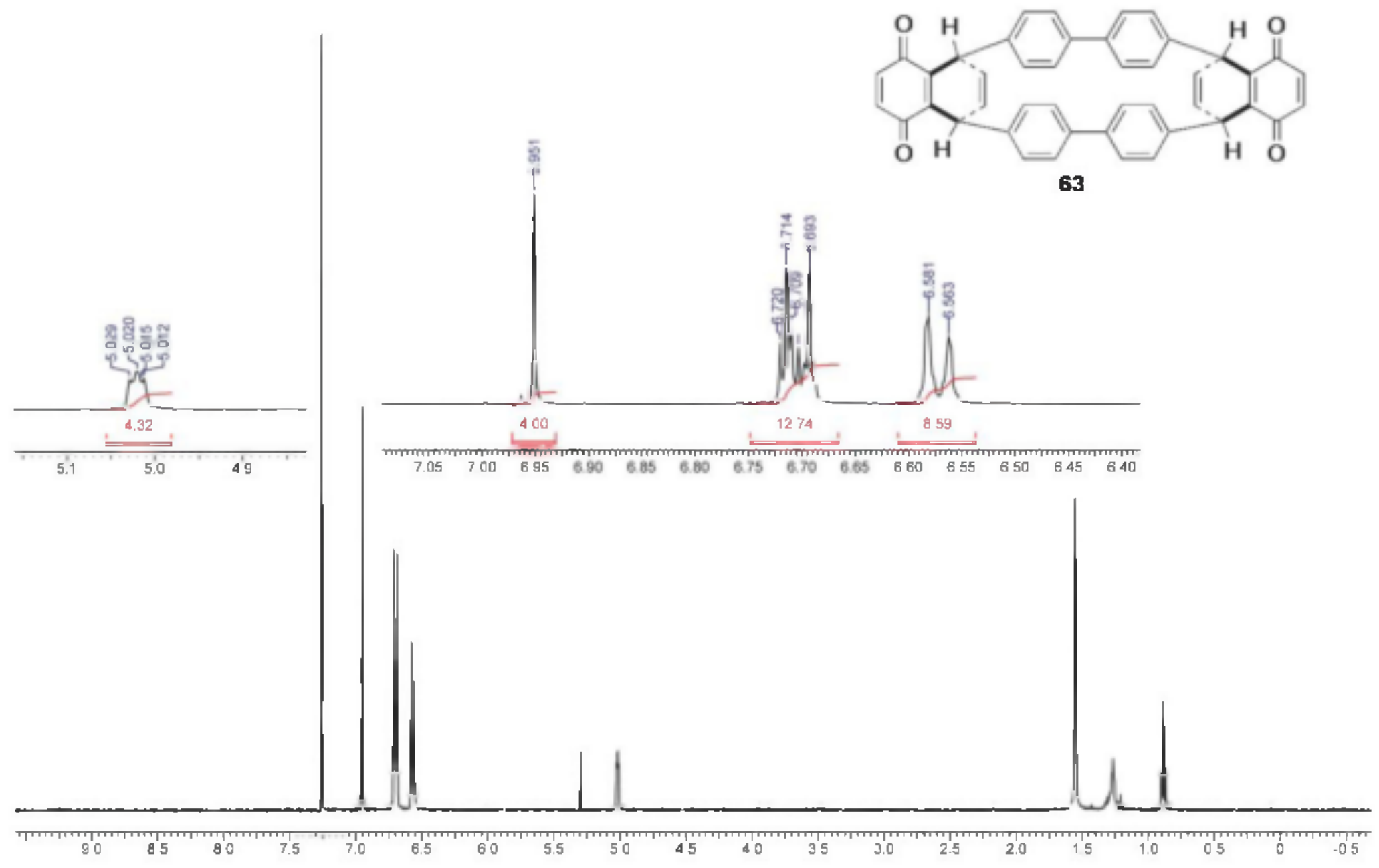




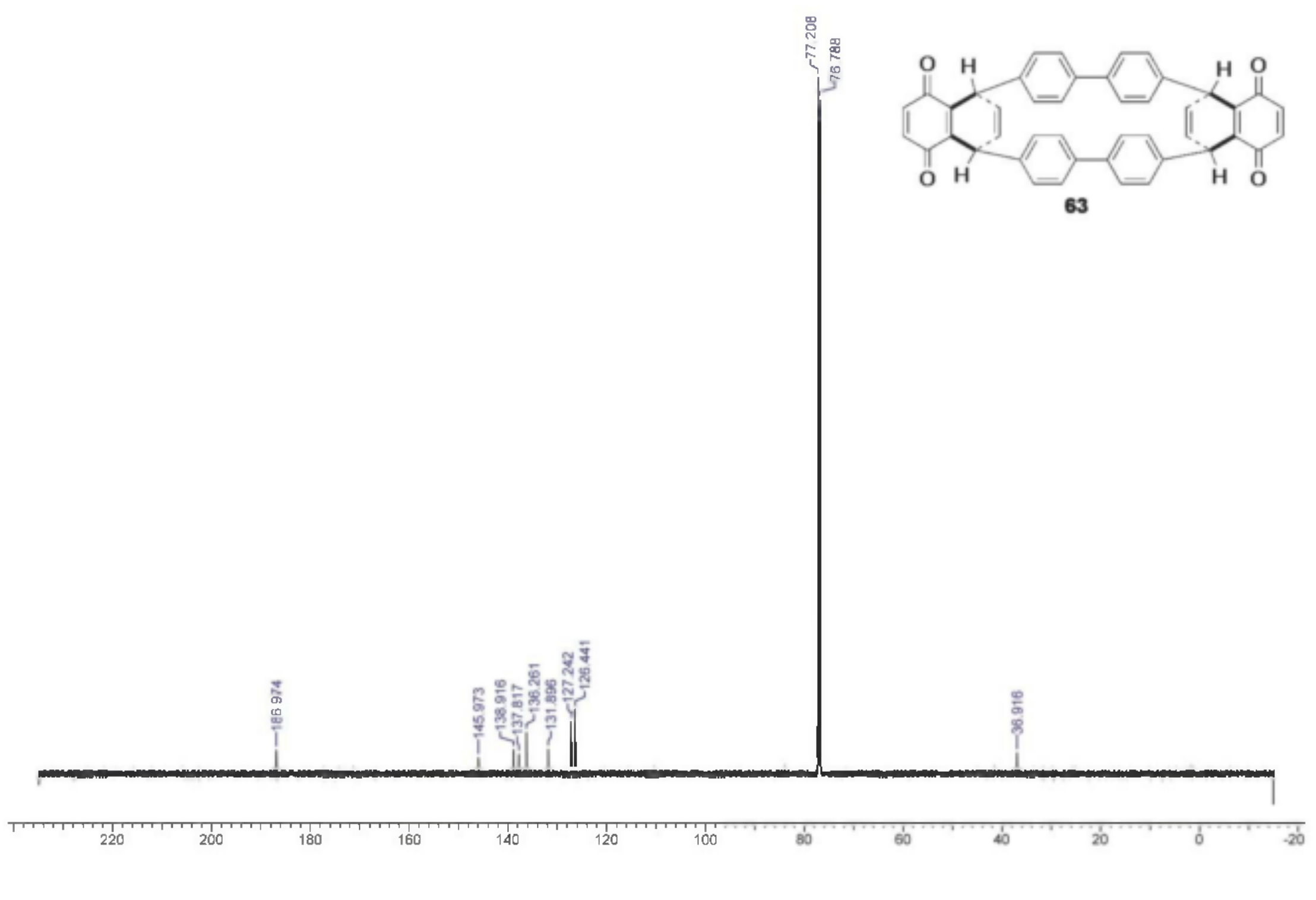




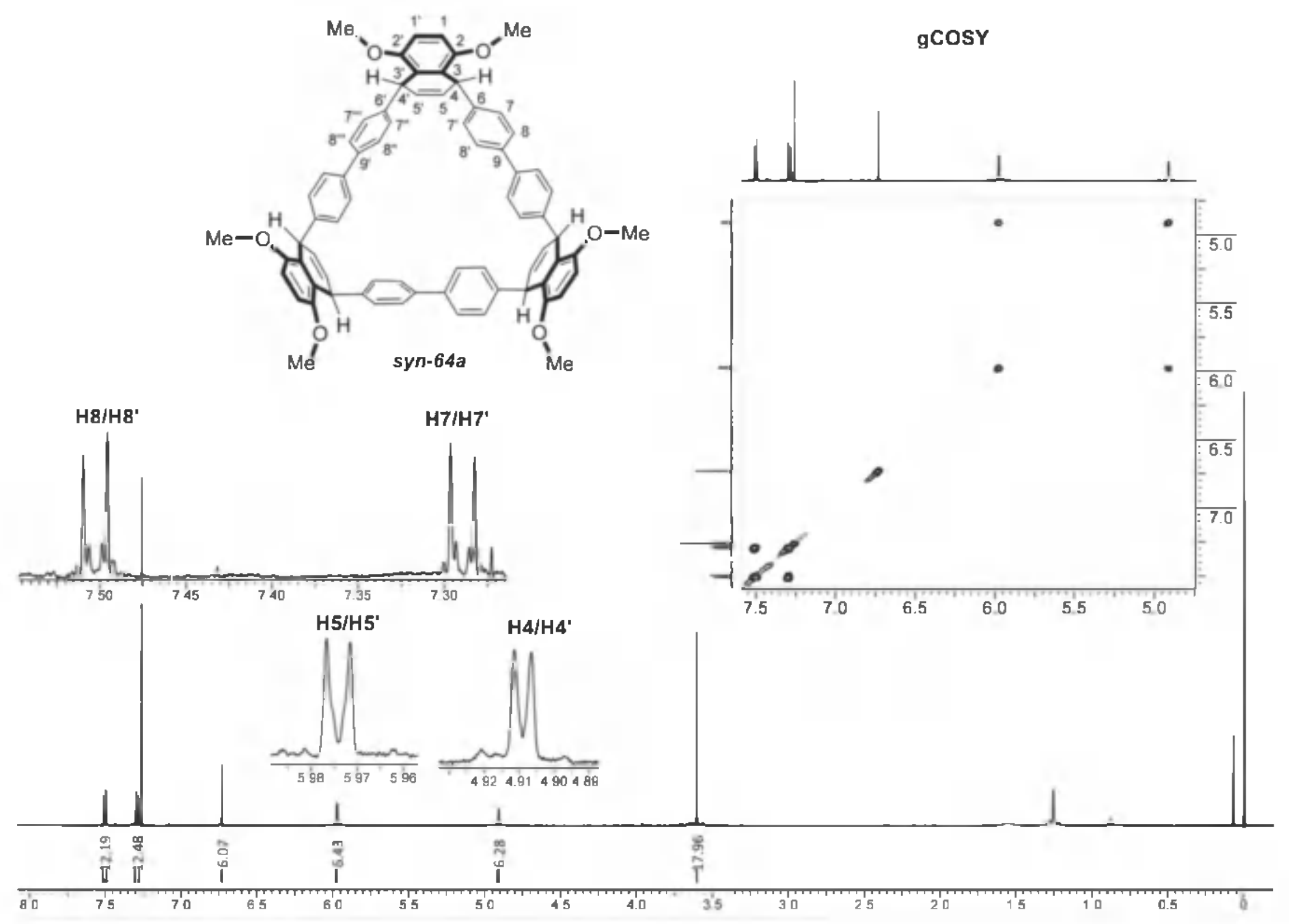




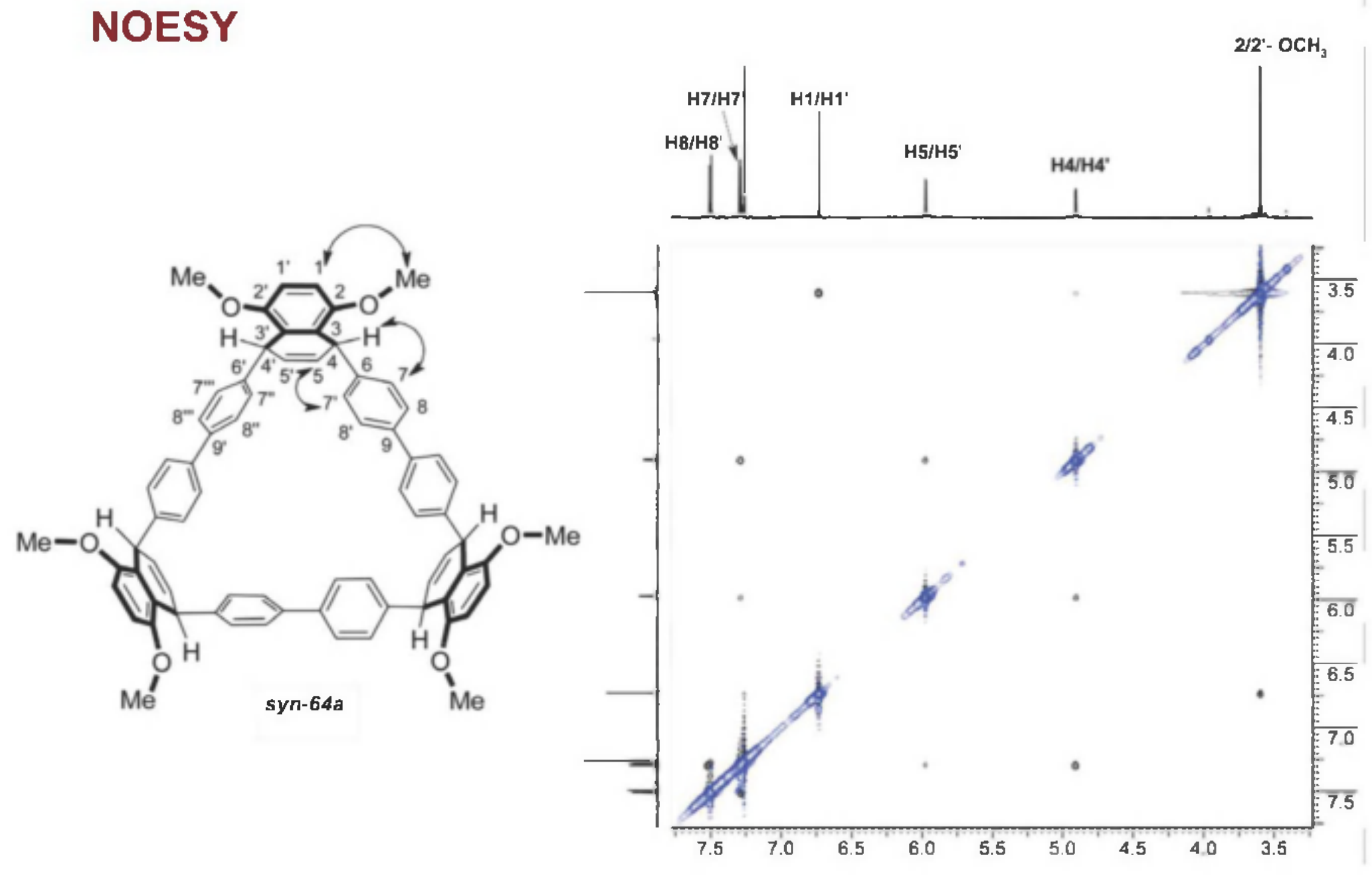


${ }^{19} \mathrm{C}$ NMR spectrum in $\mathrm{CDCl}_{3}$

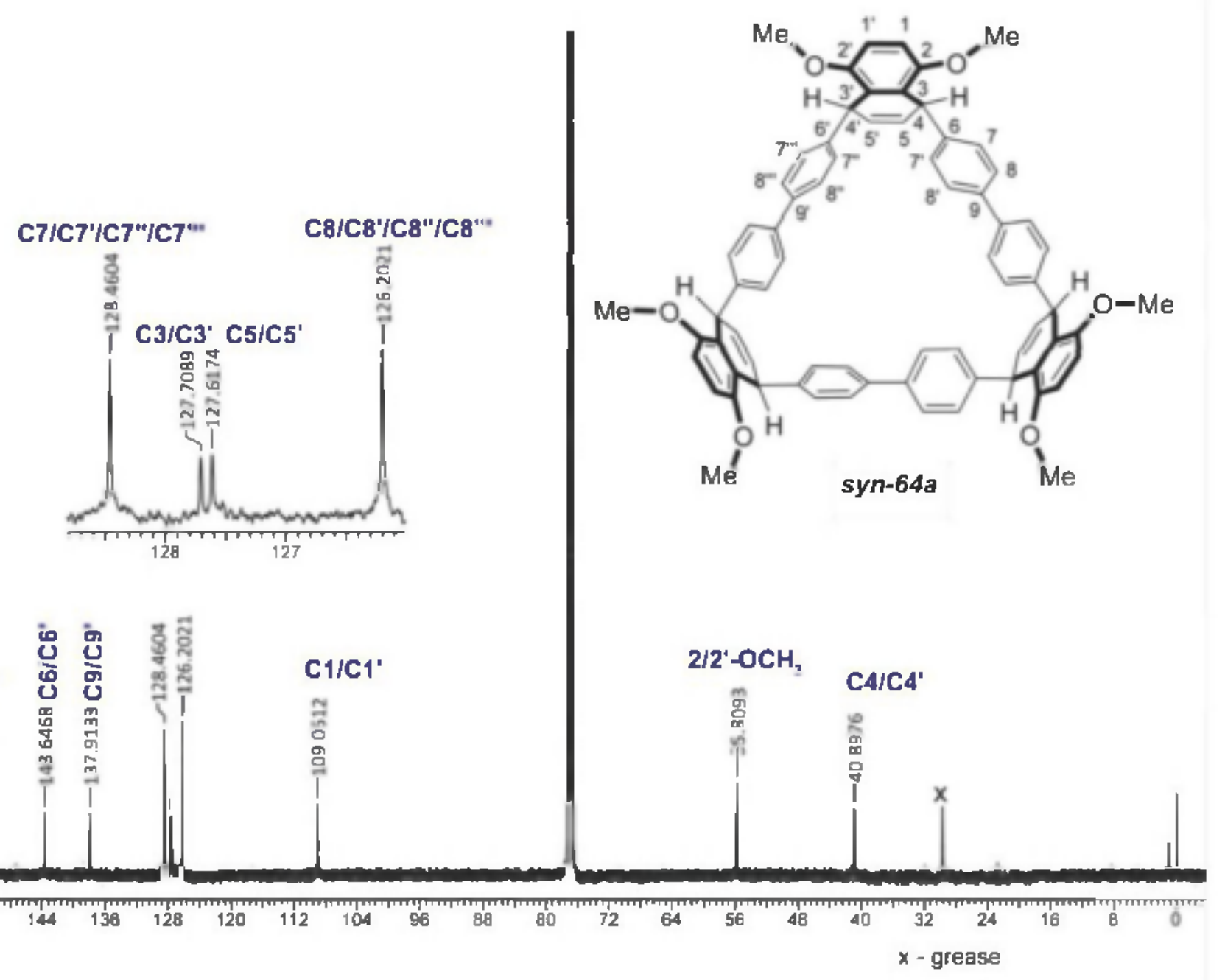


Adiabatic version of gradient selected

${ }^{1} \mathrm{H}-{ }^{13} \mathrm{C}$ gHSQCAD spectrum

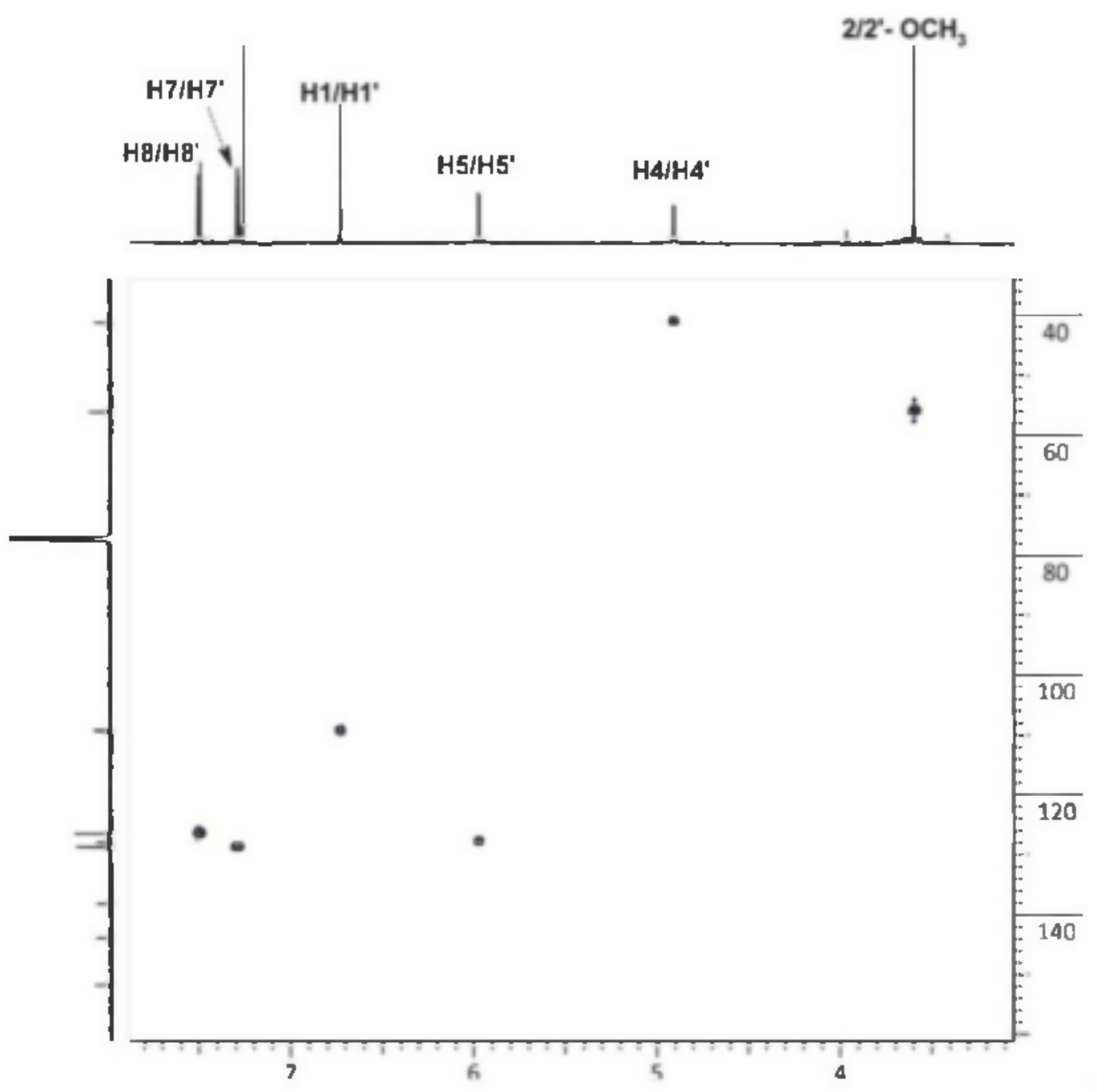




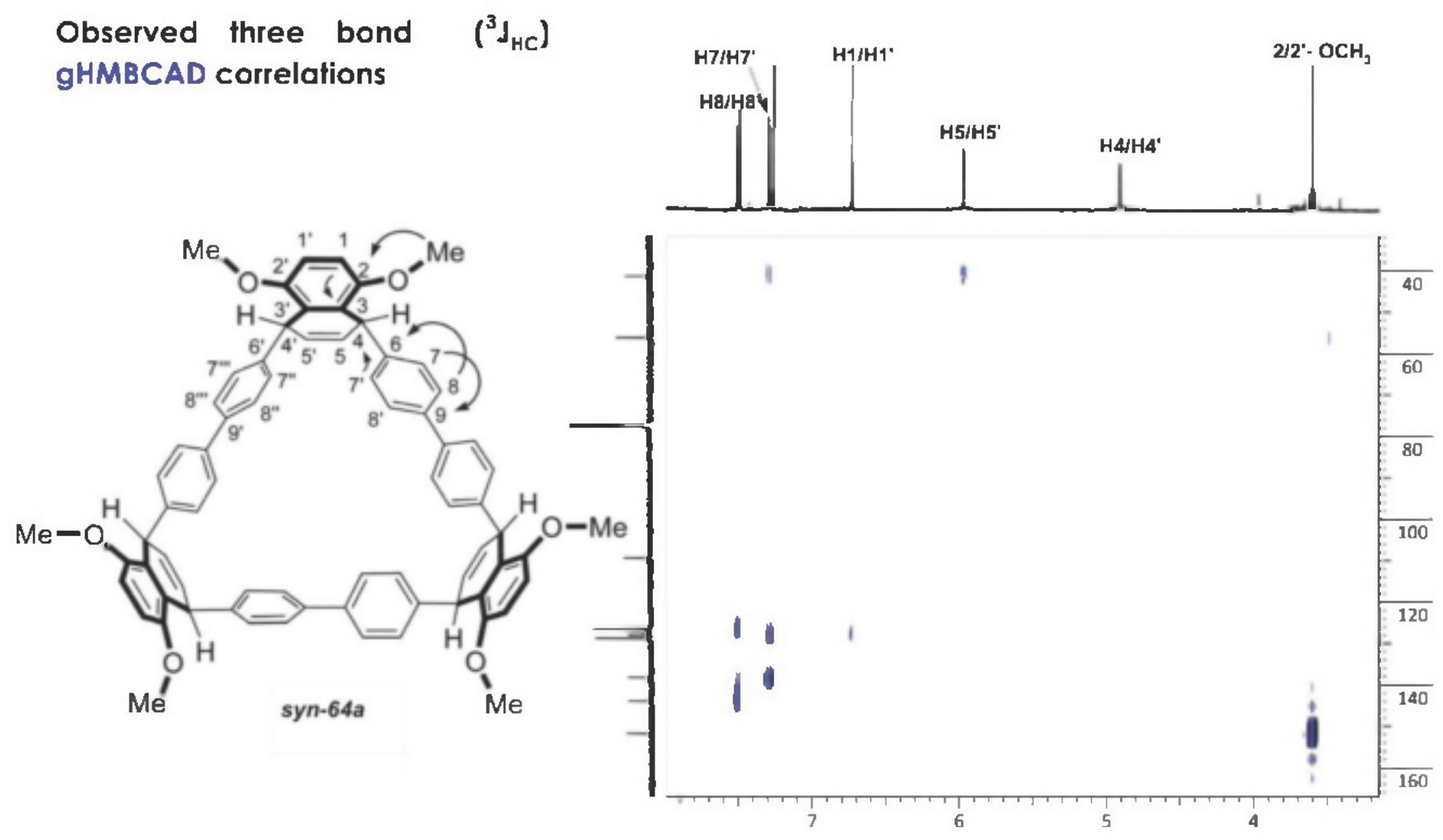




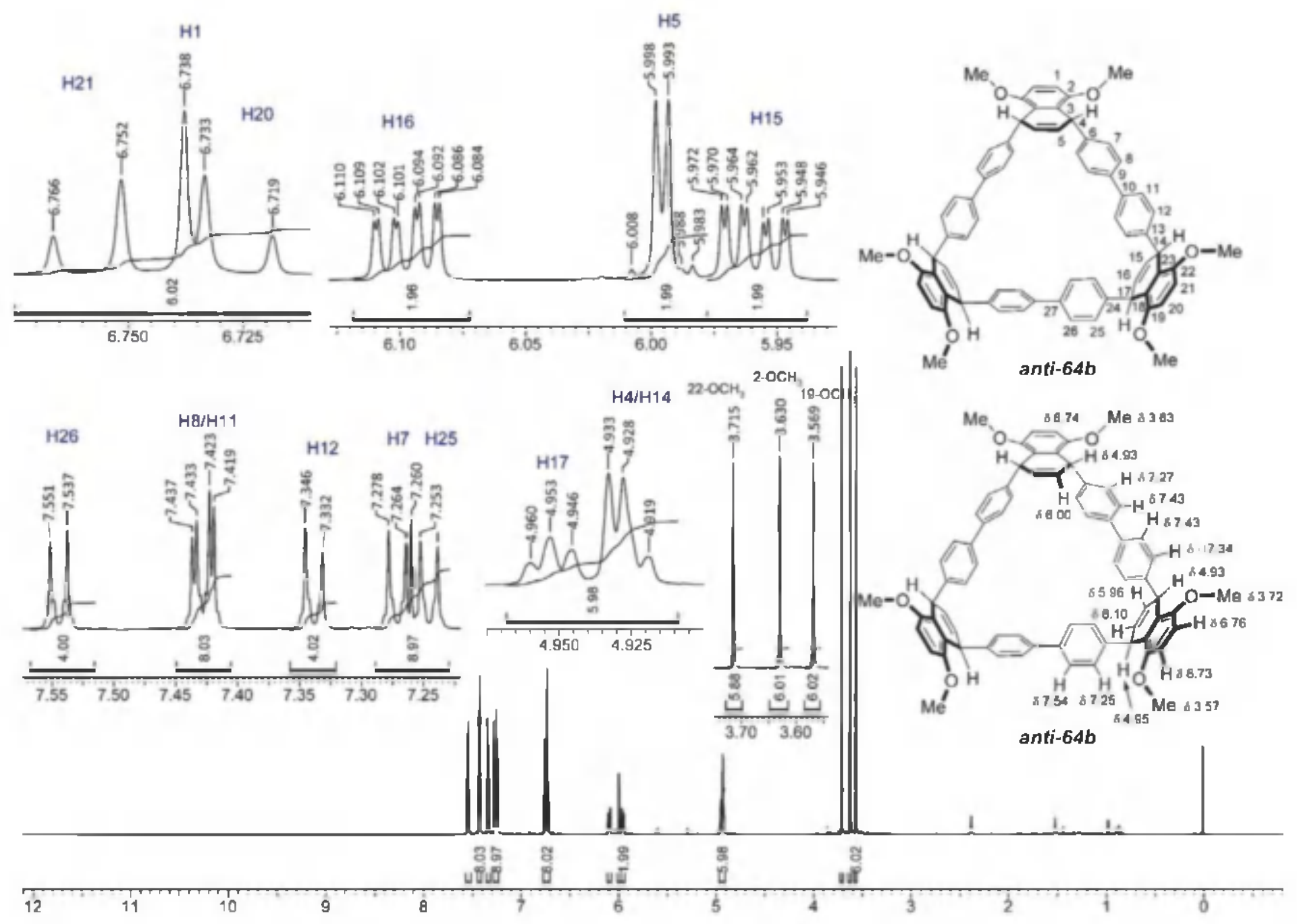


Experimental (a) and calculated

(b) splitting patterns

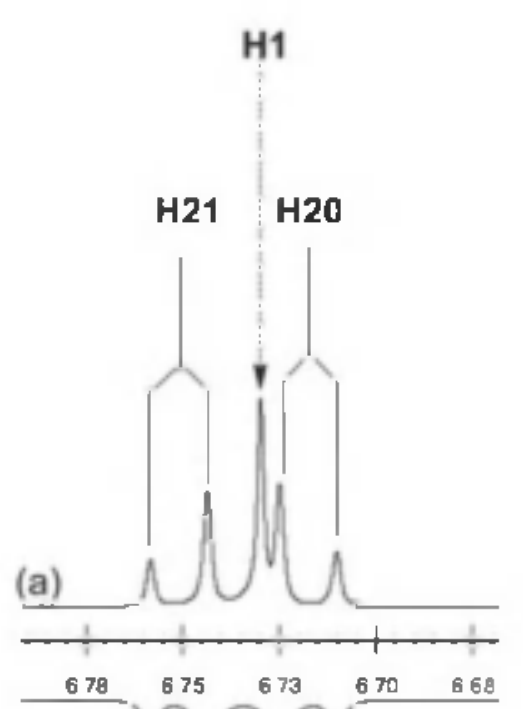

(b)

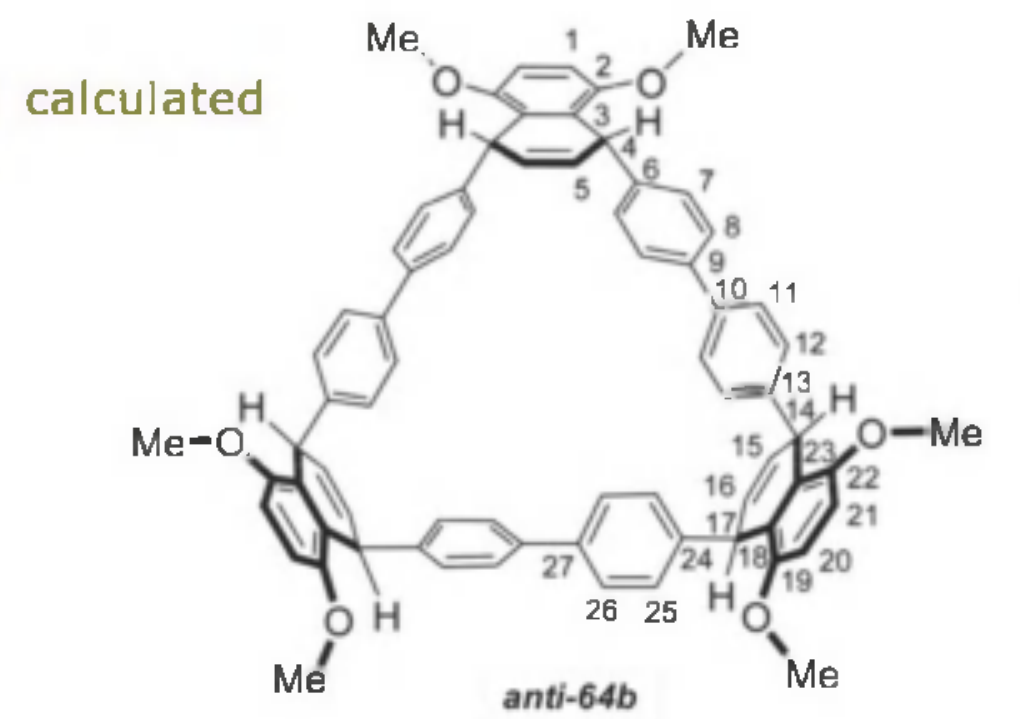

' $J(H 14, H 17)=4.17 \mathrm{~Hz}$

$3(\mathrm{H} 14, \mathrm{H} 15)=4.73 \mathrm{~Hz}$

${ }^{4} \mathrm{~J}(\mathrm{H} 14, \mathrm{H} 16)=1.13 \mathrm{~Hz}$

$33(\mathrm{H} 17, \mathrm{H} 16)=4.9 \mathrm{~Hz}$

${ }^{4} \mathrm{~J}(\mathrm{H} 17, \mathrm{H} 15)=1.21 \mathrm{~Hz}$

$3(\mathrm{H} 15, \mathrm{H} 16)=9.83 \mathrm{~Hz}$

$31\left(\mathrm{H}_{20}, \mathrm{H} 21\right)=8.90 \mathrm{~Hz}$

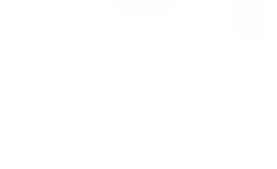

H4
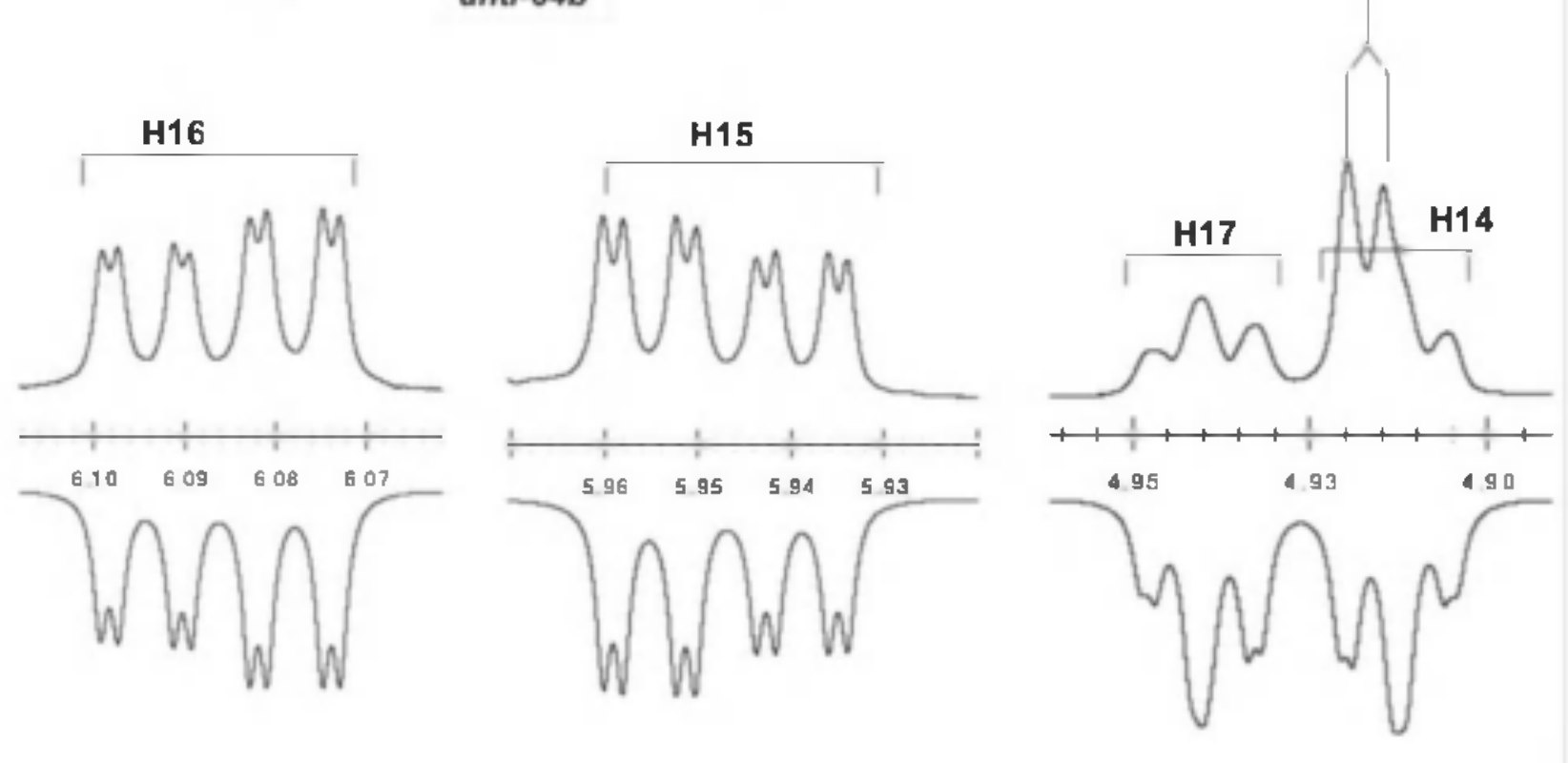
Experimental (a) and calculated

(b) splitting patterns
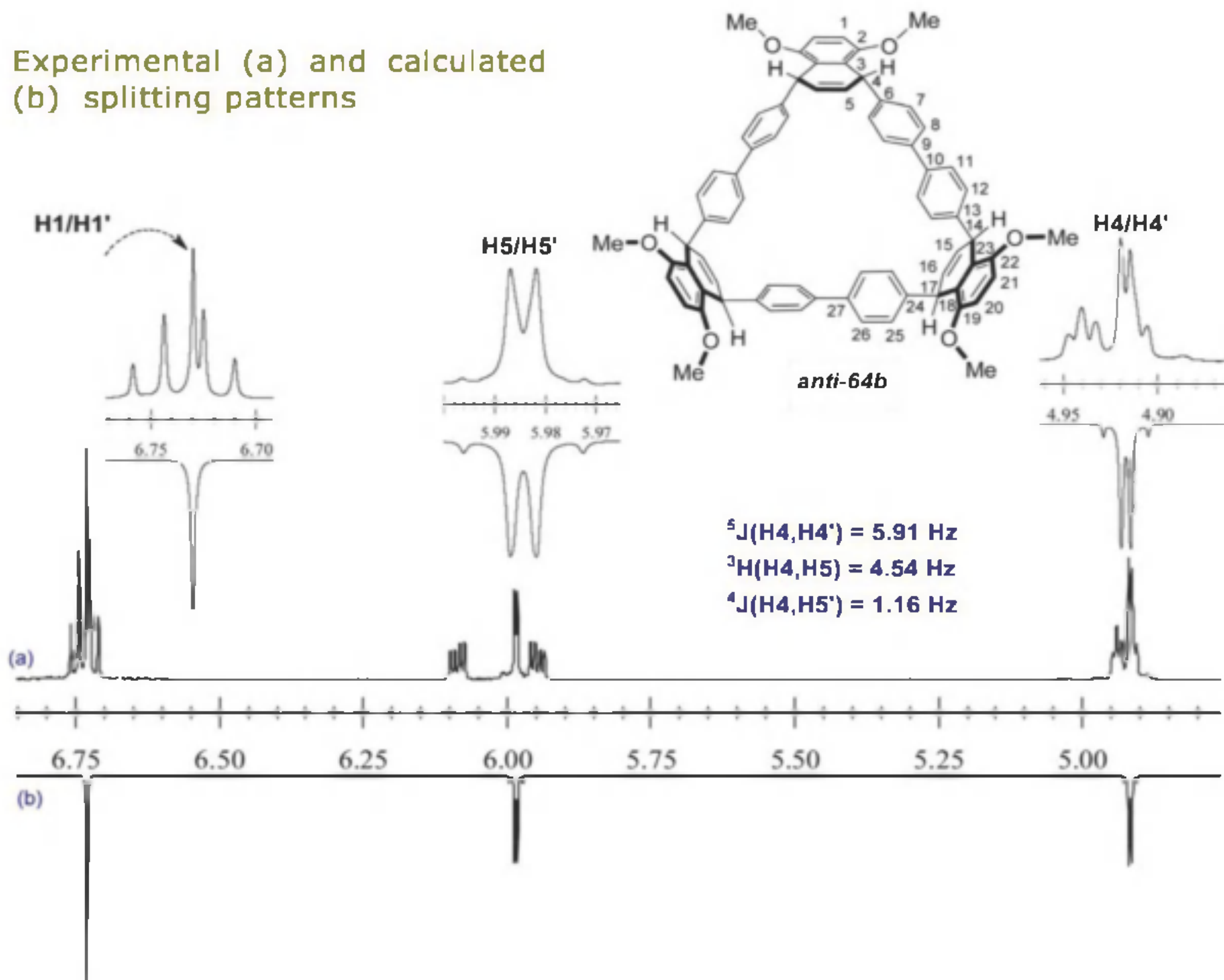


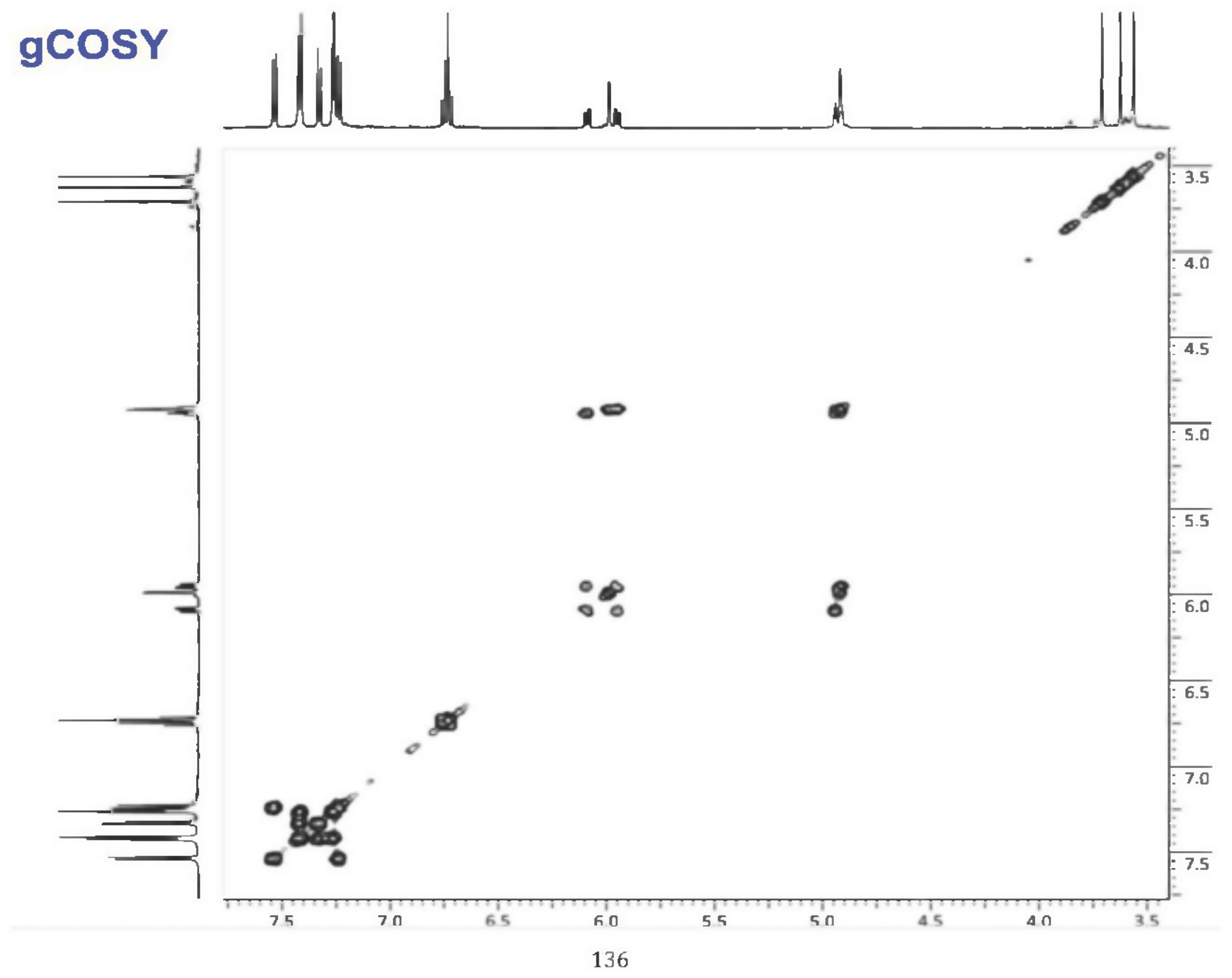




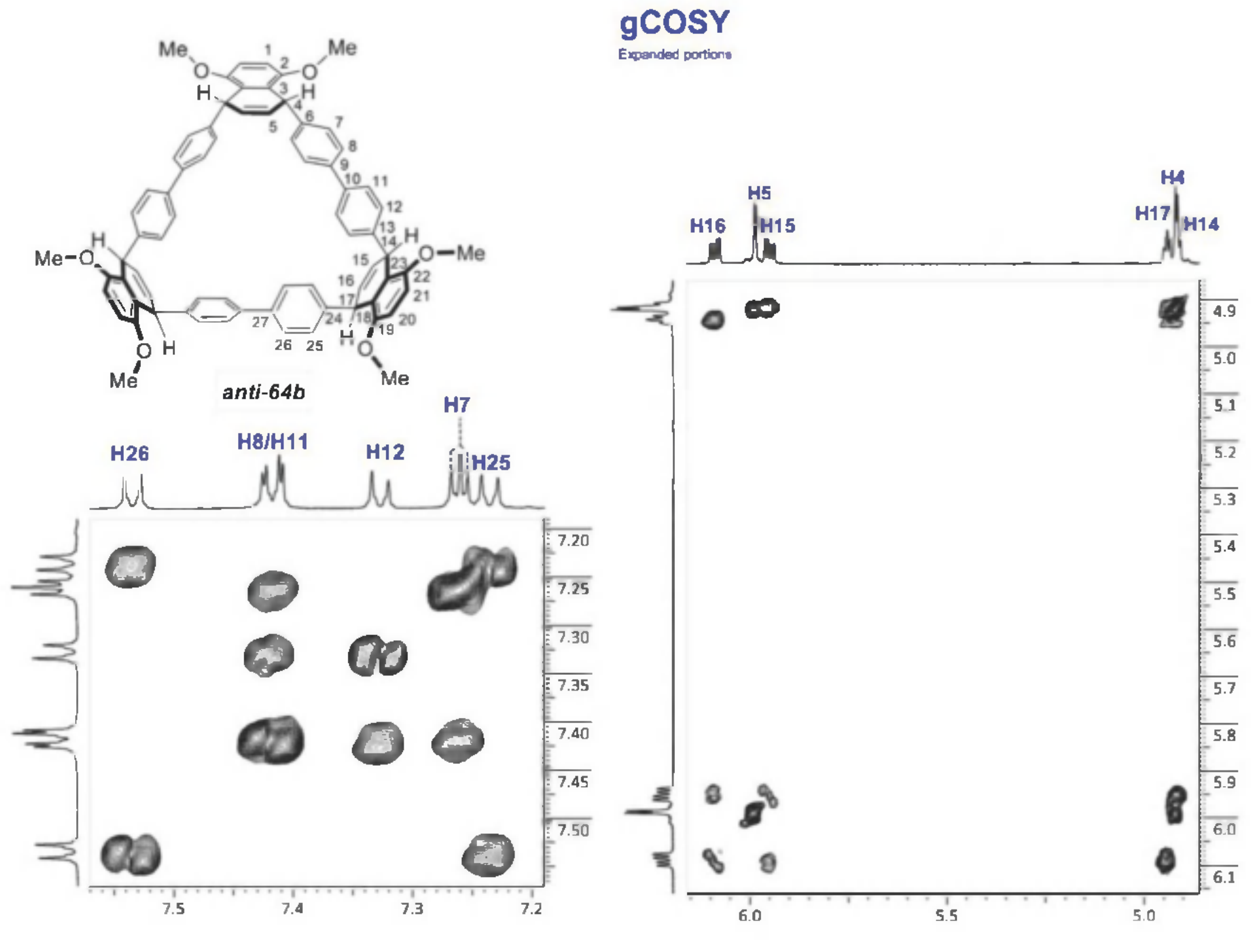


NOESY

(mix $=0.8 s$, relaxation delay $=2 s$ )
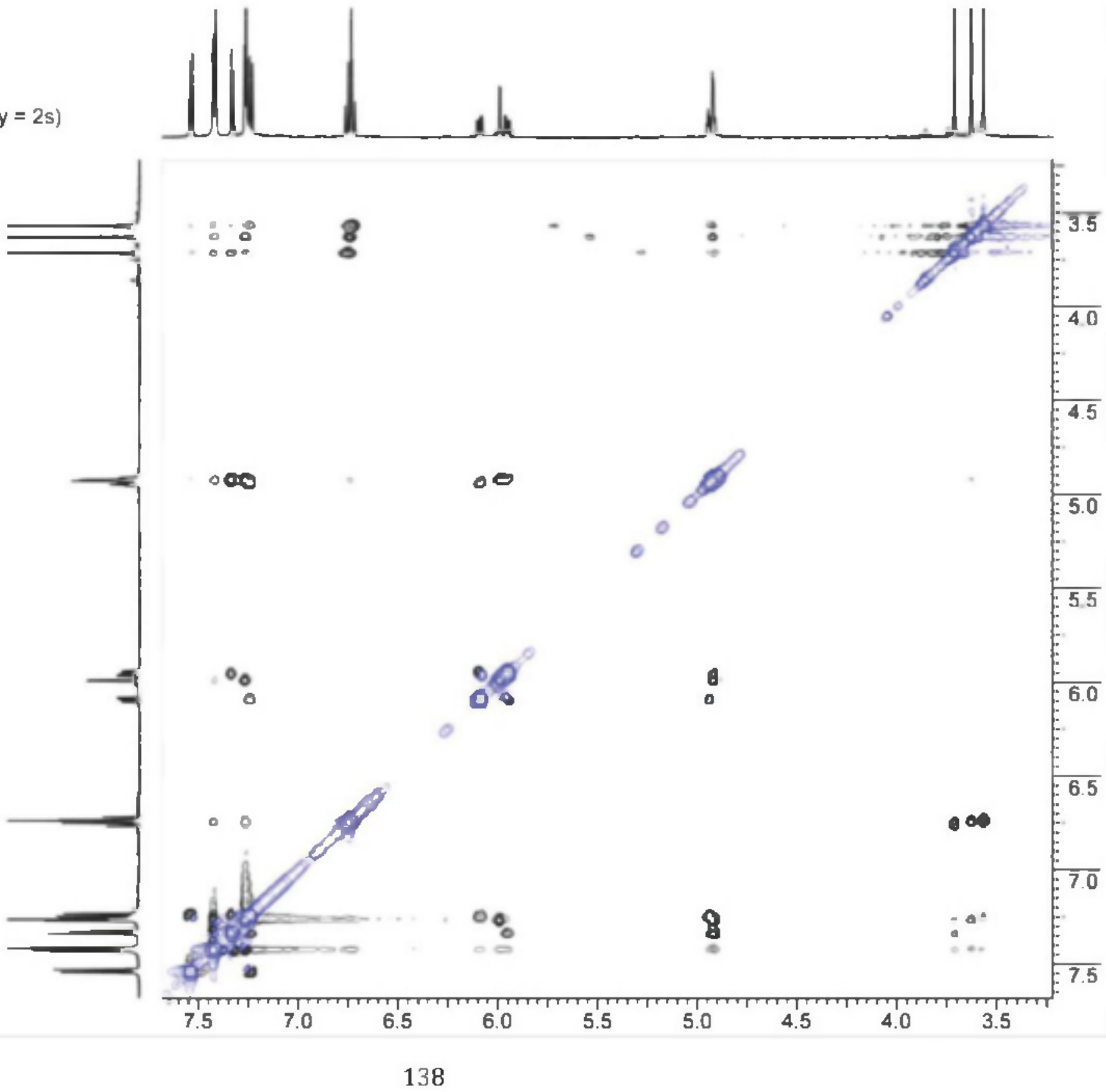


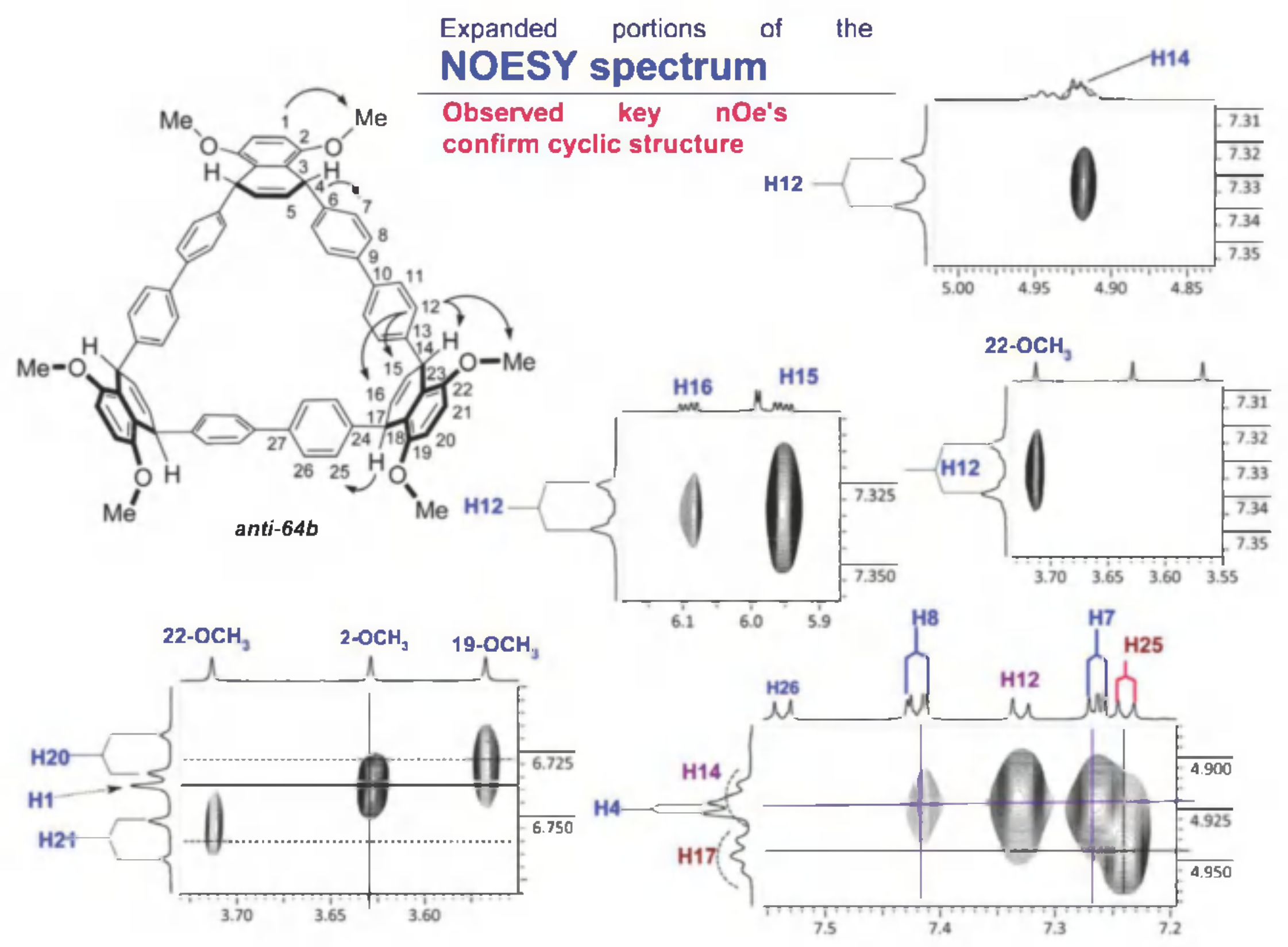


Expanded portion of the NOESY spectrum

Differentitation of $\mathrm{CH}_{3} \mathrm{O}$ groups

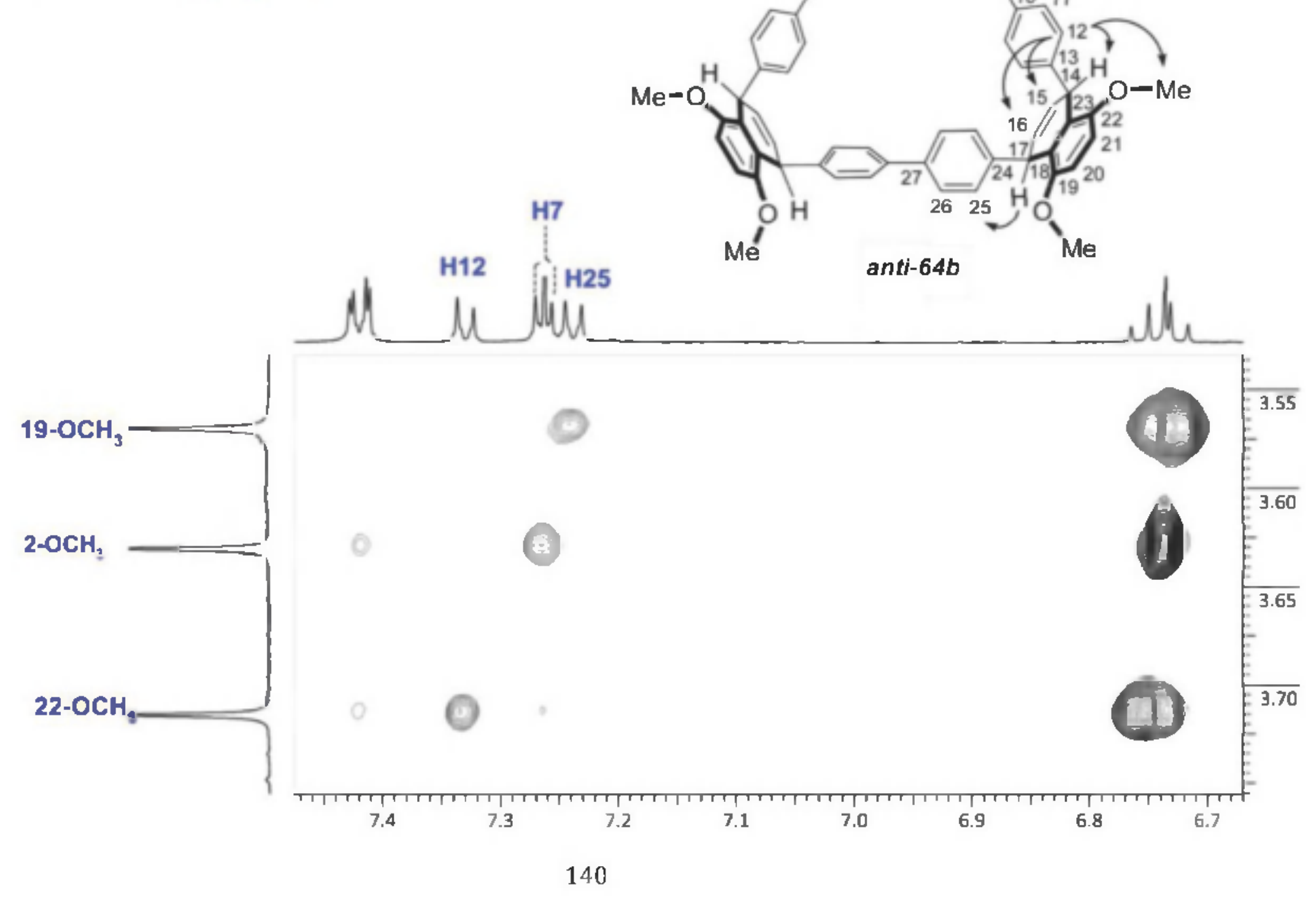



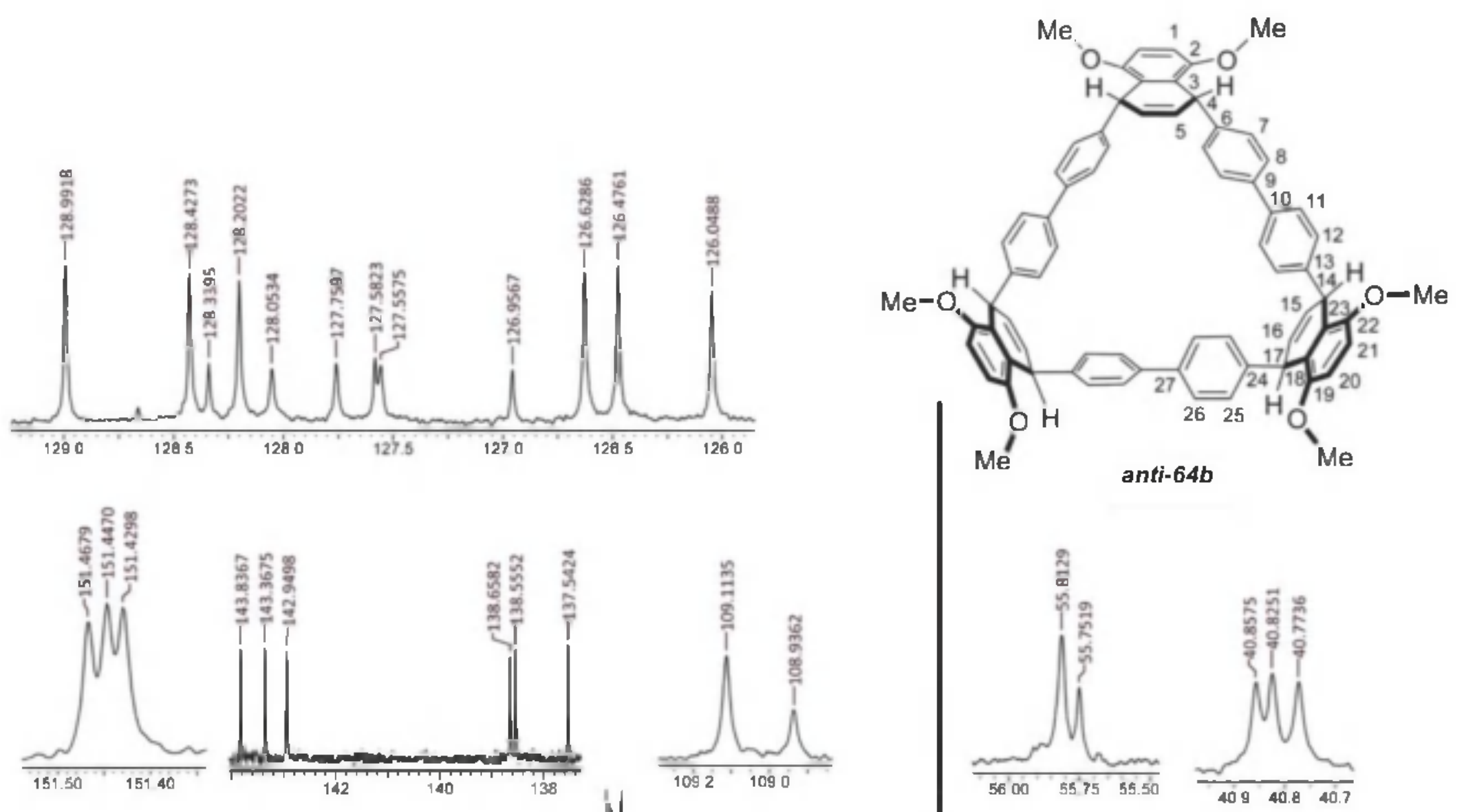
One bond correlation ( ${ }^{1} \mathrm{~J}_{\mathrm{C}-\mathrm{H}}$ )

\section{HETCOR}

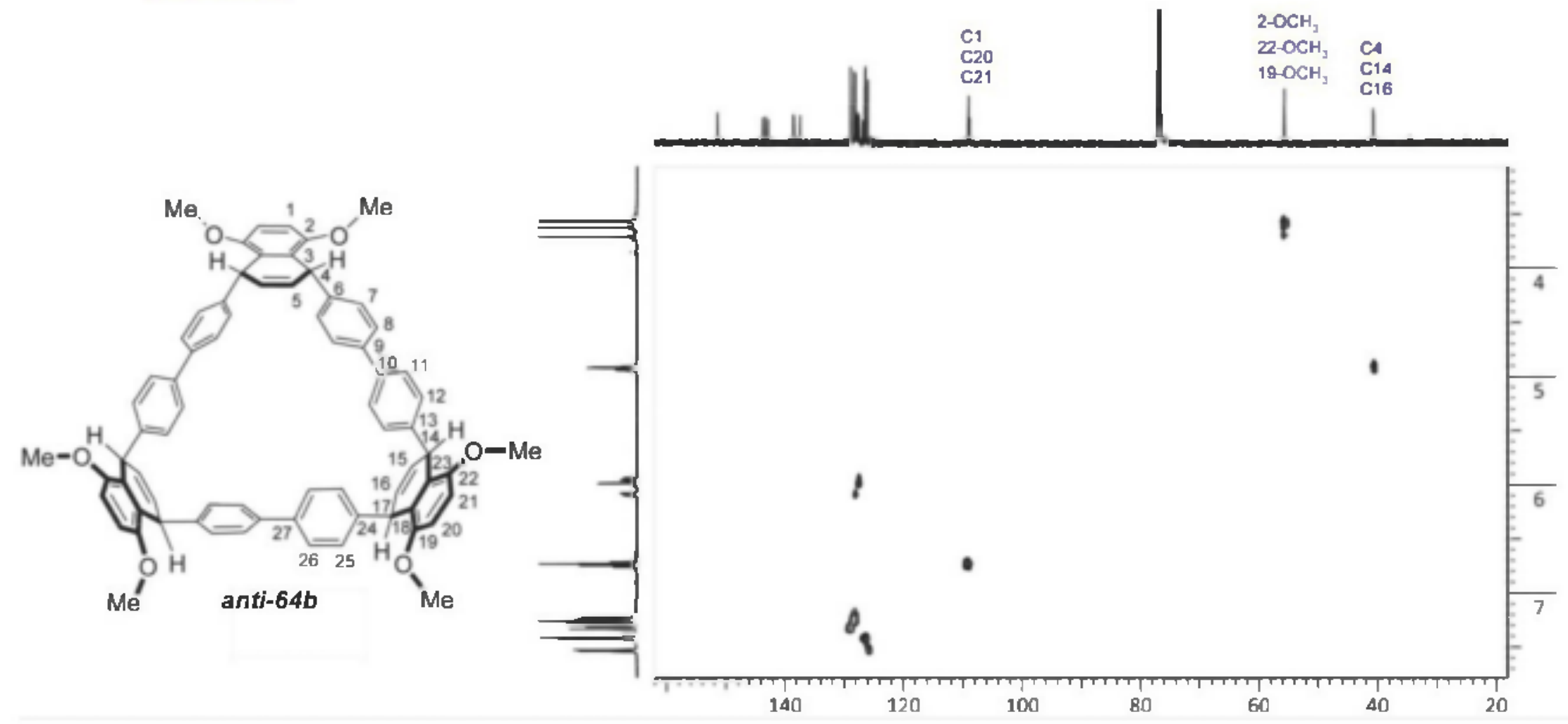


Expanded portion of the one-bond correlation

(' $\left.\mathrm{J}_{\mathrm{CH}}\right)$ HETCOR spectrum

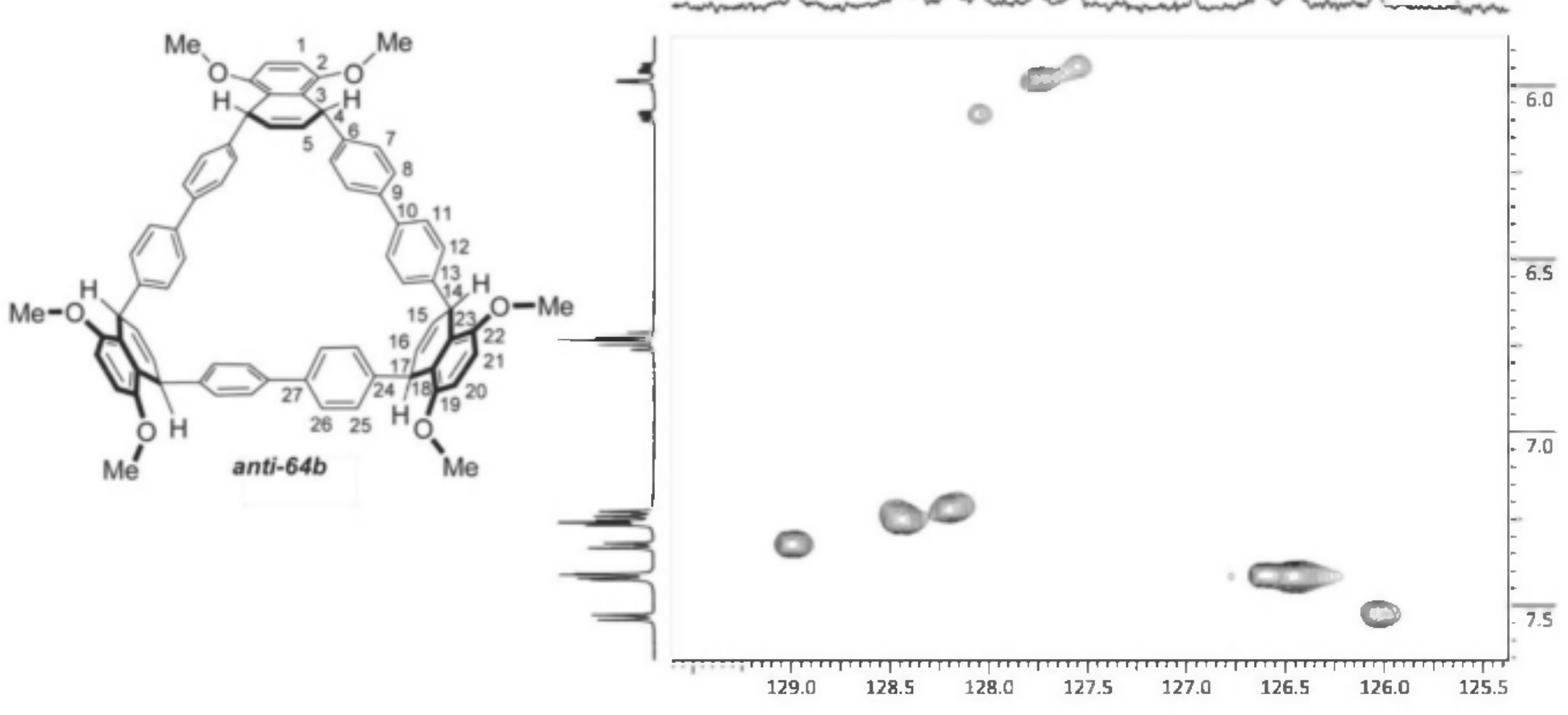




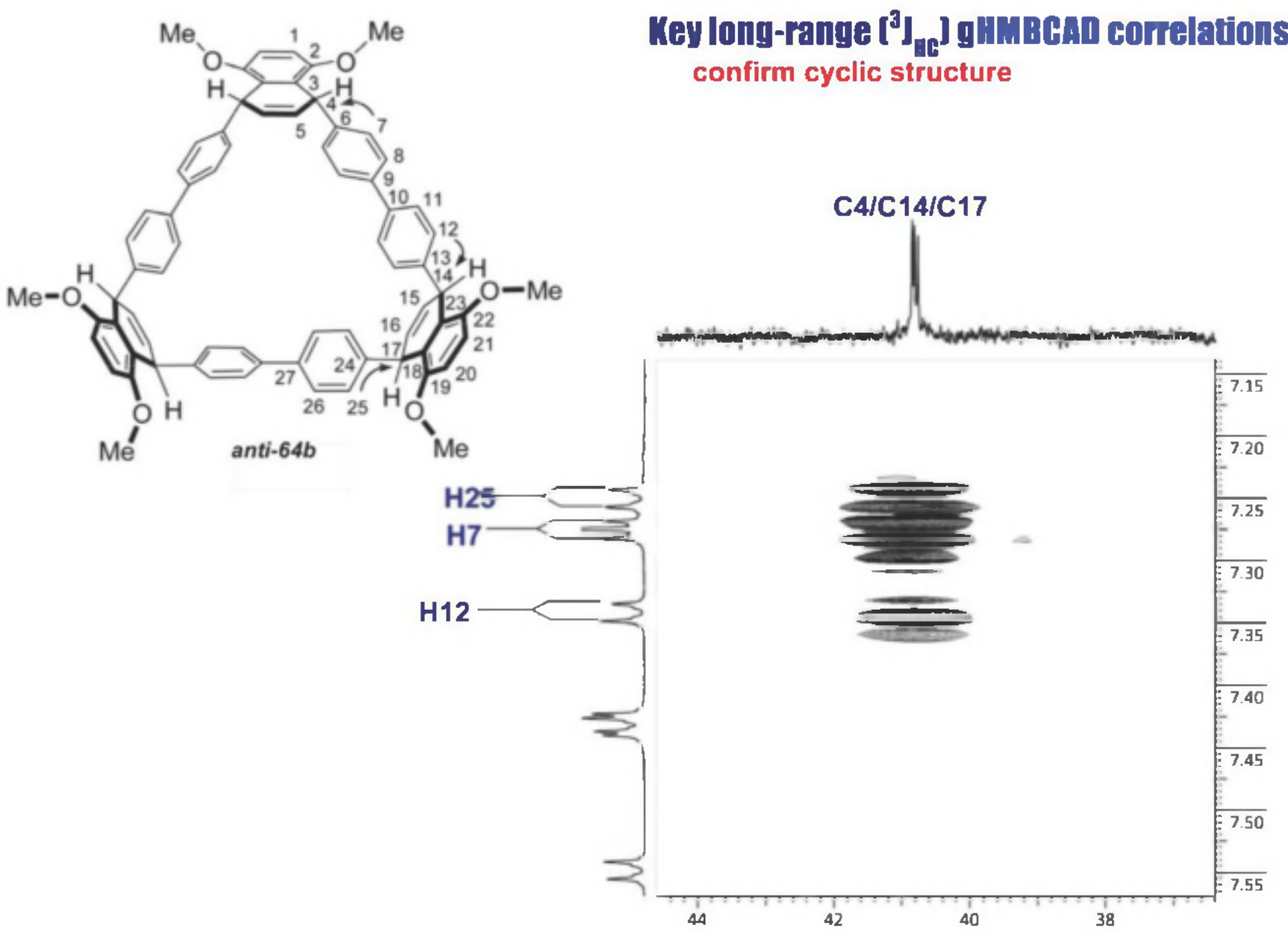


Adiabatic version of the gHMBCAD

spectrum
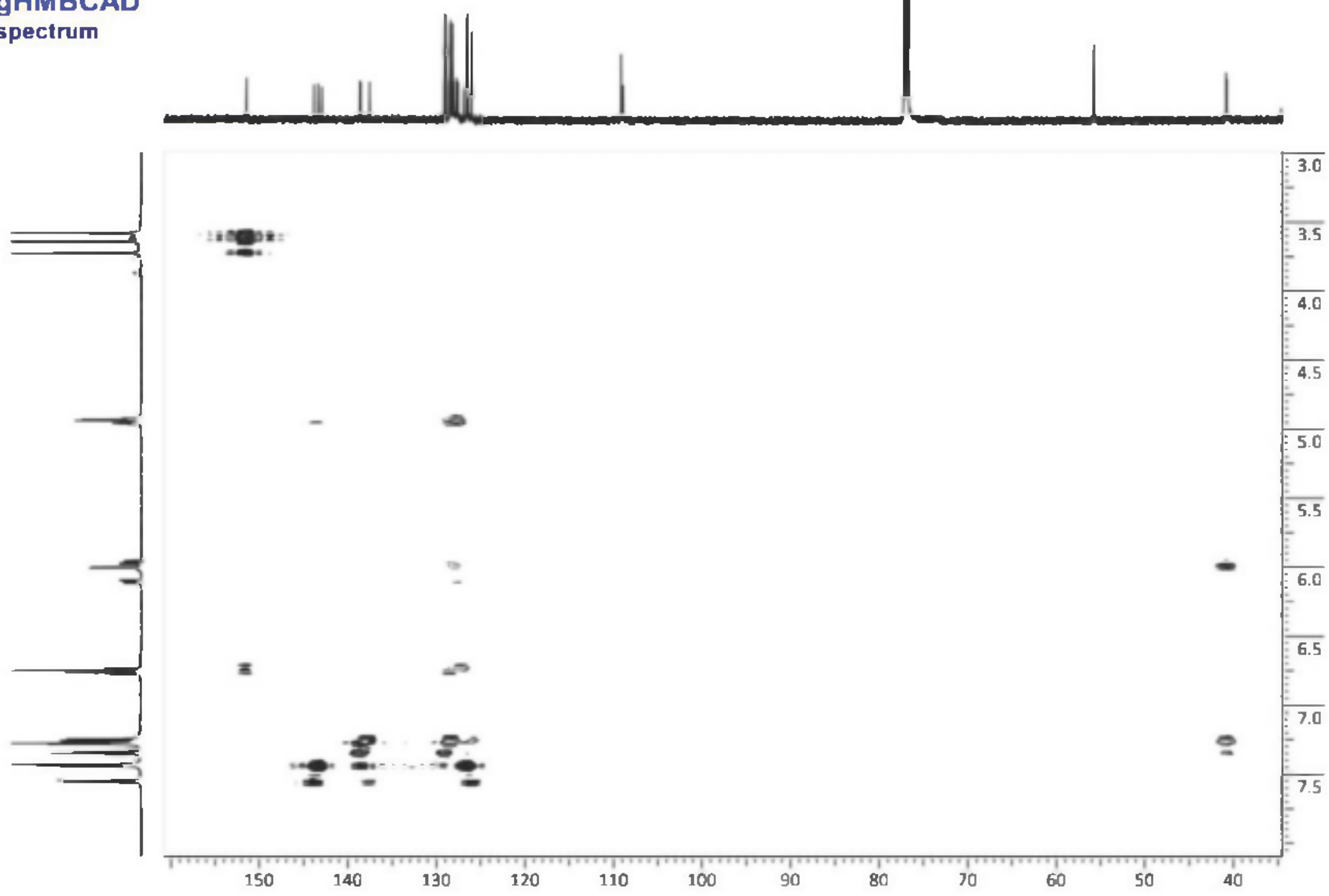
Adiabatic version of gHSQCAD

one-bond comelations
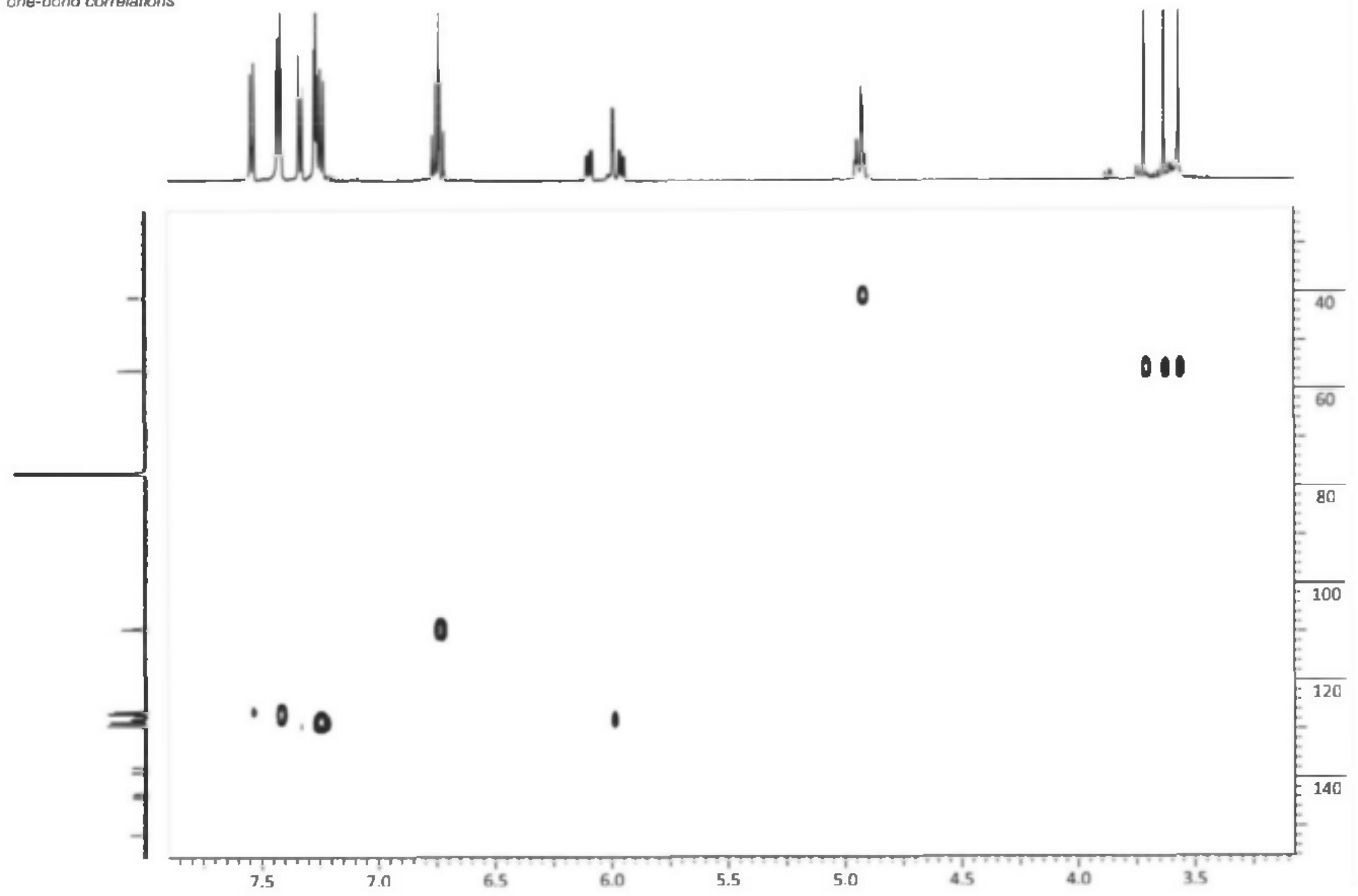


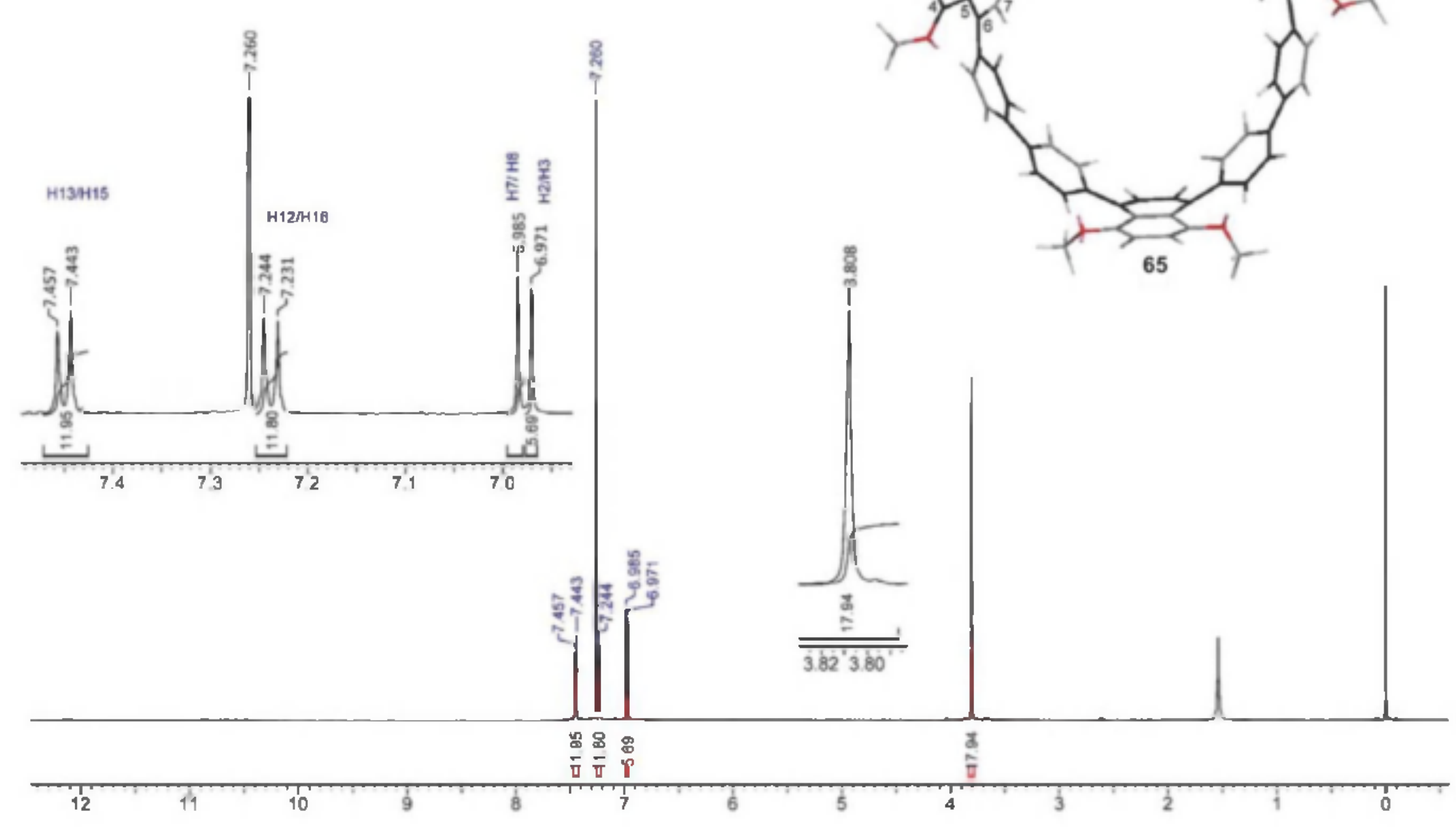


Experimental (a) and calculated (b) splitting patterns

for H12, H13, H15, and H16

\section{H13/H15 H12/H16}

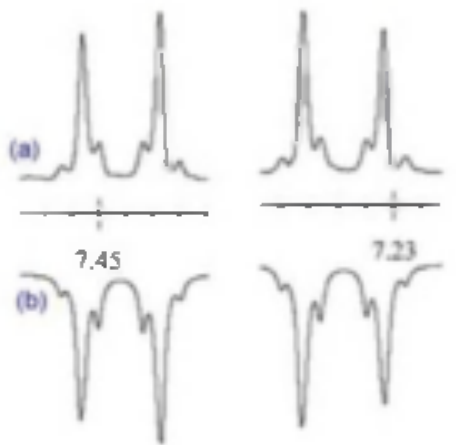

$\left.\left.{ }^{3}\right\lrcorner(\mathrm{H} 12, \mathrm{H} 13)={ }^{4}\right\lrcorner(\mathrm{H} 16,1 \mathrm{H} 15)=8.68 \mathrm{~Hz}$

$\left.{ }^{5}\right\lrcorner(H 12, H 15)=\stackrel{s}{\lrcorner}(H 13,1 H 16)=0.48 H_{2}$

$\left.\left.{ }^{4}\right\lrcorner(\mathrm{H} 12, \mathrm{H} 16)={ }^{4}\right\rfloor(\mathrm{H} 13,1 \mathrm{H} 15)=2.13 \mathrm{~Hz}$
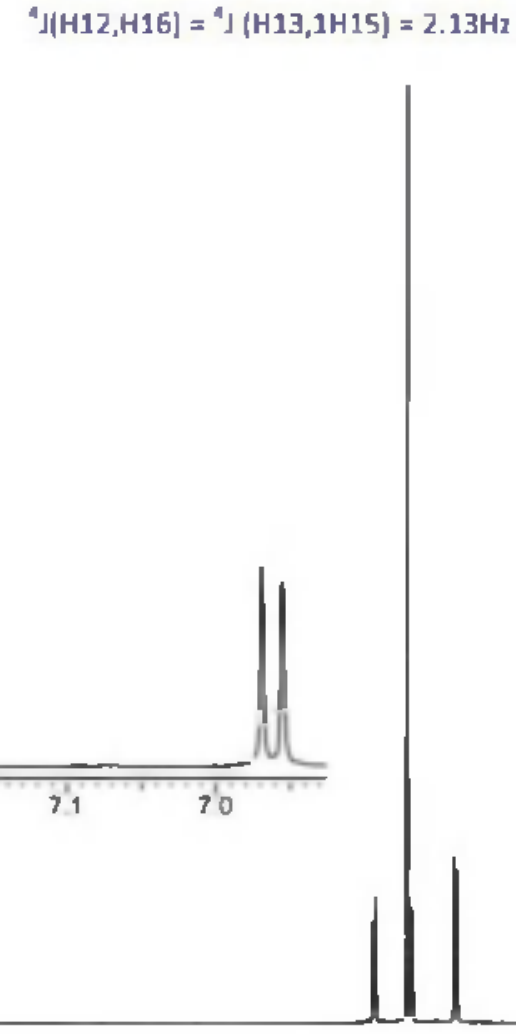

12

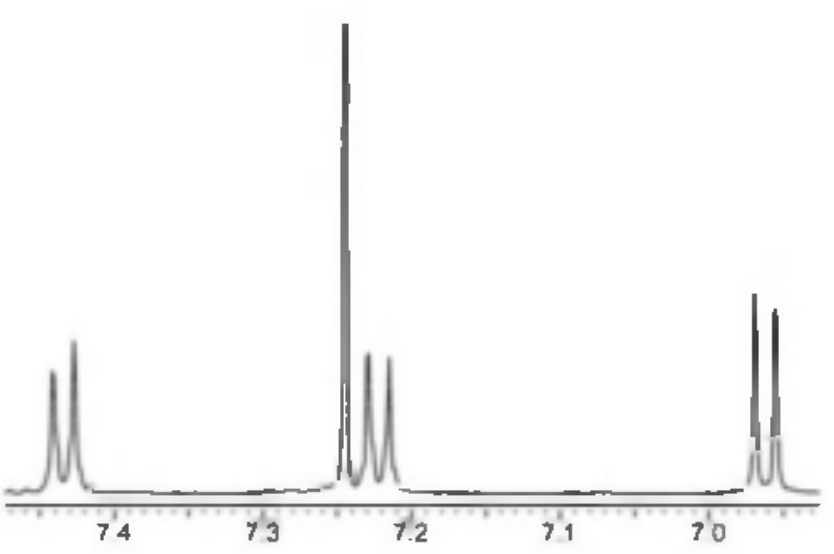

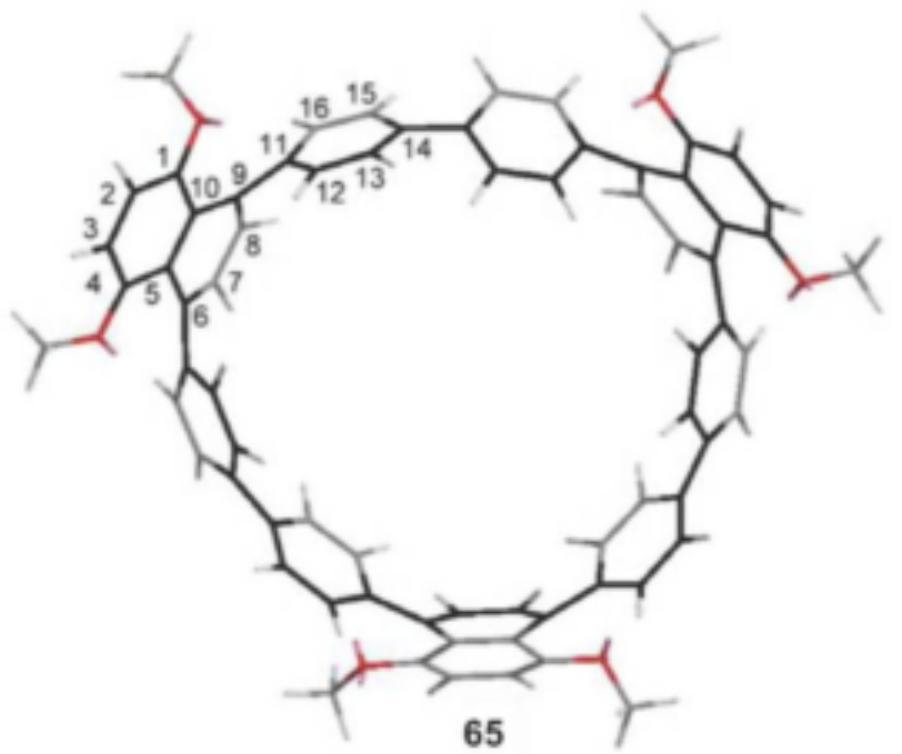

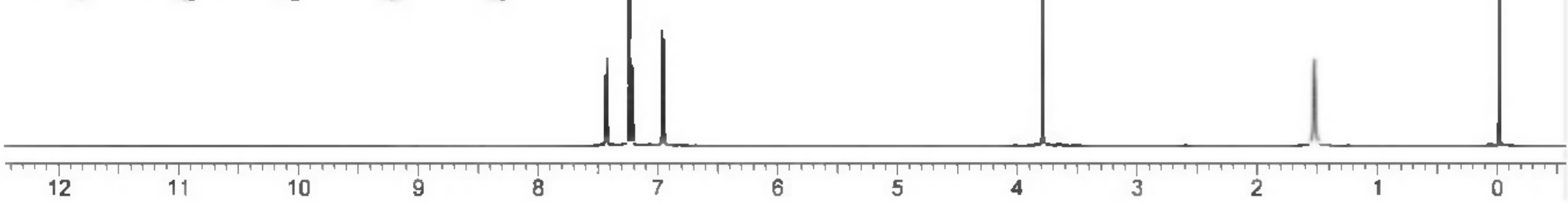


Control ${ }^{1} \mathrm{H}$ NMR spectrum (a)

1D NOESY (DPFGSENOE) spectrum (b)

Selective excitation of $\mathrm{OCH}_{\text {. }}$

H2/H3

(b)

H12/H16
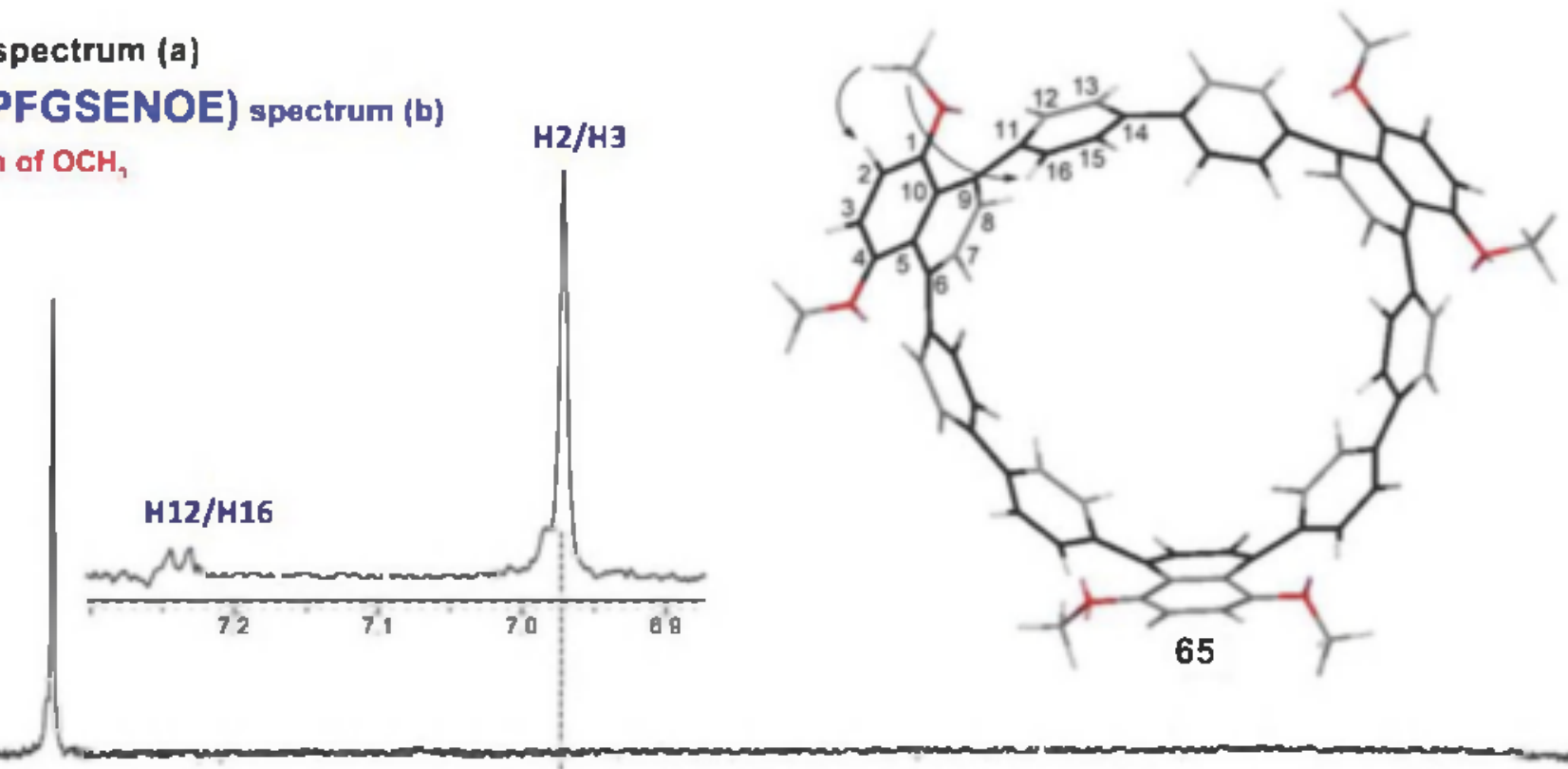

$\mathbf{O C H}_{3}$

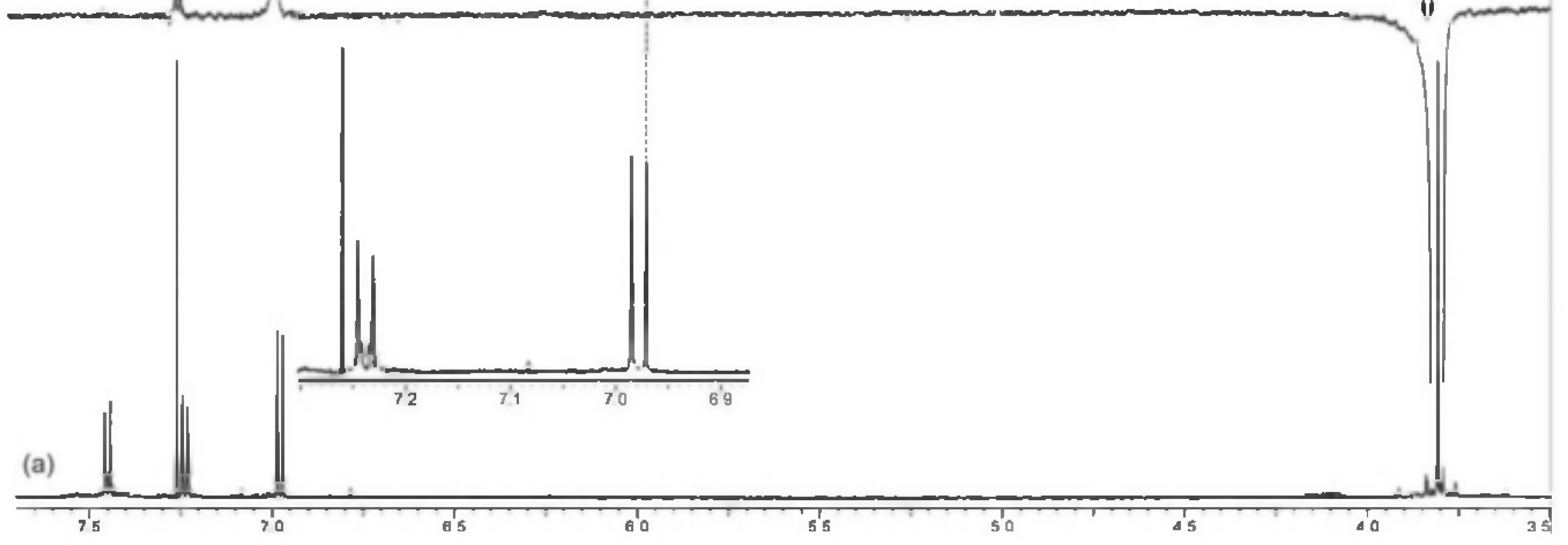


ROESYAD spectrum (a); expanded of portions of the ROESYAD spectrum $(b, c)$
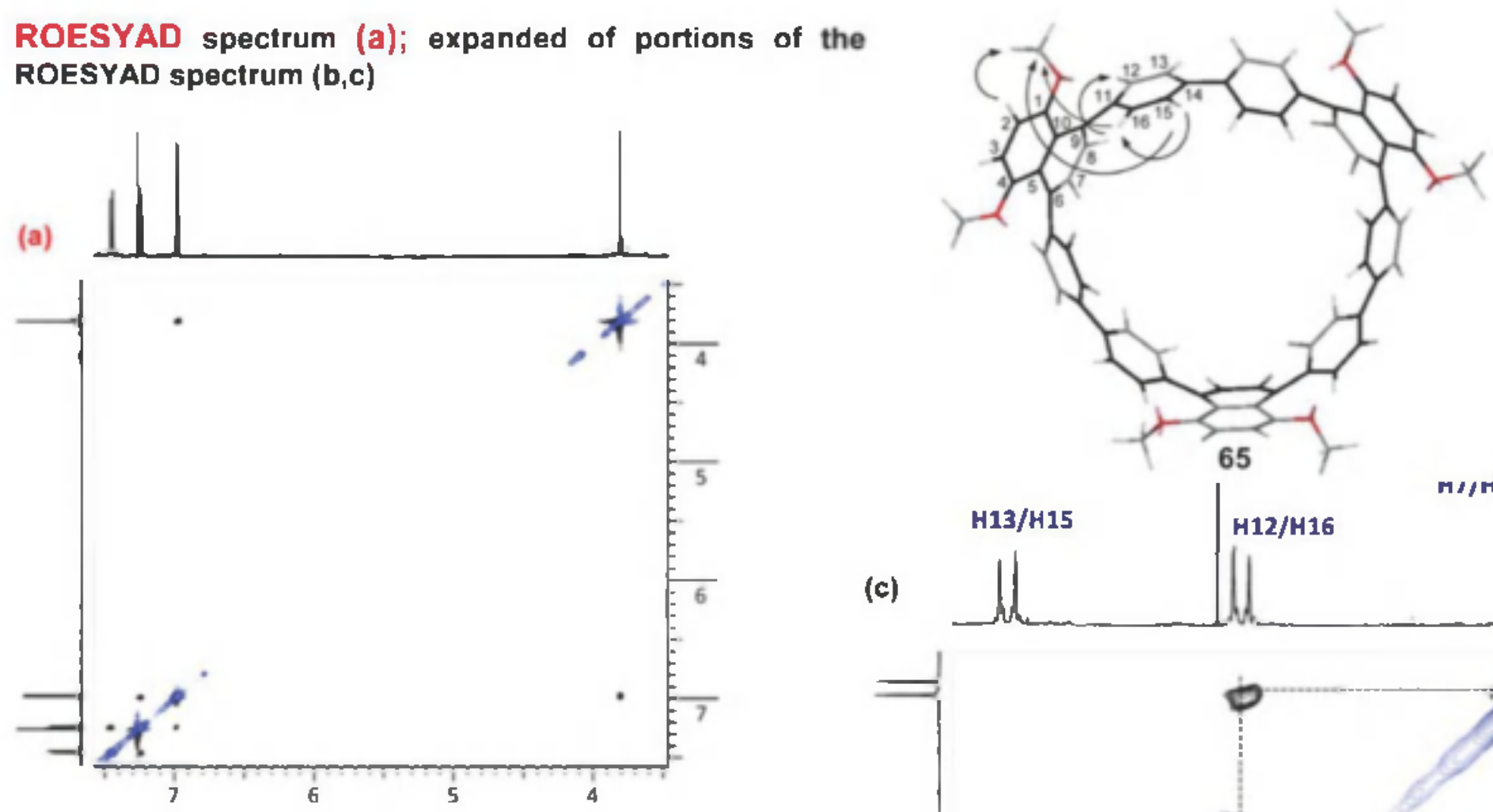

(c)

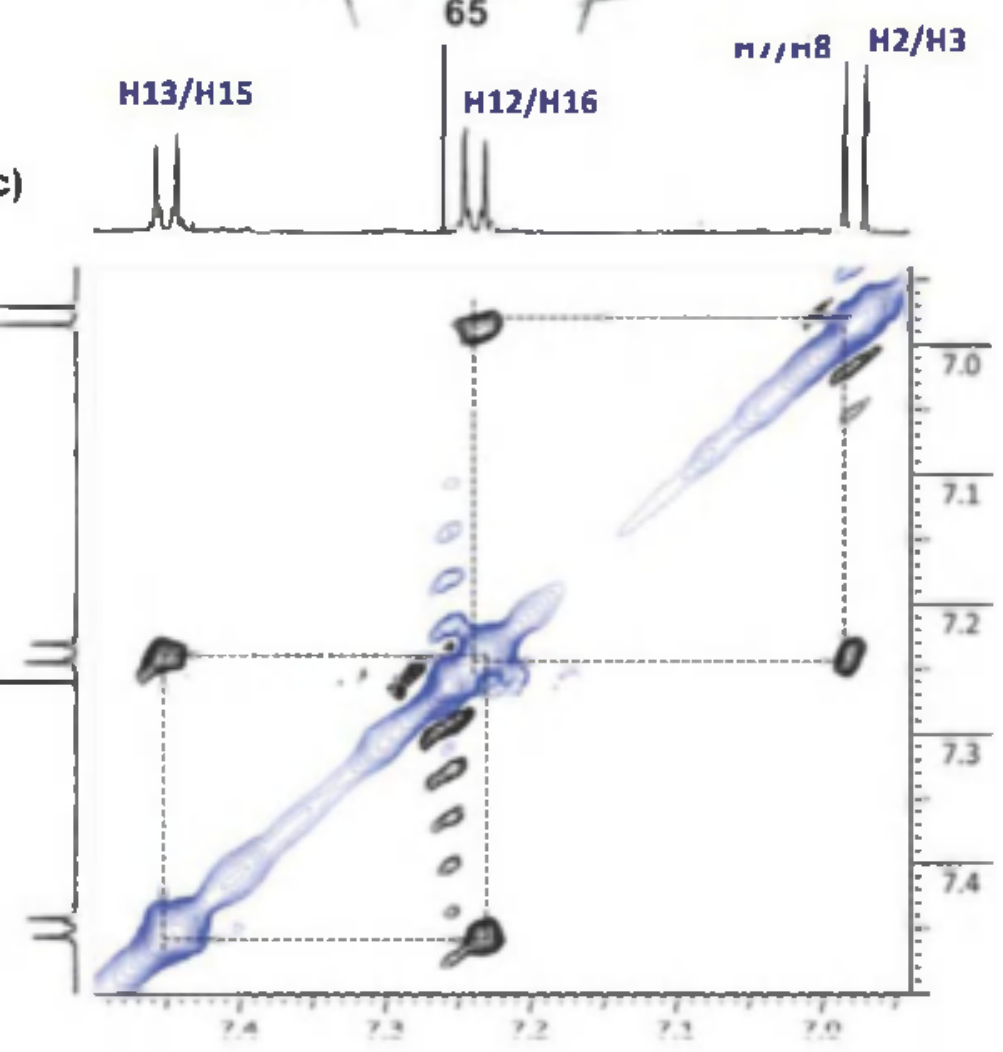


${ }^{19} \mathrm{C}$ NMR chemical shifts (8/ppm)

$\begin{array}{ll}1 / 4-\mathrm{OCH}_{3} & 55.97 \\ \mathrm{C} 2 / \mathrm{Cg} & 107.11 \\ \mathrm{C} 5 / \mathrm{C} 10 & 124.62 \\ \mathrm{C} 6 / \mathrm{Cg} & 135.80 \\ \mathrm{C} 7 / \mathrm{CB} & 132.45 \\ \mathrm{C} 11 & 142.89 \\ \mathrm{C} 12 / \mathrm{C} 16 & 127.59 \\ \mathrm{C} 13 / \mathrm{C} 15 & 126.03 \\ \mathrm{C} 14 & 137.44\end{array}$
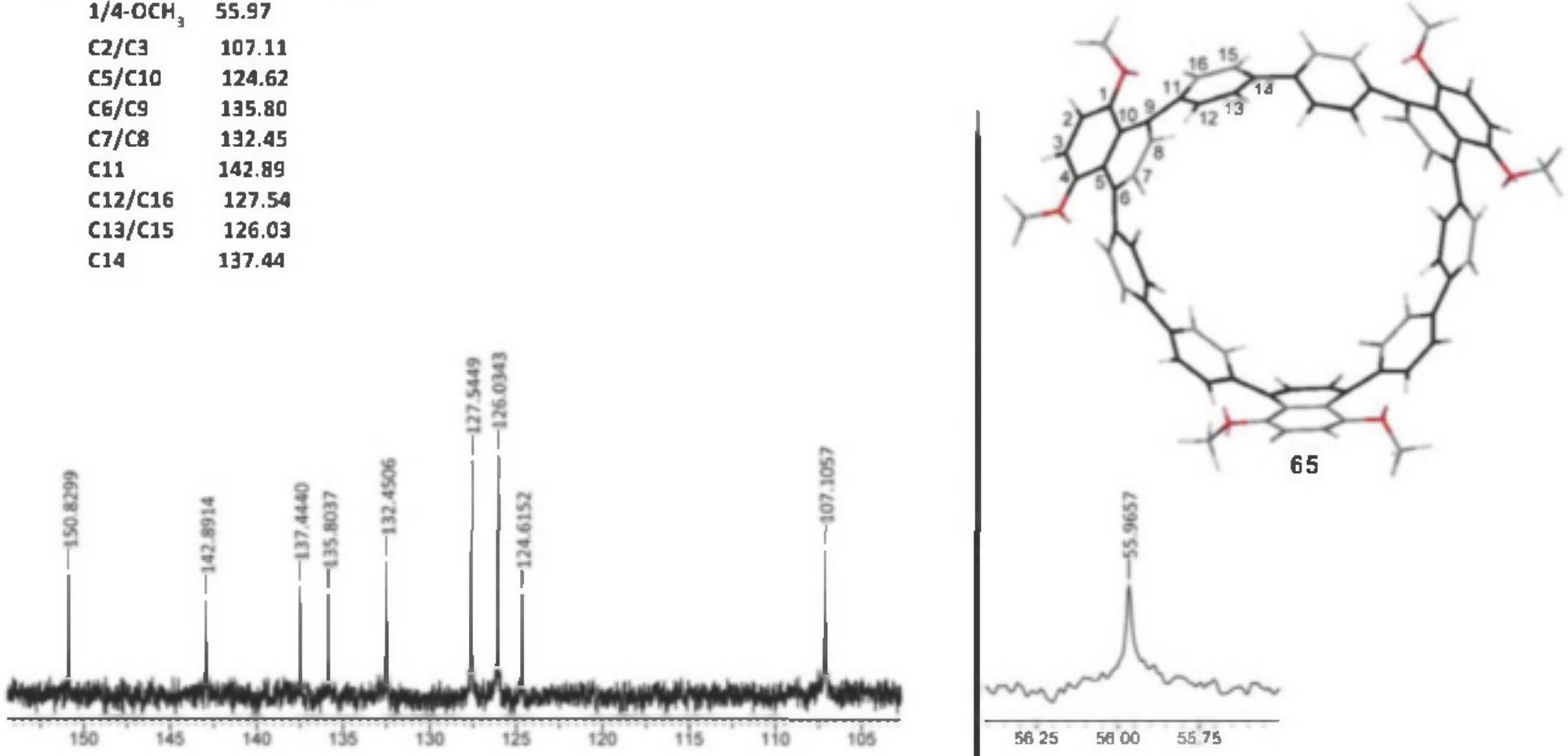


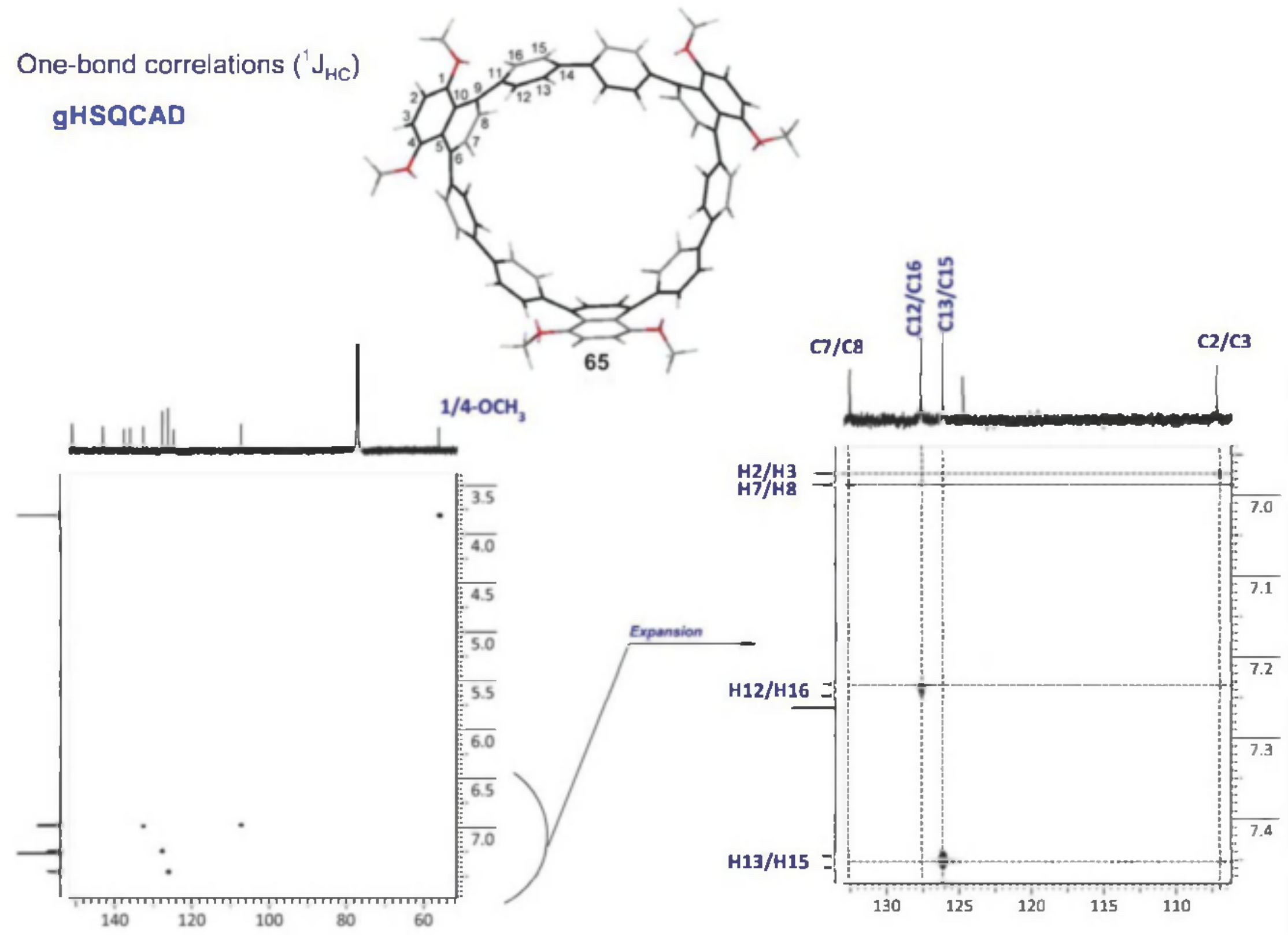


${ }^{2} \mathrm{~J}_{\mathrm{HC}}$ and ${ }^{3} \mathrm{~J}_{\mathrm{HC}}$ gHMBCAD correlations
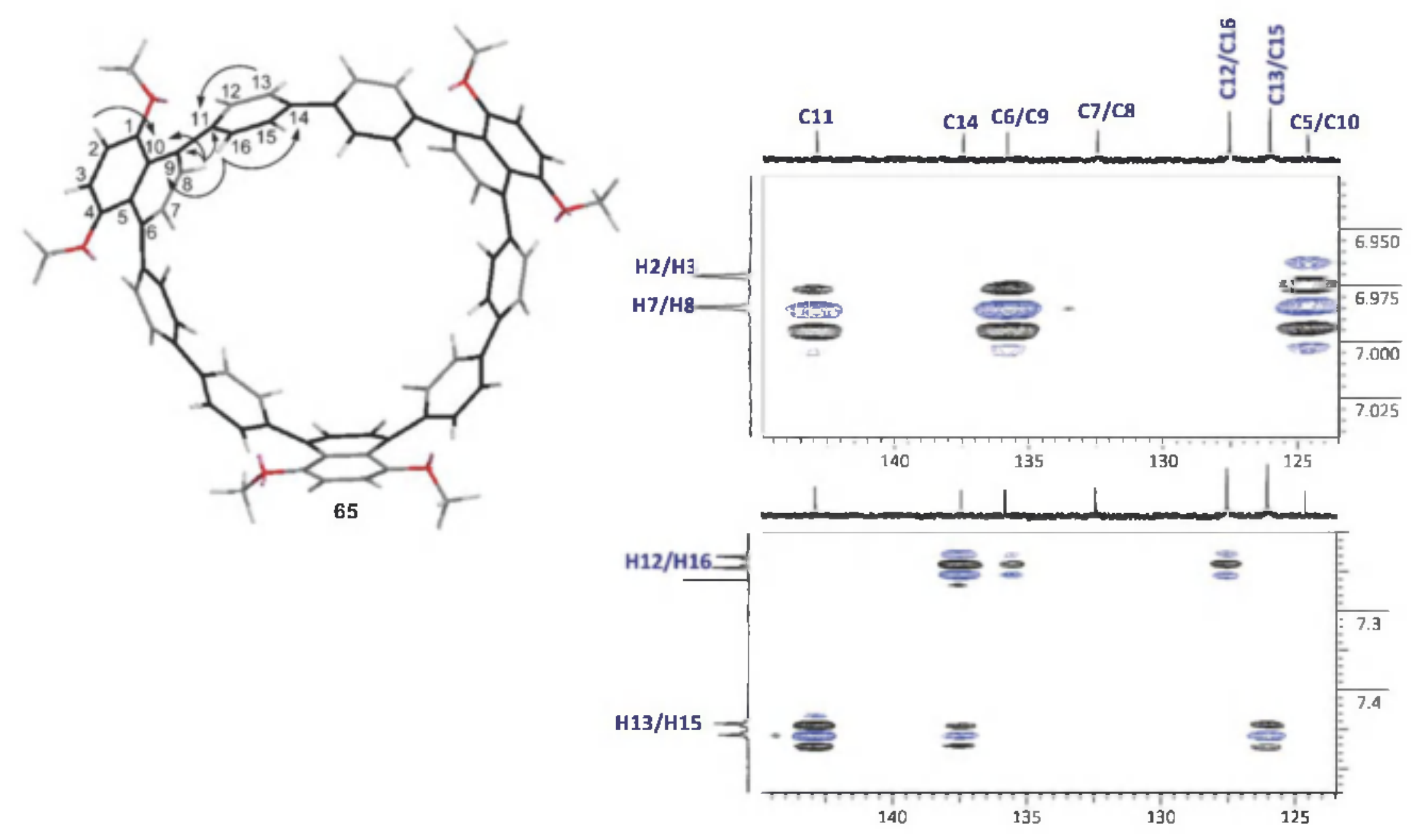


\section{UV-Vis/Fluorescence Spectra}

a)

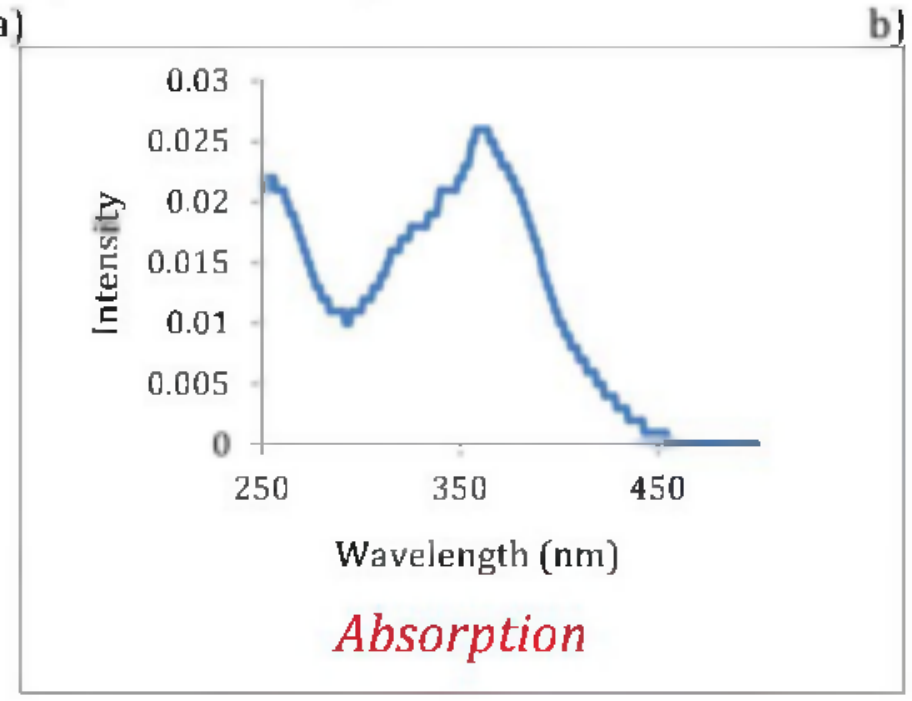

b)

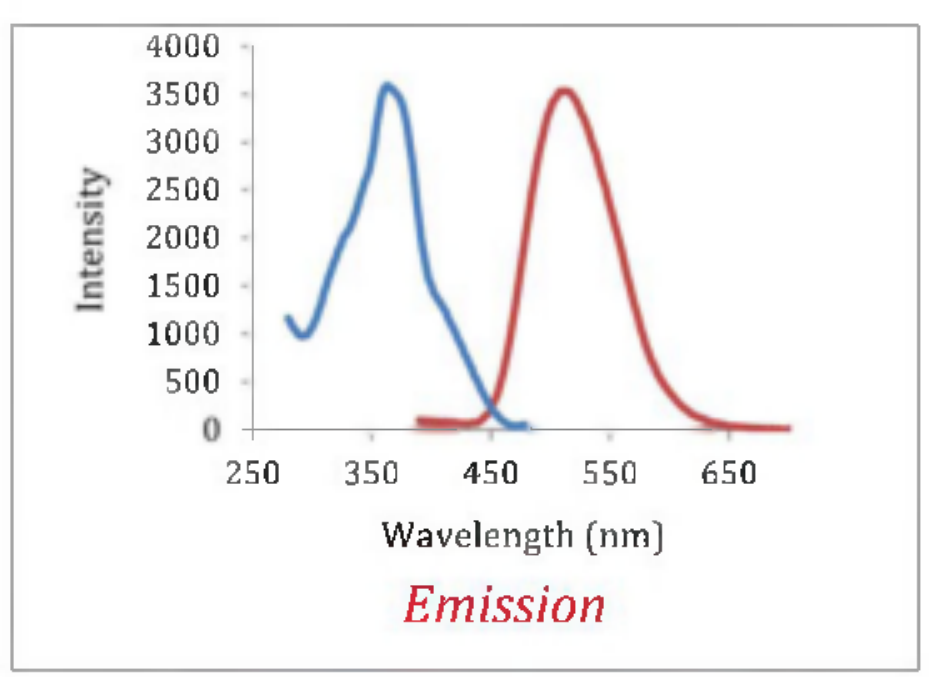

Spectra a] : UV-vis absorption spectrum of 65 at a concentration of $5^{*} 10^{-7} \mathrm{M}$ in degassed $\mathrm{CH}_{2} \mathrm{Cl}_{2}$. At absorption maximum $\lambda_{\text {abs }}=$ $362 \mathrm{~nm}, \varepsilon=5.2^{*} 10^{4} \mathrm{M}^{-1} \mathrm{CM}^{-1}$.

Spectra b): Fluorescence spectrum of 65 at a concentration of $5^{*} 10^{-7} \mathrm{M}$ in degassed $\mathrm{CH}_{2} \mathrm{Cl}_{2}$ upon excitation at $362 \mathrm{~nm}$.

Fluorescence maximum $\lambda_{\text {em }}=512 \mathrm{~nm}$.

The UV-vis spectrum of 65 in $\mathrm{CH}_{2} \mathrm{Cl}_{2}\left(5^{*} 10^{-7} \mathrm{M}\right)$ exhibited the absorption maximum ( $\left.\lambda_{\text {abs }}\right)$ at $362 \mathrm{~nm}$ with a molecular absorption coefficient ( $\varepsilon$ ] of $5.2^{*} 10^{4} \mathrm{M}^{-1} \mathrm{CM}^{-1}$, which lay between that of the parent [9] CPP (340 nm) and [9] CN (378 nm), but much red shifted compared with arene-substituted [9] CPP (310nm, 320nm). However the fluorescence maximum ( $\lambda$ en $)$ was observed at $512 \mathrm{~nm}$, which showed significant red-shift to that of [9] CPP (494 nm), [9] CN (491 nm) and arene-substituted [9] CPP $[405 \mathrm{~nm}, 400 \mathrm{~nm}]$. 


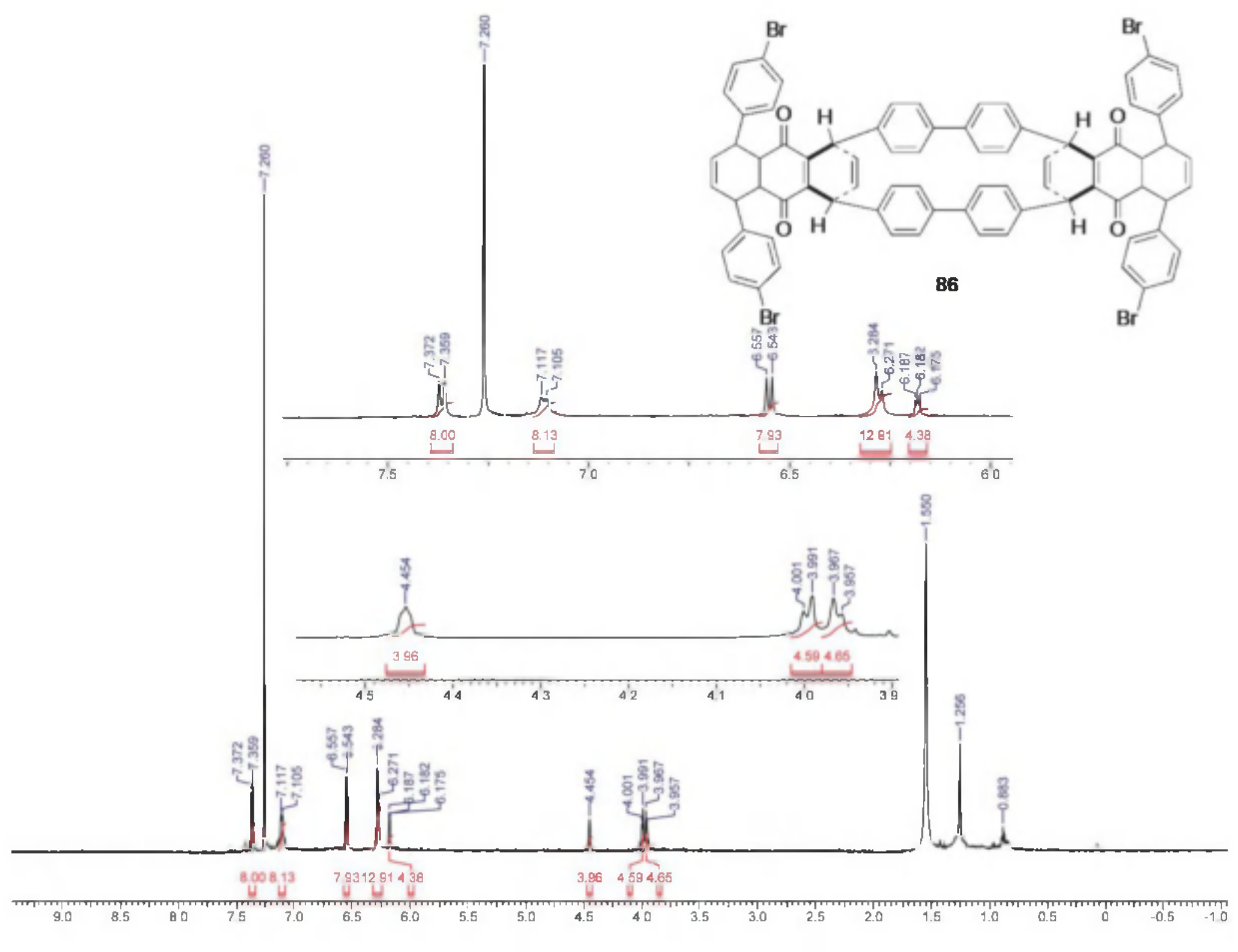




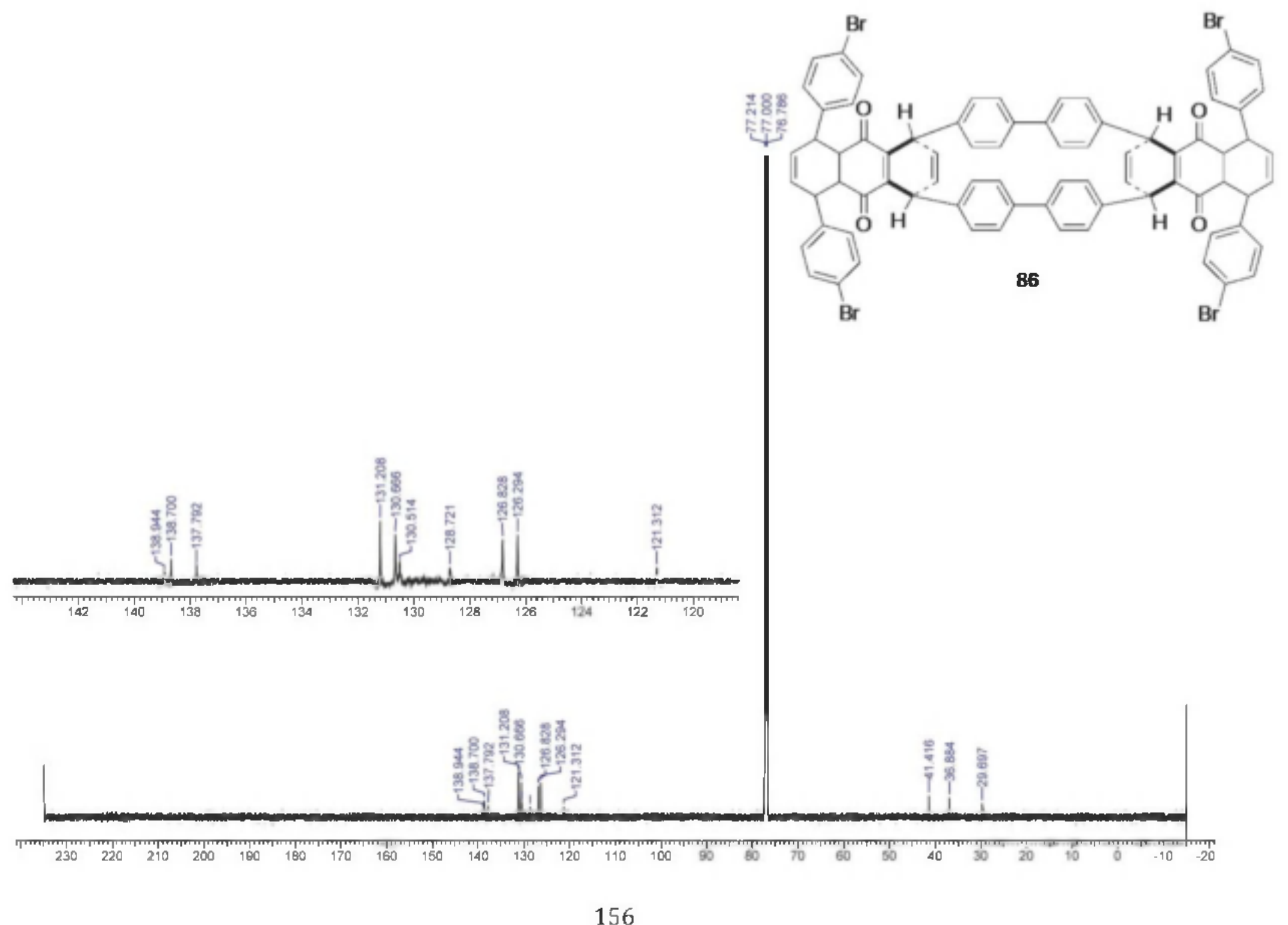




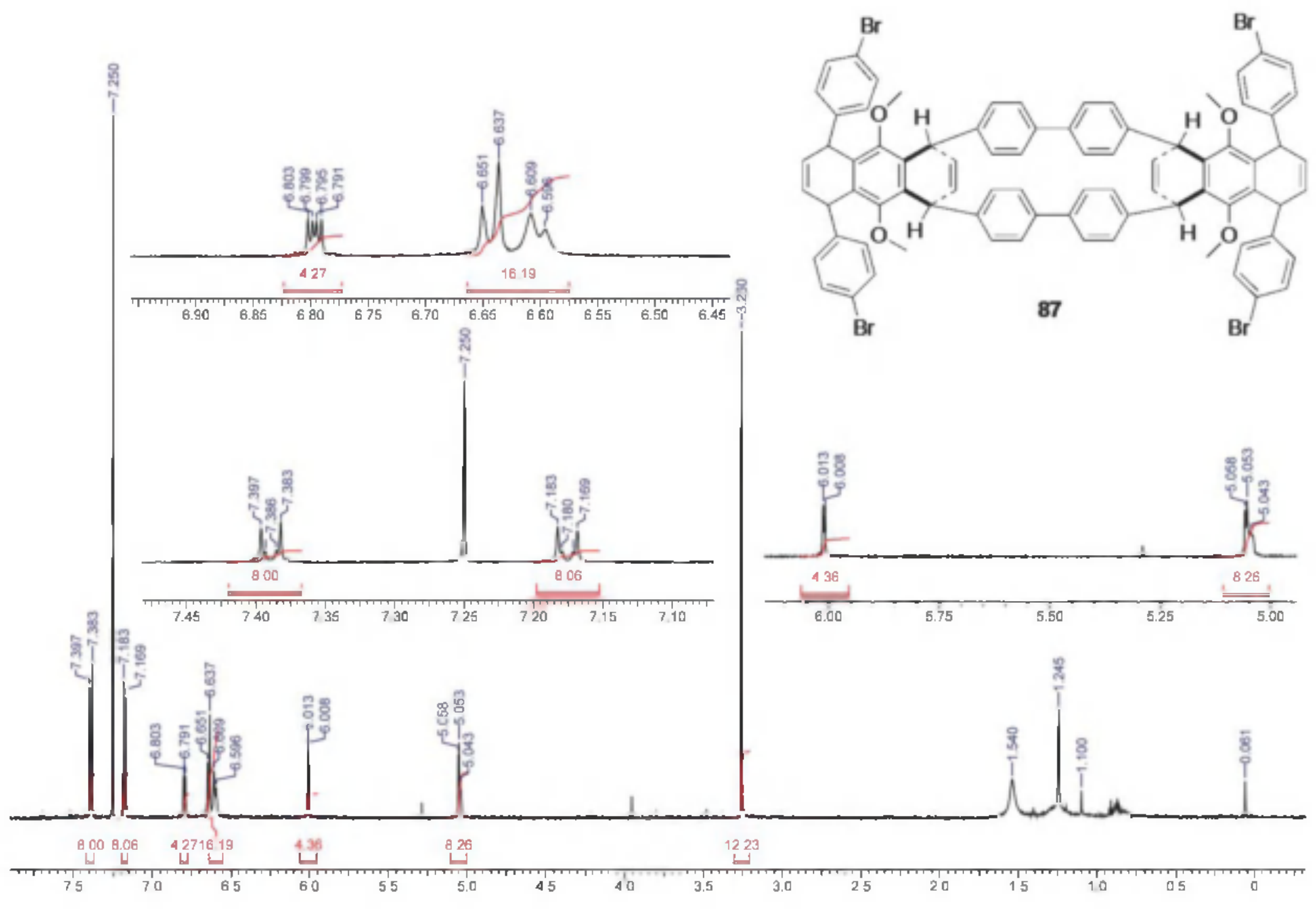




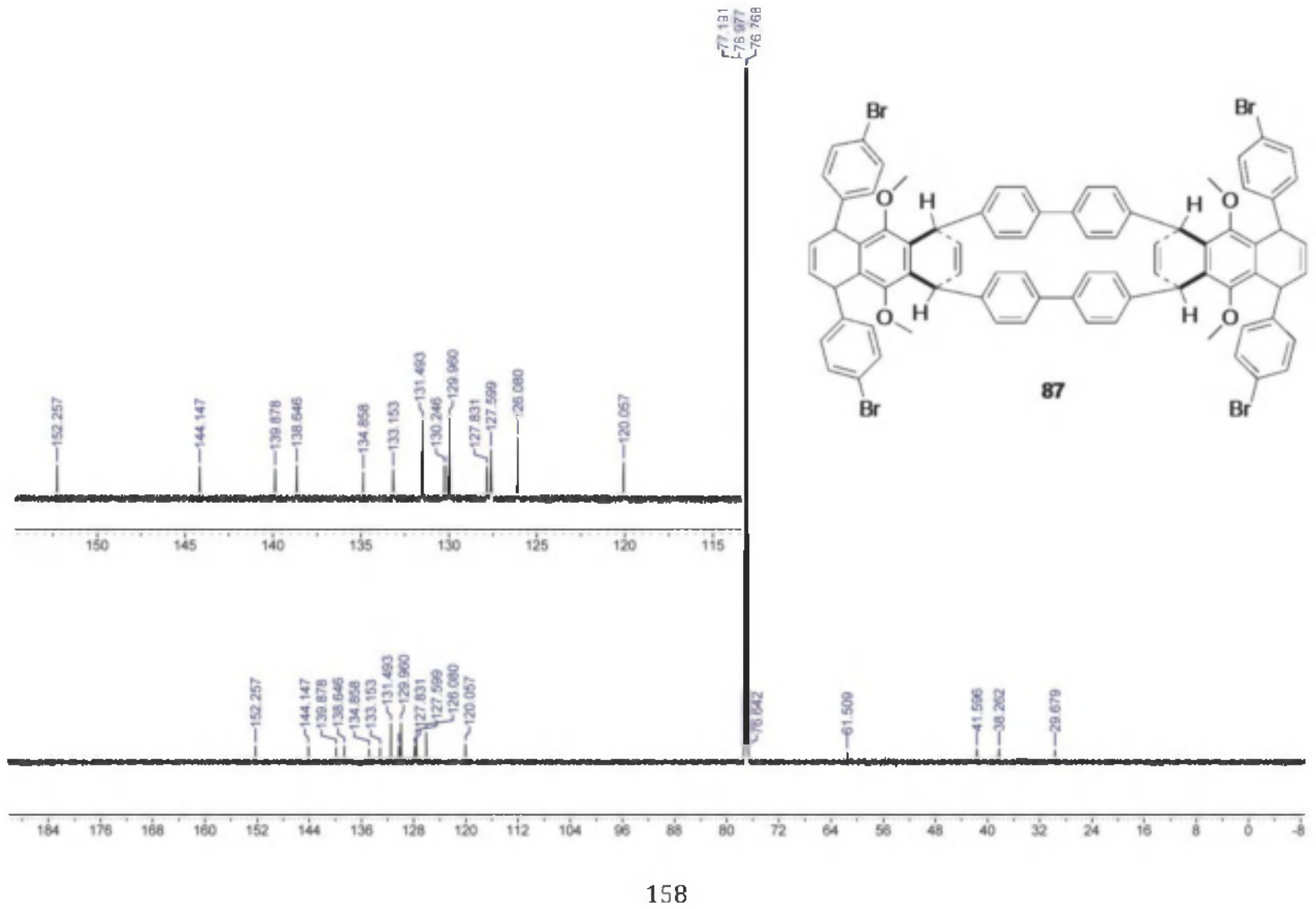




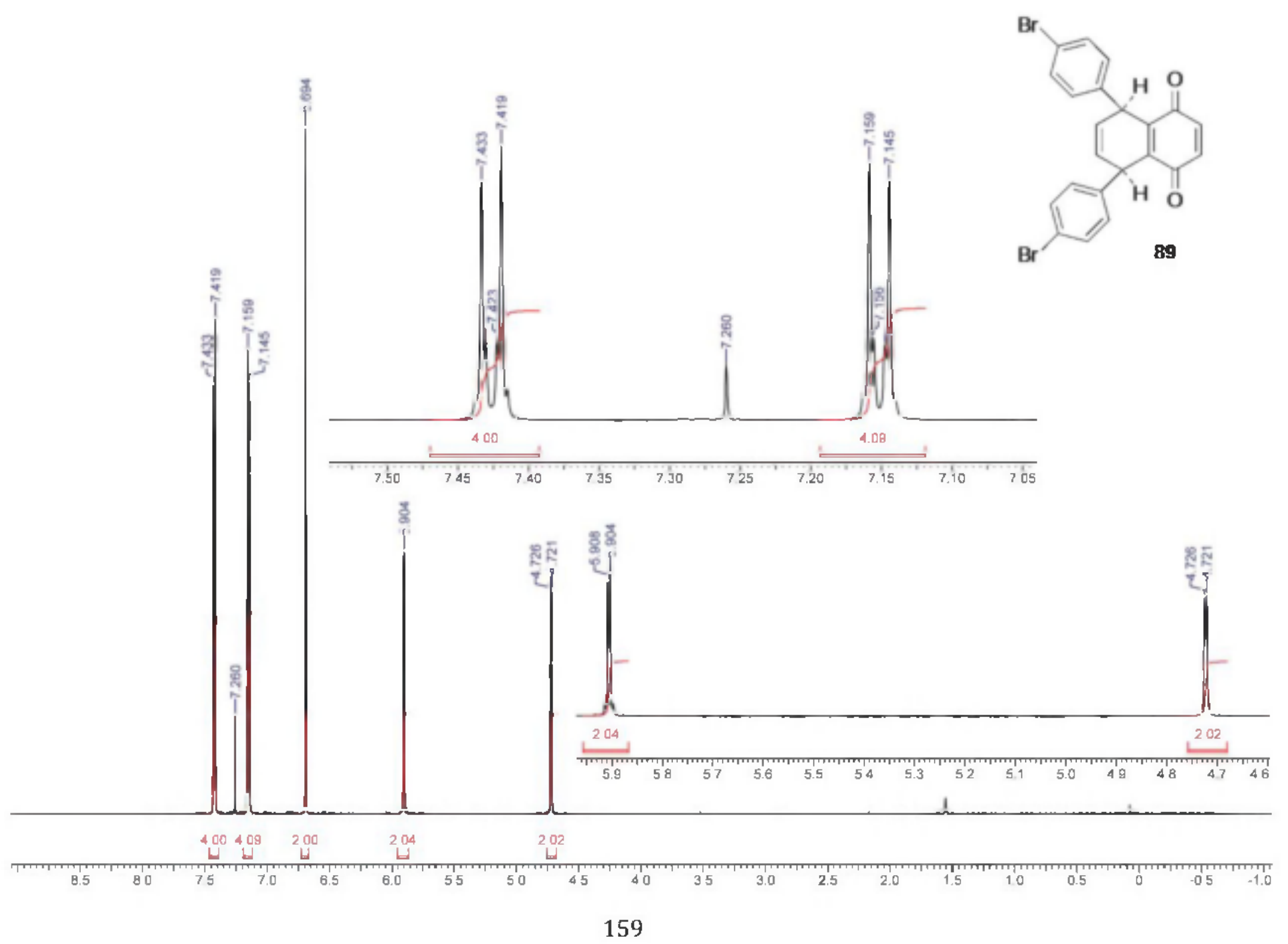




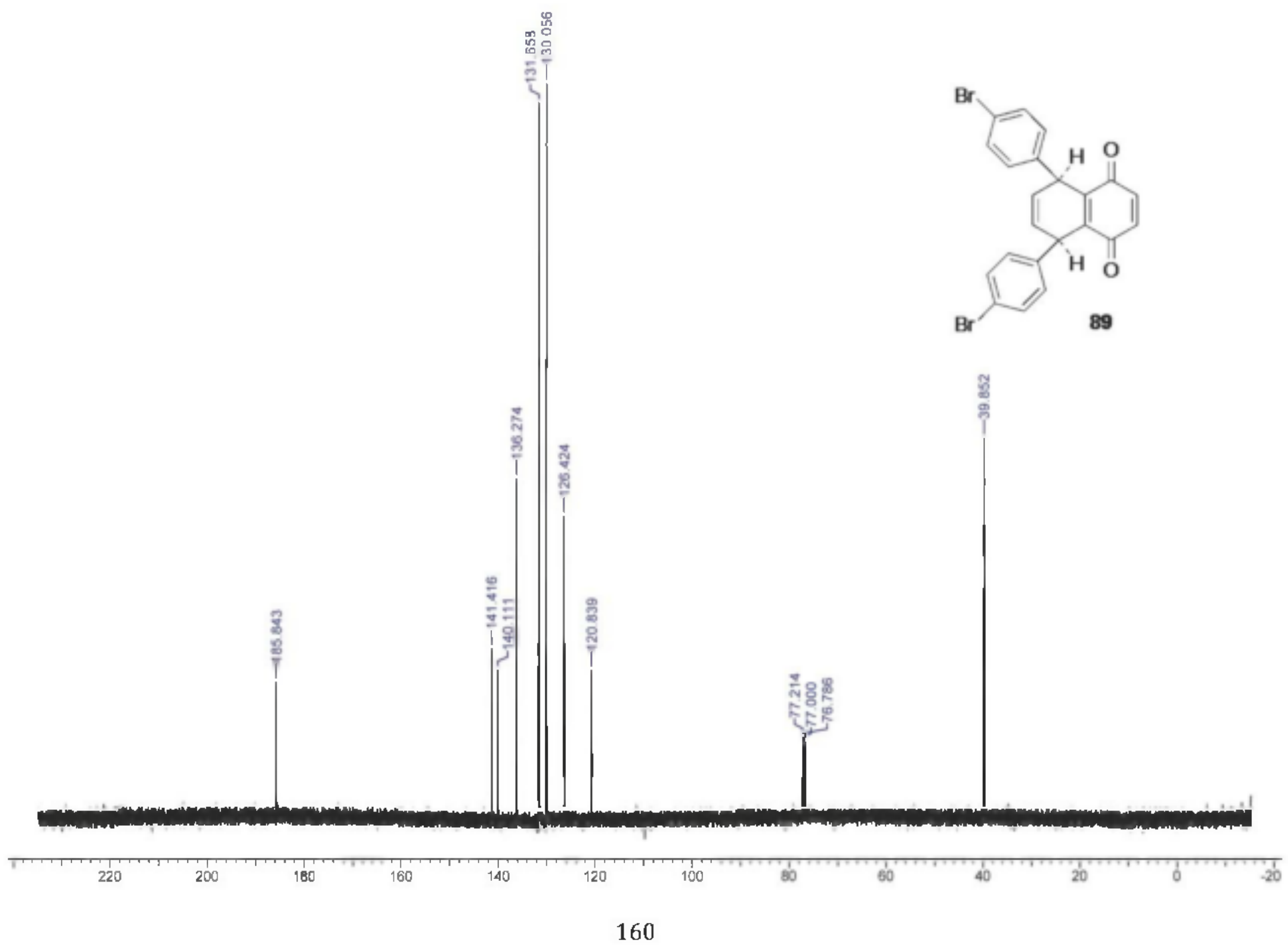




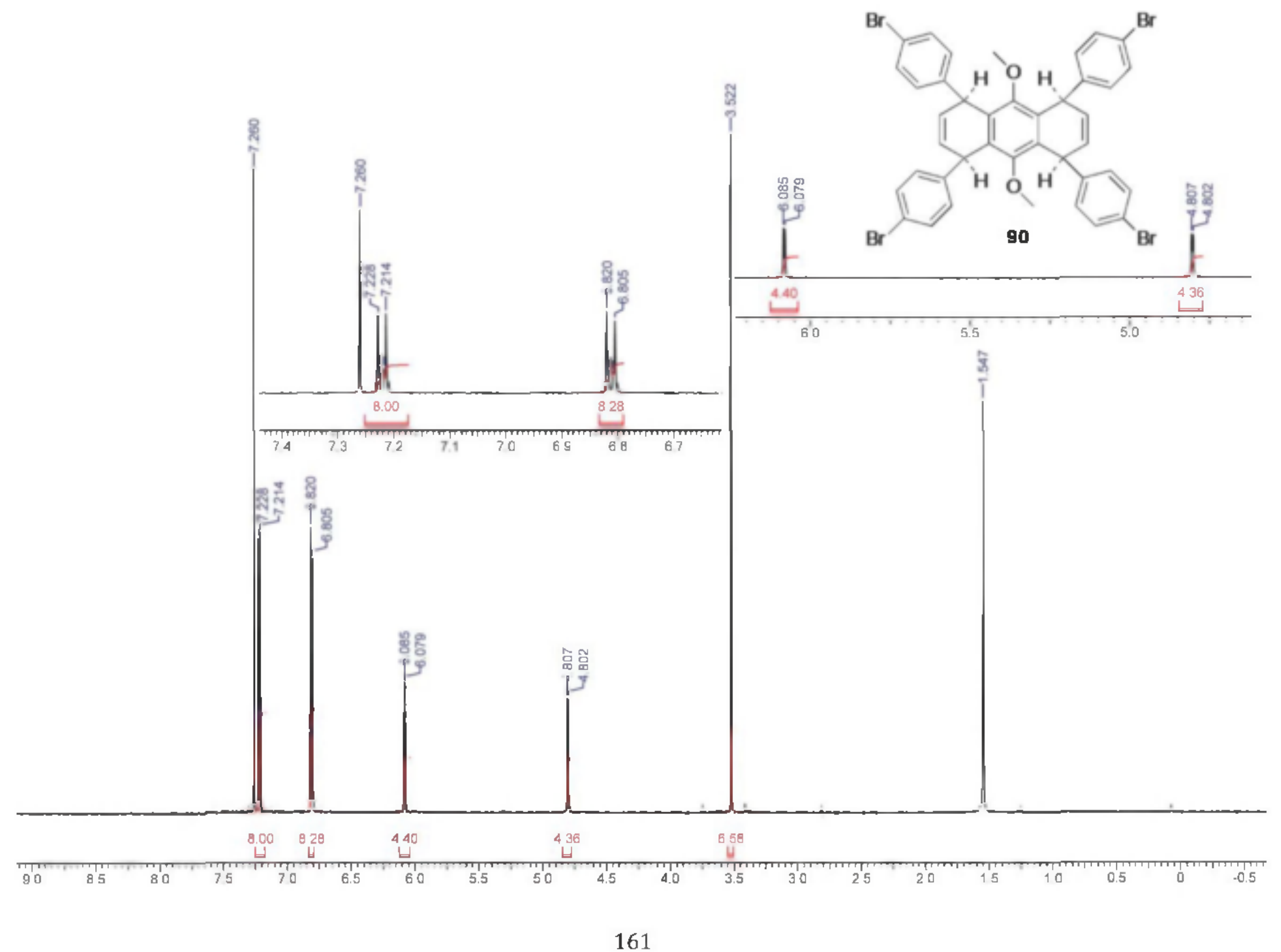




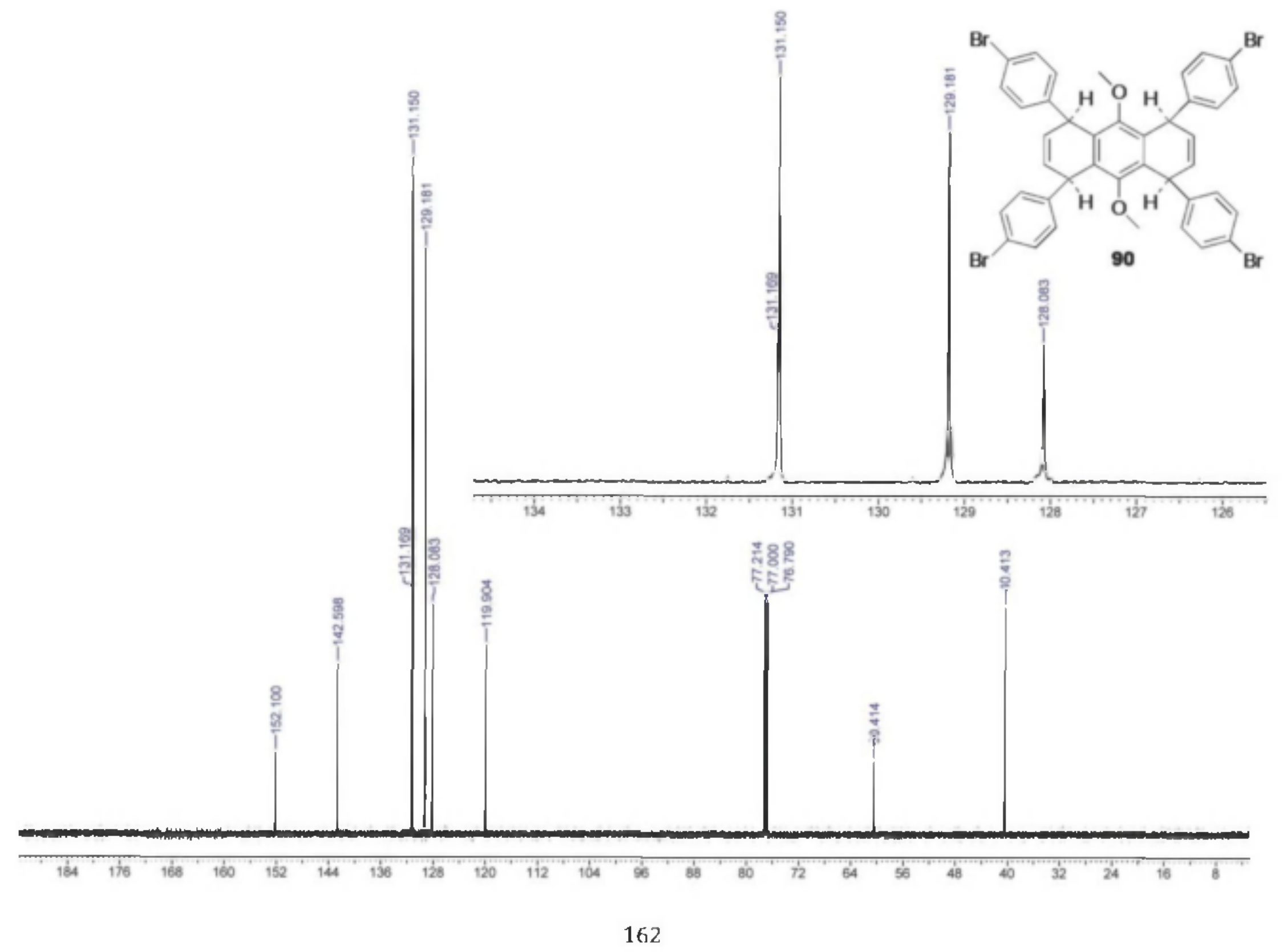




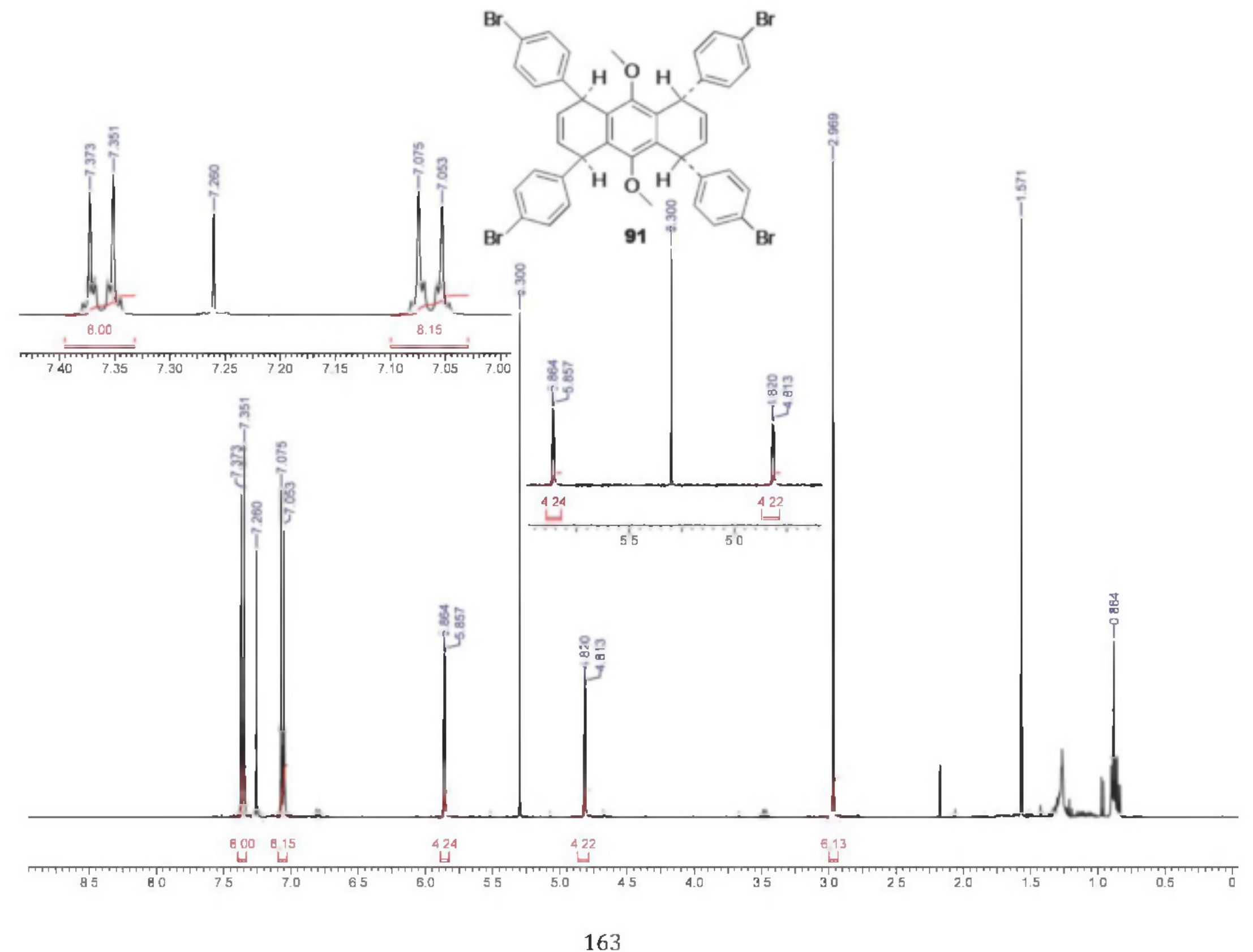



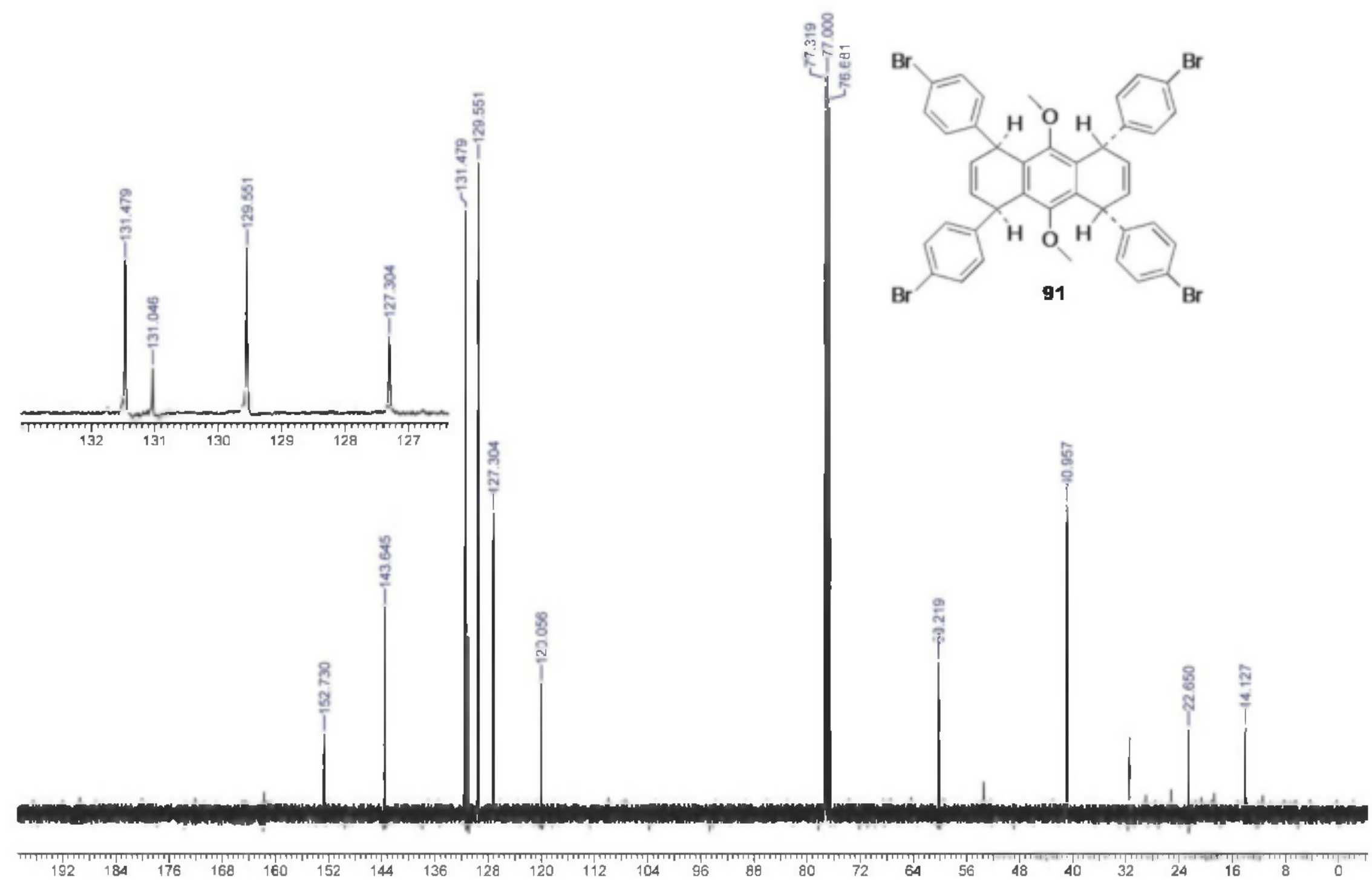
165 


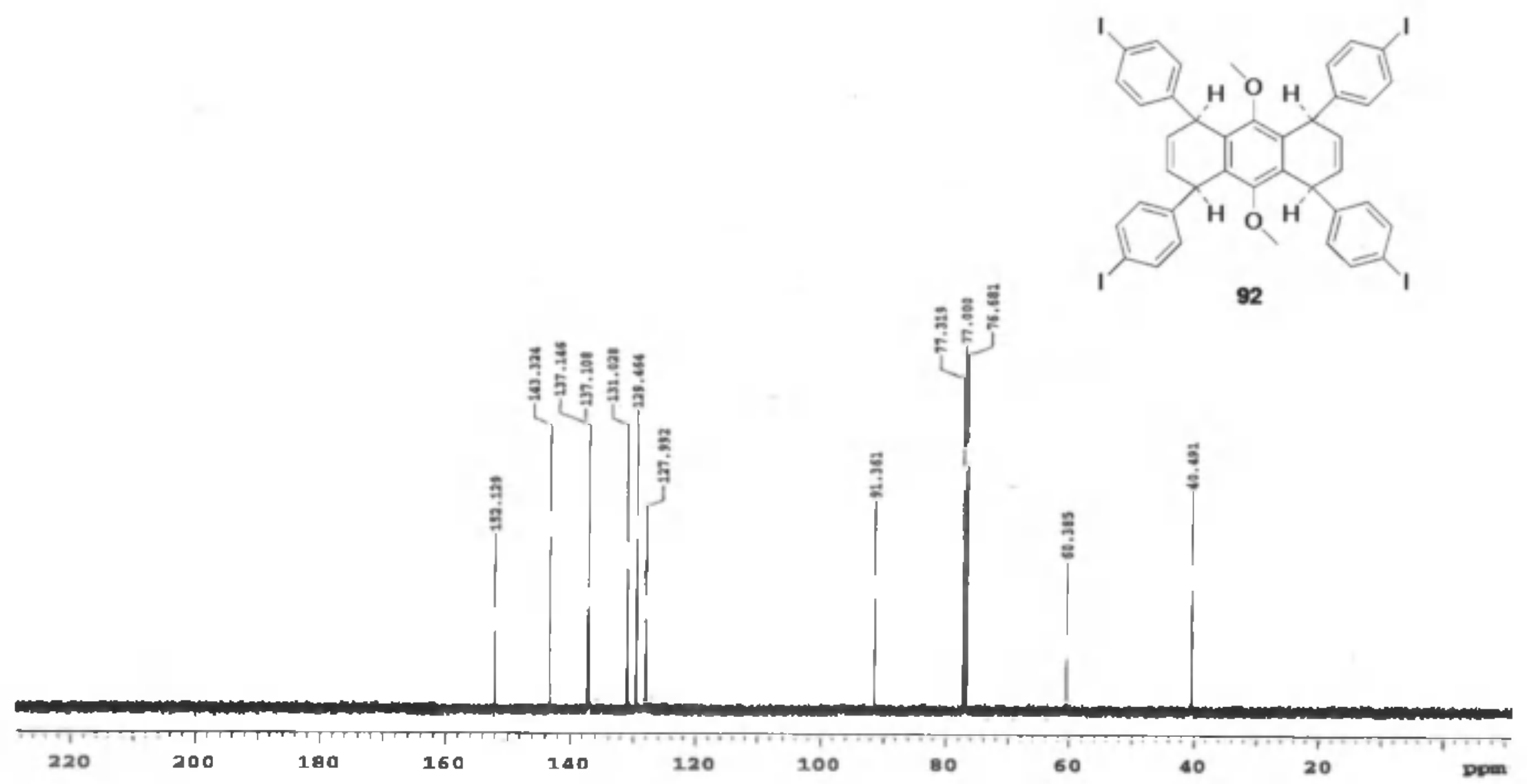




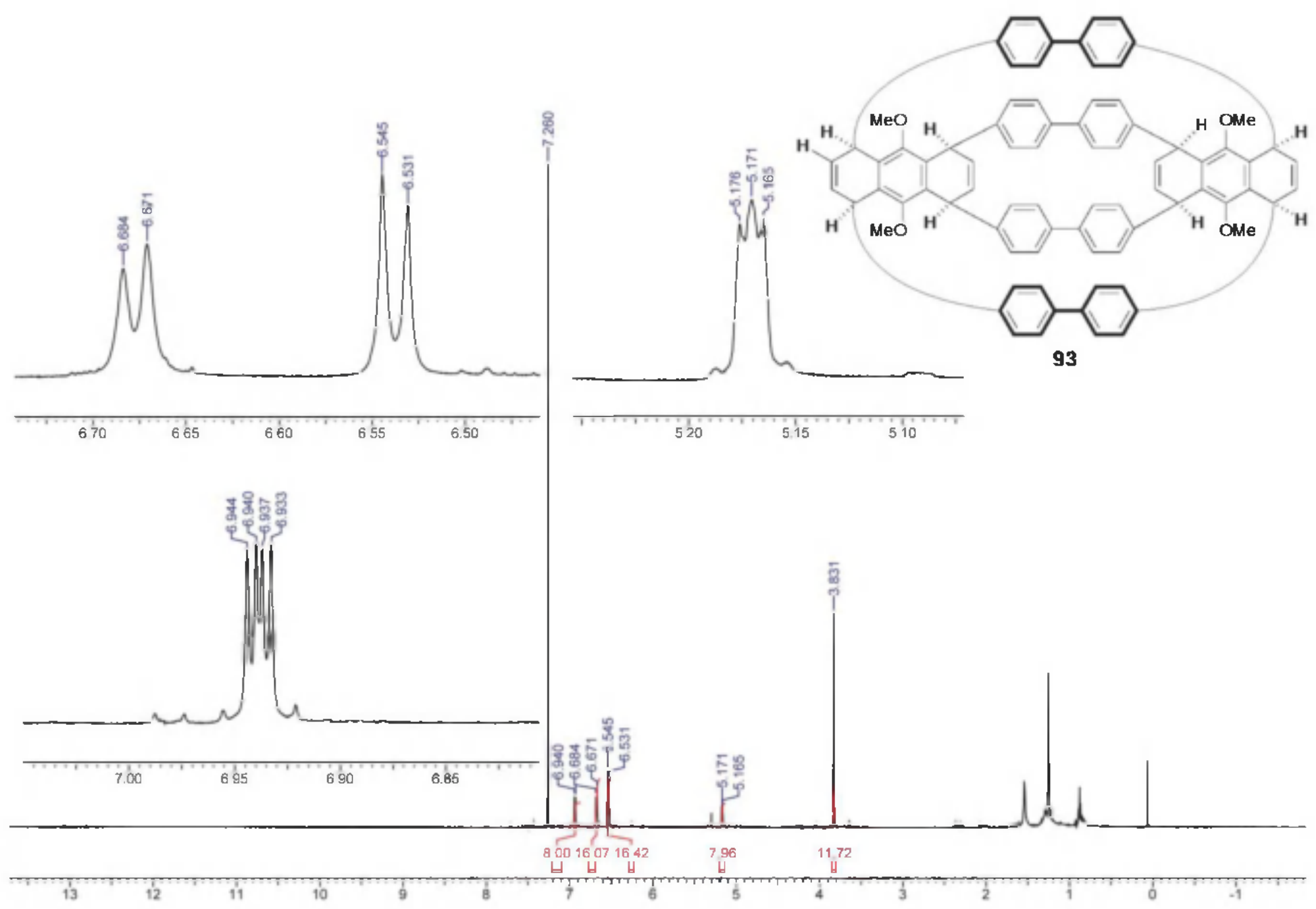




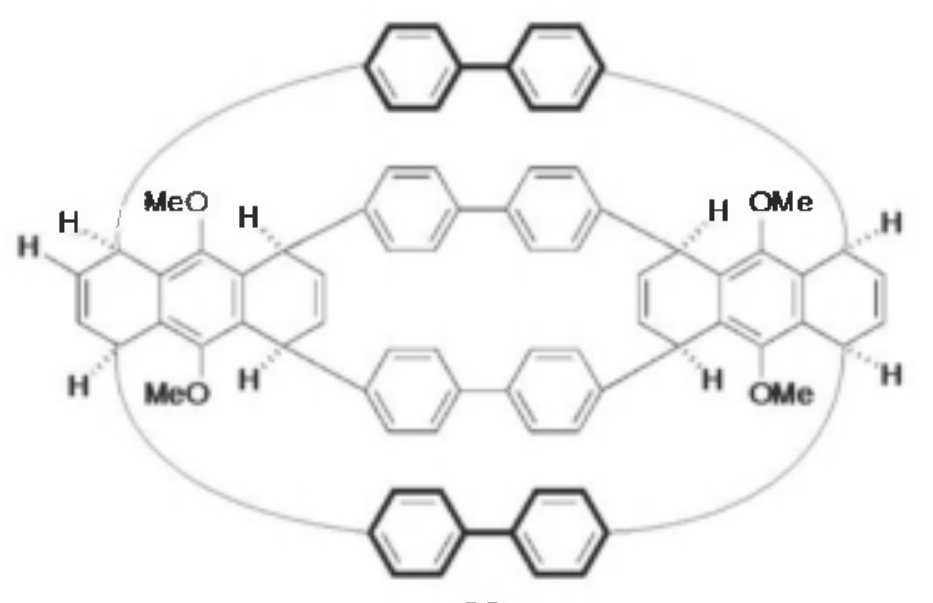

93

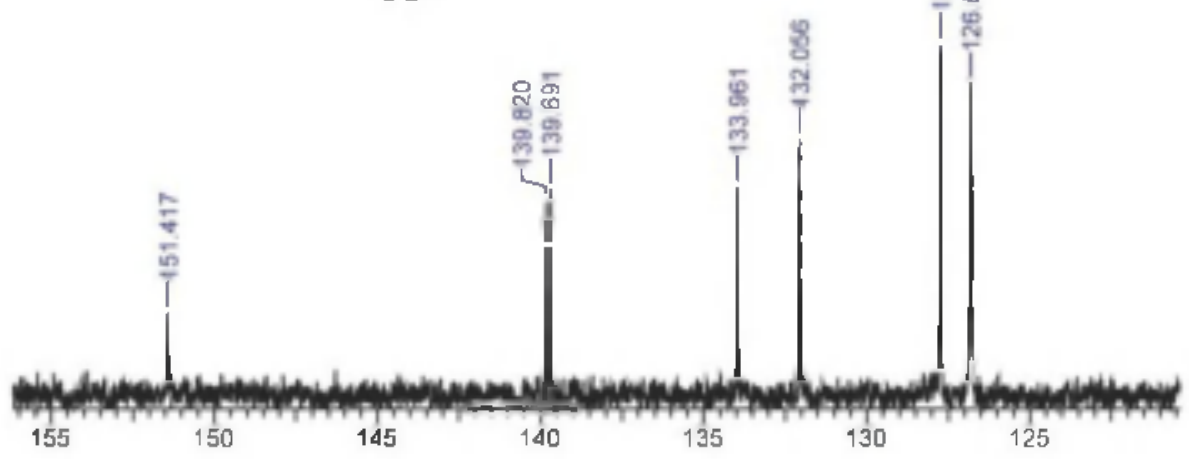

155

150

145

135

125

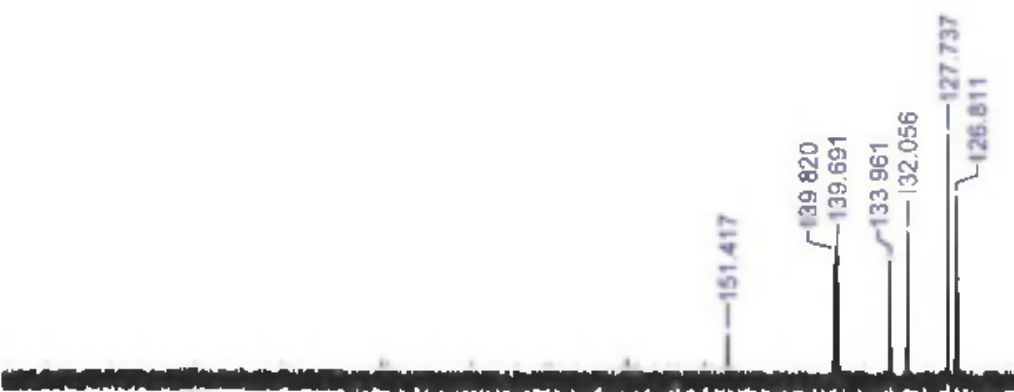




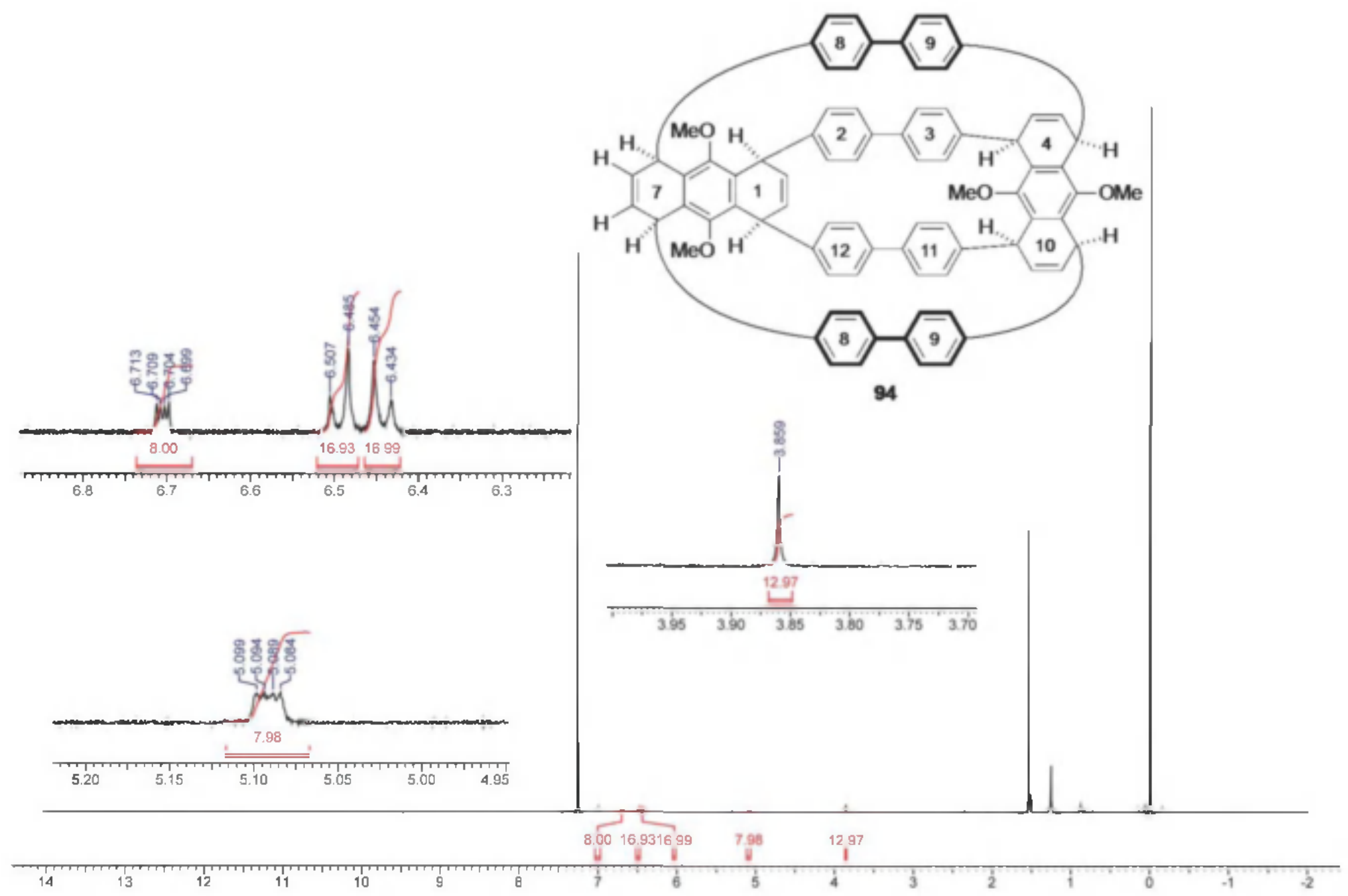




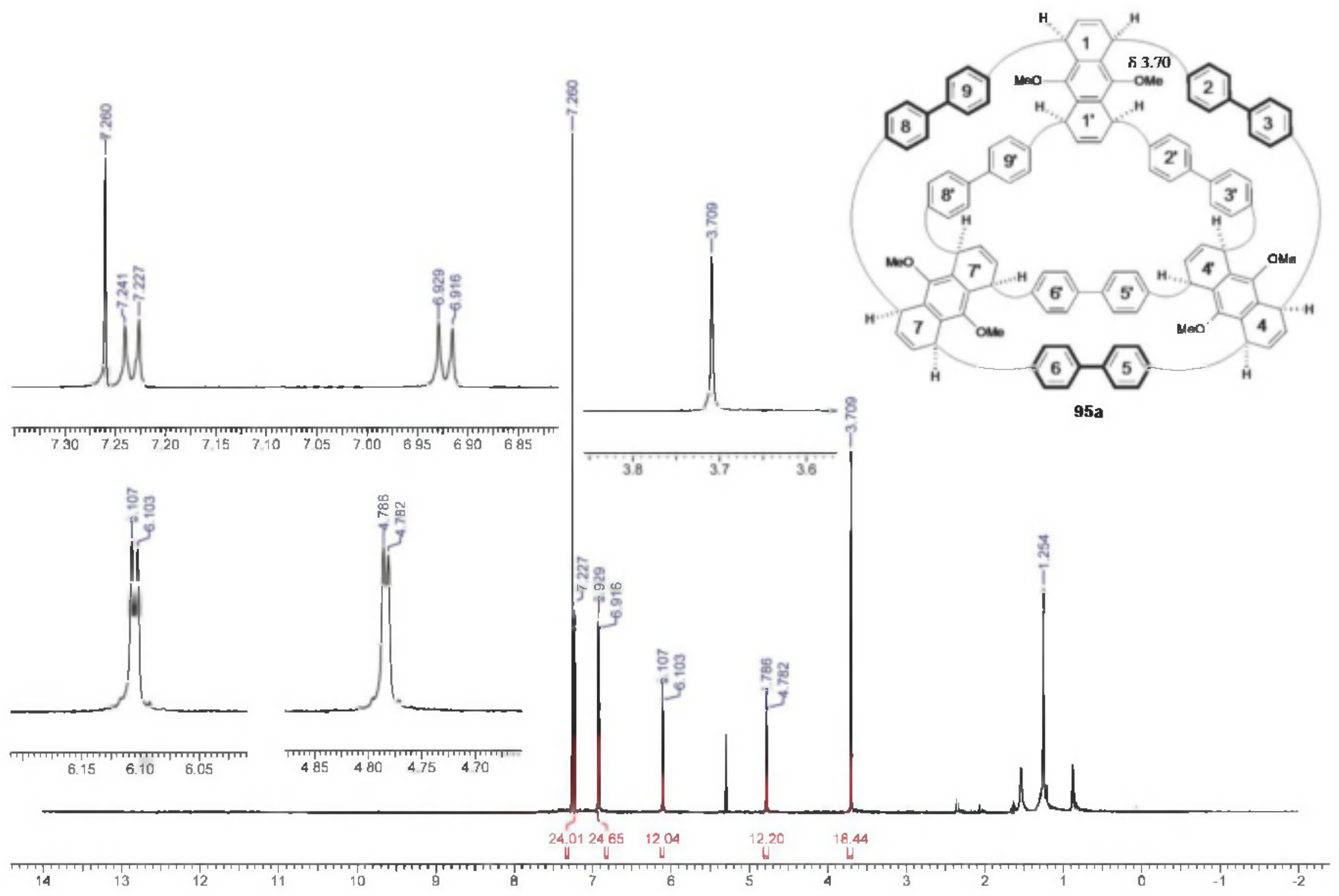




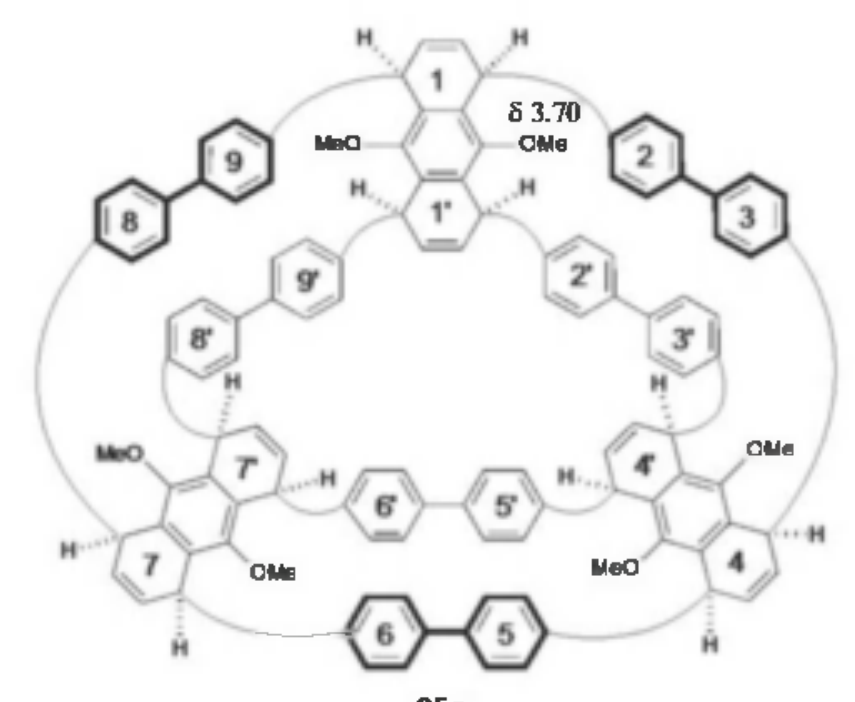

$95 a$
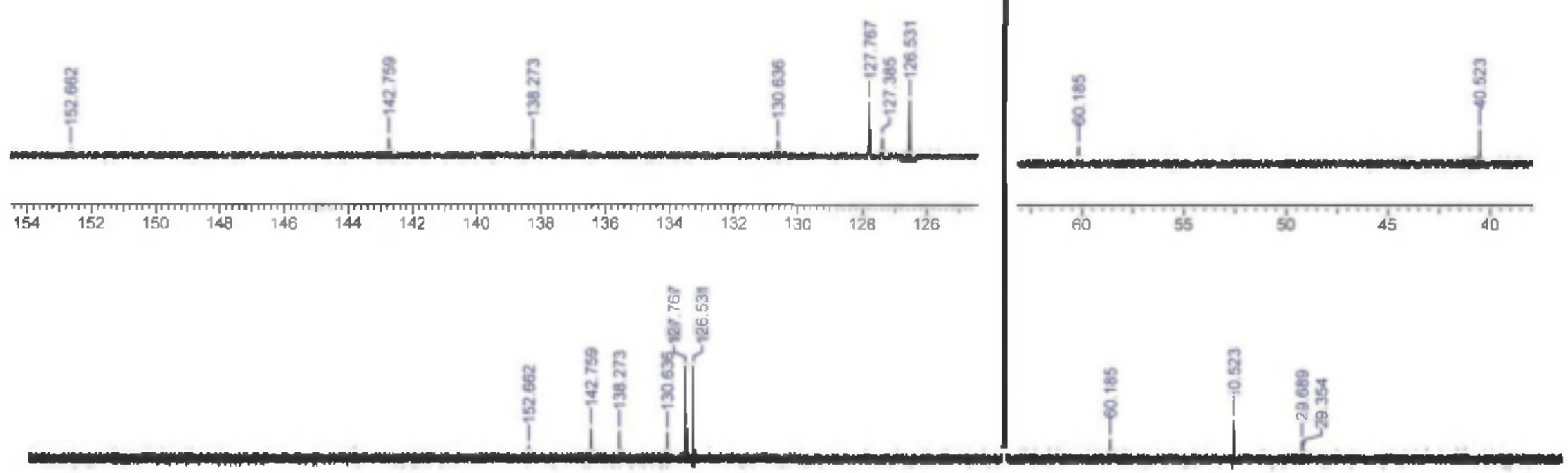

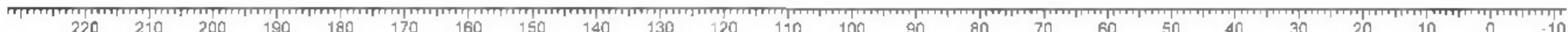




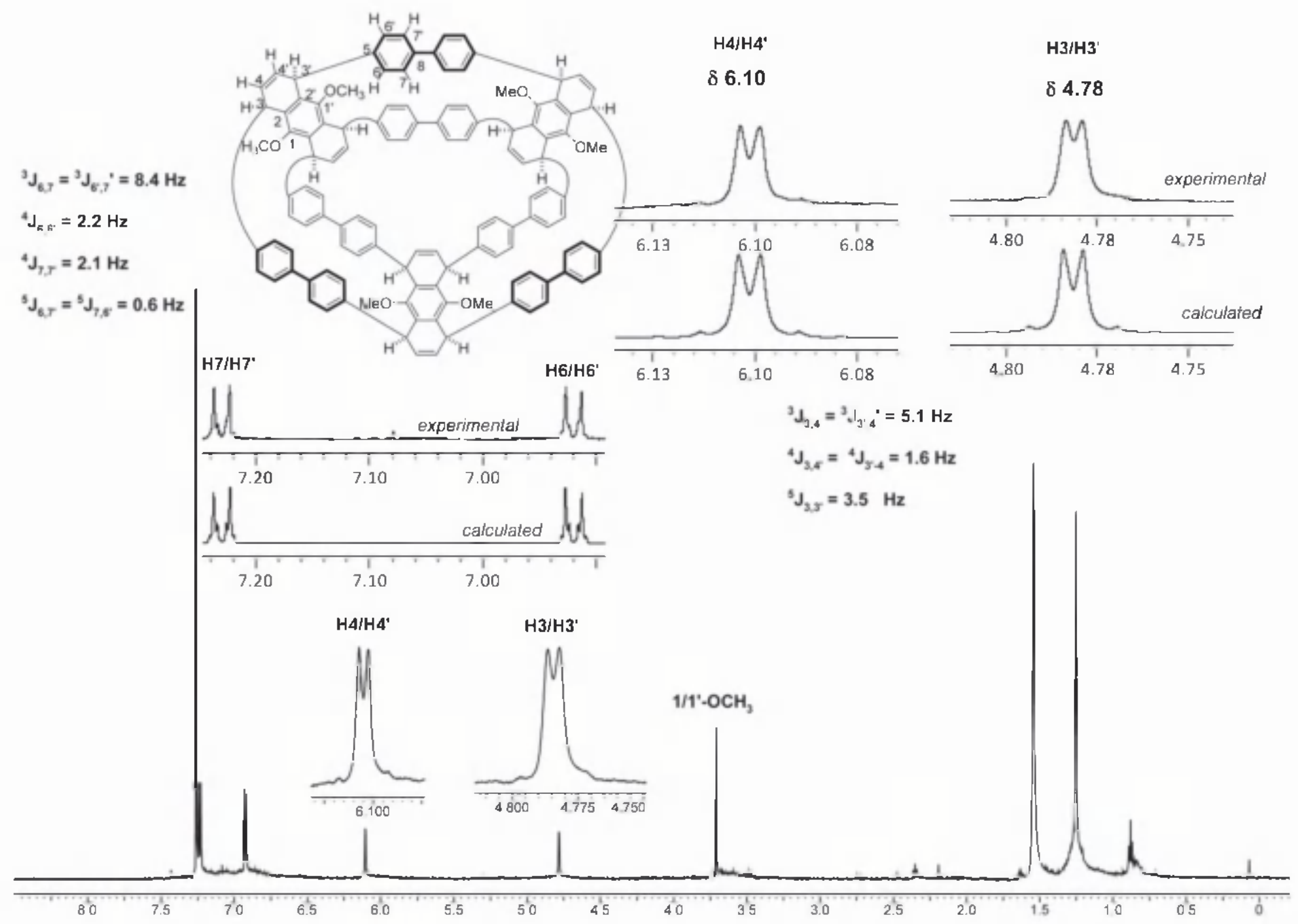




\section{ROESY}

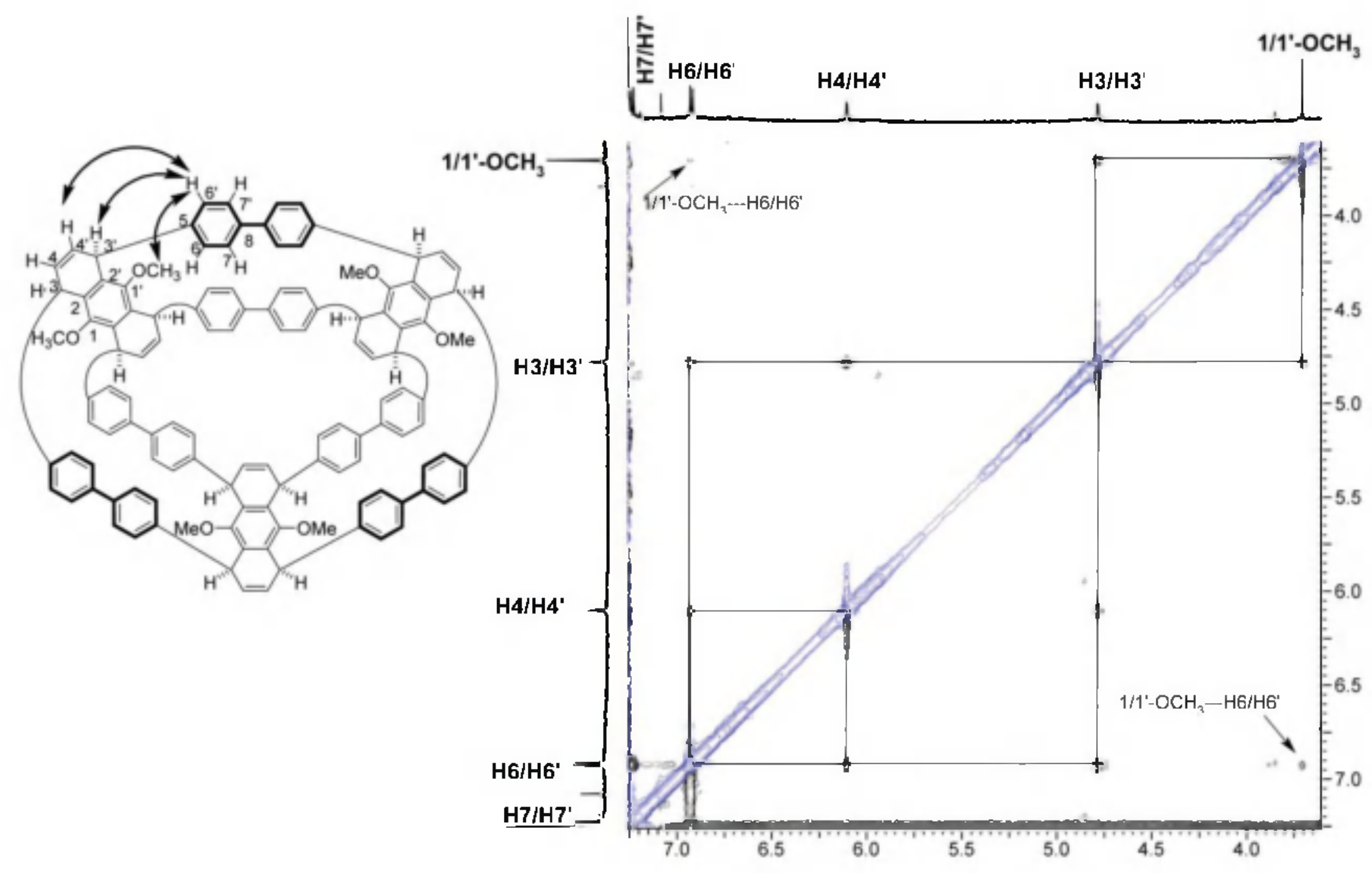




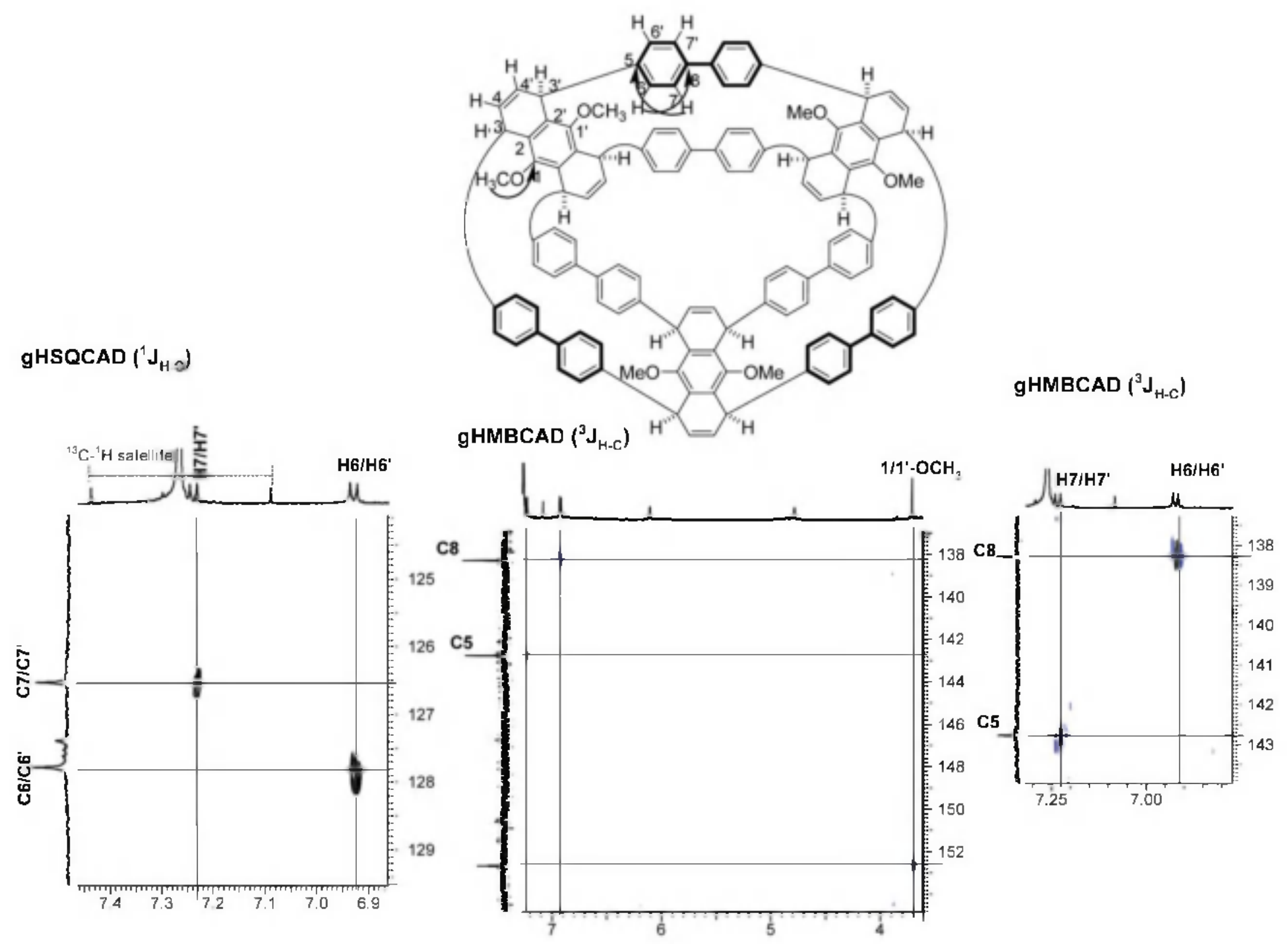




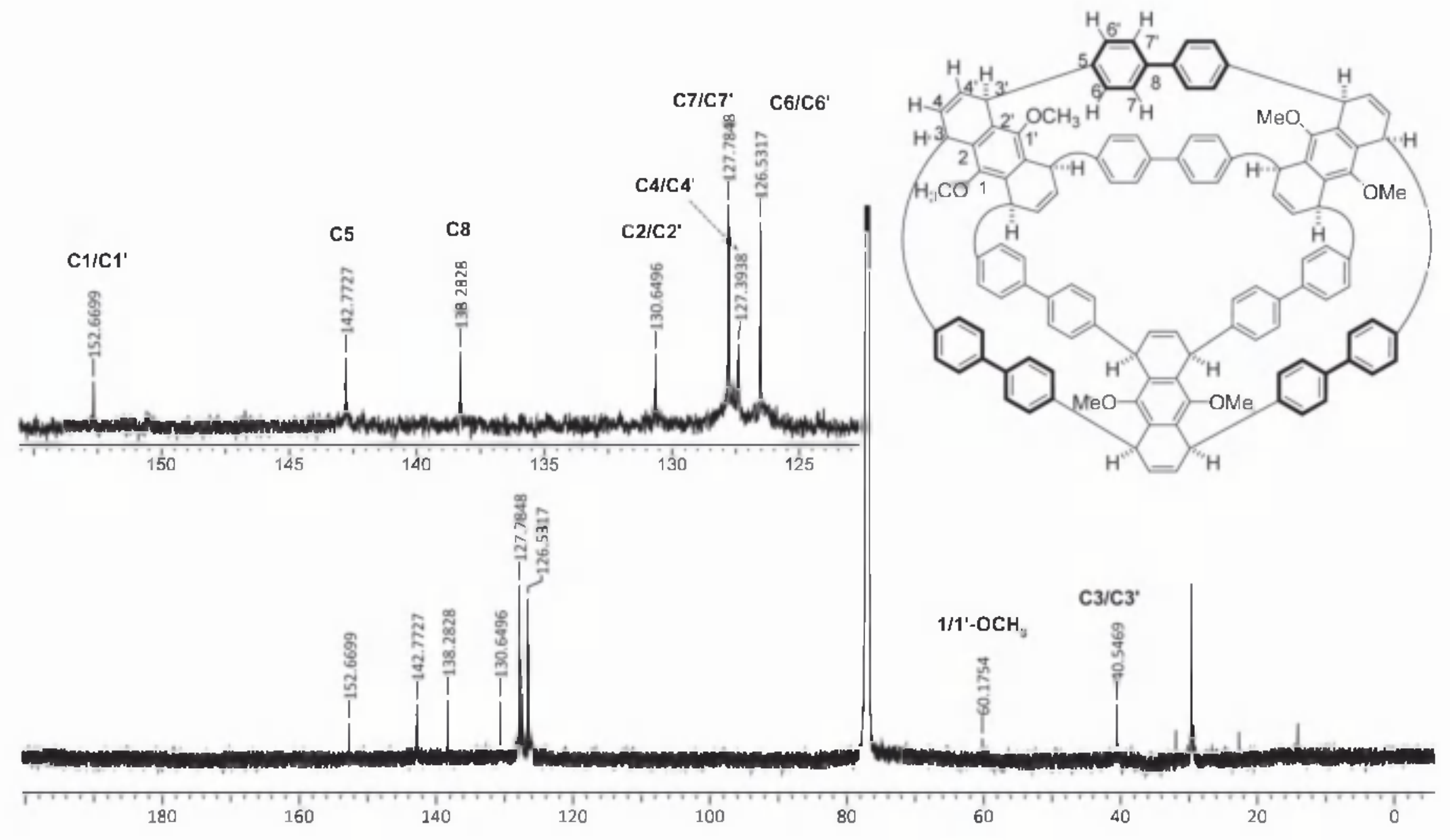




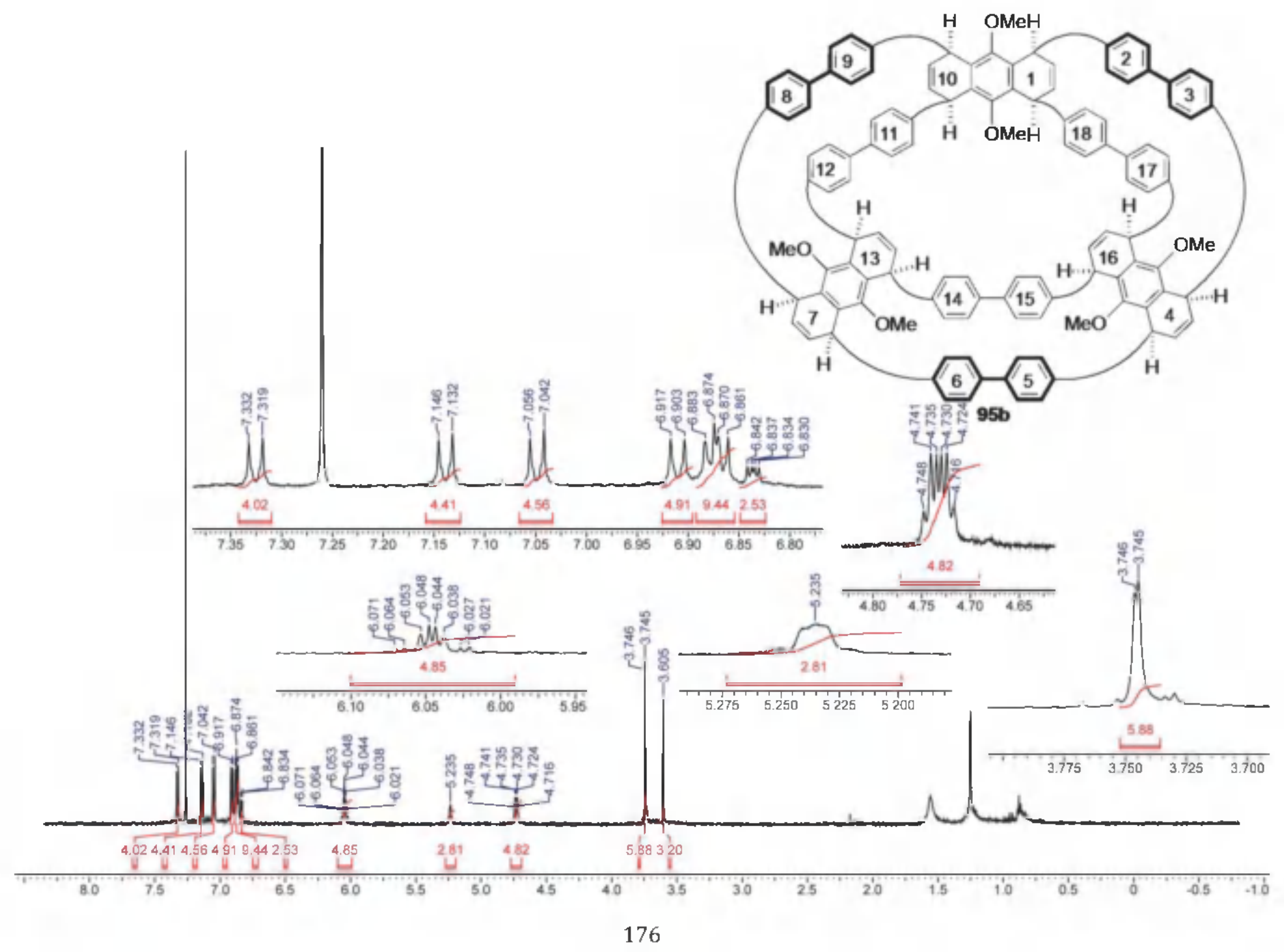




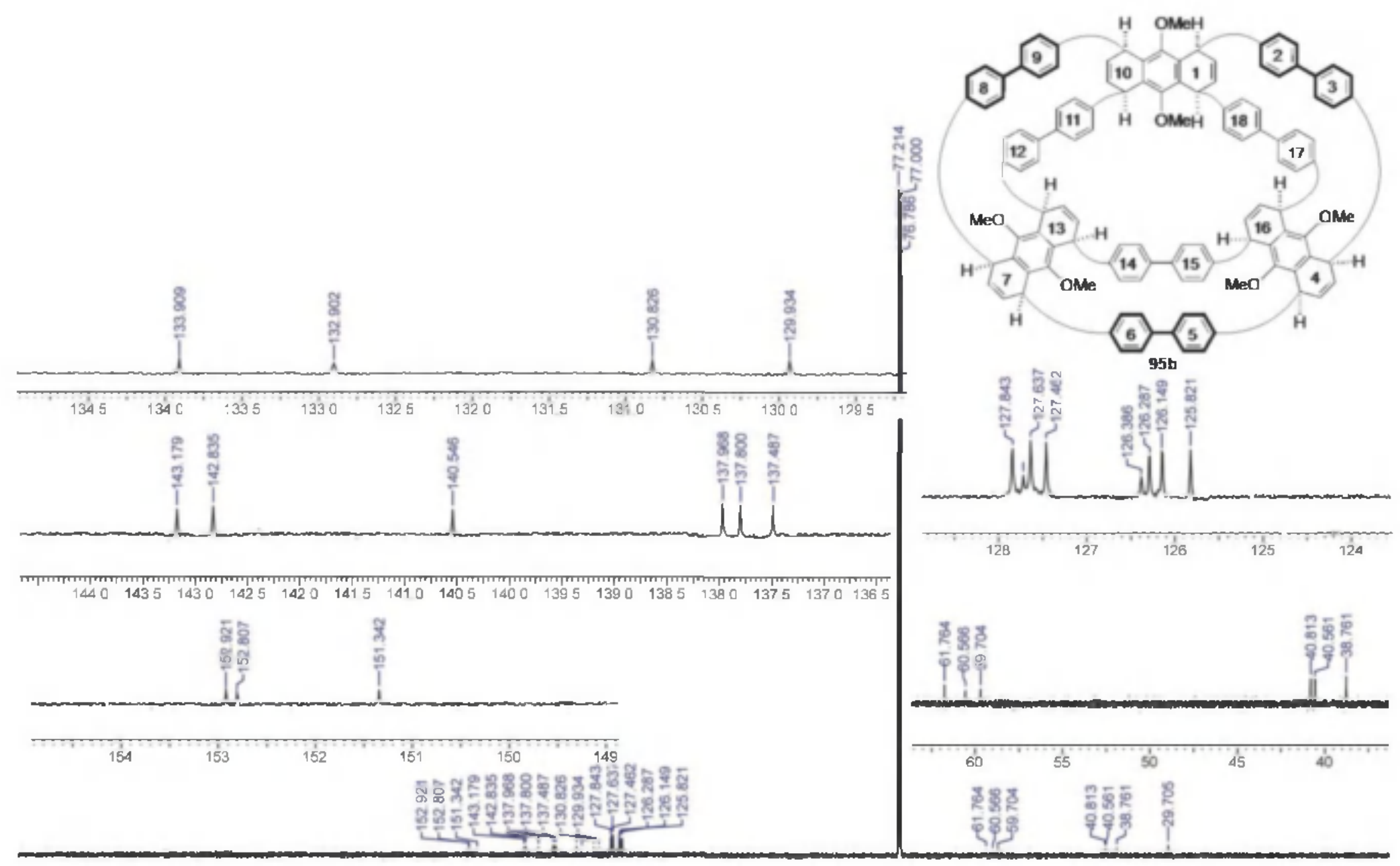

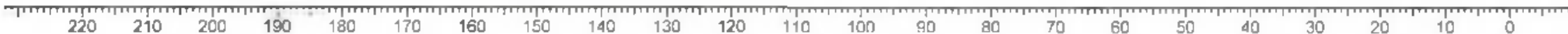


${ }^{1} \mathrm{H}$ NMR chemicals shifts $\left(\delta / \mathrm{ppm}\right.$, at $\left.-20^{\circ} \mathrm{C}\right)$

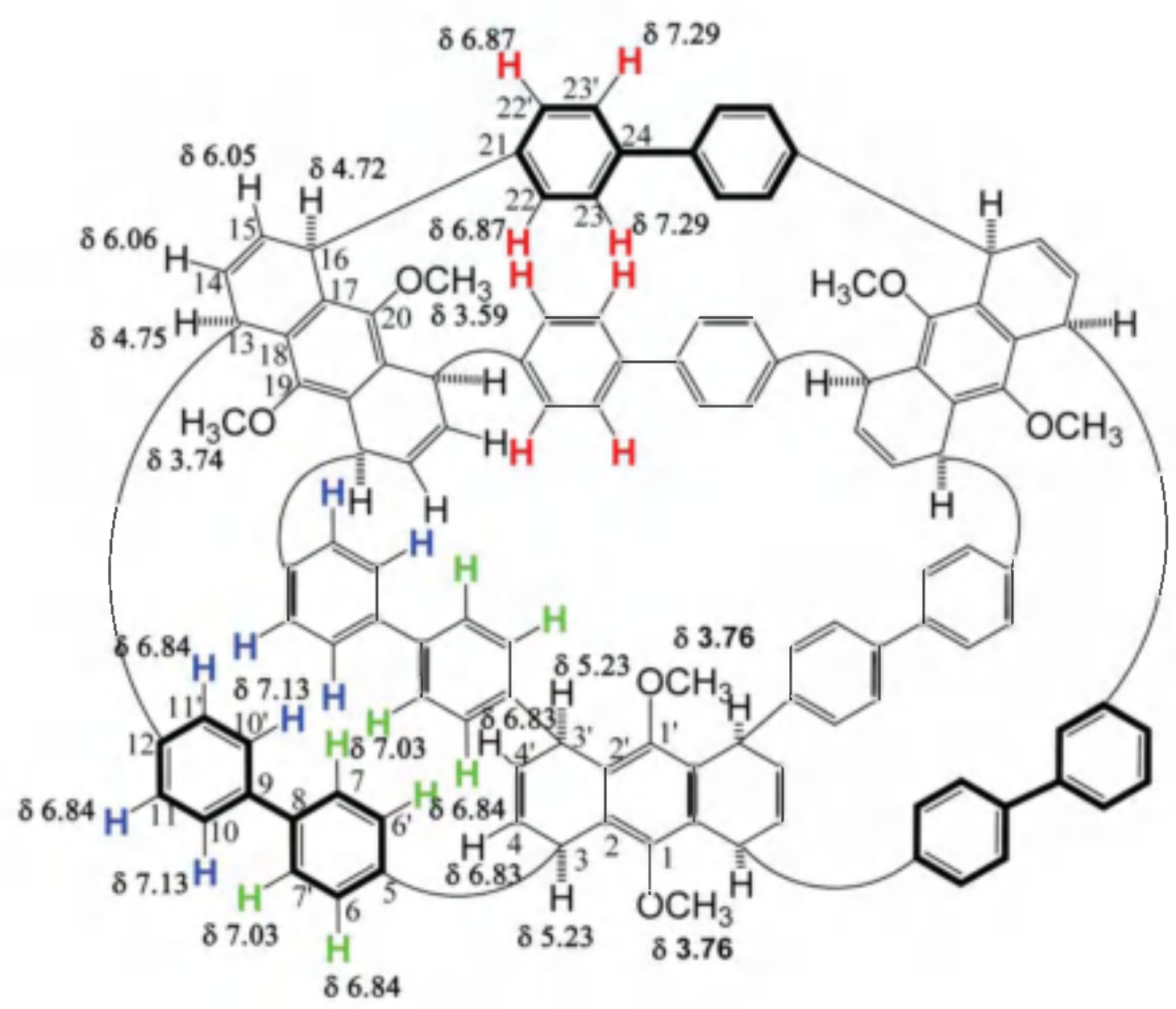

\section{${ }^{13} \mathrm{C}$ chemical shifts $\left(-20^{\circ} \mathrm{C}\right)$}

\begin{tabular}{|c|c|}
\hline $\mathrm{C} 1 / \mathrm{Cl}^{\prime}$ & 150.96 \\
\hline $1 / 1^{\prime}-\mathrm{OCH}_{3}$ & 61.80 \\
\hline $\mathrm{C} 2 / \mathrm{C}^{\prime}$ & 133.71 \\
\hline $\mathrm{C} 3 / \mathrm{C}^{\prime}$ & 38.41 \\
\hline $\mathrm{C} 4 / \mathrm{CA}^{\prime}$ & 132.72 \\
\hline $\mathrm{C} 5$ & 140.31 \\
\hline $\mathrm{C} 6 / \mathrm{CG}^{\prime}$ & 127.66 \\
\hline $\mathrm{Cl} / \mathrm{Cl}^{\prime}$ & 125.71 \\
\hline $\mathrm{Cg}$ & 137.78 \\
\hline $\mathrm{Cg}$ & 137.11 \\
\hline $\mathrm{C} 10 / \mathrm{C}^{\prime} 0^{\prime}$ & 126.61 \\
\hline $\mathrm{C} 11 / \mathrm{C} 11^{\prime}$ & 127.39 \\
\hline $\mathrm{C} 12$ & 142.53 \\
\hline $\mathrm{C} 13$ & 40.06 \\
\hline C14 & 126.15 \\
\hline $\mathrm{C} 15$ & 127.61 \\
\hline C16 & 40.49 \\
\hline $\mathrm{C} 17$ & 130.40 \\
\hline C18 & 129.70 \\
\hline C19 & 152.69 \\
\hline $19-\mathrm{CCH}_{3}$ & 61.11 \\
\hline $\mathrm{C} 20$ & 152.58 \\
\hline $20-\mathrm{OCH}_{3}$ & 59.93 \\
\hline $\mathrm{C} 21$ & 142.98 \\
\hline $\mathrm{C} 22 / \mathrm{C}^{\prime} 2^{\prime}$ & 127.24 \\
\hline $\mathrm{C} 2 \mathrm{~J} / \mathrm{C} 2 \mathrm{~J}^{\prime}$ & 126.36 \\
\hline $\mathrm{C} 24$ & 137.76 \\
\hline
\end{tabular}


${ }^{1} \mathrm{H}$ NMR spectrum in $\mathrm{CDCl}_{3}\left(-20^{\circ} \mathrm{C}\right)$
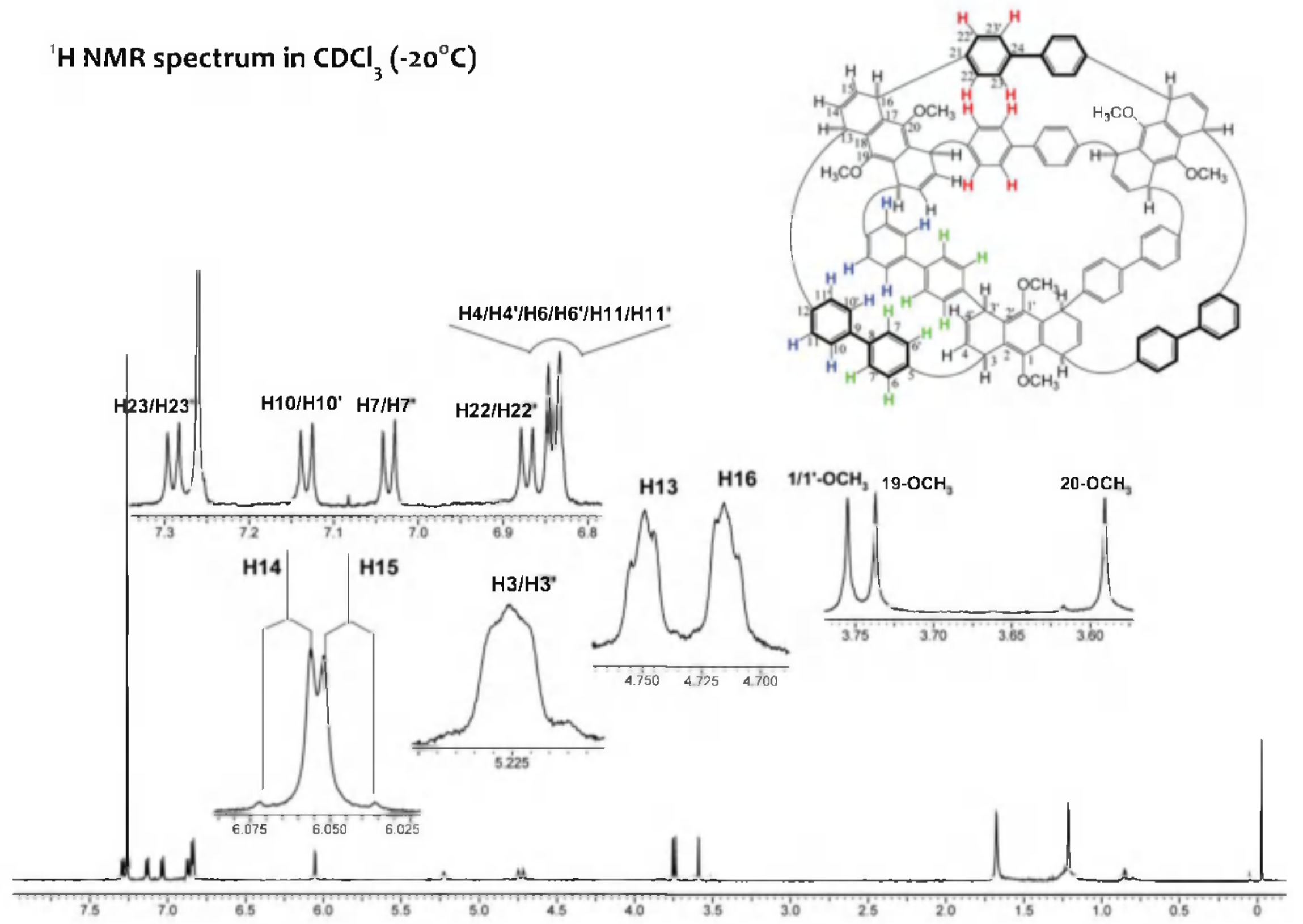
${ }^{13} \mathrm{C}$ NMR spectrum in $\mathrm{CDCl}_{3}\left(-20^{\circ} \mathrm{C}\right)$

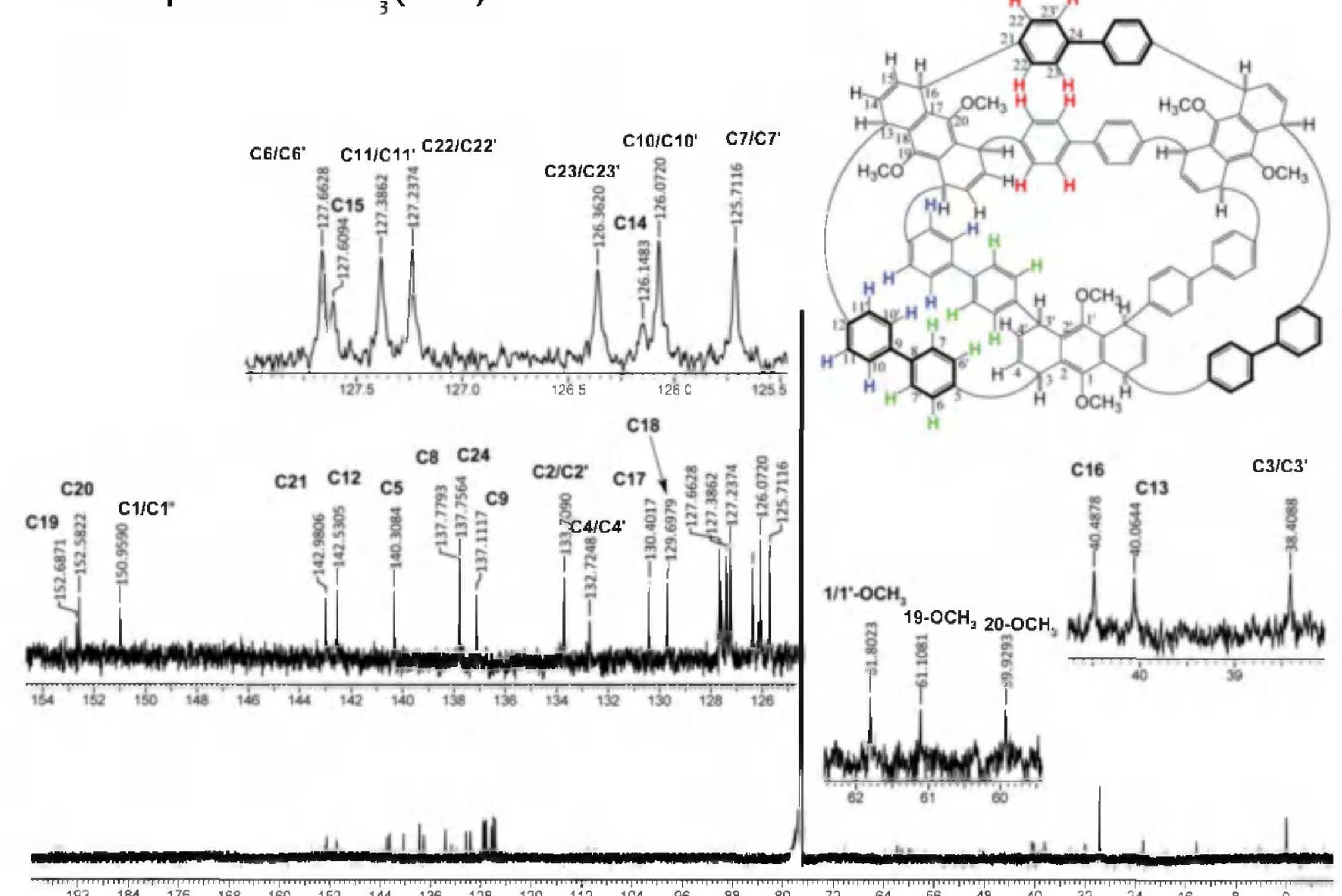




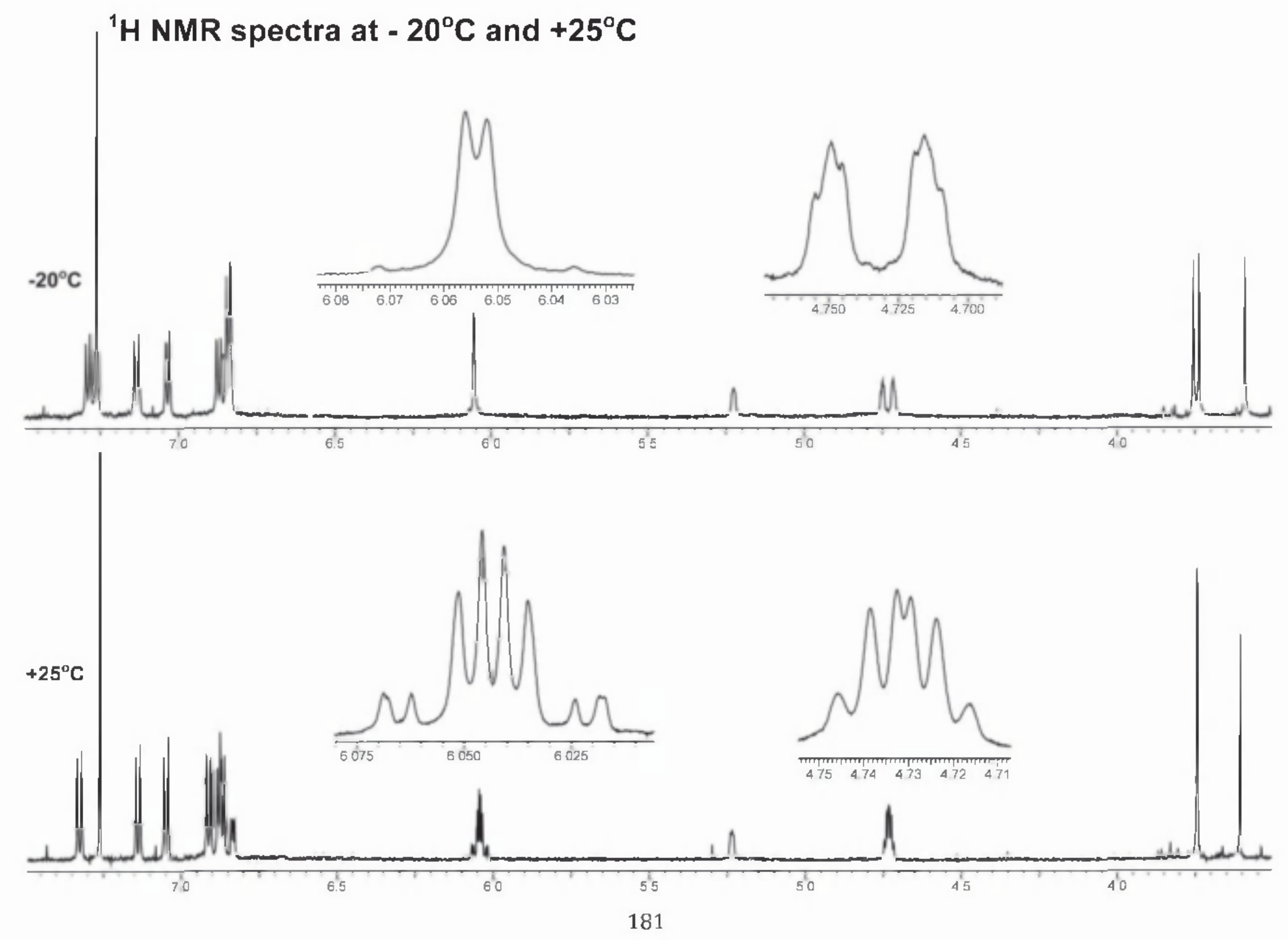


'H NMR spectrum, Double Resonance at $4.73 \mathrm{ppm}$, at $+25^{\circ} \mathrm{C}$.

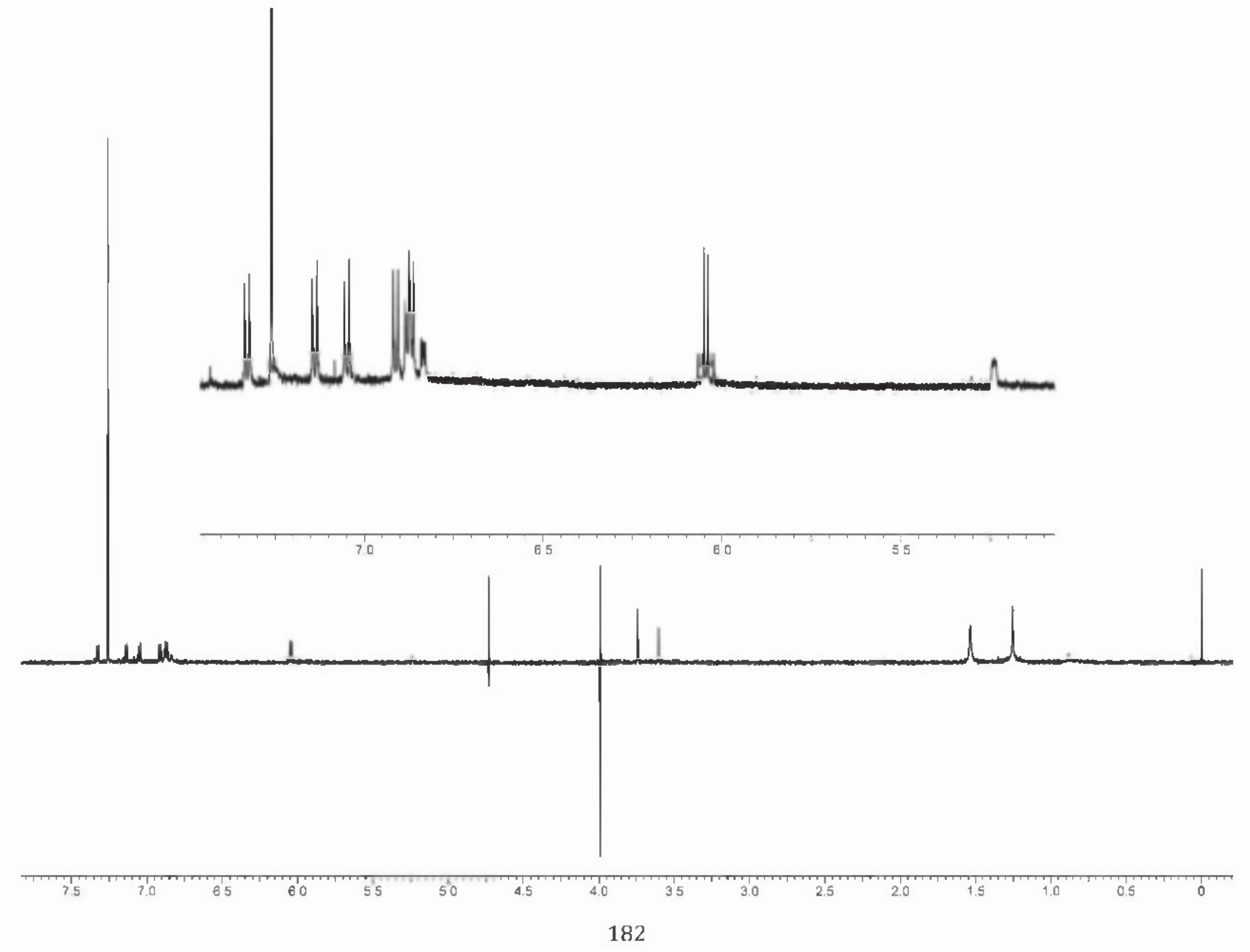


'H NMR spectrum, Double Resonance at $5.24 \mathrm{ppm}$, at $+25^{\circ} \mathrm{C}$.

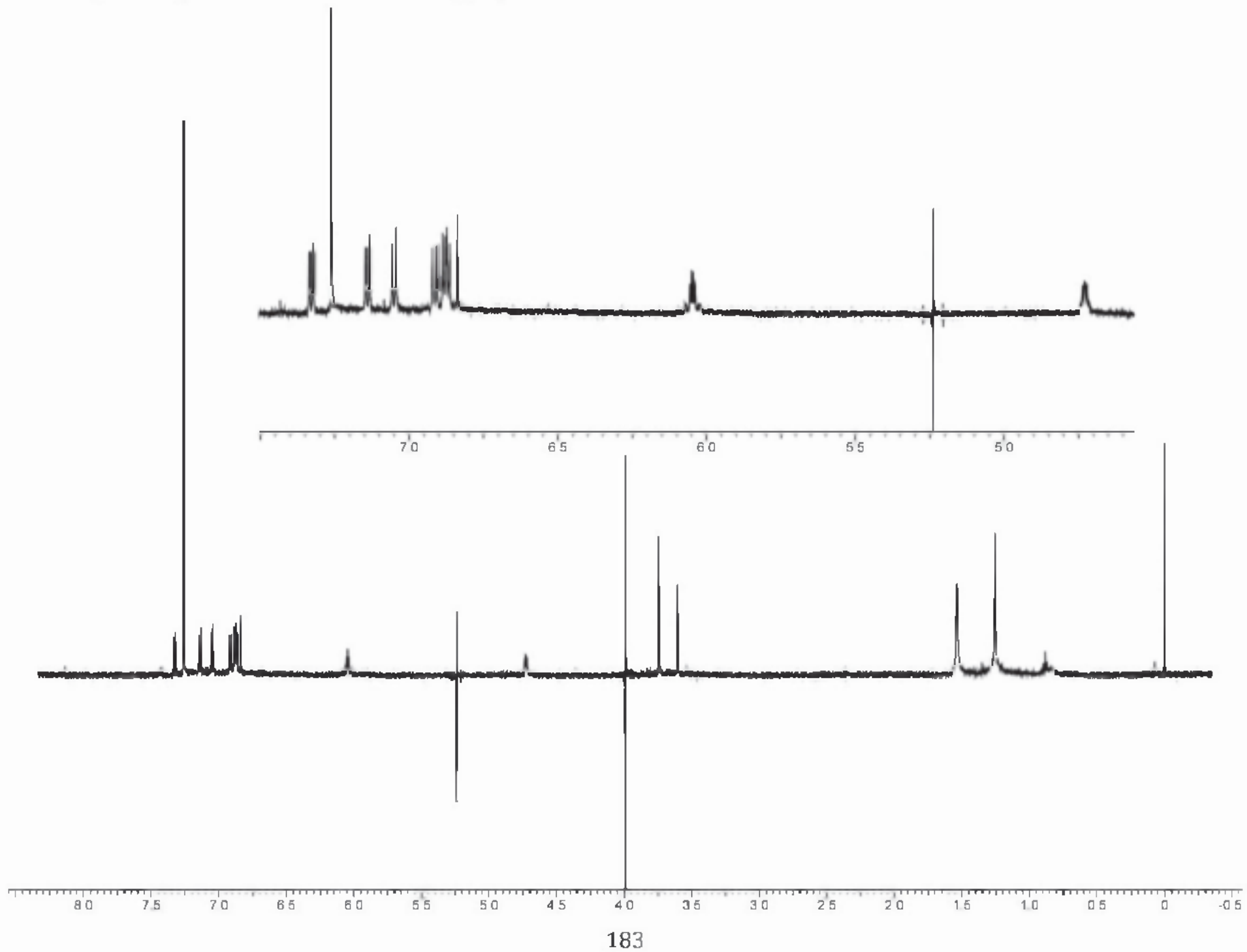


'H NMR spectrum, Double Resonance at $6.05 \mathrm{ppm}$, at $+25^{\circ} \mathrm{C}$.

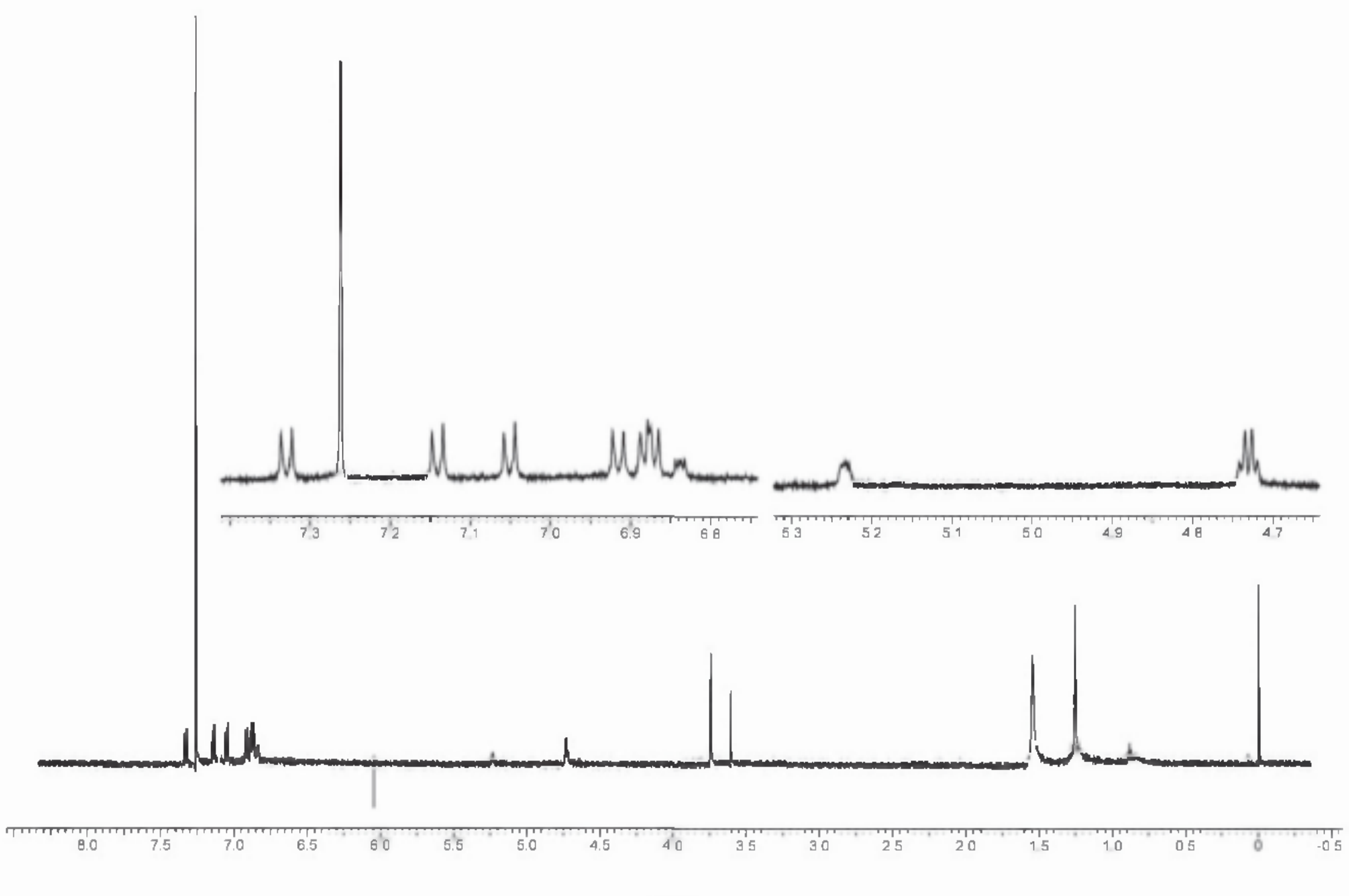


'H NMR spectrum, Double Resonance at $4.75 \mathrm{ppm}$, at $+60^{\circ} \mathrm{C}$.

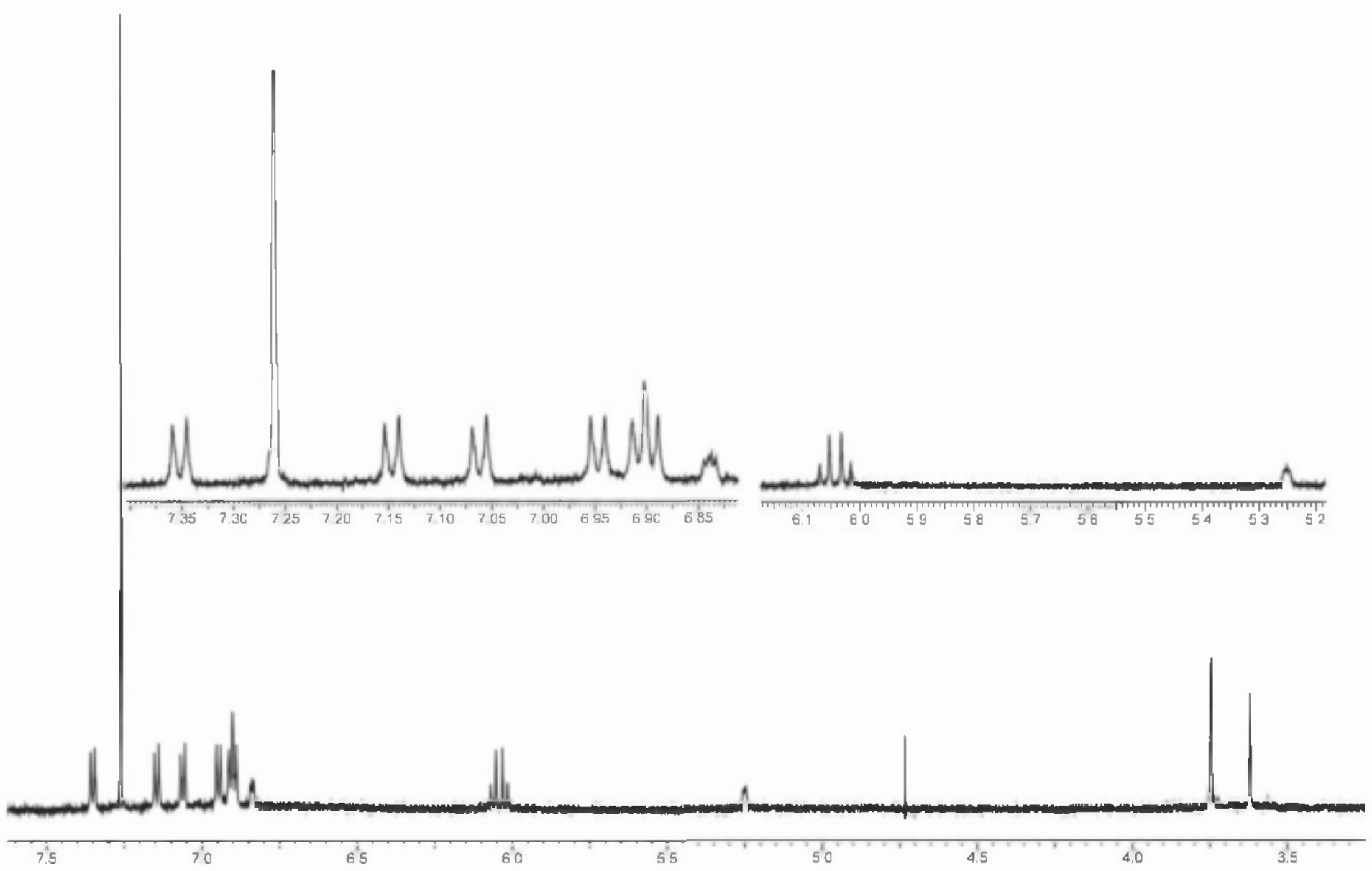


'H NMR spectrum, Double Resonance at $5.21 \mathrm{ppm}$, at $+60^{\circ} \mathrm{C}$.

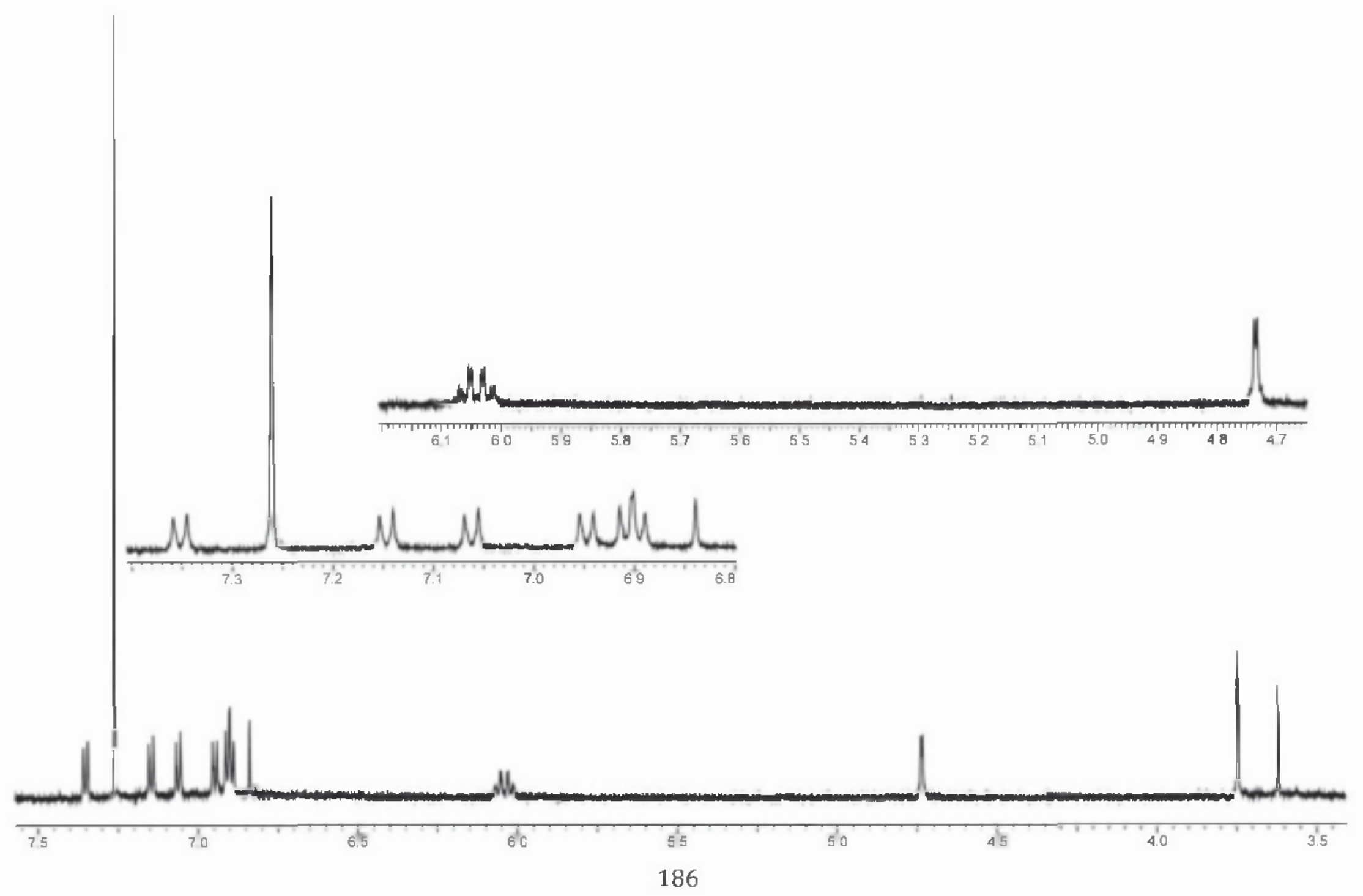


'H NMR spectrum, Double Resonance at $6.05 \mathrm{ppm}$, at $+60^{\circ} \mathrm{C}$.

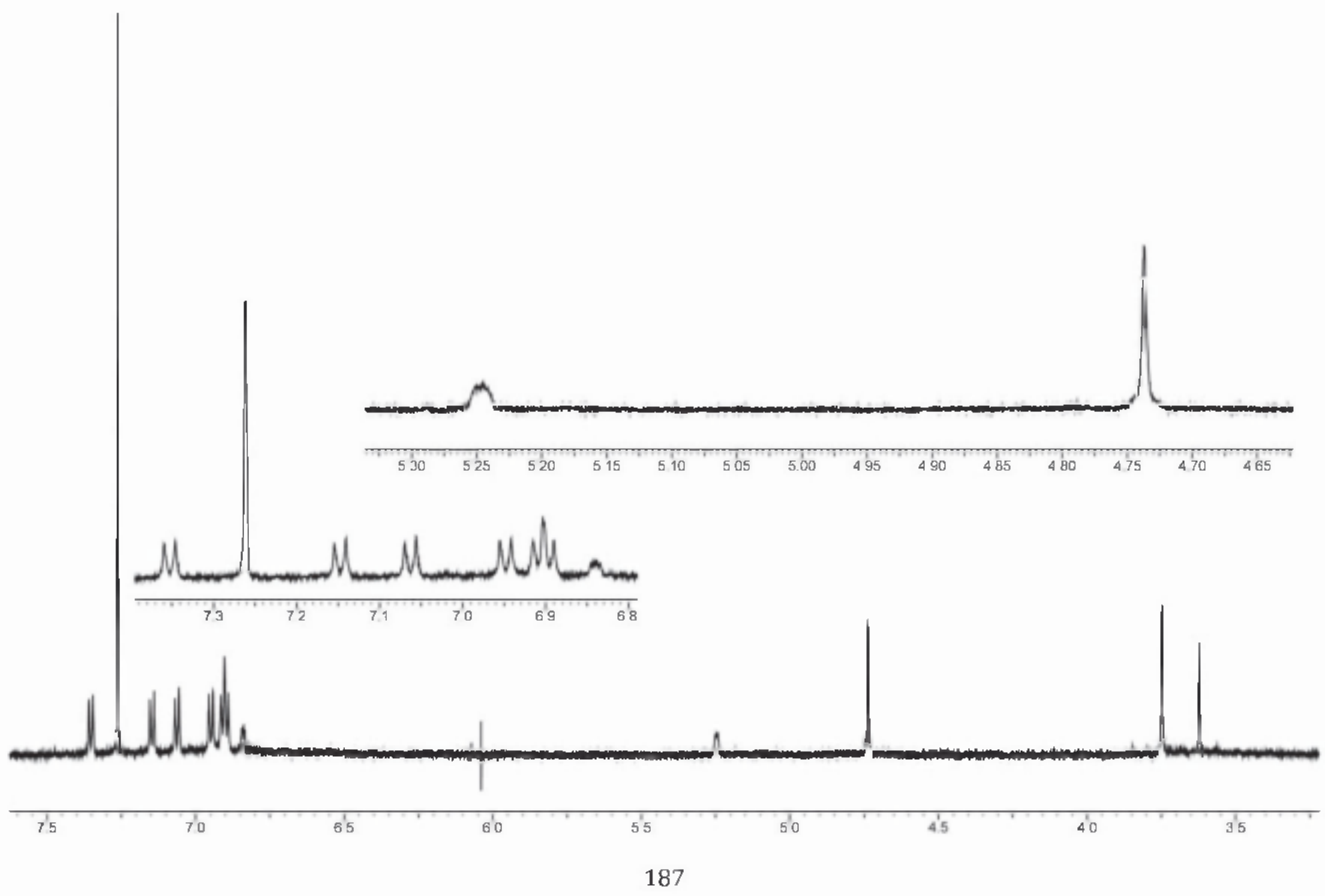


'H NMR spectrum, Double Resonance at $6.8 \mathrm{ppm}$, at $+60^{\circ} \mathrm{C}$.

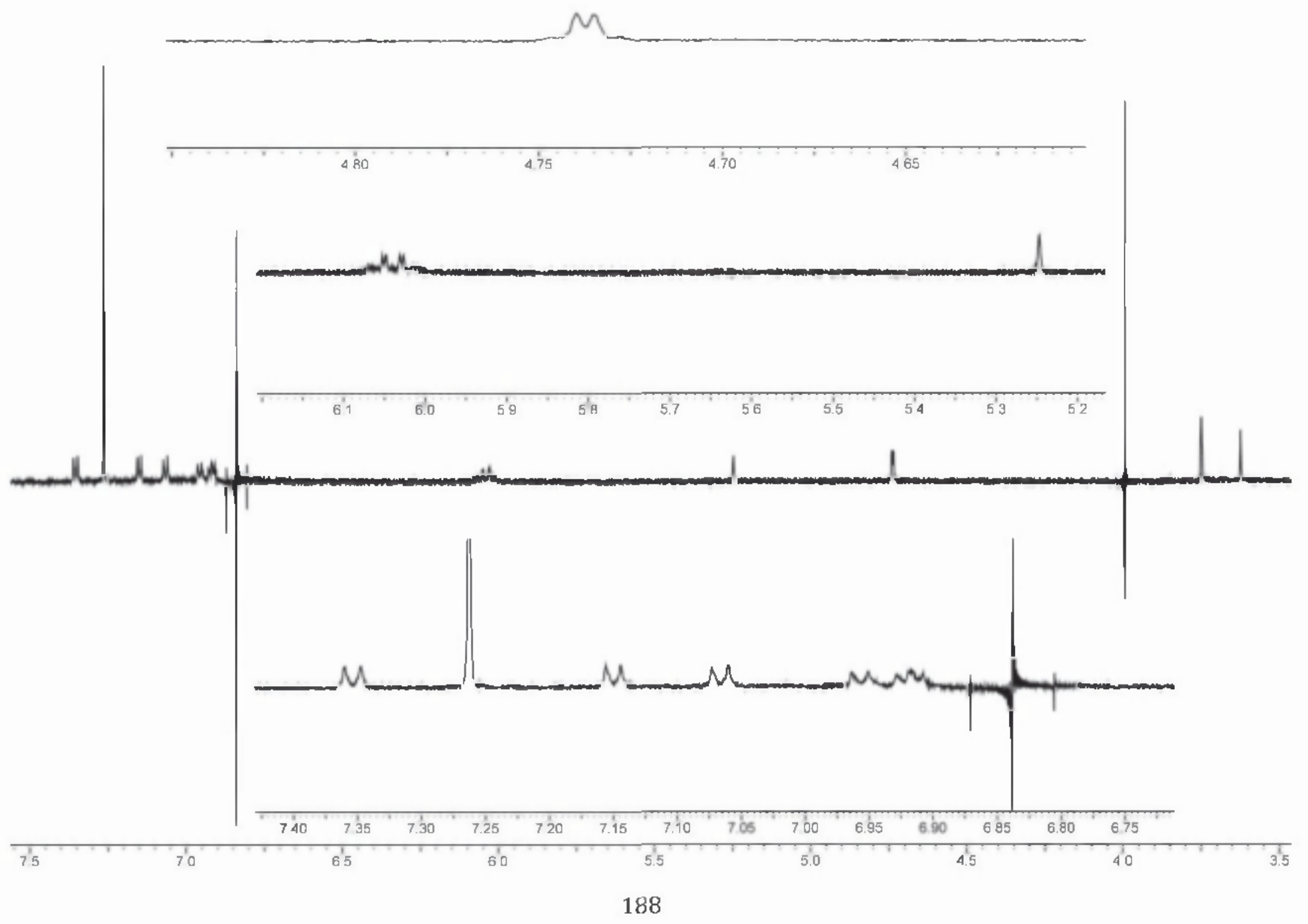




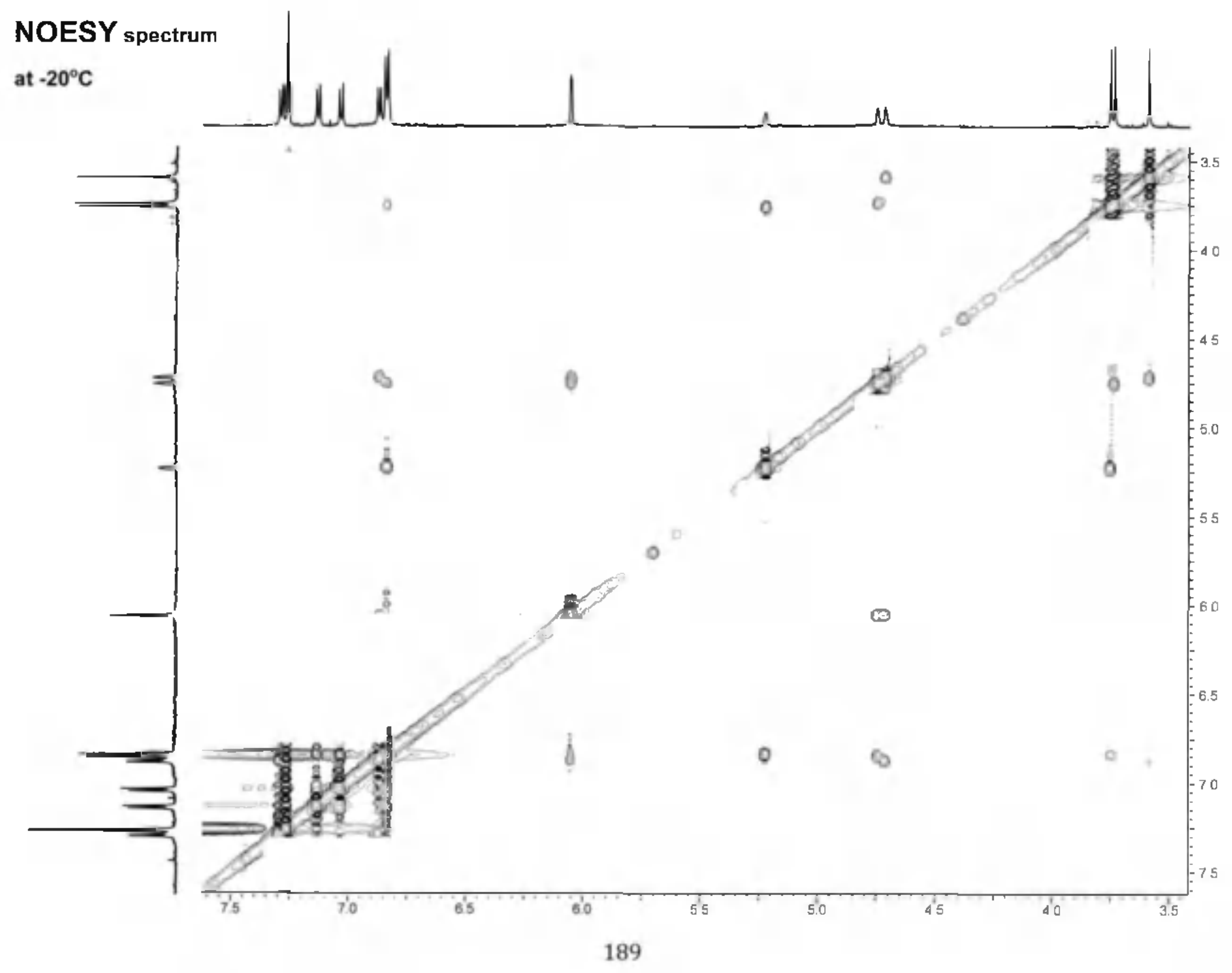




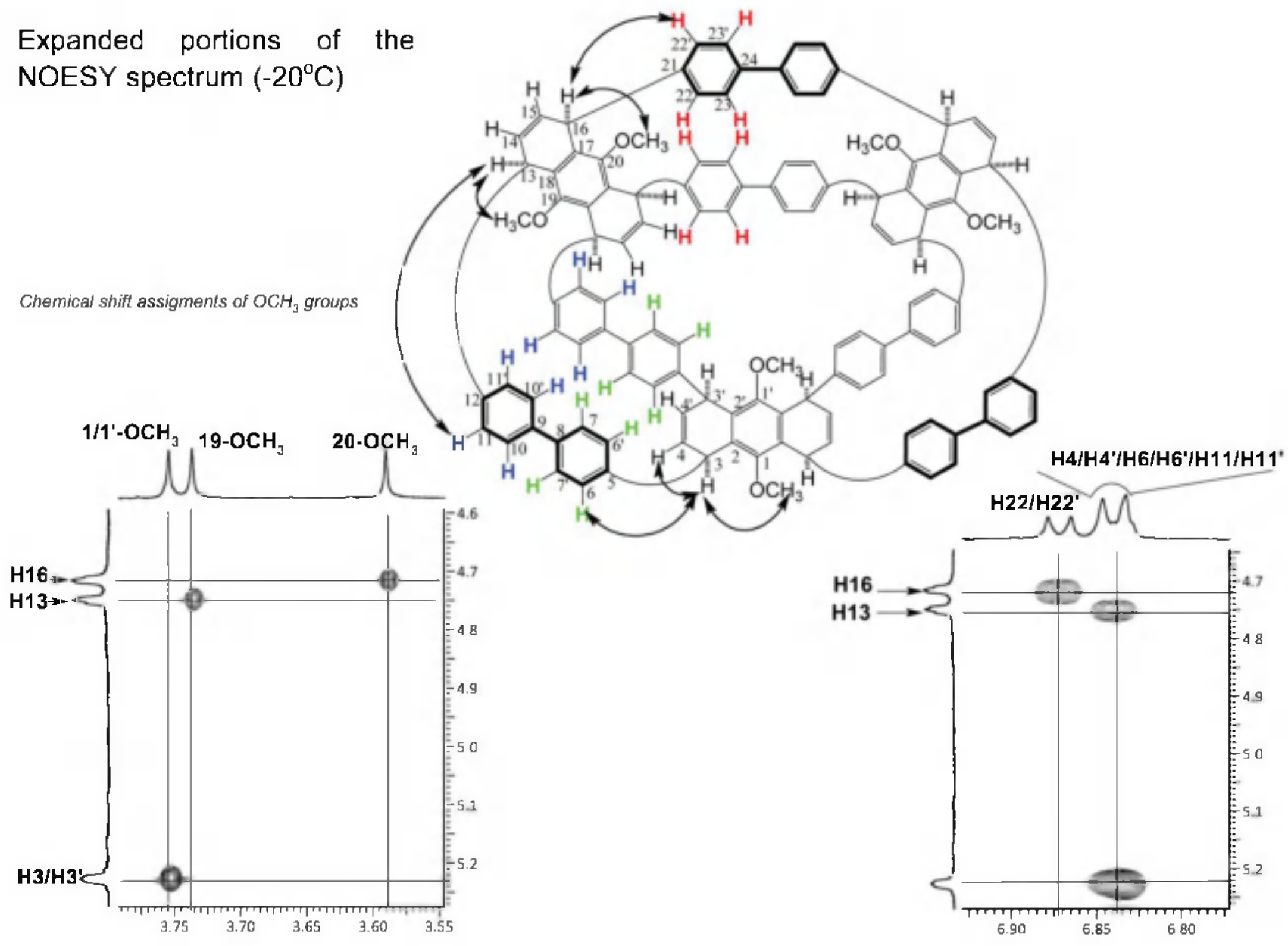


Aromatic portion of the NOESY spectrum $\left(-20^{\circ} \mathrm{C}\right)$

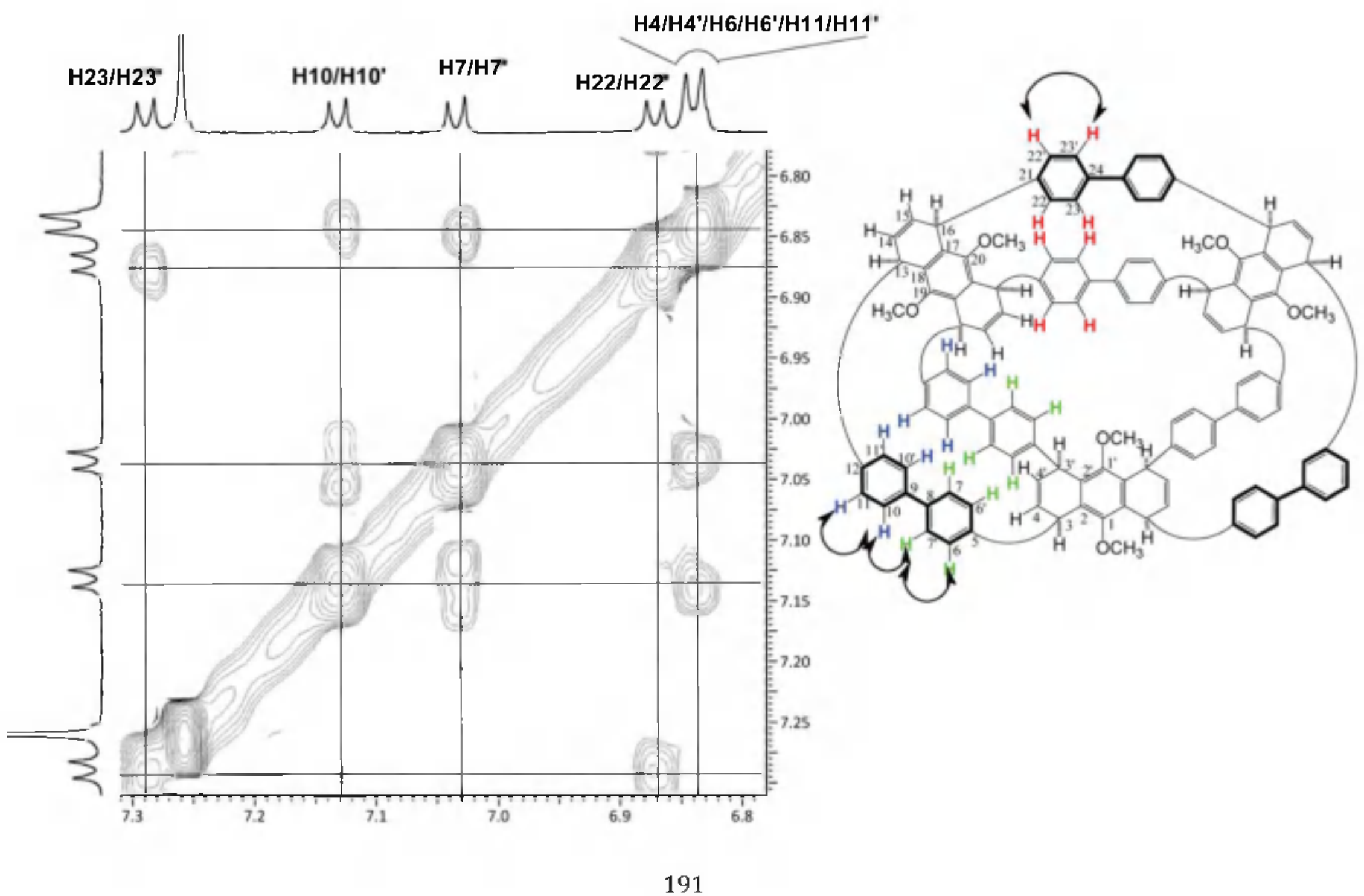



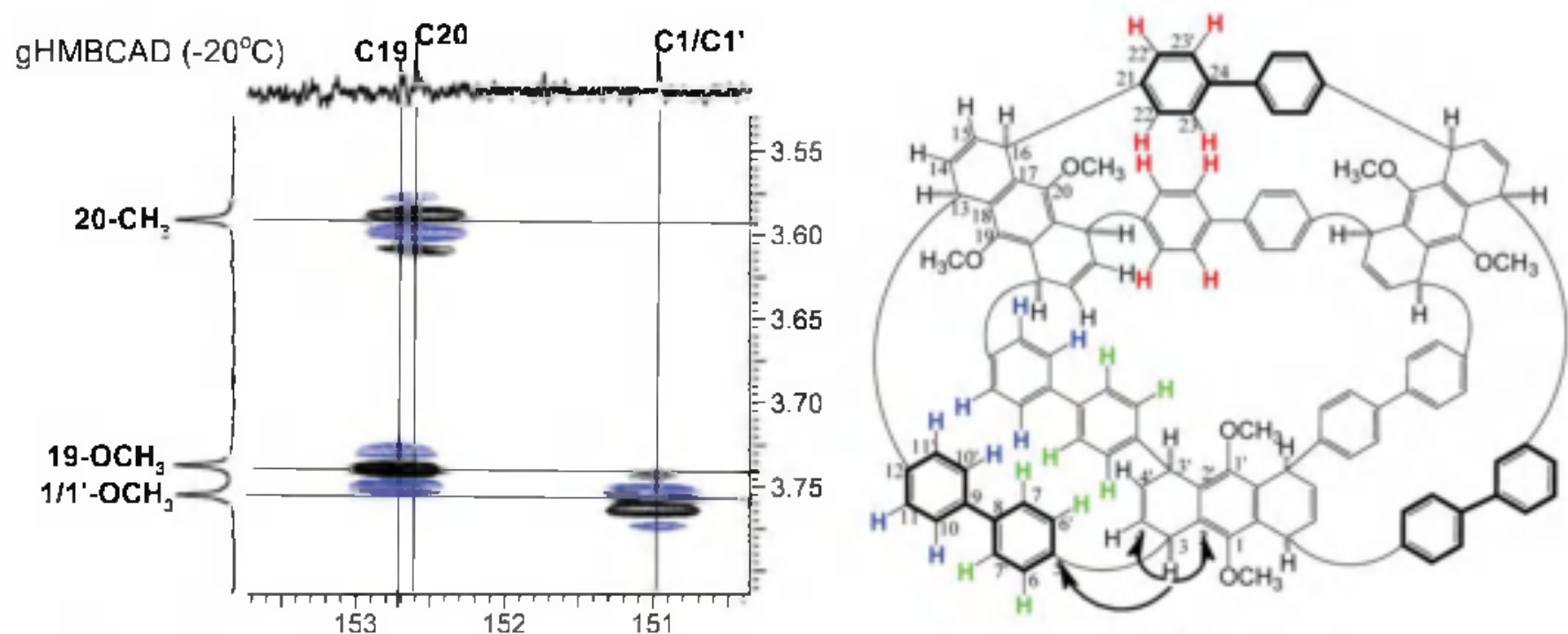

$\operatorname{gHMBCAD}\left(+25^{\circ} \mathrm{C}\right)$

gHSQCAD $\left.\left({ }^{1}\right\rfloor_{H-C}\right)$

gHMBCAD $\left(-20^{\circ} \mathrm{C}\right)$

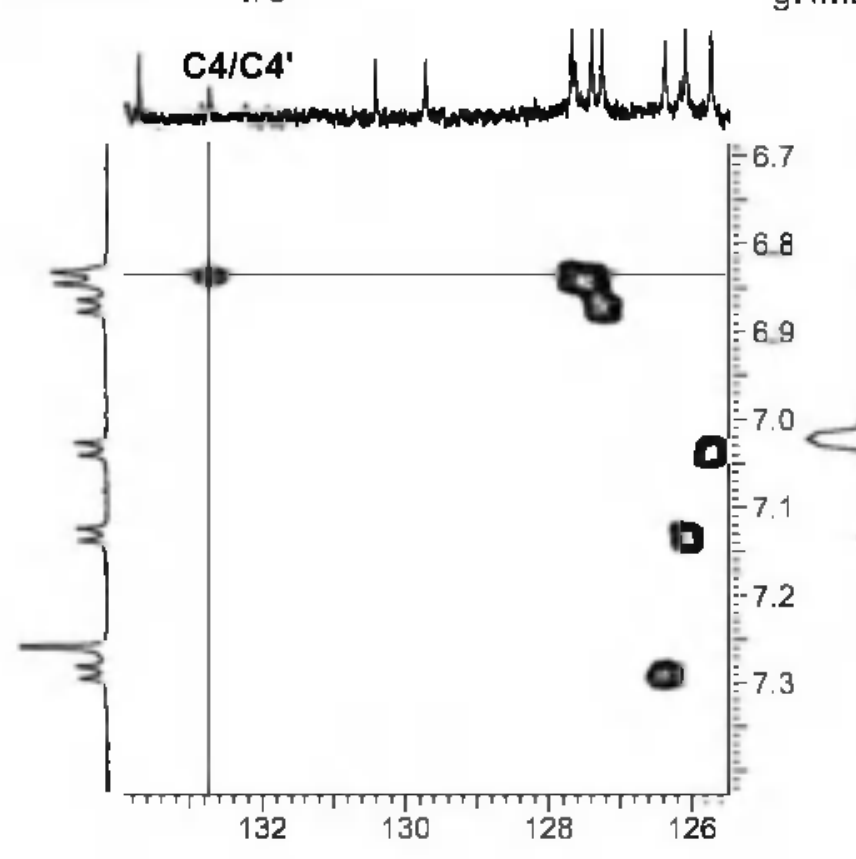

约

ปู่
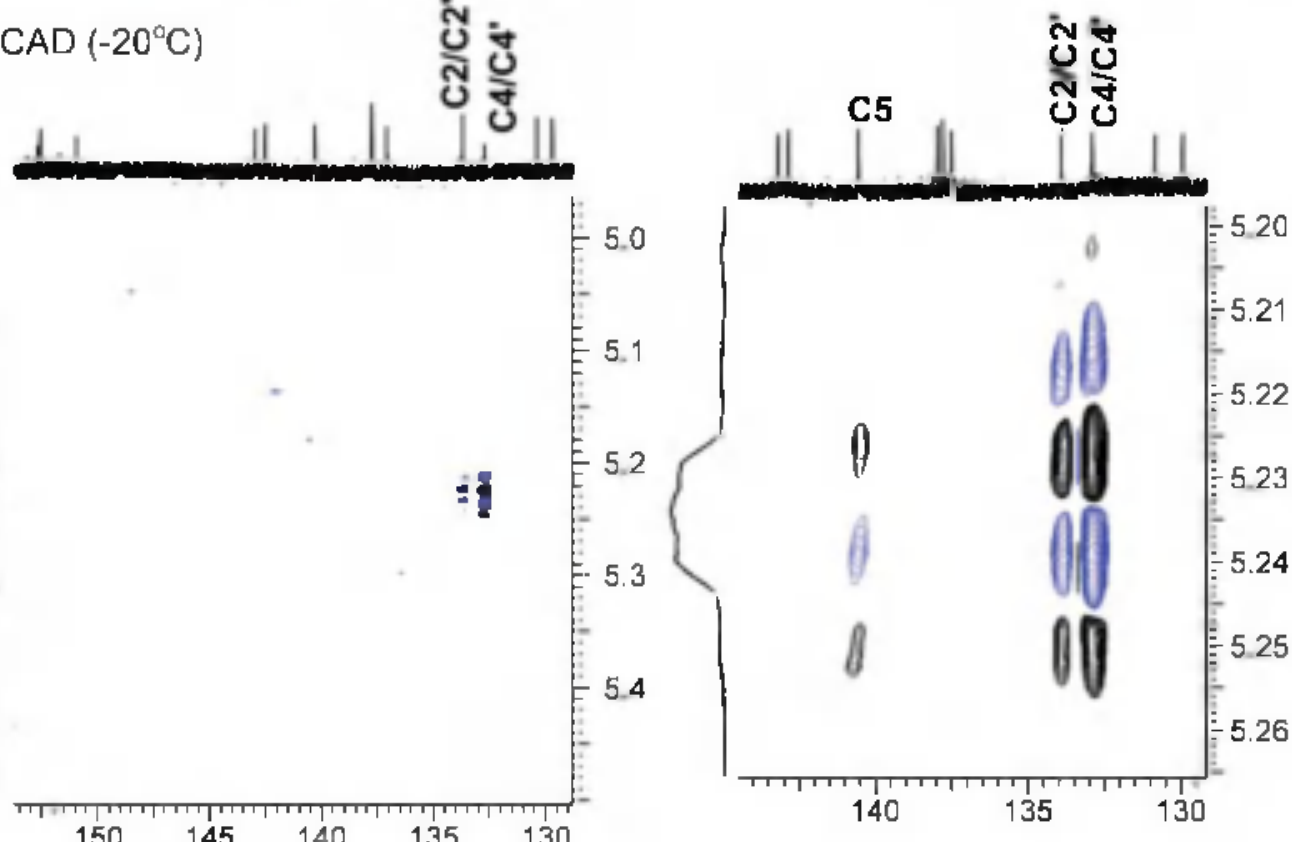

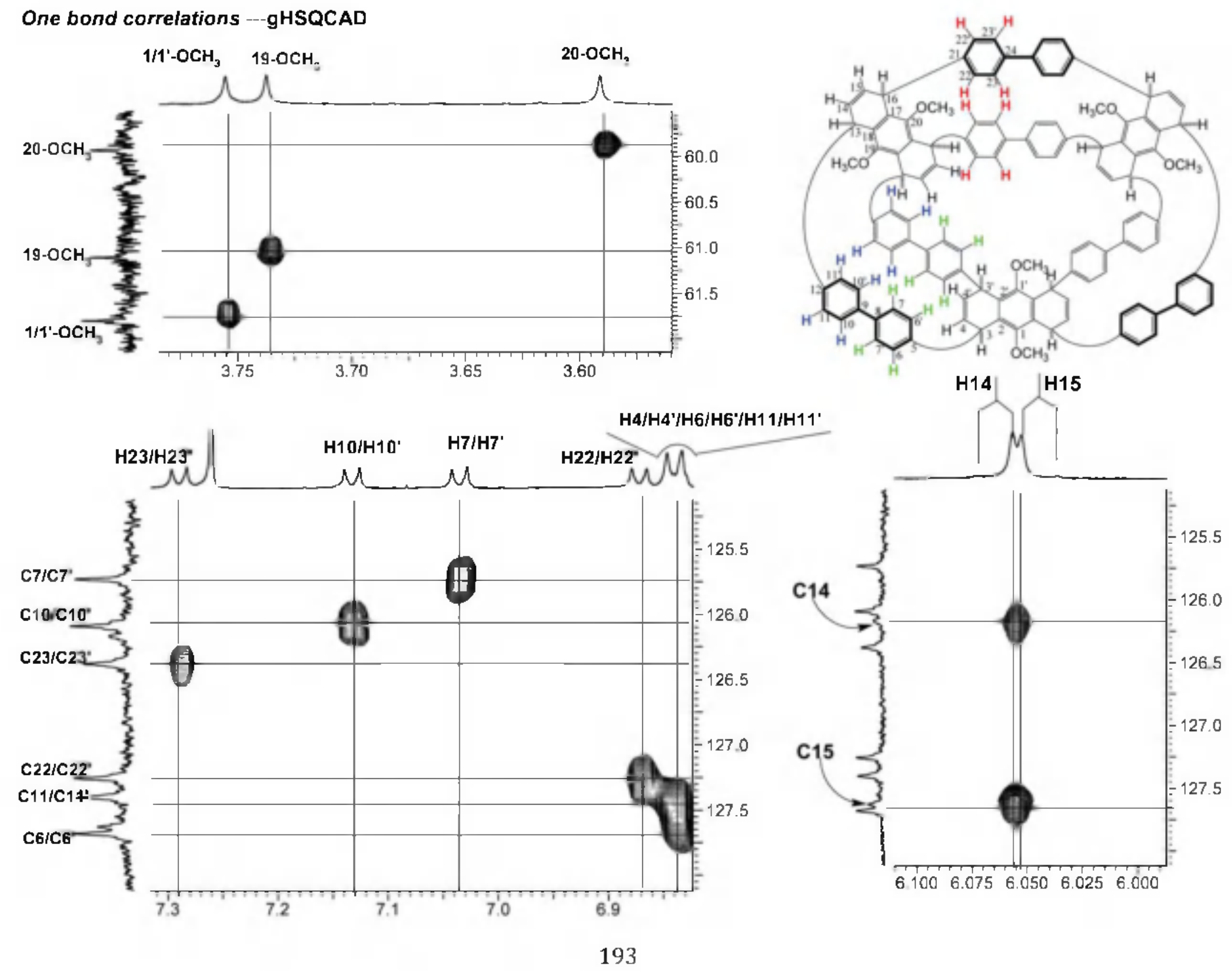


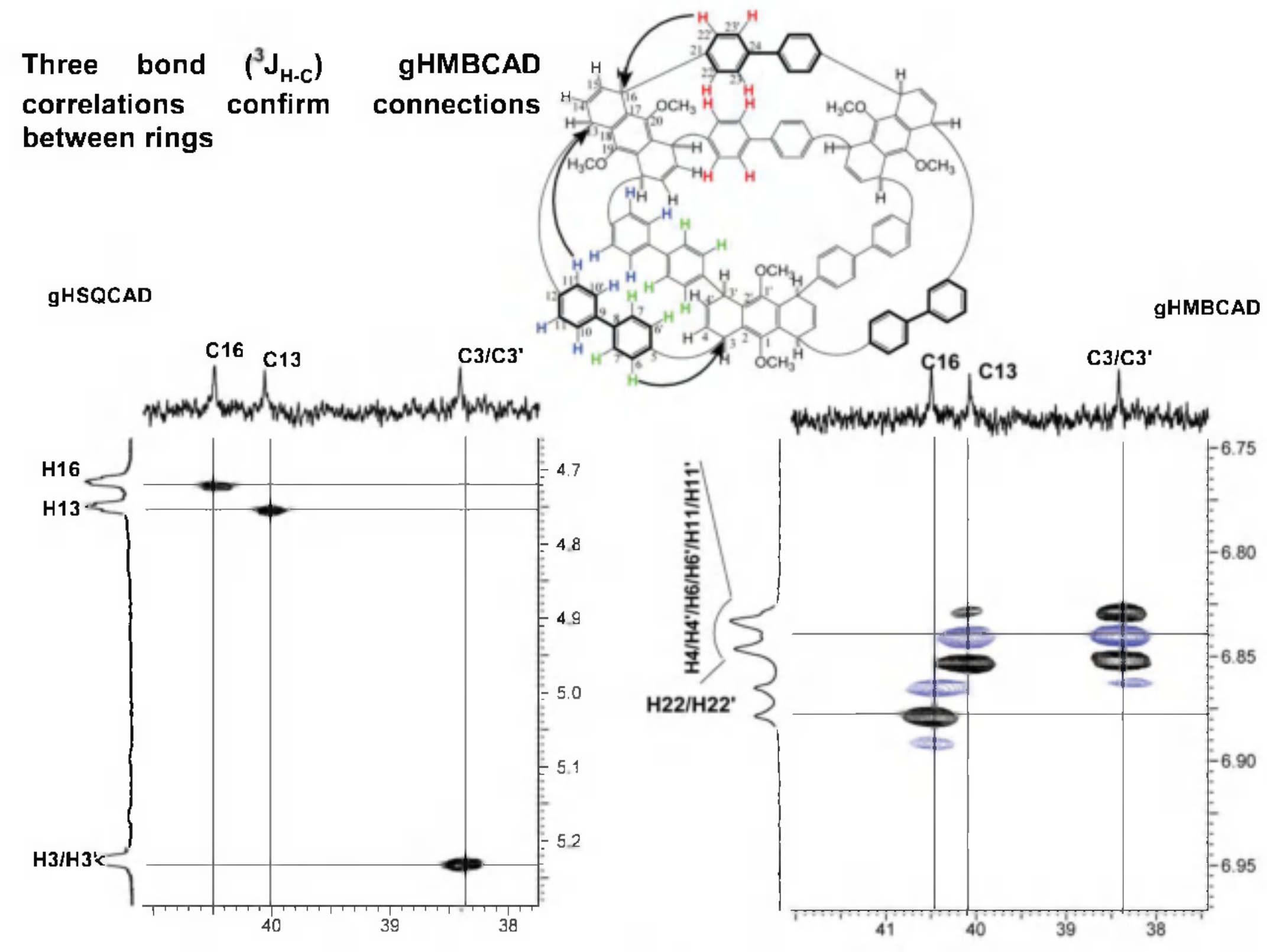




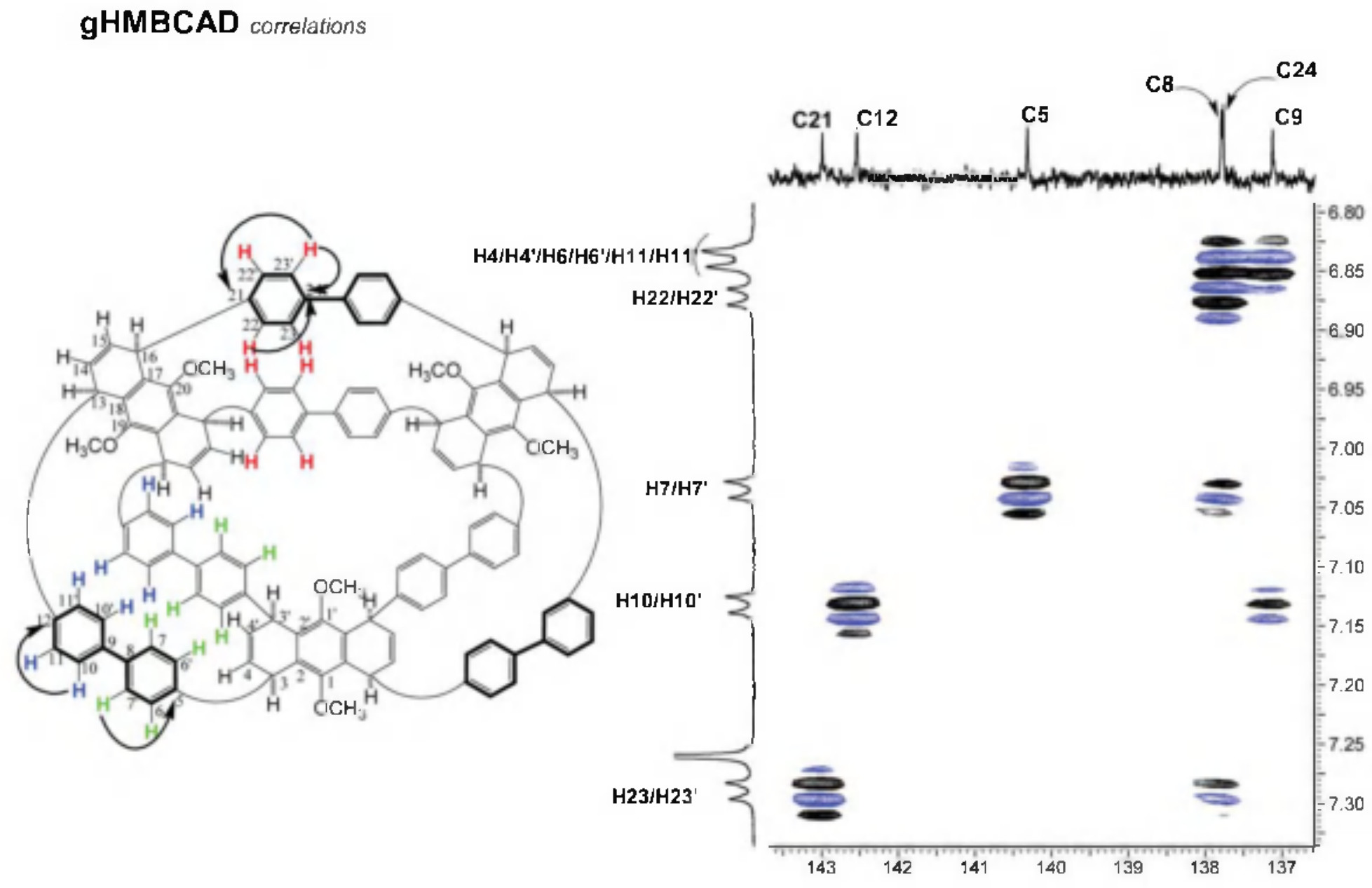



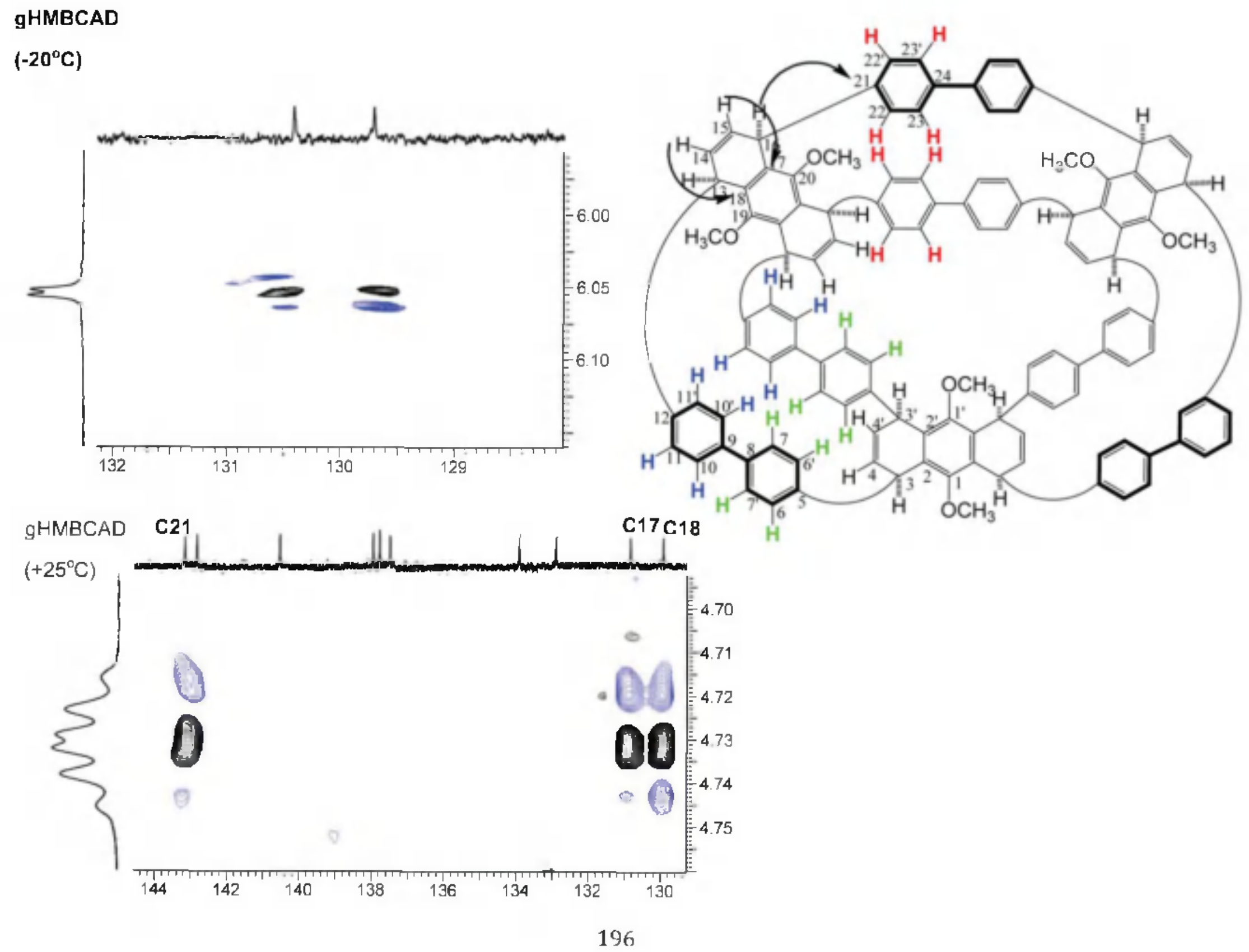
${ }^{13} \mathrm{C}$ NMR spectra

$-20^{\circ} \mathrm{C}$

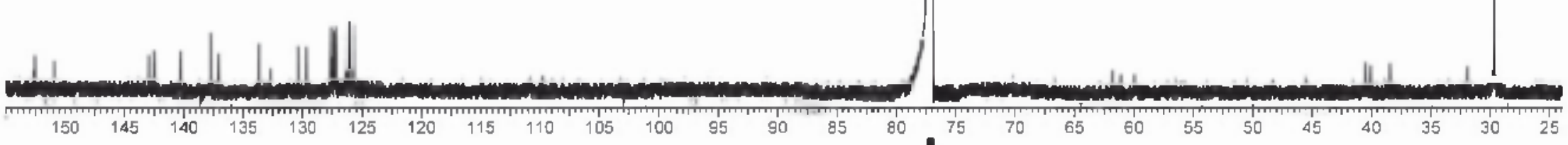

$+25^{\circ} \mathrm{C}$

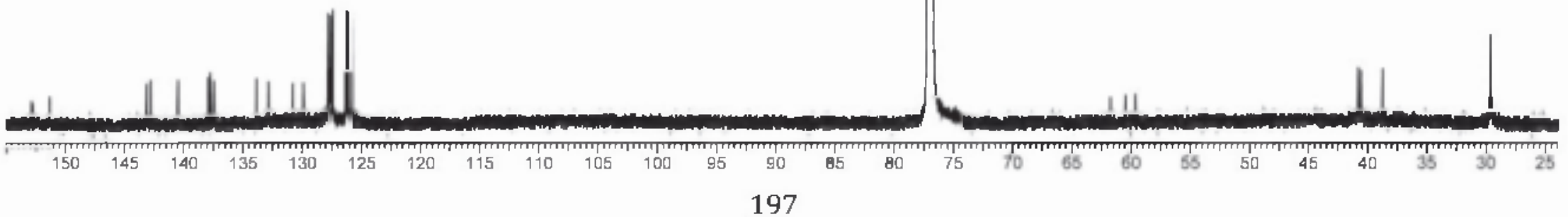


Expanded portions of the ${ }^{13} \mathrm{C}$ NMR spectra

$-20^{\circ} \mathrm{C}$

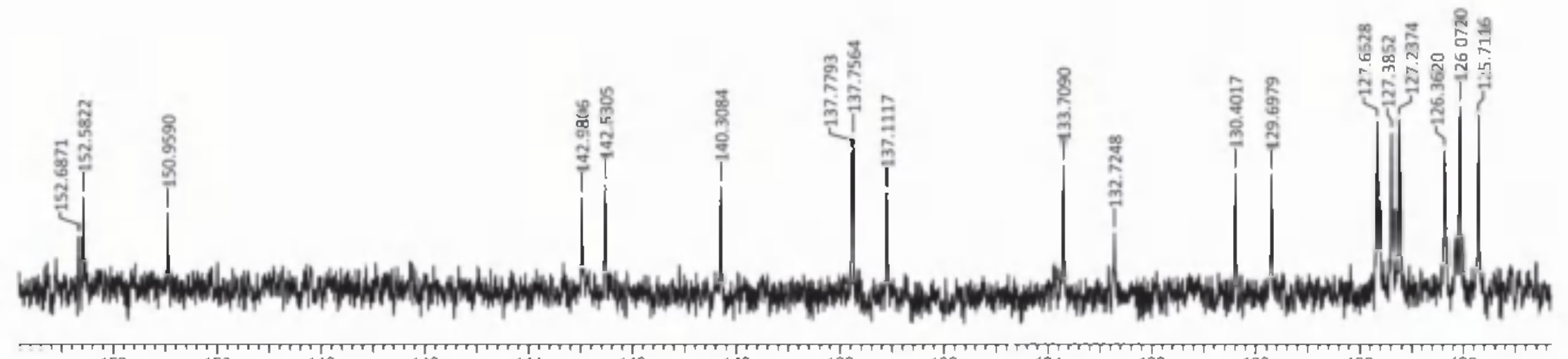

$+25^{\circ} \mathrm{C}$

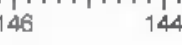

140

136

134

132

130

128

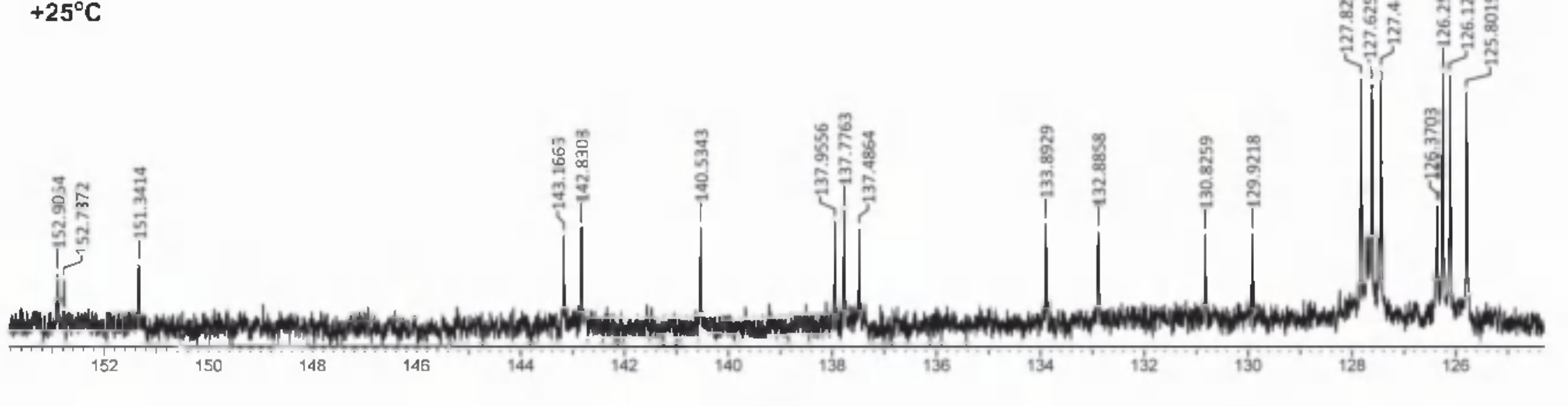




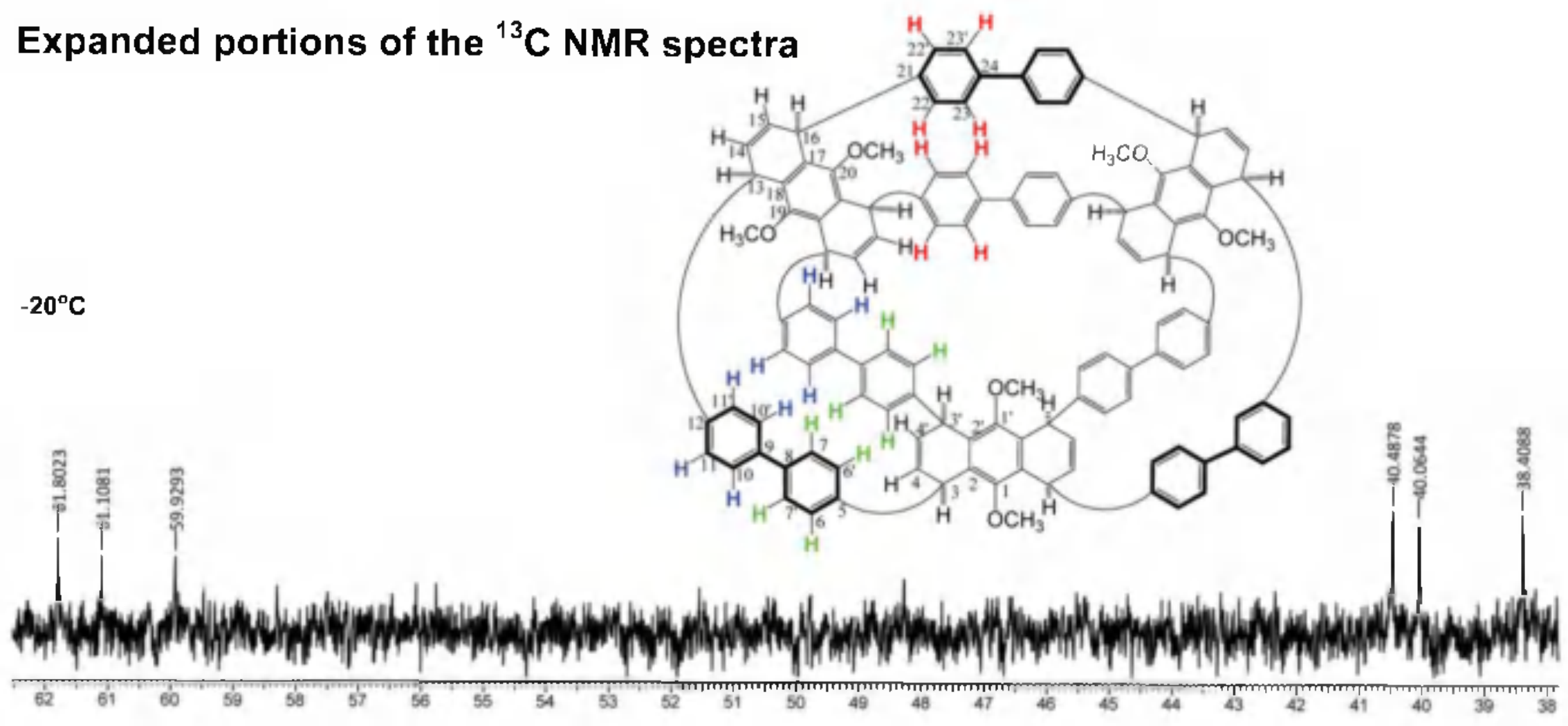

$+25^{\circ} \mathrm{C}$

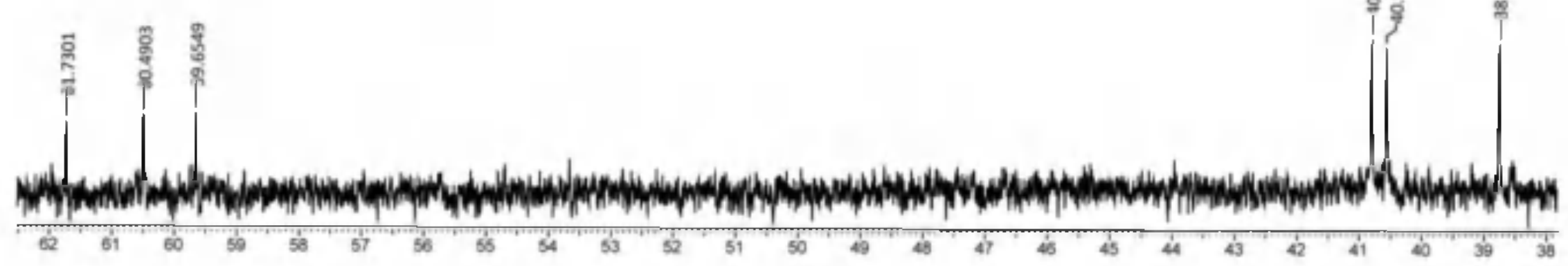




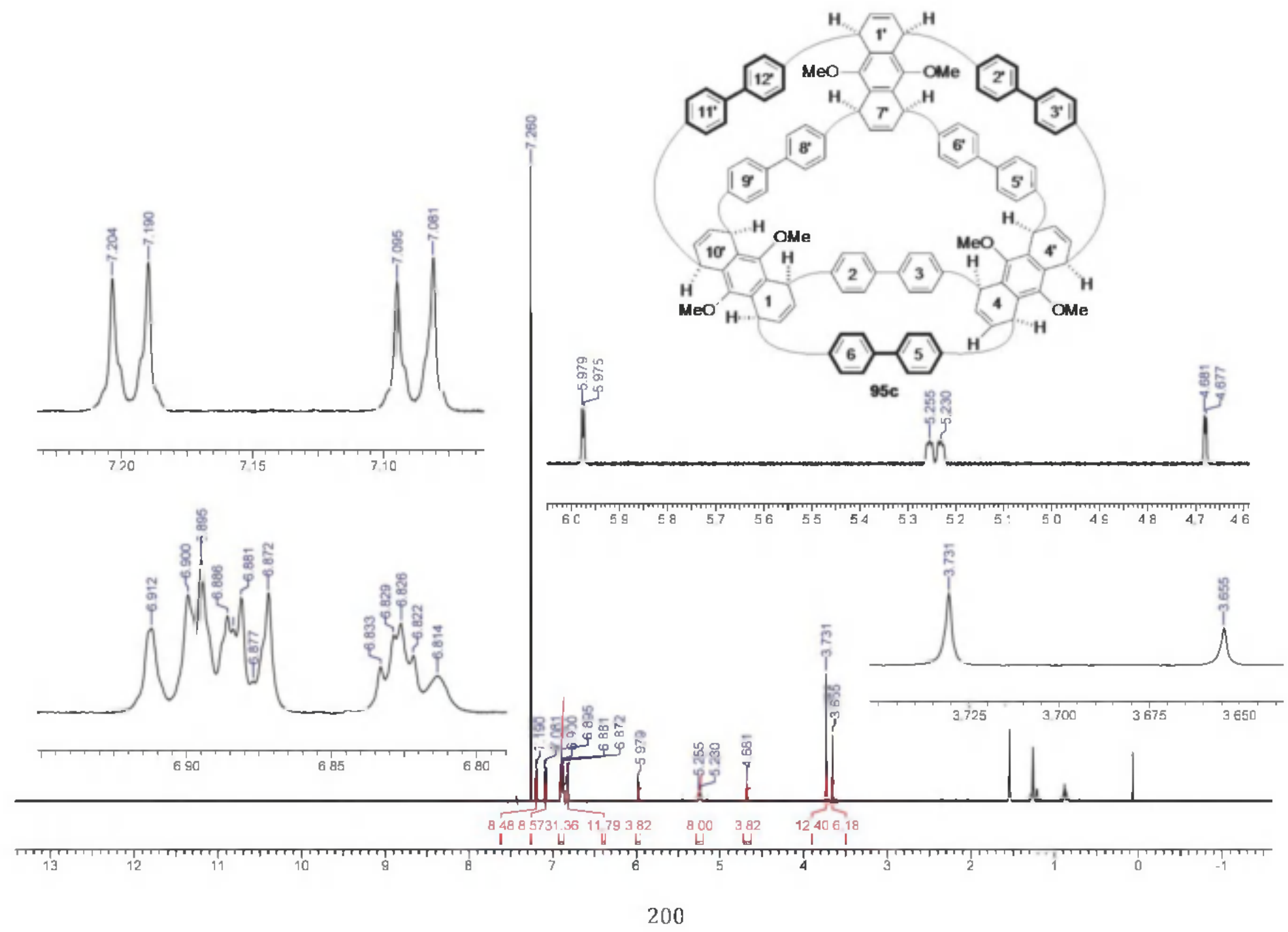




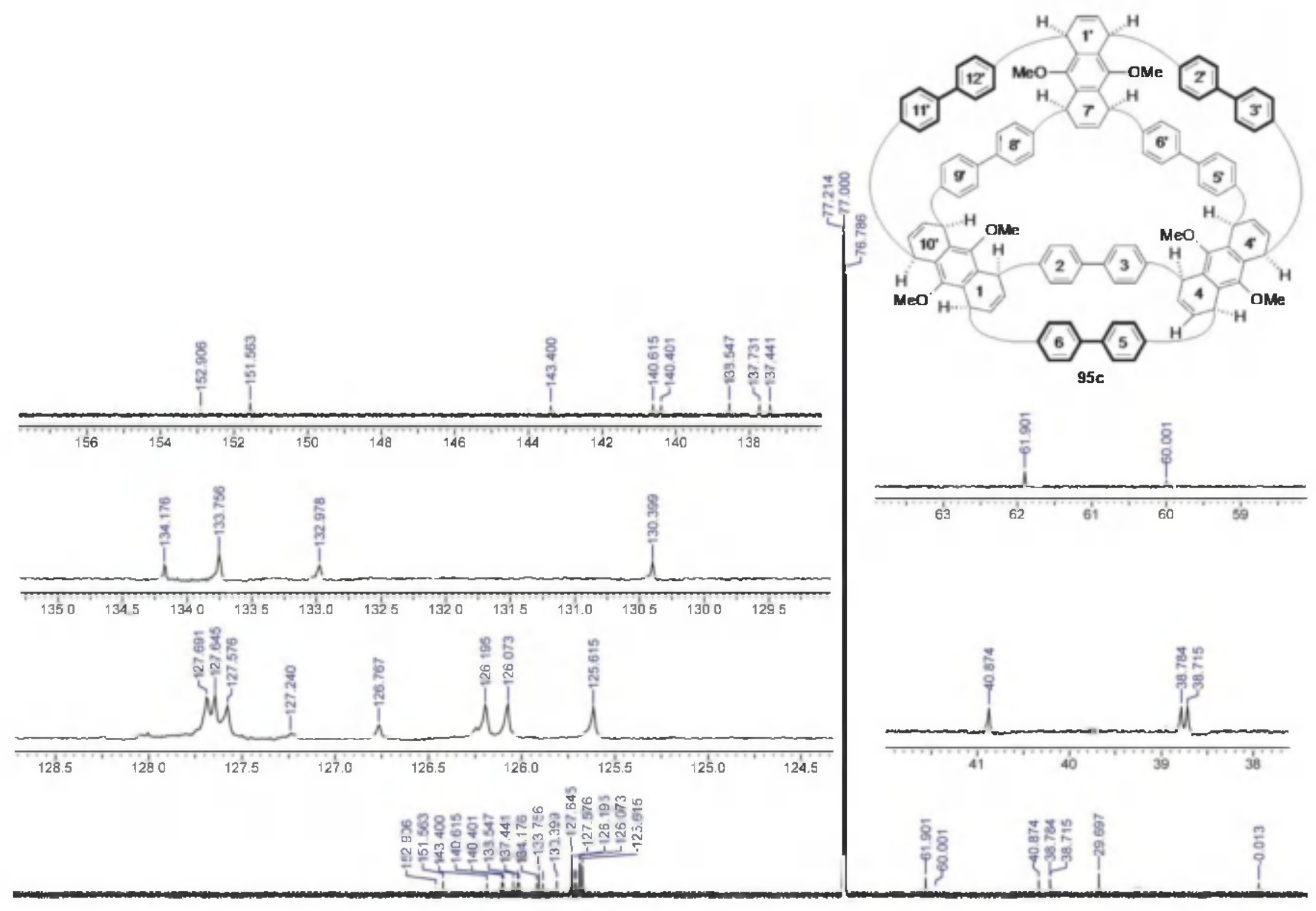

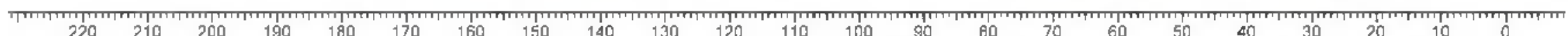




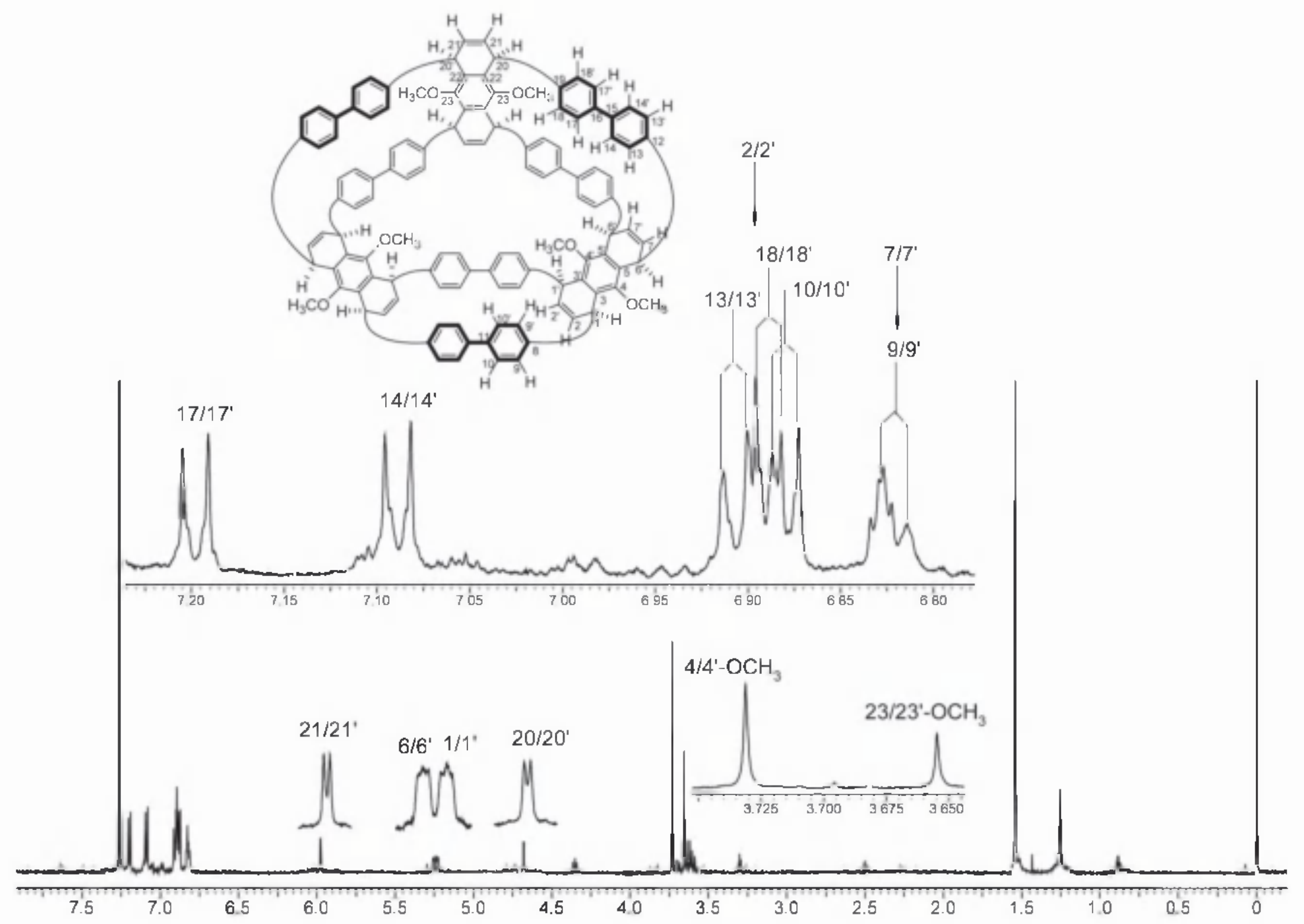




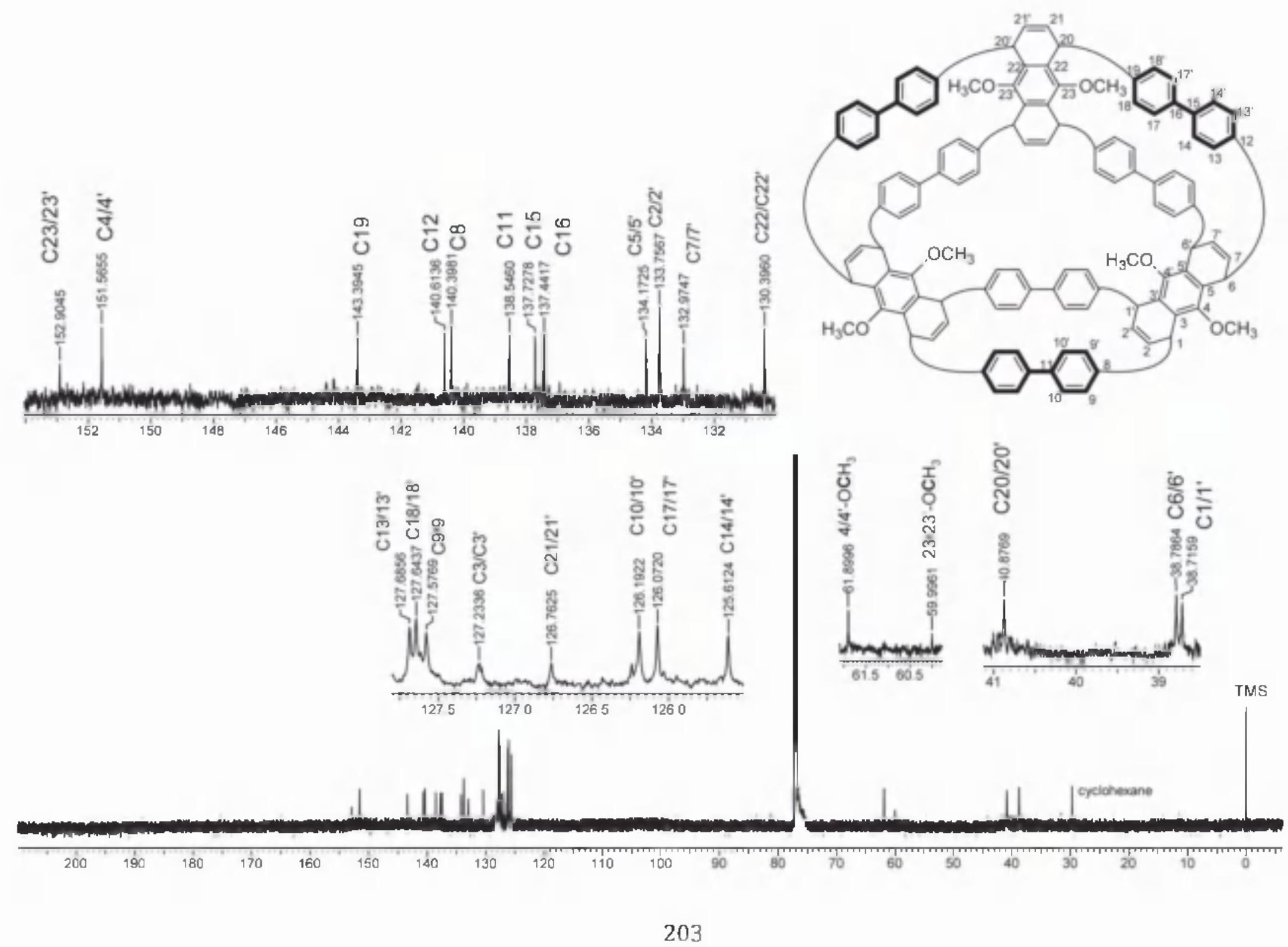




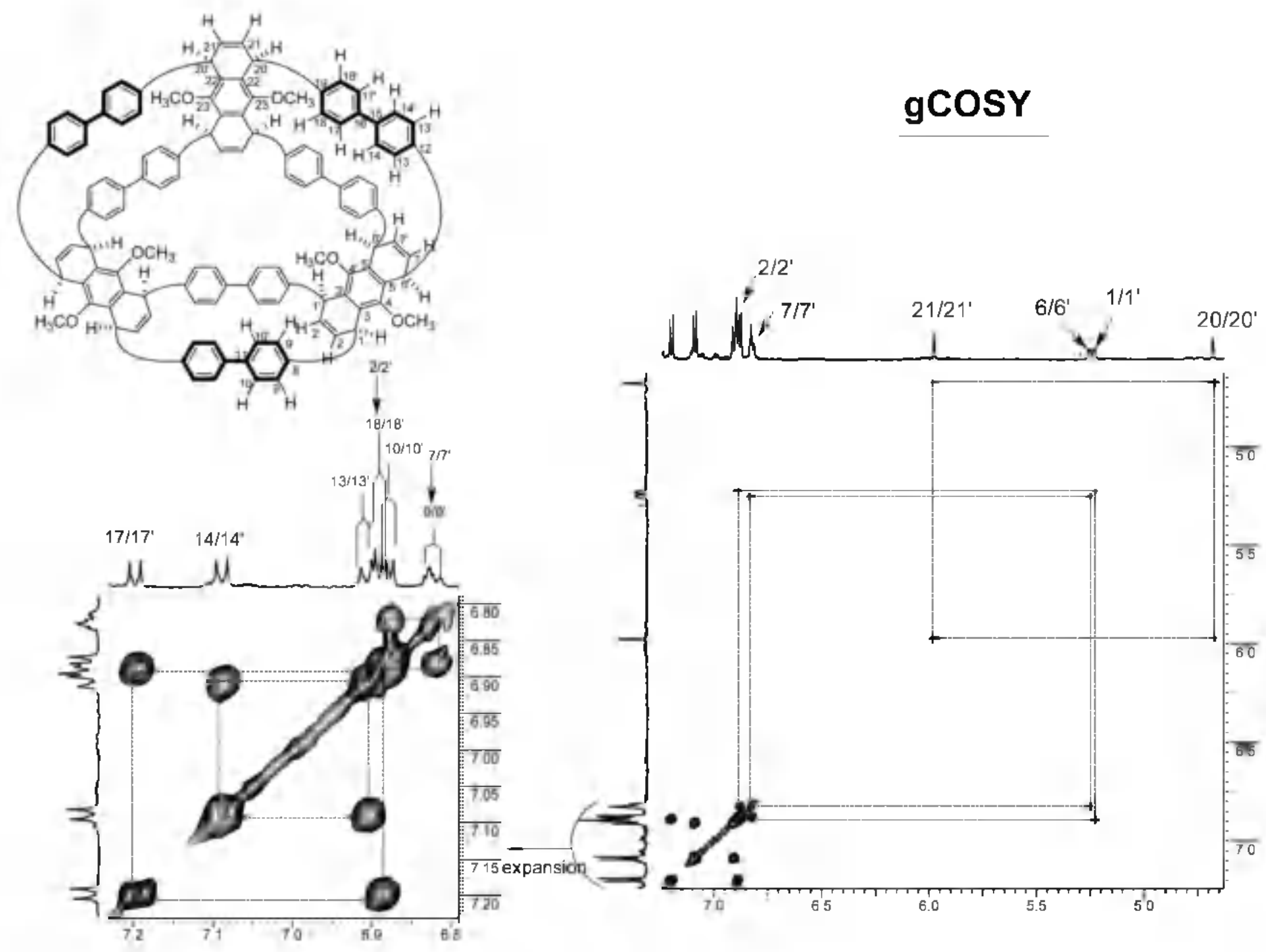


Expanded portions of the 2D NMR spectra

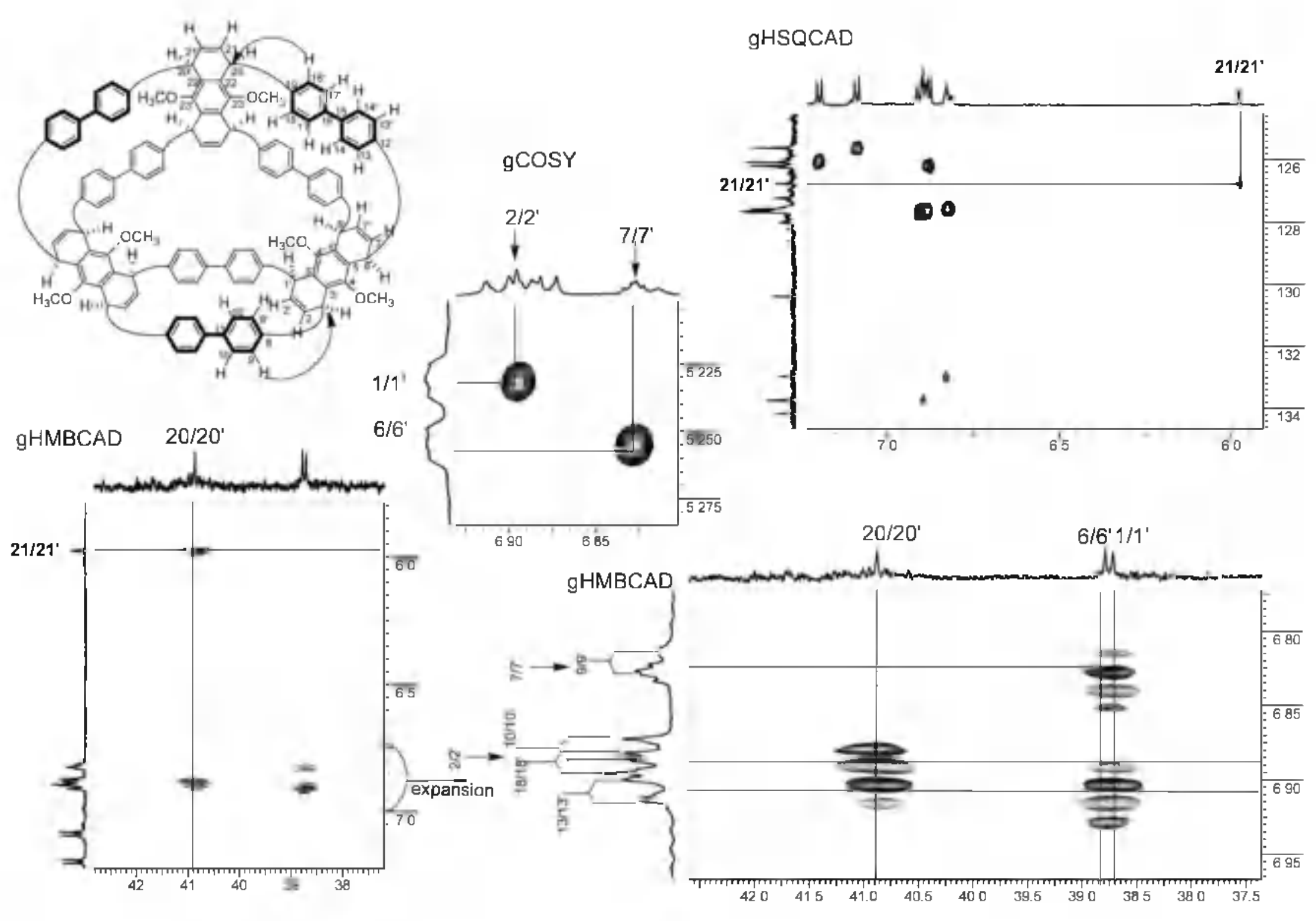




\section{Expanded portions of the ROESYAD spectrum}

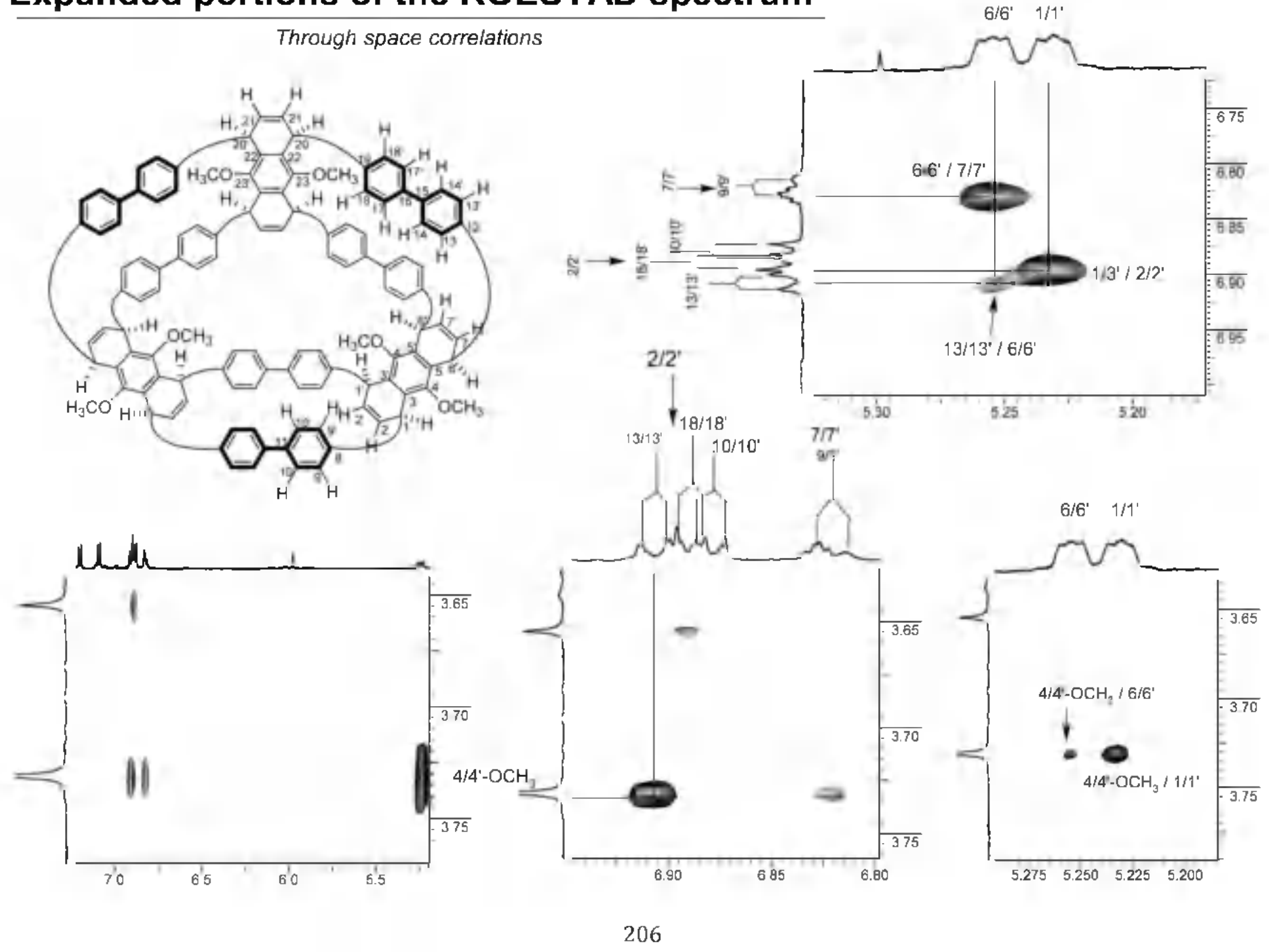




\section{ROESYAD}

Through space correlations

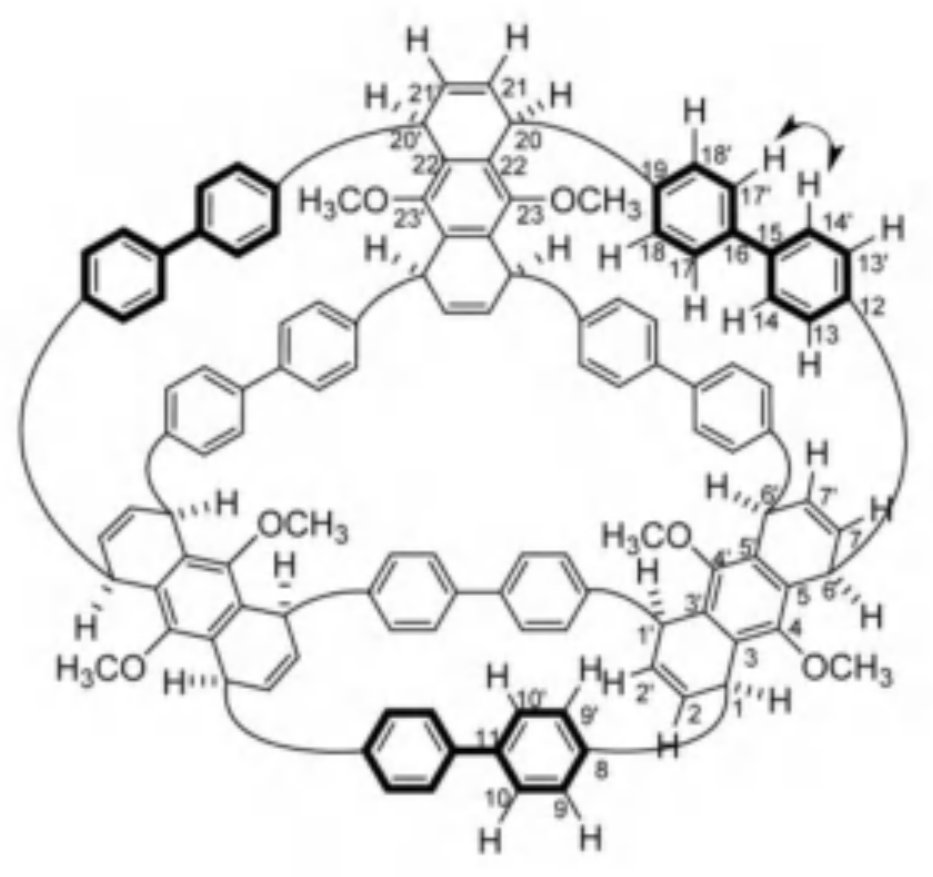

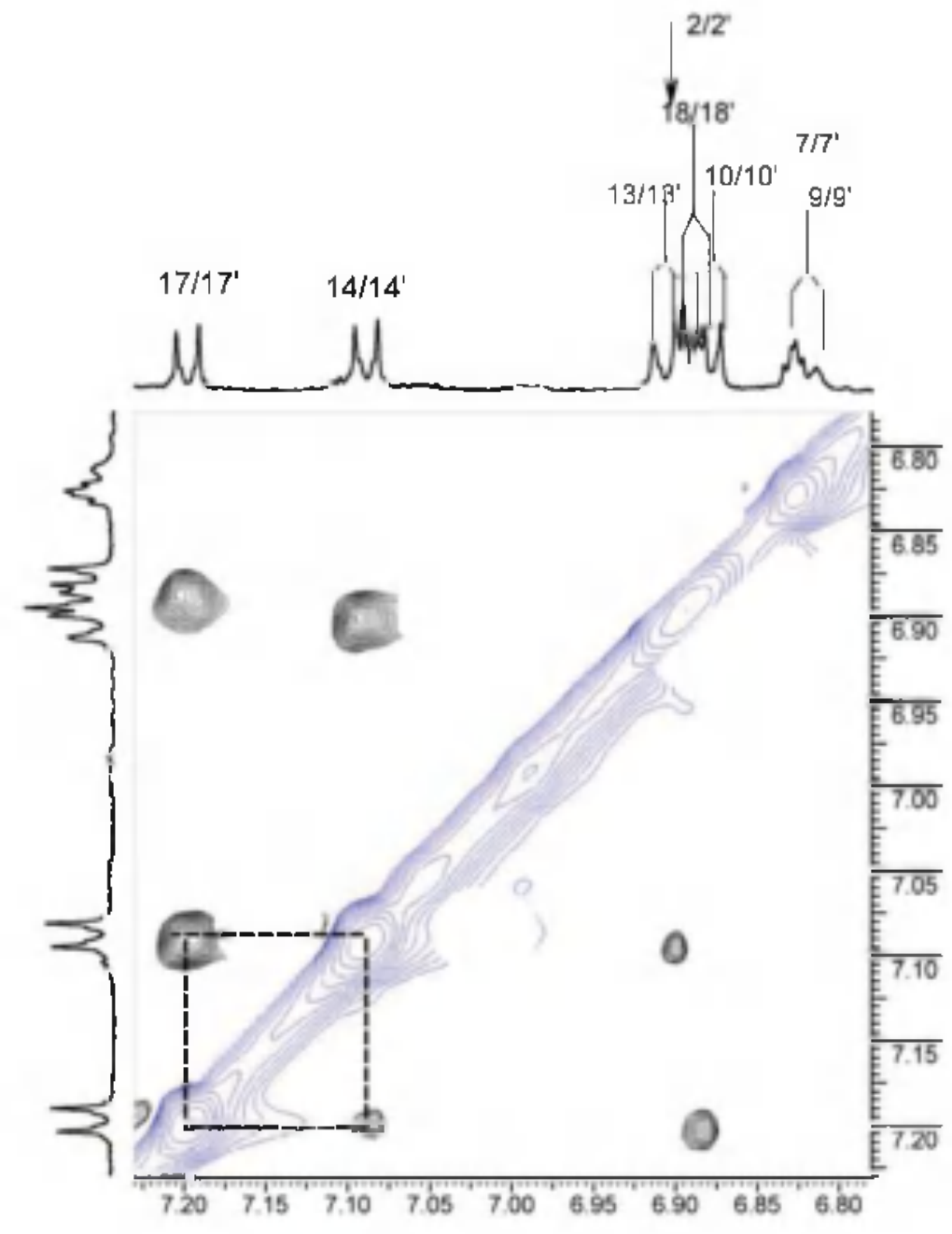

Aromatic region 
Expanded portions of the gHSQCAD $\left({ }^{1} \mathrm{~J}_{\mathrm{HC}}\right)$ spectrum

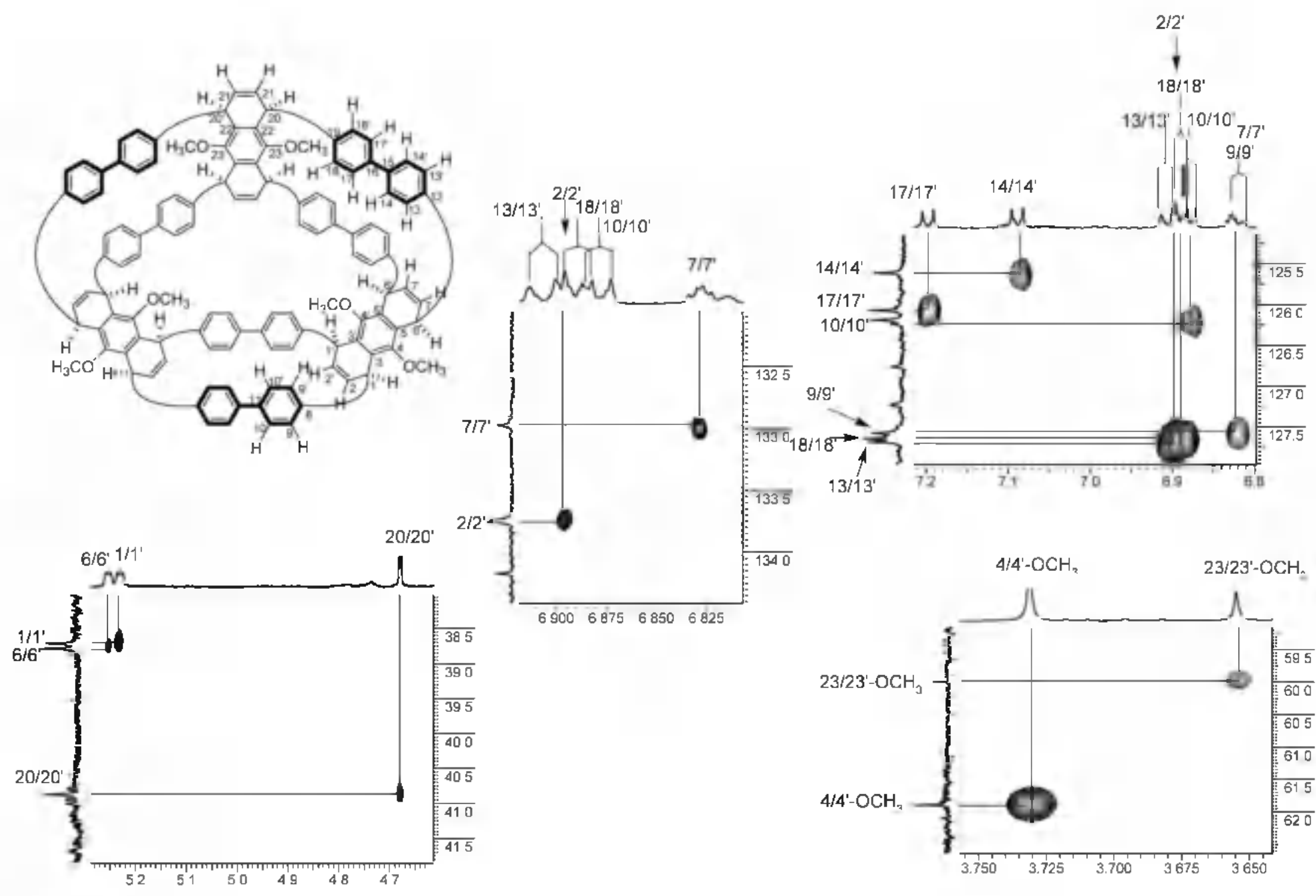


${ }^{2} J_{H C}$ and ${ }^{3} J_{H C}$ HMBC correaltions confirm interring linkage
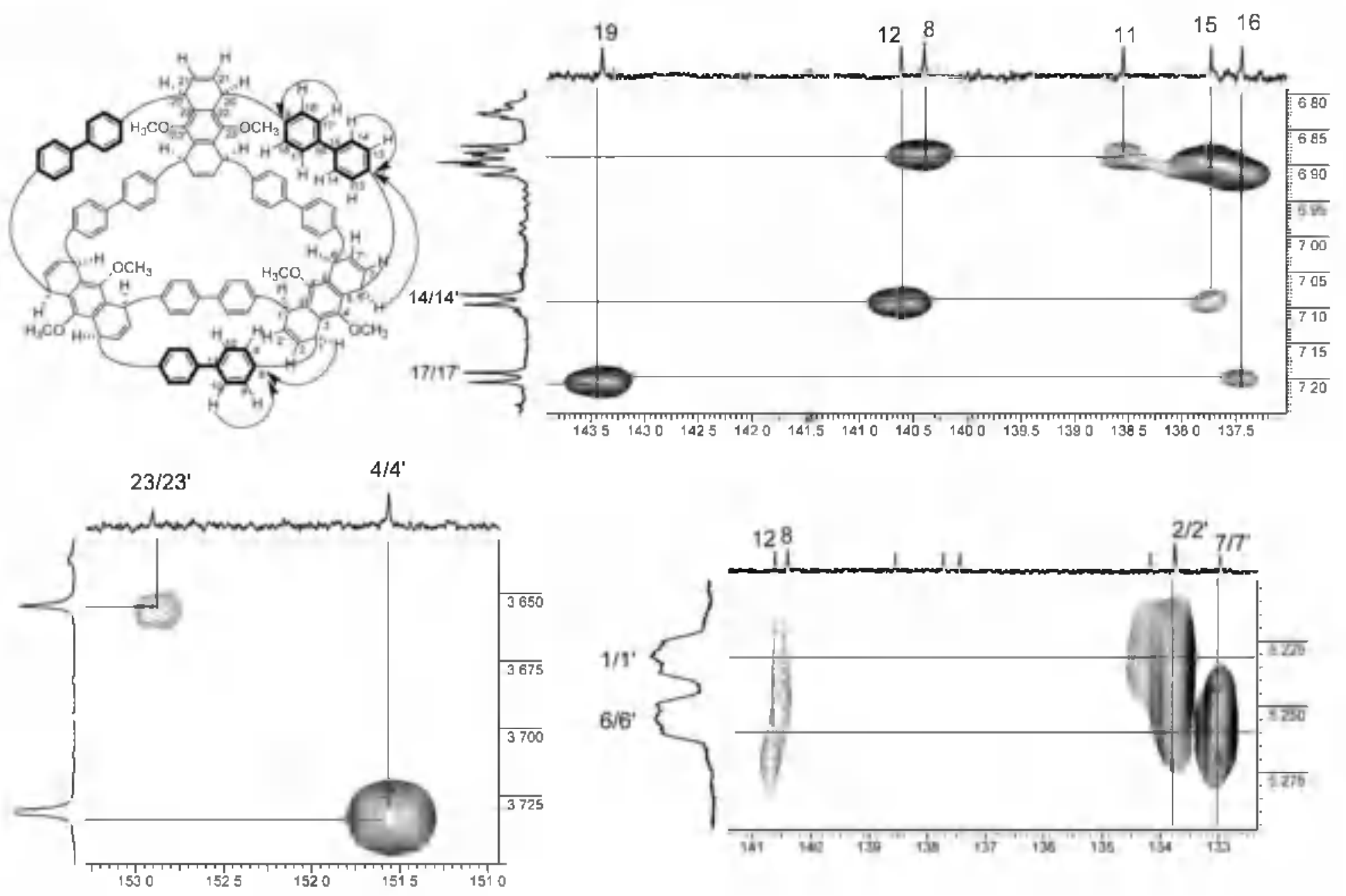

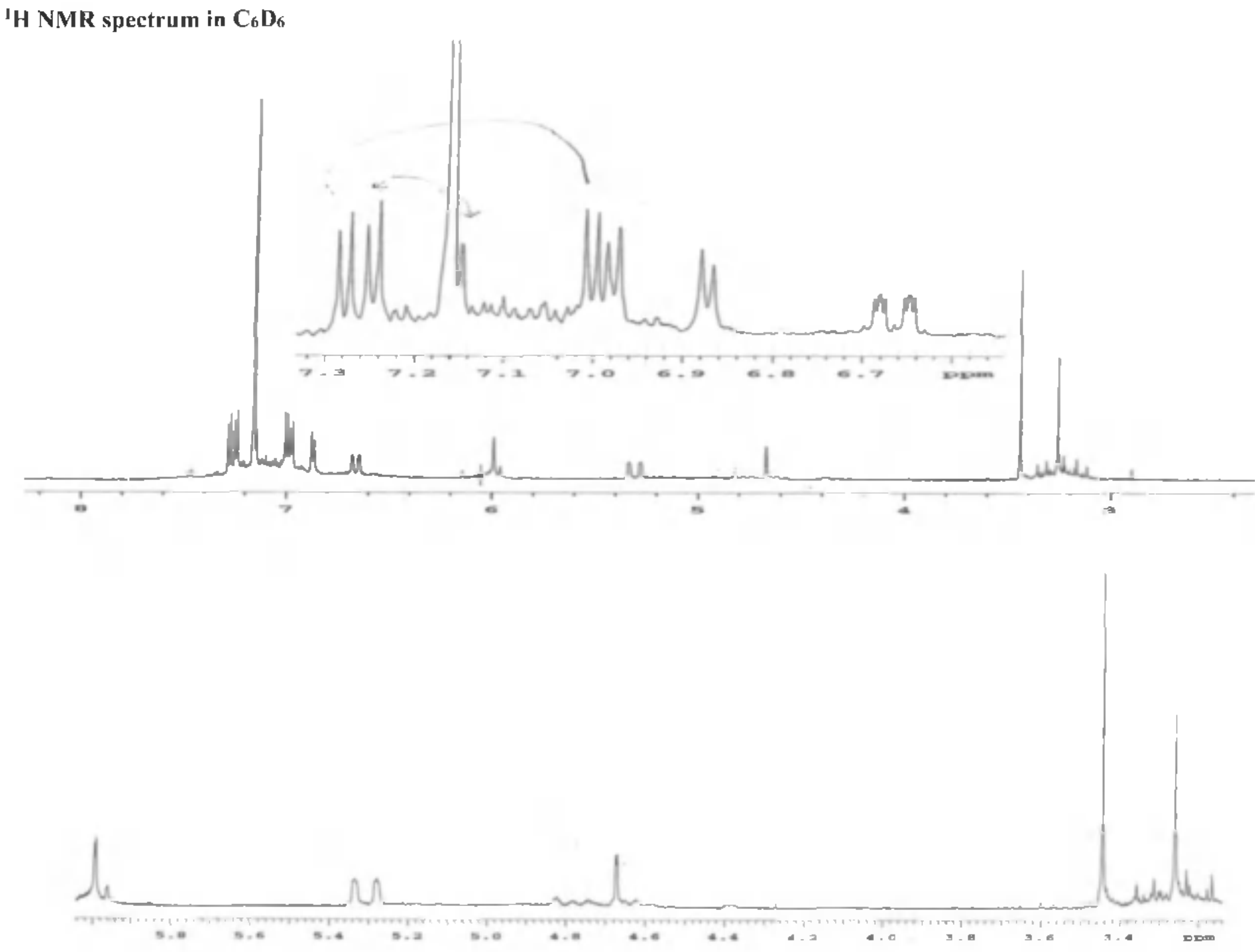
${ }^{1} \mathrm{H}$ NMR spectrum in $\mathrm{C}_{6} \mathrm{D}_{\mathrm{t}}$, Double Resonance at $7.16 \mathrm{ppm}$, at $+25^{\circ} \mathrm{C}$

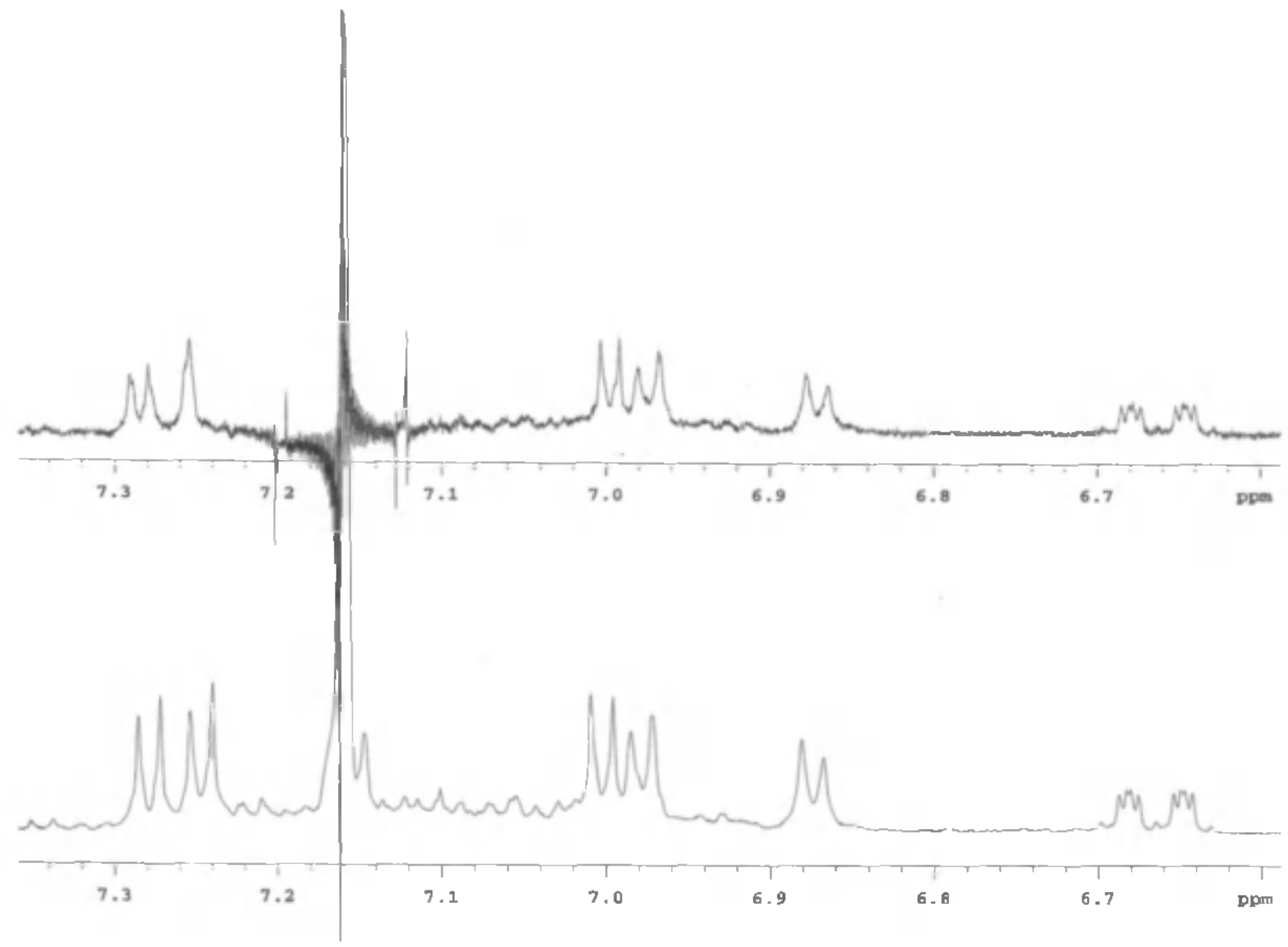

211 
${ }^{1} \mathrm{H}$ NMR spectrum in $\mathrm{C}_{6} \mathrm{D}_{6}$, Double Resonance at $4.68 \mathrm{ppm}$, at $+25{ }^{\circ} \mathrm{C}$.

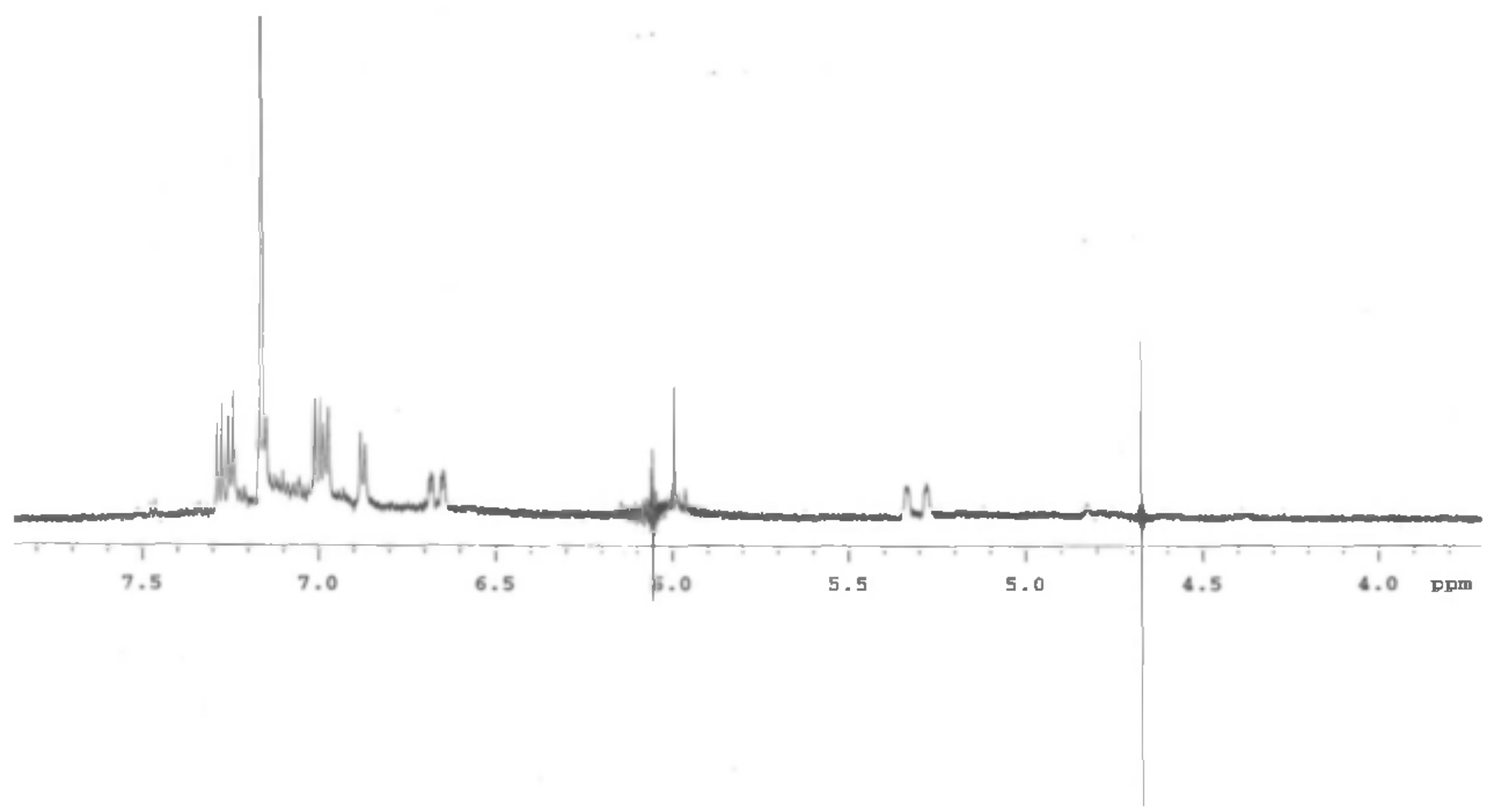


${ }^{1} \mathrm{H}$ NMR spectrum in $\mathrm{C}_{6} \mathrm{D}_{6}$, Double Resanance at $5.28 \mathrm{ppm}$, at $+25{ }^{\circ} \mathrm{C}$

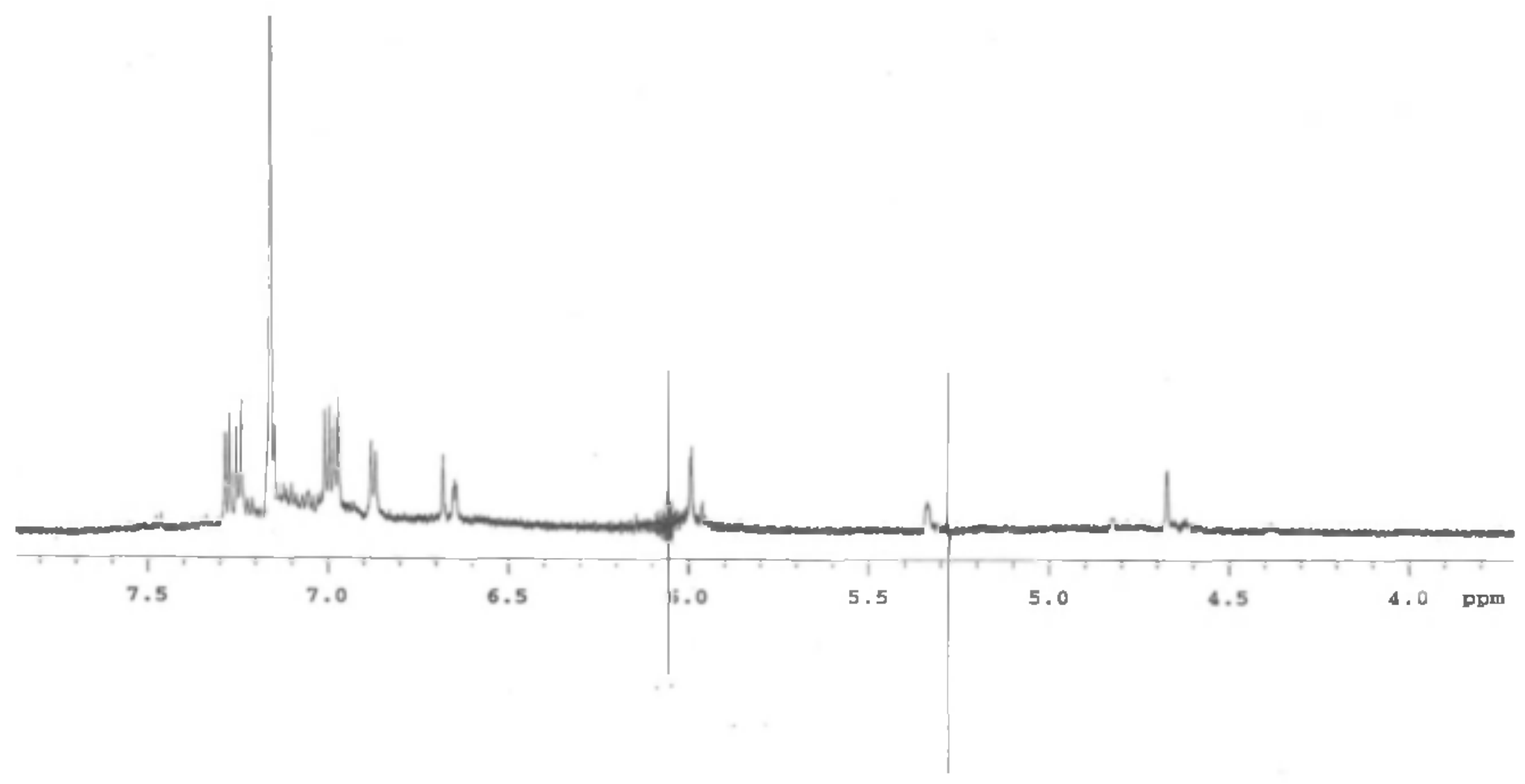


${ }^{1} \mathrm{H}$ NMR spectrum in $\mathrm{C}_{6} \mathrm{D}_{6}$, Double Resonance at $5.34 \mathrm{ppm}$, at $+25{ }^{\circ} \mathrm{C}$

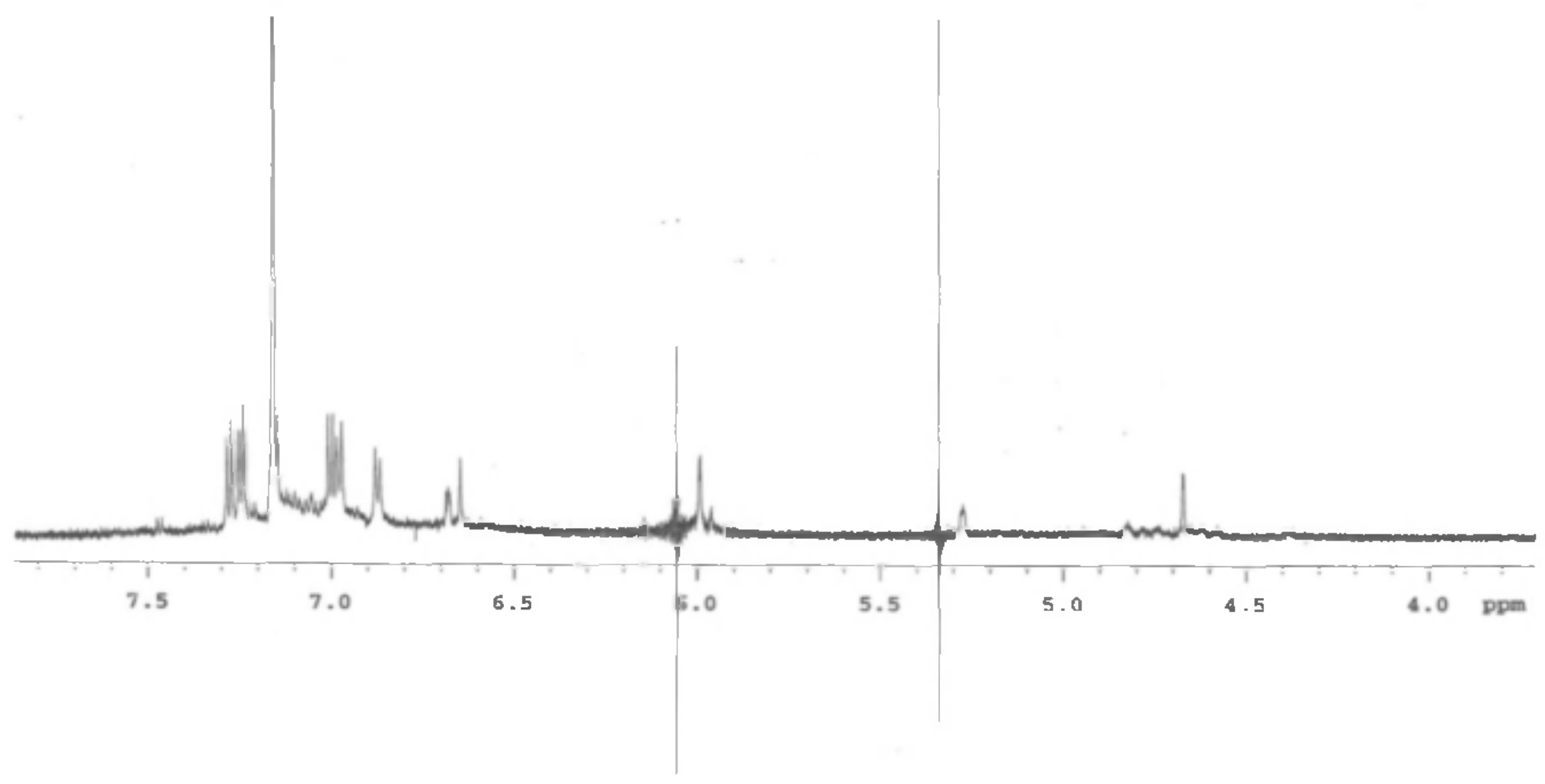




$$
t
$$


216 


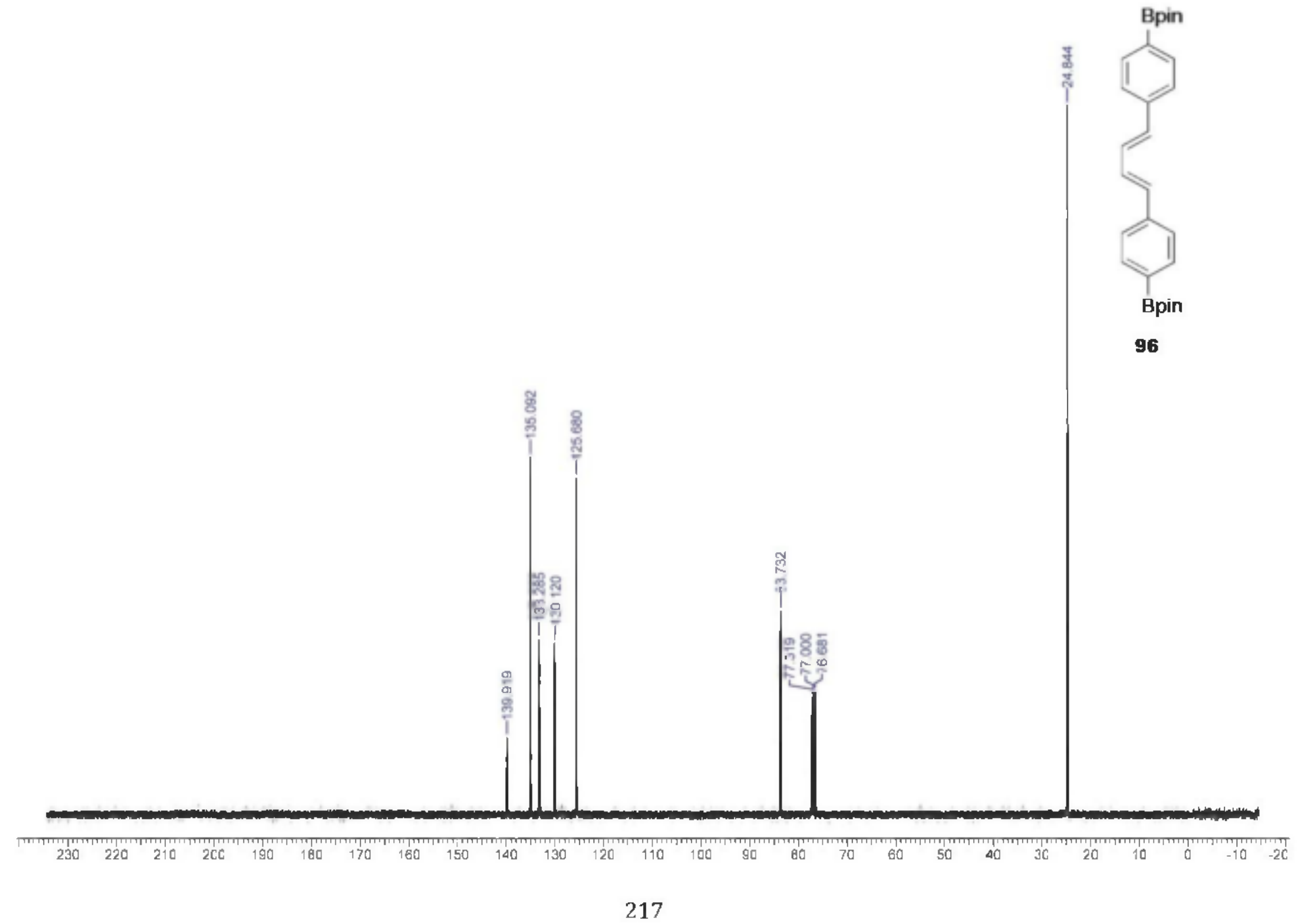




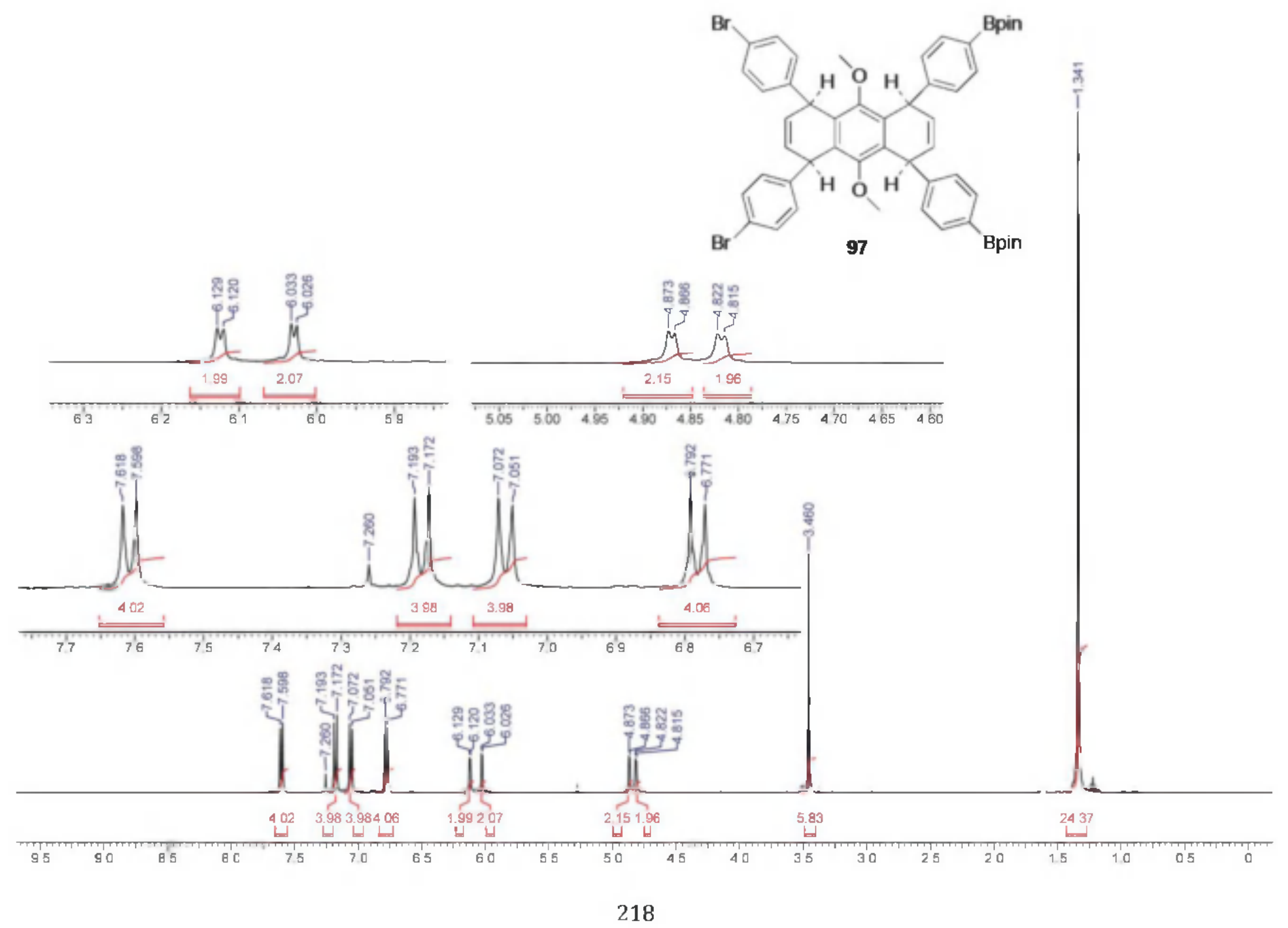




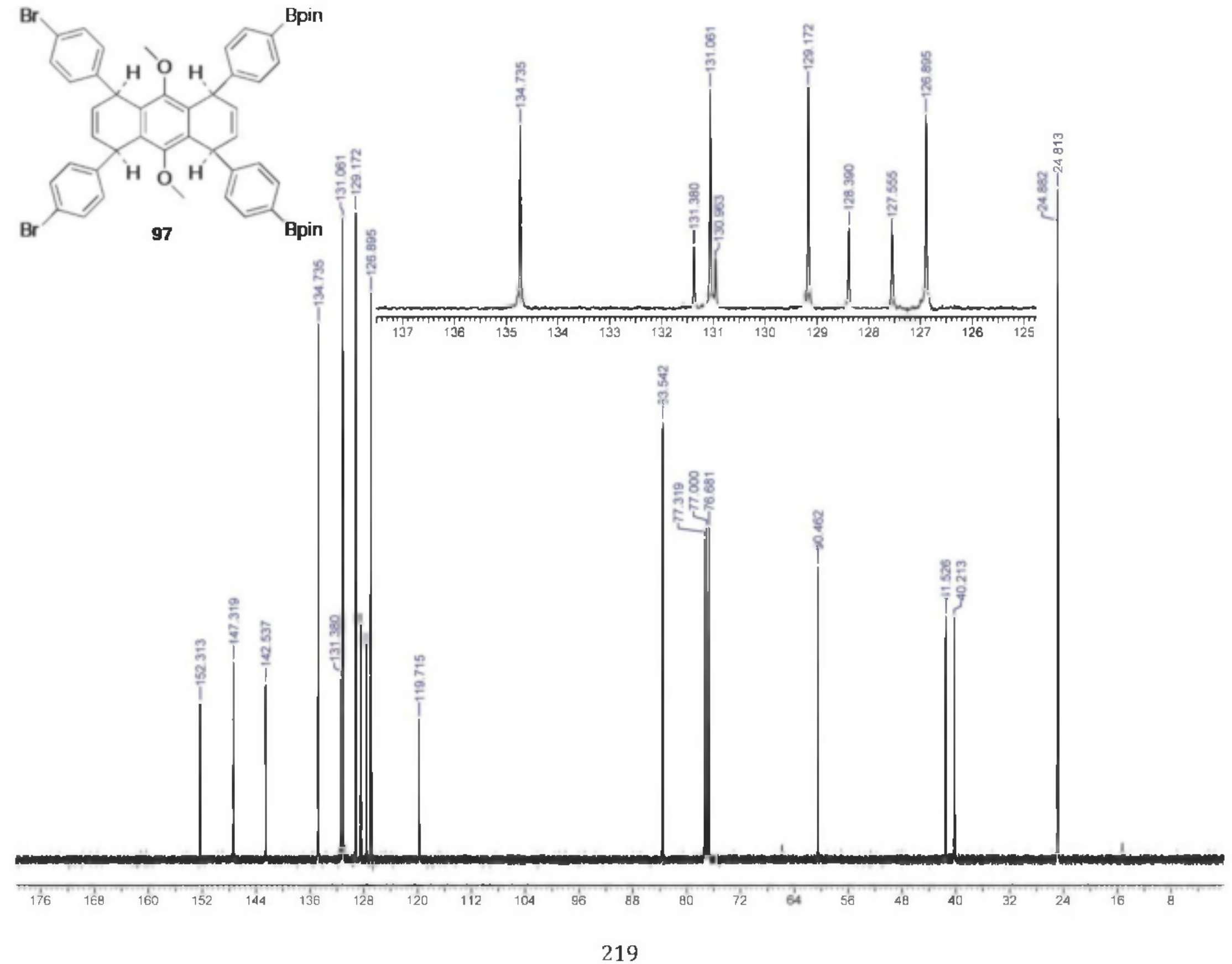




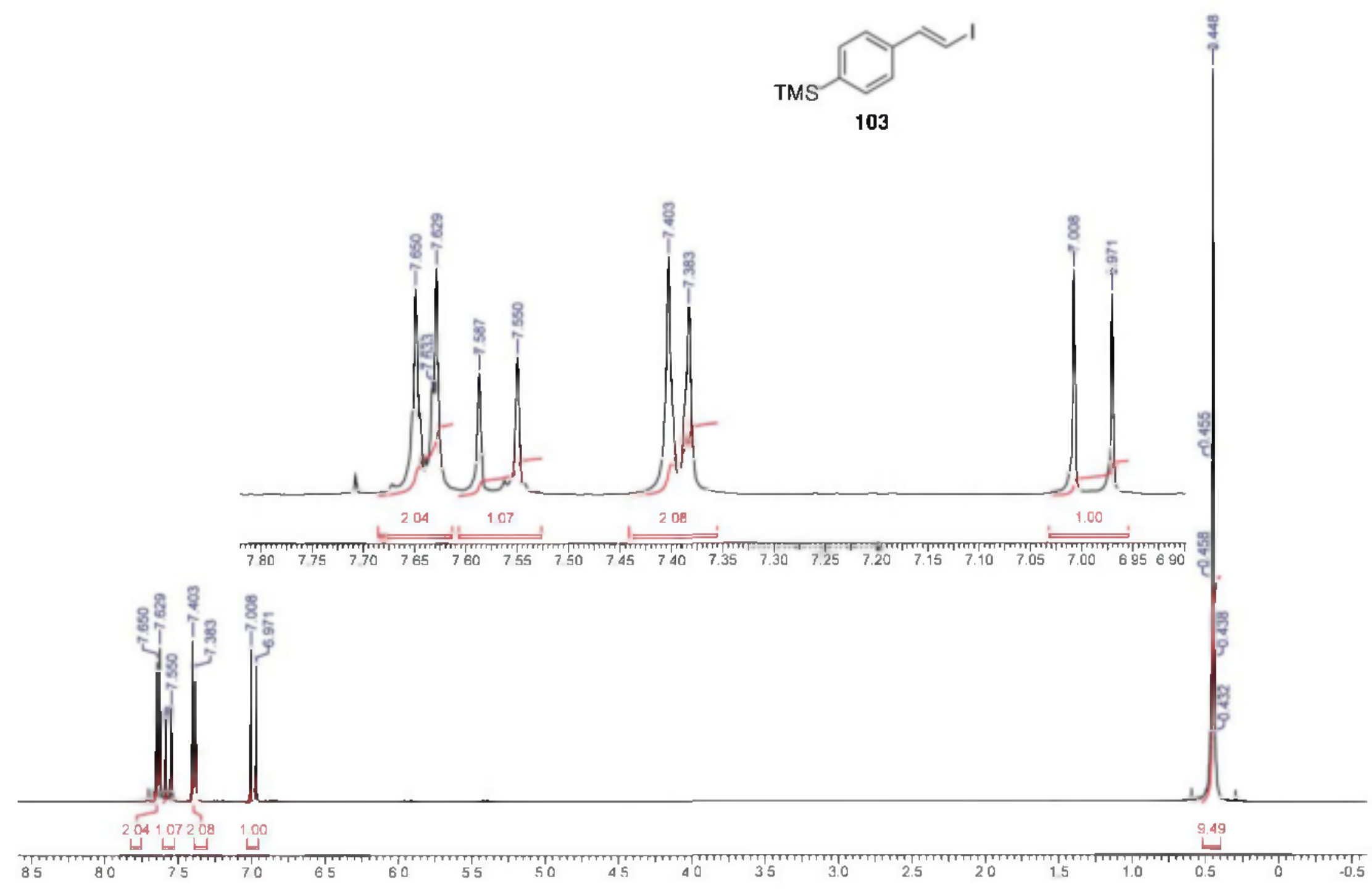




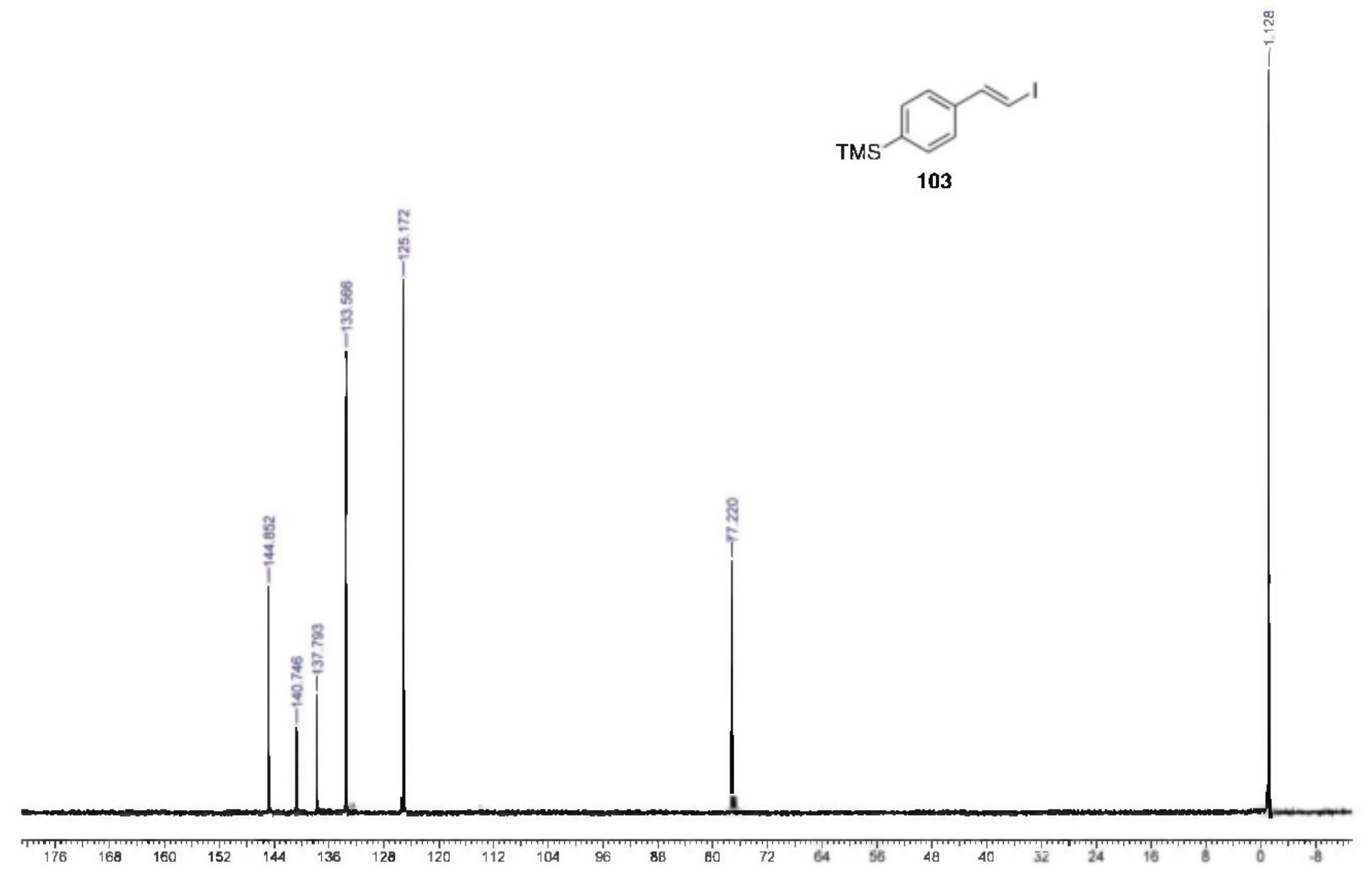




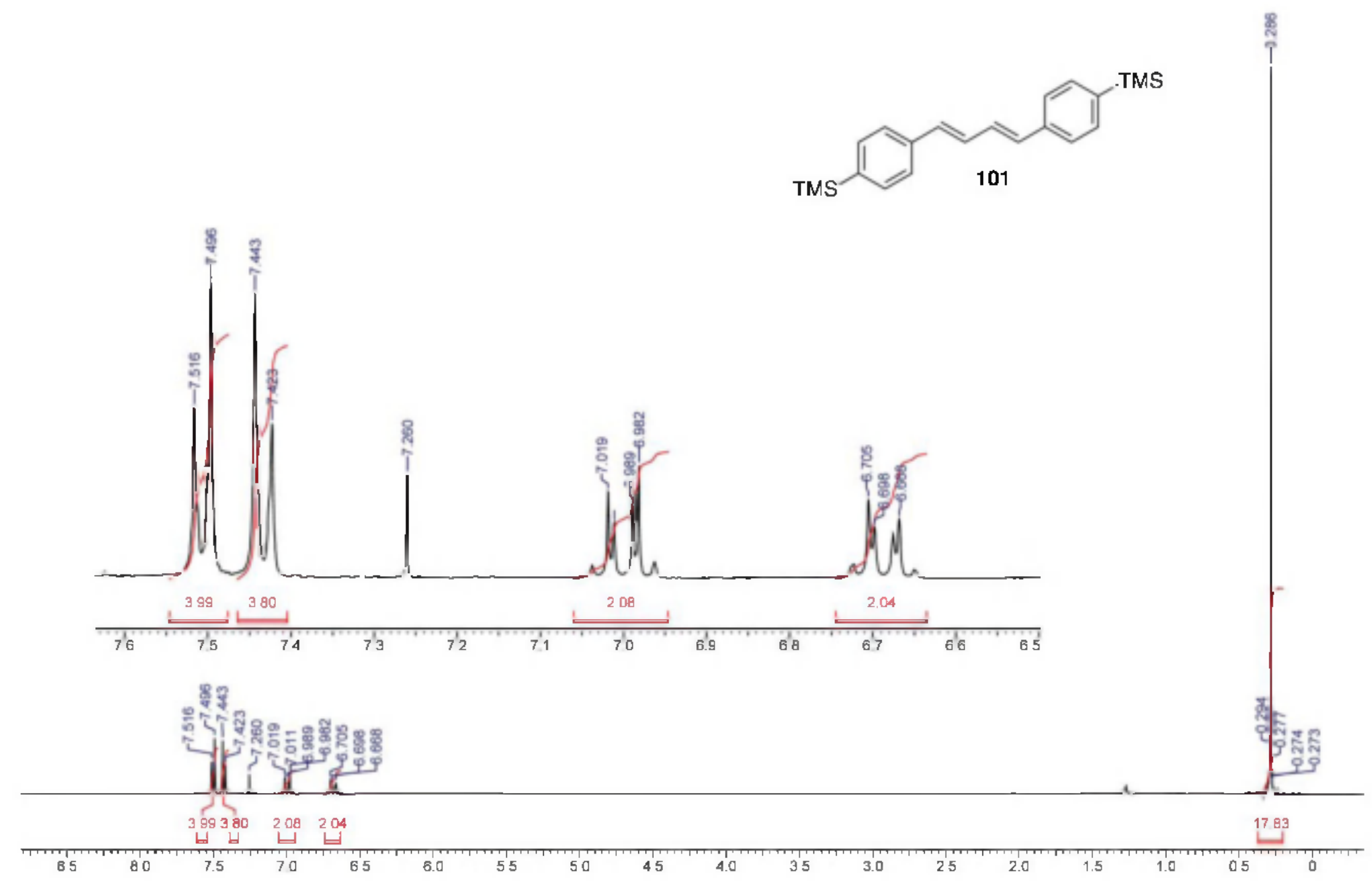




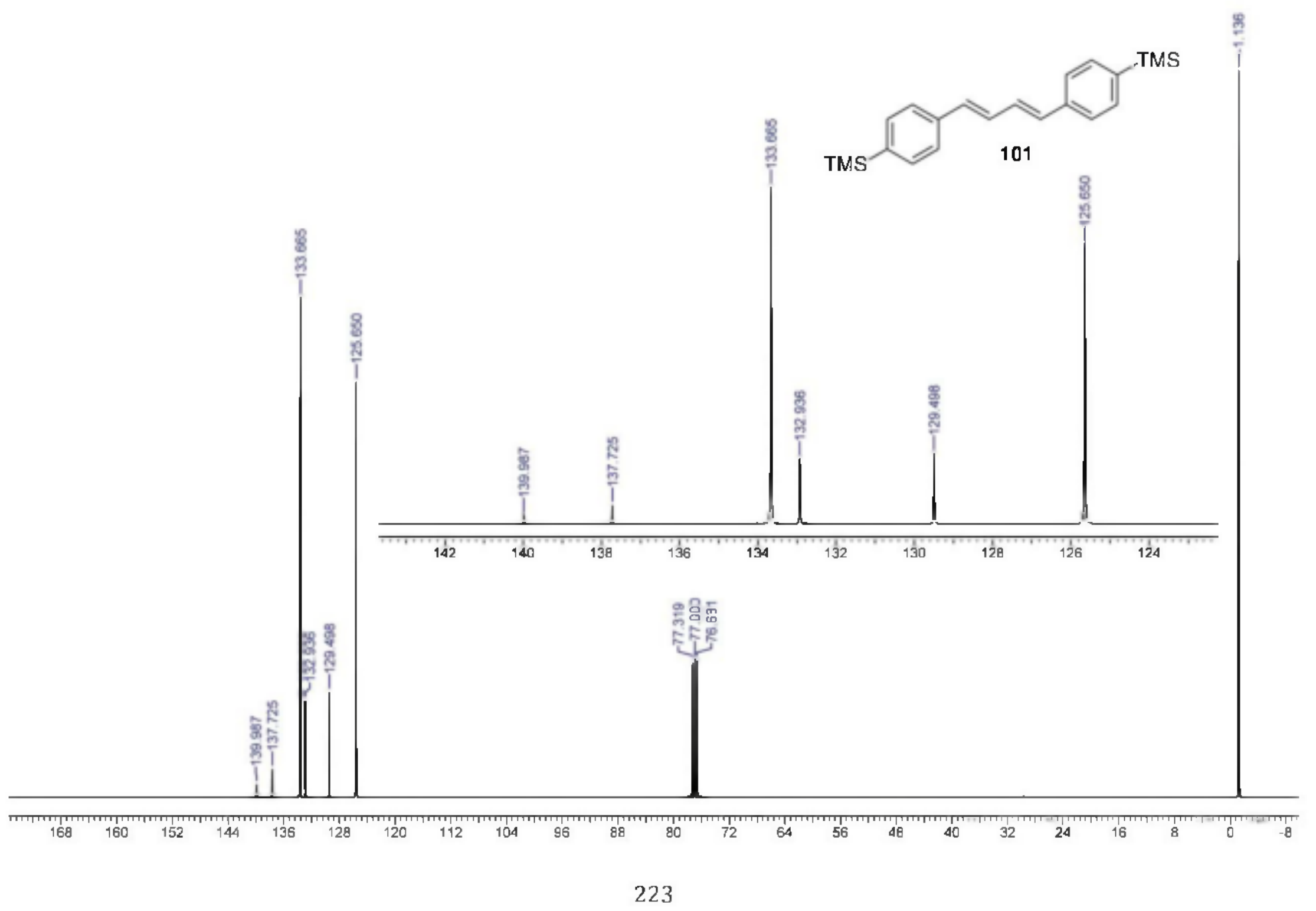



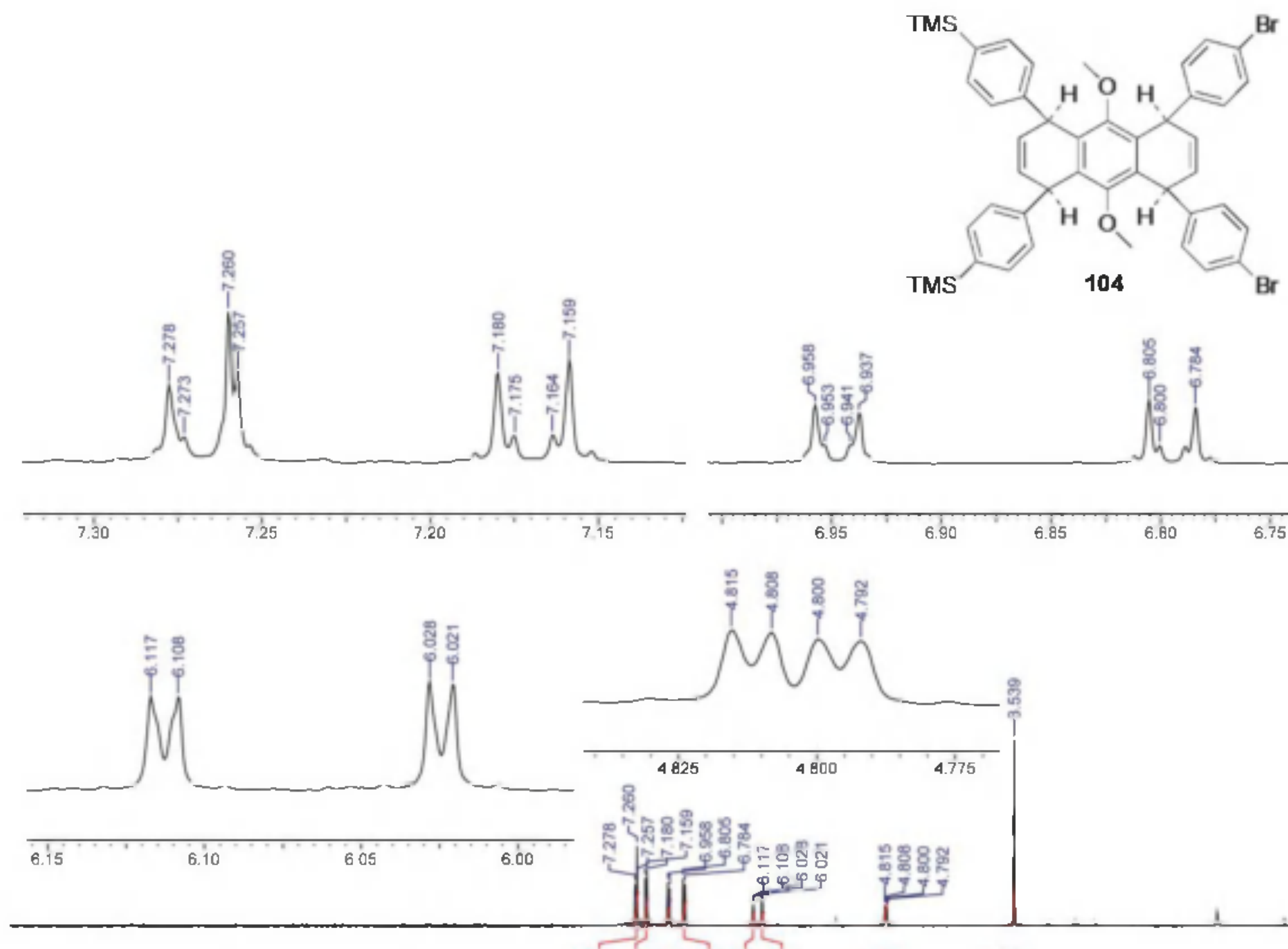

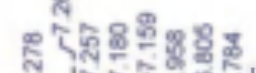

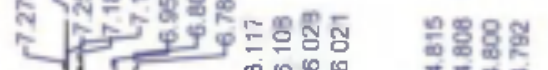
10 III 11 . 5424.354264 .01193213 $7 y$

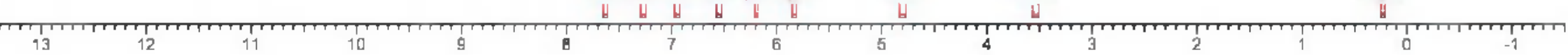

$13 \quad 12$ 


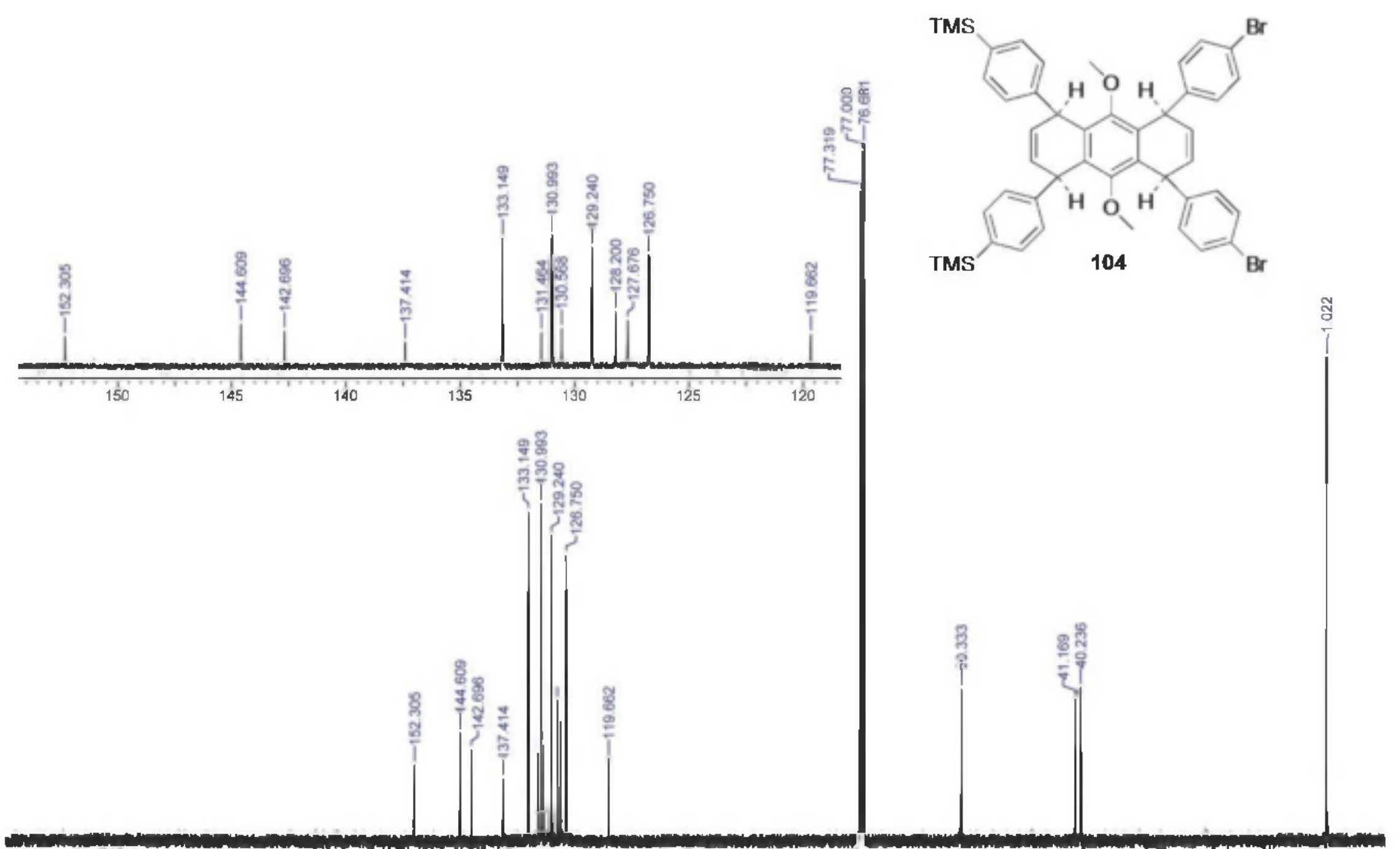

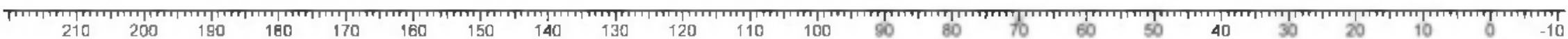




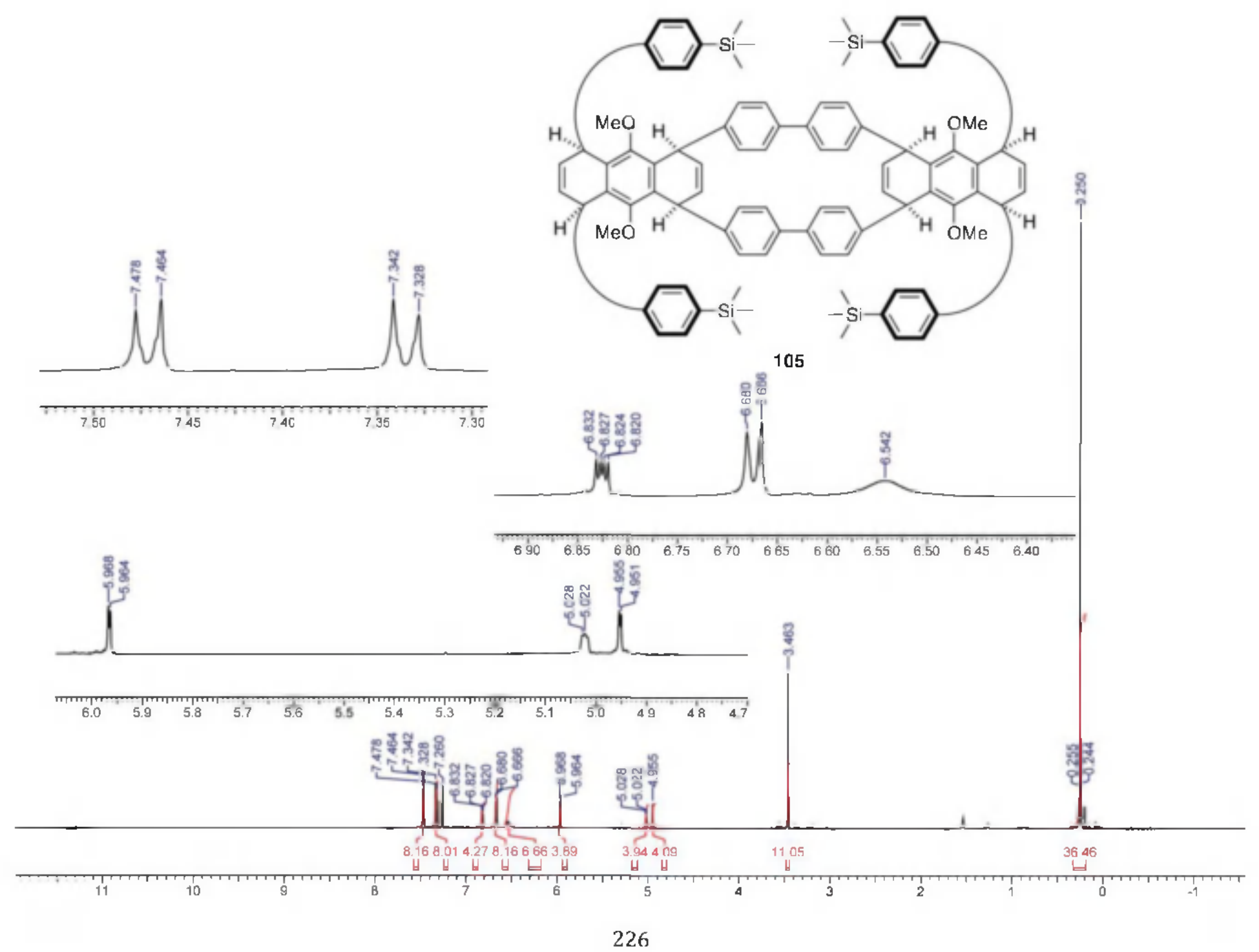




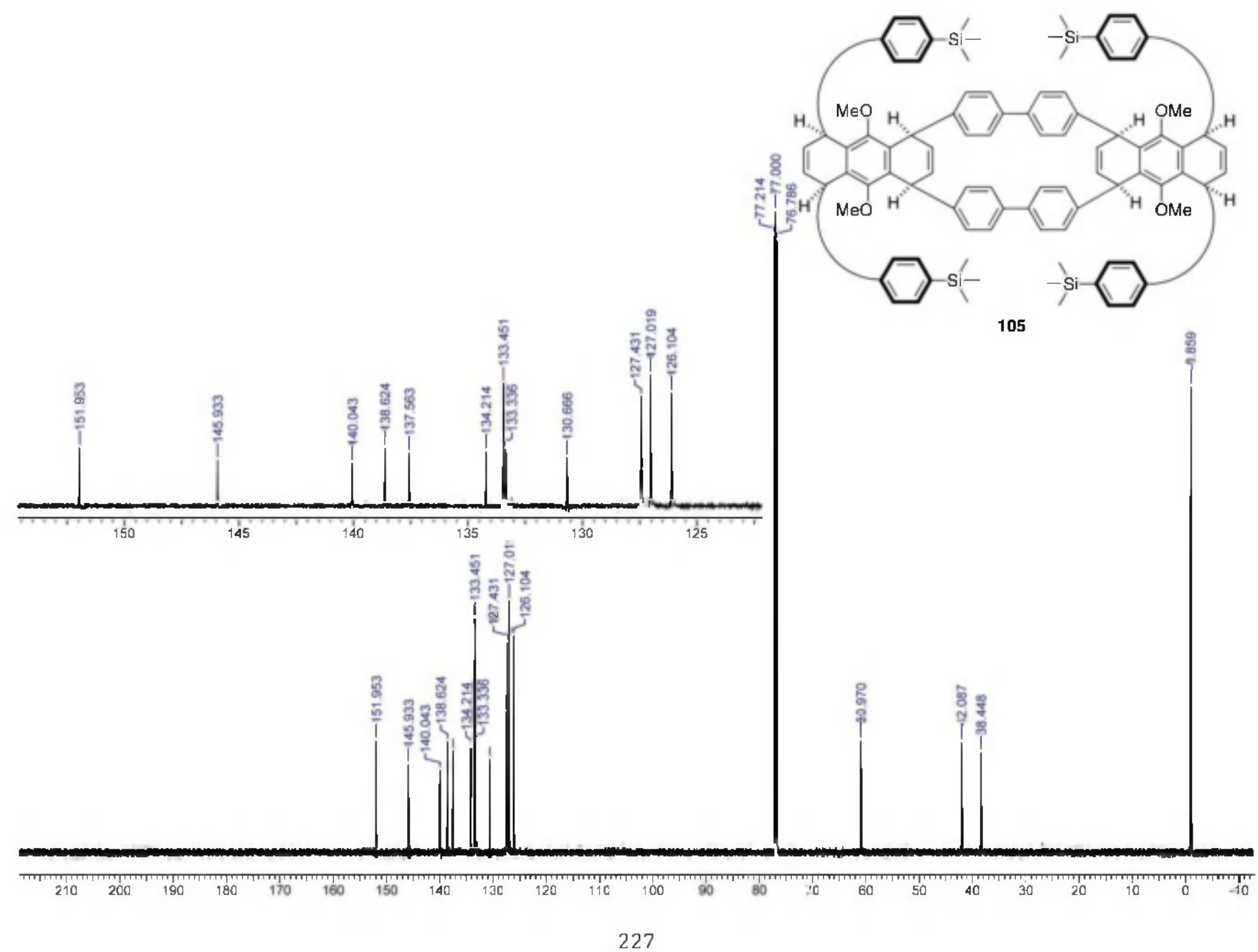




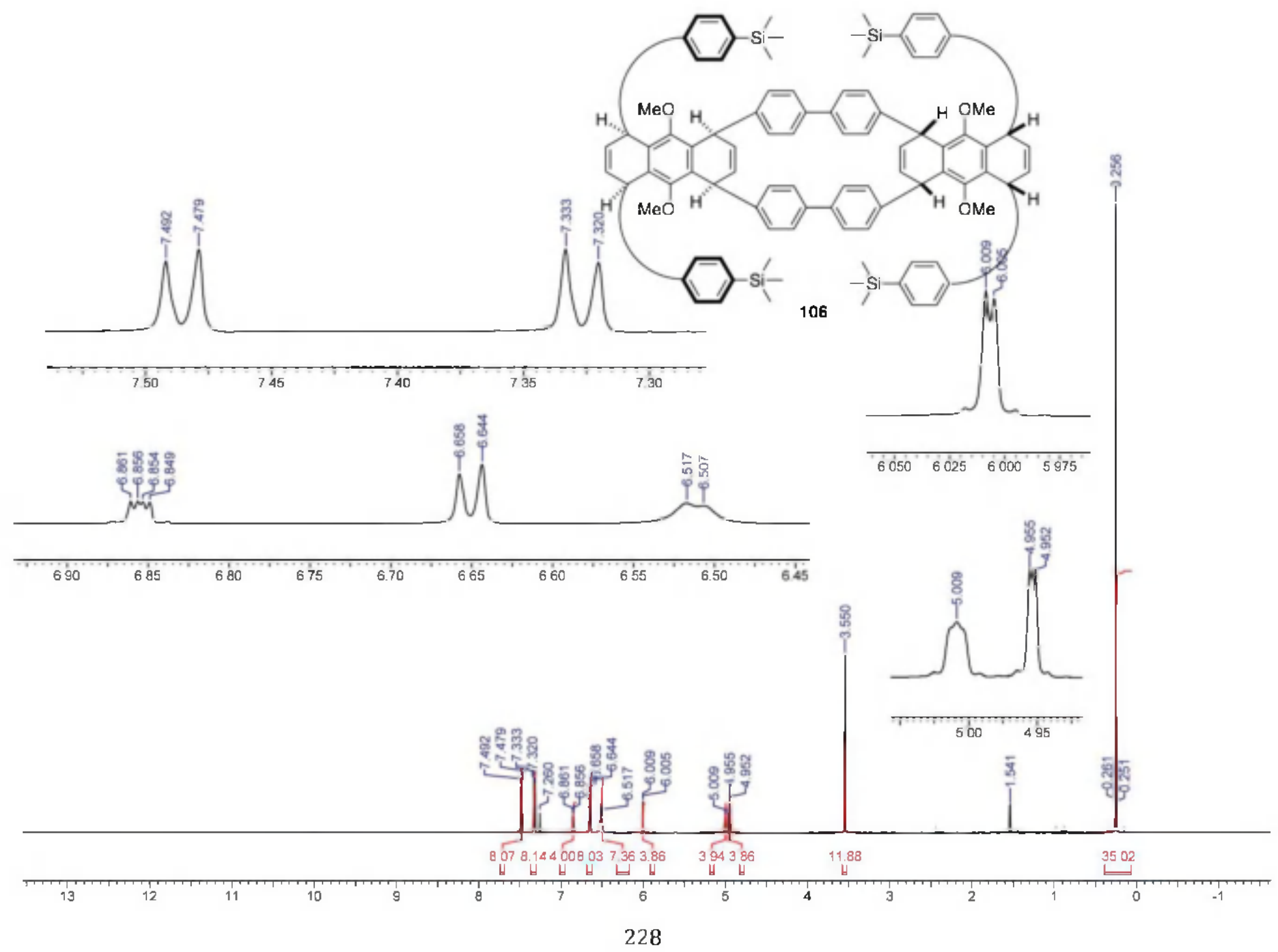




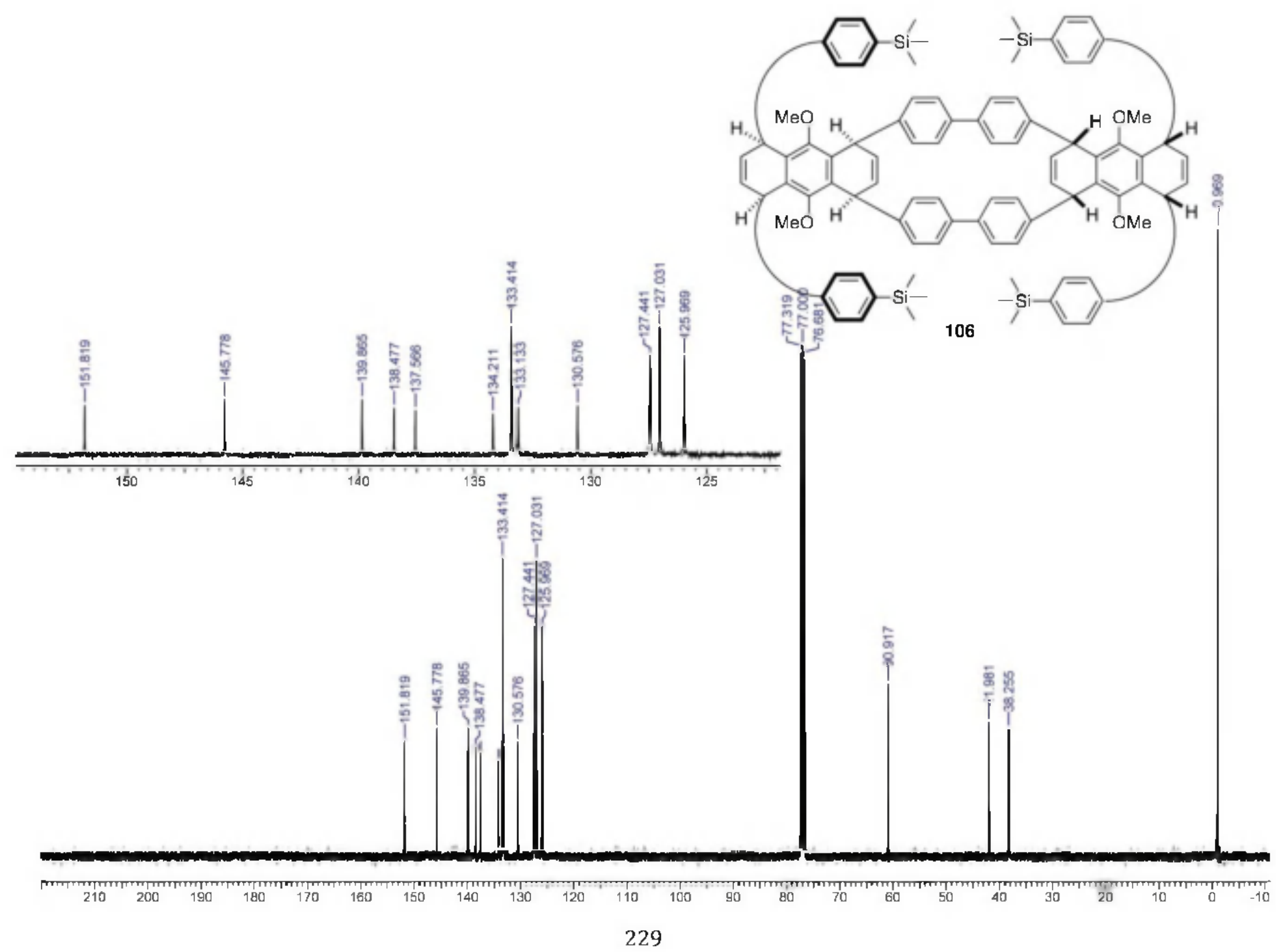


The Crude 'H NMR Spectrum of Iodination of 106 with $\mathrm{ICI}$

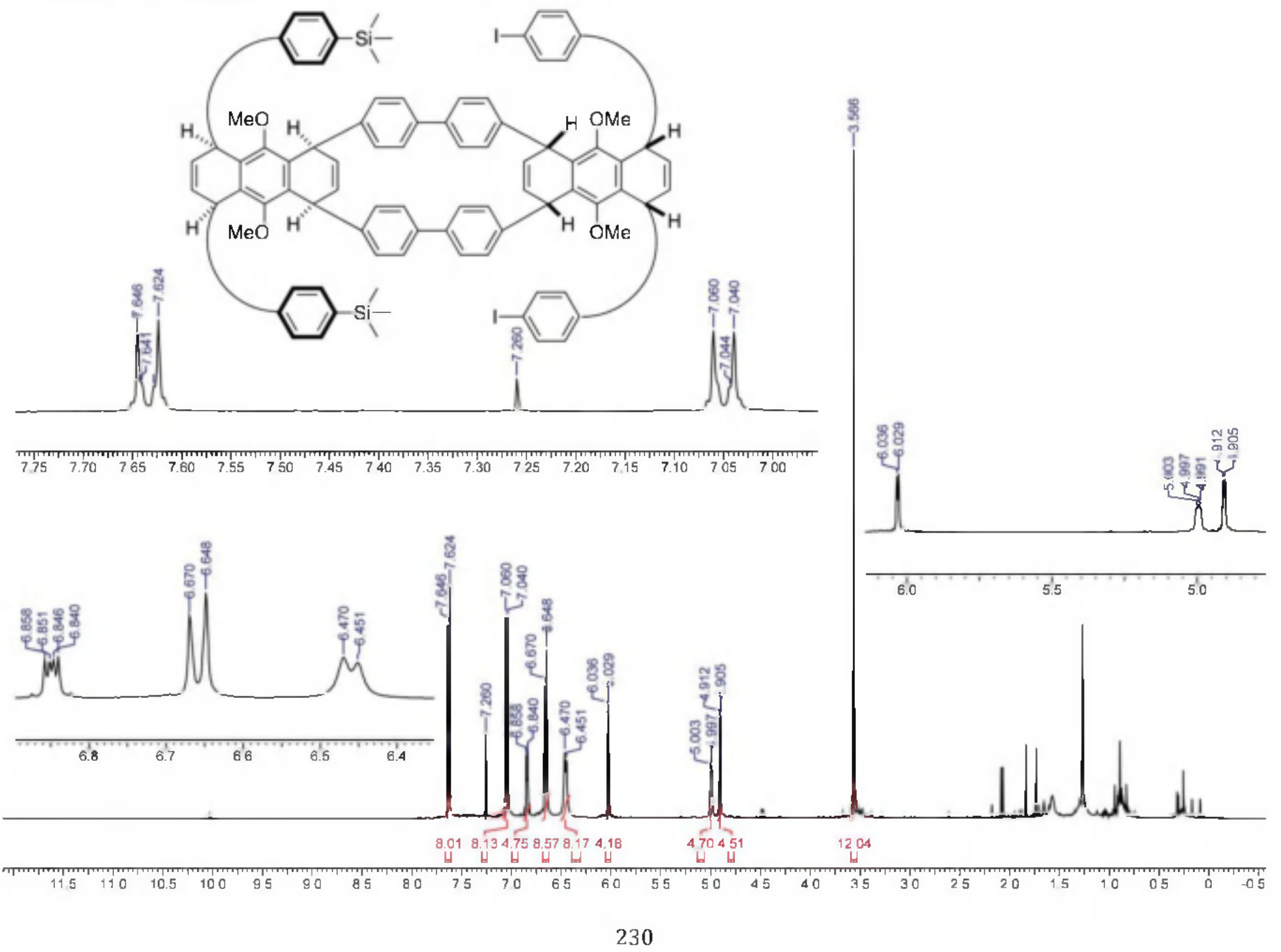


The Crude ${ }^{13} \mathrm{C}$ NMR Spectrum of Iodination of 106 with ICI

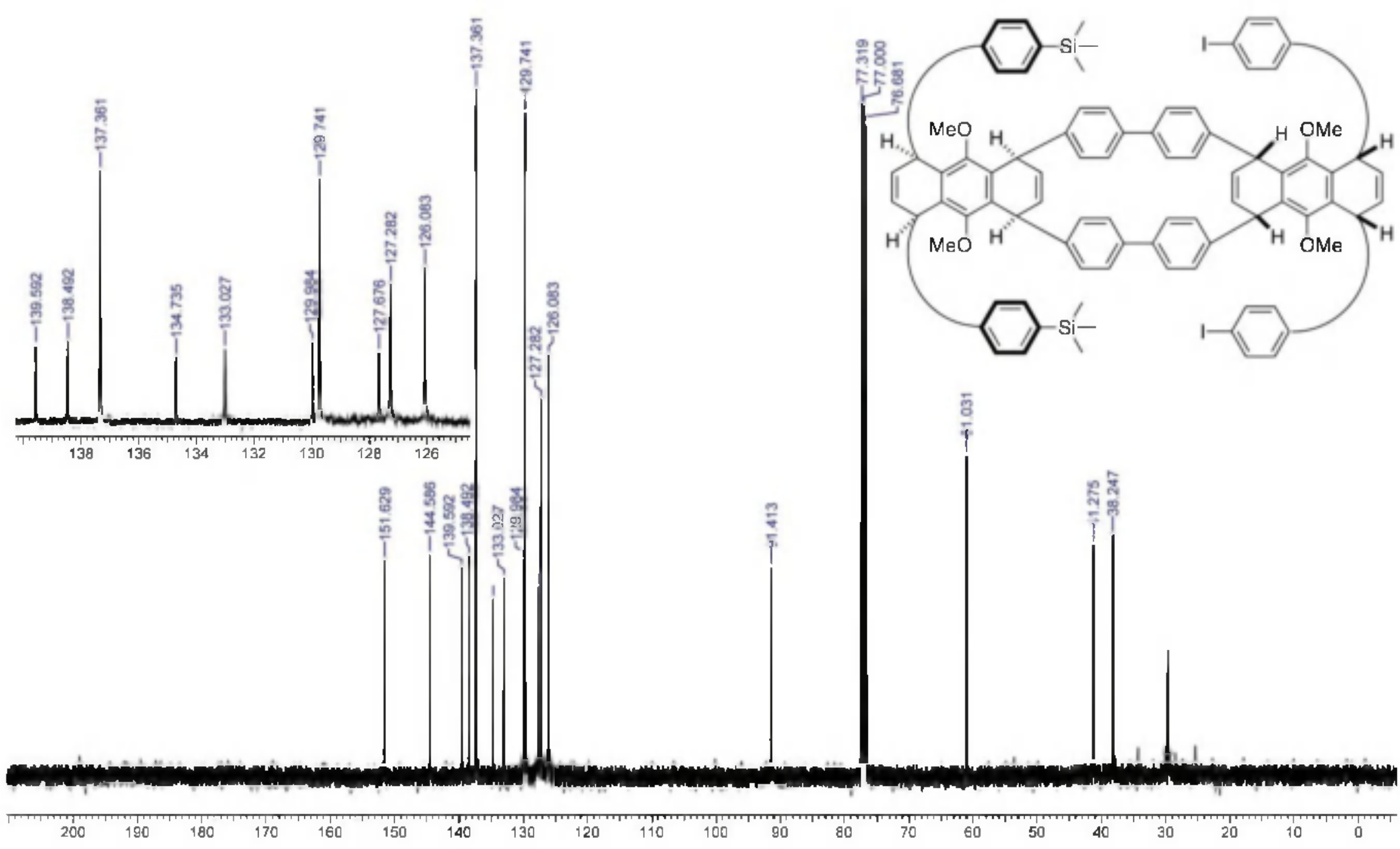


The Crude 'H NMR Spectrum of Iodination of 105 with ICI

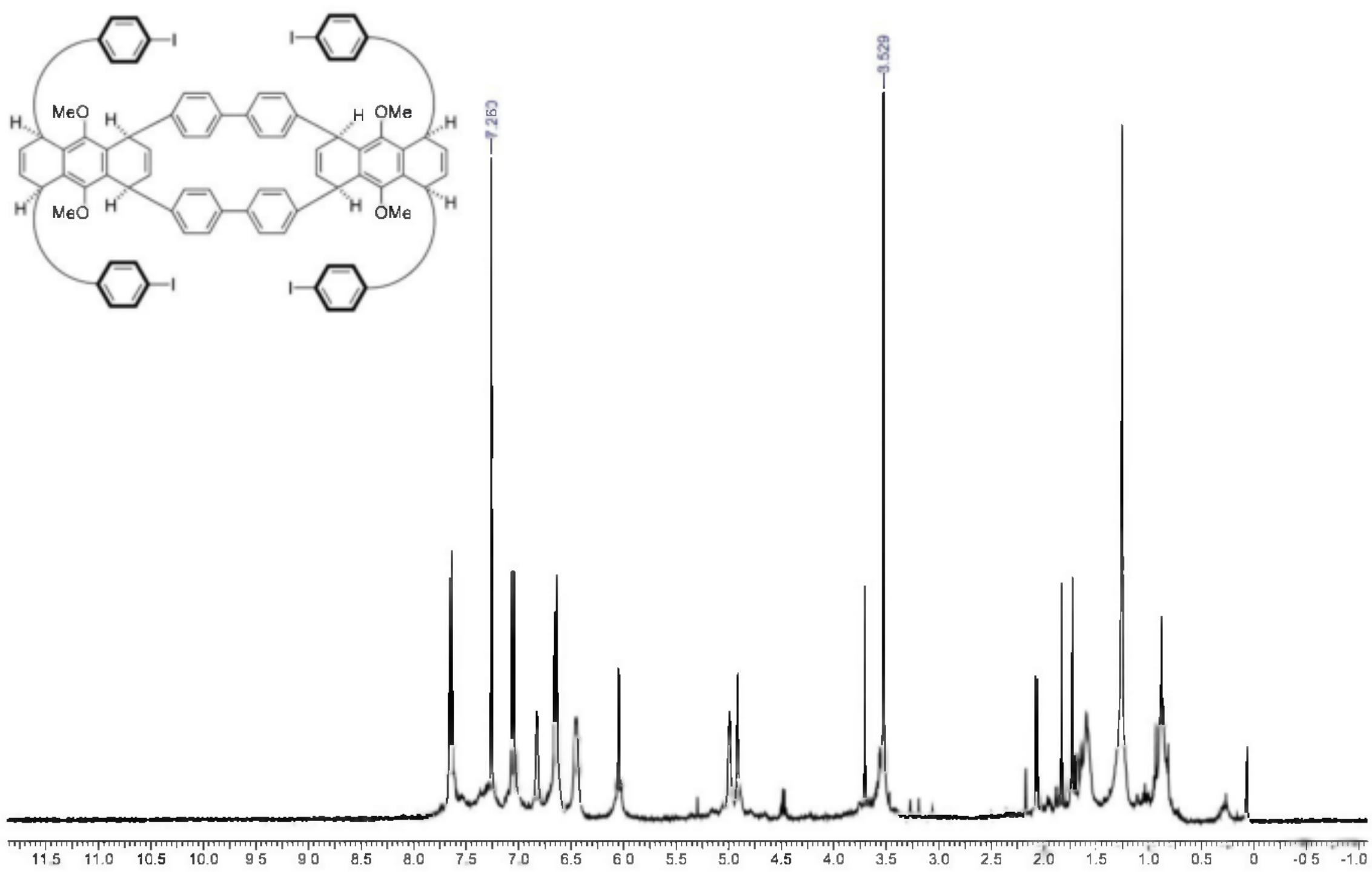


The Crude ${ }^{13} \mathrm{C}$ NMR Spectrum of lodination of 105 with ICI
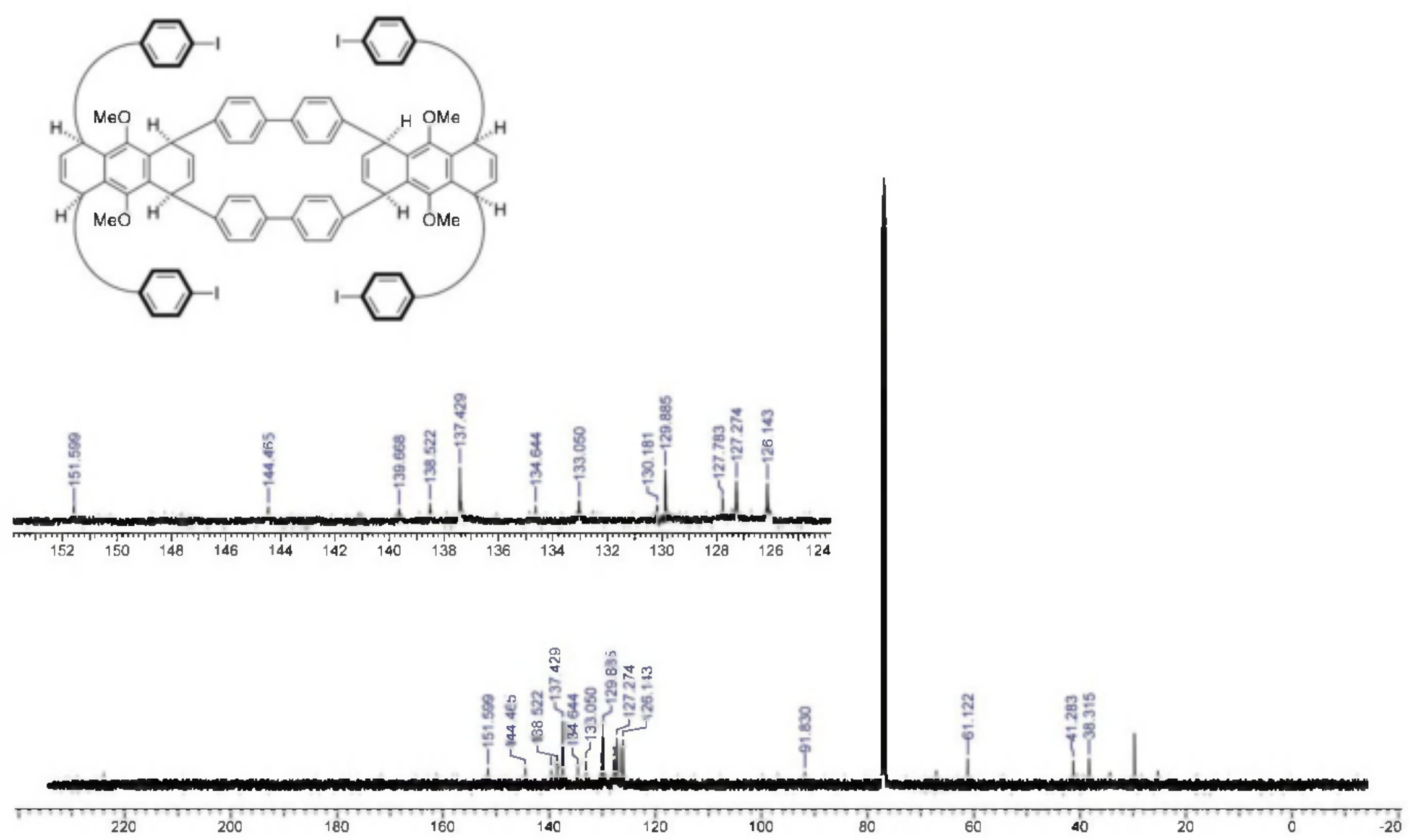
The Crude 'H NMR Spectrum of the Ni(cod)2-mediated homocoupling reaction with the crude sample from Iodination of 105 with ICl.

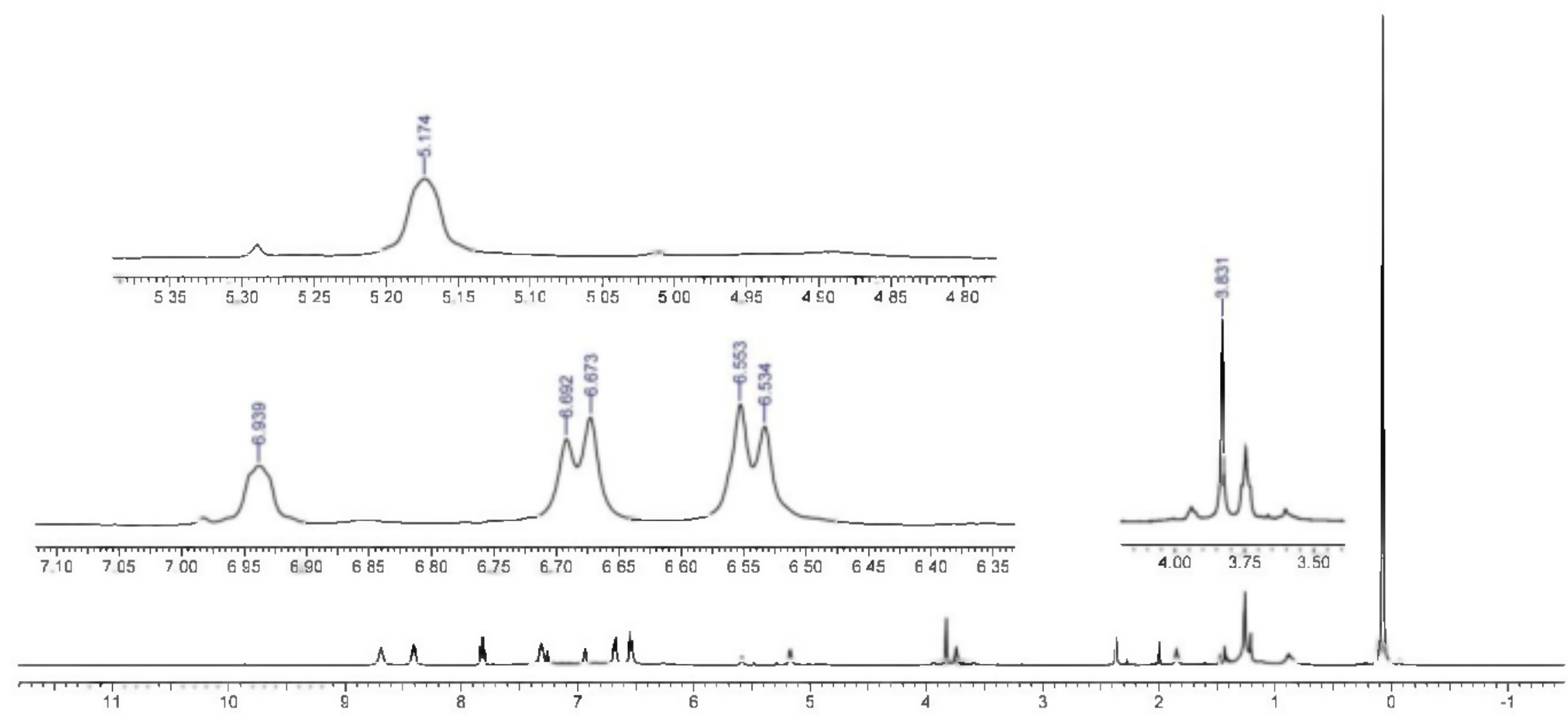




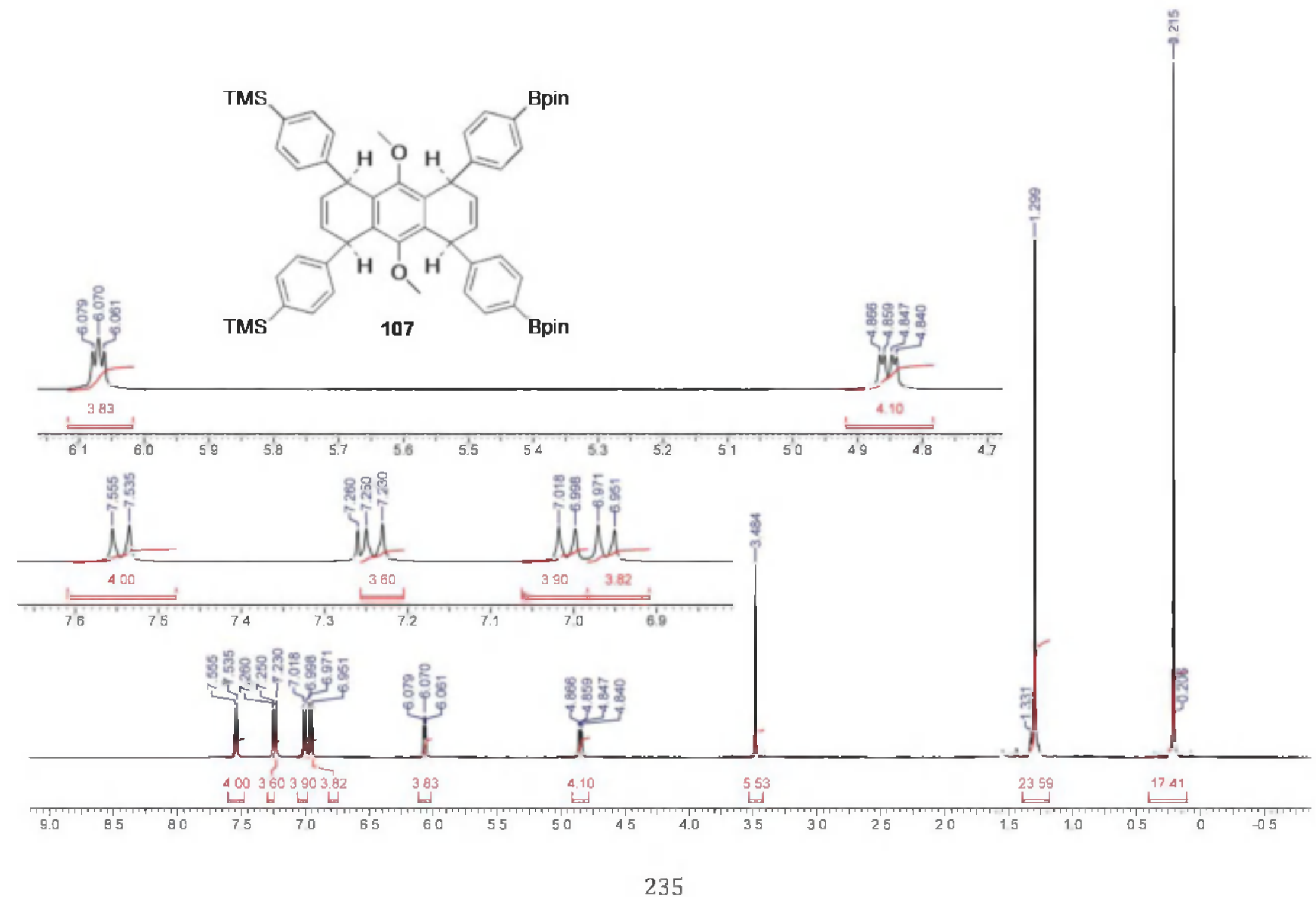




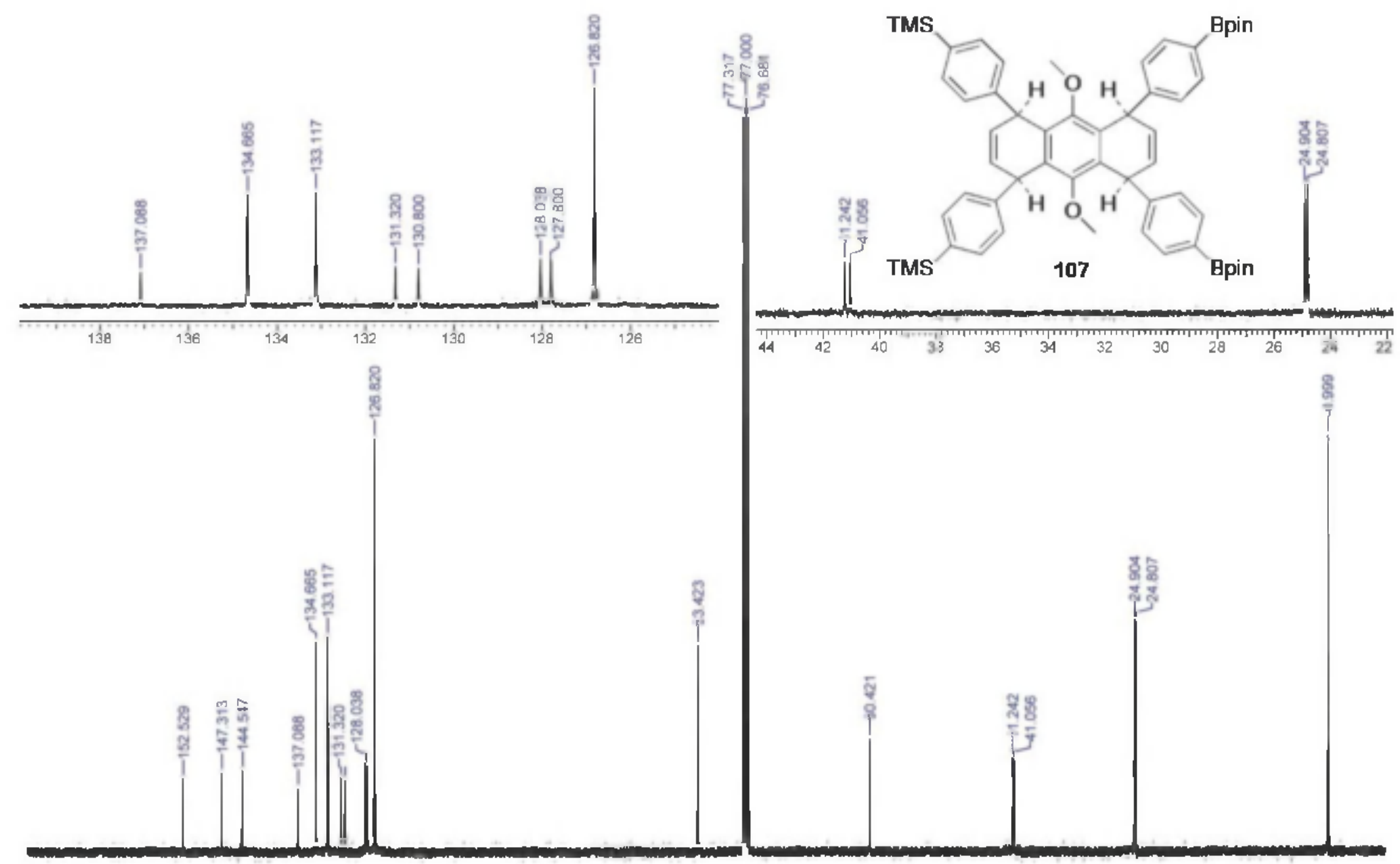

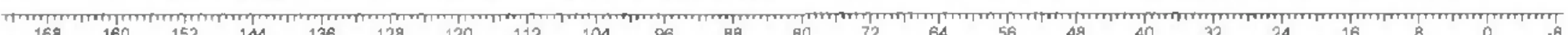




\section{Unsymmetrical Quinone}

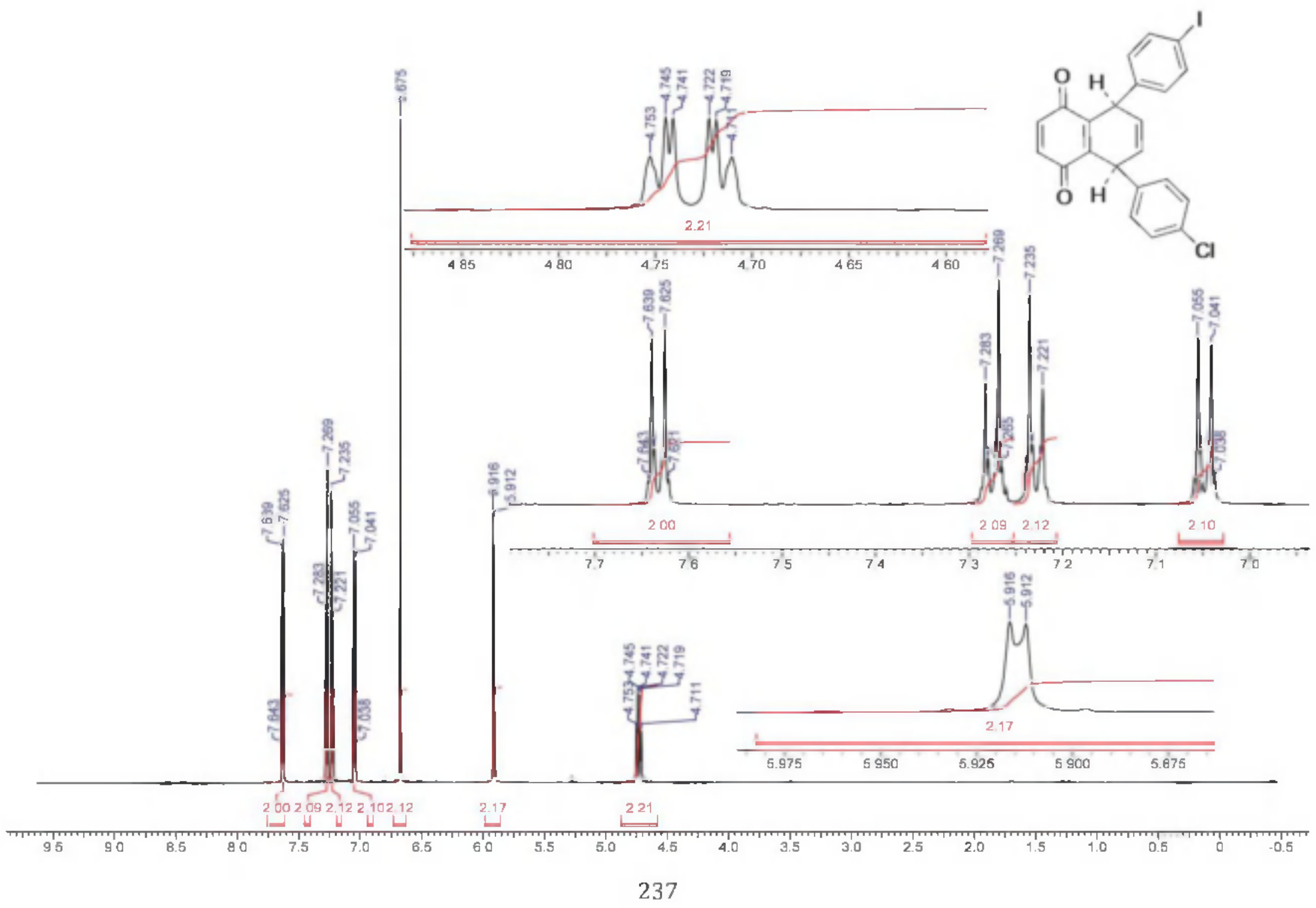




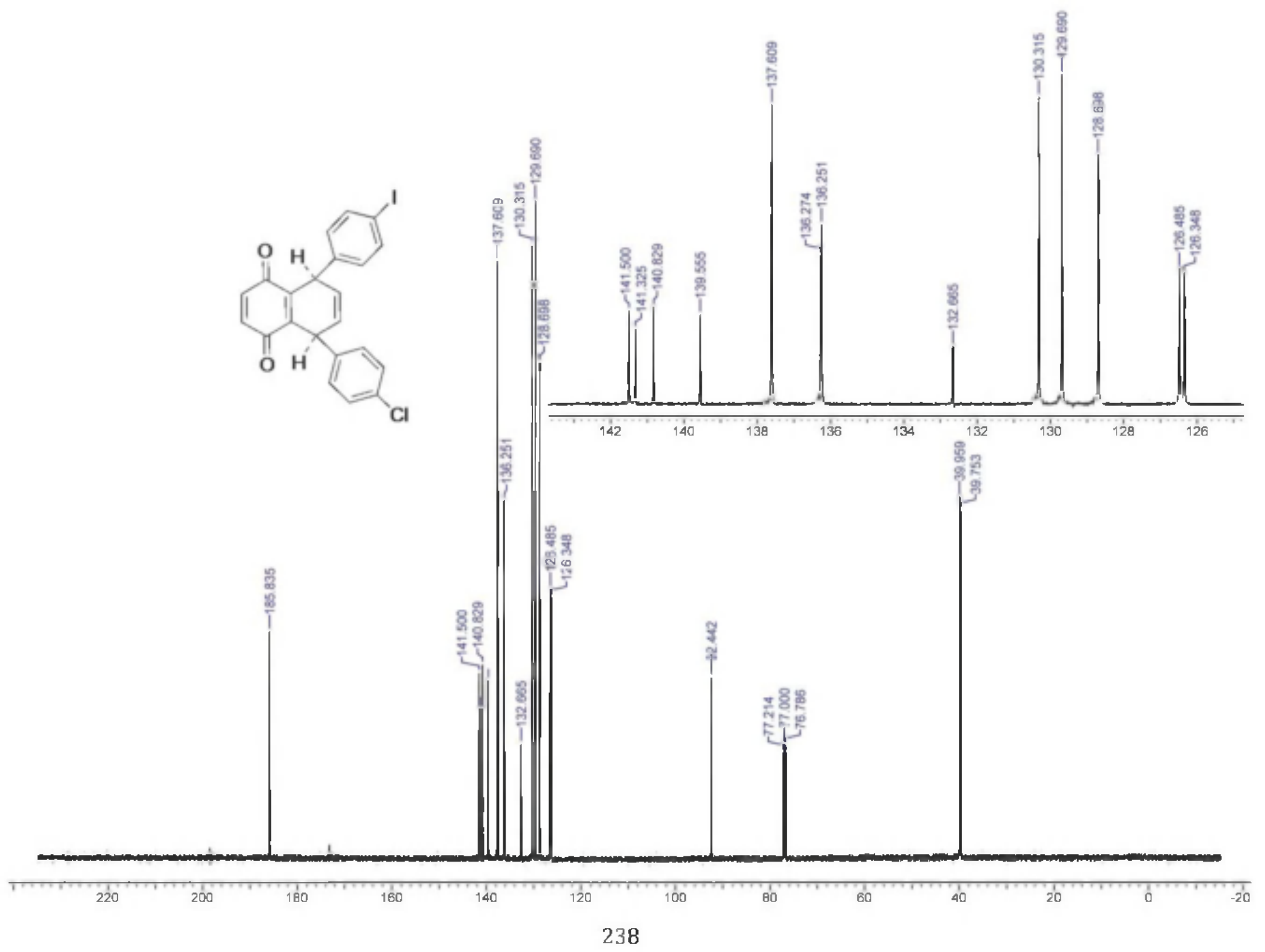




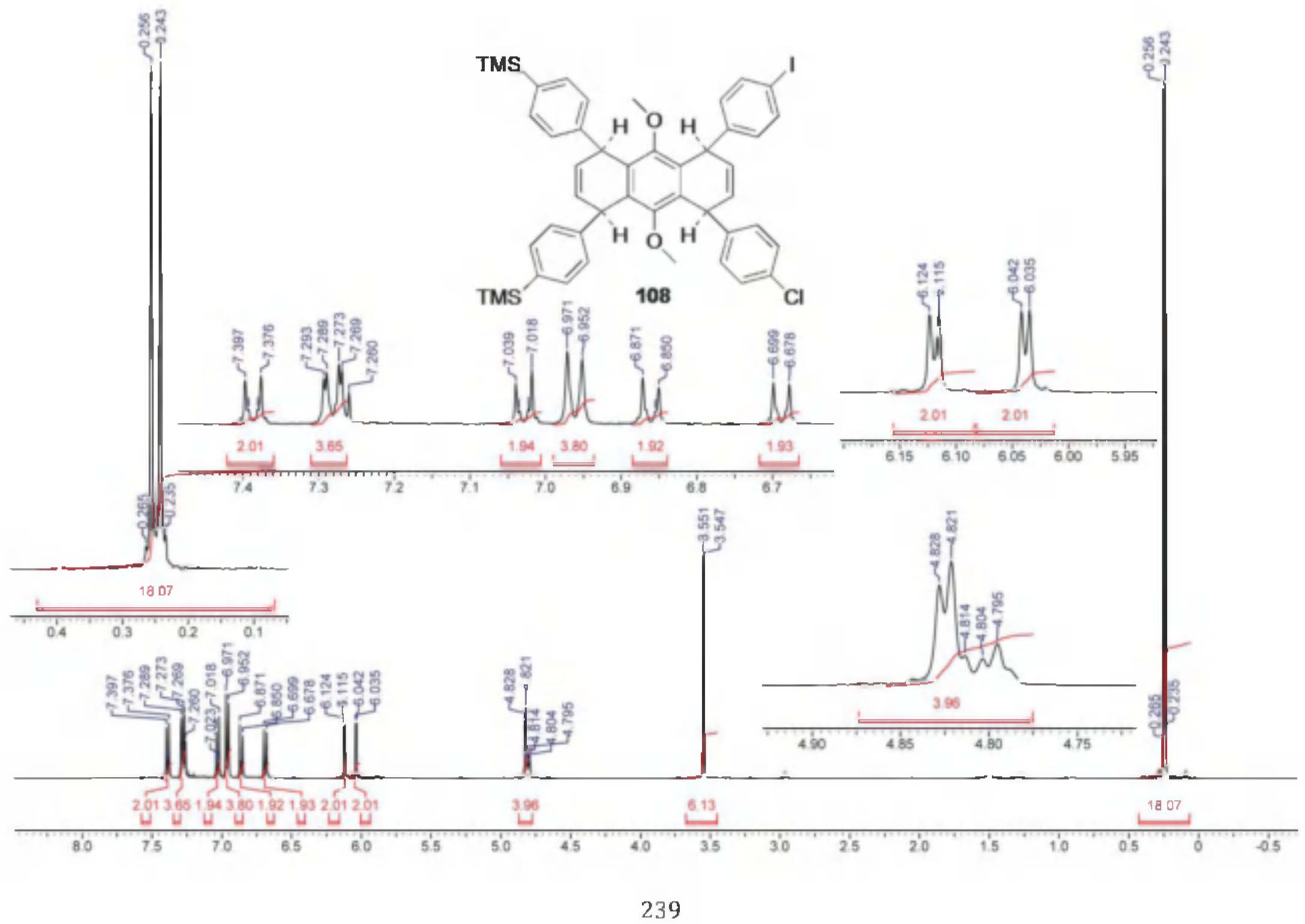




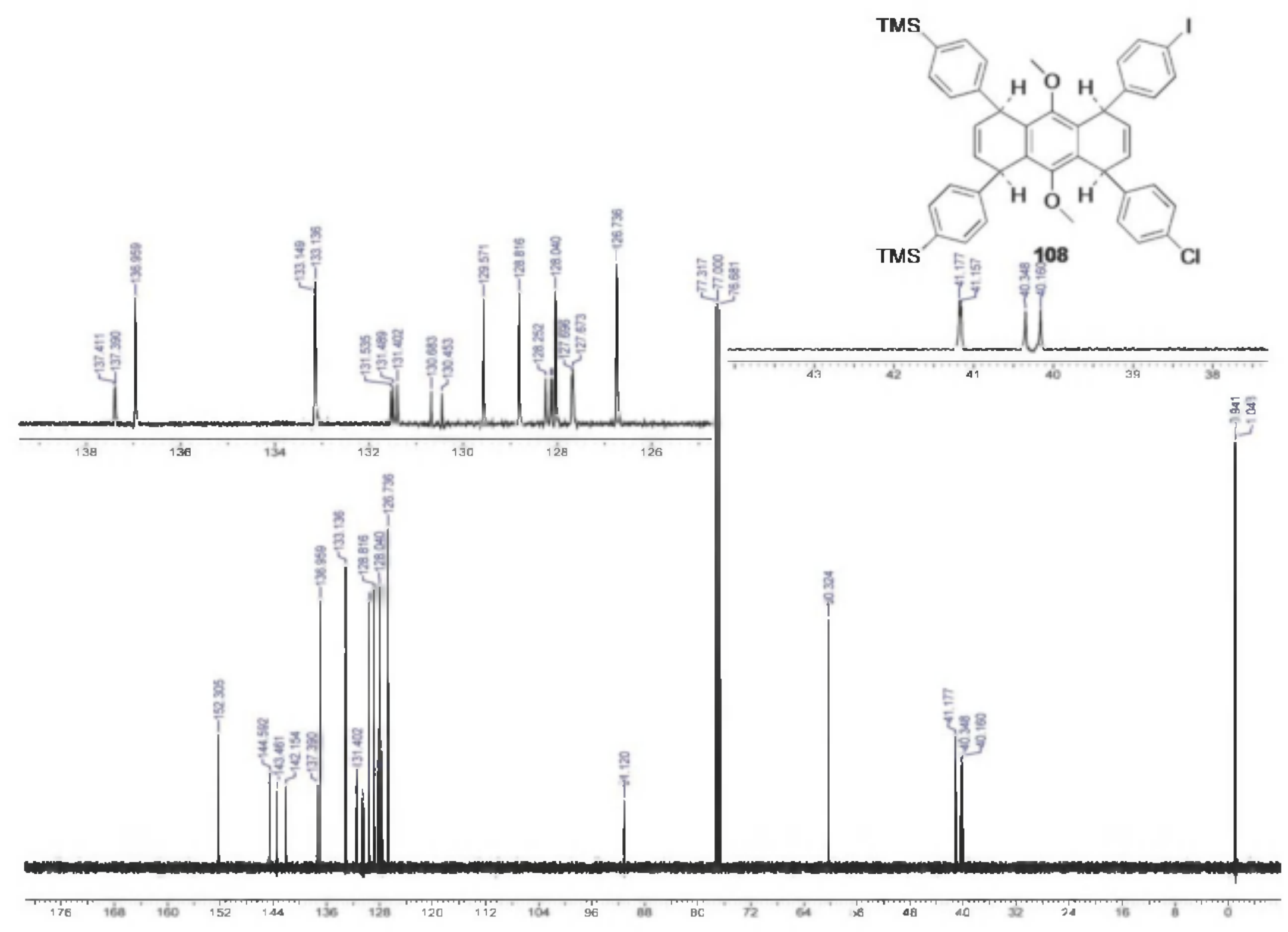




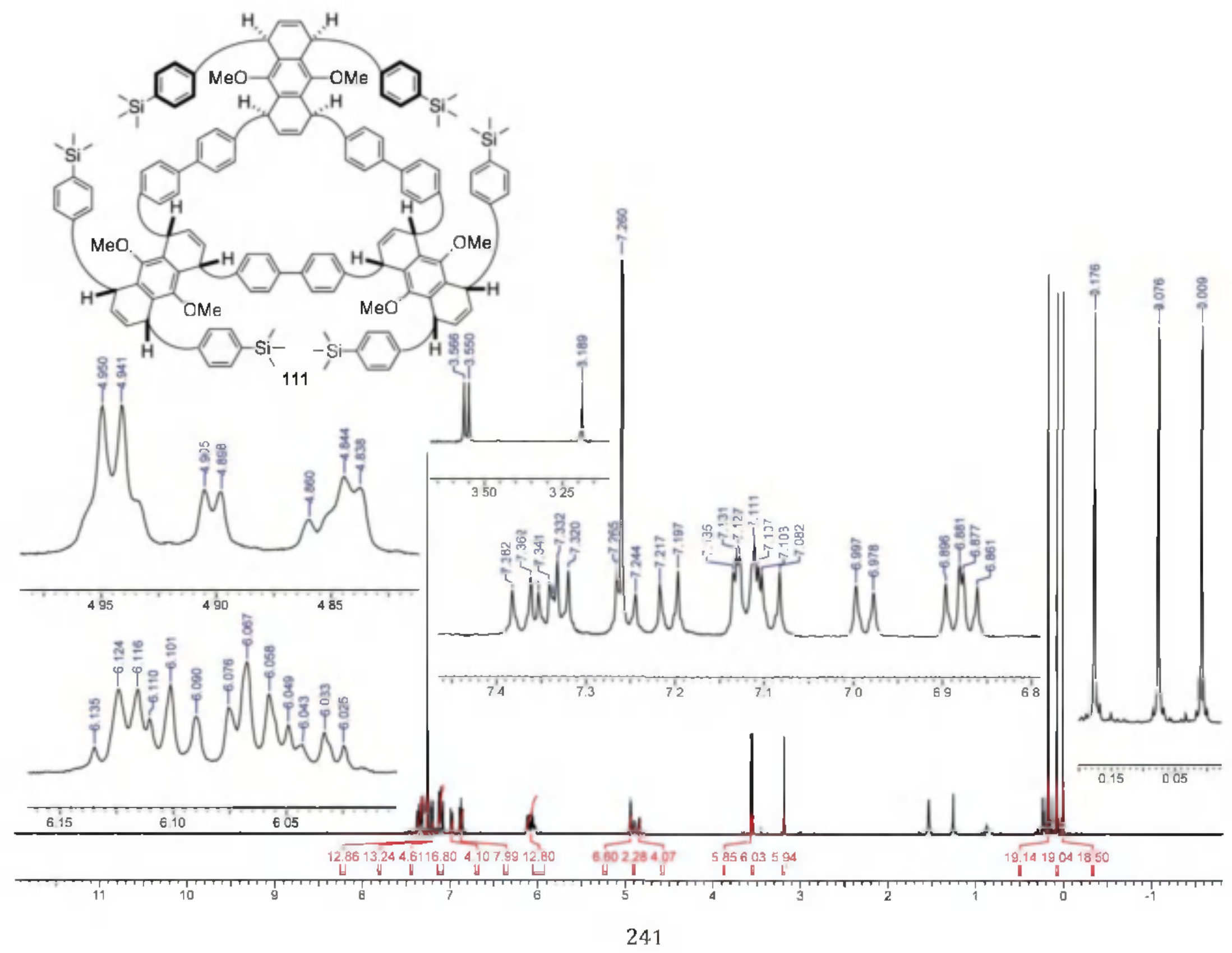




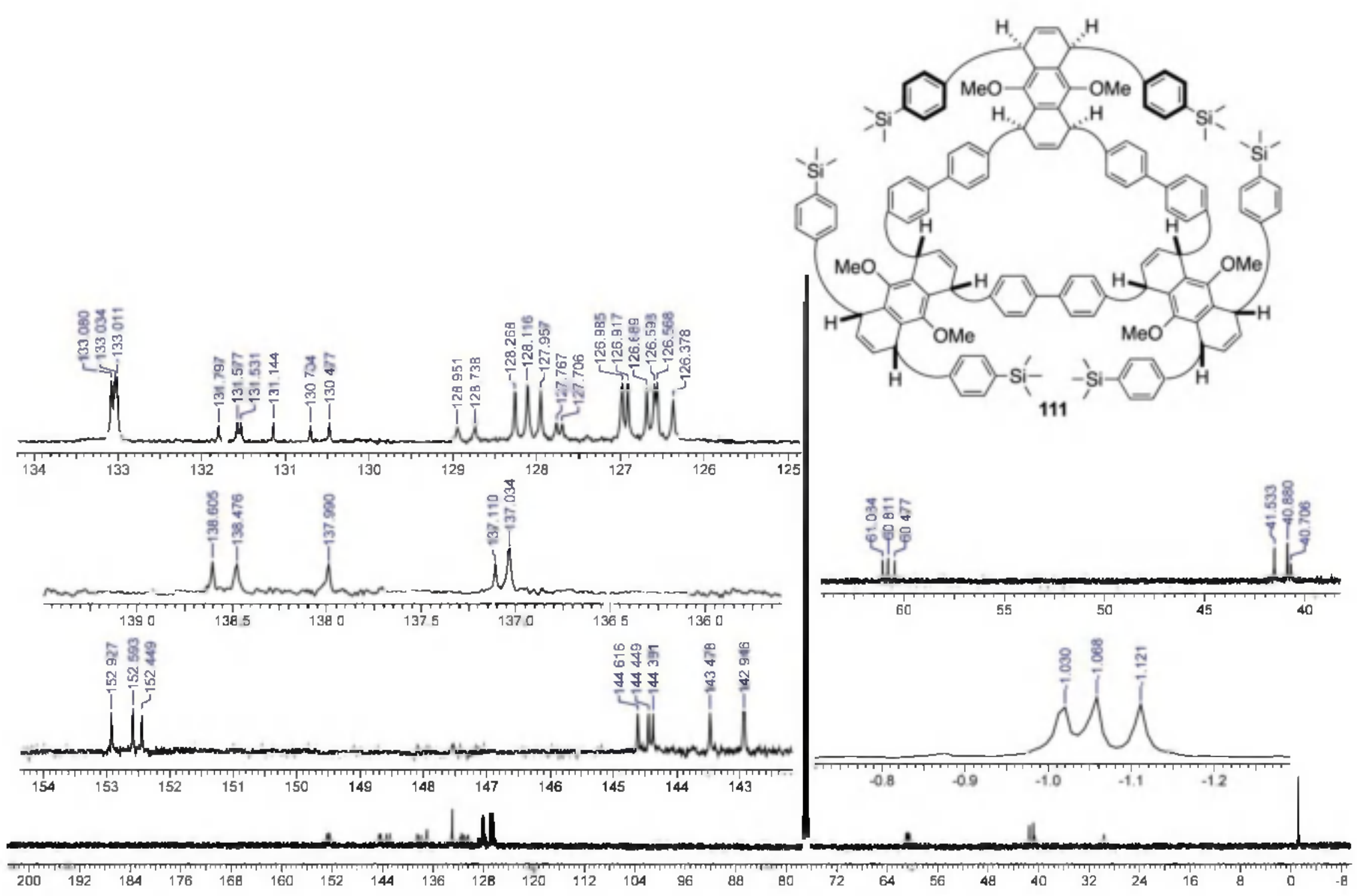




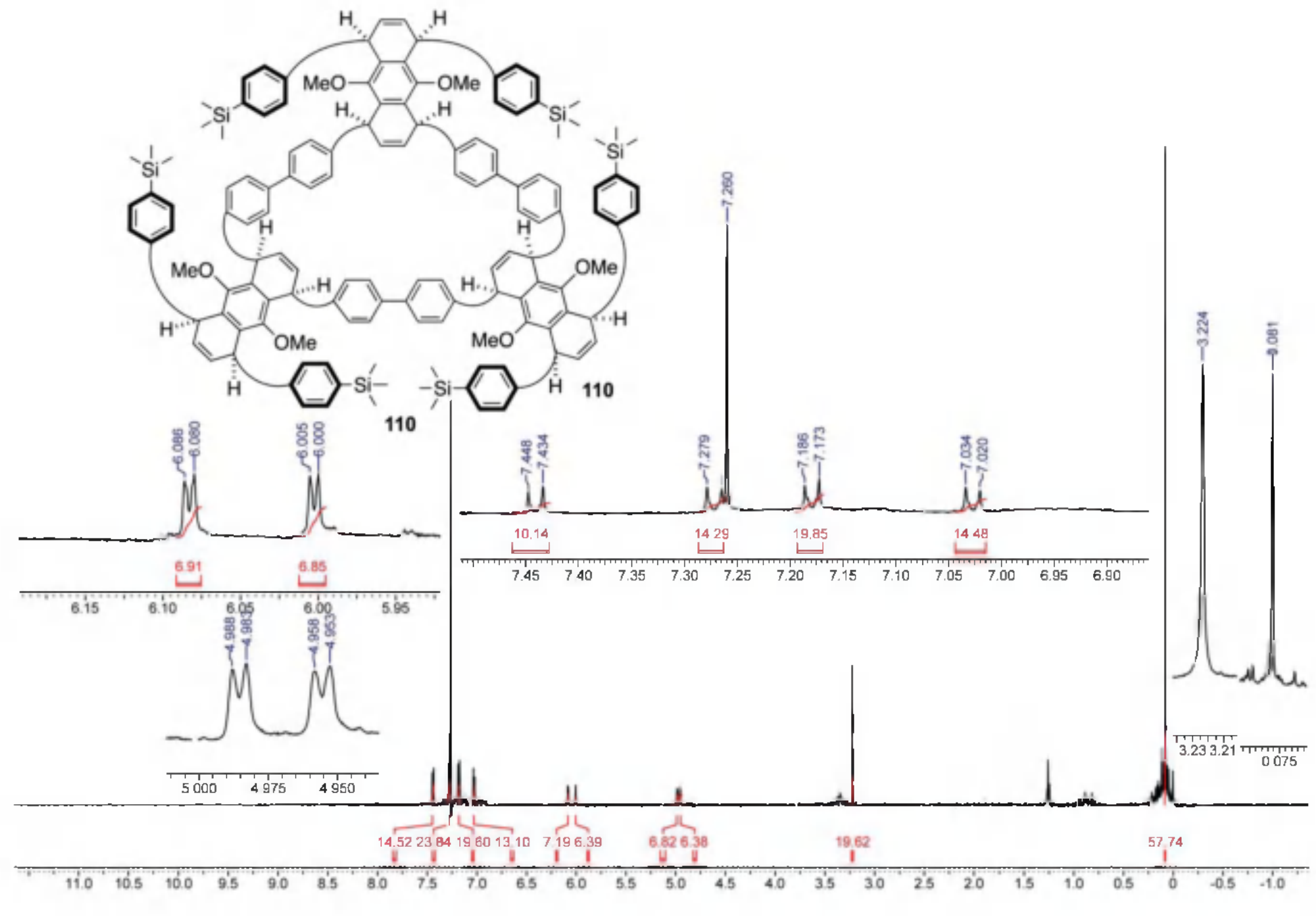



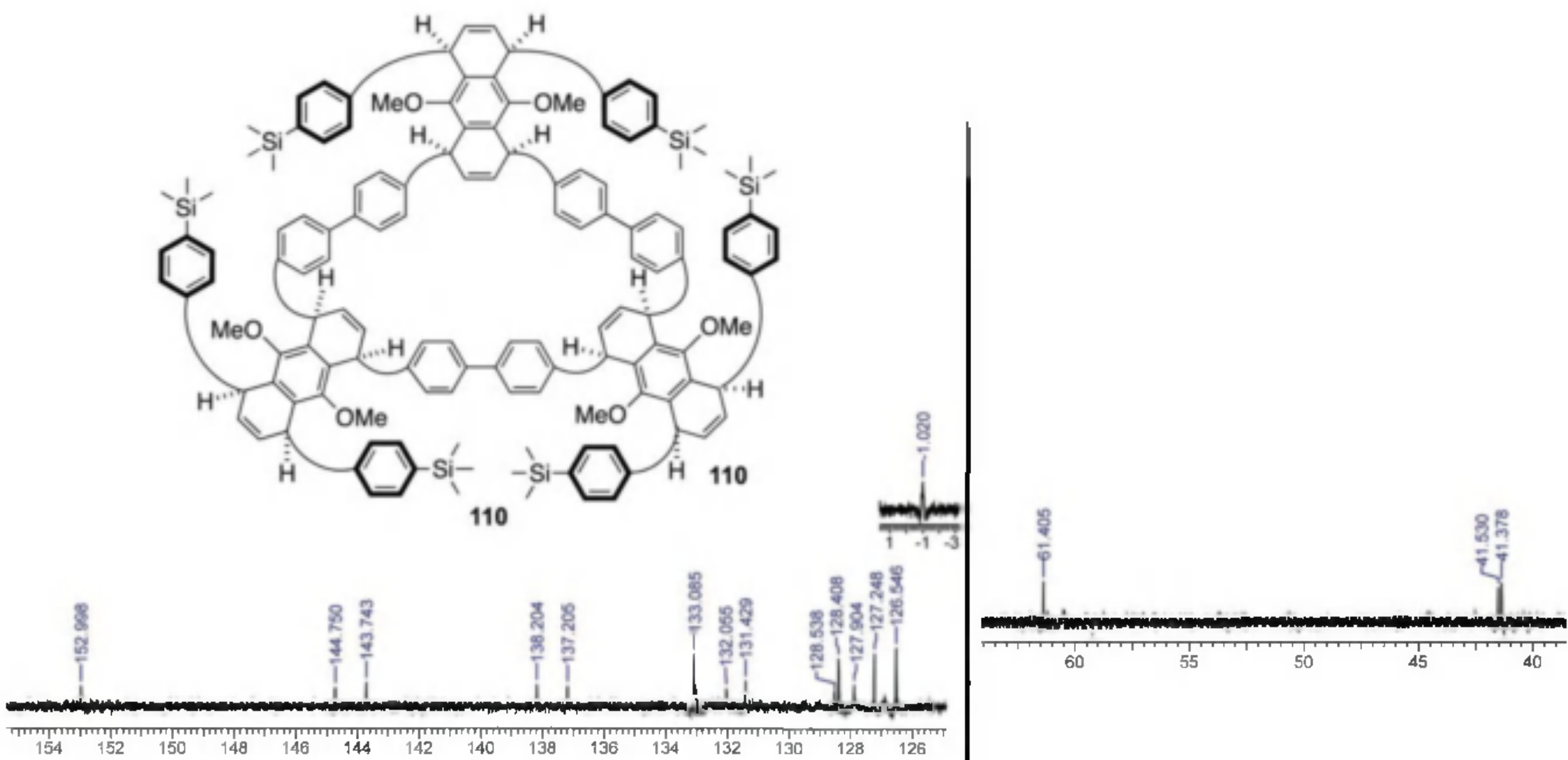

1. 1111 
The Crude 'H NMR Spectrum of Ni(cod)2-Mediated Homocoupling Reaction of 110 after Treated with ICl

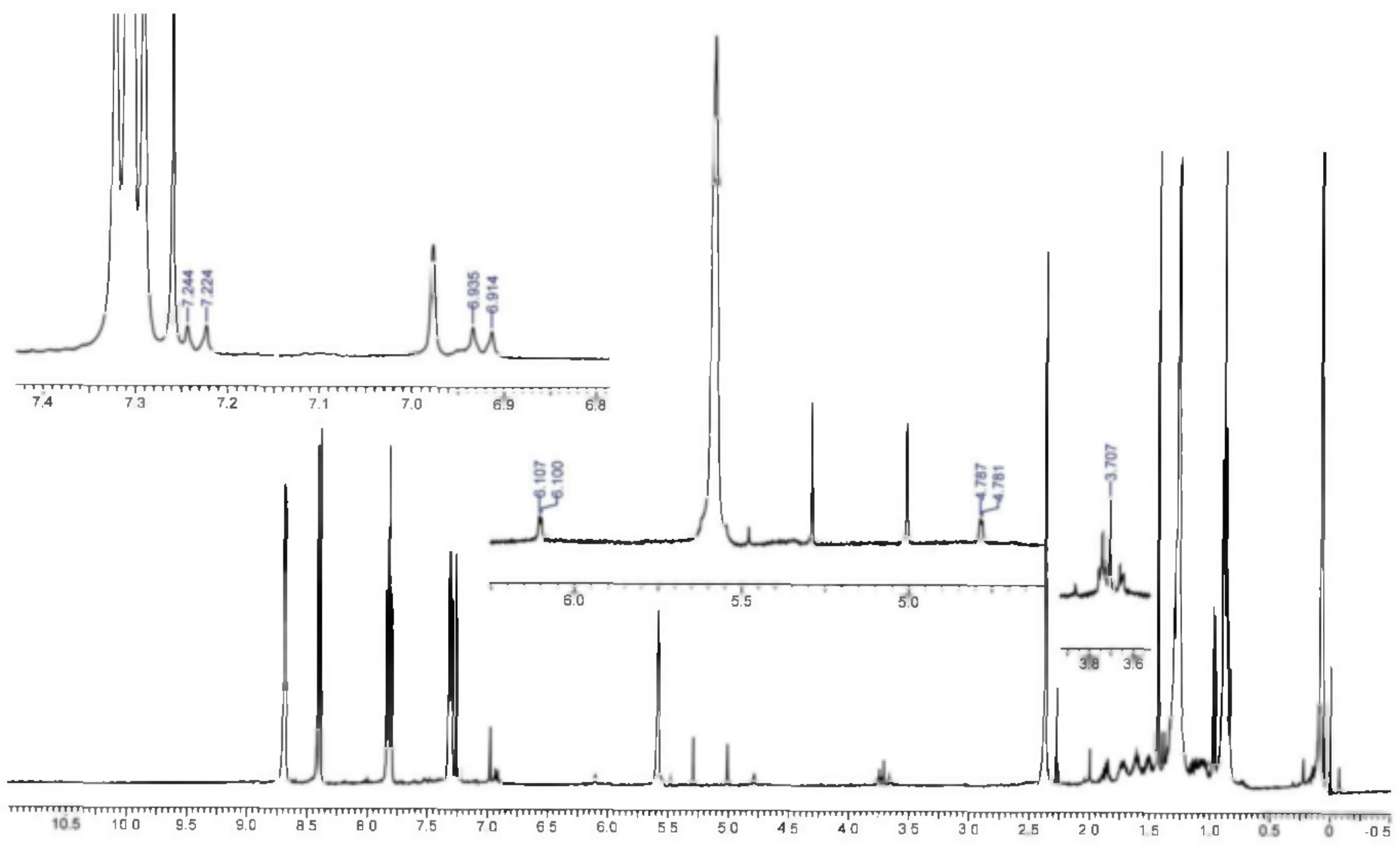




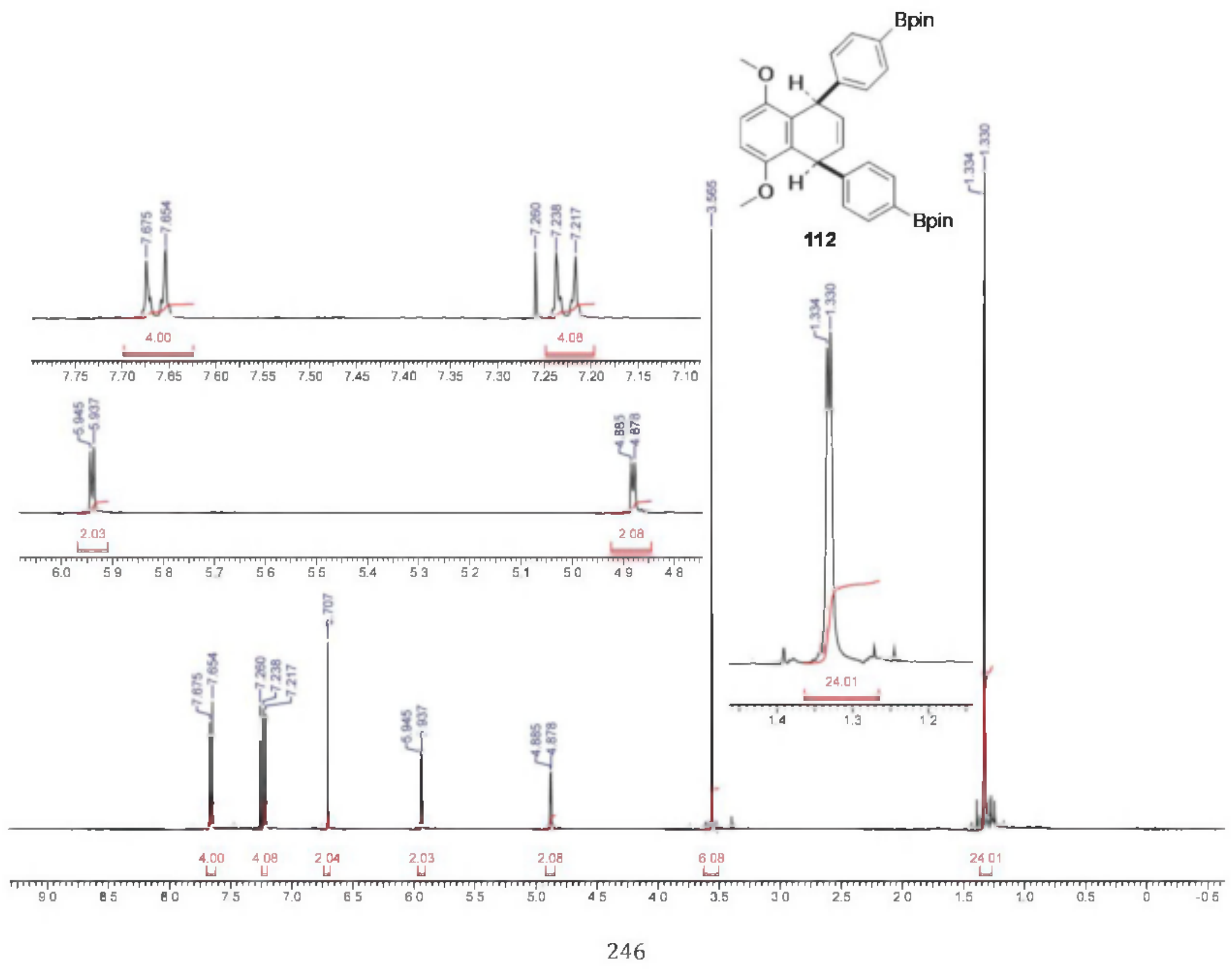




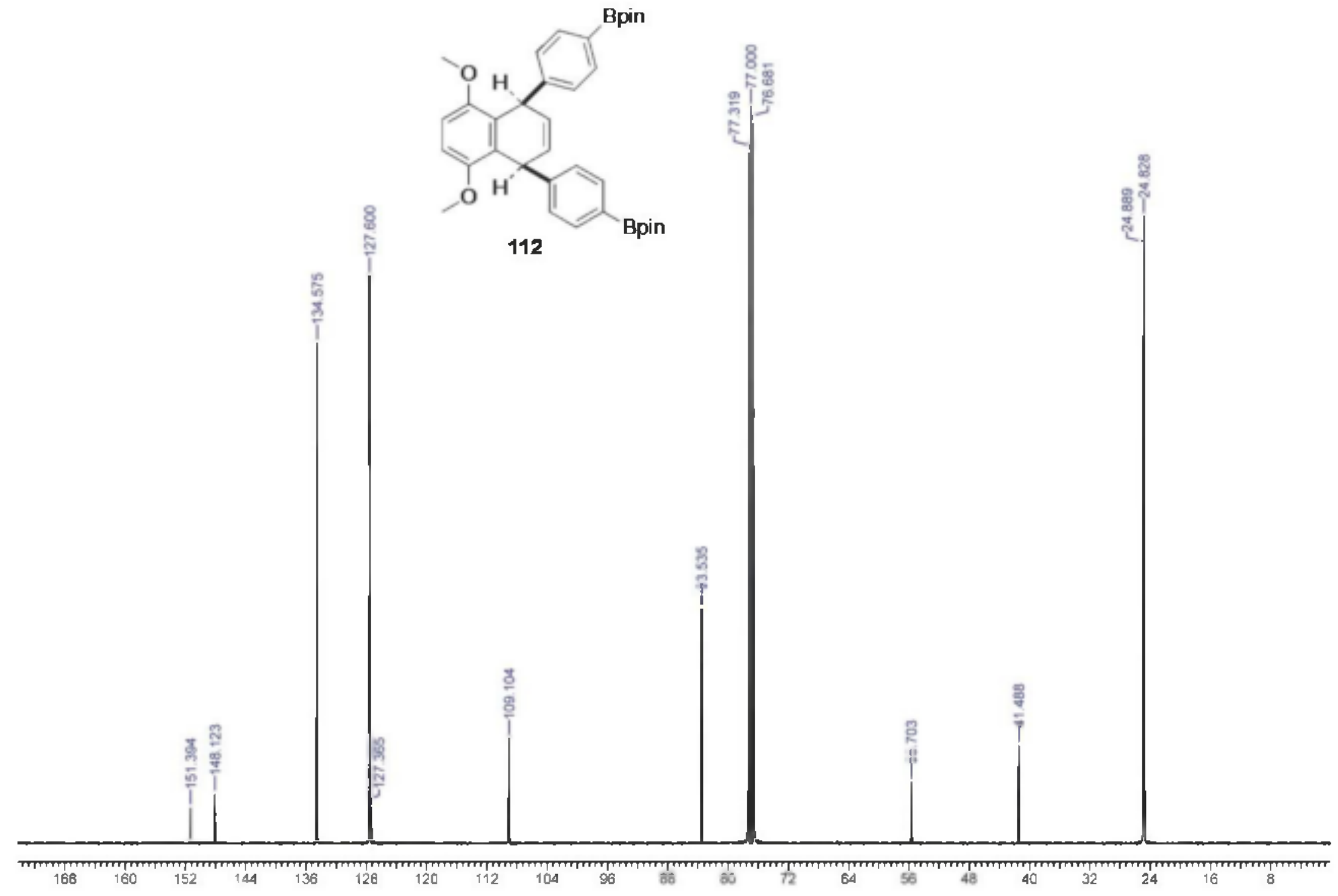




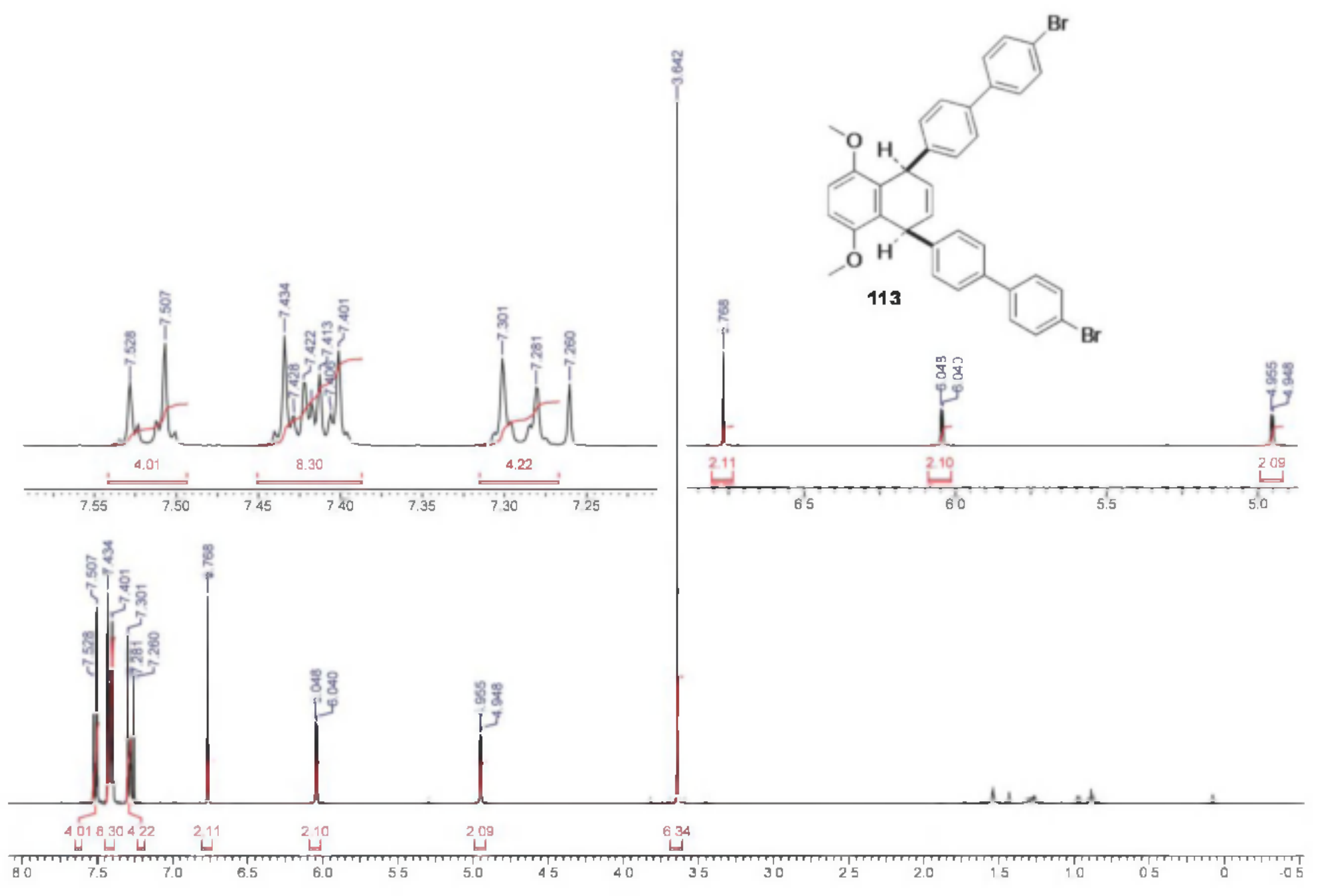




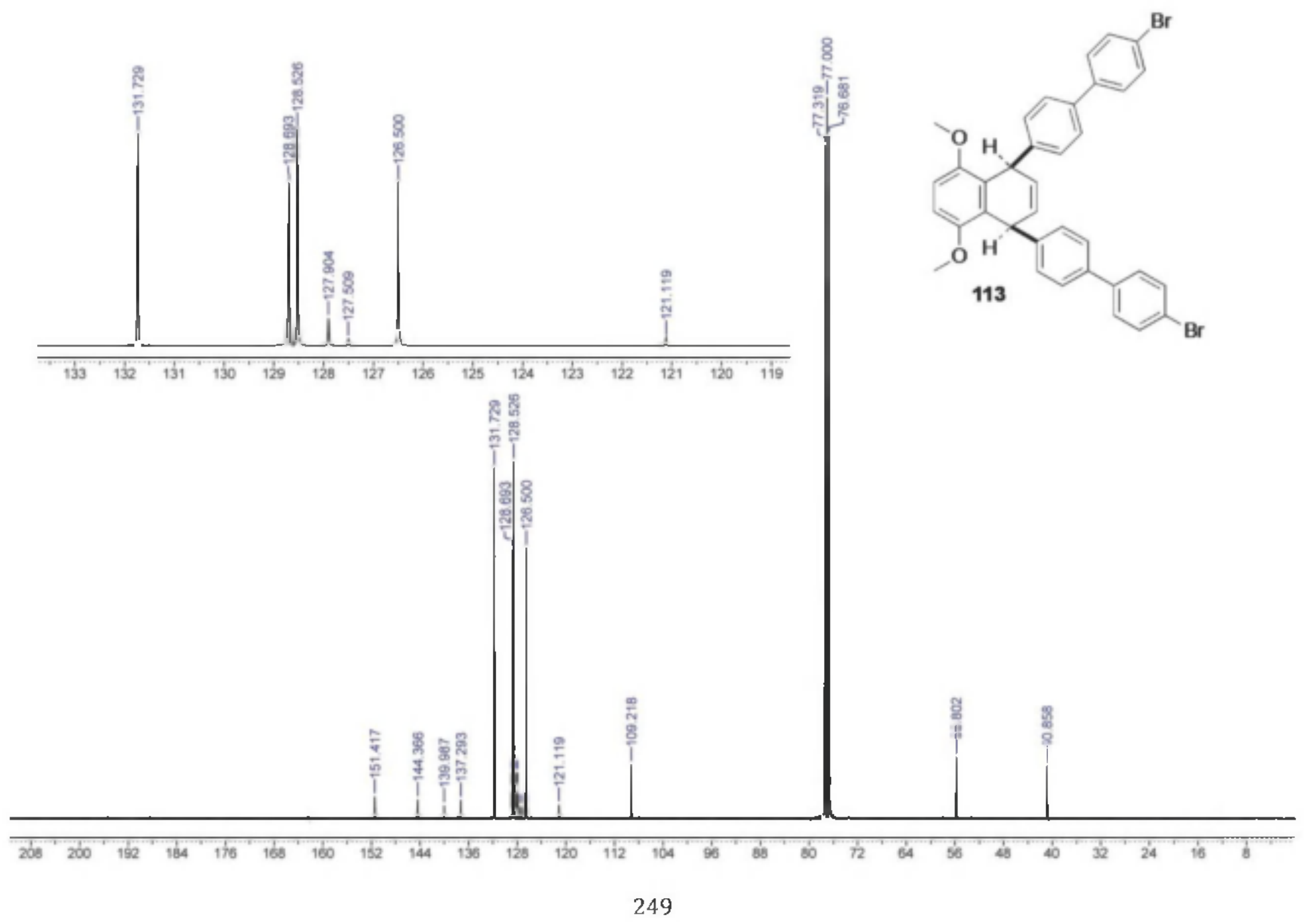




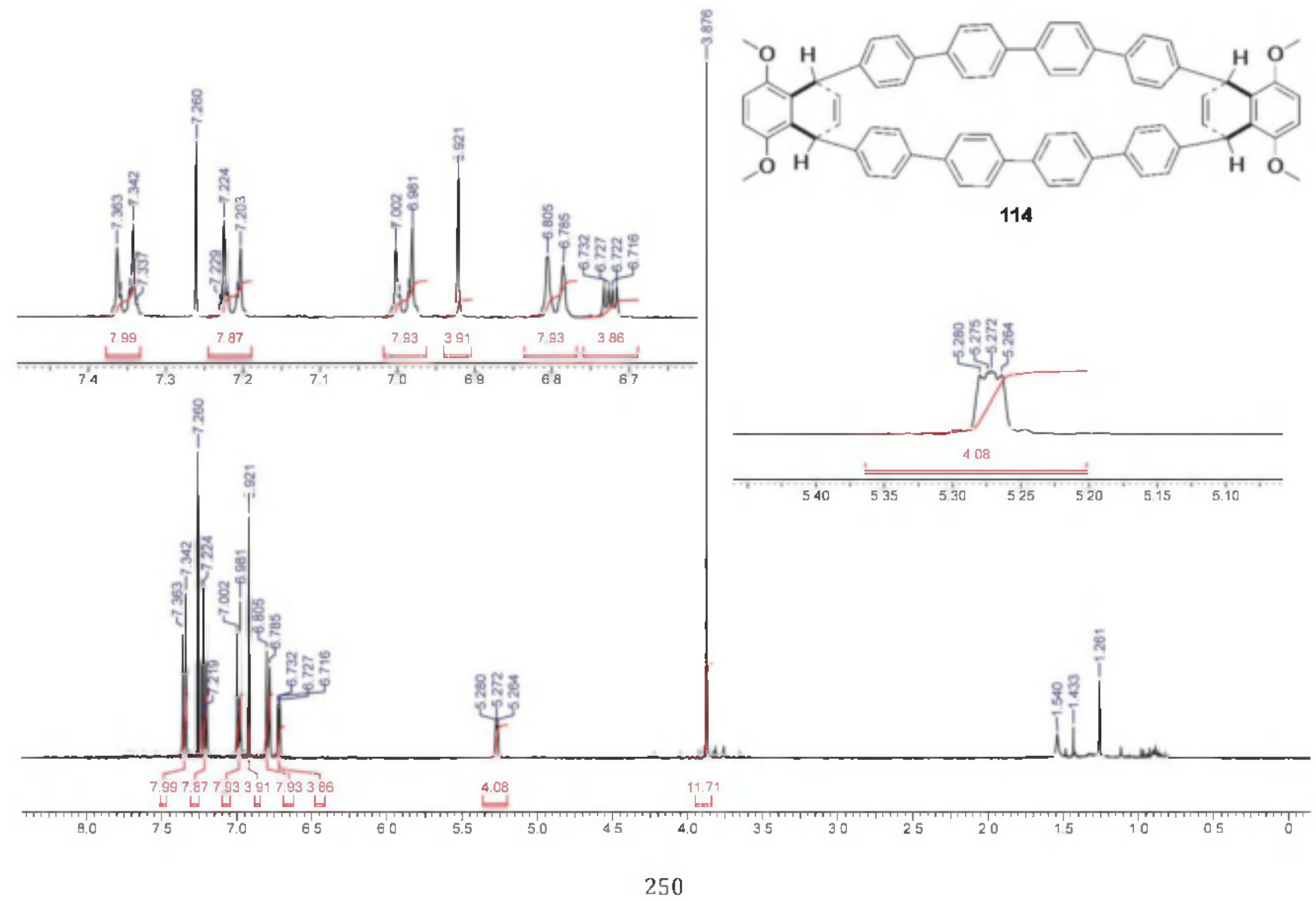




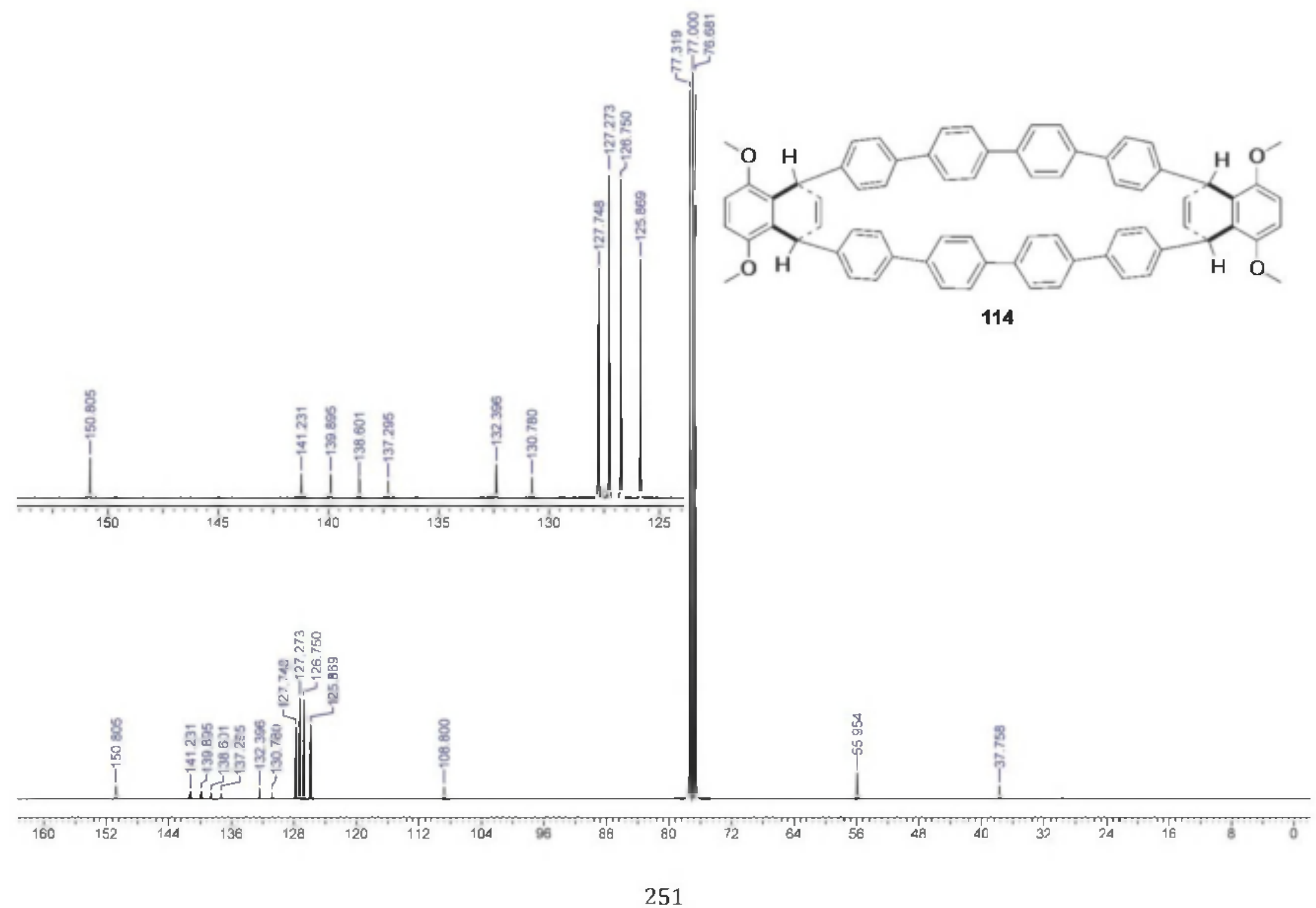


${ }^{1} \mathrm{H}$ NMR spectrum in $\mathrm{C}_{6} \mathrm{D}_{6}$

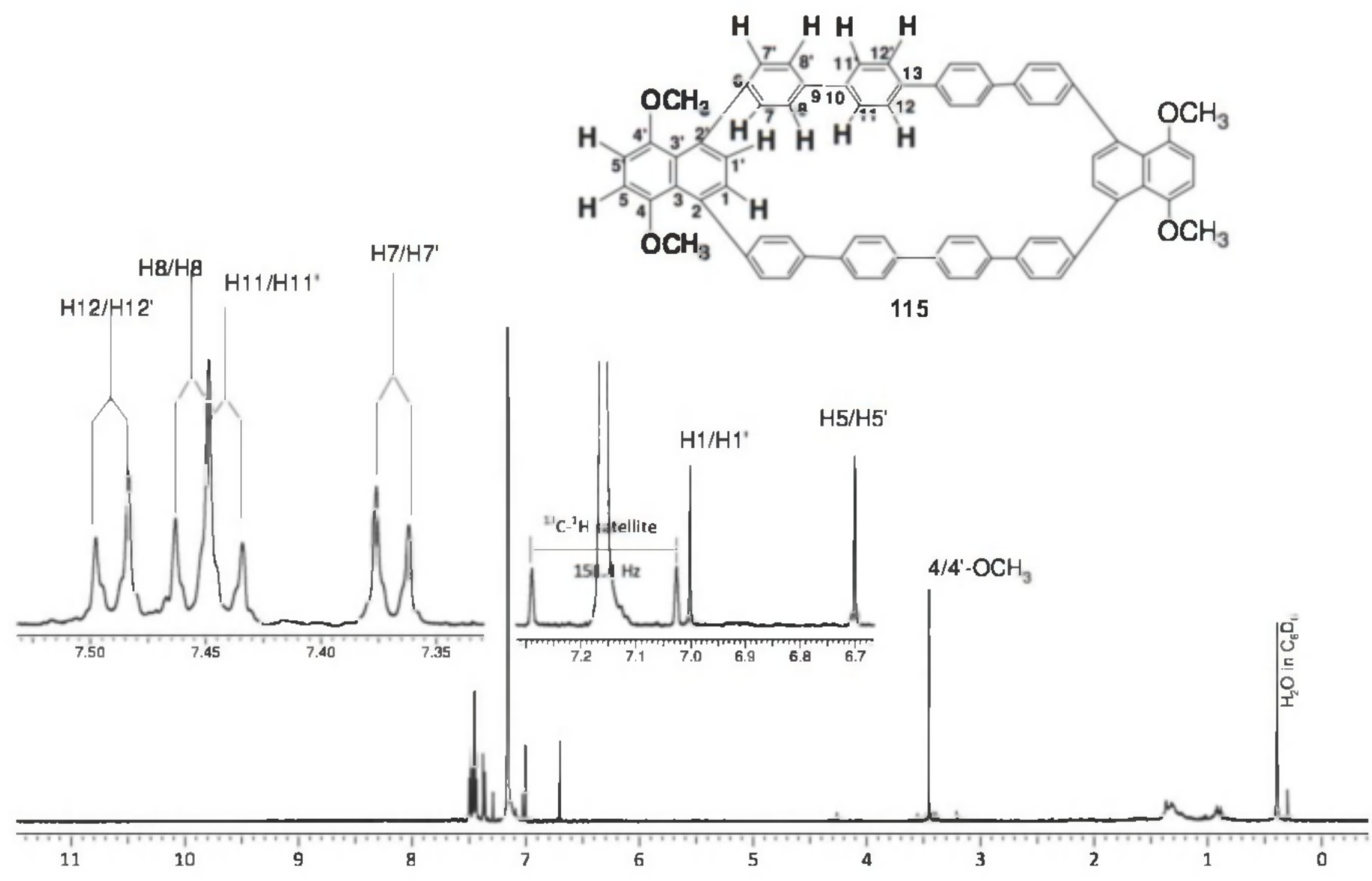


1D NOESY spectra (b-c)

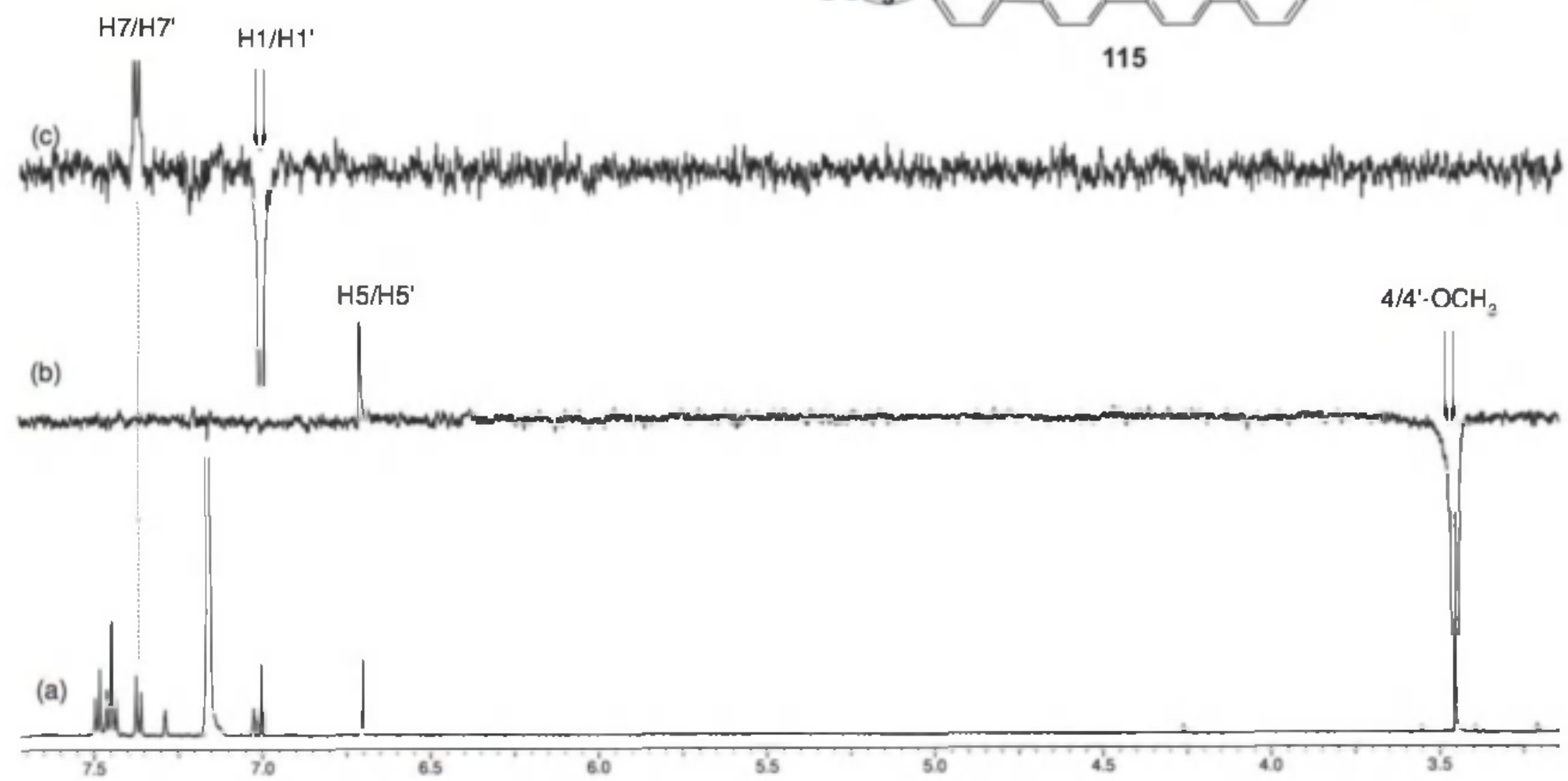




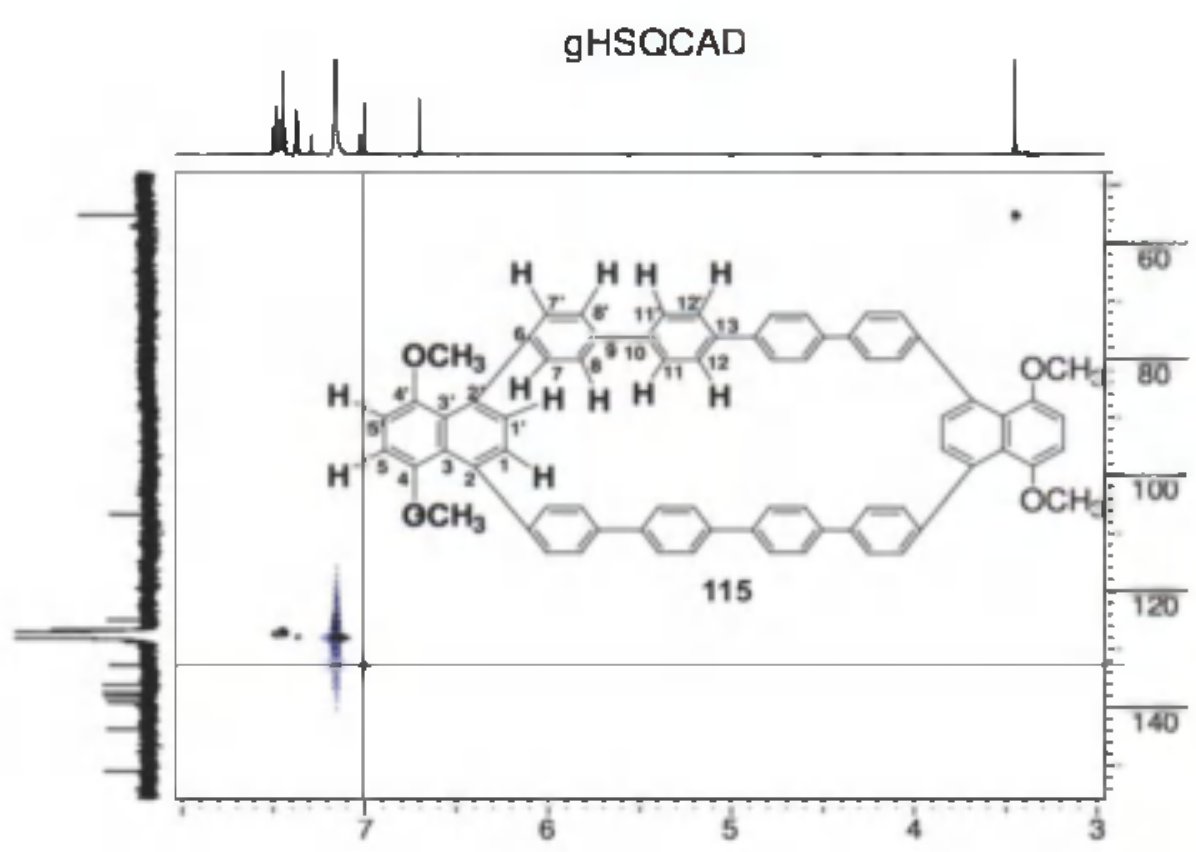

Expanded portion of the gHSQCAD spectrum
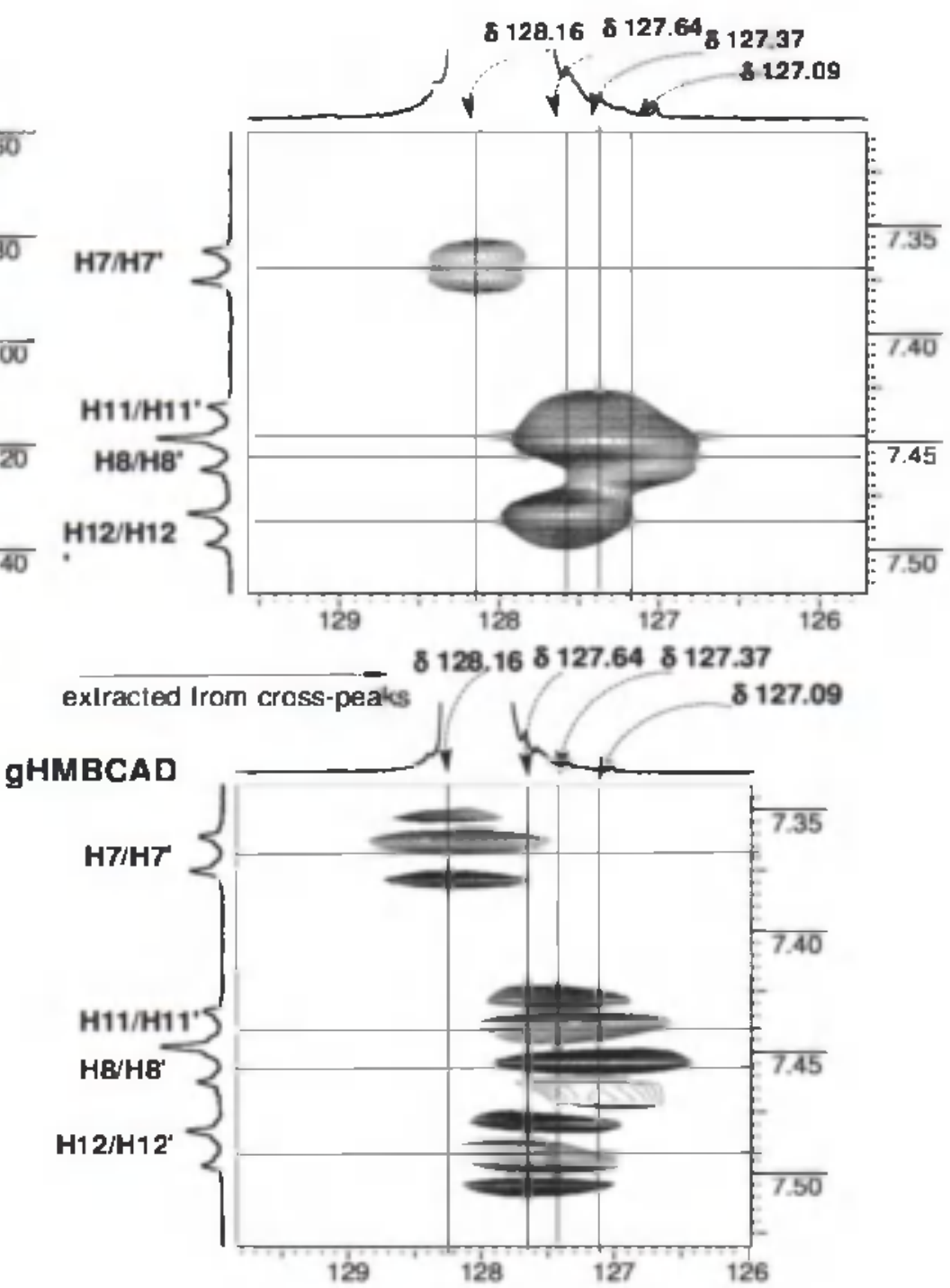

spectrum in $\mathrm{CD}_{2} \mathrm{Cl}_{2}$ 


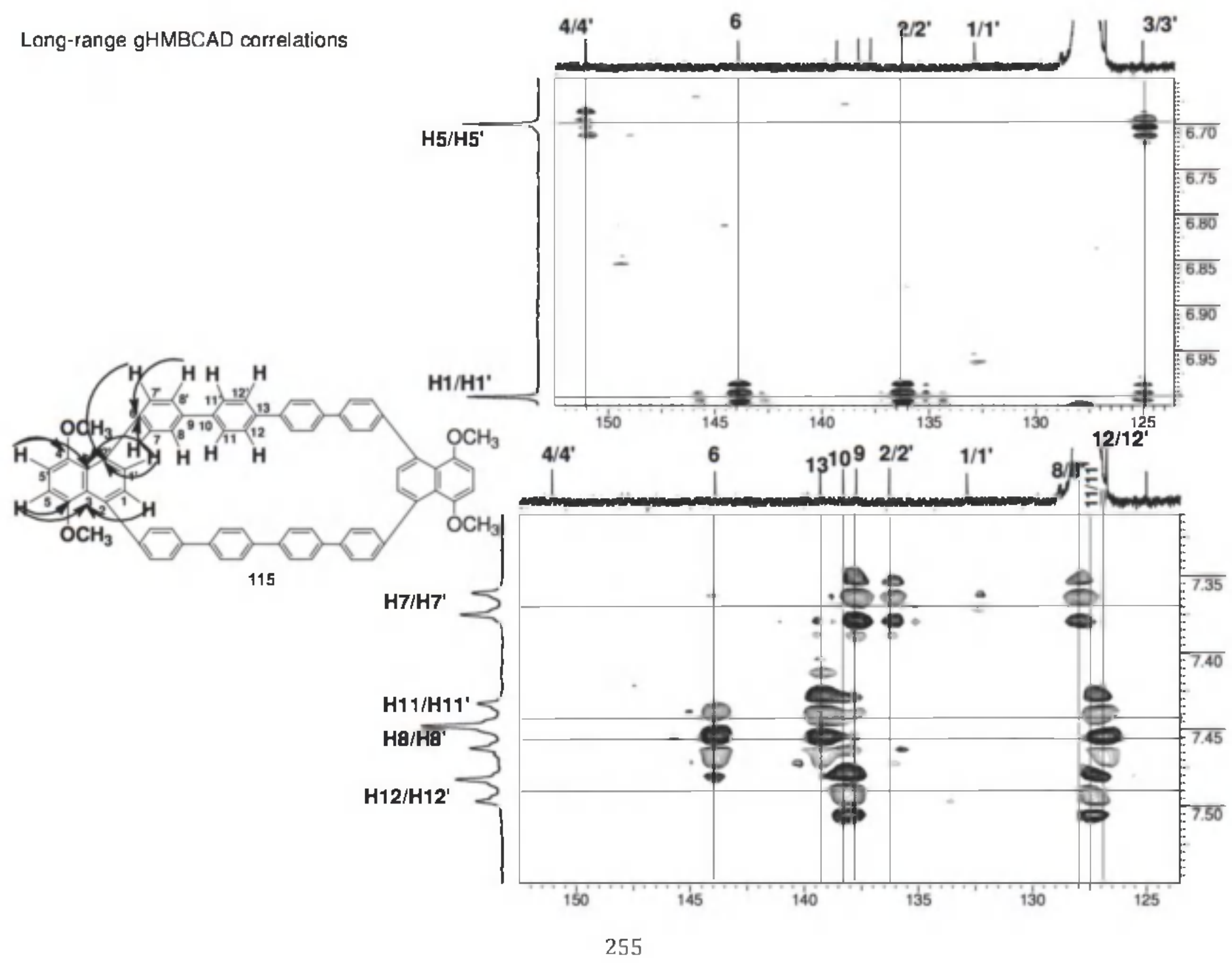




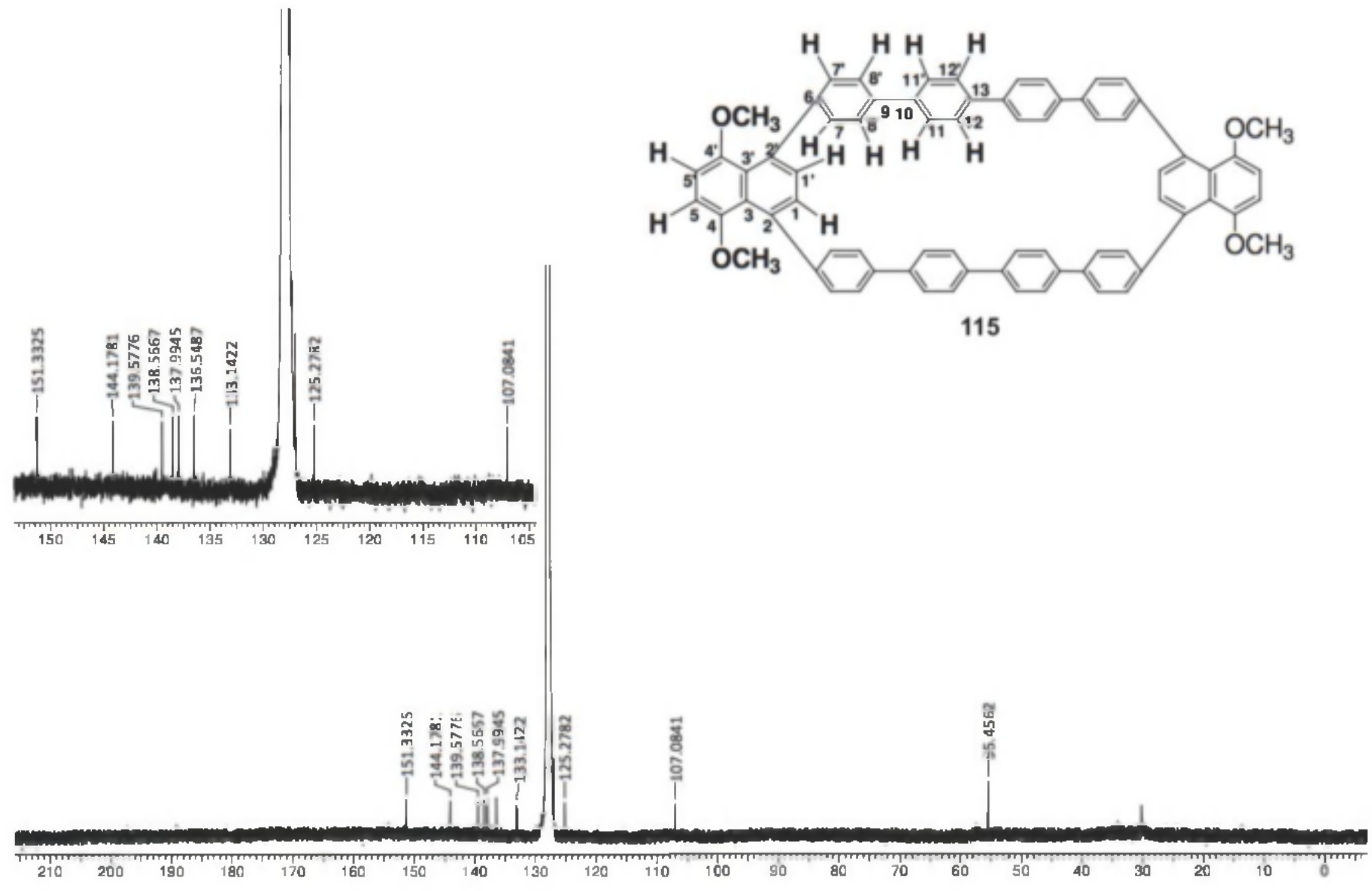




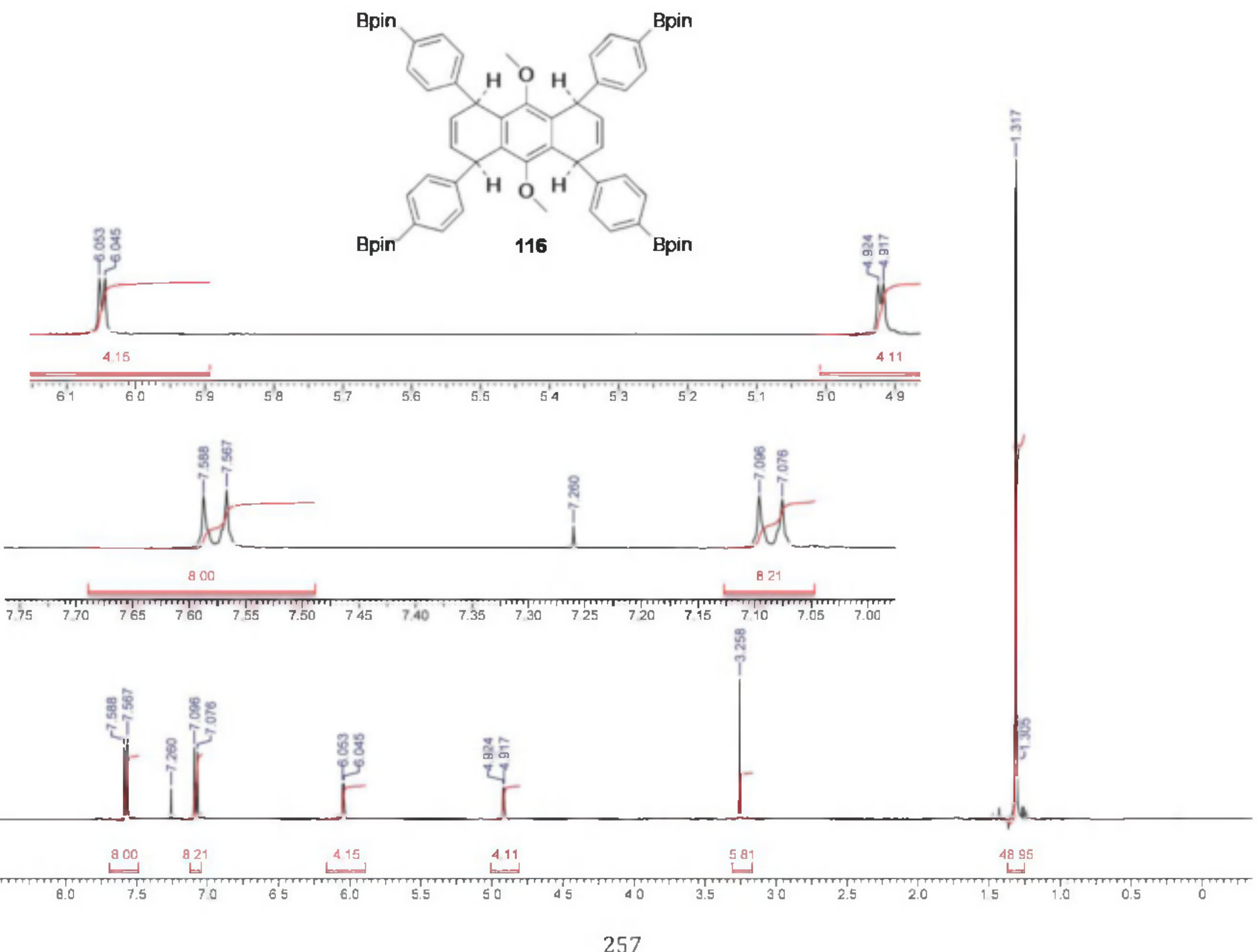




$$
\frac{511}{511}
$$




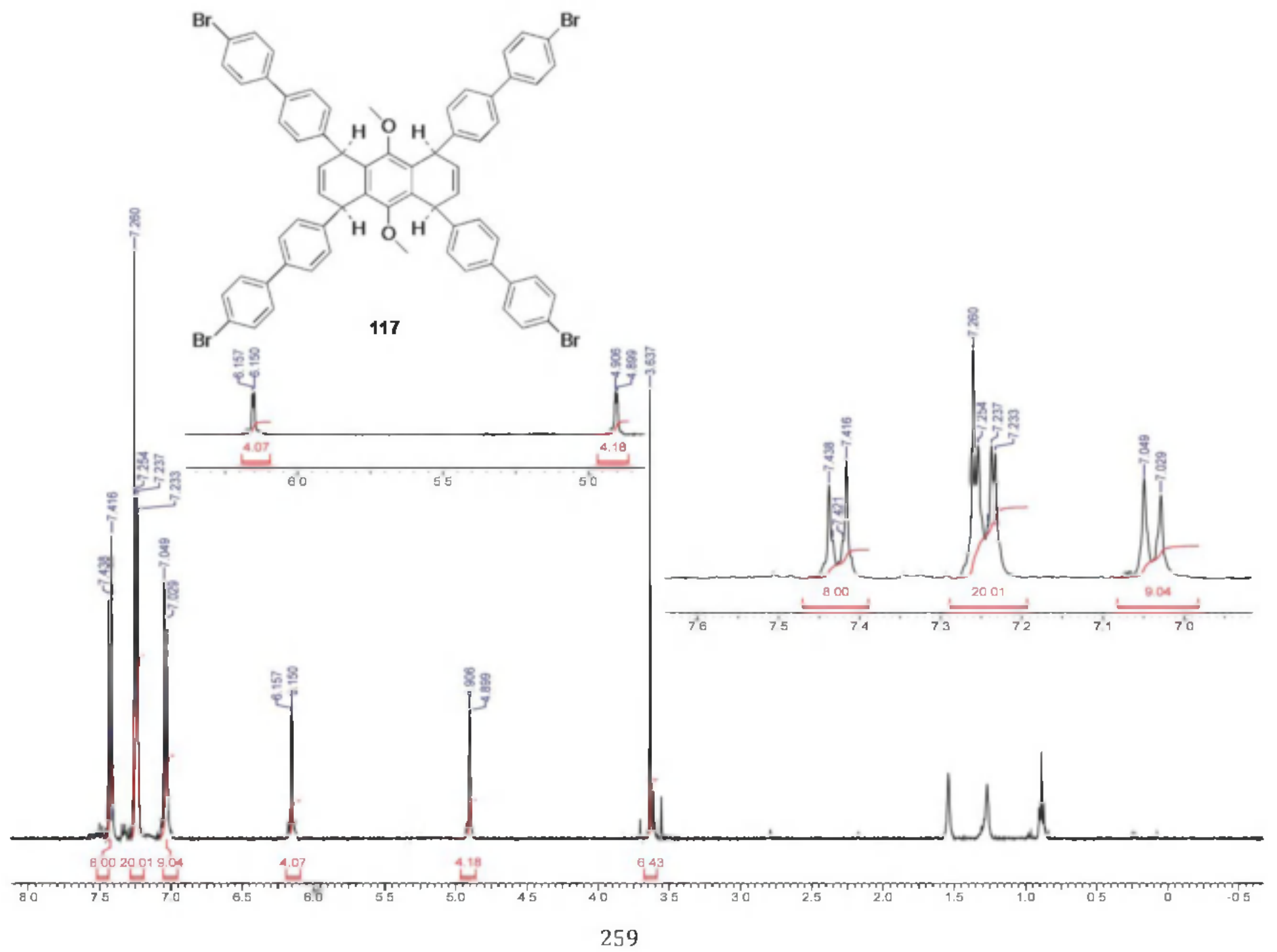




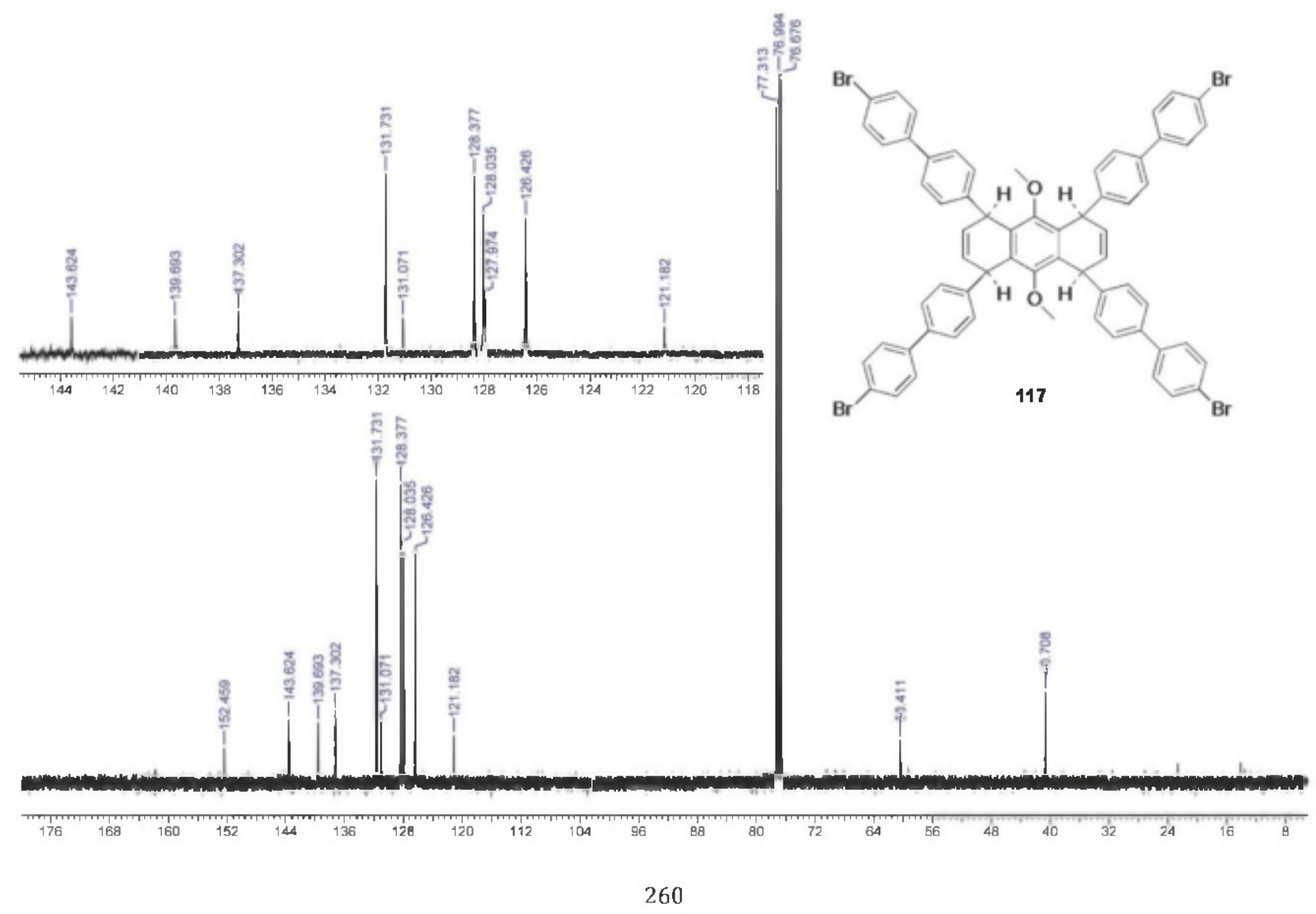




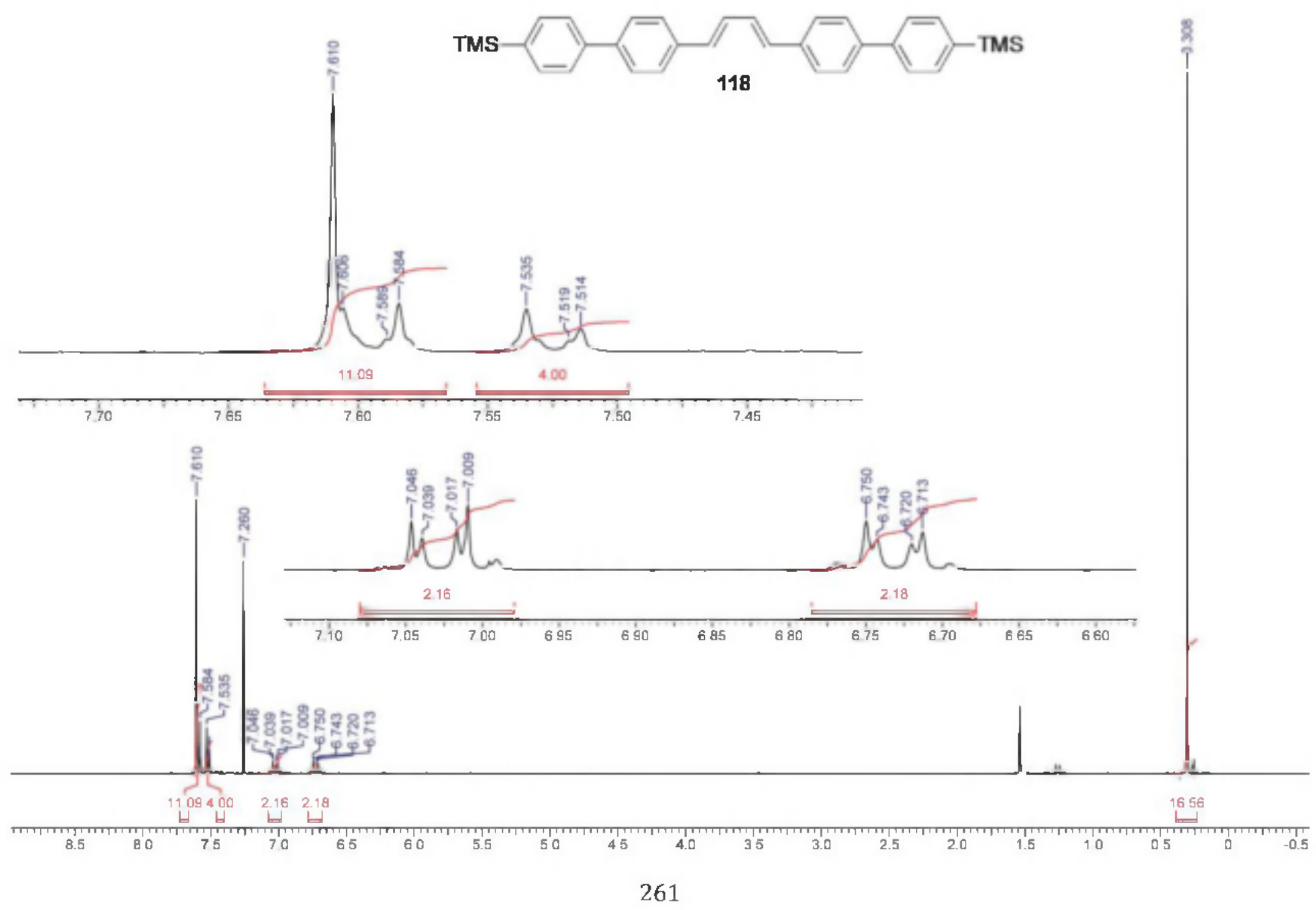




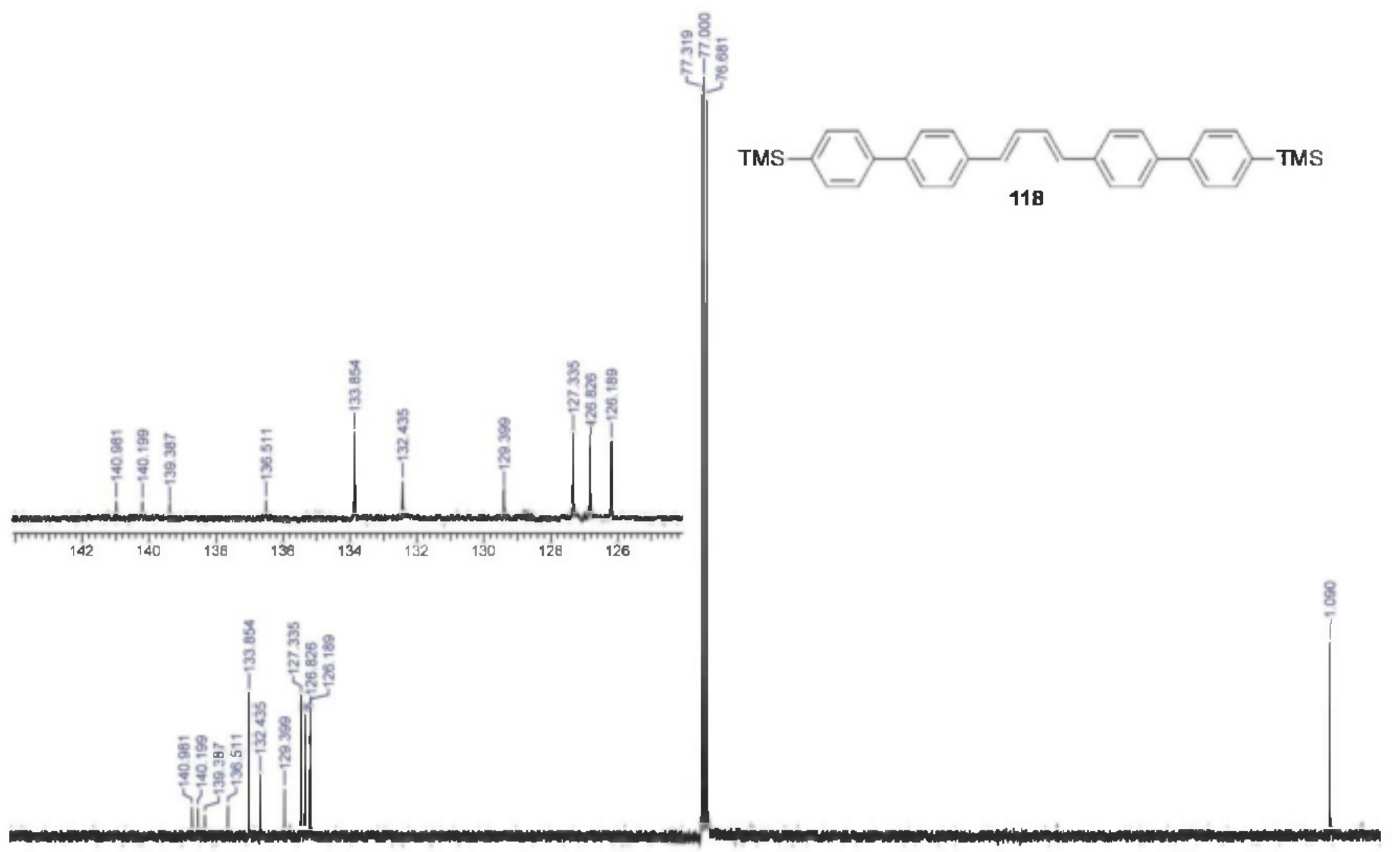

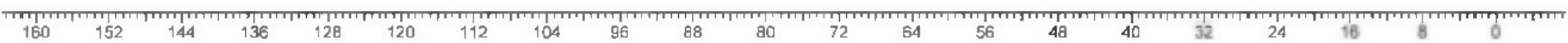




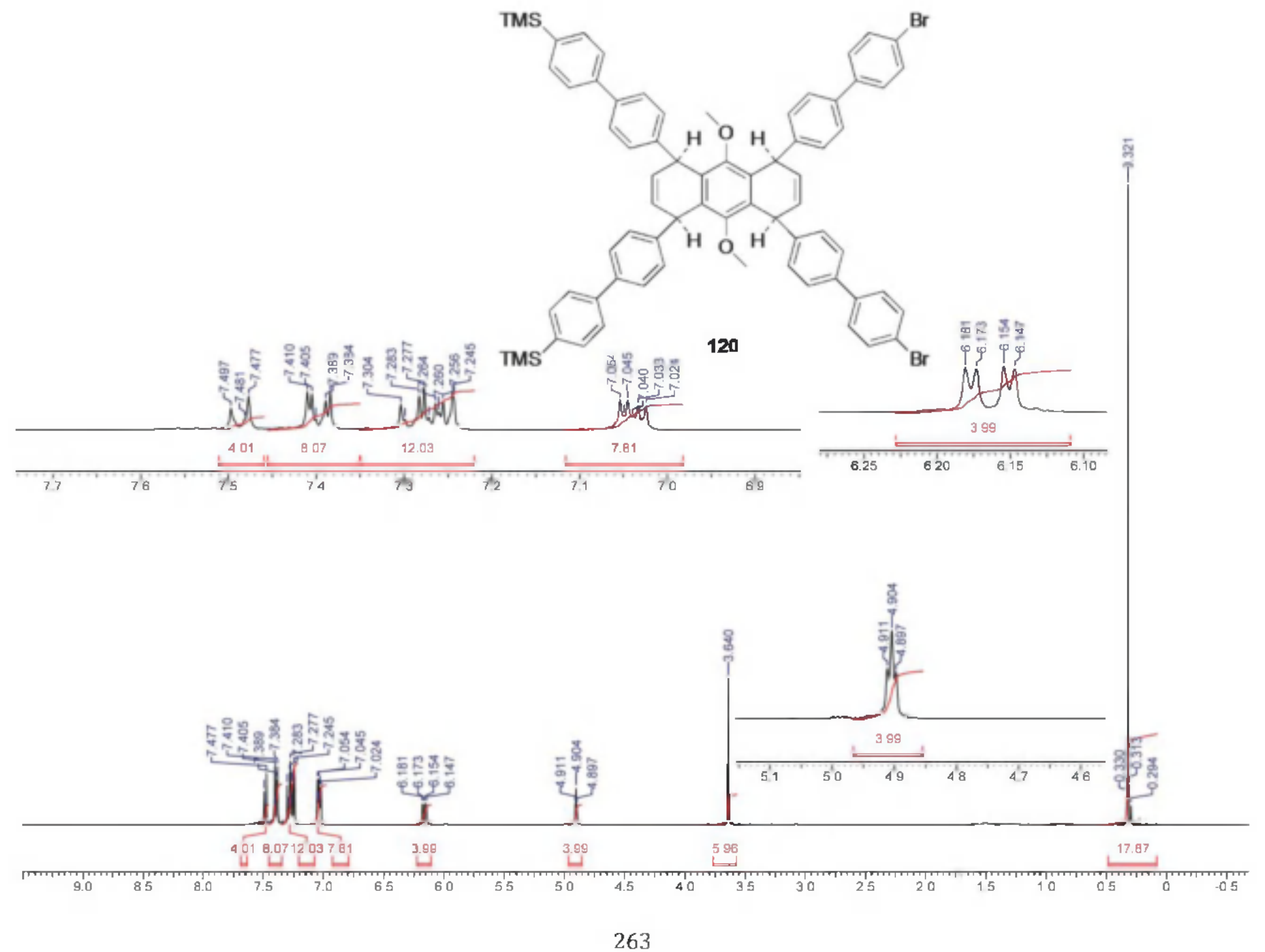




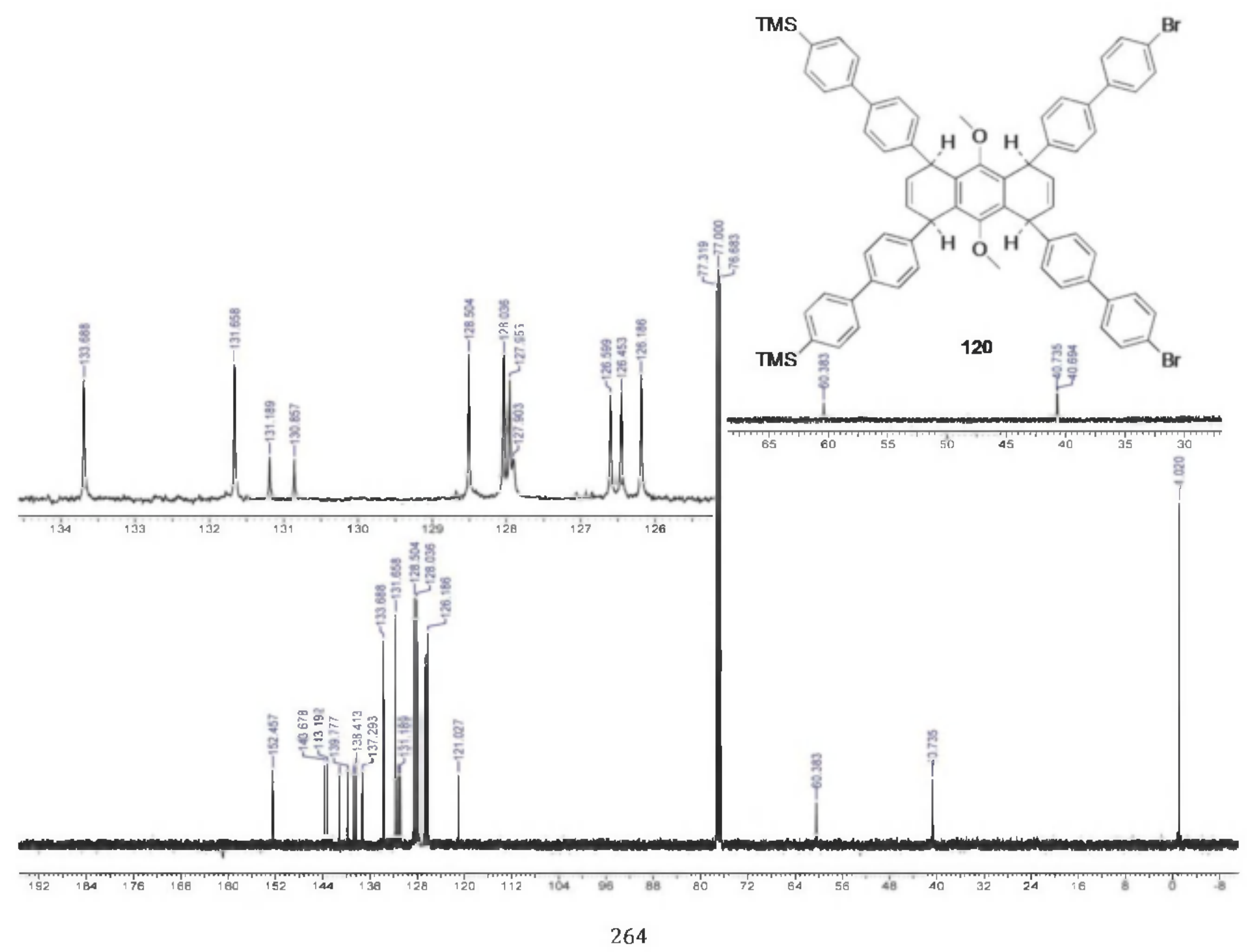




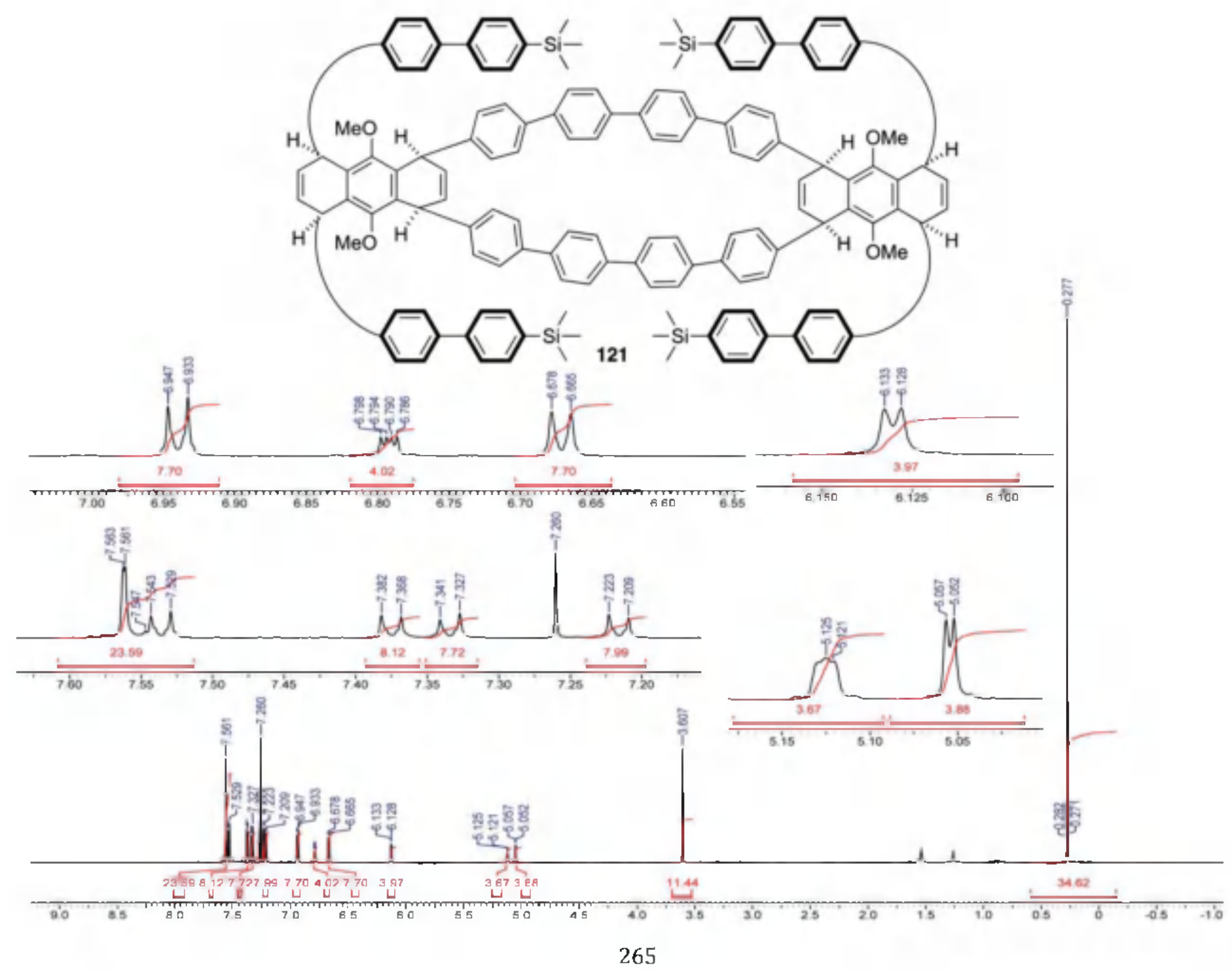




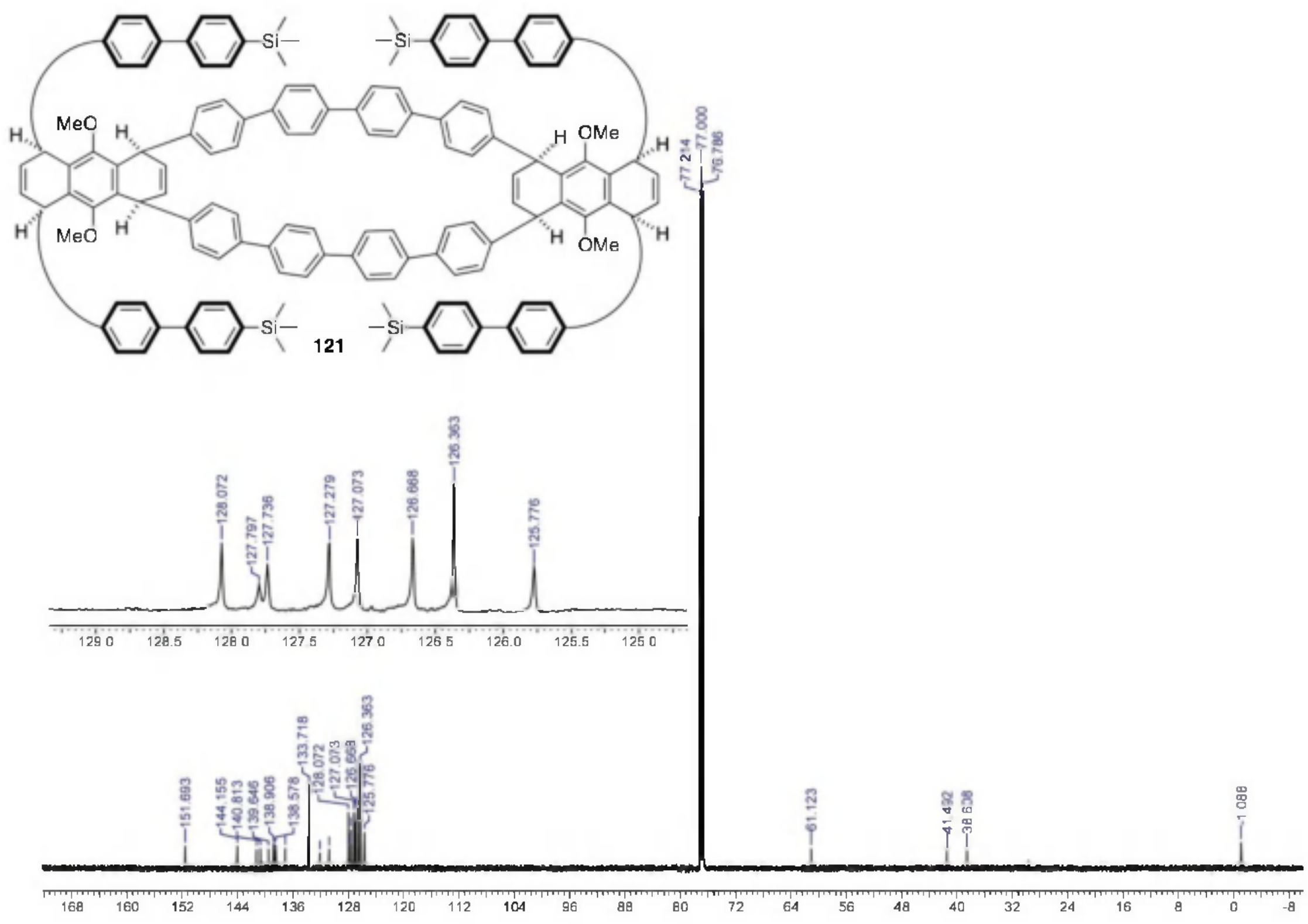




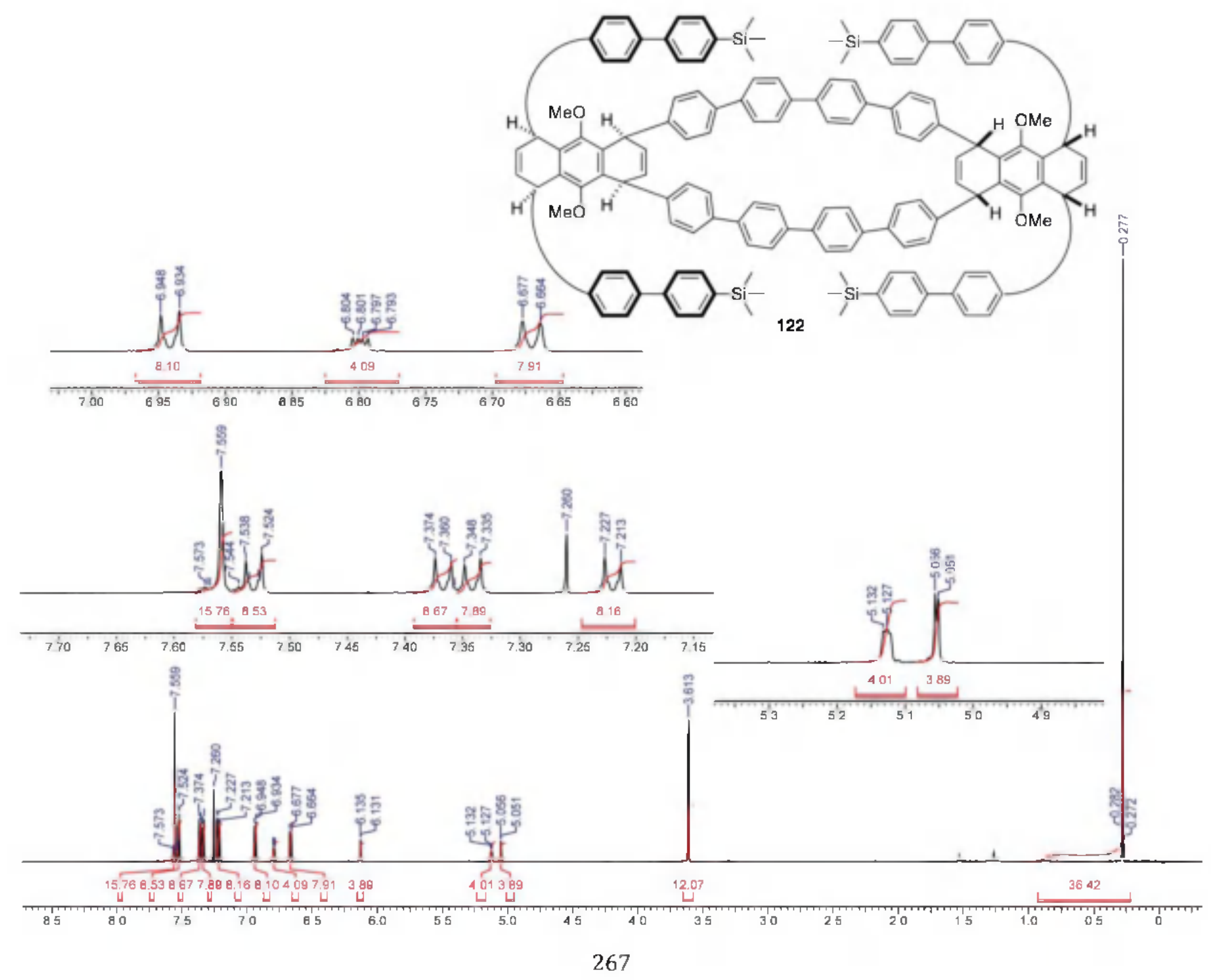




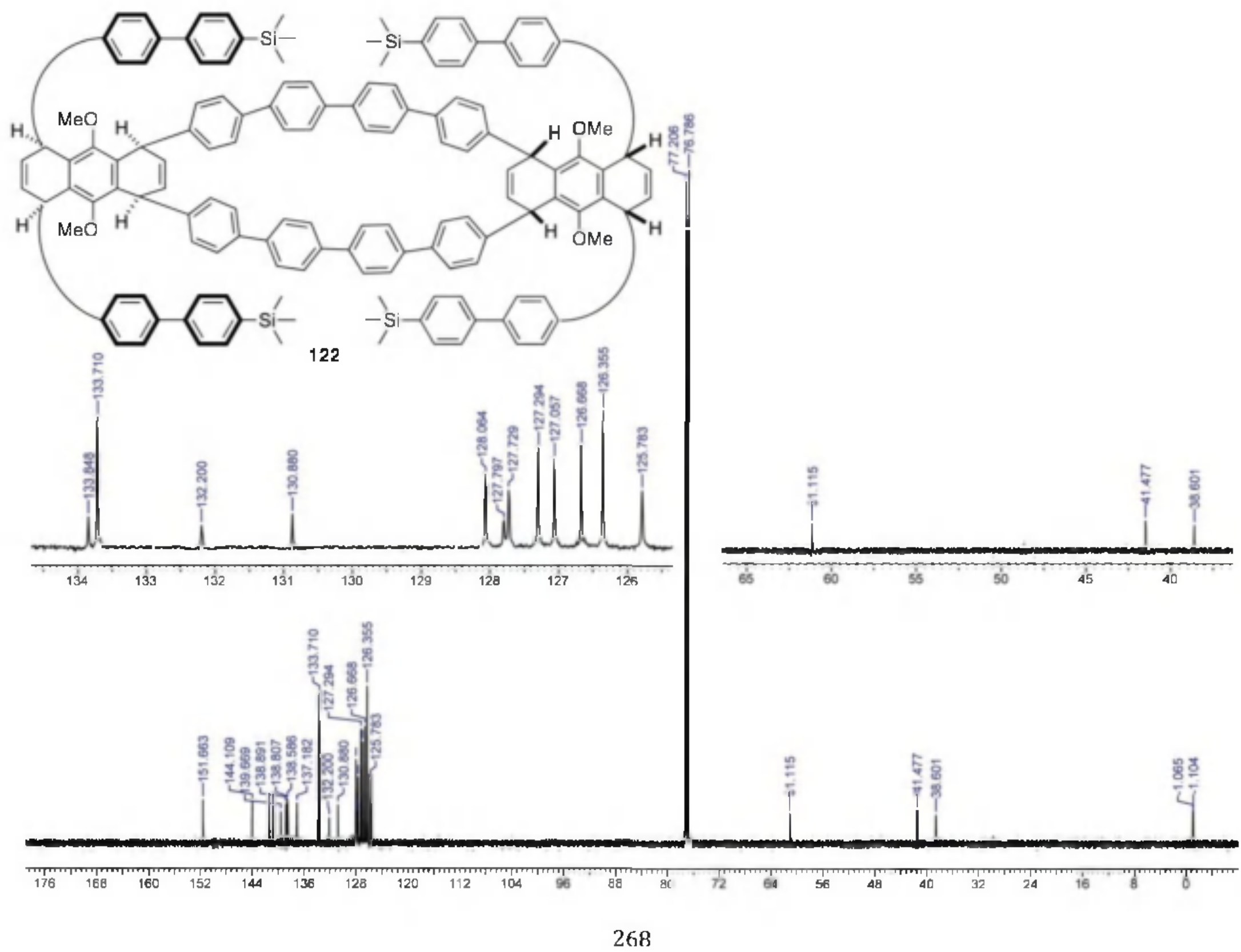


Syntheses and Structures of Functionalized Cycloparaphenylenes and Fused Cycloparaphenylene Precursors

\section{Changfeng Huang}

Dissertation submitted to the Eberly College of Arts and Sciences at West Virginia University in partial fulfillment of the requirements for the degree of

Doctor of Philosophy in Organic Chemistry

C. Eugene Bennett Department of Chemistry

APPROVAL OF THE EXAMINING COMMITTEE

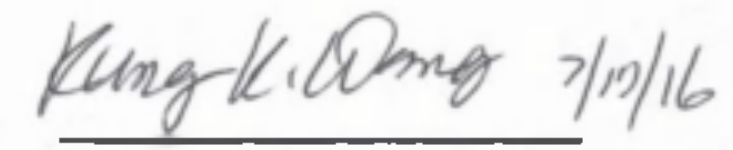

Kung K. Wang, Ph.D., Chair
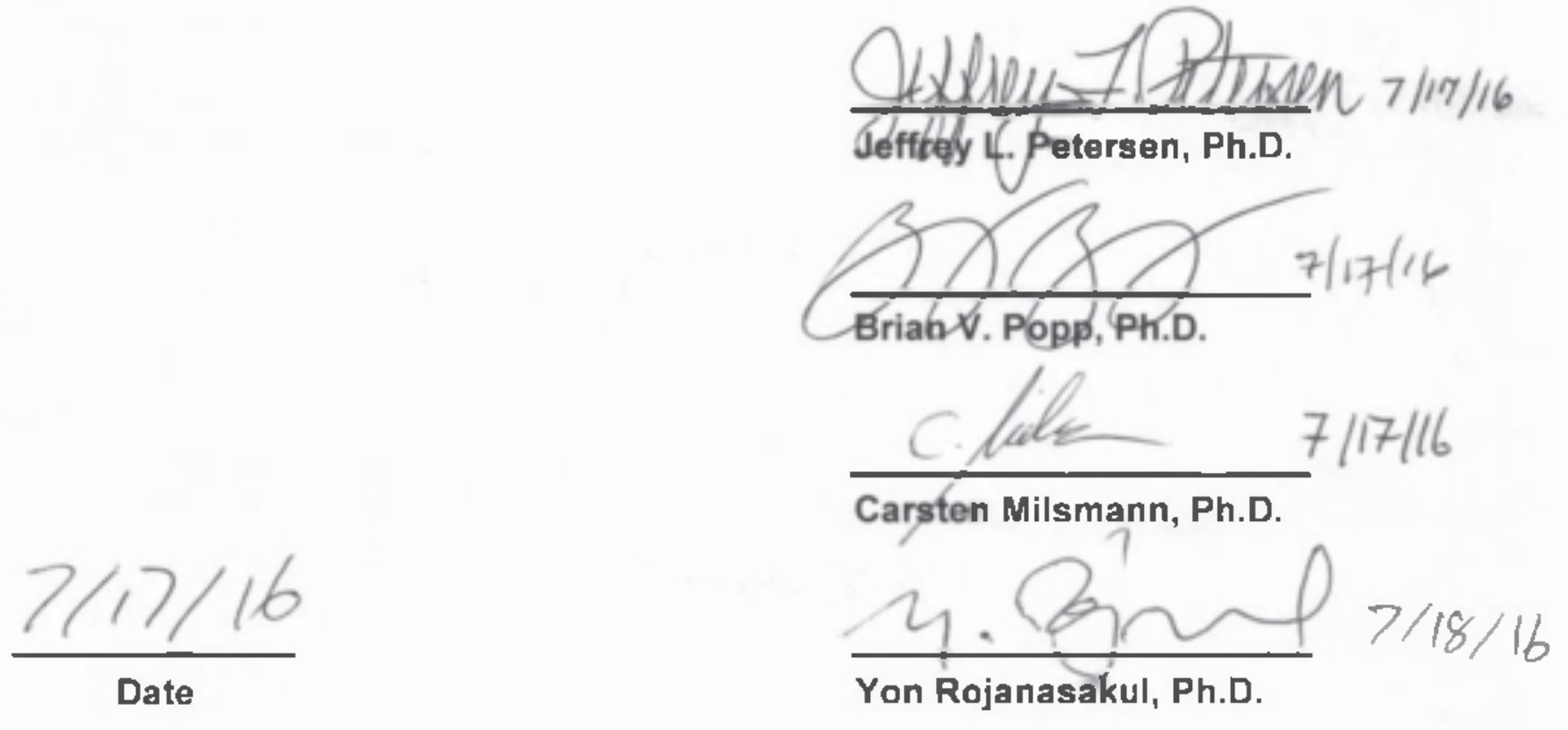